\title{
Nano-Carrier Synthesis via Z-RAFT Star Polymerisation
}

\author{
Dissertation \\ zur Erlangung des mathematisch-naturwissenschaftlichen Doktorgrades \\ „Doctor rerum naturalium" \\ an der Georg-August-Universität Göttingen
}

im Promotionsprogramm Catalysis for Sustainable Synthesis (CaSuS)

vorgelegt von

Nadja Förster

aus Berlin

Göttingen 2012 
Betreuungsausschuss

Prof. Dr. Philipp Vana

Makromolekulare Chemie, Institut für Physikalische Chemie, Georg-August-Universität Göttingen

Prof. Dr. Dietmar Stalke Institut für Anorganische Chemie, Georg-August-Universität Göttingen

Prof. Dr. Michael Buback Technische und Makromolekulare Chemie, Institut für Physikalische Chemie, Georg-August-Universität Göttingen

\section{Mitglieder der Prüfungskommission}

Referent:

Prof. Dr. Philipp Vana

Makromolekulare Chemie, Institut für Physikalische Chemie, Georg-August-Universität Göttingen

Korreferent:

Prof. Dr. Michael Buback

Technische und Makromolekulare Chemie, Institut für Physikalische Chemie, Georg-August-Universität Göttingen

Weitere Mitglieder der Prüfungskommission:

Prof. Dr. Dietmar Stalke Institut für Anorganische Chemie, Georg-August-Universität Göttingen

Prof. Dr. Peter Botschwina Theoretische Chemie, Institut für Physikalische Chemie, Georg-August-Universität Göttingen

Prof. Dr. Burkhard Geil Biophysikalische Chemie, Institut für Physikalische Chemie, Georg-August-Universität Göttingen

Jun.-Prof. Dr. Ricardo Mata Computerchemie und Biochemie, Institut für Physikalische Chemie, Georg-August-Universität Göttingen 


\section{Meinen Eltern}





\section{Table of Contents}

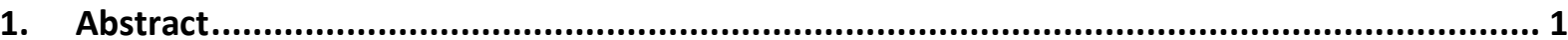

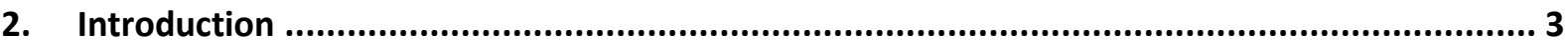

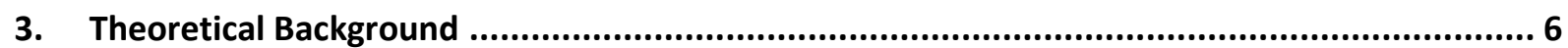

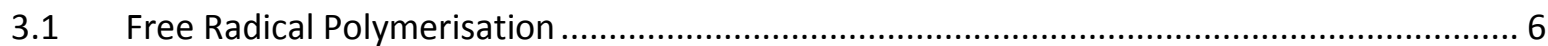

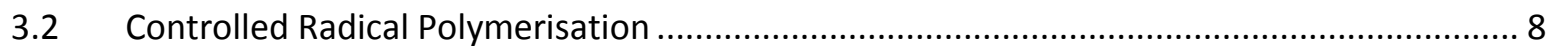

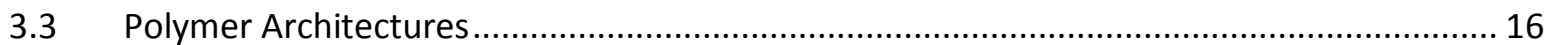

3.4 Characterisation of Polymers via their Molecular Mass ...................................................... 21

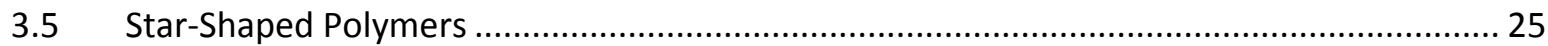

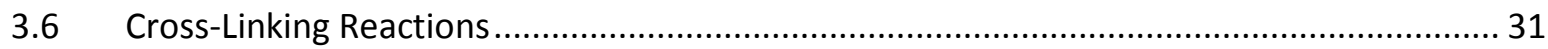

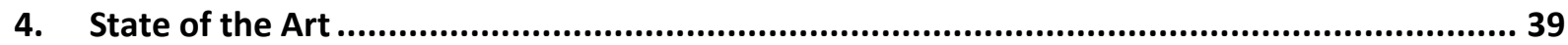

4.1 Nano-Carrier Synthesis via Cross-Linked Micelles................................................................ 39

4.2 Nano-Carrier Synthesis via Template-Assisted RAFT Polymerisation ................................. 40

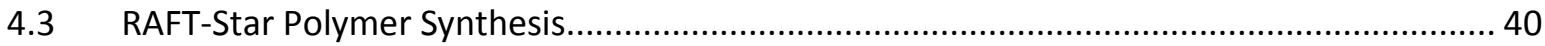

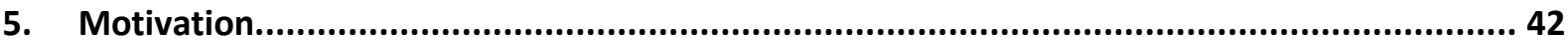

6. Shielding Effects in Polymer-Polymer Reactions ............................................................. 45

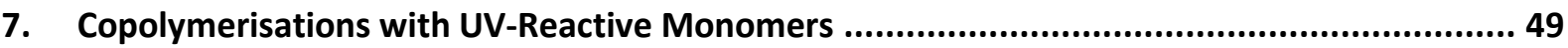

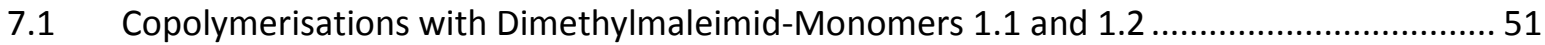

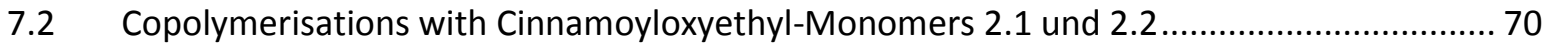

7.3 Copolymerisation with Courmarin-based Monomers.................................................... 83

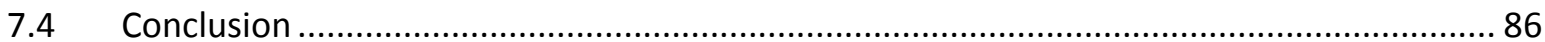

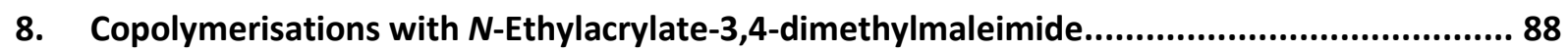

8.1 Copolymerisation of BA and 1.1

8.2 Determination of the Cross-Linkable Units in a $p$ BA Star................................................ 106 


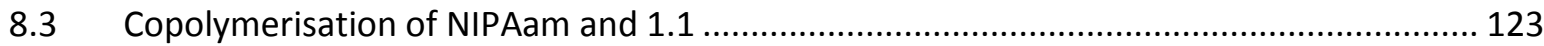

8.4 Determination of the Cross-Linkable Units in a $p$ NIPAam Star ........................................ 132

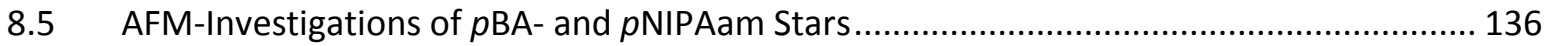

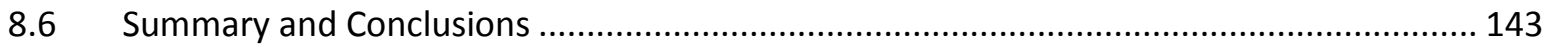

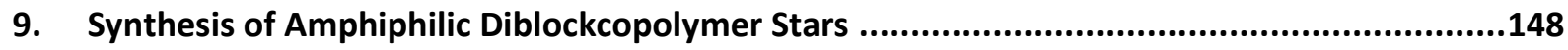

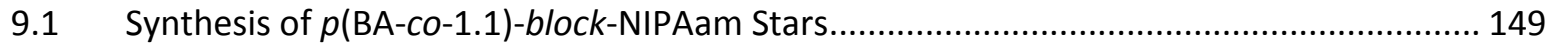

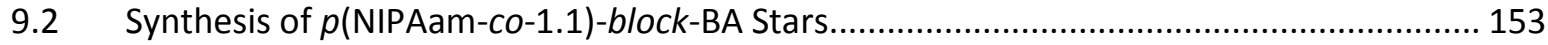

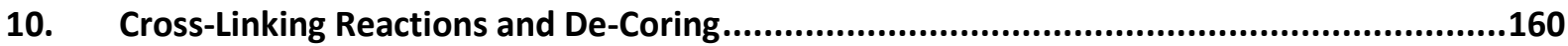

10.1 UV-Induced Cross-Linking of Dimethymaleimido Units in Star Polymers ........................... 160

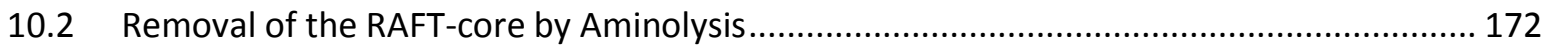

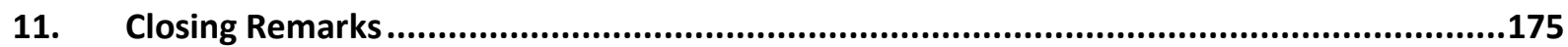

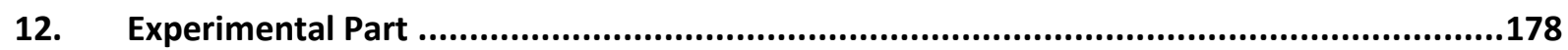

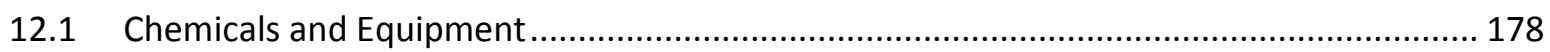

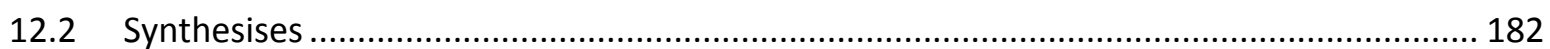

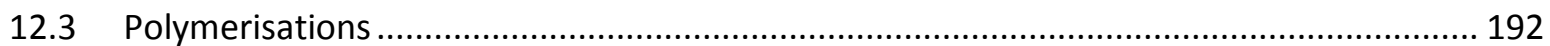

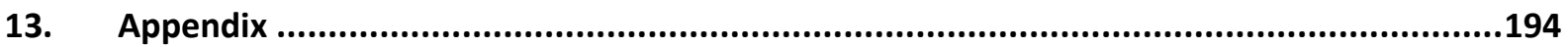

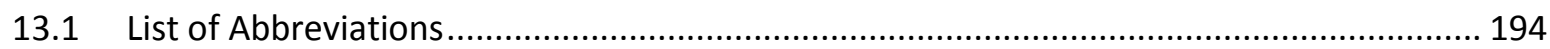

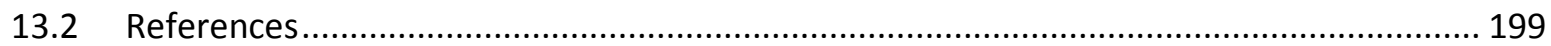

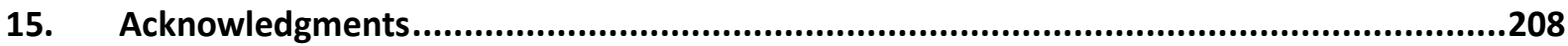

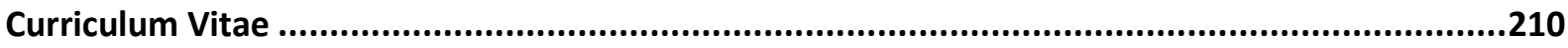




\section{Abstract}

In this project the establishment of nano-scaled and monomolecular objects working as carrier system was investigated. Such nano-carriers find application in drug delivery, catalysis or as templates for hybrid nanomaterials.

As globular platform for these nano-carriers, amphiphilic diblock star-shaped copolymers were used. Star polymers containing a central core surrounded by radiating arms provide various requirements requested to yield the proposed nano-carriers: they are unimolecular, equal sized and monodispersed, chemically stable in different modification steps and easily synthesised by means of radical Reversible Addition-Fragmentation Transfer (RAFT) polymerisation. In contrary to selfassembling systems, e.g. micelles, stability in various solvents and dilution, at different $\mathrm{pH}$-values and temperatures as well as in the presence of charges is given. ${ }^{(1)}$ The arms of the polymer stars can be tuned for the different fields of application as RAFT is versatile regarding monomers and solvents and enables the defined synthesis of polymers in size and composition. By cross-linking the arms, the carrier's shell should be formed. Removal of the RAFT-template enables the storage of the desired compounds within the shell.

Within this project RAFT-agents with six RAFT-groups represented the template for the star polymer. The number of RAFT-units corresponded to the number of arms the star consisted of. The Z-group approach was applied which enabled the arms to grow from the centre and thus prevent star-star coupling. To form the carrier's shell, [2+2]-cycloaddition by UV-irradiation was chosen. Therefore the outer block of the diblock copolymer arms had to consist of units of a basic monomer and a monomer carrying a unit which undergoes a [2+2]-cycloaddition. At first a suitable polymerisation system had to be found: monomers based on $\mathrm{N}$-alkyl-3,4-dimethylmaleimid (DMI), cinnamic acid (CA) and courmarins were tested in firstly in RAFT copolymerisations with $n$-butylacrylate (BA), N-isopropylacrylamide (NIPAam), methylmethacrylate (MMA) and methylacrylate (MA). It was found that BA and NIPAam along with the DMI-based monomer $\mathbf{1 . 1}$ yield copolymers with well-defined molecular weight and narrow polydispersities. The existence of sixarmed polymer stars could be proven by AFM-measurements. The composition of the polymers and the copolymerisation parameters for both systems were determined by NMR-spectroscopy indicating that a gradient polymer was formed. Two kinds of amphiphillic star polymers were investigated: stars with a hydrophobic corona made of $p$ (BA-co-1.1) and a hydrophilic $p$ NIPAam-core and the reversed case with a hydrophilic shell. Different UV-lamp systems were tested for cross-linking reactions induced by UV-irradiation. The success of the reaction was monitored by SEC-measurements. It was found that the RAFT centres of the star polymers were destroyed by energy entries above $150 \mathrm{~W}$ 
resulting in linear polymers, i.e. loose arms. Lower irradiation energies yielded star polymers with a closed shell. As a last step the removal of the RAFT-template by aminolysis was investigated and verified by SEC-measurements. 


\section{Introduction}

Polymers are widely used materials in the daily life of modern society. They therefore have their own field of research within the material sciences. Due to the large variety of basic compounds (monomers) and different methods to create polymer materials, it became possible to tune the properties of these materials for nearly every application. The best known examples of such materials usally used are polystyrene, called Styropor ${ }^{\circledR}$, for packing, polytetrafluorethylene (PTFE) in form of Teflon ${ }^{\circledR}$ (1946 by DuPont) as non-sticky surfaces or as water-repellent substance in GoreTex ${ }^{\circledR}$ membranes, polyethylene with low or high density for food packing or construction material and polymethylmethacrylate as Plexiglas ${ }^{\circledR}$ (1928 by Röhm).

In the beginning, the discovery of polymer substances was pure coincidence. In 1838 Henri Victor Regnault left an amount of vinylchlorid in sun and open air to gain polyvinylchlorid unintentionally. It took over 10 years until the industrial usage of rubber and latex was introduced by Charles Nelson Goodyear. ${ }^{(2 ; 3)}$ First only caoutchouc and other natural products were used in material synthesis. With the beginning of the $20^{\text {th }}$ century substances sourced from the oil industry were introduced. Plastics became replacements for building materials due to their fracture strength, low weight, high resistance against corrosion and low costs. With the developments of the last decades, the application margin of polymer materials now reaches from low-cost building and packing materials to high-cost functional polymer materials for aerospace and car industry, solar and microchip technology, medical purposes, high-tech clothing and many more specialised applications. In $201039 \%$ of the european plastic demand went into the field of packing, ca $21 \%$ into the fields of building and construction, $7.5 \%$ into the automotive field and ca $6 \%$ into electrical and electronic equipment. Other application fields such as agriculture, sport, health, leisture, safety, furniture, machinery engineering and household appliances claimed $27 \%$ of the plastic demand. (4) Furthermore, basic compounds from oil research are replaced with regenerative sources, such as starch, ${ }^{(5)}$ cellulose and lignin. ${ }^{(6)}$

To establish all these polymer materials many different technologies have been invented:

In general one can classify polymerisation reactions in two large groups, describing the basic mechanisms involved. On the one hand these are step growth reactions. Here polycondensation, e.g. used to synthesise polyethylenterephthalat (PET) or Nylon 6.6 - a polyamid (1931 by DuPont), and polyaddition, e.g. used in the production of foams, should be mentioned. On the other hand there are the chain growth reactions. In this field belong the ionic, radical and metal-catalysied reactions. Important are the Controlled Radical Polymerisations (CRP). Different major breakthroughs in the field of CRPs in the end of the $20^{\text {th }}$ century enable planning of the polymerisation beforehand to yield definite compositions in the material. The best known representatives of CRP should be mentioned 
here: After a first paper and a patent by Solomon and Rizzardo in the field of controlling free radical polymerisation reactions via stable nitroxides in $1984,{ }^{(7 ; 8)}$ a large progress in Nitroxide Mediated Polymerisation (NMP) was made by Georges et al. in 1993. ${ }^{(9)}$ Two years later the Atom-Transfer Radical Polymerisation (ATRP) was presented by Matyjaszewski and Sawamoto. ${ }^{(10 ; 11)}$ Moad, Rizzardo and Thang reported about a different mechanism to controll radical polymerisations in 1998, the Reversible Addition-Fragmentation Transfer (RAFT). ${ }^{(12)}$ As one of the latest representatives of CRPs, the Reversible Chain Transfer Catalysed Polymerisation (RCTP) needs to be mentioned, which was published by Goto in 2007. ${ }^{(13)}$ Further details of NMP, ATRP and RAFT are given in chapter 3.2.

With this control in hand one can tune the material properties exactly for the various applications. Besides the different methods the reaction medium has a large impact on the resulting material. These reactions can be carried out in bulk, solvent, melt, interfacial between liquids or liquid and gas, in emulsion, suspension or dispersion. In addition to standard organic solvents ionic liquids ${ }^{(14)}$ or supercritical gases ${ }^{(15-17)}$ can also be used.

In this work the RAFT polymerisation is used to synthesise star-shaped block-copolymers with amphiphilic arms which should establish the basis of nano-scaled objects able to carry poorly watersoluble compounds to specific targets, i.e. nano-carriers. These nano-carriers could be applied as drug delivery systems, nano-reactors or phase-transfer substrates. Figure 2-1 shows schematically the five steps to achieve this nano-particles.

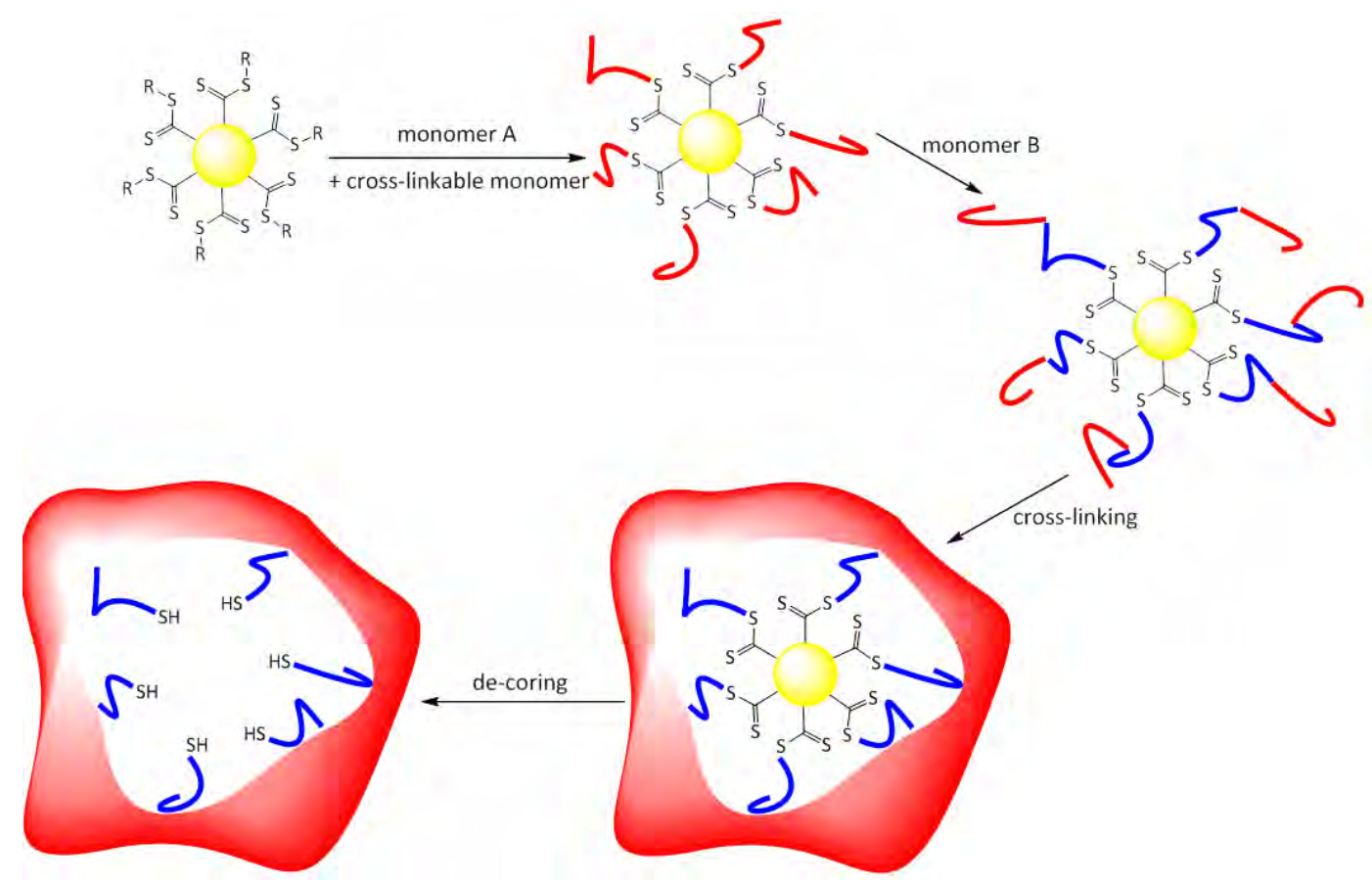

Figure 2-1: The five step approach to establish nano-scaled containers. 
The process starts with a RAFT-agent consisting of six trithioester groups, which work as controlling units during the polymerisation (chapter 3.2.3). In a first polymersation step a statistical copolymer (here red; more details are given in chapter 3.3) is synthesised from two different monomers. One of the monomers carries a functional group that enables cross-linking between themselves. In a second polymerisation step a homopolymer (here blue) is synthesised onto the first block (red). As the Z-group approach (chapter 3.5) of RAFT-polymerisation is applied, the first block (red) is the outer sphere of the polymer star. The second (blue) polymer block will be the part of the carrier in which the loaded material is soluble in. In the fourth step a cross-linking reaction is carried out to form the carrier's shell (chapter 3.6). After cross-linking and core release the carrier is ready to fulfil its purpose. Depending on the choice of monomers the properties of both blocks can be adjusted for different applications.

The following chapters clarify the basics of the steps involved to synthesise such a nanocarrier. Finally a detailed description of the procedure along with the main emphasises of investigations of this thesis will be outlined. 


\section{Theoretical Background}

On the way of establishing polymeric nano-carriers the principles of radical polymerisation processes have to be discussed as well as these of polymeric architectures and molecular mass determination. The main aspect will lie in the mechanism of the RAFT reaction as this is the chosen pathway to synthesise the backbone of the aimed carrier. The following chapters show the differences between Free Radical Polymerisation (FRP) and Controlled Radical Polymerisation (CRP) as well as these in the various systems of CRP.

\subsection{Free Radical Polymerisation}

The FRP is a chain growth reaction. A chain growth reaction is assembled of different reactions taking place in parallel, which are in competition to each other. In the FRP these are the initiation step, the chain growth itself - the propagation, chain transfer and termination reactions. The system of the FRP consists of monomer, initiator and, if necessary, solvent. Figure 3-1 presents an overview of these different reactions.

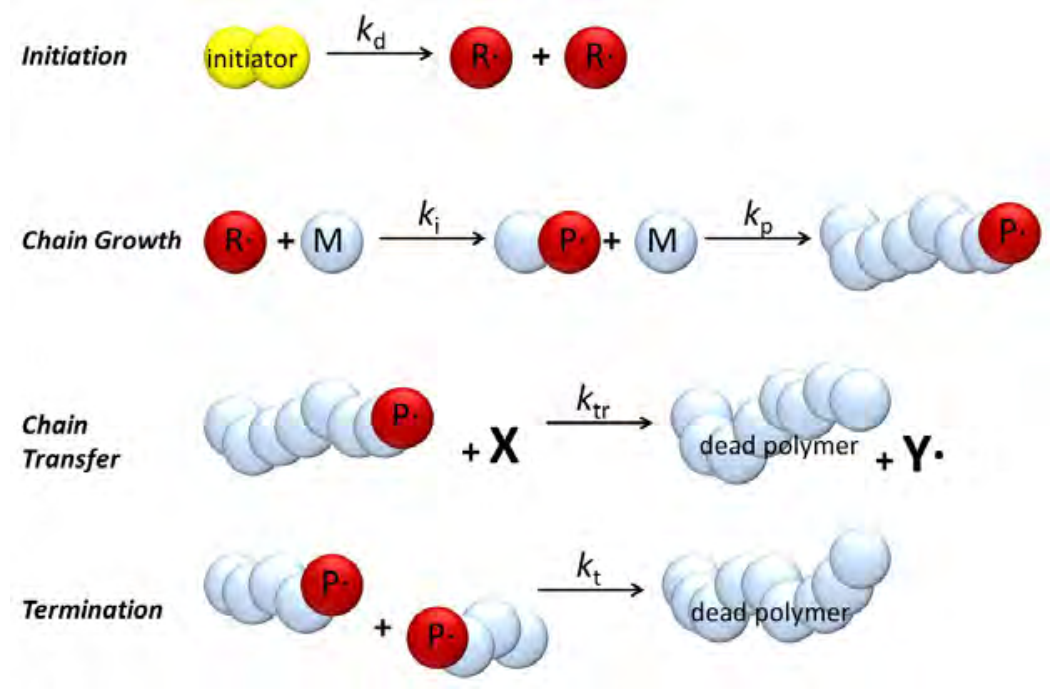

Figure 3-1: Reaction Scheme of a FRP.

To describe the ideal polymerisation kinetics four assumptions have to be made: ${ }^{(18)}$

- All reactions are irreversible.

- Monomeric species are exclusively consumed in propagation steps.

- All macroradicals show the same reactivity. The chain length has no influence.

- Termination proceeds only by disproportionation or bimolecular radical combination.

Monomers are molecules which bear a double bond in a reactive and terminal position. It is split up in the initiation step by radicals formed by the decomposition of the initiator. In the initiation step 
the initiator is decomposed thermally, photochemically or chemically into primary radicals. Azo- or peroxo-compounds are the most common initiators as they are relatively unstable. ${ }^{(19)}$ Furthermore, besides the addition of an initiator to a system, self-initiation of monomers at high temperatures is also possible. The radical functionality remains and with addition of further monomer molecules the polymer strand is formed.

In case of thermal starters the reaction is a first order reaction related to the initiator concentration. Due to the high reactivity of the formed radicals, besides the addition of monomer, the backwards reaction as well as the termination with other radicals is possible. Therefore, the initiator effectiveness $f$ describes the probability with which half of the initiator molecule start chains. This effectiveness depends on the system parameters like the initiator itself, used monomer and solvent. ${ }^{(20)}$ The addition of monomer is a very fast reaction in comparison to the decomposition reaction that it can be disregarded. Accordingly, the rate law for the initiation reaction can be described, as follows:

$$
r_{\mathrm{d}}=-\frac{1}{2} \cdot \frac{\mathrm{d}[\mathrm{In}]}{\mathrm{d} t}=\frac{\mathrm{d}[\mathrm{I} \cdot]}{\mathrm{d} t}=2 \cdot k_{\mathrm{d}} \cdot f \cdot[\mathrm{In}]
$$

where $t$ is the reaction time, $[\mathrm{In}]$ is the initiator concentration, $[I \cdot]$ the primary radical concentration and $k_{\mathrm{d}}$ the rate coefficient of the decomposition.

With the addition of monomer to the primary radical the chain growth is started. The radical functionality remains until a termination reaction takes place. The propagation rate depends on the radical and monomer concentration $\left(\left[\mathrm{R}^{\circ}\right]\right.$ and $\left.[\mathrm{M}]\right)$. In an ideal case the rate coefficient $k_{\mathrm{p}}$ is independent on the length of the chain radical, monomer concentration and conversion.

$$
r_{\mathrm{p}}=-\frac{\mathrm{d}[\mathrm{M}]}{\mathrm{d} t}=k_{\mathrm{p}} \cdot[\mathrm{M}] \cdot\left[\mathrm{R}^{\cdot}\right]
$$

There are different possibilities for the termination of the reaction. On the one hand there is the combination reaction: two chain radicals react directly via their radical functions to form one polymer strand with a saturated bond. On the other hand in the disproportionation, two chain radicals react under $\beta$ - $\mathrm{H}$-abstraction to form two polymer strands, one with a double bond and one saturated. Termination is a diffusion-driven process as the radicals have to be next to each other for the reaction. As a summary the rate law for the termination can be stated, as follows:

$$
r_{\mathrm{t}}=-\frac{\mathrm{d}[\mathrm{R} \cdot]}{\mathrm{d} t}=-2 \cdot k_{\mathrm{t}} \cdot\left[\mathrm{R}^{\cdot}\right]^{2}
$$

As can be seen the propagation reaction is always faster than the termination.

Aside from the two termination reactions which stop the reaction completely, there is also the possibility of transfer reactions. In that case one growing chain is stopped but the radical 
functionality is transferred to a chain transfer agent (CTA), another monomer, solvent or polymer molecule. The reaction then continues via the addition of a new monomer to form a polymer chain. As transfer to polymer chains does not only occur on the chain ends, it is possible to grow side chains on existing polymer chains. These transfer reactions can proceed via intra- (so called back biting) and intermolecular mechanisms. ${ }^{(21)}$ The rate law for the transfer reaction is described, as follows:

$$
r_{\mathrm{tr}}=-\frac{\mathrm{d}[\mathrm{X}]}{\mathrm{d} t}=2 \cdot k_{\mathrm{tr}} \cdot[\mathrm{R} \cdot] \cdot[\mathrm{X}]
$$

where $[\mathrm{X}]$ is the concentration of the transferring agent. If special CTAs are used, the transfer constant $C_{\mathrm{tr}}$ describes the effectiveness of the agent:

$$
C_{\mathrm{tr}}=\frac{k_{\mathrm{tr}}}{k_{\mathrm{p}}}
$$

The transfer reaction has no influence on the propagation or termination rate. The main change occurring because of transfer reactions is in the length of the polymer chains. CTAs give an opportunity to control the molar weight and with that control over material properties. ${ }^{(22)}$

To receive the rate of polymerisation as a whole the rate of initiation and the rate of termination are equated with the assumption that the radical concentration is quasi-stationary:

$$
2 \cdot k_{\mathrm{d}} \cdot f \cdot[\operatorname{In}]=2 \cdot k_{\mathrm{t}} \cdot[\mathrm{R} \cdot]^{2}
$$

Solving for $\left[R^{\bullet}\right]$ and insert in the rate law for polymerisation reveals the brutto-polymerisation rate:

$$
r_{b}=-\frac{\mathrm{d}[\mathrm{M}]}{\mathrm{d} t}=k_{\mathrm{p}} \cdot\left(f \cdot \frac{k_{\mathrm{d}}}{k_{\mathrm{t}}} \cdot[\mathrm{In}]\right)^{0.5} \cdot[\mathrm{M}]
$$

\subsection{Controlled Radical Polymerisation}

A disadvantage of the FRP is the very broad distribution of molecular weights. Due to the different reactions taking place simultaneously, as described in the previous chapter, there is a large variety in chain lengths and with that in the molecular mass of the chains. These broad distributions are adversarial for some applications; often equal sized polymer chains are preferable.

To gain control over FRP, which enables planning of molecular weight and the resulting material properties, there is the possibility to add controlling agents. These agents react reversibly with the radical species and form the so called dormant species. It is required that this species is stable enough to keep the radical species dormant, but also reactive enough for re-initiation. The resulting reaction equilibrium is shown in Figure 3-2. 


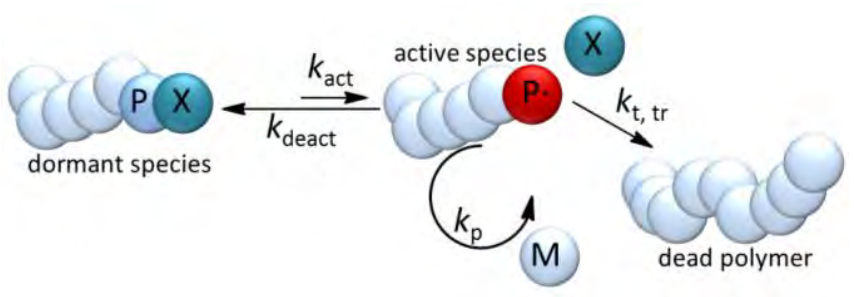

Figure 3-2: Reaction equilibrium in a CRP.

It is assumed that

$$
k_{\text {act }} \cdot[\mathrm{P}-\mathrm{X}]=k_{\text {deact }} \cdot[\mathrm{P} \cdot]
$$

The radical concentration is approximately $10^{5}$ times smaller than the concentration of the dormant species. Therefore the equilibrium is mainly on the side of the dormant species. Termination and transfer reactions are taking place but the probability for these is strongly minimized due to the low radical concentration. With that it is possible to prolong the life span of the growing polymer chains. Due to a synchronized start and the controlled equilibrium, the chains are growing simultaneously. This yields equal sized chains and therefore narrower molecular weight distributions (MWD) compared to the FRP-case as shown in Figure 3-3.

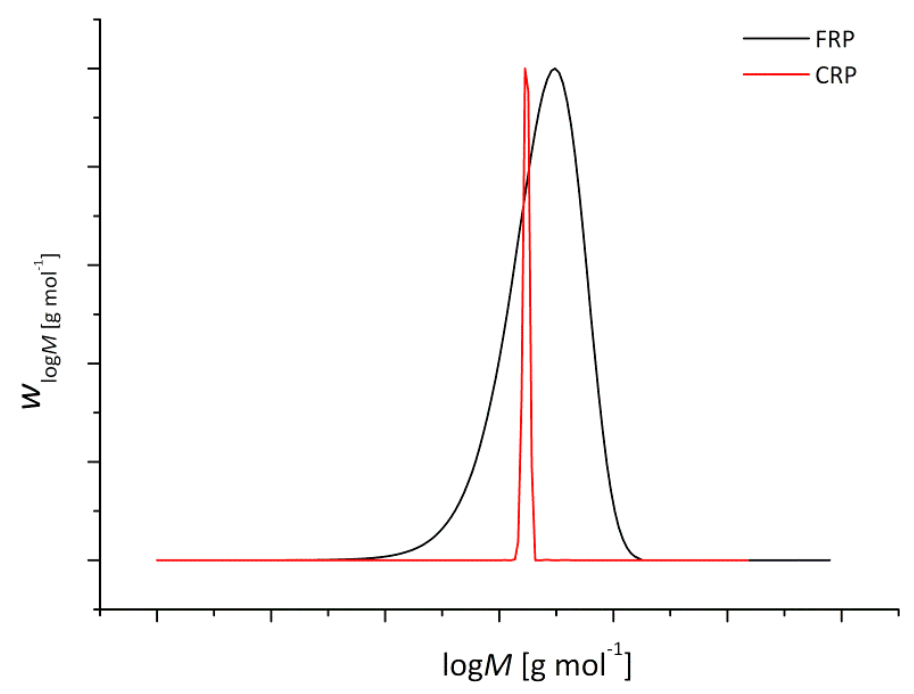

Figure 3-3: Molecular weight distributions resulting from a FRP (black) and a CRP (red). ${ }^{\text {(23) }}$

Compounds which are usually applied as controlling agents are shown in the following chapters. Here only three polymerisation techniques of Controlled Radical Polymerisations (CRP; IUPAC: Deactivation-Activation Radical Polymerisation) that currently receive the greatest attention will be presented. 


\subsubsection{Nitroxide Mediated Polymerisation}

In 1982 large success was reported by Moad, Rizzardo and Solomon in controlling radical polymerisation reactions via stable nitroxides ${ }^{(24)}$ which led to a patent in $1984 .{ }^{(7)}$ The so called Nitroxide Mediated Polymerisation (NMP) was invented.

NMP is based on a dissoziation-combination mechanism. With heat or light the bond of the dormant species is broken and a radical is released to start a chain growth reaction (Figure 3-4).

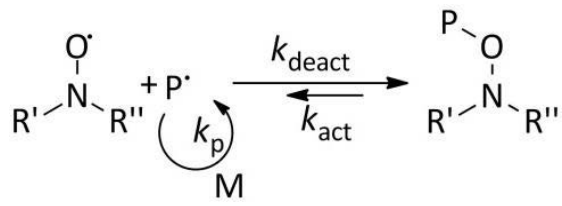

Figure 3-4: Equilibrium step of NMP.

It is important that the nitroxide radical has a stable $\mathrm{N}-\mathrm{O}$-bond, does not terminate with itself, does not initiate polymerisation and does not disproportionate with the chain radical. Due to the different $\mathrm{R}^{\prime}$ and $\mathrm{R}^{\prime \prime}$ groups the nitroxide can be tuned for certain monomer systems. The initiator is often a nitroxide-based on the structure of the monomer used.

A disadvantage of that kind of reaction is the occurance of the persistent radical effect. ${ }^{(25)}$ Both radicals (nitroxide and chain radical) are generated at the same rate, but only the polymer radical is terminated over time. The stable nitroxide radical will be accumulated in the system which leads to a slower reaction that will stop at some point.

\subsubsection{Atom-Transfer Radical Polymerisation}

A second method of CRP is the Atom-Transfer Radical Polymerisation (ATRP). Here a redox equilibrium is used to manage the transfer between the dormant species and the growing radical. The first results in this field of polymerisation were published by Sawamoto and Matyjaszewski in 1995. ${ }^{(10 ; 11)}$ In 2001 Matyjaszewski summarised the main contributions on the field of ATRP in an extensive review. ${ }^{(26)}$

In ATRP the controlling agent is a catalyst consisting of a transition metal (Me in Figure 3-5) with two oxidation states, which can be easily achieved and are only one electron apart, and ligands (L), which stabilise both oxidation states. The initiators are organohalogen or pseudo-organohalogen compounds, where the R-X bond is weakend by the correct choice of R. Stabilising groups for the resulting radical like aryl, carbonyl or ally groups as well as polyhalogenated groups are favoured. Often a group similar to the monomer used, in form of a halogen compound, is chosen to simplify 
the system and overcome difficulties in the progress of polymerisation. Figure 3-5 shows the general equilibrium of an ATRP.

$$
\mathrm{Me}^{\mathrm{l} / \mathrm{L}_{\mathrm{n}}}+\mathrm{P}_{\mathrm{m}}-\mathrm{X} \underset{k_{\text {deact }}}{\stackrel{k_{\text {act }}}{\longrightarrow}} \mathrm{X}-\mathrm{Me}^{\prime \prime} / \mathrm{L}_{\mathrm{n}}+\mathrm{P}_{\mathrm{m}}^{\cdot}\left(k_{\mathrm{D}}\right)_{\mathrm{M}}
$$

Figure 3-5: Equilibrium step of ATRP.

The catalysts most often used are copper centres with nitrogen ligands. The ligands as well as reaction conditions have a large impact on the equilibrium. ${ }^{(26)}$ With the right choice of the ligands it is possible to adapt the catalyst to the polymerisation system.

A disadvantage of ATRP is the remaining metal in the product. For applications in life sciene a expensive cleaning process has to be adjoined. These polymers are often not suitable for medical purposes.

\subsubsection{Reversible Addition-Fragmentation Chain Transfer Polymerisation}

As shown by the examples in proceeding chapters, lowering the radical concentration with capping agents is one way to control the polymerisation. Another possibility to gain control is by a degenerative chain transfer. In this case a transfer agent (TA) is added and the conventionel FRP is overlapped by a fast transfer equilibrium. The kinetics stay the same as in the FRP due to not induced lowered radical concentration. The main advantage of this concept is the prevention of the persistent radical effect. Another major benefit of this type of CRP is the application of FRP-like reaction conditions as well as monomers.

Since the middle of the 1990s the Reversible Addition-Fragmentation Chain Transfer (RAFT) polymerisation is a growing field in CRP. At least three reviews from Moad et al. summarise the developments and achievements of the different working groups researching RAFT. ${ }^{(27-29)}$ In this polymerisation concept dithioesters are used as TAs. The general structure of a RAFT-agent is shown in Figure 3-6. The agent consists of a stabilising group $\mathrm{Z}$ and a leaving group $\mathrm{R}$.

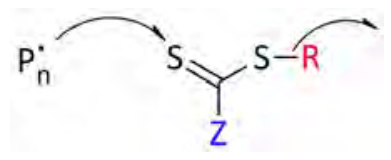

Figure 3-6: Basic structure of a RAFT-agent.

The reaction involves, like in the FRP-case, the initiation, the propagation and the termination since free radical species are included. In addition to that the transfer equilibrium between the transfer 
agent and the growing radicals takes place. Figure 3-7 illustrates the reaction scheme resulting from these reactions.

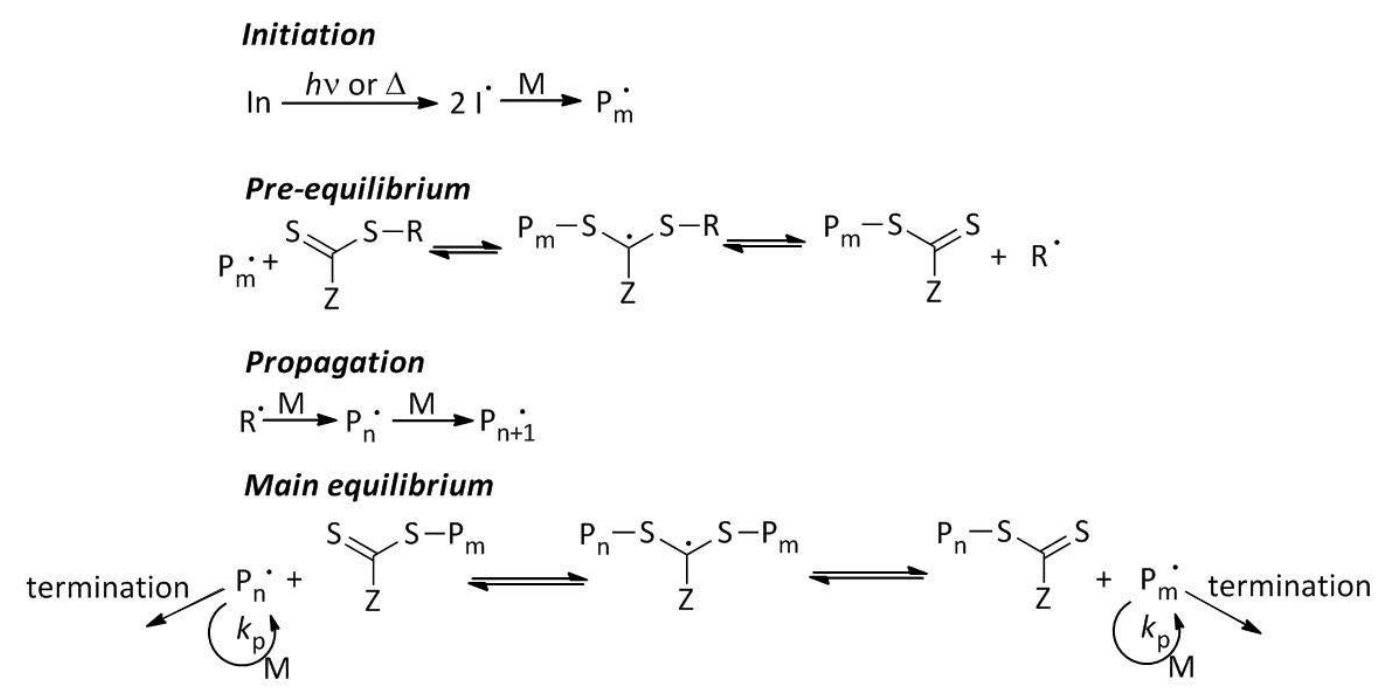

Figure 3-7: Reaction scheme for RAFT polymerisation.

After the initiation step the addition of the marco radical $P_{m} \cdot$ to the TA occurs first. Because of this attack an intermediate is formed and quickly another radical is released which starts a new chain. The following requirements have to be fulfilled for an efficient RAFT polymerisation:

- The initial RAFT as well as the macroRAFT species should have a reactive $\mathrm{C}=\mathrm{S}$ double bond.

- The intermediate radicals should fragment quickly and should not undergo side reactions.

- Also the expelled radical $R^{\bullet}$ should re-initiate effectively and the intermediate of the preequilibrium has to favour the right-hand side of the reaction.

The effectiveness of the whole agent depends on the transfer coefficient which is determined by the R- and the Z-group. ${ }^{(12 ; 30)}$ As in conventional chain transfer reactions, equation 3-5 is valid. The different transfer constants for the various RAFT-agents have been found in a range of five orders of magnitudes. This constant is highly depended of the used groups in R-and Z-position as well as of the particular monomer. Even the RAFT-agent's backbone has an influence as not only dithioesters but also xanthates (then called MADIX - Macromolecular Design by Interchange of Xanthate), trithiocarbonates and dithiocarbamates can be used. The transfer constant in dependence of the RAFT-backbones decreases from trithiocarbonats to dithioesters to xanthates to dithiocarbamates. ${ }^{(27 ; 29)}$ To plan the conditions for a RAFT-polymerisation it is important to consider beforehand the transfer constants of the starting RAFT-agent and the macroRAFTs during the reaction. Figure 3-8 gives a small overview of suitable RAFT-agent groups for different monomers, published by Moad et al. ${ }^{(27 ; 31)}$ 


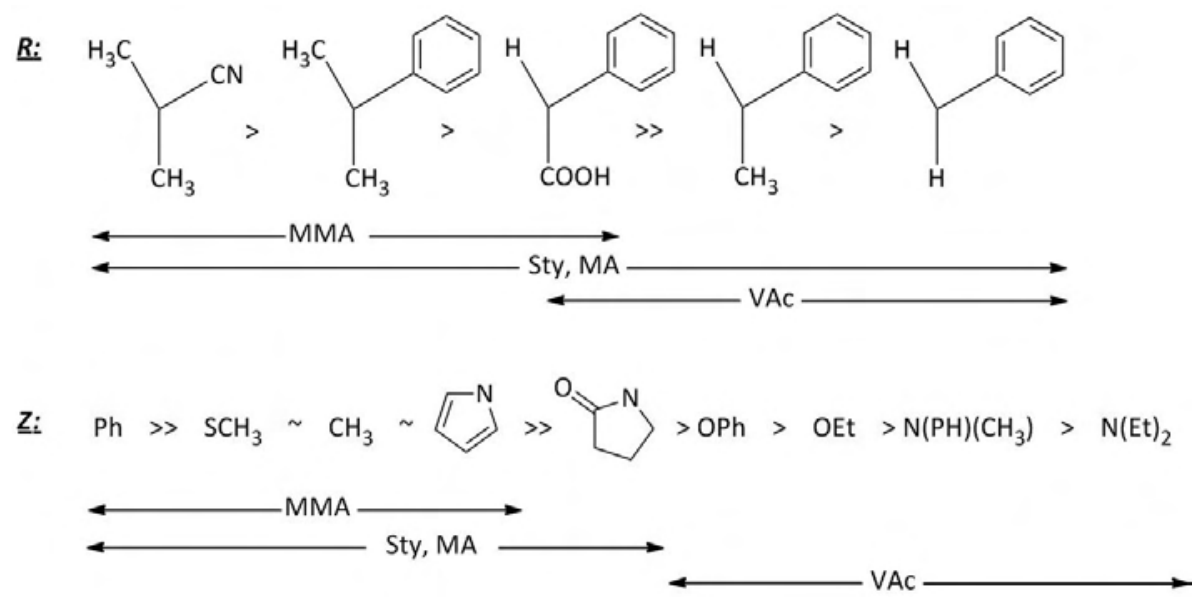

Figure 3-8: Overview of suitable RAFT-groups for different monomers (MMA - methylmethacrylate, Sty - styrene, MA methacrylate, VAc - vinyl acetate), redrawn after ref. (31).

The leaving group $R$ is released when a radical attacks the dithio unit. $R$ has to cleave off easily and form a relatively stable radical that initiates a new chain and does not directly undergo the reaction back to the intermediate, shown in Figure 3-7. To achieve the stabilisation of the radical $R$ electron withdrawing groups, delocalising systems as well as bulky substituents for sterical hinderance can be introduced. Sometimes the perfect fit for the polymerisation system is provided by a monomer-like R. In the ideal case the radical $R$ inititiates as fast as the polymer chain grows. ${ }^{(32)}$

The Z-group stabilises the intermediate formed in the equilibrium (Figure 3-7). The degree of stabilisation, which has to be adjusted to the used monomer, has great importance for the whole process: If the stabilisation of the intermediate is too weak, it is not possible to form the intermediate at all. Chain transfer cannot proceed and the reaction control is lost. If the stabilisation is too strong, the intermediate is unreactive, the dissoziation does not take place and the reaction is stopped at that point. ${ }^{(33-37)}$

The control of RAFT polymerisations results from the fast equilibrium between controlling agent and growing radical chains $\left(\mathrm{P}_{\mathrm{m}}{ }^{\cdot}\right.$ and $\left.\mathrm{P}_{\mathrm{n}}{ }^{\circ}\right)$. The kinetics of the reaction should not be affected by the new introduced equilibrium. The only effect to be seen is a differing in the molecular mass due to control over the high equilibrium velocity. With that the probability of growing is equal for all chains, i.e. narrow molecular weight distributions can be achieved. ${ }^{(27)}$ As a result the chain length is proportional to the monomer conversion, as shown in Figure 3-9. 


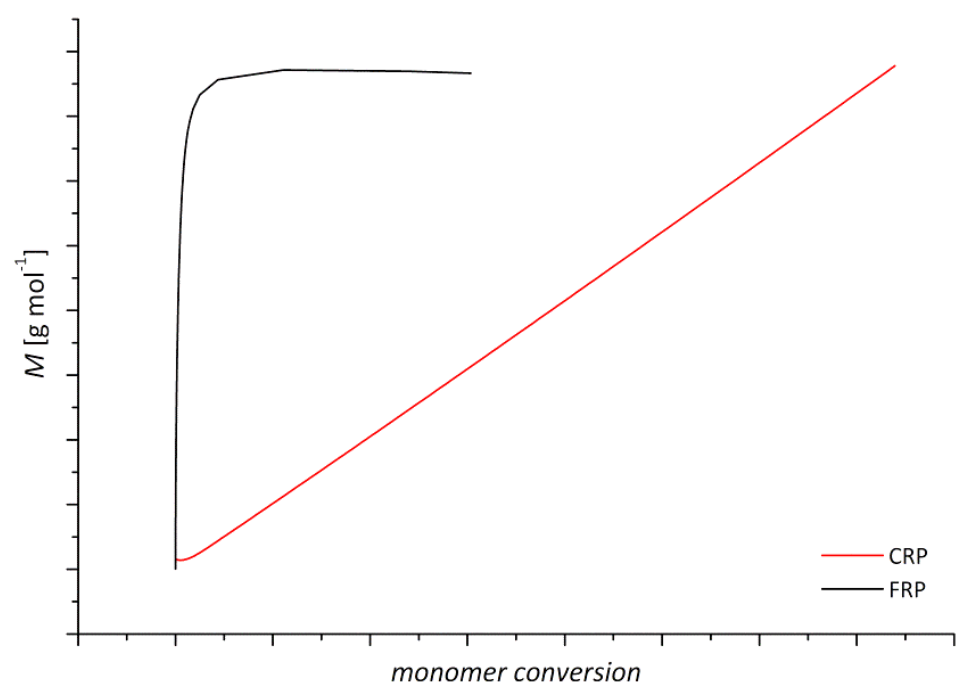

Figure 3-9: Comparison of the developments of the molecular mass of the polymers with consumed monomer in the FRPcase (black) and the CRP-case (red). ${ }^{(23)}$

During the process the RAFT-agent concentration stays constant. When the polymerisation is completed or stopped, the chains retain the RAFT-units and can be isolated as stable materials. Chains with RAFT endgroups are called living chains, whereas the ones resulting from termination are called dead chains. The ratio of living to dead polymer chains can be determined by the concentrations of RAFT-agent and initiatior $\left(\frac{C_{\text {RAF }}}{c_{\text {In }}}\right)$. ${ }^{(28)}$ The living chains can be employed as marcoRAFT-agents because they can be reactivated for further polymerisation. ${ }^{(27)}$ So a pathway to block-copolymers is open.

As the ideal behaviour of the polymerisation is just an approximation, the influence of the R-group has to be discussed. The initialisation describes the decrease of RAFT-agent with the original R-group. ${ }^{(38)}$ In the period of initialisation all original R-groups are exchanged by propagating radicals. Two extreme cases can be outlined: In the first case the leaving group $R$ is less stabilised than the propagating radical. The probability for decomposition of the formed intermediate, back to the original RAFT-agent, and the propagating radical is higher. As a result the chains are growing unequally and polymer chains with above average high molecular mass for RAFT polymerisations are received at low monomer conversion, so called hybrid behaviour can be observed. In the second case, no preference for a direction in the pre-equilibrium or the splitting of $\mathrm{R}$ can be observed. If the rate coefficient for the propagation of the R-group is high, a fast transition towards the main equilibrium takes place. ${ }^{(38)}$

The favoured release of $\mathrm{R}$ can have a second consequence. If the release of $\mathrm{R}$ is favoured or the pre-equilibrium has no preference in direction, there is a accumulation of small radicals which terminate much faster than larger radicals. This results in a radical loss during the initial period of polymerisation with a fast transition towards the main equilibrium. The polymerisation starts 
effectively later. This effect is called induction and is reinforced by slowly propagating leaving groups. ${ }^{(39)}$

The third effect observed in RAFT polymerisations is retardation. Here the rate of polymerisation decreases with increasing radical concentration. ${ }^{(40)}$ There are three estimates to what happens in the system: One describes the reversible reaction of two RAFT-intermediats. ${ }^{(41-43)}$ The second is based on an irreversible termination of a RAFT-intermediate with a marcoradical, forming a three armed star. ${ }^{(35 ; 36 ; 43)}$ In a third concept it is predicted that such a three armed star reacts with a propagating radical to form a RAFT-intermediate and dead polymer chains, as the isolation of the three armed star was only successful under special, non-polymerisation conditions. ${ }^{(44 ; 45)}$ These three estimates are summerised in Figure 3-10.

$$
\begin{aligned}
& \mathrm{P}_{\mathrm{n}}-\mathrm{S}_{\mathrm{P}_{\mathrm{o}}}^{\mathrm{Z}} \mathrm{S}_{-\mathrm{P}_{\mathrm{m}}}^{\mathrm{S}} \quad \text { irreversible termination product } \\
& \uparrow \begin{array}{l}
+\mathrm{P}_{\mathrm{o}}^{\cdot} \\
k_{\mathrm{t}, \mathrm{int}-\mathrm{R}}
\end{array}
\end{aligned}
$$

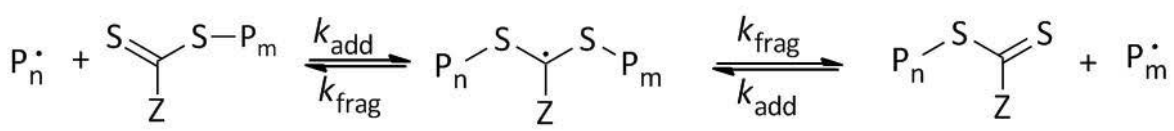

$$
\begin{aligned}
& k_{\text {reac }} \| k_{\mathrm{t}, \text { int-int }}
\end{aligned}
$$

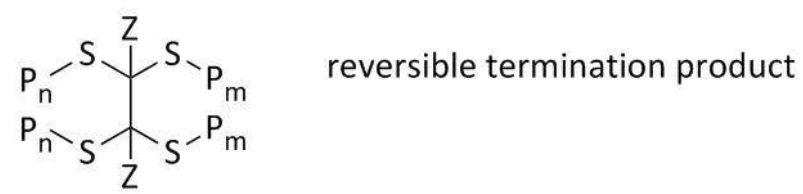

Figure 3-10: Reaction scheme of possible RAFT-termination reactions causing retardation, redrawn from ref. (46).

The concepts of RAFT-star polymerisation are outlined in chapter 3.2.3. Before going into detail of that topic some principles of polymeric architectures are given. 


\subsection{Polymer Architectures}

As previously mentioned, polymers are favoured materials for many applications in the daily life due to their versatility, low cost and weight. But how are all these different kinds of plastics achieved? As a starting point, with one single monomer type it is possible to synthesise polymers with different macroscopic properties. An example for this is polyethylene (PE). Depending on the method of the synthesis there are high density PE (HD-PE) and low density PE (LD-PE). HD-PE is a rigid material, e.g. used for plastic bottles or as construction materials. LD-PE, on the other hand, is a flexible material, which can be used as plastic films in food packaging. ${ }^{(4)}$ As there is a large variety of monomers available, it is possible to combine these, as shown in Figure 3-11 (left hand column). This can lead to a large variety of materials.

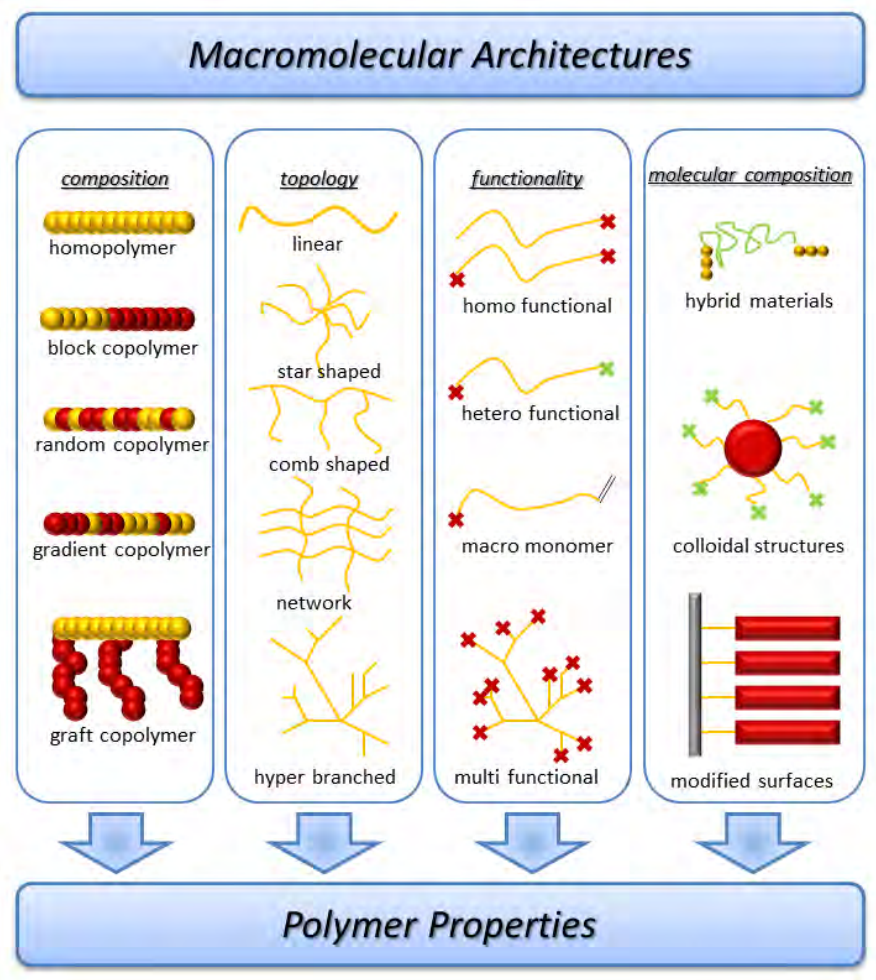

Figure 3-11: Possible architectures of polymers.

The properties of the resulting materials are the outcome of the following factors:

- The monomers used (left-hand column),

- the procedure with which the polymer is synthesised (mentioned in the introduction),

- additives (right-hand column),

- the topology of the single polymer molecules and

. the functionalities resulting from the monomers, starters or TAs (columns in the centre). ${ }^{(47)}$ 
To illustrate some structures, shown in Figure 3-11, a few examples are presented here. Subsequently some details of block copolymers and their self-assembly as well as star polymers are given.

All examples of polymer architectures shown in Figure 3-11 can be interlinked. By the choosing different functional groups the chemical nature and behaviour of the polymer can be influenced, e.g. hydrophilic end groups support a hydrophilic behaviour of the polymer. To achieve complex hybrid structures (Figure 3-11, right-hand column) polymers can be grown from the surface of, e.g. silica particles ${ }^{(48)}$ or gold foils ${ }^{(49)}$, or be connected to compounds like polyhedral oligomeric silsesquioxanes (POSS). ${ }^{(50 ; 51)}$

An example of polymers with characteristic properties originating from functional groups used in daily life is polytetrafluorethylene (PTFE), also known as Teflon ${ }^{\circledast}$ and used in Gore-Tex ${ }^{\circledR}$ membranes, as non-sticky and water-repellent compound. PTFE is a linear, non-branched homopolymer of tetrafluorethylene and stands out because of its Lotus-Effect which is a result of weak inter- and intramolecular interactions and the hydrophobic nature of fluoro polymers. ${ }^{\left({ }^{2}\right)}$

A second example from everyday life applications are super absorbers. These are insoluble copolymer networks from acrylic acid and sodium acrylate. ${ }^{(53)}$ The hydrophilicity results from the acid groups. In combination with the repulsive interactions between the acid groups and the ions an intake of water into the network can be observed. The polymer structure swells to a multiple of its own volume and can store irreversibly a hundredfold of its own weight in form of water. In a similar application, oil absorbing copolymer networks from isooctyl acrylate and cinnamic acid derivates are used. $^{(54)}$

\subsubsection{Block Copolymers and Self-Assembly}

Block copolymers are made of at least two blocks of different polymers. In the case of an amphiphilic block copolymer two antagonistic blocks are covalently connected. ${ }^{(55)}$ In selective solvents these undergo self-assembly into various nano-sized morphologies, ${ }^{(56)}$ e.g. micelles, vesicles, rods or tubes. ${ }^{(57)}$ The micelle formation starts at a compound-specific concentration, the so called Critical Micelle Concentration ( $\mathrm{CMC}$ ), which depends on the nature of the polymer blocks, block length, solvent and temperature. ${ }^{(58)}$ With rising concentration larger self-assembling structures are formed, e.g. rods. ${ }^{(57)}$ As these polymers are amphiphilic a parallel can be drawn between them and typical surfactants. ${ }^{(59)}$ In comparison, the polymeric systems of micelle formation have a larger aggregation number, i.e. the number of molecules in a micelle, and their CMC is a 1,000-fold smaller. A drawback of micellar systems is the lack of thermodynamic stability due to the dynamic exchange between micelle and unimers (single polymer molecules) in the solvent. ${ }^{(60)}$ Even though, the stability of micelles from block copolymers is higher than in the case of typical surfactants as the lower 
mobility of polymeric amphiphils slows the exchange with the environment down. ${ }^{(59)}$ Furthermore, external influences like temperature change, alteration of the $\mathrm{pH}$-value or the introduction of charges can modify or destroy the assembly. ${ }^{(60)}$

\subsubsection{Star-Shaped Polymers}

Star polymers are a special class of branched polymers. They consist of linear polymer chains which are covalently connected to a central point. ${ }^{(61-63)}$ Their synthesis can be carried out from this linking point, e.g. a RAFT-agent, or from linear chains, which can be connected to a linker, such as a silica particle. They are considered to unite colloidal and polymeric characteristics as they behave like particles in colloidal suspensions. ${ }^{(64)}$ In comparison to their linear polymer relatives of the same molecular mass, they show a lower hydrodynamic volume and a smaller size in solution due to higher segment density. ${ }^{(63)}$ This effect is more pronounced with an increasing number of arms. ${ }^{(61)}$ Besides the behaviour in solution star polymers distinguish from linear polymers in their chemical nature and macroscopic properties. This is the result of the topology and the nature of the end groups. Because polymers in star form have more end groups than linear polymers, the influence of these multiplies.

Amphiphilic star polymers can self-assemble, similarly to the block copolymers in the preceding part, but their associates are less symmetrically organised and much more complex than a core-shell micelle. ${ }^{(65)}$

Some more details of star polymers, their synthesis and application fields with respect to the topic of this project are given in following chapters.

\subsubsection{Copolymerisation Parameters}

If a copolymer is not synthesised in two steps, as it is usually done for block or grafted copolymers, but in one, there are different possibilities for the resulting polymer:

- In a statistical copolymer both monomers are distributed coincidentally.

- If one of the monomers used is favoured in the reaction the result is a gradient copolymer.

. If both monomers polymerise with the same probability an alternating copolymer is received.

In 1944 Mayo and Lewis published the basis for the comparison of the behaviour of monomers in a copolymerisation. ${ }^{(66)}$ Via so called copolymerisation parameters it is possible to predict which kind of copolymer will be synthesised. These parameters are empirical values, collected in the Polymer Handbook. Here a short explanation of these parameters is given:

In the case of a radical copolymerisation with two types of monomer $A$ and $B$ two active chain ends $A^{*}$ and $B^{*}$ have to be regarded. Therefore, resulting four different addition reactions with four corresponding rate coefficients $\left(k_{i j}\right.$ with $\left.i, j=1,2\right)$, shown in Figure 3-12: 


$$
\begin{aligned}
& \sim \sim \dot{A}+A \stackrel{k_{11}}{\longrightarrow} \sim \sim A^{\circ} \\
& \sim \sim \dot{A}+B \stackrel{k_{12}}{\longrightarrow} \sim \sim \sim \dot{B}^{\circ} \\
& \sim \sim B^{\circ}+B \stackrel{k_{22}}{\longrightarrow} \sim \sim B^{\circ} \\
& \sim \sim \dot{B}^{\circ}+A \stackrel{k_{21}}{\longrightarrow} \sim \sim B^{\circ}
\end{aligned}
$$

Figure 3-12: Four reactions of copolymerisation resulting from two monomers.

It is assumed and shown that the radical reactivity is only dependent on the nature of the active chain end and independent on the number and kind of the attached units. ${ }^{(66)}$ Therefore the decrease of $A$ and $B$ can be described as

$$
\begin{aligned}
& -\frac{\mathrm{d}[\mathrm{A}]}{\mathrm{d} t}=k_{11}[\mathrm{~A}] \sum\left[\mathrm{A}^{\cdot}\right]+k_{21}[\mathrm{~A}] \sum\left[\mathrm{B}^{\cdot}\right], \\
& -\frac{\mathrm{d}[\mathrm{B}]}{\mathrm{d} t}=k_{22}[\mathrm{~B}] \sum[\mathrm{B} \cdot]+k_{12}[\mathrm{~B}] \sum\left[\mathrm{A}^{\cdot}\right]
\end{aligned}
$$

with $\Sigma\left[\mathrm{A}^{*}\right]$ or $\Sigma\left[\mathrm{B}^{*}\right]$ equals all active centres with an $\mathrm{A}$ - or $\mathrm{B}$-unit at the end. The ratio of integration between $A$ and $B$ is described, as follows

$$
\frac{\mathrm{d}[\mathrm{A}]}{\mathrm{d}[\mathrm{B}]}=\frac{[\mathrm{A}]}{[\mathrm{B}]}\left[\frac{\left(k_{11} \sum[\mathrm{A} \cdot] /[\mathrm{B} \cdot]\right)+k_{21}}{\left(k_{12} \sum[\mathrm{A} \cdot] /[\mathrm{B} \cdot]\right)+k_{22}}\right] .
$$

The ratio of the radical centres can be simplified by the assumption of steady state conditions for $A^{\circ}$. This stationary state describes that equal many $A^{\circ}$-chains are produced and consumed.

$$
\begin{aligned}
& \frac{\mathrm{d} \sum\left[\mathrm{A}^{\cdot}\right]}{\mathrm{d} t}=k_{21}[\mathrm{~A}] \sum\left[\mathrm{B}^{\cdot}\right], \\
& -\frac{\mathrm{d} \sum[\mathrm{A} \cdot]}{\mathrm{d} t}=k_{12}[\mathrm{~B}] \sum\left[\mathrm{A}^{\cdot}\right]
\end{aligned}
$$

which leads to

$$
\begin{gathered}
k_{21}[\mathrm{~A}] \sum\left[\mathrm{B}^{\cdot}\right]=k_{12}[\mathrm{~B}] \sum\left[\mathrm{A}^{\cdot}\right] \\
\rightarrow \frac{\sum\left[\mathrm{A}^{\cdot}\right]}{\sum\left[\mathrm{B}^{\cdot}\right]}=\frac{k_{21}[\mathrm{~A}]}{k_{12}[\mathrm{~B}]}
\end{gathered}
$$

With this approximation it is value that

$$
\frac{\mathrm{d}[\mathrm{A}]}{\mathrm{d}[\mathrm{B}]}=\frac{[\mathrm{A}]}{[\mathrm{B}]}\left[\frac{k_{11}[\mathrm{~A}] / k_{12}+[\mathrm{B}]}{[\mathrm{A}]+k_{22}[\mathrm{~B}] / k_{21}}\right] .
$$


The reaction ratios are given with

$$
\begin{aligned}
& r_{1}=\frac{k_{11}}{k_{12}} \\
& r_{2}=\frac{k_{22}}{k_{21}} .
\end{aligned}
$$

So the copolymerisation equation can be described as

$$
\frac{\mathrm{d}[\mathrm{A}]}{\mathrm{d}[\mathrm{B}]}=\frac{[\mathrm{A}]}{[\mathrm{B}]}\left[\frac{r_{1}[\mathrm{~A}]+[\mathrm{B}]}{r_{2}[\mathrm{~B}]+[\mathrm{A}]}\right]
$$

With equation 3-17 it is possible to determine the composition of the synthesised polymer for any moment of the polymerisation. As the concentrations of $A$ and $B$ are changing during the polymerisation process the composition of the polymer changes too, along with the reaction time. For an ideal statistical copolymer $r_{1}$ and $r_{2}$ have values of 1 , for a strictly alternating copolymer $r_{1}$ and $r_{2}$ are 0 .

To avoid strong dissimilarity in the parameters of the used monomers and with that in the polymerisation behaviour, the monomeric backbones should be as equal as possible. In this project the following monomers were used.

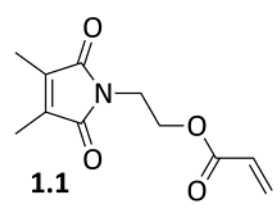<smiles>[R1]COC(=O)/C=C/C(=O)OCCOC(=O)/C=C/c1ccccc1</smiles>

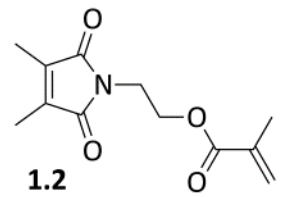<smiles>C=C(C)C(=O)OCCOC(=O)/C=C/c1ccccc1</smiles>
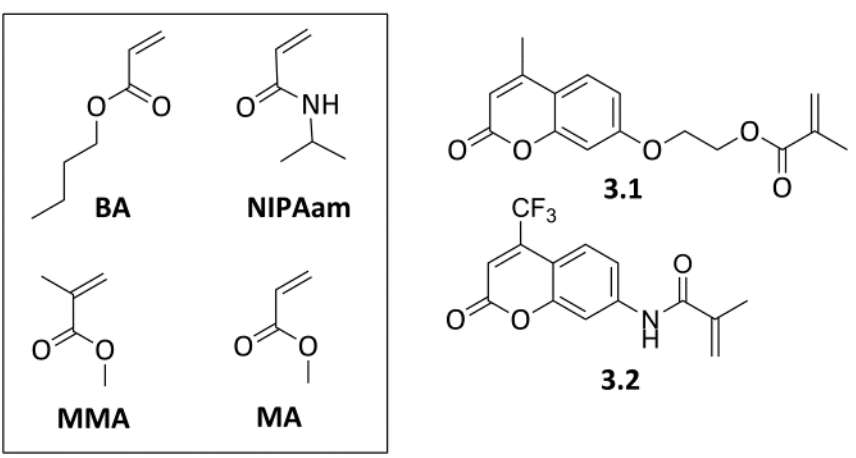

Figure 3-13: UV-reactive monomers and basis monomers used in copolymerisations.

It is assumed that a C2-bridge is enough to separate the reactive double bond for polymerisation from the UV-reactive rest of the monomer; hence the copolymerisation with 
butylacrylate (BA) should have a satisfying outcome. In the case of monomer 3.2, a commercially available monomer was chosen. It should be tested in copolymerisation with $\mathrm{N}$-isopropylacrylamide (NIPAam). For this project it was only important that the cross-linkable monomer is integrated in a sufficient amount to enable a complete cross-linking. It was less important if the resulting polymer is a strongly alternating or a gradient copolymer.

\subsection{Characterisation of Polymers via their Molecular Mass}

In contrast to biological polymers, e.g. proteins, or low molecular compounds that are uniform and have a similar molar mass, synthetic polymer chains do not have equal weights. Due to the way of the synthesis and the usage of many monomer units to build up the chains, a distribution of their weights was found as a result of statistical reasons: Chains resulting from a long-living radical have naturally higher molar masses than chains which are radically terminated after just a few growing steps (chapters 3.1 and 3.2). To characterise polymer samples some key figures are introduced here.

The numeric average molar mass $\overline{M_{\mathrm{n}}}$ represents the mass of the most frequently occurring chain. It corresponds with the frequency of amount $x$ of molecules with the chain length $i$ and the molecular weight of these $M_{i}$. The amount of molecules can also be described as number $N$ of molecules with the chain length $i$, as follows:

$$
\overline{M_{\mathrm{n}}}=\sum_{i=1}^{\infty} x_{i} M_{i}=\frac{\sum_{i=1}^{\infty} N_{i} M_{i}}{\sum_{i=1}^{\infty} N_{i}}
$$

The average molecular weight $\overline{M_{\mathrm{w}}}$ describes which molecular mass has the highest share in the polymer sample. It is formed analogously to $\overline{M_{\mathrm{n}}}$ with the weight share $w$ of the molecules with the chain length $i$.

$$
\overline{M_{\mathrm{w}}}=\sum_{i=1}^{\infty} w_{i} M_{i}=\frac{\sum_{i=1}^{\infty} N_{i} M_{i}^{2}}{\sum_{i=1}^{\infty} N_{i} M_{i}}
$$

The more equal the chain lengths of the molecules are and with that the more equal the molecular masses of these chains are, the closer do the values $\overline{M_{\mathrm{n}}}$ and $\overline{M_{\mathrm{w}}}$ lie together (Figure 3-14). 


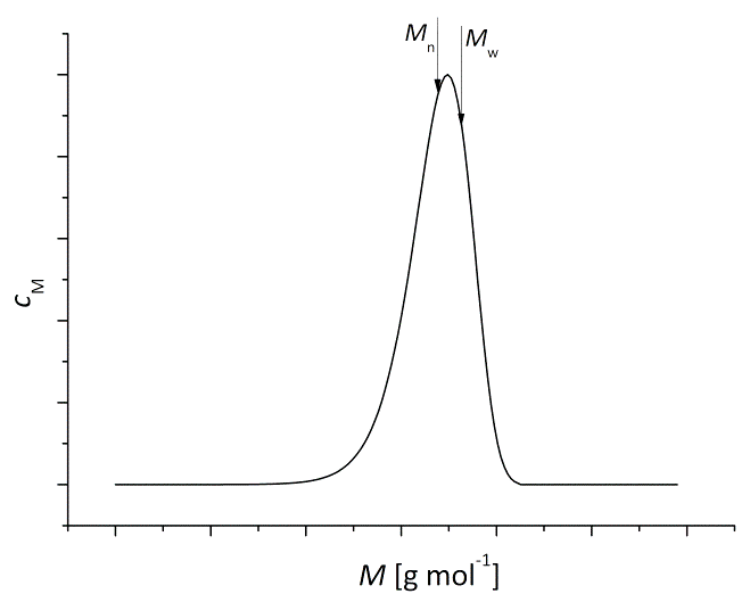

Figure 3-14: Comparison of $M_{n}$ and $M_{w}$ in the MWD. ${ }^{(23)}$

The polydispersity index (PDI) describes this difference. It is a measure for the width of the distribution curve.

$$
P D I=\frac{\overline{M_{w}}}{\overline{M_{n}}}
$$

A PDI-value of 1 is a signal for one molecular mass only in the sample, as e.g. for biological macromolecules. A value near 1 shows a narrow distribution. The larger the value the broader is the distribution. It is also possible to describe the PDI with the degree of polymerisation $\bar{P}_{\mathrm{n}}$ if the distribution function corresponds with a Poission distribution, e.g. for controlled polymerisation systems. The degree of polymerisation gives the chain length in monomer units

$$
\overline{P_{\mathrm{n}}}=\frac{X \cdot[M]_{0}}{[I]_{0}}
$$

with $X$ as monomer conversion, $[\mathrm{I}]_{0}$ and $[\mathrm{M}]_{0}$ as initial concentration of the initiator and monomer, respectively.

$$
P D I=1+\frac{1}{\overline{P_{n}}}
$$

The type of termination has a large influence on the resulting PDI. In case of FRP, for termination via combination a PDI of 1.5 is expected, via disproportionation a PDI of $2 .{ }^{(41 ; 67)}$ As it is nearly impossible to keep the conditions in a FRP stationary, i.e. the monomer and initiator concentrations as well as the temperature are only approximately constant during the process of a FRP, a PDI of larger than 1.5 is expected as none of the two termination reactions can be ruled out. Due to reduced termination reactions in controlled processes, the degree of polymerisation is heading to infinity. So for an ideal CRP the distribution function corresponds with a Poission distribution, PDI values smaller than 1.5 
can be expected. With the PDI in hand a criterion to evaluate controlled systems is found. ${ }^{(67)}$ For typical RAFT-polymerisations PDIs between 1.1 and 1.2 are usual. ${ }^{(12)}$

For ideal, controlled systems a theoretical value is computable. ${ }^{(28)}$ As the number of chains is given by the concentration of the controlling agent, the increase of the molecular mass is direct proportional to the conversion.

$$
\overline{M_{\mathrm{n}}^{\text {calc. }}}=\frac{X \cdot M_{\text {monomer }} \cdot[\mathrm{M}]_{0}}{[\mathrm{RAFT}]_{0}}
$$

A part of the polymer chains gets lost via termination. This part depends on the radical concentration and the effectiveness of the initiator. Insertion of these parameters in equation 3-23 leads to:

$$
\overline{M_{\mathrm{n}}^{\text {calc. }}}=\frac{X \cdot M_{\text {monomer }} \cdot[\mathrm{M}]_{0}}{[\mathrm{RAFT}]_{0}+[I]_{0} \cdot d \cdot f \cdot\left(1-e^{-k_{\mathrm{d}} \cdot t}\right)}+M_{R A F T}
$$

with $d$ describing the type of termination ( $d \approx 1$ for acrylates) ${ }^{(68)}, t$ the reaction time, $f$ as effectiveness of the initiator and $k_{\mathrm{d}}$ corresponding the rate of initiator decomposition. As every chain carries a RAFT-unit, the molecular mass of this compound has to be added, too. Often the ratio of initiating radical to RAFT-concentration is really small that the share of dead polymer is negligible. In that case the use of equation $3-25$ is sufficient. But especially in case of slowly propagating monomers, such as styrene, the term of termination has a large impact as a significant amount of dead chains are produced before higher monomer conversions are achieved, ${ }^{\left({ }^{(6)}\right)}$ here equation 3-26 has to be used.

$$
\begin{gathered}
\overline{M_{\mathrm{n}}^{\text {calc. }}}=\frac{X \cdot M_{\text {monomer }} \cdot[\mathrm{M}]_{0}}{[\mathrm{RAFT}]_{0}}+M_{R A F T} \\
\overline{M_{\mathrm{n}}^{\text {calc. }}}=\frac{X \cdot M_{\text {monomer }} \cdot[\mathrm{M}]_{0}}{[\mathrm{RAFT}]_{0}+[\mathrm{I}]_{0} \cdot d \cdot f \cdot\left(1-e^{-k_{\mathrm{d}} \cdot t}\right)}+M_{R A F T}
\end{gathered}
$$

If two monomers are going to be copolymerised, the equation has to be adapted as follows:

$$
\overline{M_{\mathrm{n}}^{\text {calc. }}}=\frac{X \cdot\left[\left(M_{\mathrm{A}} \cdot\left[\mathrm{M}_{\mathrm{A}}\right]_{0}\right)+\left(M_{\mathrm{B}} \cdot\left[\mathrm{M}_{\mathrm{B}}\right]_{0}\right)\right]}{[\mathrm{RAFT}]_{0}}+M_{R A F T}
$$

with the indices $A$ and $B$ corresponding with the two different monomers. 
The Size Exclusion Chromatography (SEC) is a standard method to determine the average molecular weight or rather the molecular weight distributions of polymer samples. In contrast to mass spectrometry, it is possible to measure wider mass ranges and higher molecular masses, as long as correct calibrations, standards and Mark-Houwink-parameters (see below) are applied.

In SEC, dilute solutions of polymer samples are passed through columns packed with porous filler at a constant flow rate and temperature. These fillers are cross-linked polymer materials of defined pore sizes, e.g. polystyrene cross-linked with divinyl benzene. Due to the different pore sizes the polymeric molecules are separated via their hydrodynamic volume. The volume of the pores corresponds with the molecular expansion in the dissolved state. The hydrodynamic volume of the macromolecules is inversely proportional to its elution time and volume, i.e. smaller molecules fit into the more pores of the column material, so they are stronger detained on their way through the column. Larger molecules are eluated faster as they are less detained. The hydrodynamic volume $V_{\mathrm{h}}$ is correlated with the molecular mass via the intrinsic viscosity $[\eta]$.

$$
V_{\mathrm{h}}=[\eta] \cdot M
$$

Different detectors which register, e.g. the change in the refractive index (RI) or in the absorbance of UV-light, can be applied to record the gradation of the eluated fractions. The retention times depend on the experimental set up, polymer, solvent, temperature and filling material. Therefore it is necessary to calibrate the system with appropriate standards.

If there are no calibration standards available, a universal calibration can be applied. ${ }^{(70)}$ Here the polymer in question is compared with a known polymer where a calibration is possible by the use of the Kuhn-Mark-Houwink-Sakurada equation 3-29 with the Mark-Houwink (MH) parameters $K$ and $a$.

$$
[\eta]=K M^{a}
$$

This equation gives a description of the dissolved state of the polymers independent from pseudoideal dilution conditions. It describes the molecular weight via the ratio of intrinsic viscosity $[\eta]$ with $K$ as the $\mathrm{MH}$ coefficient, empirically determined because it is depending on the monomer used and $a$, an empirically determined constant that describes the hydrodynamic interactions between polymer and solvent. The $a$-value outlines the topology that a polymer in the dissolved state will take. For rigid rods $a$ lies in a range of 2, for small spheres $a$ is zero. Well dissolved and expanded polymers have an $a$-value of 0.5 to 1 , where $a_{\text {linear }}>a_{\text {branched }}$ is valid. ${ }^{(71)}$

From these two assumptions the following equation can be derived: ${ }^{(72)}$ 


$$
\log M_{2}=\frac{1}{1+a_{2}} \log \frac{K_{1}}{K_{2}}+\frac{1+a_{1}}{1+a_{2}} \log M_{1}
$$

where the index 1 corresponds with the known polymer and the index 2 with the polymer of interest. This method assumes known MH-parameters for the polymers. If these are unknown, the molecular mass of the polymer may be determined via light scattering detection and correlated viscosity or a SEC set up coupled to a soft ionization mass spectrometry. ${ }^{(73)}$

\subsection{Star-Shaped Polymers}

Star polymers are an interesting field in material science since they behave different compared to their linear relatives. ${ }^{(74-76)}$ Their viscosity is much lower since entanglements are ruled out. ${ }^{(63 ; 77 ; 78)}$ More details concerning the hydrodynamic volume in comparison to linear polymers with the same molecular weight are given in the sections 3.3.2 and 4.3.

The large number of end groups in the outer sphere determines the properties decisively; in linear polymers this effect is negligible as only two end groups are present. These functional groups have an influence on the solubility, the glass transition temperature, the conformation and shape of the molecule. Via modification of these groups these parameters can be changed. ${ }^{(79 ; 80)}$ Hence a pathway for other modifications as well as applications is open, e.g. loading with guest-molecules ${ }^{(81)}$ or as additives and blends. ${ }^{(82)}$ They are used as nano-reactors, storage media and carriers for catalytical units. ${ }^{(80 ; 81)}$

One important kind of star-shaped polymers are dendrimers (dendron, greek: tree). ${ }^{(79 ; 83)}$ Dendrimers are compounds which are perfectly monodispersed since they are synthesised via stepgrowth reactions from a perfect core. ${ }^{(60 ; 84)}$ Figure 3-15 shows an example of a dendritic core (left) and the three generations (G3) of polymer surrounding (right).

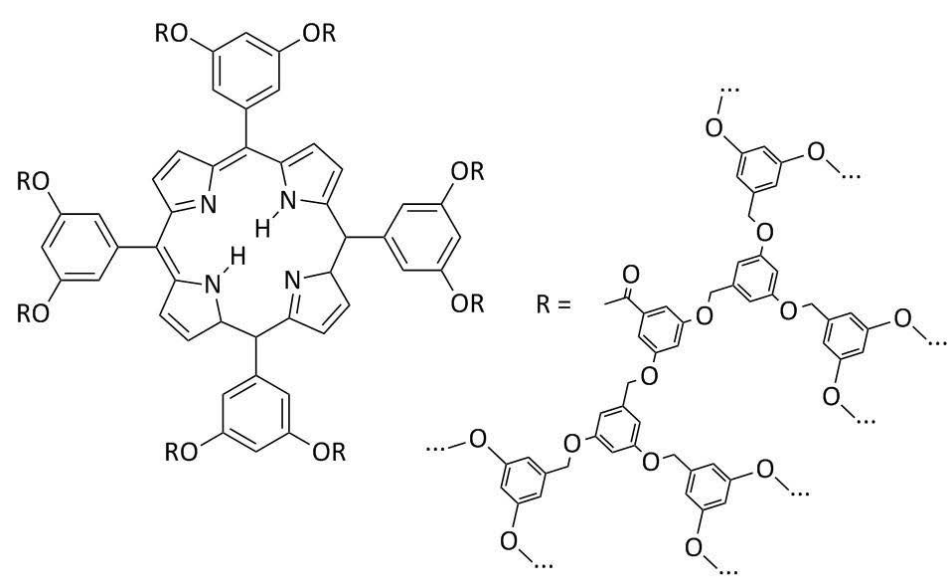

Figure 3-15: A G3-dendrimer. ${ }^{(85)}$ 
The main disadvantage of dendrimer preparation is the complex and time consuming synthesis of these compounds. ${ }^{(80)}$ Star-shaped polymers, on the other hand, show defects. These are a consequence of the polymerisation process, but these defects have no influence on the application. ${ }^{(86)}$

\subsubsection{Approaches to Synthesise Star-Shaped Polymers}

All of the in chapter 3.2 presented CRPs allow the synthesis of many different polymer architectures. The arm-first or core-first approaches, described in this chapter, for star-shaped polymers are also valuable in ATRP or NMP processes. Figure 3-16 represents known agents for ATRP (left) and NMP star-polymers (right) from the literature.
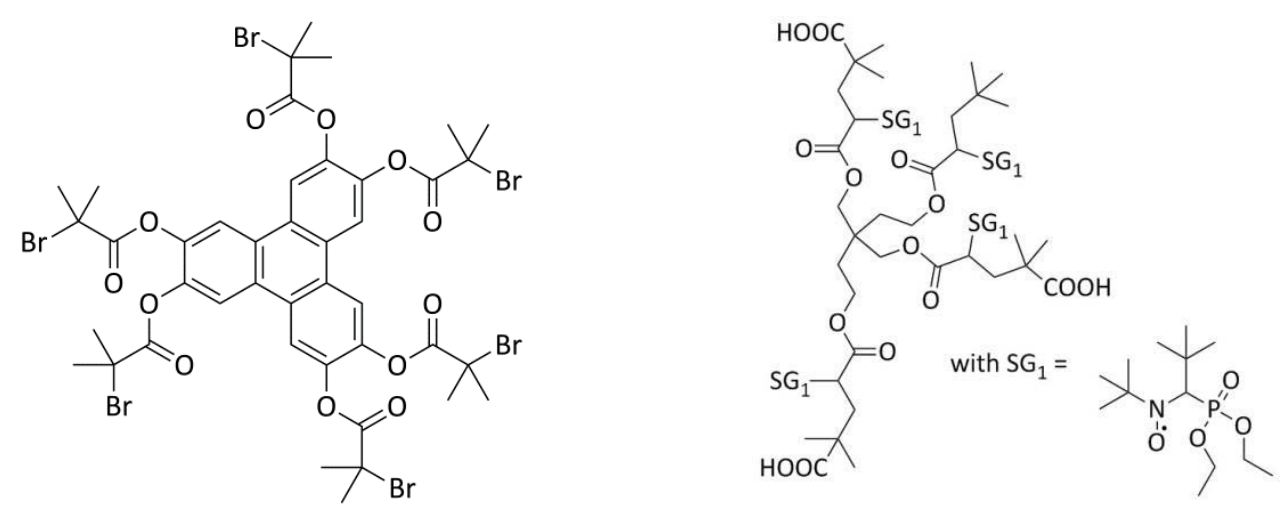

Figure 3-16: Literature known starters for star polymers by ATRP and NMP.

Left: a core-first ATRP-starter published by Xue et al. ${ }^{(87)}$

Right: a NMP-agent for a core-first approach synthesised by Dufils et al. ${ }^{\left({ }^{(8)}\right.}$

In this work star-shaped polymers are the basis to gain nano-scaled containers. The chosen CRP is RAFT as it has many advantages: RAFT-systems are versatile in combination with all kinds of monomers and solvents, robust against water as well as impurities and no metals have to be used during the synthesis. ${ }^{(29)}$ The RAFT-agents are easily synthesised and it is possible to cleave off the RAFT-unit in the end to gain a colour- and odourless polymer.

There are two ways to synthesise star-shaped polymers, shown in Figure 3-17, the arm-first and the core-first approach. ${ }^{\left({ }^{89)}\right.}$ In the arm-first approach linear polymer chains are synthesised and these are connected to a multifunctional core. The disadvantage of this method is an incomplete occupancy of all functional groups on the surface. The coiled polymer chains cause steric hindrance; hence there is a high probability that not all functionalities of the core's surface are occupied. 


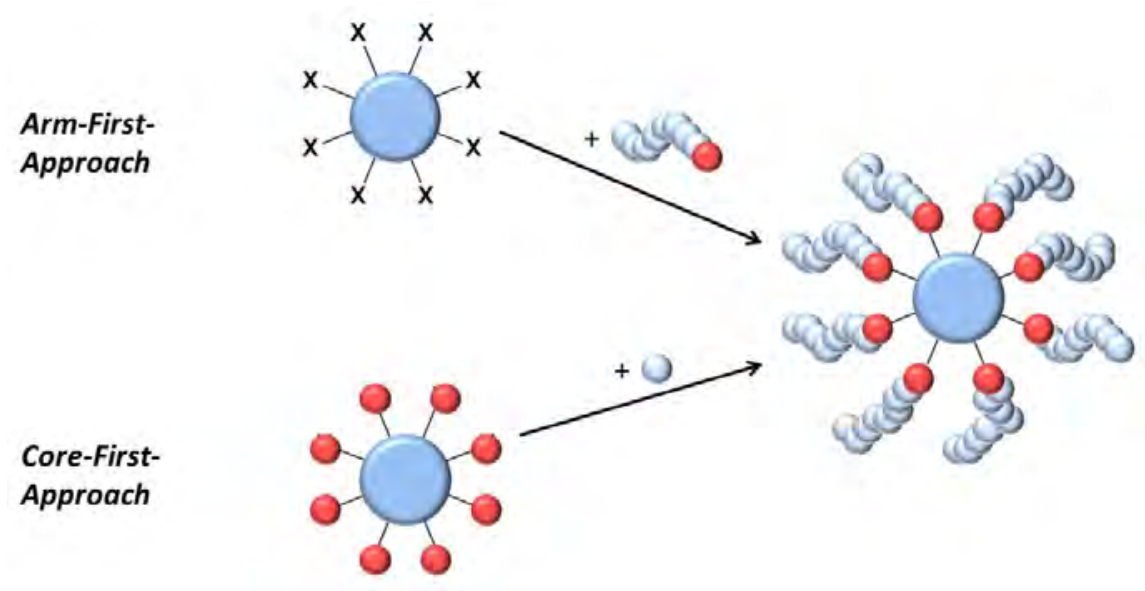

Figure 3-17: Two approaches to yield star-shaped polymers.

The second approach is the core-first approach. Here the core carries the controlling functionalities. The polymerisation takes place on the core's surface. As all chains start at the same time, the core is evenly covered with polymer chains of similar lengths and the final structure is predetermined. ${ }^{(69 ; 90)}$ This strategy of synthesis provides a predefined number of arms and enables manipulation of the end groups at the arm's tips. ${ }^{(47)}$ In case of RAFT polymerisation the favoured core-first approach is split up in the R-group- and the Z-group approach. ${ }^{(91)}$ The Figures 3-18 and 3-20 illustrate these two concepts with red as free radical functionalities, blue symbolising the Z-groups and yellow the R-groups.

In the R-group approach the leaving group $\mathrm{R}$ represents the core. ${ }^{(92)}$ Connected to it are dithioester units with the stabilising Z-group.

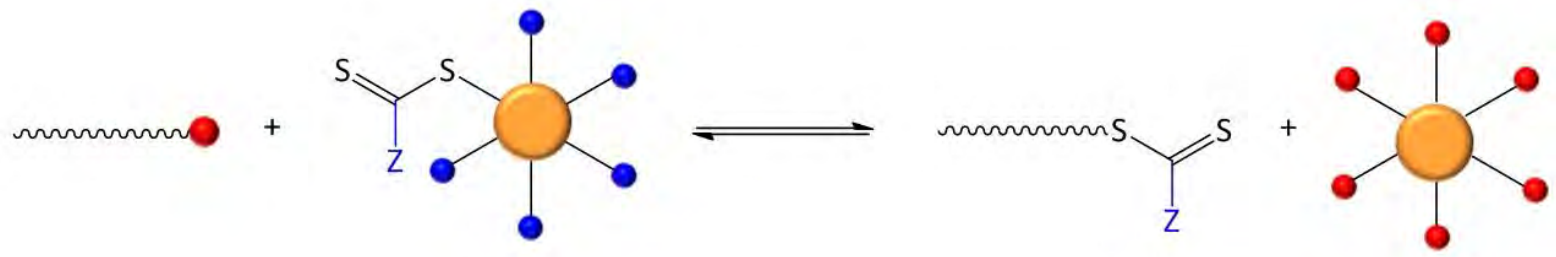

Figure 3-18: R-group approach.

During the polymerisation the RAFT-unit with the Z-group is split from the core and transferred to the chain. At this point the radical functionality is on the core which can lead to starstar-coupling via termination by combination. At the end of the reaction the products are dead chains, living chains, single- and double-star polymers as well as stars with a mixture of dead and living arms. This mixture is difficult to separate which makes this approach unfavourable for the progress to synthesise equal sized nano-scaled containers. ${ }^{(93)}$ Figure 3-19 shows literature-known RAFT-agents for the R-group approach. 

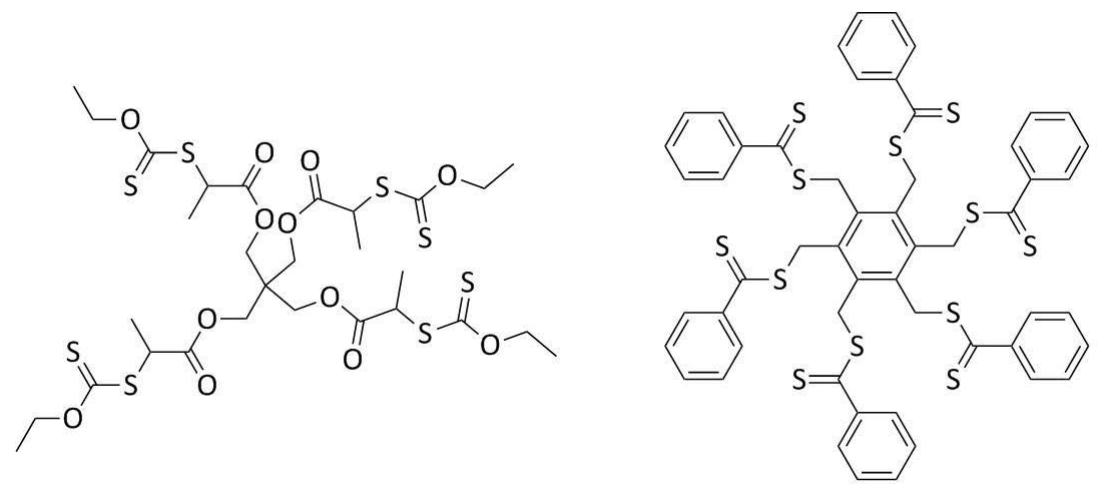

Figure 3-19: R-RAFT-agents: tetrafunctional xanathate agent ${ }^{(94)}$ and

1,2,3,4,5,6-Hexakis(thiobenzoylthiomethyl)benzene. ${ }^{\text {(95) }}$

When applying the Z-group approach (Figure 3-20) star-star-coupling is avoided. ${ }^{(89 ; 93)}$ Here the radical functionality is never at the core since the core is the stabilising group; therefore, a high homogeneity of the generated stars is given. ${ }^{(93)}$ The resulting products of this process are single-star polymers and dead chains. Since the RAFT-units remain on the core throughout the reaction, the whole star stays in a living state. Therefore, it can be used as a macroRAFT-agent for block copolymer synthesis.

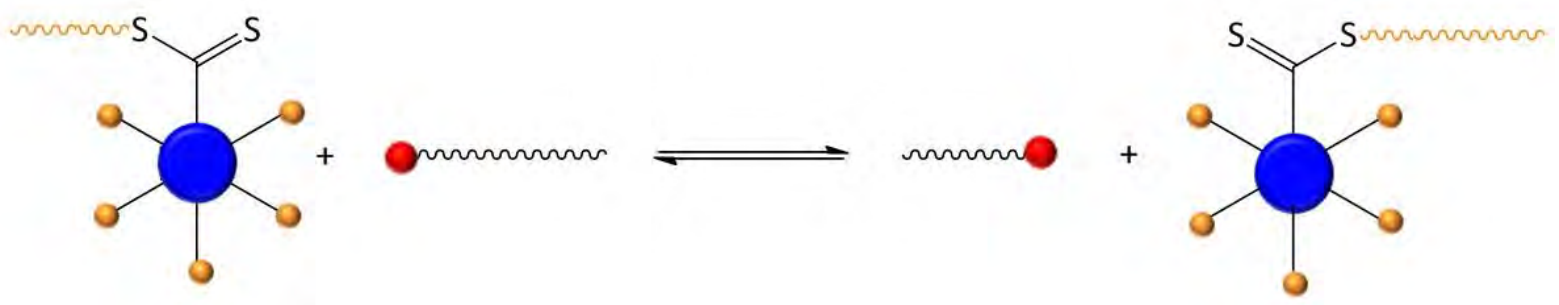

Figure 3-20: Z-group approach.

Two examples of RAFT-agents for the Z-group approach, used by Boschmann and Mänz in preceding projects, are shown in Figure 3-21. Mayadunne et al. firstly introduced such pentaerythriol-based multifunctional RAFT-agents in $2003 .{ }^{(96)}$ 


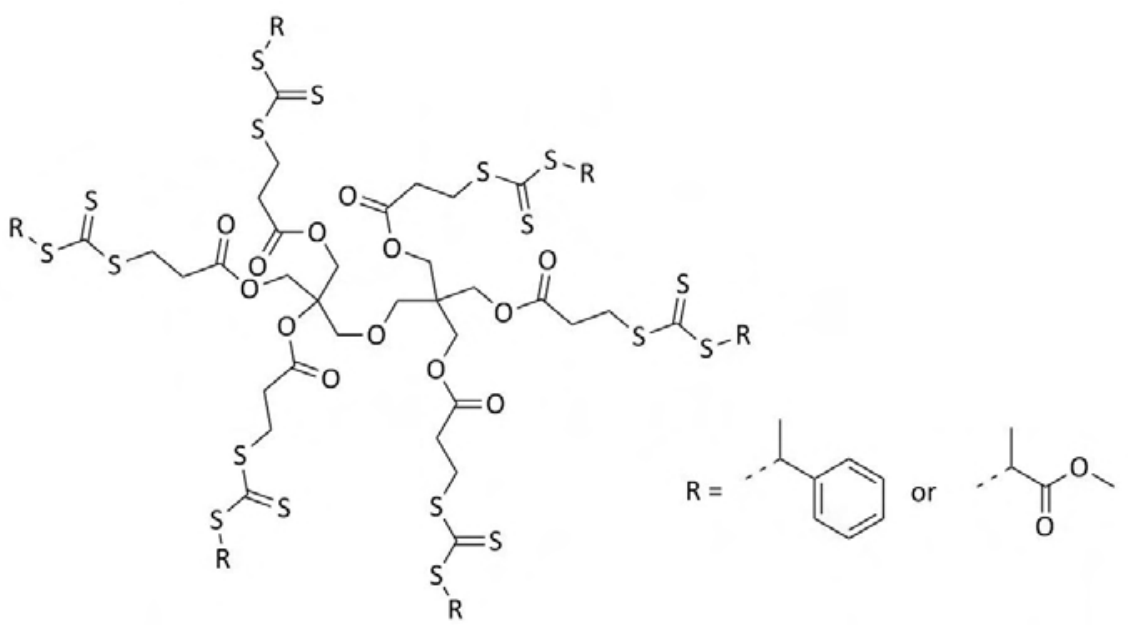

Figure 3-21: Z-RAFT-agents: Dipentaerythriol-hexakis(3-S-phenylethyl-tricarbonylpropanoate) and Dipentaerythriolhexakis(3-S-(methyl-2-propanoatotrithiocarbonyl)propanoate). ${ }^{(47 ; 90)}$

\subsubsection{Molecular Mass-Determination of Star-Shaped Polymers}

In case of star polymers the via SEC experimentally found molecular weights deviate from theoretical values (equation 3-26). For increasing number of arms this deviation increases systematically. ${ }^{(69)}$ This effect is a result of smaller hydrodynamic volumes of star polymers compared to linear polymers of the same type and same molecular weight as $[\eta]$ decreases with increasing number of arms ${ }^{(77)}$ and equation 3-28 is valid. This effect is a consequence of greater ternary chain interferences with an increased chain density. ${ }^{(75)}$ So the star polymer is more dense than a chain polymer of the same molecular weight. Figure 3-22 shows this schematically.

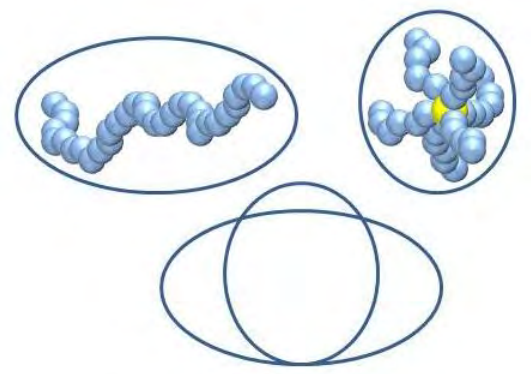

Figure 3-22: Comparision of the hydrodynamic volumes (blue outline) of six-armed star and chain polymer with the same number of monomer units, i.e. the same molecular mass.

A triple detector system for the SEC set up consisting of a refractive index detector, a light scattering detector and a viscometer would bring complete clarification of the polymer's topology, mass and dissolved state, but is not found easily in laboratories.

For a usual SEC set up linear polymers are used as the standard for calibration. Therefore, the obtained molecular weights for star polymers are much too low as the star polymers eluate later due 
to their smaller hydrodynamic volume. Consequently a method had to be found to determine the correct values. Boschmann designed an experiment to overcome the problem by taking advantage of the RAFT-equilibrium. ${ }^{(69)}$ RAFT-polymerisations are performed with a mixture of mono- and multifunctional RAFT-agents. The two RAFT-equilibria are interconnected via the growing macro radicals (left in Figure 3-23).

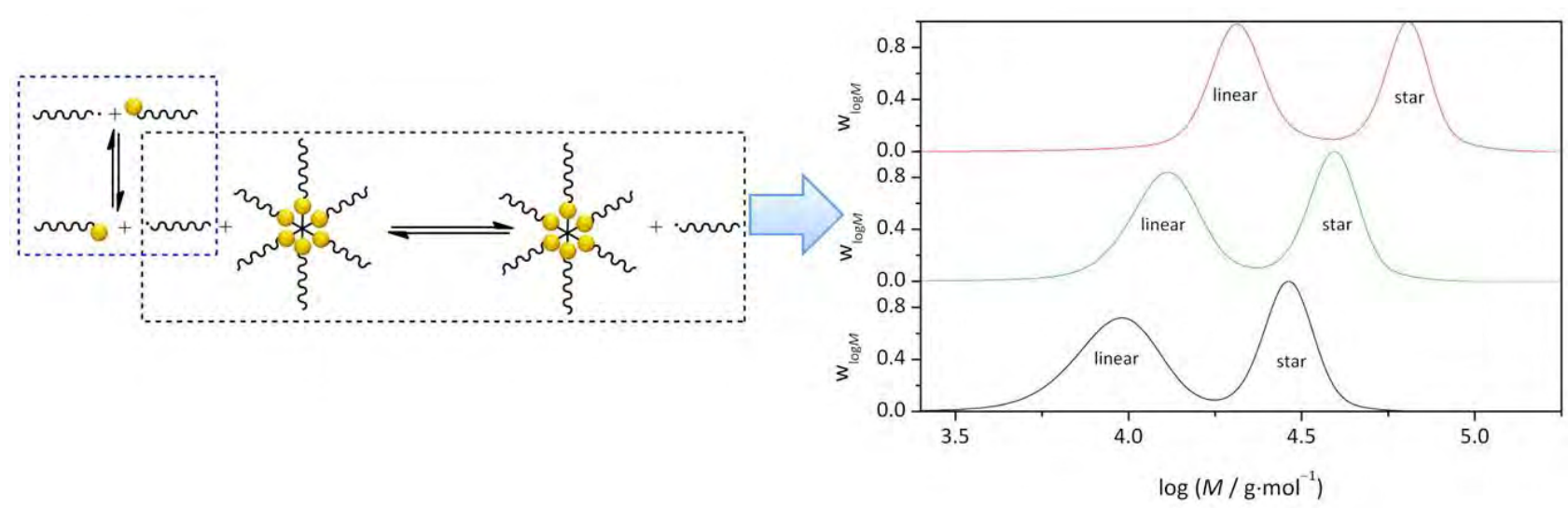

Figure 3-23: Left: The interlinked equilibria of simultaneously proceeding linear and Z-RAFT star polymerisation, redrawn after ref. (69). Right: The resulting molecular weight distributions for different conversions of a styrene polymerisation with mono- and multifunctional RAFT-agents (black $29 \%$, green $43 \%$ and red $81 \%$ monomer conversion). ${ }^{\text {(21) }}$

During the polymerisation the radicals do not distinguish between the mono-RAFT- and the star-RAFT-agent as these are chemically equal. Due to the controlling nature of the process all macro radicals have the same chain length. The resulting molecular weight distributions obtained by the SEC measurements are distinctively bimodal. An example is shown right in Figure 3-23. The actual number average molecular weight of the star polymer is calculated by multiplying the number average value of the linear polymer by the number of arms of the star polymer.

Also an apparent number of arms can be calculated by dividing the peak molecular weight of linear and star polymer. It is always smaller than the expected one, again the result of the much more compact nature of the star polymers in comparison to the linear polymers. It was found that six-armed stars seem to have 3.94 , four-armed stars 3.05 and three-armed stars 2.58 arms. The apparent number of arms is independent from the monomer conversion and stays constant during the whole process. With the apparent number of arms and the theoretical number of arms it was possible to establish a correction factor $K$ to determine the true molecular weight of star polymers without applying RAFT-agent combination in all polymerisations:

$$
K=\frac{f_{\text {theor. }}}{f_{\text {app. }}}
$$


with $f_{\text {theor. }}$ being the number of RAFT-functionalities of the star and $f_{\text {app. }}$ the apparent number of arms. For six-armed stars a molar mass correction factor of 1.52 was found. ${ }^{(69)}$

This enables a determination of the real number average molar weights for star-shaped polymers without a lavish SEC-detector system.

\subsection{Cross-Linking Reactions}

As discussed in the introduction, in this project the aimed nano-carrier should be established by using multifunctional RAFT-agents in block copolymerisations and cross-linking of monomer units to form the carrier's shell.

There will be two steps of polymerisation. The general idea is depicted in Figure 3-24. In the first polymerisation a statistical copolymer is synthesised with a main monomer and a co-monomer carrying a linkable unit. In Figure 3-24 these two kinds of monomers are symbolised by red and orange spheres. As the polymer from the main monomer has defined and desired properties, the integration of the co-monomer should not alter these. In a second polymerisation step another block is synthesised onto the first one, the blue block in Figure 3-24. This is a homopolymer with different polarity than the first one as amphiphilic stars and carriers are aimed for.

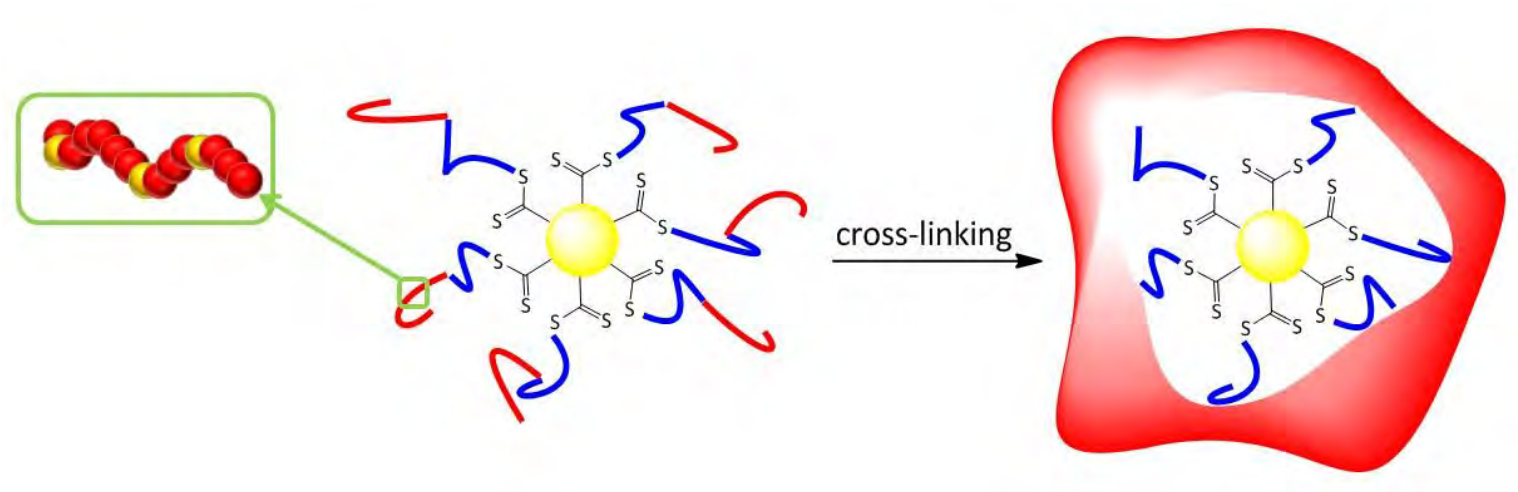

Figure 3-24: General scheme of the formation of the carrier's shell.

There are different reaction mechanisms of cross-linking reactions which can be used to conduct the formation of the carrier's shell. Some of them are shortly introduced here. In the second part of this chapter more details are given on the topic of cross-linking reactions under UV-irradiation and the classes of UV-sensitive monomers chosen for this project. For all cross-linking processes in star polymers to form single spherical particles the dilution of the solution is important to avoid intermolecular coupling, e.g. star-star coupling. 


\subsubsection{Cross-Linking via Chemical Linkers}

On the one hand, functional groups introduced into the polymer by appropriate monomers can be linked via additional cross-linkers. Esterfication reactions between hydroxy-groups in the polymer and acid chlorides or dichlorosilane compounds ${ }^{(97)}$ as well as de-epoxydation of glycidyl methacrylate (GMA) units with the bifunctional cystamine are options. ${ }^{(98)}$ Also the 1,3-dipolar Huisgen cycloaddition (so called Click-reaction) between alkyenes and azid-groups is a possibility. ${ }^{(99 ; 100)}$ Here a large drawback is the addition of metal salts as a catalyst, which makes it difficult to use the synthesised materials for medical purposes. Stenzel and co-workers reported about cross-linking via chain extension of $p$ NIPAam blocks using hexamethylene diacrylate. ${ }^{(101)}$ Another way to cross-link pNIPAam was investigated by Singh et al. using $N, N^{\prime}$-methylene bisacrylamide (BIS) as bifunctional linker. ${ }^{(102)}$

In all of these cases the right stoichiometry is essential because additional compounds, such as bases, can destroy the polymer. It is rather difficult to enable this exact stoichiometry as there are uncertainties in a polymer's molecular mass and linkable co-monomer content. Also the system has to be perfectly dry and clean for chemical cross-linking, as e.g. esterfication reactions, to assure complete cross-linking and avoid side reactions.

\subsubsection{Cross-Linkage between Monomers}

On the other hand, cross-linking among the monomers themselves by their functional groups is plausible. The coupling reaction takes place without additional chemicals. The reaction is triggered by favourable conditions, e.g. heat, light or change of the $\mathrm{pH}$-value. These kinds of reactions have some advantages towards cross-linking reactions with additional chemical linkers. Here for the crosslinking reactions the right stoichiometry is given from the beginning because the linkable unit is integrated in the polymer during the polymerisation as a co-monomer. Also side reactions with additional substances are ruled out as well as chemical destruction or alternation of the polymer. Three reaction variations are introduced here:

The monomer ethylene glycol dimethylacrylate (EGDMA) cross-links during the polymerisation step itself. The basic monomer, e.g. MMA and EGDMA are polymerised together. Depending on the MMA:EGDMA ratio cross-linking to form networks or star-shaped polymers is favoured. ${ }^{(103)}$ For a styrene system divinyl benzene can be used in the same way. ${ }^{(104)}$ Another method using polymerisation was published by Du Prez and co-workers: Preparing star polymers via ATRP they substituted the remaining bromine functionality with a methacrylic unit and polymerise these intramolecularly. ${ }^{(105)}$

At high temperatures $\left(T \approx 250{ }^{\circ} \mathrm{C}\right)$ the cyclobutene ring of monomers such as 4-vinylbenzocyclobutene is opened and two extended double bonds are formed. From this 
intermediate there are two pathways for the next reaction step but under these conditions the formation of an eight membered ring is favoured over the back reaction to the cyclobutane ring. ${ }^{(106)}$ In this kind of cross-linking procedure the danger of polymer destruction by heat is given.

A destruction-free pathway is provided by cross-linking via UV-irradiation since wavelength and applied energy entry can be easily controlled. In the literature many UV-cross-linkable monomers can be found. They are based on $N$-alkyl-3,4-dimethylmaleimid (DMI) (107), anthracene ${ }^{(108 ; 109)}$, stilbene ${ }^{(110)}$, cinnamic acid ${ }^{(54 ; 111)}$, uracil derivatives ${ }^{(112)}$ or courmarin ${ }^{(113)}$ and cross-link via [2+2]-cycloaddition. Sometimes this cycloaddition has to be assisted by sensitizers, e.g. acetophenone or benzil, depending on the amount of UV-sensitive units and the polymer surrounding. These are substances which enable the transition toward the excited state. ${ }^{(107 ; 114 ; 115)}$ In the next section the monomers and their corresponding cross-linking reactions used within this project are introduced.

\subsubsection{Cross-Linking via UV-Sensitive Units}

These monomers consist of a UV-sensitive unit and the monomer unit itself where the polymerisation takes place. These should be chemically different and spatially separated to evade consumption of the UV-unit during the polymerisation. The monomer unit is adapted to the main monomer used to avoid large differences in the polymerisation behaviour, represented by different copolymerisation parameters (for further information, see chapter 3.3.3). This adaption has to be made so that the UV-sensitive monomer is integrated in the polymer in sufficient quantities, otherwise a complete cross-linking of the shell cannot be guaranteed. In the experimental section (chapter 12) the syntheses procedures for these monomers can be found.

\subsubsection{N-Alkyl-3,4-Dimethylmaleimids}

A large group of so called photodimers are the DMIs. These were thoroughly investigated by Kuckling et al. ${ }^{(102 ; 107 ; 114 ; 116-118)}$ The monomers consist of an $N$-substitued dimethylmaleimide unit which undergoes a [2+2]-cycloaddition under UV-irradiation of wavelengths above $300 \mathrm{~nm} .^{(107)}$ In Figure 3-25 the resulting reaction scheme is shown for the in this project used DMIs. 


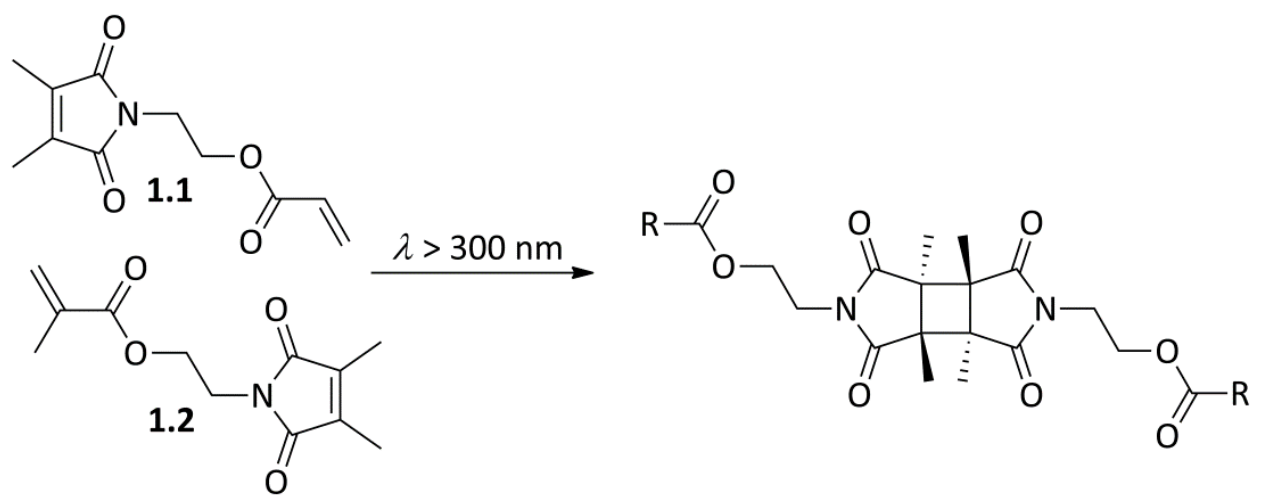

Figure 3-25: N-ethylacrylate-3,4-dimethylmaleimide (1.1) and N-ethylmethacrylate-3,4-dimethylmaleimide (1.2).

1.2 was first introduced by Baumann in 1978. ${ }^{(119)}$ Berger and Zweifel started investigations in homo- and copolymerisation in $1983 .{ }^{(120)}$ They aimed for photoactive polymers which do not undergo side reactions known from other photosensitive monomers, e.g. cinnamic acid derivatives, which are prone to cyclopolymerisation at the UV-sensitive double bond and also to E-Zisomerisation under UV-irradiation at this double bond. ${ }^{(121)}$ DMIs were chosen because they carry two differently stimulatable double bonds to divide clearly the polymerisation from the cross-linking unit. ${ }^{(116 ; 120)}$ The vinyl double bond is only active for the step of radical polymerisation. The second double bond is part of the maleimido moiety and is therefore a tetra-substituted olefin which does not undergo radical polymerisation reactions. ${ }^{(122)}$ Furthermore these rings dimerise under UVirradiation and due to the ring structure and E-Z-isomerisation is prevented. ${ }^{(123 ; 124)}$ It was found that DMI moieties act as regulators in the FRP-case, resulting in smaller yields of the polymerisation. ${ }^{(117)}$ During the polymerisation no cross-linking is observed. ${ }^{(114 ; 118)}$ Copolymerisations were carried out with ethylacrylate (EA), MMA and MA. For these combinations the following copolymerisation parameters were found: 1.2-co-MA $r_{1.2}=1.36, r_{\mathrm{MA}}=0.77 ; 1.2$-co-MMA $r_{1.2}=1.16, r_{\mathrm{MMA}}=0.47$ and 1.2-co-EA $r_{1.2}=1.60, r_{\mathrm{EA}}=0.44 .{ }^{(120)}$

In 2008 the reaction mechanism and product ratios of the photodimerisation of different DMIs were reported by Kuckling et al. ${ }^{(107)}$ They found that besides the known cyclobutane-fused cyclic dimer a single bonded dimer is formed. The proposed mechanism is shown in Figure 3-26. 


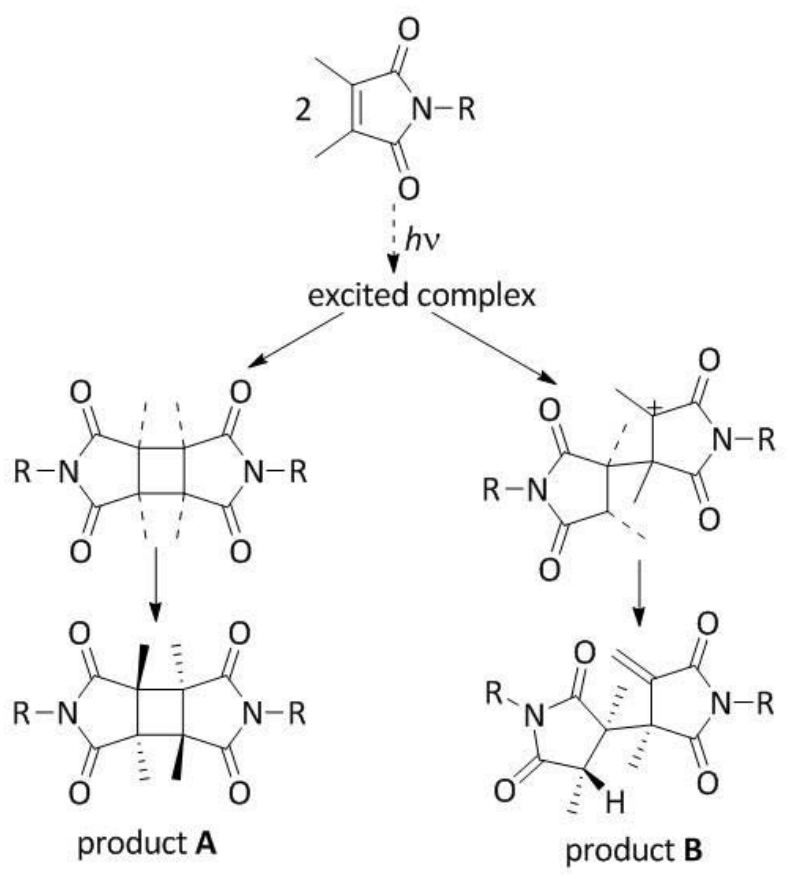

Figure 3-26: Proposed cross-linking mechanism by Kuckling et al., redrawn from (107).

A high dependence on the solvent but only low dependencies on the temperature and the wavelength $(\lambda>300 \mathrm{~nm})$ were determined. The ratio between the two products differs from 5 to $50 \%$ depending on the solvent polarity. It was found that product $\mathbf{A}$ is the major product in nearly all cases. Steady state irradiation experiments showed a decrease of $\mathbf{A}$ with an increase of the solvent polarity, e.g. as the product ratio is up to $98: 2$ for some DMIs in cyclohexane, it can get to 50:50 in solvents like methanol or acetonitrile. Also a faster dimerisation in solvents of poorer polarity is reported. The process of photodimerisation proceeds through a triplet state mechanism. A linear increase of the photochemical conversion via irradiation time is reported, too. For polymers with a high DMI-load it is not necessary to apply sensitizer as the DMI-moieties are close enough for efficient self-quenching of the triplet state. In case of polymers with a low DMI-load sensitizers, e.g. benzophenone or thioxanthone, ${ }^{(115)}$ can be added to increase the yield of the dimerisation reaction. ${ }^{(107)}$

In case of the copolymerisation of DMI-NIPAam with NIPAam it is reported that the introduction of DMI-units shifts the hydrophilic-hydrophobic balance towards the hydrophobic regimes. ${ }^{(116)}$ That effect leads to a decrease of the phase transition temperature $\left(T_{c}\right)$ of the polymer solution in water with increasing amount of DMI in the polymer until a DMI-share of 15.9 mol\% when $T_{\mathrm{c}}$ disappears. ${ }^{(114)}$ Also a decrease of the glass transition temperature $\left(T_{\mathrm{g}}\right)$ is observed. Due to the integration of maleimido side groups the spacing between the polymer chains is raised, as a result there are less interactions and the system is more flexible. ${ }^{(117)}$ 


\subsubsection{Cinnamoyloxyethyl Monomers}

Another group of UV-sensitive monomers are cinnamic acid (CA) derivates. Homopolymers from CA-based monomers are known since the middle of the $1950 \mathrm{~s}^{(125)}$ and stand out because of their ability to cross-link photo-reversible. ${ }^{(111)}$ As in the case of the DMIs, a [2+2]-cycloaddition takes place under UV-irradiation (here: $\lambda>260 \mathrm{~nm}$ ). This can be reversed by application of wavelengths below $260 \mathrm{~nm}$. The following figure shows the two CA-based monomers tested within this project.

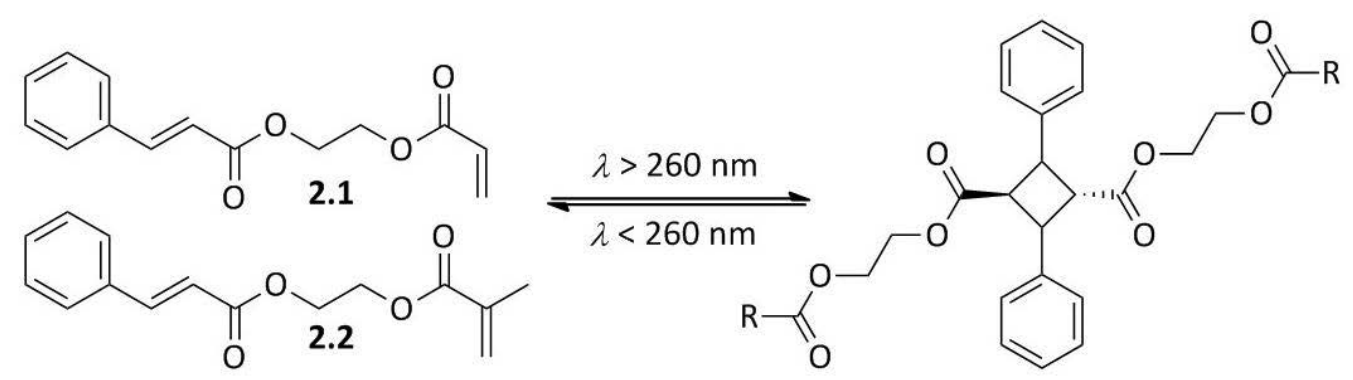

Figure 3-27: Cinnamoyloxyethyl acrylate (2.1) and cinnamoyloxyethyl methacrylate (2.2).

This cross-linking was subject of research of different working group since the 1960s. Tsuda studied the photosensitivity of $p$ (vinyl cinnamate) under the influence of molecular weight and the employment of sensitizers. ${ }^{(126)}$ Tanaka and Honda were running investigations to study the crosslinking in solution and at cryogenic temperatures. ${ }^{(127)}$ Egerton et al. extended these experiments to measurements in the solid state of crystal CA and films of $p$ (vinyl cinnamate). ${ }^{(128)}$ It was found that cross-linking in solids is not complete and that freezing the solution is just another way to achieve a fixed state during the reaction where the cross-linking conversion stops at $40 \%$. Due to the decreasing encounter probability between the chromophore units the conversion in the solution also decreased with time but reaches values over 80 \%. ${ }^{(128)}$ In 1967 Nakamur and Kikuchi published results of ESR-investigations which showed that the linking step proceeds via a radial mechanism. ${ }^{(129)}$ The photosensivity and with that the reactivity under irradiation of these monomers is influenced by the substituent in para-position of the benzyl ring. Investigations by Ali and Srinivasan were carried out with methoxy, chlorine and nitro groups in this position. They revealed that a methoxy group improves the photosensivity considerably in comparison towards the non-substituted ring. Substituents like chlorine increase the sensitivity, contrary to this a nitro group in para-position leads to a decrease in sensitivity. ${ }^{(130)}$

In the end of the $20^{\text {th }}$ century the investigations on CA-derivatives in polymer science got more application-orientated. In copolymerisation with other hydrophobic monomers oil absorbing networks are established where a three-dimensional structure is achieved through the cross-linking. ${ }^{(54 ; 131)}$ Atta et al. reported a random distribution of the monomers in such polymer 
networks and determined copolymerisation parameters for $\mathbf{2 . 2}$ in copolymerisation with isooctyl acrylate $(\mathrm{IOA}): r_{2.2}=1.04, r_{\mathrm{IOA}}=2.58 .{ }^{(54)}$

Another application field was found as smart materials. These are materials with the capacity to response to environmental stimuli by change of their morphological parameters. ${ }^{(132)}$ Stimuli can be chemically, physically or photochemically. ${ }^{(133)}$ In case of materials containing CA-units this effect is induced by UV-irradiation. The combination of photodimerisation and E-Z-isomerisation leads to a change in the shape of a polymer film. These so called shape-memory polymers were investigated by Lendlein et al. ${ }^{(111)}$ They synthesised polymer films with fixed pre-determined shapes from $\mathbf{2 . 1}$ with BA and hydroxyethylmethacrylate (HEMA) that change shape under irradiation with different wavelength. The process can be carried out several times and the fixed forms are also stable under treatment with higher temperatures.

The main advantage to use CA-based monomers in the medical applications is their biocompatibility. Cinnamic acid and its derivates are intermediate metabolites found in plant cell walls. ${ }^{(134)}$ Therefore, it is possible to synthesise biologically based polymers that are biocompatible, biodegradable, nontoxic and photo responsive which can enable a controlled drug release. ${ }^{(135)}$ In 2007, 2008 and 2011 Shi and co-workers published reports about polycondensation of 4-hydroxycinnamic acid and 3,4-dihydroxycinnaminc acid to form hyper branched copolymers which could be used to establish nano-carrier systems. ${ }^{(135-137)}$

\subsubsection{Courmarin-Based Monomers}

The third group of in this project used monomers are courmarin based monomers. The courmarin group consists of more than 800 derivates and were highly investigated in the last 110 years. Trenor and others summarised the main characteristics of courmarins and achievements on this field of research in a review in 2004. ${ }^{(138)}$ Some of these are nowadays commercially available, e.g. 3.2. Like in the case of CA-derivatives, the [2+2]-cycloaddition is photo-reversible. The cycloaddition takes place due to UV-irradiation $\lambda>310 \mathrm{~nm}$, whereas the cleavage of the cyclobutene ring is trigged at wavelength below $260 \mathrm{~nm} .{ }^{(113)}$ The photodimerisation of courmarins was firstly discovered by Chiamician and Silber in 1902. ${ }^{(139)}$ Besides the photodimerisation, the fluorescence of these compounds is a field of interest and finds application in electrical devices, e.g. light-emitting diodes (LED), ${ }^{(140 ; 141)}$ as the wavelength of fluorescence is determined via substituents in 4-position. ${ }^{(142)}$

Figure 3-28 shows the two courmarin derivates used within this project and the reversible dimerisation under UV-irradiation. The replacement of hydrogen with fluorine at the methyl group in 4-position should support the cycloaddition step due to the electron withdrawing effect. 


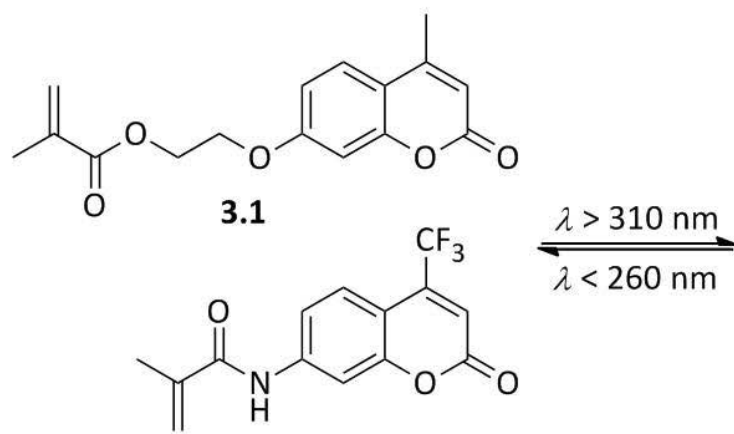

3.2

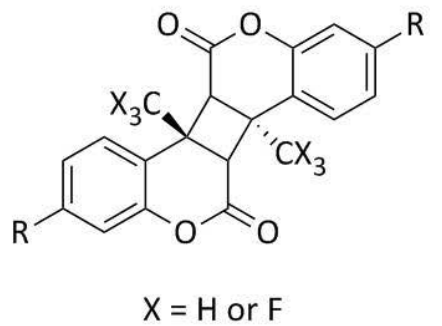

$\mathrm{X}=\mathrm{H}$ or $\mathrm{F}$

Figure 3-28: 7-(4-methylcourmarin)methacrylate (3.1) and 7-[4-(trifluoromethyl)coumarin]methacrylamide (3.2).

Studies by Lewis and Barancyk revealed three products of dimerisation: a syn head-to-head, an anti head-to-head and a syn head-to-tail dimer. They also reported that the mechanism of this photo reaction works for high courmarin concentrations via a singlet state of the courmarin and leads to the syn head-to-head dimer. For low concentrations the reaction proceeds via a triplet state and yields the anti head-to-head product. Also the application of sensitizers and the nature of the solvent used have an influence on the outcome. Polar solvents favor the singlet state and therefore the syn-products whereas non-polar solvents or the addition of sensitizer lead to anti-products. ${ }^{(143 ; 144)}$

7-(4-methylcourmarin)acrylate (MCA) has been copolymerised with $\mathrm{N}$-(1-phenylethyl)acrylamide (NPeAa) by Chen and Wu to investigate the reactivity ratios and the kinetics of the reversible photodimerisation. ${ }^{(145)}$ The photocycloaddition was carried out at $350 \mathrm{~nm}$ and the cleavage at $254 \mathrm{~nm}$. It was shown that both reactions proceed via first-order kinetics. The photo cross-linking rate constant increases with increasing mole fraction of the UV-sensitive unit in the polymer, whereas the cleaving constant decreases in this case. The kinetics and reaction rates are affected by the solubility of the polymer, the molecular mass and the polymer composition. It was shown that in the case of cleavage side reactions take place which influence a repeated crosslinking. They determined the copolymerisation parameters via four different methods and determined: $r_{\mathrm{MCA}}=1.13-1.28, r_{\mathrm{NPeAa}}=0.12-0.16$. Further investigations by Chen and co-workers have been carried out with different courmarin derivates and vinylacetate. ${ }^{(146)}$

The reversibility of the photo reaction was explored by Jiang et al. who copolymerised 3.1 with MMA in an ATRP process. ${ }^{(113)}$ They synthesised amphiphilic micelles of $p$ (ethyleneoxide)-block(3.1-co-MMA) where the cross-linking of $\mathbf{3 . 1}$ achieves the stabilisation of the micelle under different solvent conditions to yield versatile applicable nano-containers which can be stimulated to release loaded compounds. Their investigations revealed complete dimerisation and cleavage over at least five cycles. Furthermore, $\mathbf{3 . 1}$ was used in a FRP-copolymerisation with MMA for the synthesis of polymeric drug depots, published in 2010 by Sinkel et al. ${ }^{(147)}$ 


\section{State of the Art}

When this project started in spring 2009, there was hardly any literature published on the concept of nano-scaled and hollow-spaced objects synthesised via star-shaped polymers.

Other methods to synthesise nano-scaled container systems were already known, e.g. from emulsion polymerisation, ${ }^{(148)}$ polymer coating of inorganic or organic templates ${ }^{(149 ; 150)}$ or via selfassembling polymers forming micelles. ${ }^{(151 ; 152)} \mathrm{Xu}$ and Liu summarised many results of research in the field of polymeric micelles from self-assembling amphiphilic copolymers with application in drug delivery in a highlight in 2008. ${ }^{(153)}$ All examples base on storage of hydrophobic drugs in the hydrophobic core of such a micelle. The drug release is trigged by external or internal stimuli effecting the hydrophilic corona. pNIPAam or NIPAam-copolymers are the most often used hydrophilic parts. For the hydrophobic core styrene, $n$-butyl methacrylate, $D, L$-lactide or $\varepsilon$-caprolactone polymers are applied.

The following subchapters should give a short insight of recently published procedures to use RAFT polymerisation for nano-carrier establishment by self-assembling systems and for templateassisted synthesis of nano-capsules. Also the known facts of Z-RAFT star polymerisation as a pathway to nano-scaled vessels will be summarised.

\subsection{Nano-Carrier Synthesis via Cross-Linked Micelles}

The idea of Shell Cross-linked Micelles (SCM) is known since the middle of the 1990s. ${ }^{(154 ; 155)}$ These are micelles consisting of amphiphilic block copolymers which have cross-linkable units integrated. After micelle formation the cross-linking is triggered, as explained in the examples in chapter 3.6, resulting in more stable aggregates due to the covalent interconnections. ${ }^{(103)}$ Their application in the field of compound storage began in the 1990s. Nowadays they are known and used media for drug delivery and drug storage.

In 2011 Chang et al. published results of the investigations in thermo-responsive SCMs for drug delivery. Using RAFT polymerisation, they synthesised amphiphilic, linear diblock copolymers from MMA and NIPAam with $N$-acroxysuccinimide as cross-linkable co-monomer. ${ }^{(156)}$ In aqueous solution these polymer chains form micelles. Also in aqueous solution drug loading takes place and afterwards the chemical cross-linking via esterification with ethylenediamine. Taking advantage of the thermo-responds of $p$ NIPAam the drug is set free. The drug-loading capacity and the cumulative drug release where tested with prednisone acetate, a hydrophobic anti-inflammatory drug. At $20^{\circ} \mathrm{C}$ $33 \%$ of the drug is released over 120 hours, increasing temperature accelerates the delivery.

Xu et al., also published in 2011, synthesised thermo-responsive ABC-triblock copolymers from $\alpha$-methoxy(ethyleneoxide), $N$-(3-aminopropyl)methylacrylamide (APMA) and NIPAam via the 
RAFT process in aqueous solution. (157) The pAPMA-block is reversibly cross-linked with terephthaldicarboxaldehyde at a pH-value of 9 and a temperature of $50{ }^{\circ} \mathrm{C}$. A "core-shell-corona" system is gained. By dropping temperature and $\mathrm{pH}$-value the micelle is de-cross-linked. Drug loading and release were tested with prednisolone 21 -acetate by dissolving drug and polymer in water. By changing the $\mathrm{pH}$-value to 9 and heating slowly to $50{ }^{\circ} \mathrm{C}$ the micelles are formed and cross-linking can be carried out. At $50{ }^{\circ} \mathrm{C}$ and dropping the $\mathrm{pH}$-value to $580 \%$ of the drug are released after two hours. Under conditions adapted to the human body $\left(\mathrm{pH} 7.4\right.$ and $\left.37^{\circ} \mathrm{C}\right) 80 \%$ of the drug are released after 10 hours.

\subsection{Nano-Carrier Synthesis via Template-Assisted RAFT Polymerisation}

By the group of Huang and Voit a different approach of establishing nano-scaled hollow particles was published in 2011. ${ }^{(48)}$ Here functionalised silica particles of defined sizes were used as templates to graft block copolymers onto their surface. The whole procedure consists of five steps: The functionalisation of the silica particles with amino silane agents, anchoring RAFT-agents via their R-group onto the surface, a copolymerisation of $t$-butyl methylacrylate with $\mathbf{1 . 2}$, a second polymerisation with 2-hydroxypropyl methacrylamide onto the first block, a photo cross-linking step and the etching of the silica core with a $\mathrm{NH}_{4} \mathrm{~F} / \mathrm{HF}$ buffer. The size of the nano-capsule is defined by the size of the silica particles used which also leads to monodisperse particles. By the choice of the size, the RAFT-agent and the monomers the nano-carriers can be tuned for their purpose. Aimed was a medical application but also the use as catalyst carrier is conceivable.

In 2012 the research group of Sun et al. presented a paper about the synthesis of nanocarriers by means of RAFT polymerisation with silica nanoparticle templates for the controlled release of rhodamine B. Here macroRAFT agents were grafted onto the silica surface and used to built-up temperature-responsive shells from 2-(dimethylamino)ethylmethacrylate as thermosensitive part and 3-dimethylmethacroyloxyethyl(ammonium propanesulfonate) to achieve biocompatibility. ${ }^{(158)}$

\subsection{RAFT-Star Polymer Synthesis}

With the beginning of the new century the interest in star polymer synthesis via RAFT polymerisation grew rapidly. In 2001 one of the first papers regarding this topic was published by Stenzel et al. Here the star polymers were generated via the R-group approach. The disadvantage of this method regarding the star-star-coupling was reported, too. ${ }^{(92)}$ One year later the same group reported about star polymerisation via the Z-approach with a RAFT-agent basing on $\beta$-cyclodextrin. ${ }^{\left({ }^{89}\right)}$ It was found that a broadening of the molecular weight distribution, as found in experiments of the R-approach, is avoided due to the location of all living centres at the core. 
Furthermore, the synthesis of $p$ (vinyl alcohol) star polymers ${ }^{(159)}$ prepared via xanthate based pentaerythritol derivates, as introduced by Mayadunne et al. ${ }^{(96)}$

RAFT-agents from pentaerythritol derivates are the basis of the research in the field of star polymer synthesis in the group of Vana et al. Since 2004 projects were carried out to find a better understanding in polymerisation with multifunctional RAFT agents using the Z-group approach with the long-term goal to establish nano-carriers finding application in medical treatment.

24 different RAFT-agents were synthesised and tested in Sty-, MA-, BA-, dodecyl acrylate (DA)-,VAc-, vinyl propionate (VPr)- and $\mathrm{N}$-acryloylmorpholine (NAM)-polymerisations as well as in copolymerisation with various cross-linkable monomers. ${ }^{(21 ; 160)}$

By Boschmann was found that four-armed xanthates effectively control vinyl ester polymerisations $(P D I=1.2)$ but shielding effects occur at higher conversions in bulk polymerisations that hinder the addition reaction which leads to a reduction in control in these regimes. ${ }^{(93)}$ This was supported by a paper from Stenzel et al. ${ }^{(94)} \mathrm{He}$ also determined kinetic coefficients $k_{\mathrm{tr}}$ for the polymerisations of BA and DA with a hexa-functional RAFT-agent. Polymerisations of these monomers are well-controlled by RAFT-agents with methyl acrylate as R-groups. ${ }^{(47)}$ Furthermore, besides the in section 3.5 .2 explained method to determine the molecular mass of star polymers, ${ }^{(69)}$ investigations on the preequilibrium of RAFT-star-polymerisations via Nuclear Magnetic Resonance (NMR) spectroscopy, Monte Carlo simulations and the apparent number of arms as well as in dependence to the nature of the leaving group have to be taken into his account. ${ }^{(21 ; 90 ; 161)}$

The investigations regarding the pre-equilibrium with respect to the leaving groups were continued by Mänz. ${ }^{(160 ; 161)}$ Furthermore the main target of this dissertation was the establishment of amphiphil Sty-NAM block copolymer stars and the search for an efficient cross-linking method by chemical means. On trial were esterification and click reactions as well as imide formation. Due to problems with the correct stoichiometry and dilution, the chemical cross-linking reactions could not be carried out in a satisfactory way; therefore first few tests in the cross-linking via UV-irradiation were done. A drawback was the lack of characterisation methods to prove the successful formation of nanoparticles besides SEC-analysis. ${ }^{(160)}$ 


\section{Motivation}

Aim of this project was the controlled synthesis of uni-molecular and therefore equal- and nano-sized containers via a simple synthetic pathway applying RAFT chemistry. RAFT was chosen as it stands out because of its versatility regarding the reaction conditions as well as the use of solvents and monomers. ${ }^{\left({ }^{93}\right)}$ Furthermore, the resulting polymer is free from contaminations of metal catalysts. The envisaged goal for these containers is an application for medical purposes as drug delivery and depot units. Also the usage as phase transfer agents, nano-reactors or storage units for catalysts in chemical reactions is conceivable. In this work these carriers ought to be established via RAFT-star polymers and an efficient cross-linking procedure. As described by Schubert et al., star polymers have the same ability to fulfil the above-mentioned applications as nano-carriers from dendrimers. ${ }^{\left({ }^{(6)}\right.}$ For medical purposes, theses assemblies should consist of a hydrophobic core and a hydrophilic corona. Then it would be possible to deliver lipophilic drugs, which would be stored in the hydrophobic core of the carrier, through aqueous media and gain at the same time the necessary stability in the biological environment. ${ }^{(65 ; 98 ; 153)}$

A main drawback of establishing nano-carriers from the self-assembly of linear amphiphilic copolymers, as explained in the previous chapter, is the fragile nature of micellar systems. The micelle formation, the size and form of these assemblies are strongly dependent on different factors regarding the nature of the amphiphil and the surrounding conditions. The $\mathrm{CMC}$ is a characteristic value for the micelle formation of a surfactant type, i.e. a molecule consisting of two phase separable parts. Above the $\mathrm{CMC}$ micelles are formed, below the surfactant molecules are distributed and mainly adsorbed at the air-liquid interface. The CMC depends on the nature of the copolymer and the individual block lengths of both parts. As a result from one set of monomers and defined block length only one kind of micelles can be obtained and there is no possibility to broaden the application margin by introducing other properties via different monomers. Micelle formation is also strongly associated with the surrounding conditions as temperature, $\mathrm{pH}$-value or introduced charges, as outlined in chapter 3.3.1. Small disturbances lead to changes in the system, hence alternation in the micelles or the dissociation of the micelles into unimers are results. ${ }^{(1 ; 100 ; 157)}$ Therefore the combination of a micelle based system with chemical cross-linking is risky. Also it will be difficult to maintain constancy of the procedure in an up-scaled measure which is obligatory for technical processes. 
Contrary to this, the use of star polymers can avoid these problems:

- Firstly, a core-first approach is used. The copolymer arms are synthesised from the core, so they are covalently attached. Even by changing the surrounding conditions the covalent bonding remains.

- As RAFT-agents are versatile compounds, many monomers can be applied and used together to synthesise star block copolymers. That opens up the possibility of tuning the carriers for different applications.

Furthermore, star polymers fulfil all requirements compared to micelles: They are nano-scaled, uniform and monodisperse with a large specific surface area. They have a spherical shape independent from the concentration. ${ }^{(103)}$

In Figure 5-1 the five steps of the in this project used synthesis-pathway to establish nanoscaled hollow spheres basing on star[(A-co-UV)-block-B]-polymers are given. Below the essential tasks arising from these different steps for this thesis are explained.

(1)
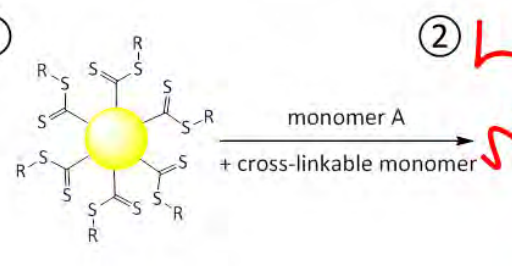

(2)

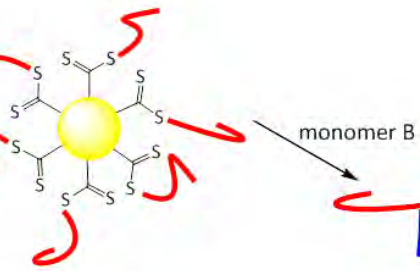

(3)

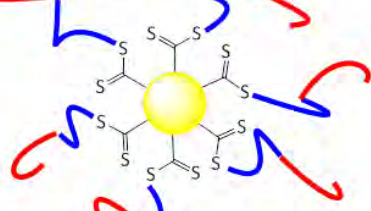

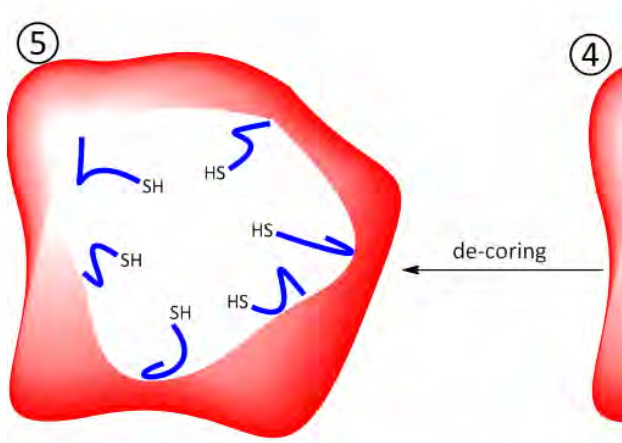

(4)

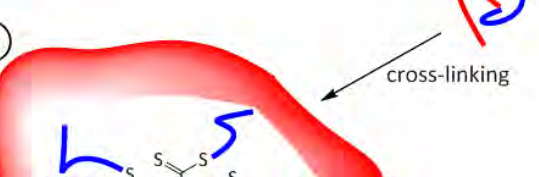

Figure 5-1: Five steps from a multifunctional RAFT-agent to a nano-scaled container. 
1) The multi-functional RAFT-agent has to be synthesised as well as the UV-reactive monomer.

2) A copolymerisation with the multifunctional RAFT agent, a main monomer and the UV-cross linkable monomer is carried out to yield a statistical star copolymer (the red block in Figure 5-1). Here it is important to use the Z-group approach to avoid star-star coupling. The resulting polymer has to be either hydrophobic or hydrophilic. Integration of the UV-sensitive monomer should not alter the properties originating from the main monomer.

3) Onto the first block of polymer (red) a second block (blue) of opposite polarity to achieve an amphiphilic character of the star is synthesised. This second block does not contain any comonomer. As a consequence of the applied Z-group approach the new formed block is the inner part of the nano-carrier, i.e. the storage unit.

4) Under UV-irradiation of appropriate wavelengths the cross-linking reaction is carried out.

5) At last the RAFT-core is decomposed via aminolysis ${ }^{(162)}$ to gain hollow sphered particles and the particle volume is checked by loading with test compounds.

From these different steps arise a multitude of tasks to cope with: New multifunctional RAFTagents with different R- and Z-groups as well as different backbones have to be synthesised and tested in polymerisations with different monomers. The syntheses of a scope of UV-reactive monomers have to be improved and carried out on large scale. These monomers ought to be copolymerised with different monomers. Methods have to be found to determine the UV-monomer share in the copolymer. After successful integration of the UV-sensitive monomer into the star polymer and synthesis of the second polymer block, the cross-linking reaction has to be carried out. For this purpose a selection of UV-lamps are at disposal. Also methods have to be found and tested which enable the characterisation of the star polymers before and after cross-linkage in size and shape. Quantitative determinations can be carried out via NMR spectroscopy, SEC and UV-Vis spectroscopy. As a depicting device Atomic Force Microscopy (AFM) is available. 


\section{Shielding Effects in Polymer-Polymer Reactions}

\section{Styrene Polymerisation under Various Solvent Conditions}

A disadvantage of synthesising star-shaped polymers in a "core-first" fashion, as introduced in chapter 3.5 , is the shielding effect of the reactive centres by surrounding polymer segments, which may lead to a reduced contact probability of these reactive sites. In case of the Z-RAFT polymerisation, applied here, the effect may be especially pronounced: The growing chain has to penetrate the star polymer to undergo the activation-deactivation step with the trithio-unit which is the fundamental step to achieve control in the RAFT polymerisation. Consequently, a loss in reaction control leads to a loss in control over molecular weight and PDI especially at higher monomer conversions. ${ }^{(89 ; 93)}$ This shielding effect was investigated by Zifferer et al. ${ }^{(163 ; 164)}$ Computer simulation methods based on the exact numeration of star- and linear polymer pairs prepared by Monte-Carlo techniques were used to study the influence of the surrounding arms on a contact pair of reactive sites located in the centre of the star and the end of the linear chain. Figure 6-1 illustrates this system.
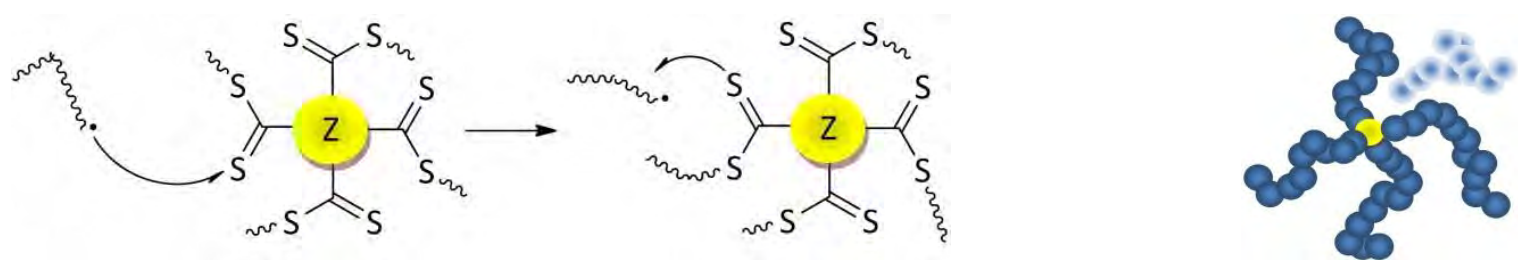

Figure 6-1: Left: Radical attack at the RAFT-unit. Right: Penetration of the growing chain (light blue) through the dormant arms of the star polymer (dark blue).

It was found that the shielding factors, i.e. the contact probability between growing chain and trithiounits at the star's centre, decreases with increasing arm lengths. The influence of the core's size could also be determined: A slight expansion of the core leads to a significantly reduced steric hindrance. ${ }^{(164)}$ Furthermore the number of arms has an impact on this matter. Star polymers with two to six arms were investigated. As expected, the contact probability is lessened with an increasing number of trithio-units at the core, i.e. a larger number of arms. ${ }^{(163)}$ Hence, it was concluded that the addition rate is lowered and the control over molecular weight and PDI is reduced. All these studies were carried out under the assumption of athermal, i.e. good, solvent conditions. Therefore, the shielding effect has been investigated for good and poor solvents via different simulations ranging from athermal to theta conditions. ${ }^{(165)}$ It was found that the shielding factor for theta conditions is much higher and the chain-length dependence of the shielding factor is less pronounced in theta solvents compared to athermal solvents. In conclusion, the addition reaction under theta conditions should be faster than under good solvent conditions and the control over the reaction should be 
increased. To confirm these theoretical data within the course of the present thesis, Z-RAFT polymerisations were carried out with styrene (Sty) and penta-erythritol-tetrakis-(3-(S-benzyltrithiocarbonate))-propanoate $\mathbf{R}_{\mathbf{4}} \mathbf{1}$ (Figure 6-2) as a four-functional RAFT-agent under athermal and theta conditions, using toluene as good and cyclohexane as poor solvent. To achieve ideal reaction conditions in cyclohexane the reaction had to be carried out at or near its theta temperature $\left(37.5^{\circ} \mathrm{C}\right)$. In a theta solvent polymer chains behave like ideal chains, i.e. the energy balance resulting from interactions between polymer segments and solvent molecules is zero. At theta temperature, a critical, solvent-specific temperature, this state is achieved for polymer chains in non-solvents. As there is no initiator which decomposes sufficiently at $37.5^{\circ} \mathrm{C}$, di(2-ethylhexyl)peroxydicarbonate (DEPC) was chosen. It has a half-life of 10 hours at $47^{\circ} \mathrm{C}$, which corresponds to the conditions when using $\mathrm{N}, \mathrm{N}$-azobisisobutylnitrile (AIBN) as initiator at $60{ }^{\circ} \mathrm{C}$ and is therefore suitable for the polymerisation of styrene. It has to be noted that a deviation from the non-solvent character of cyclohexane is created by the here applied ratio of monomer to solvent (3:1) as the monomer itself is of course a good solvent for itself and the resulting polymer.

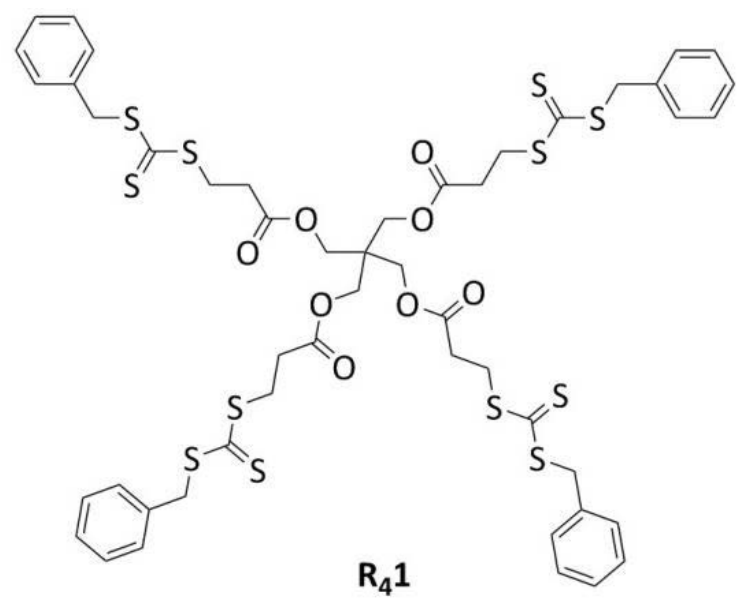

Figure 6-2: Penta-erythritol-tetrakis-(3-(S-benzyl-trithiocarbonate))-propanoate $\boldsymbol{R}_{\mathbf{4}} \mathbf{1}$.

As shown in Figure 6-3, the molecular mass increases linearly with monomer conversion. This demonstrates that both solvent polymerisations proceed, as expected, in a controlled manner. Nevertheless, the PDI-values of the polymerisation in toluene, i.e. under good solvent conditions, are higher than those resulting from the polymerisation in a poor solvent. For higher monomer conversions the PDI-values under theta conditions are at 1.2 which is characteristically for RAFT polymerisations (chapter 3.4). Contrary to this, the scattering of PDI-values above 1.2 in the athermal case indicates a reduction of control. 


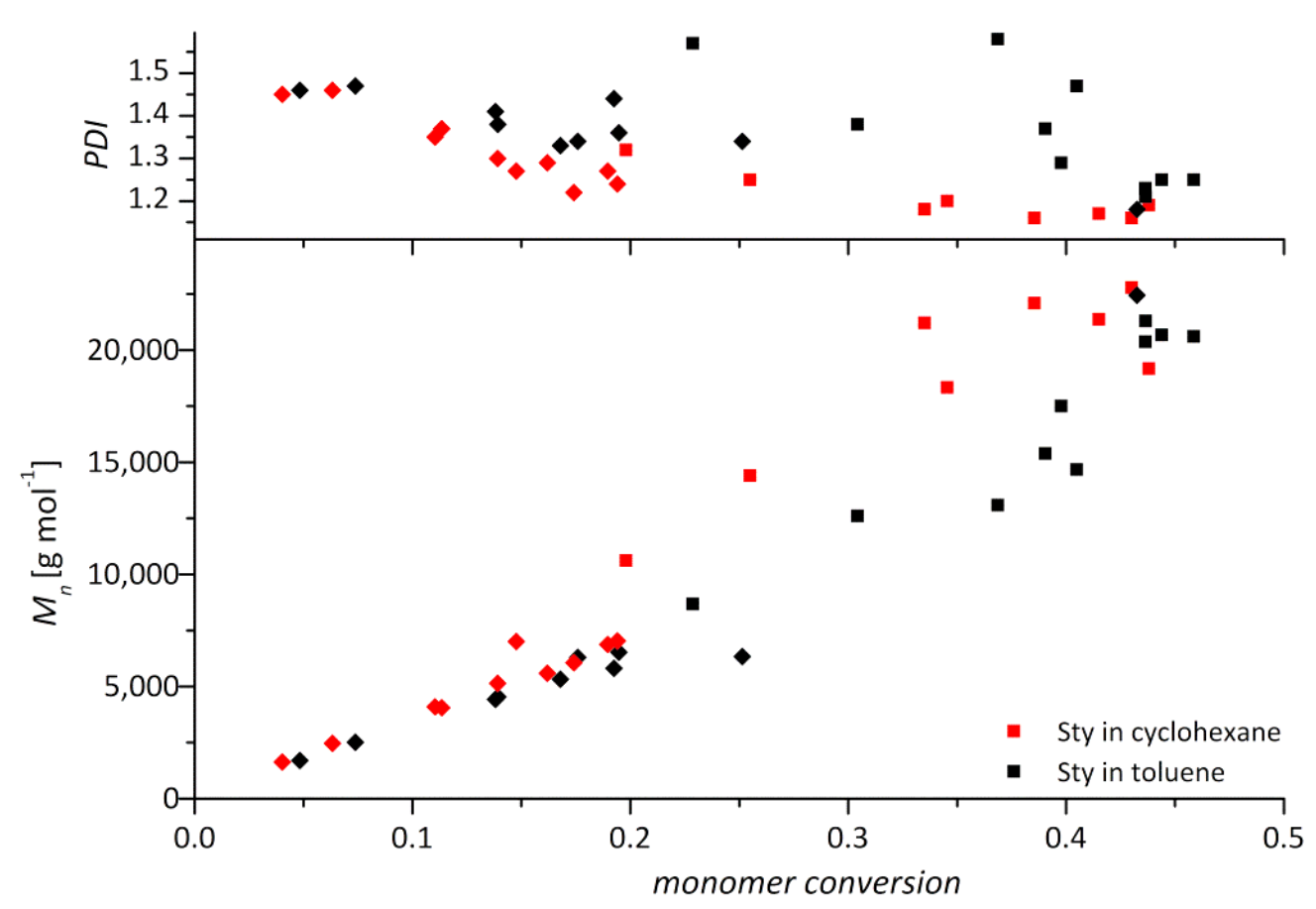

Figure 6-3: Development of the molecular mass and PDI with monomer conversion for solvent polymerisations of Sty in toluene (black) and cyclohexane (red) at $47^{\circ} \mathrm{C}$. Solvent:monomer 3:1, series: squares with $2.5 \mathrm{mmol} \cdot \mathrm{L}^{-1} \boldsymbol{R}_{\mathbf{4}} \mathbf{1}$ and $2.5 \mathrm{mmol} \cdot \mathrm{L}^{-1} D E P C$, diamonds with $5.0 \mathrm{mmol} \cdot \mathrm{L}^{-1} \boldsymbol{R}_{4} \mathbf{1}$ and $1.4 \mathrm{mmol} \cdot \mathrm{L}^{-1} D E P C$.

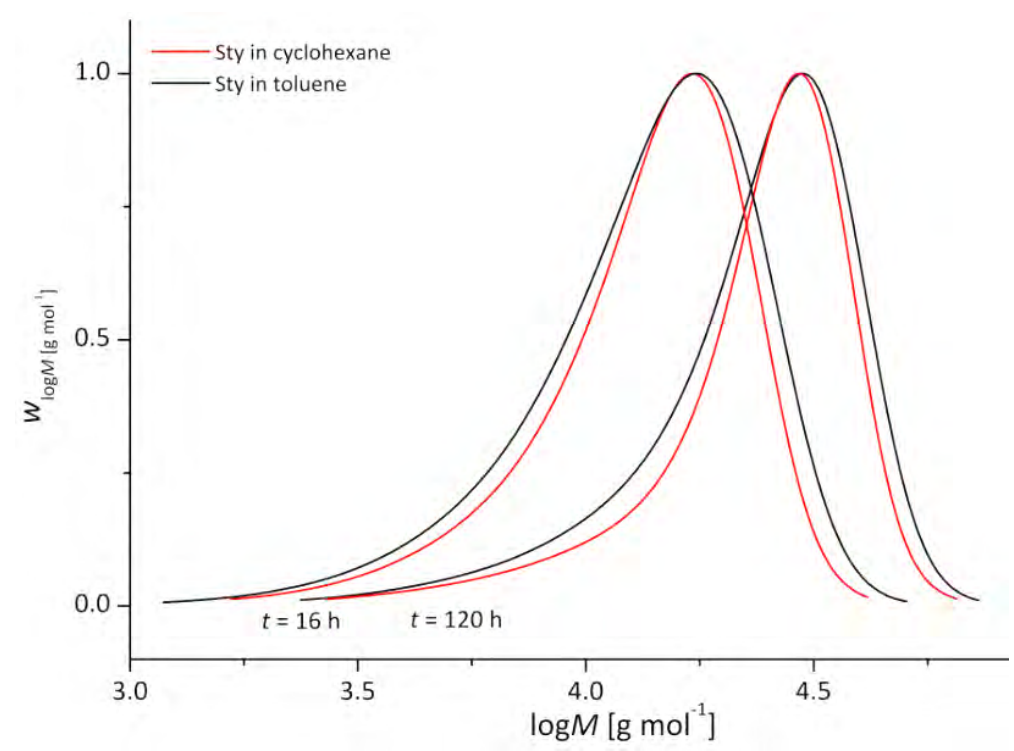

Figure 6-4: Molecular weight distributions of pSty resulting from solvent polymerisations in cyclohexane (red) and toluene (black), $t=16 \mathrm{~h}$ and $20 \%$ monomer conversion, $t=120 \mathrm{~h}$ and $45 \%$ monomer conversion. 
Furthermore, the molecular weight distributions (MWD), shown in Figure 6-4, of two $p$ Sty samples with different monomer conversions illustrate the decrease in polydispersity, i.e. broadness of the curve, with decreasing thermodynamic solvent quality even up to high monomer conversions. This is in agreement with the theoretical predictions. ${ }^{(165)}$ The assumption is reasonable that in the cyclohexane case the monomer and polymer molecules are collected in the region of the star polymer as this is a chemically equal environment in contrary to the solvent itself. Consequently, the growing polymer chains do not need to penetrate the chains surrounding the star as the reactants are spatially close to one another which enables a fast reaction. In case of solvent polymerisation in toluene the solubility is equally good in the entire system. Hence, there is no concentration of reactants in one spot. Diffusion pathways are much longer and the penetration of the growing chain through the polymer shell of the star polymer has a much larger consequence. 


\section{Copolymerisations with UV-Reactive Monomers}

In this chapter the results of the different copolymerisations with the monomers shown in Figure 7-1 are presented. The aim was to find a good match between a basic monomer and a monomer carrying a UV-active unit, i.e. the reaction should take place in a controlled way, in a manageable period of time and should result copolymers with statistically distributed UV-units. The monomers 1.1, 1.2, 2.1, 2.2 and 3.1 were synthesised in accordance to the in chapter 12.2.2 described procedures. As basic monomers butyl acrylate (BA), methyl methacrylate (MMA) and methyl acrylate (MA) as hydrophobic representatives and $\mathrm{N}$-isopropylacrylamide (NIPAam) as a hydrophilic representative were chosen. The acrylic and the methacrylic groups of the UV-monomers should support a good copolymerisation between those UV-monomers and the corresponding basic monomer. ${ }^{(166)}$ Monomer $\mathbf{3 . 2}$ is a commercially available compound and should copolymerise well with NIPAam due to the similarity in their monomeric unit.

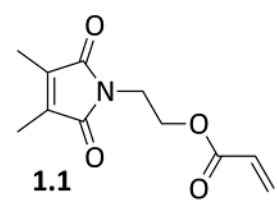<smiles>C=CC(=O)OCCOC(=O)/C=C/c1ccccc1</smiles>

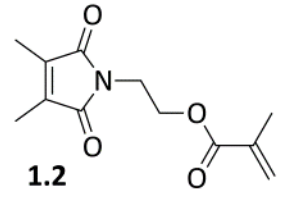<smiles>C=C(C)C(=O)OCCOC(=O)/C=C/c1ccccc1</smiles>
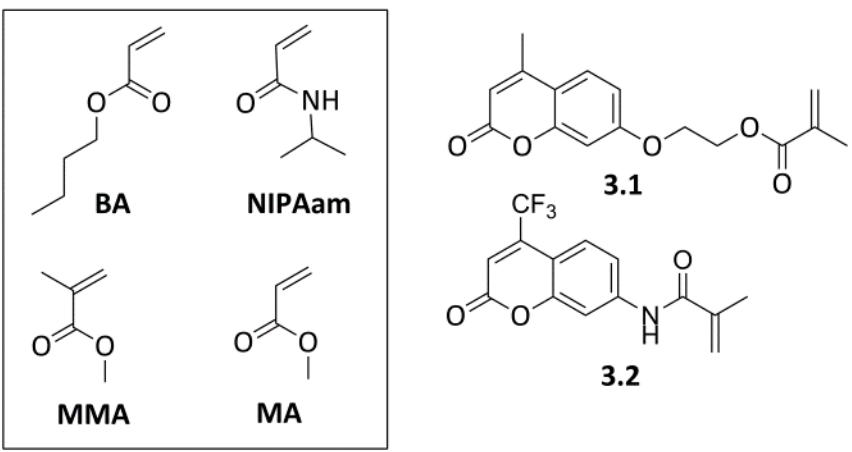

3.2

Figure 7-1: Different monomers carrying a UV-reactive unit to be copolymerised with various monomers.

Literature searches showed that these UV-reactive monomers (Figure 7-1, except of $1.2^{(48)}$ ) have not been used in RAFT-polymerisation of any kind. Therefore, tests were performed to find the best fit in copolymerisations with BA, MMA, MA or NIPAam for an efficient and quick establishing synthesis of polymeric stars. The six-functional RAFT-agents $\mathbf{R}_{6} \mathbf{2}$ and $\mathbf{R}_{\mathbf{6}} \mathbf{3}$ shown in Figure 7-2 along with the corresponding linear RAFT-agents were applied as mediating agents in the various polymerisations. 


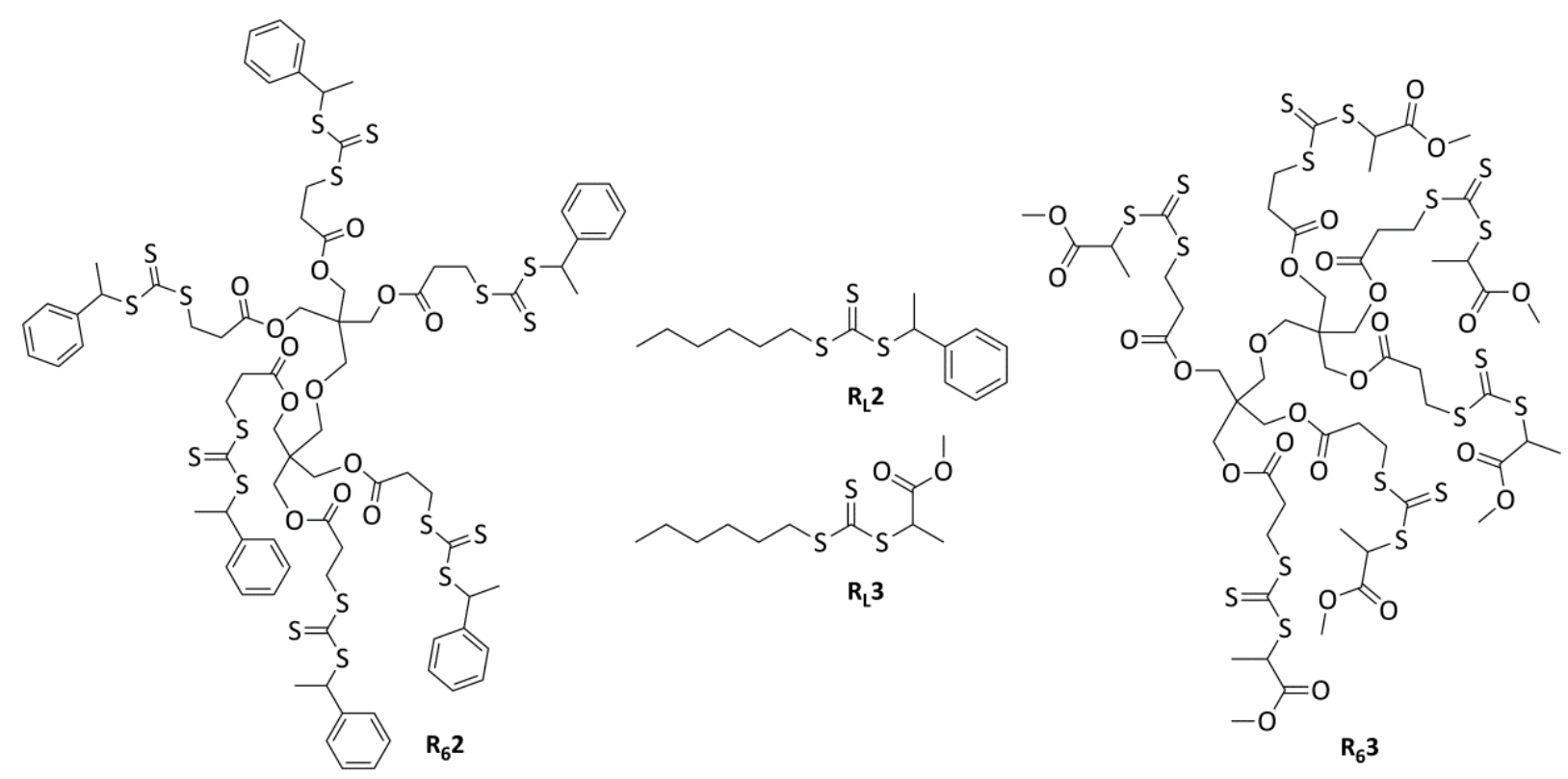

Figure 7-2: Dipentaerythriol-hexakis-(3-(phenylethyltricarbonate))-propanoate $\boldsymbol{R}_{6} \mathbf{2}$, (Phenylethyl)hexyltricarbonate $\boldsymbol{R}_{L} \mathbf{2}$,

Dipentaerythriol-hexakis-(3-(S-methoxopropionate-trithiocarbonate))-propanoate $\boldsymbol{R}_{6} \mathbf{3}$ and

(Methoxopropionate)hexyltricarbonate $\boldsymbol{R}_{\mathbf{L}} \mathbf{3}$.

Besides the investigations into the copolymerisation behaviour of the different monomers, a method had to be found to help determining the amount of co-monomer in the polymer, as this will help to evaluate the effectiveness of the cross-linking step. As options, UV-Vis and NMR spectroscopy will be discussed in chapter 8 . These investigations have to be considered in order to determine the adequate amount of co-monomer in the polymer, regarding a complete closure of the carrier's shell. By introducing a large amount of co-monomer there is the danger of altering the polymer's properties and with that the risk to turn the material ineffective for its purpose. Therefore, it is necessary to find this characteristic concentration of UV-reactive units in the polymer where the properties are not changed and the shell formation is completed. To consider the success of the cross-linking step, UV-Vis spectroscopy as a method for quantitative definition and Atomic Force Microscopy (AFM) as a depicting method should be combined. 


\subsection{Copolymerisations with Dimethylmaleimid-Monomers 1.1 and $\mathbf{1 . 2}$}

The polymerisations can be carried out in bulk or in solution. In the case of these first experiments bulk polymerisations were executed with the hexa-functional RAFT-agent $\mathbf{R}_{\mathbf{6}} \mathbf{2}$ (Figure 7-2). The linear RAFT-agent $\mathbf{R}_{\mathbf{L}} \mathbf{2}$ was added to help determining the molecular mass in accordance to the procedure described in chapter 3.5.2.

\subsubsection{Copolymerisation of BA and $N$-Ethylacrylate-3,4-dimethylmaleimide}

The experiments were all conducted as bulk polymerisations to avoid the influence of additional solvent and to confirm the quality of the copolymerisation between the monomers. It was found that the solubility limit of $\mathbf{1 . 1}$ is $220 \mathrm{mmol} \cdot \mathrm{L}^{-1}$ in $\mathrm{BA}$ at room temperature. Polymerisation mixtures were prepared with

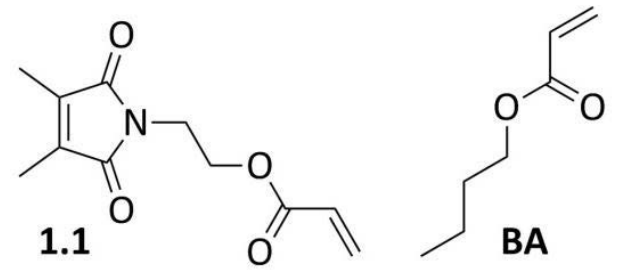
0.5, 1 and 3 mol\% of $\mathbf{1 . 1}$ compared to the initial amount of BA. To compare the polymerisation behaviour, homopolymerisations of BA under the same conditions were performed.

In Figure 7-3 below the development of the molecular weight with the monomer conversion is shown for the copolymerisation of BA with $0.5 \mathrm{~mol} \%$ of 1.1 using $2 \mathrm{mmol} \cdot \mathrm{L}^{-1} \mathrm{AIBN}, 1 \mathrm{mmol} \cdot \mathrm{L}^{-1} \mathbf{R}_{6} \mathbf{2}$ and $3 \mathrm{mmol} \cdot \mathrm{L}^{-1} \mathbf{R}_{\mathbf{L}} \mathbf{2}$ at $60^{\circ} \mathrm{C}$. The upper part of the diagram presents the development of the PDIvalues with monomer conversion for this polymerisation. The black symbols correspond to the SECdata obtained from SEC measurements in tetrahydrofuran (THF) using the UV-Vis detector with a wavelength of $310 \mathrm{~nm}$. In these plots only the experimental data for the star polymer are shown. The red, filled symbols are the resulting molecular weight after correction with the factor $1.52{ }^{(93)}$ and the empty red symbols are the correlated theoretical molecular weight calculated with equation 3-25 and only for star-shaped polymer. 


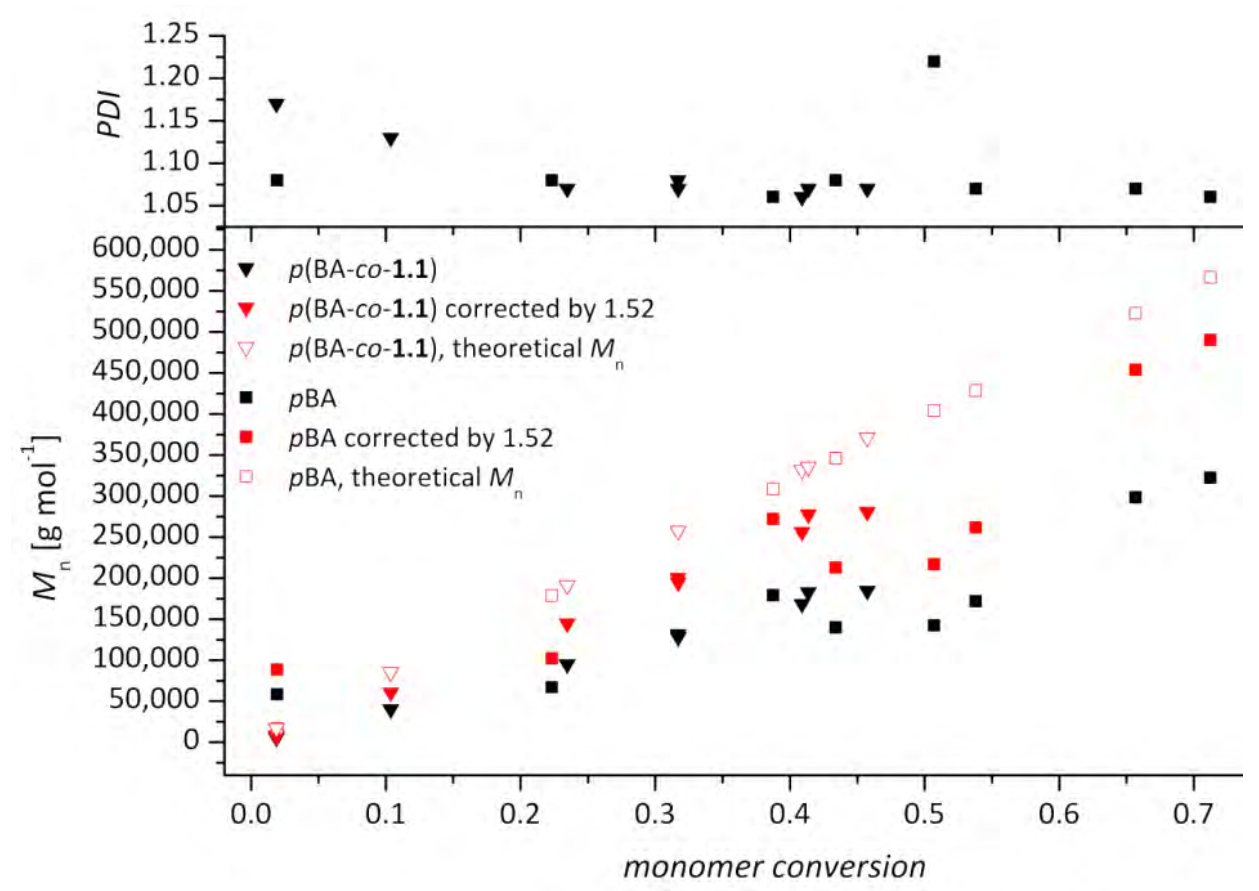

Figure 7-3: Development of molecular mass and PDI with monomer conversion in a homopolymerisation of BA (squares) and a copolymerisation of BA with $0.5 \mathrm{~mol} \% 1.1$ (triangles) using $2 \mathrm{mmol} \cdot \mathrm{L}^{-1} \mathrm{AlBN}, 1 \mathrm{mmol} \cdot \mathrm{L}^{-1} \boldsymbol{R}_{6} \mathbf{2}$ and $3 \mathrm{mmol} \cdot \mathrm{L}^{-1} \boldsymbol{R}_{L} \mathbf{2}$ at $60{ }^{\circ} \mathrm{C}$. Shown is only the data of the star-shaped polymer determined by UV-detection on a THF-SEC.

The developments of the molecular weights confirm a linear increase with monomer conversion. This indicates a controlled behaviour of the polymerisation, as explained in chapter 3.2.3. Due to the addition of the mediating agent, the probability of growing is equal for all chains in the system. Out of that, the chain length is proportional to the monomer conversion which leads to a linear slope in the plot of the molecular weight versus monomer conversion. The corrected values of the molecular weight agree well with the theoretical data. The deviation between both plots results from the determination of the molecular weight via the UV-signal. The UV-detection obtains slightly smaller values as the RI-detection as a consequence of the measuring process. The PDI-values support the observation of controlled reaction behaviour as these are all below 1.2 which is the characteristic value for CRPs (chapter 3.4). Nevertheless it has to be noticed that the copolymerisation yields smaller molecular weights and monomer conversions than the homopolymerisation. 

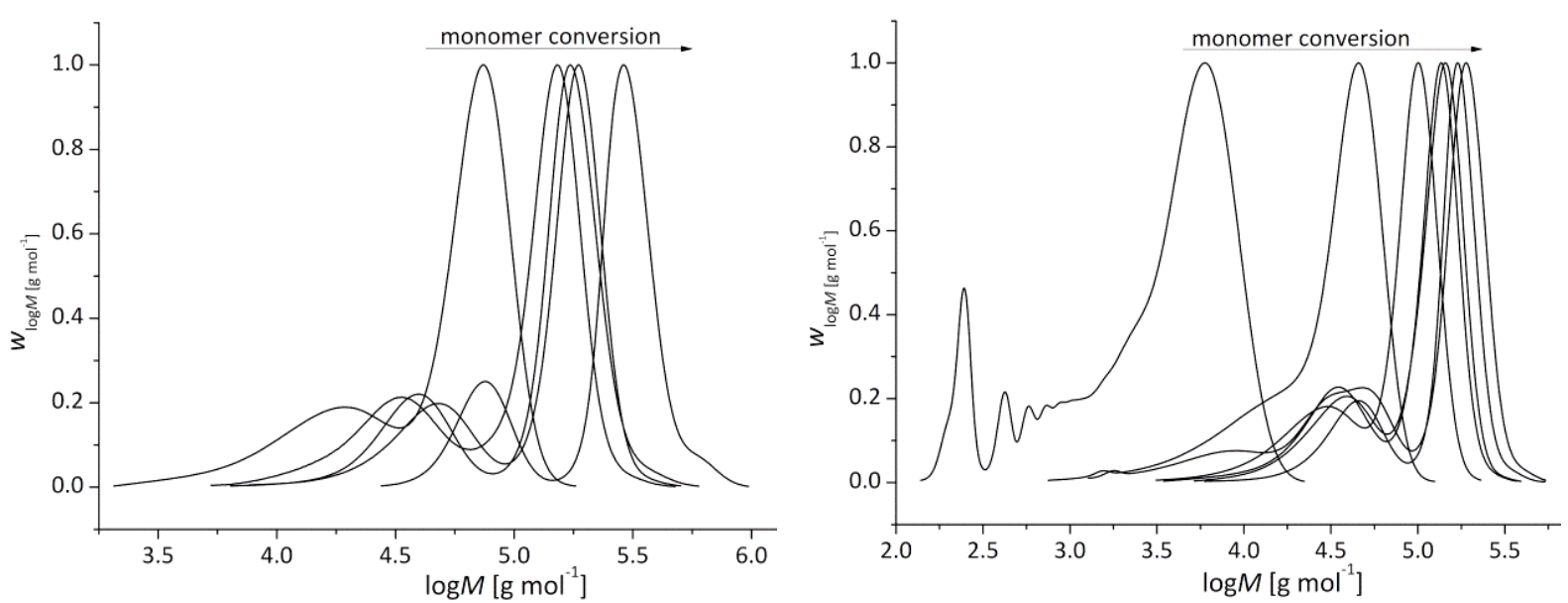

Figure 7-4: SEC-UV-data for a homopolymerisation of BA (left) and a copolymerisation of BA with 0.5 mol\% 1.1 (right) using $2 \mathrm{mmol} \cdot L^{-1} A I B N, 1 \mathrm{mmol} \cdot L^{-1} \boldsymbol{R}_{6} \mathbf{2}$ and $3 \mathrm{mmol} \cdot L^{-1} \boldsymbol{R}_{L} \mathbf{2}$ at $60{ }^{\circ} \mathrm{C}$.

In the corresponding SEC-traces (Figure 7-4) bimodal distributions are observed which result from the addition of the linear RAFT-agent. The intensity of the signal resulting from the linear polymer, i.e. in the regions of smaller molecular weights, is low, compared to the signal of the higher molecular star polymers. It was expected that the difference in intensity between both peak maxima corresponds to a ratio of $1: 2$ as $3 \mathrm{mmol} \cdot \mathrm{L}^{-1}$ linear RAFT-agent and $1 \mathrm{mmol} \cdot \mathrm{L}^{-1}$ six-functional RAFTagent, which equals $6 \mathrm{mmol} \cdot \mathrm{L}^{-1}$ RAFT-units, were applied. It can be assumed that the absorbance of $\mathbf{R}_{\mathbf{6}} \mathbf{2}$ is higher than expected due to spatial proximity of the trithio groups and free electron pairs of the oxygen atoms. In chapter 8 this phenomenon will be discussed further. From the first SEC-curve of the copolymerisation in Figure 7-4 (right) the initial step of the reaction can be observed through the multitude of peaks in the regime of smaller molecular weights. These different signals result from stars in various states of growing. The initial step takes some time until the RAFT-equilibrium is adjusted. Afterwards the expected bimodal distribution is found. The SEC-curves support the statement of controlled reaction behaviour as the increase of the molecular weights with monomer conversion is shown. If these data are compared with a FRP of BA with 1.1, as expected, the difference is observed easily. In the Figure 7-5 the growth of the molecular weight as well as the PDIvalues of this reaction are plotted versus the monomer conversion for a copolymerisation of BA with $1 \mathrm{~mol} \% 1.1$ using $0.9 \mathrm{mmol} \cdot \mathrm{L}^{-1} \mathrm{AIBN}$ at $60^{\circ} \mathrm{C}$. Contrary to the RAFT-polymerisation shown above no linear increase in the molecular weights is observable and the PDI-values are comparatively high. 


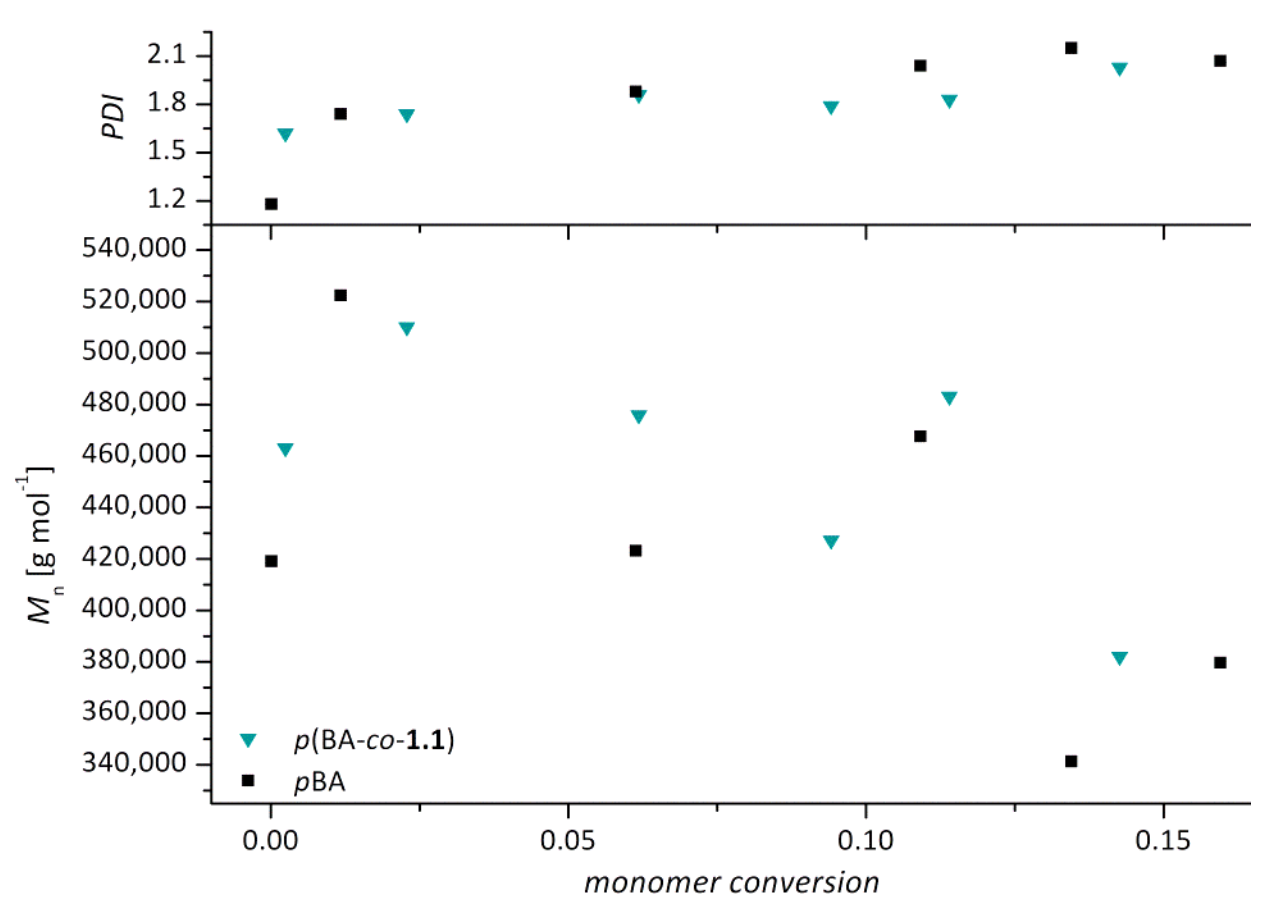

Figure 7-5: Development of the molecular weight and the PDI, determined by RI-detection on a THF-SEC, versus the monomer conversion of a FRP of BA (black) and BA with $1 \mathrm{~mol} \% 1.1$ (aqua) using $0.9 \mathrm{mmol} \cdot \mathrm{L}^{-1} \mathrm{AlBN}$ at $60^{\circ} \mathrm{C}$.
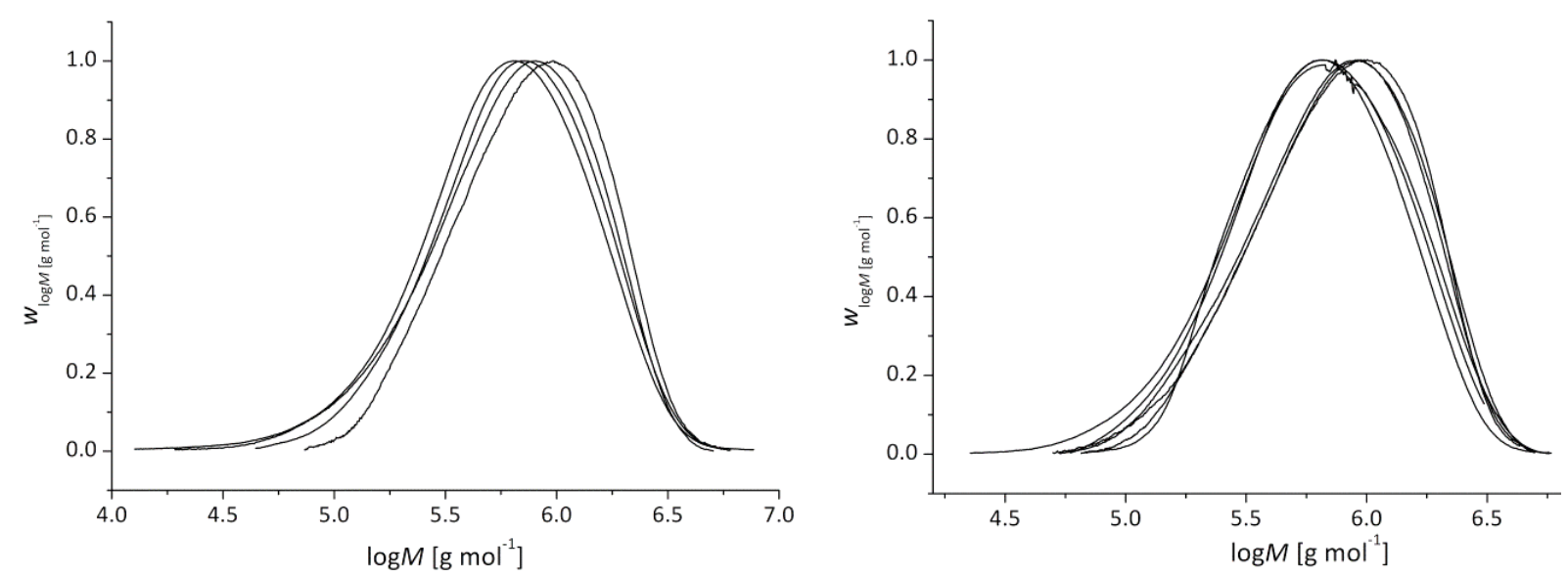

Figure 7-6: SEC-RI-data of a FRP of BA (left, sample-order: 4-2-1-5) and BA with 1 mol\% 1.1 (right) using $0.9 \mathrm{mmol} \cdot \mathrm{L}^{-1}$ AIBN at $60^{\circ} \mathrm{C}$.

Furthermore, the SEC-data (Figure 7-6) show no significant difference in the molecular weights of the polymers. For the FRP of BA without any additional co-monomer (left) a development could be suspected but the first shown spectrum belongs to the aliquot with the reaction time of 60 minutes, followed by 35 minutes, then 20 minutes and the spectrum with the highest molecular weight corresponds with 70 minutes of reaction time. The difference between them is negligible small. 
The following figures present the developments of molecular weight and PDI with monomer conversion and the SEC-curves for the copolymerisations of BA and 1 mol\% of $\mathbf{1 . 1}$.

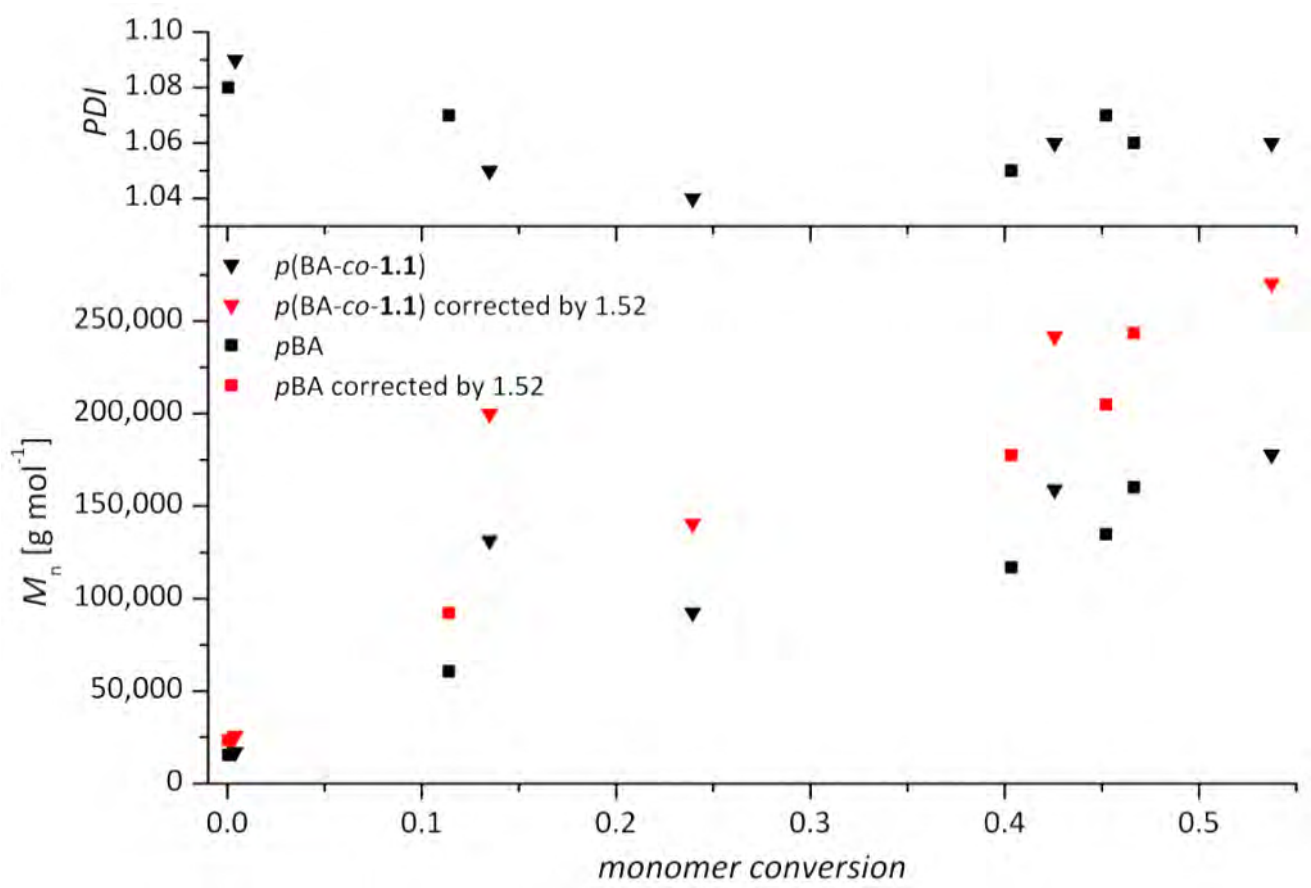

Figure 7-7: Development of molecular mass and PDI with monomer conversion in a homopolymerisation of BA (squares) and a copolymerisation of BA with $1 \mathrm{~mol} \% 1.1$ (triangles) using $2 \mathrm{mmol} \cdot \mathrm{L}^{-1} \mathrm{AlBN}, 1 \mathrm{mmol} \cdot \mathrm{L}^{-1} \boldsymbol{R}_{6} \mathbf{2}$ and $13 \mathrm{mmol} \cdot \mathrm{L}^{-1} \boldsymbol{R}_{L} \mathbf{2}$ at $60^{\circ} \mathrm{C}$. Shown are only the data of the star-shaped polymer determined by UV-detection on a THF-SEC.
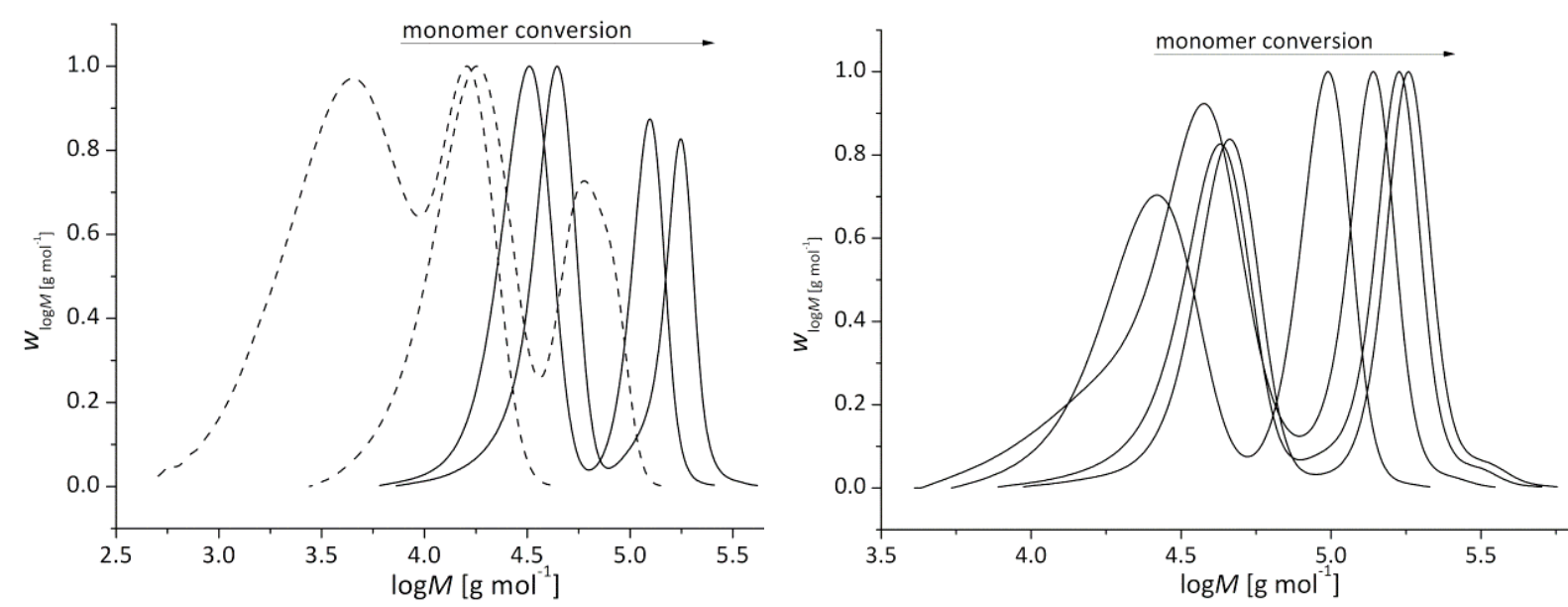

Figure 7-8: SEC-UV-data for a homopolymerisation of BA (left) and a copolymerisation of BA with 1 mol\% 1.1 (right) using $2 \mathrm{mmol} \cdot \mathrm{L}^{-1} \mathrm{AlBN}, 1 \mathrm{mmol} \cdot \mathrm{L}^{-1} \boldsymbol{R}_{6} \mathbf{2}$ and $13 \mathrm{mmol} \cdot \mathrm{L}^{-1} \boldsymbol{R}_{L} \mathbf{2}$ at $60{ }^{\circ} \mathrm{C}$.

In general, a linear increase in the growth of the molecular weight and PDI-values below 1.10 were observed, as in the case of the copolymerisation with 0.5 mol\% 1.1 (Figure 7-7). The SEC-curves support those findings as the increase of the molecular weight with monomer conversion is shown 
(Figure 7-8). Due to the higher concentration of the linear RAFT-agent the signal resulting from the linear polymer is nearly as intense as the signal of the star polymer.

With the exception of the following copolymerisation with $3 \mathrm{~mol} \%$ of $\mathbf{1 . 1}$, the polymerisations of BA and $\mathbf{1 . 1}$ by means of RAFT polymerisation proceed well controlled as expected. In the polymerisation with the high co-monomer concentration a strong, non-linear increase in molecular weight is found at small monomer conversions (Figure 7-9).

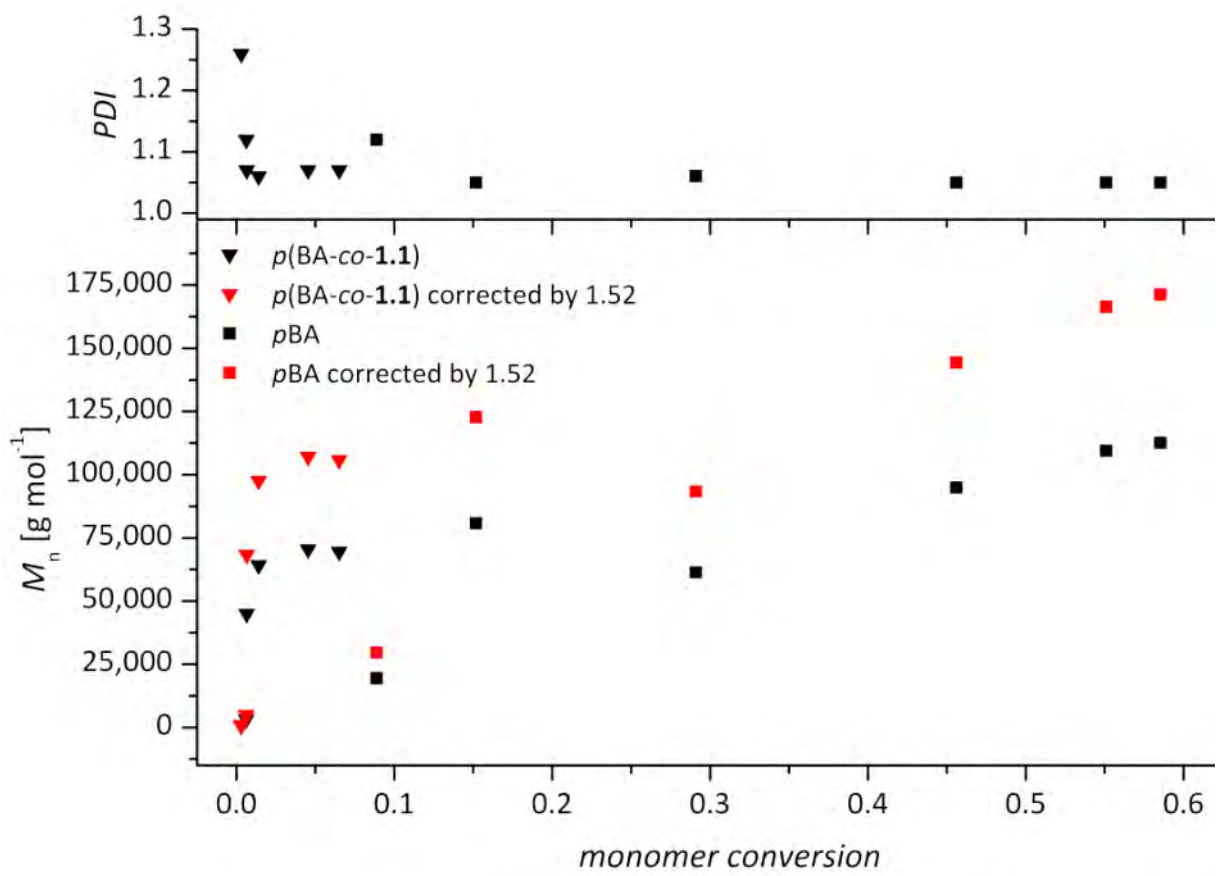

Figure 7-9: Development of molecular mass and PDI with monomer conversion in a homopolymerisation of BA (squares) and a copolymerisation of BA with $3 \mathrm{~mol} \% 1.1$ (triangles) using $2 \mathrm{mmol} \cdot \mathrm{L}^{-1} \mathrm{AlBN}, 3 \mathrm{mmol} \cdot \mathrm{L}^{-1} \boldsymbol{R}_{6} \mathbf{2}$ and $4 \mathrm{mmol} \cdot \mathrm{L}^{-1} \boldsymbol{R}_{L} \mathbf{2}$ at $60^{\circ} \mathrm{C}$. Shown are only the data of the star-shaped polymer determined by UV-detection on a THF-SEC. 

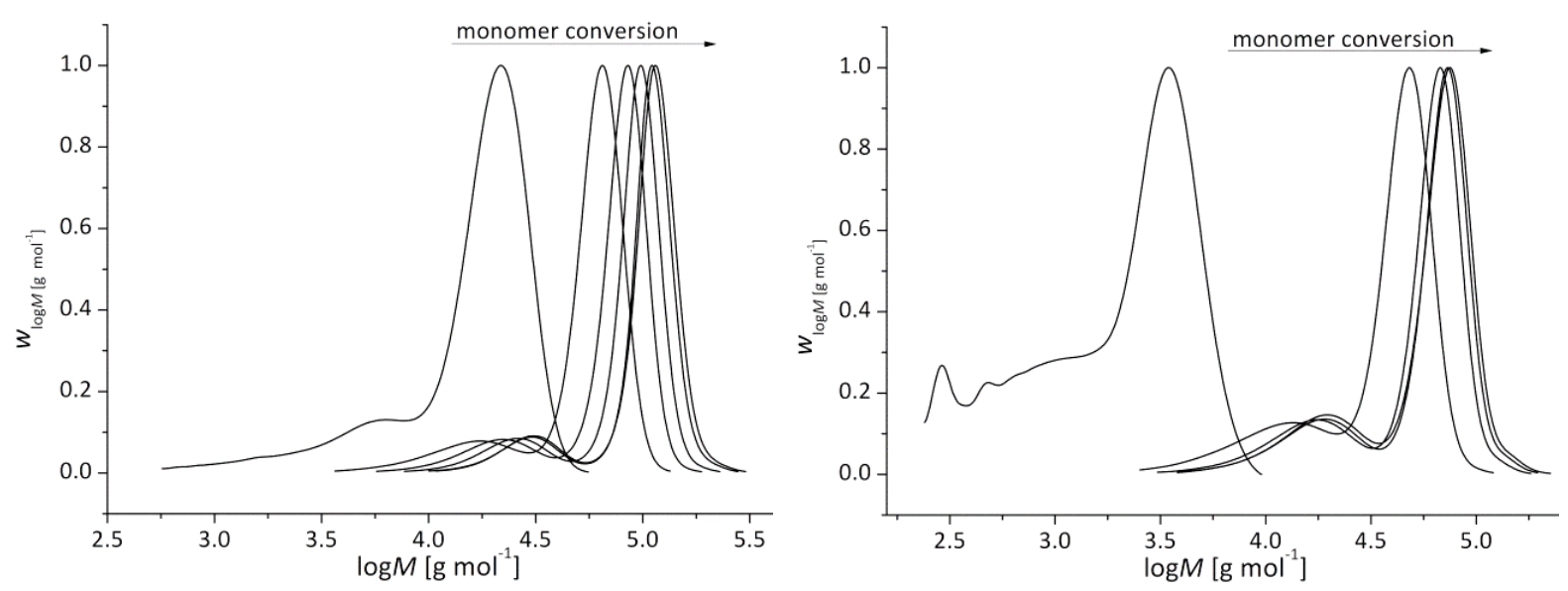

Figure 7-10: SEC-UV-data for the homopolymerisation of BA (left) and the copolymerisation of BA with 3 mol\% 1.1 (right) using $2 \mathrm{mmol} \cdot \mathrm{L}^{-1} \mathrm{AIBN}, 3 \mathrm{mmol} \cdot \mathrm{L}^{-1} \boldsymbol{R}_{6} \mathbf{2}$ and $4 \mathrm{mmol} \cdot \mathrm{L}^{-1} \boldsymbol{R}_{L} \mathbf{2}$ at $60^{\circ} \mathrm{C}$.

The strong increase in the molecular weights at very small monomer conversions is observed in the copolymerisation with 3 mol\% 1.1 which stands against a controlled behaviour of this reaction (Figures 7-9 and 7-10). The decrease of the PDI-values with increasing monomer conversion indicates that the controlling equilibrium was established after an initial period which is excelled by strong hybrid behaviour (chapter 3.2.3). To verify whether the polymerisation proceeds in a controlled manner, the plot of monomer conversion and the plot of molecular mass versus time (Figure 7-11) are shown.

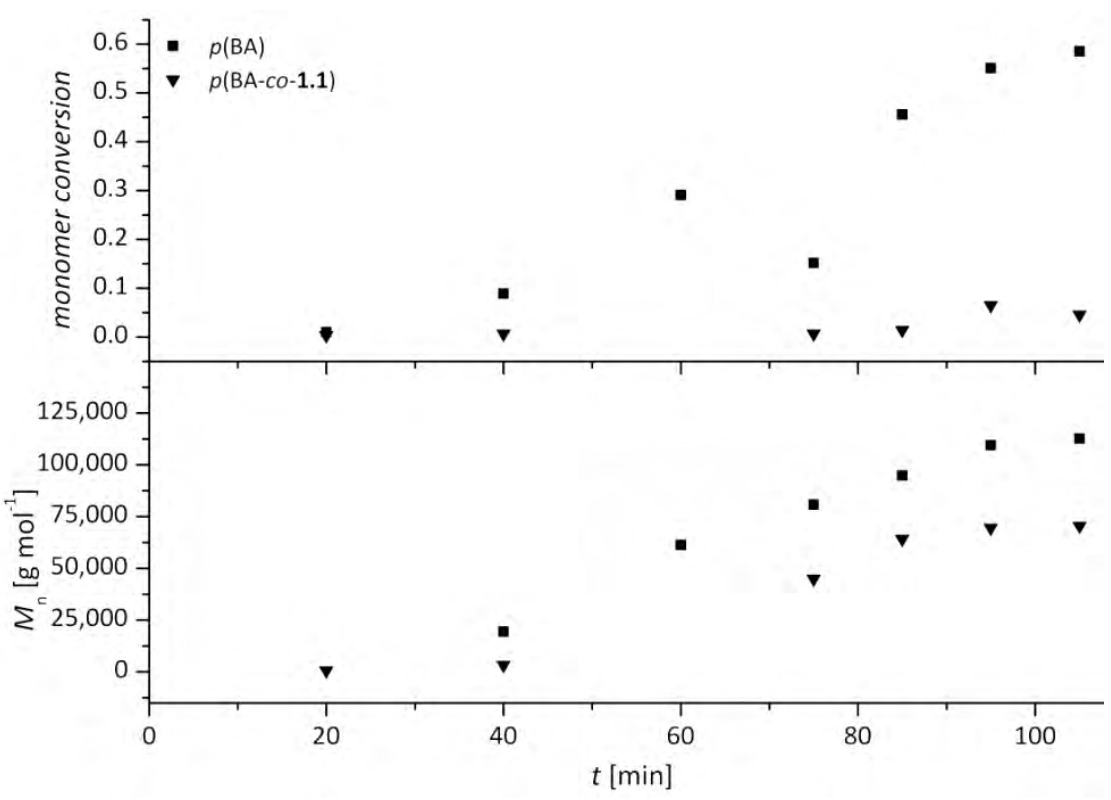

Figure 7-11: Development of monomer conversion and molecular weight (only star polymer) with reaction time in a homopolymerisation of BA (squares) and a copolymerisation of BA with $3 \mathrm{~mol} \% 1.1$ (triangles) using $2 \mathrm{mmol} \cdot \mathrm{L}^{-1} \mathrm{AlBN}$, $3 \mathrm{mmol} \cdot \mathrm{L}^{-1} \boldsymbol{R}_{6} \mathbf{2}$ and $4 \mathrm{mmol} \cdot \mathrm{L}^{-1} \boldsymbol{R}_{L} \mathbf{2}$ at $60^{\circ} \mathrm{C}$. 
The development of the molecular weight with reaction time proceeds in a linear fashion which supports the assumption of controlled reaction behaviour. Nevertheless these high molecular weights are obtained for very small monomer conversions, which indicate a minimisation of control in this reaction. As mentioned for the copolymerisations with 0.5 and 1 mol\% 1.1, a delay in the start of the copolymerisation compared to the homopolymerisation is observed. In the case that the delay is dependent on the co-monomer concentration, it can be suspected that due to the high load of comonomer a critical co-monomer concentration is passed and the polymerisation does not reach the RAFT-equilibrium. This problem has to be investigated further and the polymerisation with high comonomer concentrations has to be improved.

From the first two polymerisations, it can be concluded that with the addition of $\mathbf{1 . 1}$ the controlled character of the reaction remains. However, the addition of $\mathbf{1 . 1}$ influences the reaction. This effect will be discussed in detail in the upcoming chapter 8 .

From these first experiments it can be concluded that the copolymerisation of BA and $\mathbf{1 . 1}$ by means of RAFT-polymerisation works sufficiently. The reaction takes place in an adequate period of time and can therefore be applied as a pathway to integrate the cross-linking units in the star polymer even on large scale. Polymer samples with narrow-distributed MWDs $(P D I<1.3)$ and molecular weights up to $250,000 \mathrm{~g} \cdot \mathrm{mol}^{-1}$ could be synthesised. Detailed investigations regarding the improvement of the polymerisation by addition of solvents is to follow. Furthermore the effect of different amounts of $\mathbf{1 . 1}$ in the polymerisation mixture has to be examined to reveal the connection between amount of $\mathbf{1 . 1}$ in the mixture and a sufficient number of UV-units for shell formation in the resulting polymer. 


\subsubsection{Copolymerisation of MMA with Dimethylmaleimid-Monomers 1.1 and 1.2}

In addition to BA, MMA was tested in the copolymerisation with 1.1 and $\mathbf{1 . 2}$ using the RAFTagents $\mathbf{R}_{6} \mathbf{2}$ and $\mathbf{R}_{\mathbf{L}} \mathbf{2}$, mentioned before, as well as $\mathbf{R}_{\mathbf{6}} \mathbf{3}$ and the corresponding linear RAFT-agent $\mathbf{R}_{\mathrm{L}} \mathbf{3}$ (Figure 7-12) which should work well in polymerisations of those monomers due to the similarity of the leaving groups and the monomer. ${ }^{(21)}$ The methyl acrylate moiety as leaving group guarantees a good reinitiation for acrylate and methacrylate polymerisations. ${ }^{(47)}$ As in the experiments for BA and 1.1 the polymerisations were conducted in bulk at $60^{\circ} \mathrm{C}$.

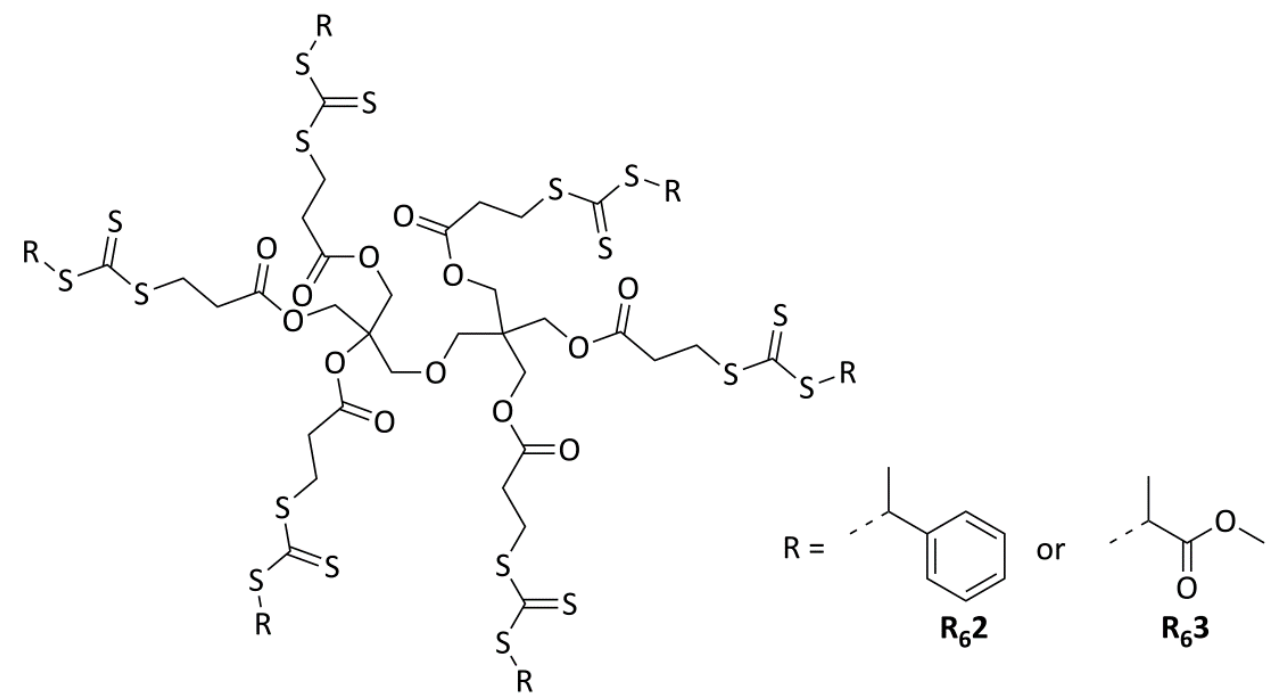

Figure 7-12: Dipentaerythriol-hexakis-(3-(phenylethyltricarbonate))-propanoate $\boldsymbol{R}_{6} \mathbf{2}$ and Dipentaerythriol-hexakis-(3-

(S-methoxopropionate-trithiocarbonate))-propanoate $\boldsymbol{R}_{6} \mathbf{3}$.

\section{Methylmethacrylate with 1.1}

The SEC-UV-spectra of the different MMA-polymer samples, synthesised with different amounts of RAFT-agent, AIBN and 1.1, where applied, do not show any absorption in regions of the polymer signals. This indicates that there are no RAFT-units integrated as the trithio units of the RAFT-agents have their UVabsorption maximum at the wavelength applied $(\lambda=310 \mathrm{~nm})$. (167) High molecular weight polymer could only be distinguished<smiles>C=C(C)C(=O)OC</smiles>

MMA<smiles>C=CC(=O)OCCN1C(=O)C(C)=C(C)C1=O</smiles>

by the detection of the refractive index. Therefore, it can be assumed that the polymerisation takes place in an uncontrolled way. The monomer conversions were low and could not be determined as the weight difference, after removal of the monomer, was within the range of the tolerance of the balance. To summarise these findings, the growth of molecular weight versus reaction time (Figure 7-13) and the SEC-spectra (Figure 7-14) of one experiment are shown here. 


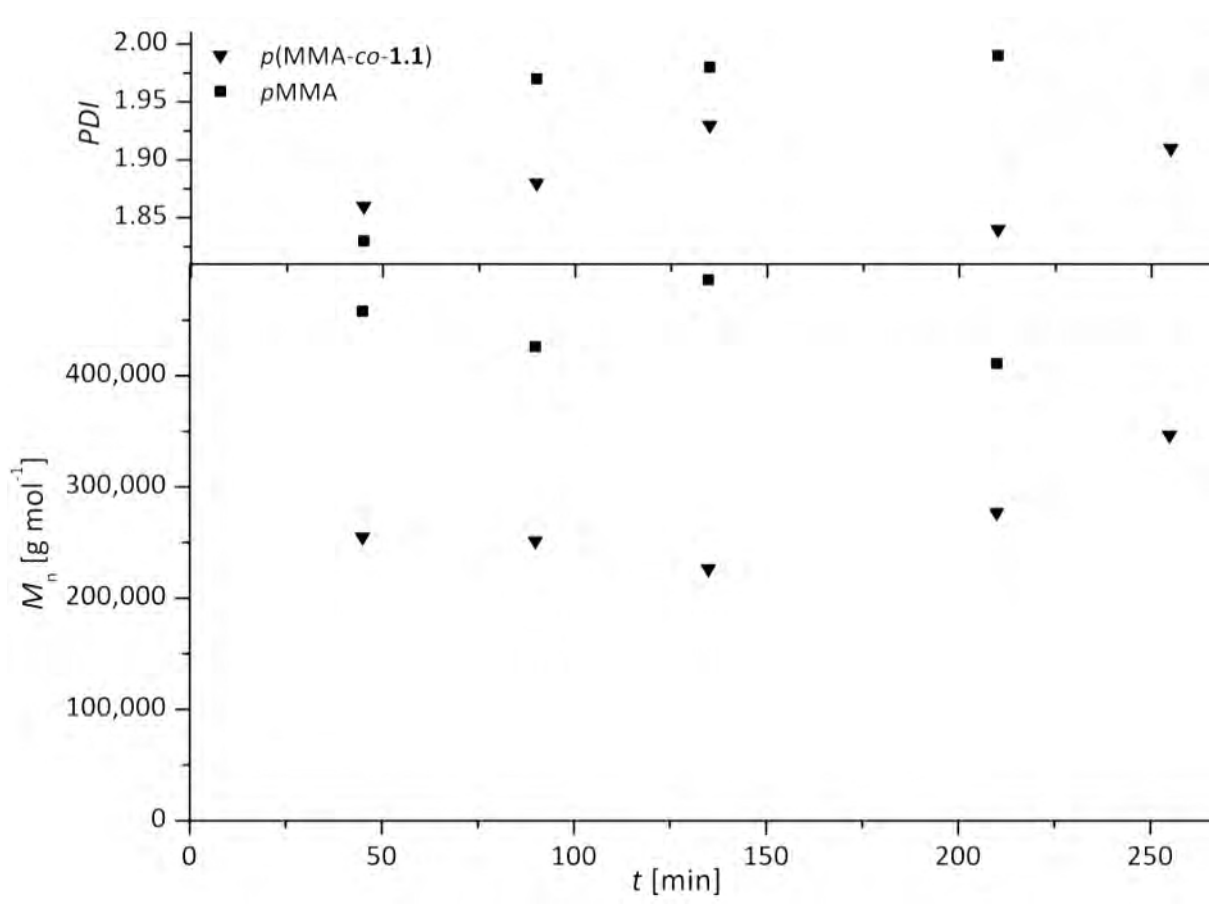

Figure 7-13: Development of molecular weight and PDI, determined by RI-detection on a THF-SEC, with time of a MMAhomopolymerisation (squares) and a copolymerisation of MMA with $1 \mathrm{~mol} \% 1.1$ (triangles) using $20 \mathrm{mmol} \cdot \mathrm{L}^{-1} \mathrm{AlBN}$, $1 \mathrm{mmol} \cdot \mathrm{L}^{-1} \boldsymbol{R}_{6} \mathbf{3}$ and $10 \mathrm{mmol} \cdot \mathrm{L}^{-1} \boldsymbol{R}_{L} \mathbf{3}$ at $60^{\circ} \mathrm{C}$.

Both the plots of molecular weight against reaction time (Figure 7-13) illustrate no linear increase as it would be expected. The resulting molecular weights are high though the monomer conversion is nearly zero. As the conversions were too low to measure, a complete consumption of monomer can be excluded. Hence, the plateau found in the plot cannot be a result of full monomer consumption. In FRP the molecular weight of the polymer increases abrupt to a maximum value and does not change much until all monomer is consumed. Also the PDI-values between 1.85 and 2.00 verify that the polymerisation takes place in an uncontrolled manner, as described in chapter 3.1. 

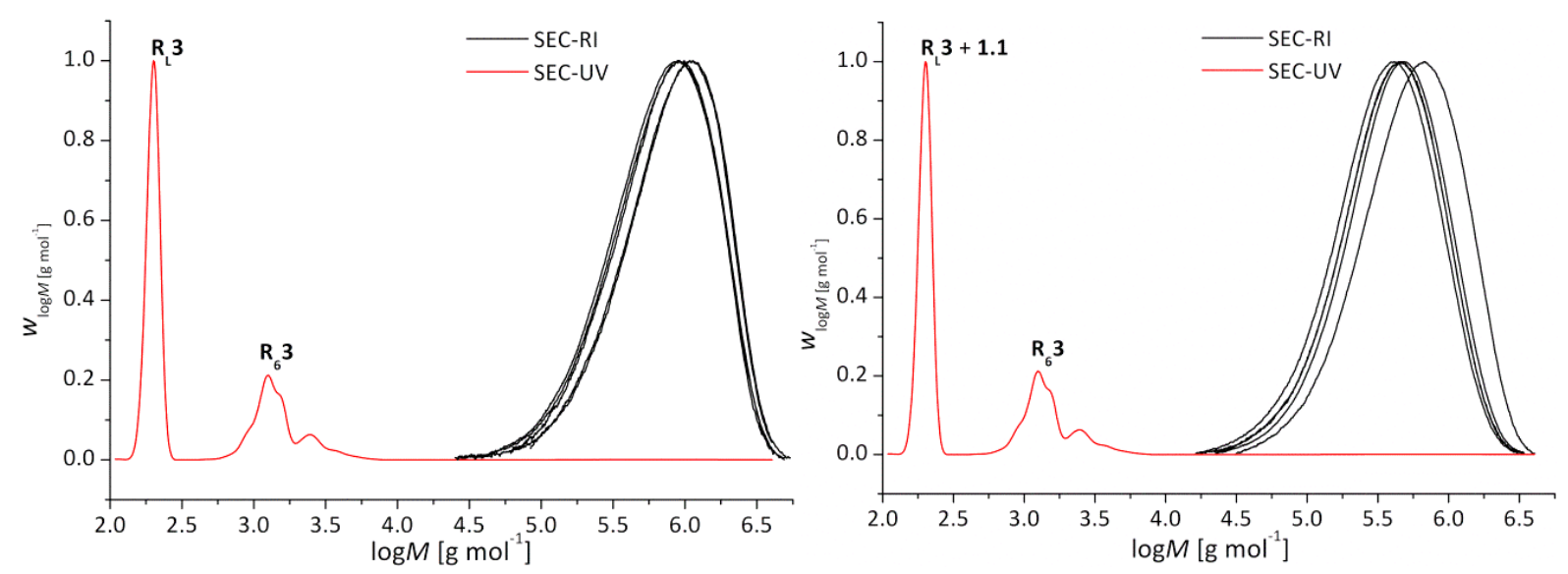

Figure 7-14: SEC-RI-data for the homopolymerisation of MMA (left) and the copolymerisation of MMA with 1 mol\% 1.1 (right) using $20 \mathrm{mmol} \cdot \mathrm{L}^{-1} \mathrm{AIBN}, 1 \mathrm{mmol} \cdot \mathrm{L}^{-1} \boldsymbol{R}_{6} 3$ and $10 \mathrm{mmol} \cdot \mathrm{L}^{-1} \boldsymbol{R}_{L} 3$ at $60^{\circ} \mathrm{C}$; in red the detected UV-signal.

The SEC-curves support the mentioned findings. The two UV-signals, shown in red in Figure 7-14, correspond to the weight of the six-armed RAFT-agent as well as the linear RAFT-agent and the UVmonomer as they have approximately the same molecular mass. The molecular weight distributions obtained by detection of the refractive index (black) show no change with time. Therefore it can be recorded that the polymerisations of MMA with and without co-monomer mediated by $\mathbf{R}_{6} \mathbf{3}$ does not work at all which is surprising as MMA is known to polymerise under RAFT-conditions. ${ }^{(27-29)}$

\section{Methylmethacrylate with 1.2}

Contrary to the finding in the MMA-1.1-copolymerisation, UV-absorbance of the polymer sample resulting from the copolymerisation of MMA and $\mathbf{1 . 2}$ in the SEC-measurement could be observed. However, the signal is noisy and low in intensity. The comparison with the recorded RI-signal with the UV-signal is shown in Figure 7-16 (left). A large difference can be seen, when comparing with an analogous copolymerisation of BA and $\mathbf{1 . 1}$<smiles>C=C(C)C(=O)OC</smiles>

MMA<smiles>C=C(C)C(=O)OCCN1C(=O)C(C)=C(C)C1=O</smiles>

(Figure 7-16, right). The amount of UV-active units originating from RAFT-agents and UV-monomers is the same in both reaction mixtures. The absorbance resulting from the RAFT-agents $\left(6 \cdot 1 \mathrm{mmol} \cdot \mathrm{L}^{-1}\right.$ RAFT-units resulting from $\mathbf{R}_{6} \mathbf{2}$ and $9 \mathrm{mmol} \cdot \mathrm{L}^{-1} \mathbf{R}_{\mathbf{L}} \mathbf{2}$ ) gives a strong signal, as can be seen in the spectrum of the BA-1.1-copolymerisation (Figure 7-16). In the spectrum of the MMA-1.2copolymerisation (Figure 7-16) this signal is missing completely. It can be concluded that the occurring UV-absorbance results only from the statistically distributed RAFT-agent in the polymer which is generated under FRP-conditions as the co-monomer does not show any absorbance at this wavelength (UV-Vis spectra see chapter 8.2). 


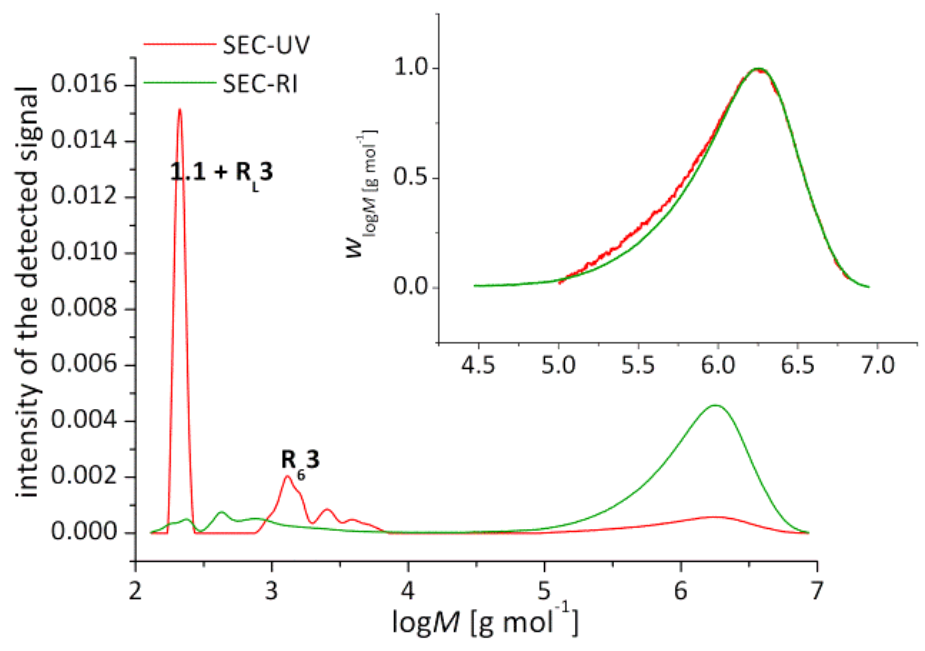

Figure 7-15: Comparison of the SEC-UV- and SEC-RI-data of the sample with the highest conversion and highest molecular weight from a copolymerisation of MMA with $1 \mathrm{~mol} \% 1.2$ using $2 \mathrm{mmol} \cdot \mathrm{L}^{-1} \mathrm{AlBN}, 1 \mathrm{mmol} \cdot \mathrm{L}^{-1} \boldsymbol{R}_{6} 3$ and $10 \mathrm{mmol} \cdot \mathrm{L}^{-1} \boldsymbol{R}_{L} 3$ at $60{ }^{\circ} \mathrm{C}$

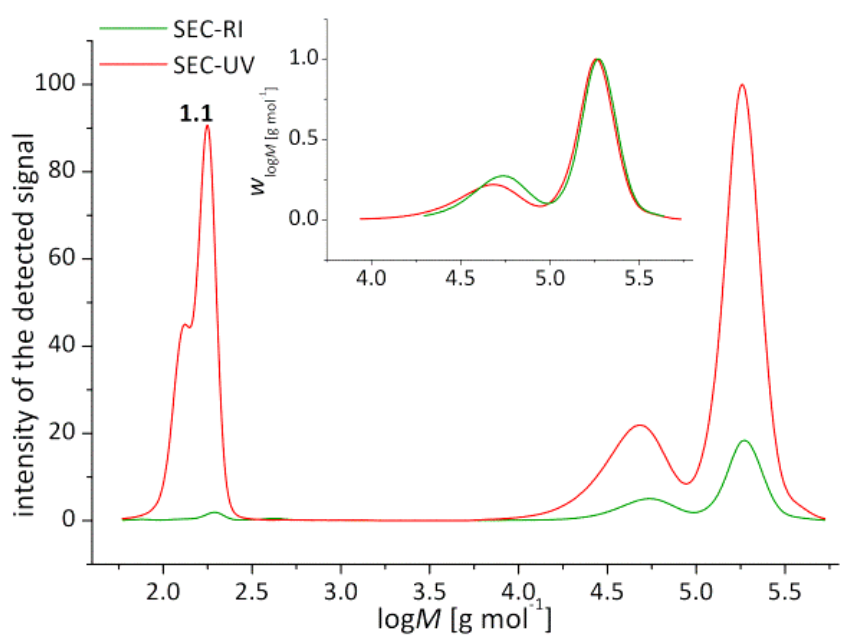

Figure 7-16: Comparison of the SEC-UV-and SEC-RI-data of the sample with the highest conversion and highest molecular weight from a copolymerisation of BA with $1 \mathrm{~mol} \% 1.1$ using $2 \mathrm{mmol} \cdot \mathrm{L}^{-1} \mathrm{AlBN}, 1 \mathrm{mmol} \cdot \mathrm{L}^{-1} \boldsymbol{R}_{6} \mathbf{2}$ and $9 \mathrm{mmol} \cdot \mathrm{L}^{-1} \boldsymbol{R}_{L} \mathbf{2}$ at $60{ }^{\circ} \mathrm{C}$

As in the previous examples, the peaks in the SEC-UV-spectrum corresponding to the substances of lower molecular weight (RAFT-agents, 1.2) could be detected. The plot of the molecular weight and PDI versus the monomer conversion (Figure 7-17) and the MWDs (Figure 7-18; samples from left to right: 3-2-1-4) prove a FRP-character of the polymerisation which is caused by the missing controlling units in the polymer. 


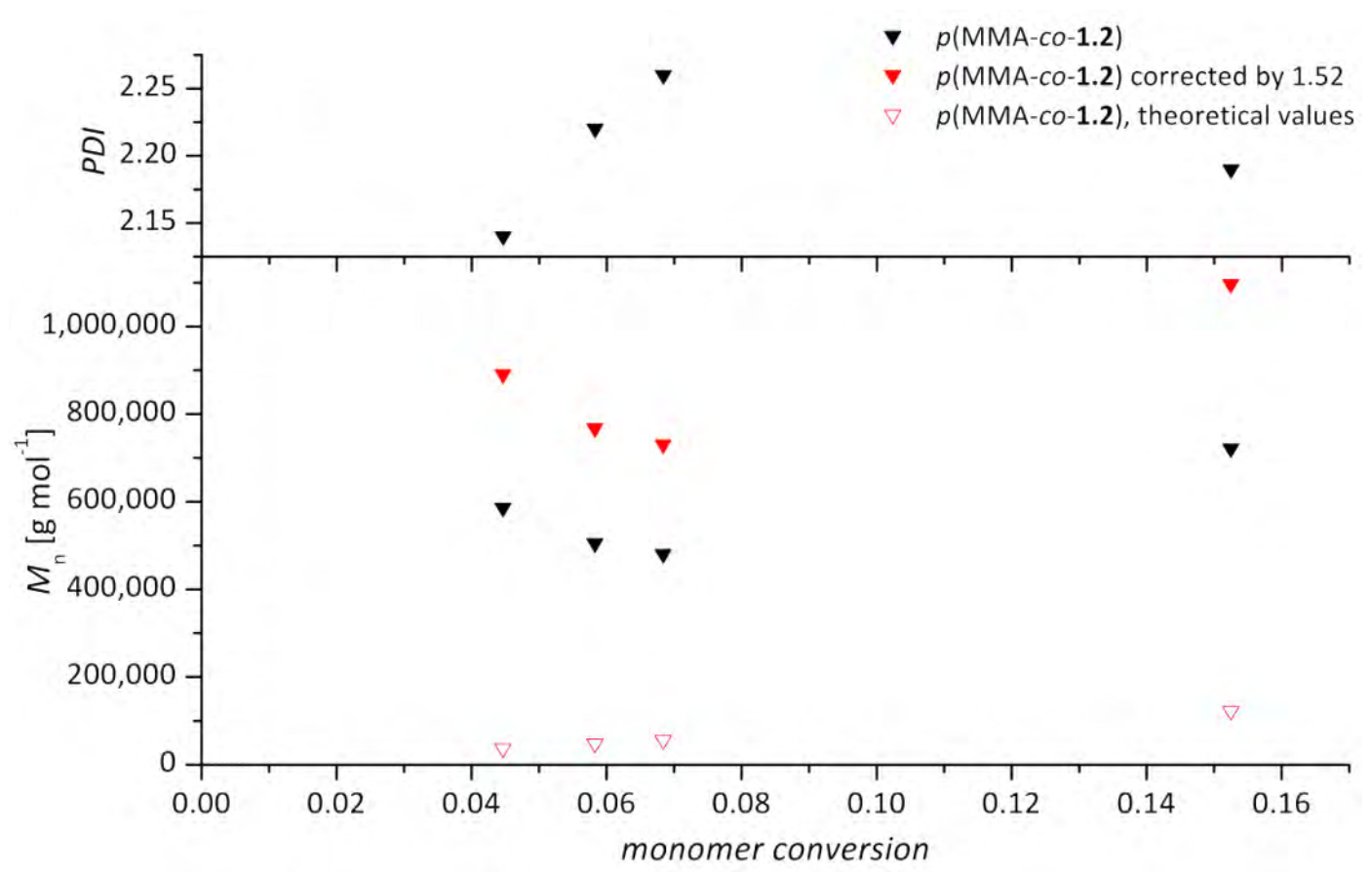

Figure 7-17: Development of molecular mass and PDI, determined by RI-detection on a THF-SEC, with monomer conversion in a copolymerisation of MMA with $1 \mathrm{~mol} \% 1.2$ using $2 \mathrm{mmol} \cdot \mathrm{L}^{-1} \mathrm{AlBN}, 1 \mathrm{mmol} \cdot \mathrm{L}^{-1} \boldsymbol{R}_{6} 3$ and $10 \mathrm{mmol} \cdot \mathrm{L}^{-1} \boldsymbol{R}_{L} 3$ at $60{ }^{\circ} \mathrm{C}$ up to 180 minutes.

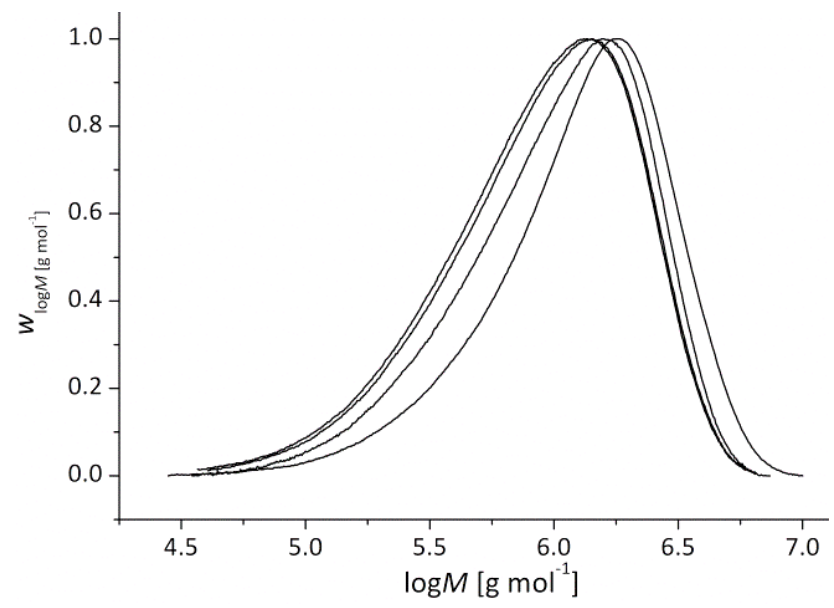

Figure 7-18: SEC-RI-data of the copolymerisation of MMA with $1 \mathrm{~mol} \% 1.2$ using $2 \mathrm{mmol} \cdot \mathrm{L}^{-1} \mathrm{AlBN}, 1 \mathrm{mmol} \cdot \mathrm{L}^{-1} \boldsymbol{R}_{6} 3$ and $10 \mathrm{~mol} \cdot \mathrm{L}^{-1} \boldsymbol{R}_{L} \mathbf{3}$ at $60^{\circ} \mathrm{C}$; sample order from left to right: 3-2-1-4.

The copolymerisation of MMA and $\mathbf{1 . 2}$ gives better results in terms of the UV-absorbance in the SECexperiment than the copolymerisation of MMA and 1.1. This can be attributed to the similar chemical nature of the vinyl bonds of the monomers. This is rather surprising as $\mathbf{1 . 2}$ is known to copolymerise with MMA, MA and EA under FRP-conditions ${ }^{(120)}$ which should be adaptable to RAFT. Nevertheless, the copolymerisation of the DMIs with MMA does not work under RAFT-conditions and will therefore not be investigated any further. 


\subsubsection{Copolymerisation of MA and $N$-Ethylacrylate-3,4-dimethylmaleimide (1.1)}

MA was tested in copolymerisation with 1 mol\% of the UV-reactive monomer 1.1 regarding the initial amount of MA. Again advantage should be taken of the similarity of the monomeric unit to perform the steady copolymerisation. In these bulk polymerisations both six-functional RAFT-agents $\left(\mathbf{R}_{6} \mathbf{2}\right.$ and $\left.\mathbf{R}_{6} \mathbf{3}\right)$ were applied along with the corresponding linear agents. Due to difficulties in the work-up of the polymers as the polymer could not be precipitated<smiles>C=CC(=O)OCCN1C(=O)C(C)=C(C)C1=O</smiles>
completely, it was not possible to determine the monomer conversion exactly. The few calculated values of the conversions do not follow the common linear trend. The monomer conversions range from $22 \%$, for the shortest reaction time, up to $75 \%$, for the longest reaction time, in the homopolymerisation and from 13 to $66 \%$ in the copolymerisation. Therefore the development of the molecular weights is plotted against time. Figure 7-19 shows this plot for the polymerisations carried out with $2 \mathrm{mmol} \cdot \mathrm{L}^{-1} \mathrm{AIBN}, 1 \mathrm{mmol} \cdot \mathrm{L}^{-1} \mathbf{R}_{6} \mathbf{2}$ and $10 \mathrm{mmol} \cdot \mathrm{L}^{-1} \mathbf{R}_{\mathbf{L}} \mathbf{2}$ at $60{ }^{\circ} \mathrm{C}$. For both polymerisations a linear increase of the molecular weight can be observed till a reaction time of 140 minutes was reached. The PDI-values for the copolymerisation increased with time but were still in the characteristic range for a CRP.

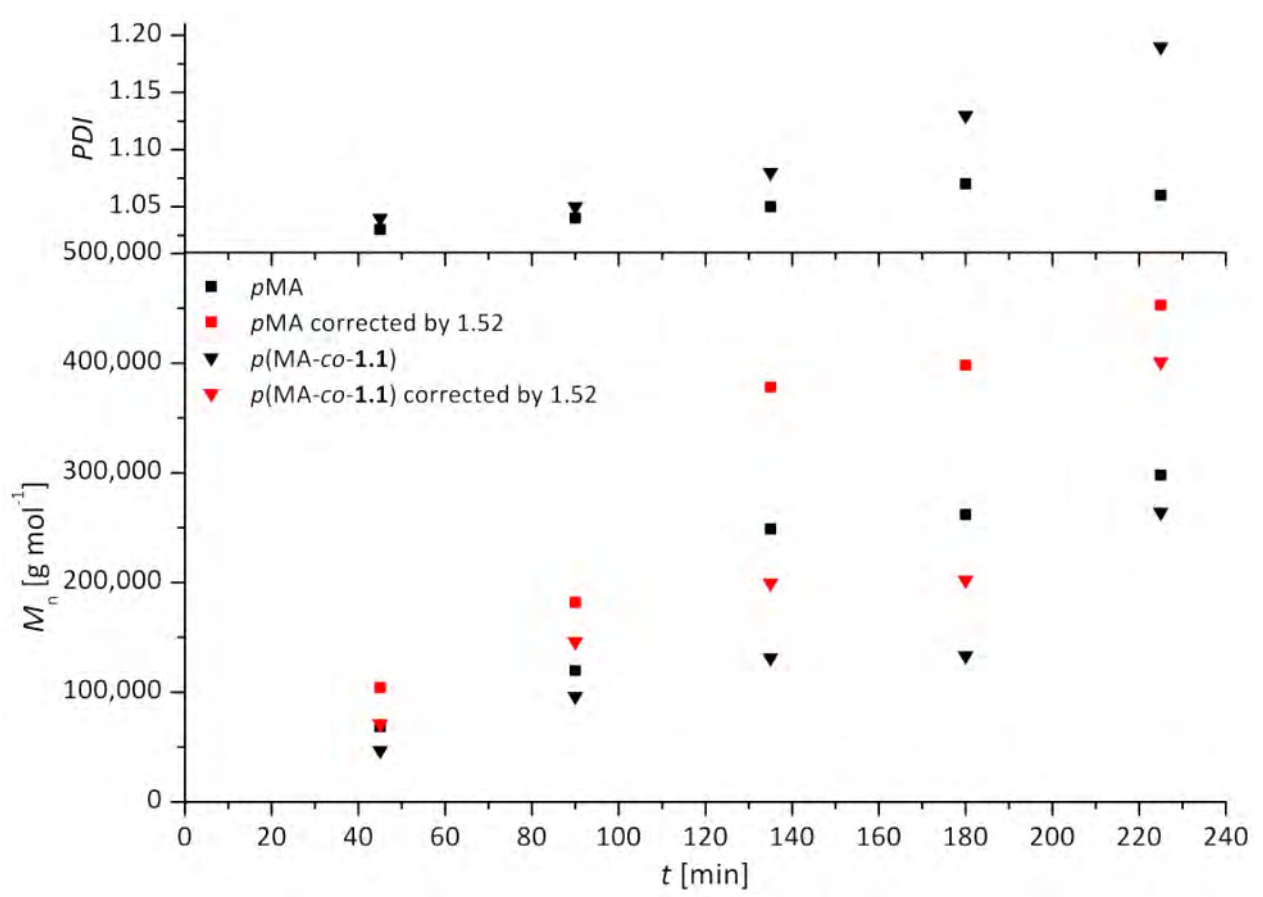

Figure 7-19: Development of molecular mass and PDI, determined by UV-detection on a THF-SEC, with time in a homopolymerisation of MA (squares) and a copolymerisation of MA with $1 \mathrm{~mol} \% 1.1$ (triangles) using $2 \mathrm{mmol} \cdot \mathrm{L}^{-1} \mathrm{AlBN}$, $1 \mathrm{mmol} \cdot \mathrm{L}^{-1} \boldsymbol{R}_{6} \mathbf{2}$ and $10 \mathrm{mmol} \cdot \mathrm{L}^{-1} \boldsymbol{R}_{L} \mathbf{2}$ at $60^{\circ} \mathrm{C}$. Shown are only the data of the star polymer. 
This is confirmed by the MWDs (Figures 7-19 and 7-20). As the spectra are confusingly crowded when plotted with linear and star polymer signals in one spectrum (left), the signal of the linear polymer was deleted. The spectra on the right show only the signals of the star polymer signals. In the case of the copolymerisation, shoulders can be seen for the higher molecular weight samples which indicate unwanted side reactions. This increase in molecular weight seems not to be a result of growing stars but star-star-coupling as the molecular weights between the two peak maxima differ by a factor of two (Figure 7-21), which destroys the monodispersity of the samples. As this is only discovered in the copolymerisation, it can be assumed that the DMI moiety takes part in these unwanted side reactions. It can be assumed that the cross-coupling is induced by chemical or thermal means.

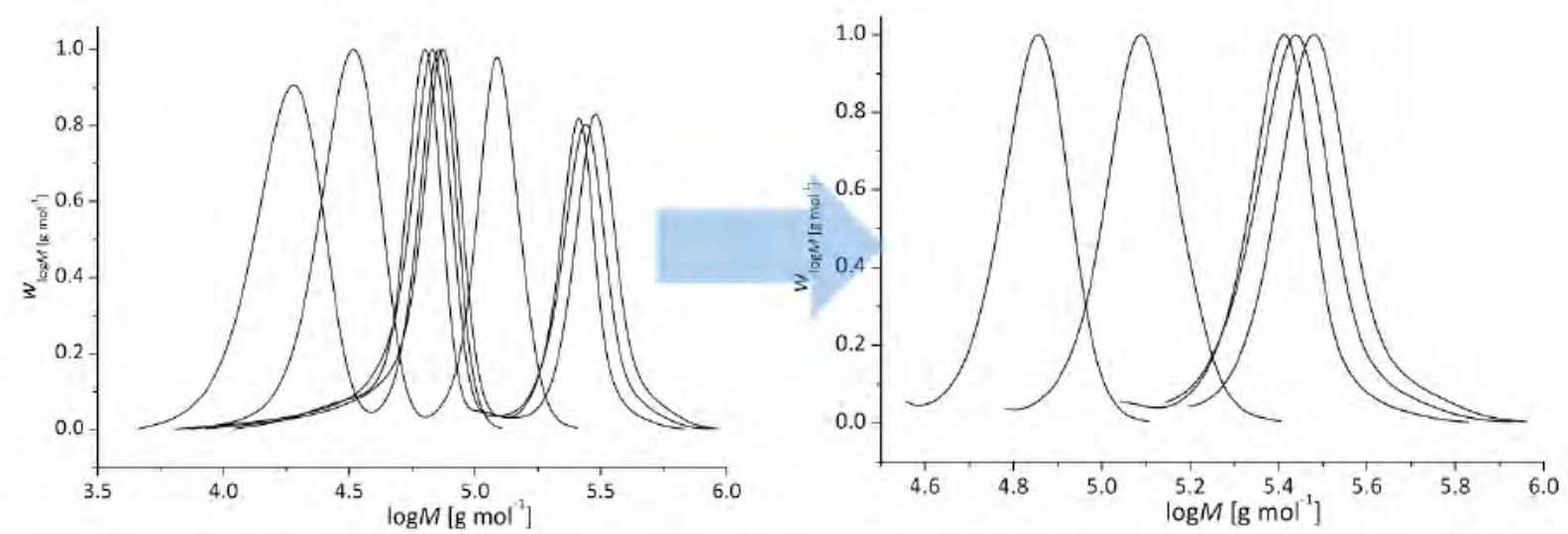

Figure 7-20: SEC-UV-data for the homopolymerisation of MA using $2 \mathrm{mmol} \cdot \mathrm{L}^{-1} \mathrm{AlBN}, 1 \mathrm{mmol} \cdot \mathrm{L}^{-1} \boldsymbol{R}_{6} \mathbf{2}$ and $10 \mathrm{mmol} \cdot \mathrm{L}^{-1} \boldsymbol{R}_{L} \mathbf{2}$ at $60{ }^{\circ} \mathrm{C}$. Left: complete SEC-spectra with linear and star polymer, right: partial SEC-spectra showing only the signals resulting from the star polymers.

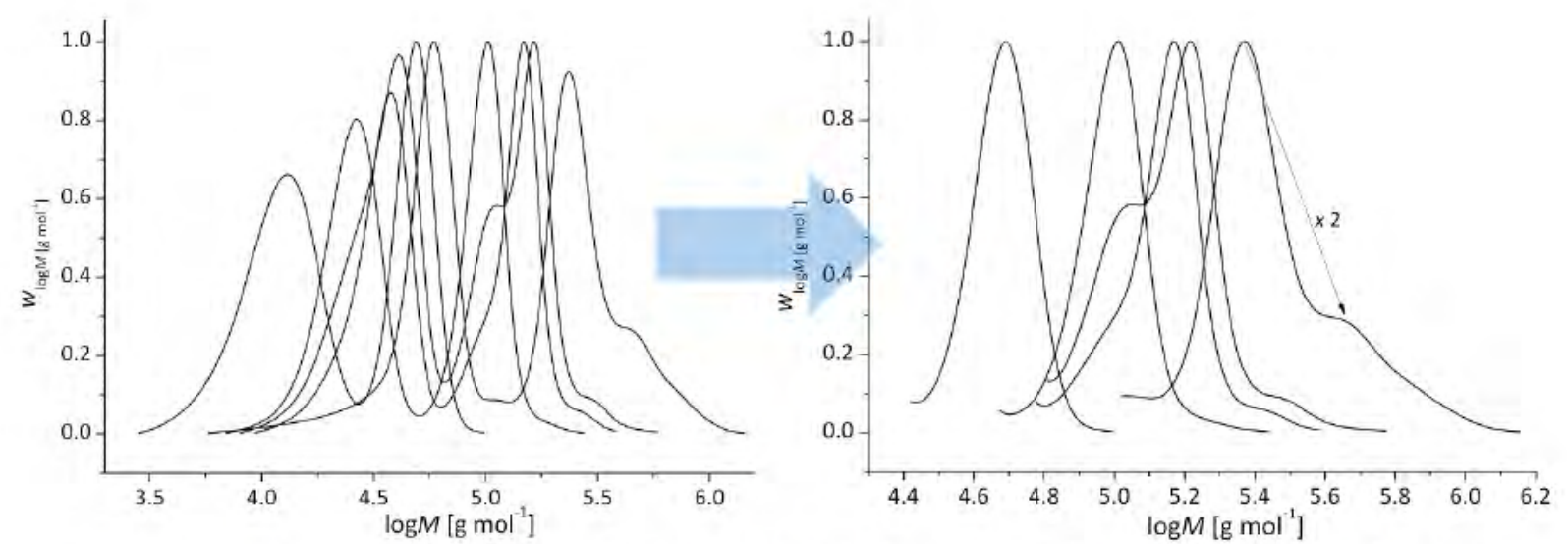

Figure 7-21: SEC-data for the copolymerisation of MA with $1 \mathrm{~mol} \% 1.1$ using $2 \mathrm{mmol} \cdot \mathrm{L}^{-1} \mathrm{AlBN}, 1 \mathrm{mmol} \cdot \mathrm{L}^{-1} \boldsymbol{R}_{6} \mathbf{2}$ and $10 \mathrm{mmol} \cdot \mathrm{L}^{-1} \boldsymbol{R}_{L} 2$ at $60^{\circ} \mathrm{C}$. Left: complete SEC-spectra with linear and star polymer, right: partial SEC-spectra showing only the signals resulting from the star polymers. 
In the case of MA-polymerisations with $\mathbf{R}_{6} \mathbf{3}$ the trend differs. The plot of molecular weight against reaction time is shown in Figure 7-22. The molecular weight in the copolymerisation shows a linear increase. The monomer conversions for these polymerisations range from $21 \%$ for the shortest reaction time to $76 \%$ for the longest reaction time in the homopolymerisation and from 33 to $55 \%$ in the copolymerisation. The SEC-spectra (Figure 7-23) reveal a well-controlled course of the polymerisation with the linear RAFT-agent. On the contrary, the signals resulting from the star polymers appear unstructured. Again it seems as unwanted side reactions change the constitution of the star polymer which leads to broadened signals.

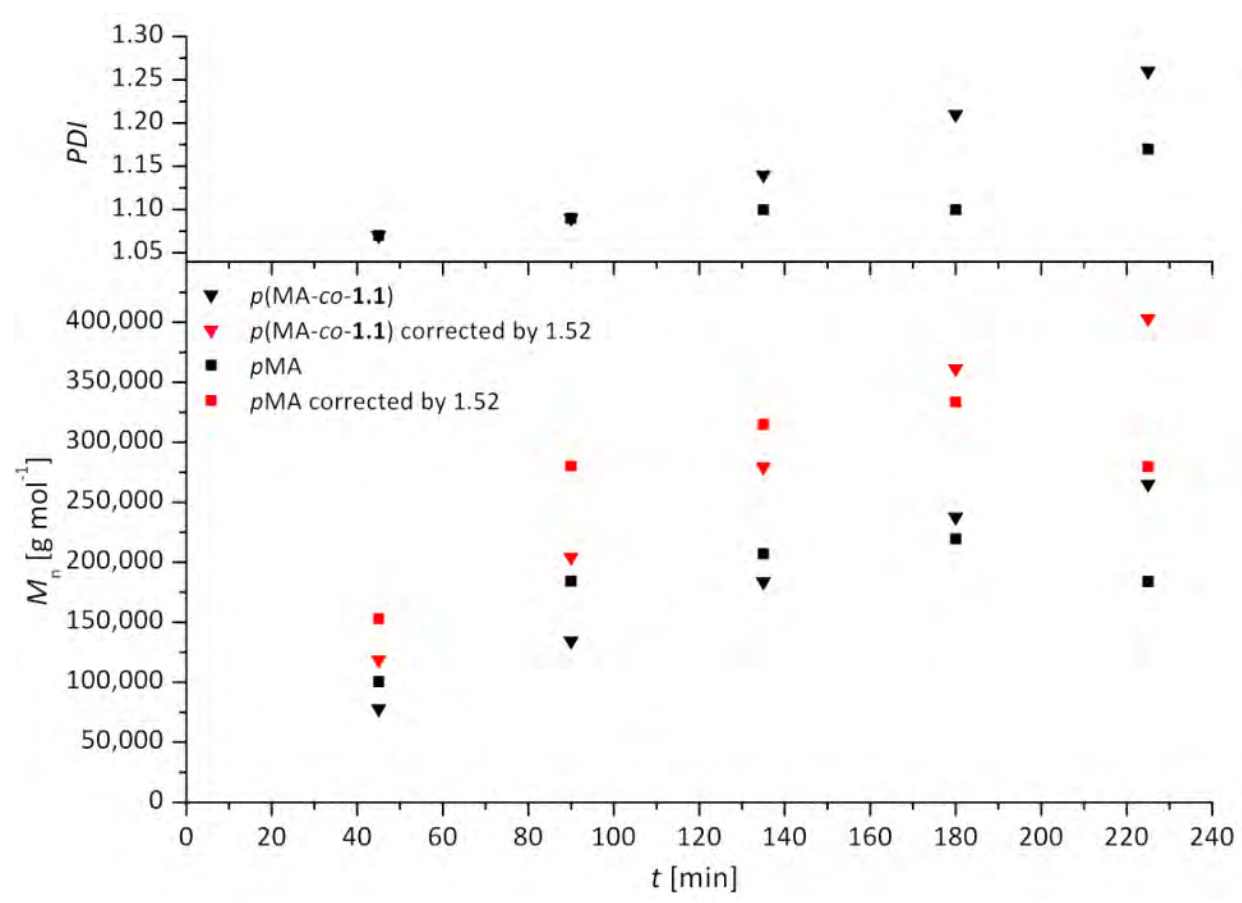

Figure 7-22: Development of molecular mass and PDI with time in a homopolymerisation of MA (squares) and a copolymerisation of MA with $1 \mathrm{~mol} \% 1.1$ (triangles) using $2 \mathrm{mmol} \cdot \mathrm{L}^{-1} \mathrm{AlBN}, 1 \mathrm{mmol} \cdot \mathrm{L}^{-1} \boldsymbol{R}_{6} 3$ and $10 \mathrm{mmol} \cdot \mathrm{L}^{-1} \boldsymbol{R}_{L} 3$ at $60{ }^{\circ} \mathrm{C}$. Shown are only the data of the star polymer. 

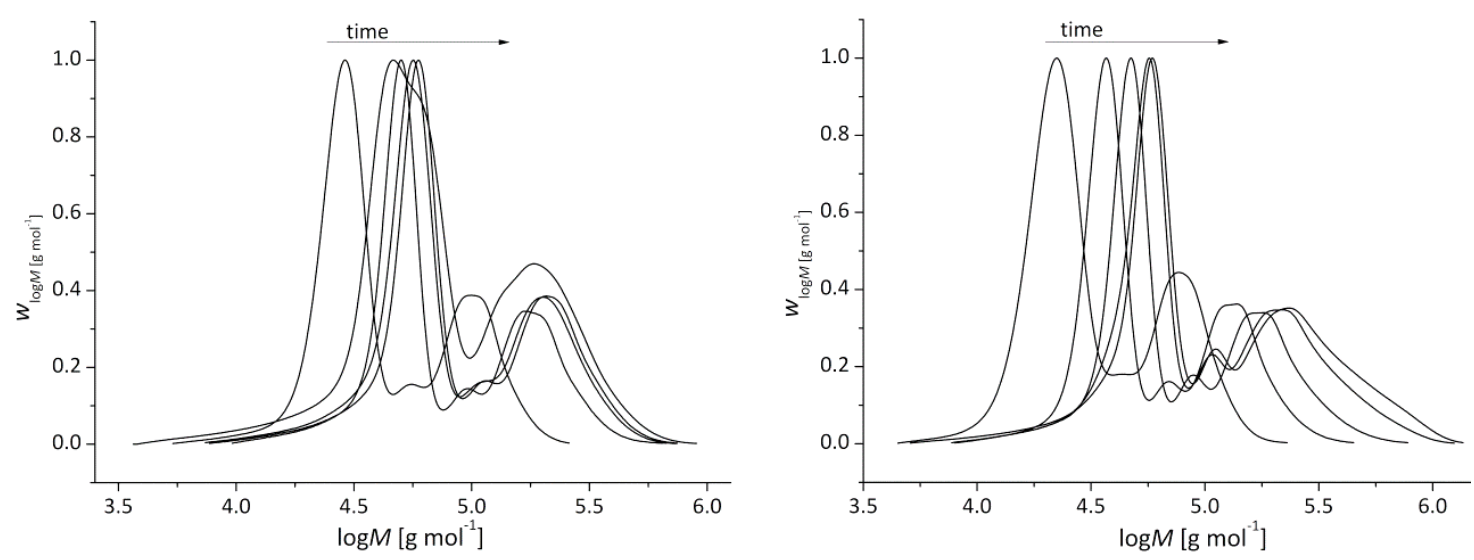

Figure 7-23: SEC-data for a MA-homopolymerisation (left) and a copolymerisation of MA with 1 mol\% 1.1 (right) using $2 \mathrm{mmol} \cdot \mathrm{L}^{-1} \mathrm{AlBN}, 1 \mathrm{mmol} \cdot \mathrm{L}^{-1} \boldsymbol{R}_{6} 3$ and $10 \mathrm{mmol} \cdot \mathrm{L}^{-1} \boldsymbol{R}_{L} 3$ at $60^{\circ} \mathrm{C}$.

It can be summarised that the copolymerisation of MA and $\mathbf{1 . 1}$ is successful but that the controlled character of the reaction could not be proven unambiguously due to the failure of the determination of the monomer conversion. The PDI-values in all MA-polymerisations indicate a controlled process but from the development of the MWDs arises doubt. As the work-up of these polymers turned out to be difficult, a use of this system on a large scale is excluded.

\subsubsection{Copolymerisation of NIPAam and N-Ethylacrylate-3,4-dimethylmaleimide}

Polymers from NIPAam are water and methanol soluble and serve here as hydrophilic polymer block. To achieve amphiphilic nano-carriers with a hydrophilic shell the UV-monomer has to be copolymerised with NIPAam. In the literature it is reported about copolymers from the DMI-NIPAam and NIPAam itself synthesised by FRP. ${ }^{(116)}$ Here the monomer 1.1 should be copolymerised with NIPAam by means of RAFT. The reaction is<smiles>C=CC(=O)NC(C)C</smiles>

NIPAam<smiles>C=CC(=O)OCCN1C(=O)C(C)=C(C)C1=O</smiles>
carried out in DMF, as the monomer is a solid, at $60{ }^{\circ} \mathrm{C}$ and is mediated by $\mathbf{R}_{6} \mathbf{3}$. As THF is a poor solvent for pNIPAam the determination of the molecular weights was performed in $\mathrm{N}, \mathrm{N}$-dimethylacrylamide (DMac) against linear pMMA-standards. The Figure 7-24 illustrates the development of the molecular weight and the PDI-values with monomer conversion. The growth of the molecular weights follows a linear trend for both polymerisations. Furthermore the PDI-values support a controlled process during the reaction. The MWDs also show the typical development with time and monomer conversion (Figure 7-25). 


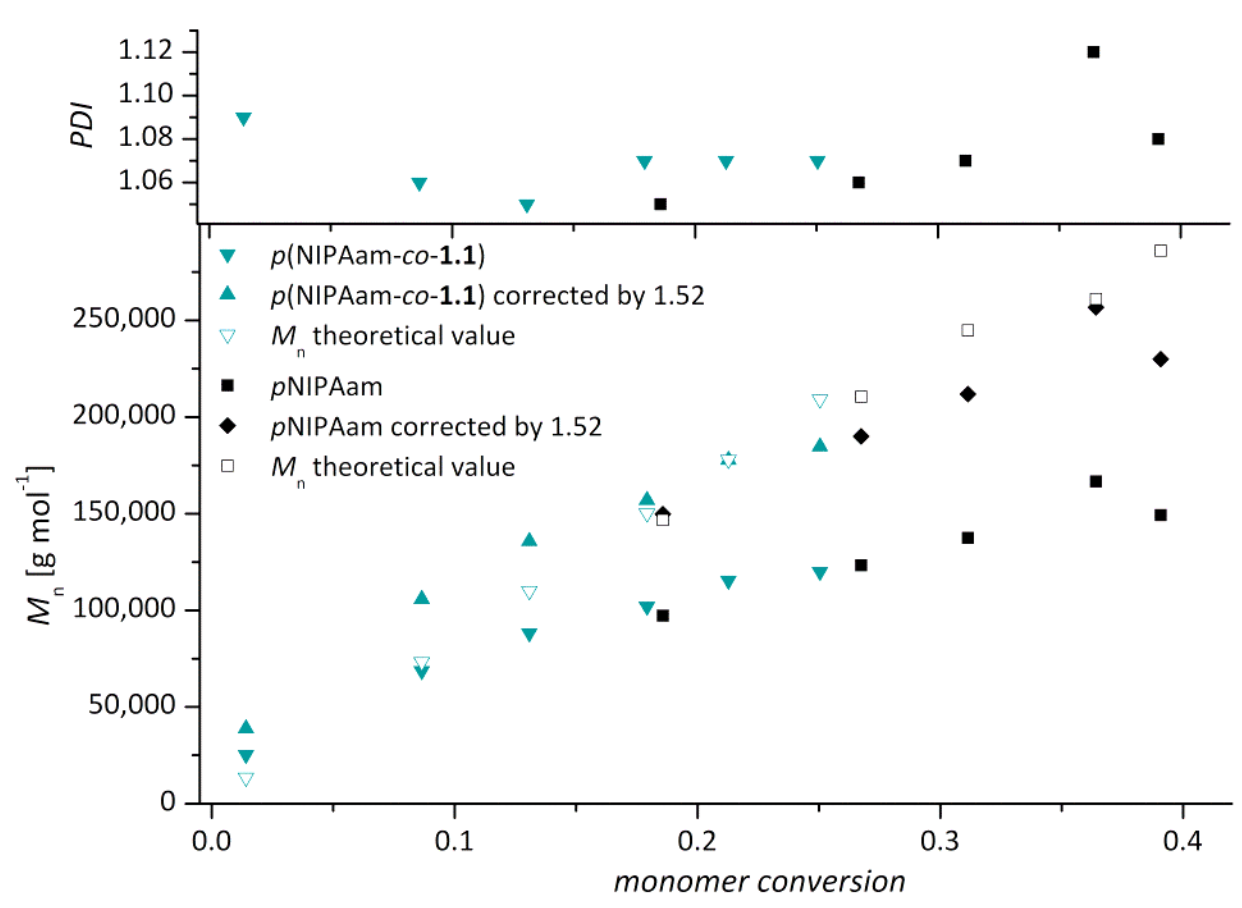

Figure 7-24: Development of molecular weight and PDI, determined by UV-detection on a DMac-SEC with a pMMA calibration, with monomer conversion for a copolymerisation of NIPAam and 3 mol\% 1.1 with $1.3 \mathrm{mmol} \cdot \mathrm{L}^{-1} \mathrm{AlBN}, 0.5 \mathrm{mmol} \cdot \mathrm{L}^{-1} \boldsymbol{R}_{6} 3$ in $D M F$ at $60^{\circ} \mathrm{C}$.
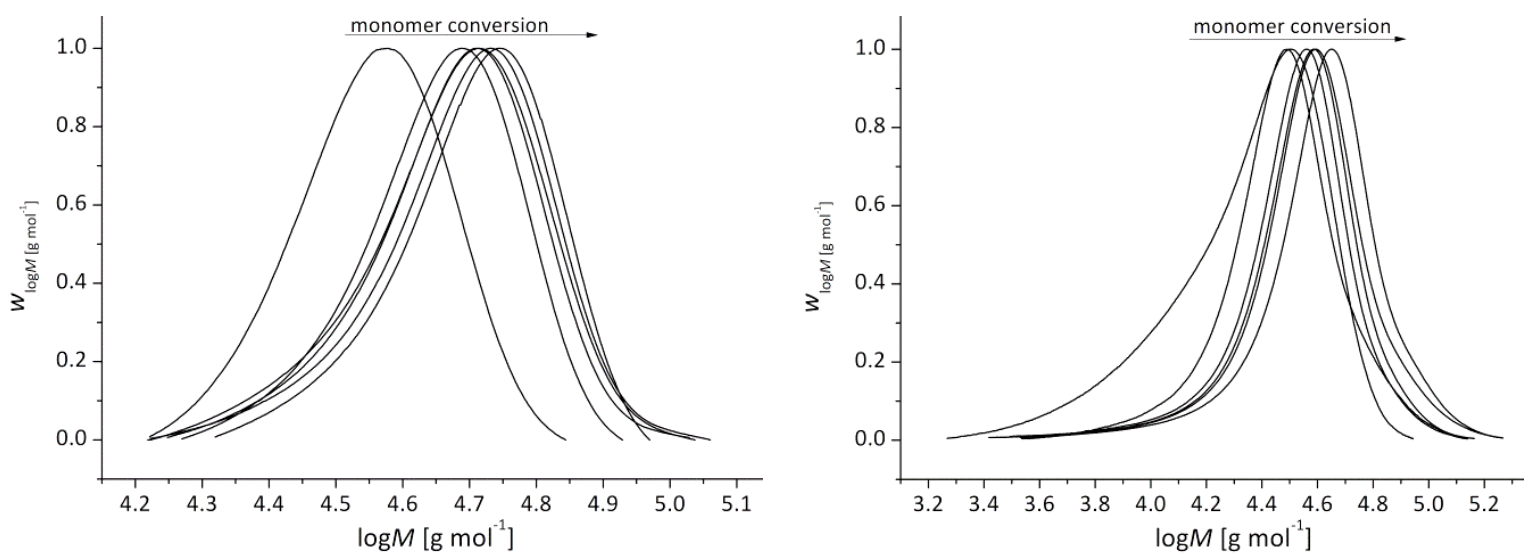

Figure 7-25: SEC-UV-data for a NIPAam-homopolymerisation (left) and a copolymerisation of NIPAam $3 \mathrm{~mol} \% 1.1$ (right) with $1.3 \mathrm{mmol} \cdot \mathrm{L}^{-1} \mathrm{AIBN}$ and $0.5 \mathrm{mmol} \cdot \mathrm{L}^{-1} \boldsymbol{R}_{6} 3$ in $\mathrm{DMF}$ at $60^{\circ} \mathrm{C}$.

It can be observed that the copolymerisation starts delayed compared to the homopolymerisation. Figure 7-26 shows this phenomenon in detail. Samples of the copolymerisation taken at the same times show smaller molecular weights than those of the pure NIPAam-polymerisation. The monomer conversion is also distinctively smaller (Figures 7-23 and 7-25). This phenomenon will be discussed in the up-coming chapter 8. 


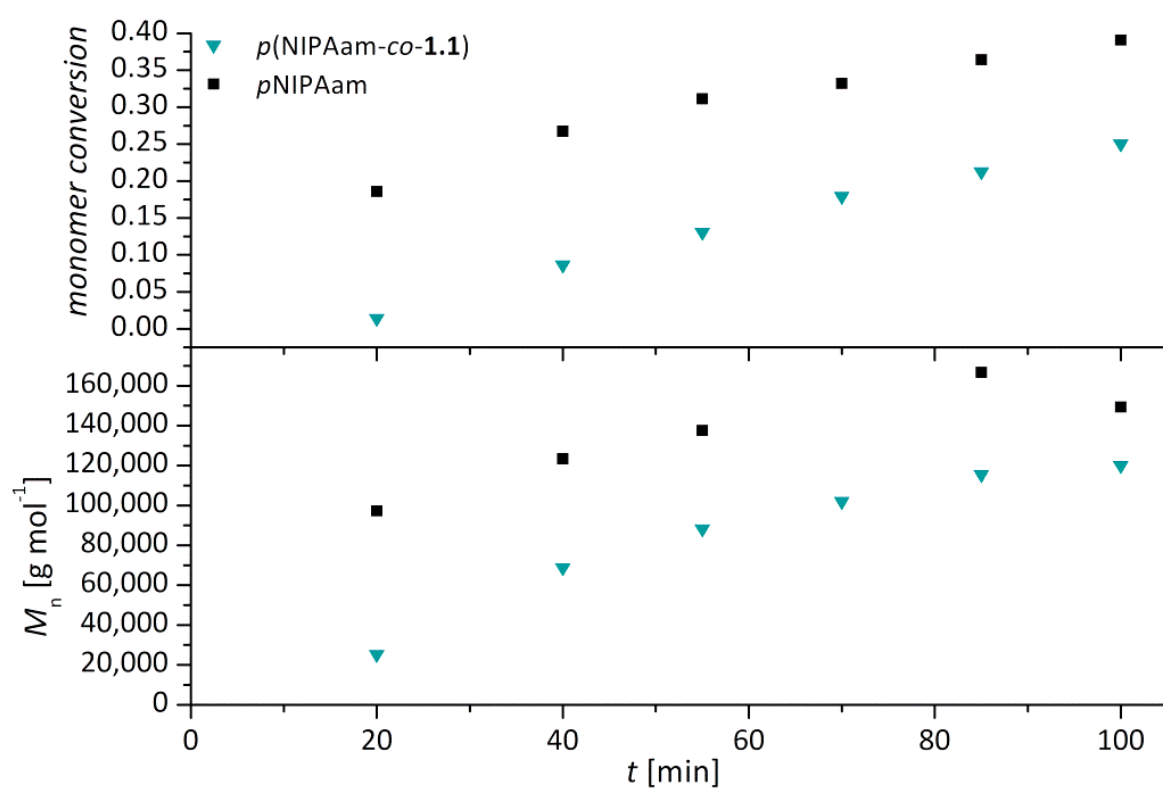

Figure 7-26: Development of monomer conversion and molecular weight with reaction time for a homopolymerisation of NIPAam (black) and a copolymerisation of NIPAam and $3 \mathrm{~mol} \% 1.1$ (aqua) with $1.3 \mathrm{mmol} \cdot \mathrm{L}^{-1} \mathrm{AIBN}$ and $0.5 \mathrm{mmol} \cdot \mathrm{L}^{-1} \boldsymbol{R}_{6} 3$ in DMF at $60^{\circ} \mathrm{C}$.

Nevertheless, the controlled copolymerisation of NIPAam and $\mathbf{1 . 1}$ by means of RAFT polymerisation works well. As in the homopolymerisation case, polymers with defined molecular weights and narrow distributions in the molecular weight can be obtained in manageable periods of time. Therefore this system will be investigated further to gain amphiphilic nano-carriers with a hydrophilic shell. 


\subsection{Copolymerisations with Cinnamoyloxyethyl-Monomers 2.1 und 2.2}

\subsubsection{Copolymerisation of BA and Cinnamoyloxyethyl-Monomers}

In this chapter the RAFT-copolymerisation of BA and cinnamoyloxyethylacrylate (CEA, 2.1) is under investigation. As mentioned previously (chapter 3.6.3), this monomer is known to copolymerise with BA in a FRP (using AIBN and HEMA for 12 hours at $80{ }^{\circ} \mathrm{C}$ ) to form shape memory polymers. ${ }^{(111)}$ It is expected that the reaction can be easily transferred to RAFT-conditions without changing the kinetics<smiles>C=CC(=O)OCCOC(=O)/C=C/c1ccccc1</smiles><smiles>[Y11]</smiles><smiles>C=C(C)C(=O)OCCOC(=O)/C=C/c1ccccc1</smiles>
(chapter 3.2.3). Furthermore the cinnamoyloxyethylmethacrylate (CEMA, 2.2) was tested. It is assumed that $\mathbf{2 . 1}$ copolymerises better with BA than $\mathbf{2 . 2}$ due to a chemically equivalent vinyl double bond. Experiments were carried out in bulk and both RAFT-agents (Figure 7-12) were tested. As in the examples before, homopolymerisations of $B A$ under the same reaction conditions were performed to compare the results.

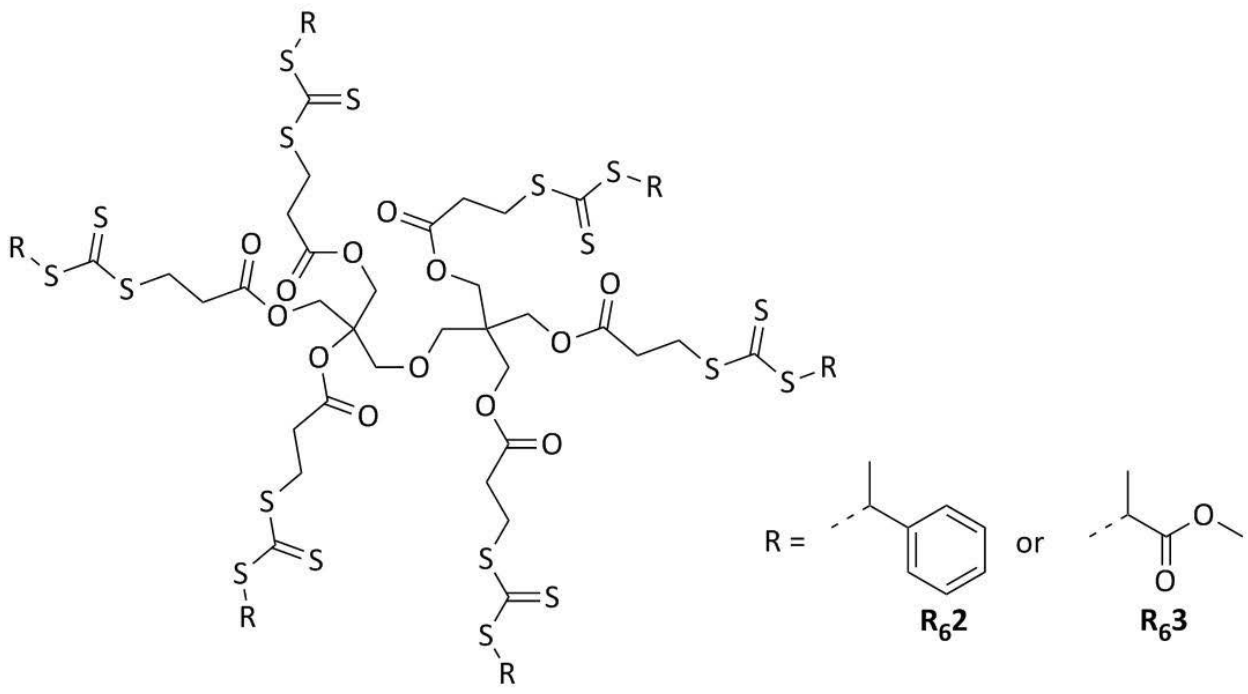

Figure 7-2: Dipentaerythriol-hexakis-(3-(phenylethyltricarbonate))-propanoate $\mathbf{R}_{6} \mathbf{2}$ and Dipentaerythriol-hexakis-(3-

(S-methoxopropionate-trithiocarbonate))-propanoate $\boldsymbol{R}_{6} 3$.

In a first experiment BA was combined with 3 mol\% $\mathbf{2 . 2}$ using $\mathbf{R}_{6} \mathbf{3}$ as mediating agent. Aliquots were taken up to a time of 120 minutes. The resulting plots of molecular weight and PDI versus the monomer conversion are shown in Figure 7-27. It can be seen that the homopolymerisation proceeds, as expected, in a controlled way but also that the addition of $\mathbf{2 . 2}$ inhibits the polymerisation. All data points of the copolymerisation are located at zero monomer conversion and have no perceptible molecular weight (black arrow). 


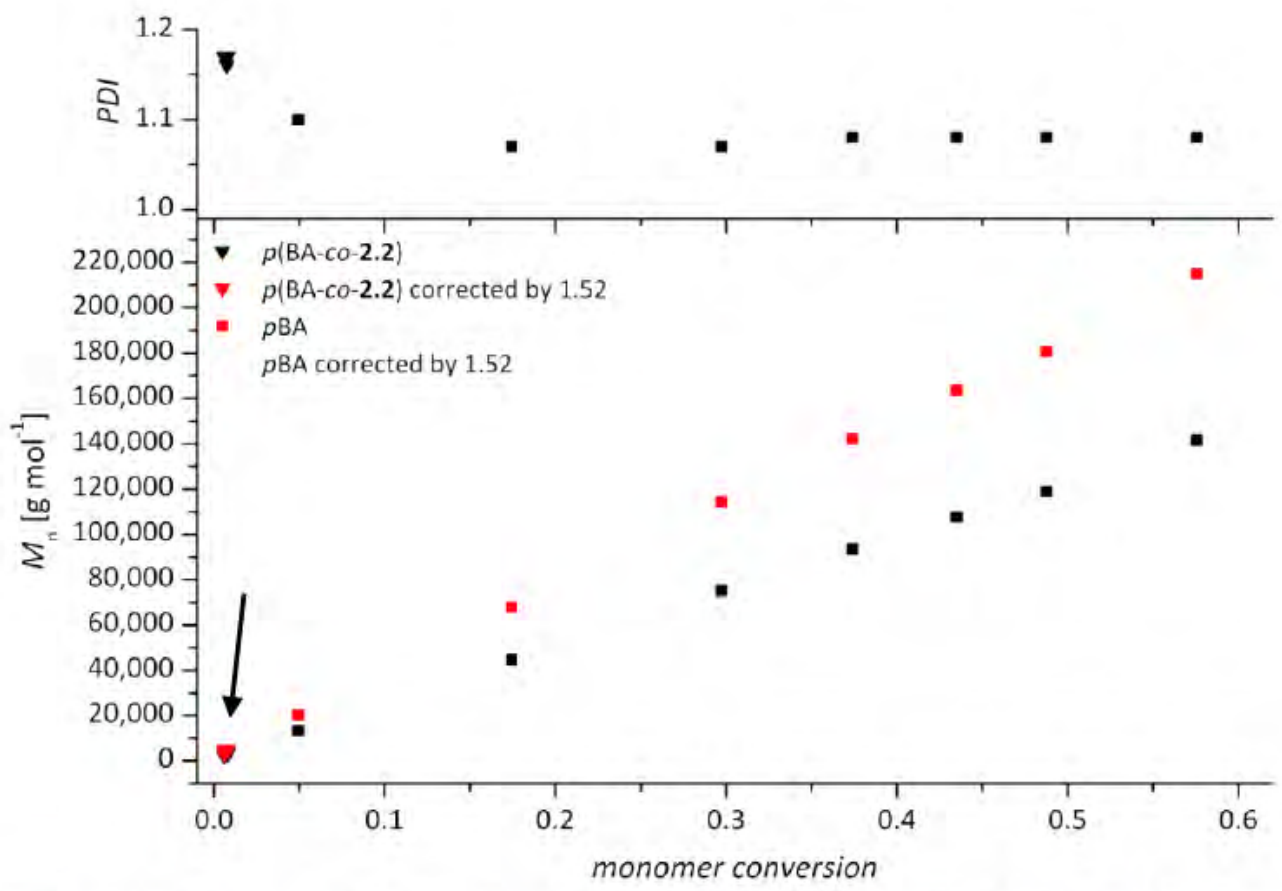

Figure 7-27: Development of molecular mass and PDI, determined by UV-detection on a THF-SEC, with monomer conversion in a homopolymerisation of BA (squares) and a copolymerisation of BA with $3 \mathrm{~mol} \% \mathbf{2 . 2}$ (triangles) using $4 \mathrm{mmol} \cdot \mathrm{L}^{-1} \mathrm{AlBN}$, $2 \mathrm{mmol} \cdot \mathrm{L}^{-1} \boldsymbol{R}_{6} 3$ and $19 \mathrm{mmol} \cdot \mathrm{L}^{-1} \boldsymbol{R}_{L} 3$ at $60^{\circ} \mathrm{C}$. Shown are only data of the star polymer.
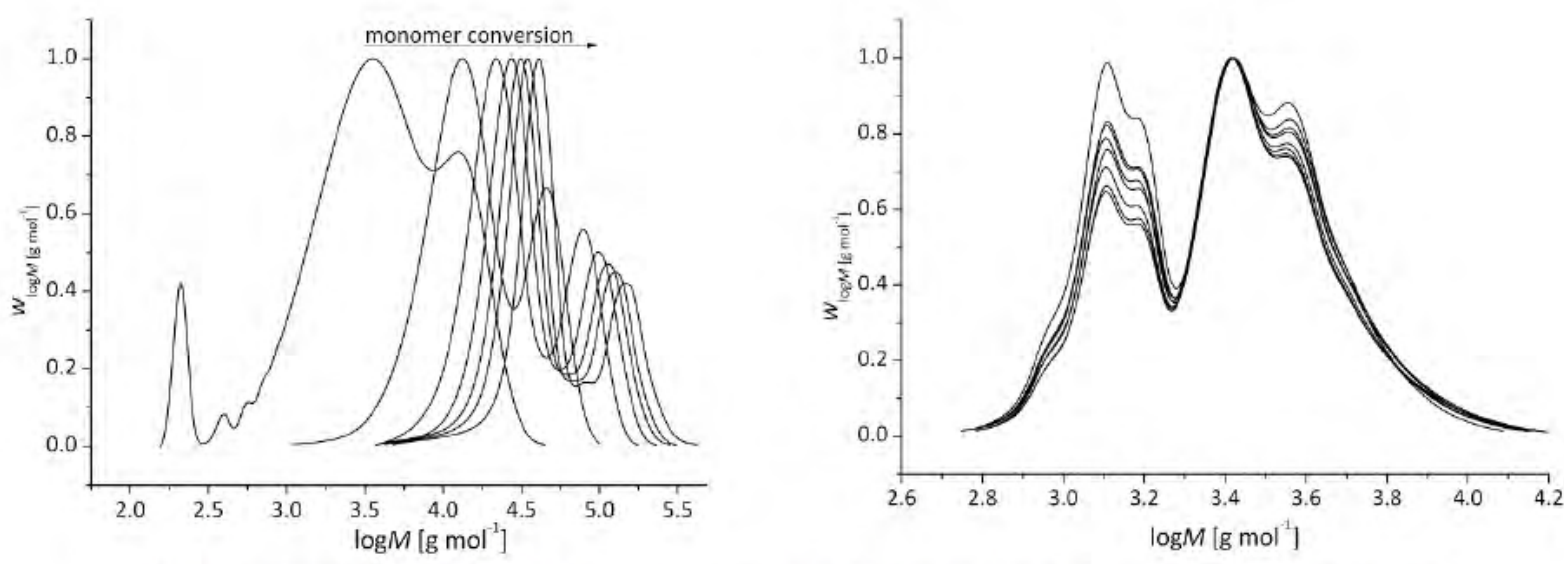

Figure 7-28: SEC-UV-data for the homopolymerisation of BA (left) and the copolymerisation of BA with $3 \mathrm{~mol} \% 2.2$ (right) using $4 \mathrm{mmol} \cdot \mathrm{L}^{-1} \mathrm{AlBN}, 2 \mathrm{mmol} \cdot \mathrm{L}^{-1} \boldsymbol{R}_{6} \mathbf{3}$ and $19 \mathrm{mmol} \cdot \mathrm{L}^{-1} \boldsymbol{R}_{\mathrm{L}} \mathbf{3}$ at $60^{\circ} \mathrm{C}$.

The SEC-spectra (Figure 7-28) confirm those findings. In the first spectrum of the homopolymerisation (left) the initial step of the polymerisation with different states of the growing stars is observable. The spectra of the copolymerisation all correspond to the same molecular weight and there is no trend in the development of these spectra. A repetition of this experiment under the same conditions and with reaction times up to 790 minutes did not show any change in monomer conversion or molecular weight. For all samples no monomer conversion was obtained and molecular weights of ca $2,500 \mathrm{~g} \cdot \mathrm{mol}^{-1}$ were measured which corresponds, in the range of the failure of the SEC set up, to the molecular weight of the RAFT-agent $\left(M=1,756.46 \mathrm{~g} \cdot \mathrm{mol}^{-1}\right)$. 
To examine whether the R-group of the RAFT-agent has an influence on this process, the experiment was also carried out with $\mathbf{R}_{6} \mathbf{2}$ and a reaction time of 75 minutes. In Figure 7-29 the molecular weight and the PDI-values are plotted against the monomer conversion. Here a small increase in the monomer conversion can be observed. The molecular masses of these samples are around $3,700 \mathrm{~g} \cdot \mathrm{mol}^{-1}$. That corresponds to $1,864 \mathrm{~g} \cdot \mathrm{mol}^{-1}$ of the RAFT-agent plus approximately 14 monomer units.

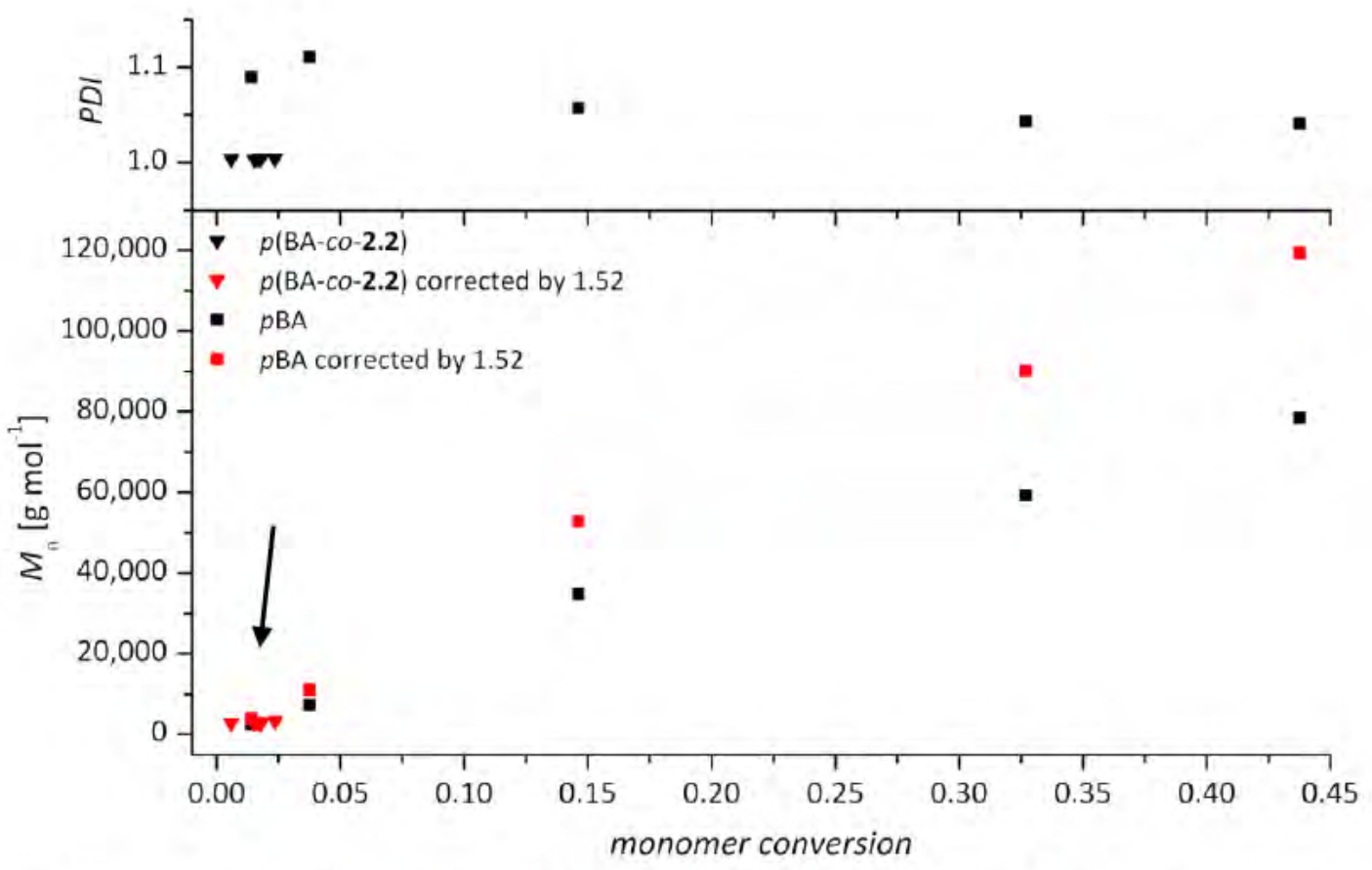

Figure 7-29: Development of molecular mass and PDI, determined by UV-detection on a THF-SEC, with monomer conversion in a homopolymerisation of BA (squares) and a copolymerisation of BA with $3 \mathrm{~mol} \% 2.2$ (triangles) using $3 \mathrm{mmol} \cdot \mathrm{L}^{-1} \mathrm{AlBN}$, $3 \mathrm{mmol} \cdot \mathrm{L}^{-1} \boldsymbol{R}_{6} \mathbf{2}$ at $60^{\circ} \mathrm{C}$. 

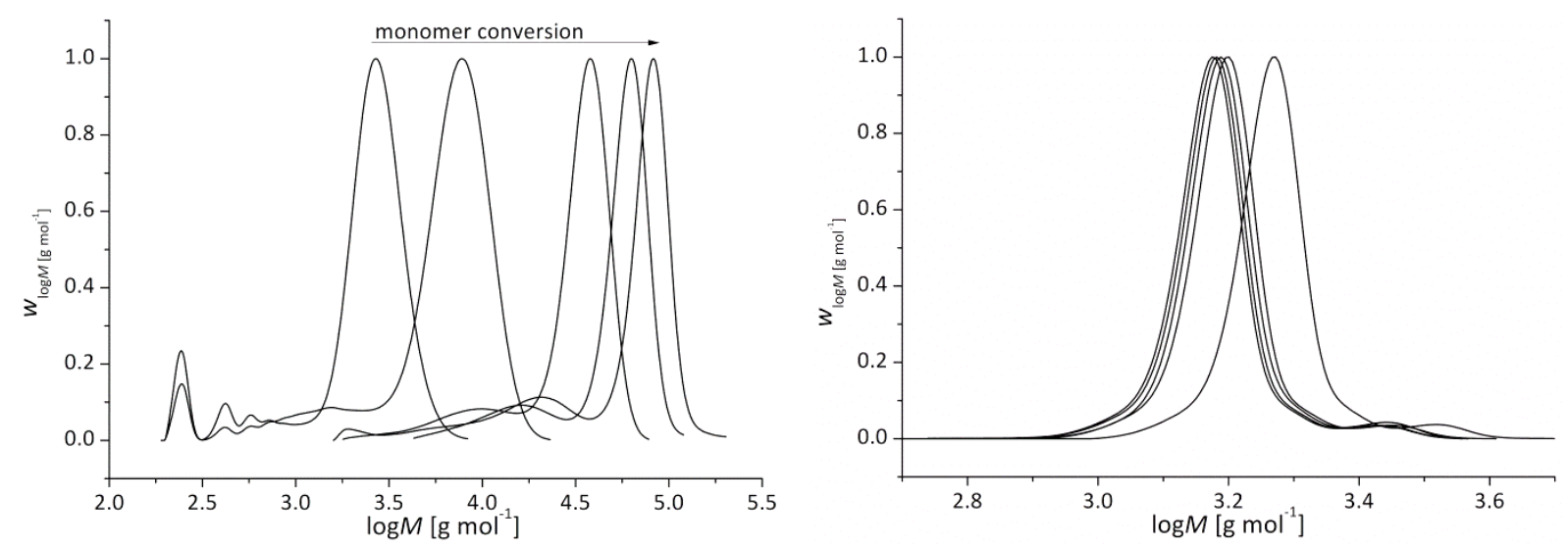

Figure 7-30: SEC-UV-data for the homopolymerisation of BA (left) and the copolymerisation of BA with 3 mol\% 2.2 (right) using $3 \mathrm{mmol} \cdot \mathrm{L}^{-1} \mathrm{~A} / \mathrm{BN}$ and $3 \mathrm{mmol} \cdot \mathrm{L}^{-1} \boldsymbol{R}_{6} \mathbf{2}$ at $60^{\circ} \mathrm{C}$.

The SEC-data confirm that the homopolymerisation works fine whereas the copolymerisation does not work under RAFT-conditions. It can be concluded that the applied R-group of the RAFT-agent has no large influence on this process.

Besides the cinnamoyl-methacrylate 2.2, the cinnamoyl-acrylate 2.1 was tested in a copolymerisation with BA. The reaction time was prolonged up to six days. Therefore no comparative BA-homopolymerisation was carried out along as the monomer conversion for that case is complete in less than a day. Furthermore, the amount of co-monomer was raised to $9 \mathrm{~mol} \%$. As can be seen in Figure 7-31 from many samples at approximately $0 \%$ monomer conversion, the reaction has a very long initial phase. After adjustment of the RAFT-equilibrium the molecular weights increase in a linear fashion. The aliquot at approximately $10 \%$ monomer conversion was taken after 24 hours, the last one of this plot after six days. Even then the monomer conversion is below $20 \%$. Quite surprising is the good fit of the theoretical values of the molecular weight to the uncorrected experimental results. 


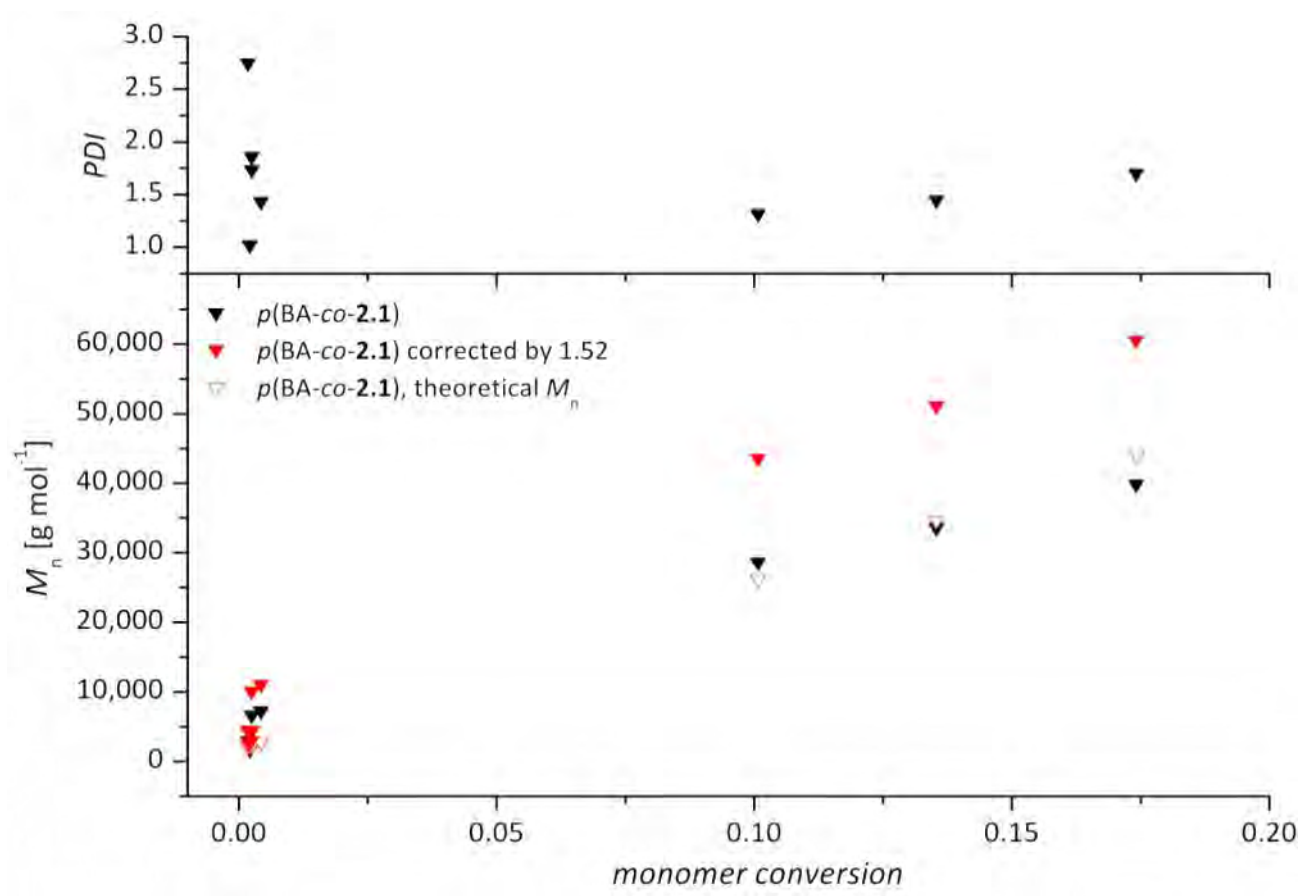

Figure 7-31: Development of molecular mass and PDI, determined by UV-detection on a THF-SEC, with monomer conversion in a copolymerisation of $B A$ with $9 \mathrm{~mol} \% 2.1$ using $10 \mathrm{mmol} \cdot \mathrm{L}^{-1} \mathrm{AlBN}$ and $4 \mathrm{mmol} \cdot \mathrm{L}^{-1} \boldsymbol{R}_{6} 3$ at $60{ }^{\circ} \mathrm{C}$.

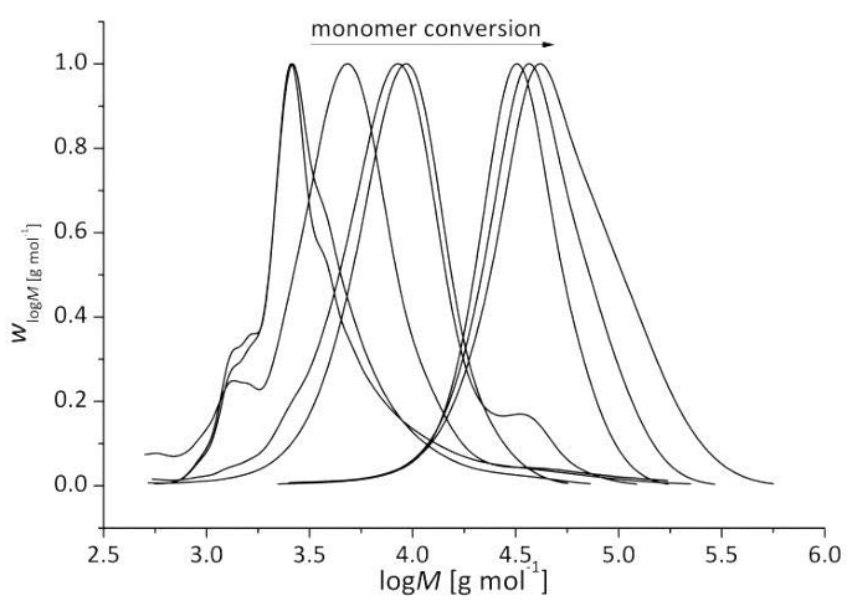

Figure 7-32: SEC-UV-data of a copolymerisation of BA with $9 \mathrm{~mol} \% \mathbf{2 . 1}$ using $10 \mathrm{mmol} \cdot \mathrm{L}^{-1} \mathrm{AIBN}$ and $4 \mathrm{mmol} \cdot \mathrm{L}^{-1} \boldsymbol{R}_{6} \mathbf{3}$ at $60{ }^{\circ} \mathrm{C}$.

As well as the PDI-values which are in the characteristic range for CRP, do the SEC-spectra of this prolonged experiment confirm the controlled polymerisation behaviour. With longer reaction times a shoulder in the higher molecular weight regime appears which result from side reactions (transfer reactions, back-biting).

In conclusion it can be said that the monomer $\mathbf{2 . 2}$ does not copolymerise with BA under RAFT-conditions in the here applied periods of time. Even after longer reaction times no change in molecular weight or monomer conversion could be observed. In the case of $\mathbf{2 . 1}$ a copolymerisation takes place which is strongly inhibited in the beginning. Long reaction times are needed to yield 
polymer material. After six days a polymer with a molecular weight of around $60,000 \mathrm{~g} \cdot \mathrm{mol}^{-1}$ and with a monomer conversion of $17 \%$ was isolated. A similar yield could potentially be recovered for the same polymerisation when using the $\mathbf{2} \mathbf{2}$ monomer. However, as the reaction times are very long the polymerisations were not further investigated for the purpose of this thesis as the application is unrealistic. In the case of a star polymer of $M_{\mathrm{n}} \approx 60,000 \mathrm{~g} \cdot \mathrm{mol}^{-1}$ one arm consists of approximately 75 monomer units. If $\mathbf{2 . 1}$ and BA react with the same probability, statistically less than one UVmonomer is integrated in a single arm which is insufficient for a successful shell-formation.

\subsubsection{Copolymerisation of MMA and Cinnamoyloxyethylmethacrylate}

Another test of a copolymerisation was the combination of $\mathbf{2 . 2}$ and MMA. The polymerisations were carried out with 1 and 3 mol\% 2.2 regarding the initial amount of MMA over 225 minutes and were mediated by $\mathbf{R}_{6} \mathbf{3}$. The resulting plots from molecular mass and PDI against monomer conversion for the polymerisations with $2 \mathrm{mmol} \cdot \mathrm{L}^{-1}$<smiles>C=C(C)C(=O)OC</smiles>

MMA<smiles>[R2]COC(=O)/C=C/c1ccccc1</smiles>
AIBN, $1 \mathrm{mmol} \cdot \mathrm{L}^{-1} \mathbf{R}_{6} \mathbf{3}$ and $10 \mathrm{mmol} \cdot \mathrm{L}^{-1} \mathbf{R}_{\mathbf{L}} \mathbf{3}$ are shown in the following figures. As in the examples before, the black symbols correspond to the obtained values from the THF-SEC-measurements; the filled red symbols equal the corrected values $\left(M_{n}{ }^{\text {corr. }}=M_{n} \cdot 1.52\right) .{ }^{(93)}$ The plots of the corresponding MWDs follow subsequently.

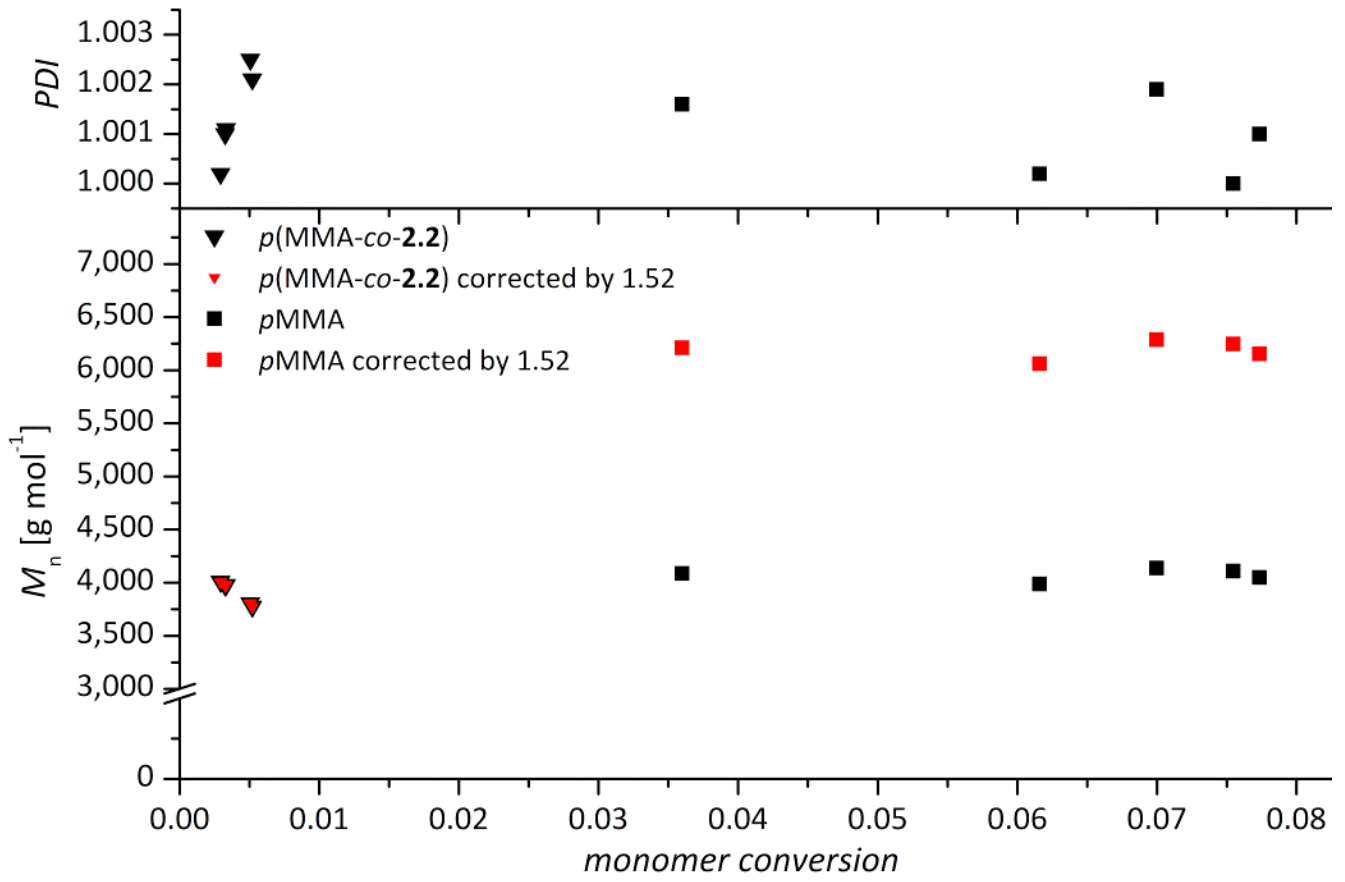

Figure 7-33: Development of molecular mass and PDI, determined by UV-detection on a THF-SEC, with monomer conversion in a homopolymerisation of MMA (squares) and a copolymerisation of MMA with 1 mol\% 2.2 (triangles) using $2 \mathrm{mmol} \cdot \mathrm{L}^{-1} \mathrm{AlBN}, 1 \mathrm{mmol} \cdot \mathrm{L}^{-1} \boldsymbol{R}_{6} 3$ and $10 \mathrm{mmol} \cdot \mathrm{L}^{-1} \boldsymbol{R}_{L} 3$ at $60^{\circ} \mathrm{C}$. 

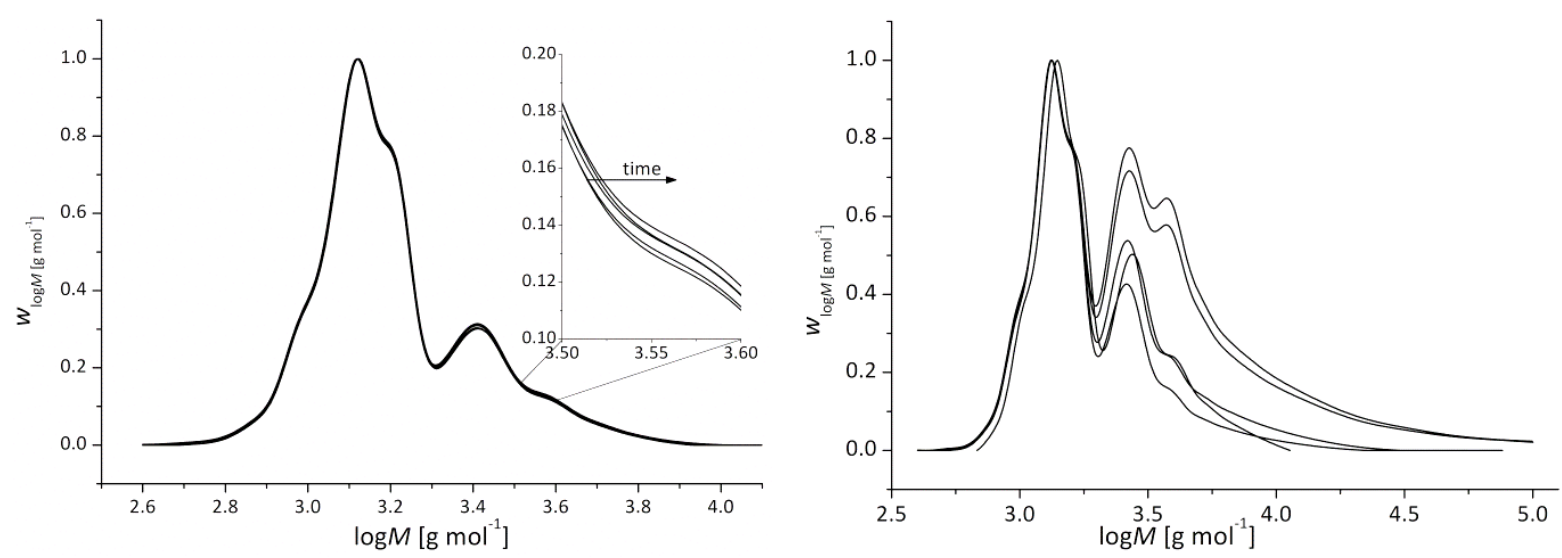

Figure 7-34: SEC-UV-data for the homopolymerisation of MMA (left) and the copolymerisation of MMA with 1 mol\% 2.2 (right) using $2 \mathrm{mmol} \cdot \mathrm{L}^{-1} \mathrm{AIBN}, 1 \mathrm{mmol} \cdot \mathrm{L}^{-1} \boldsymbol{R}_{6} 3$ and $10 \mathrm{mmol} \cdot \mathrm{L}^{-1} \boldsymbol{R}_{L} 3$ at $60{ }^{\circ} \mathrm{C}$

The MWDs for both polymerisations show no increase in the molecular weights. As can be seen in the zoom of the diagram of the homopolymerisation (Figure 7-34, left), the change between the different spectra with time (225 minutes) is insignificant small. For the copolymerisation with 3 mol\% a similar behaviour could be observed, as the following figures show.

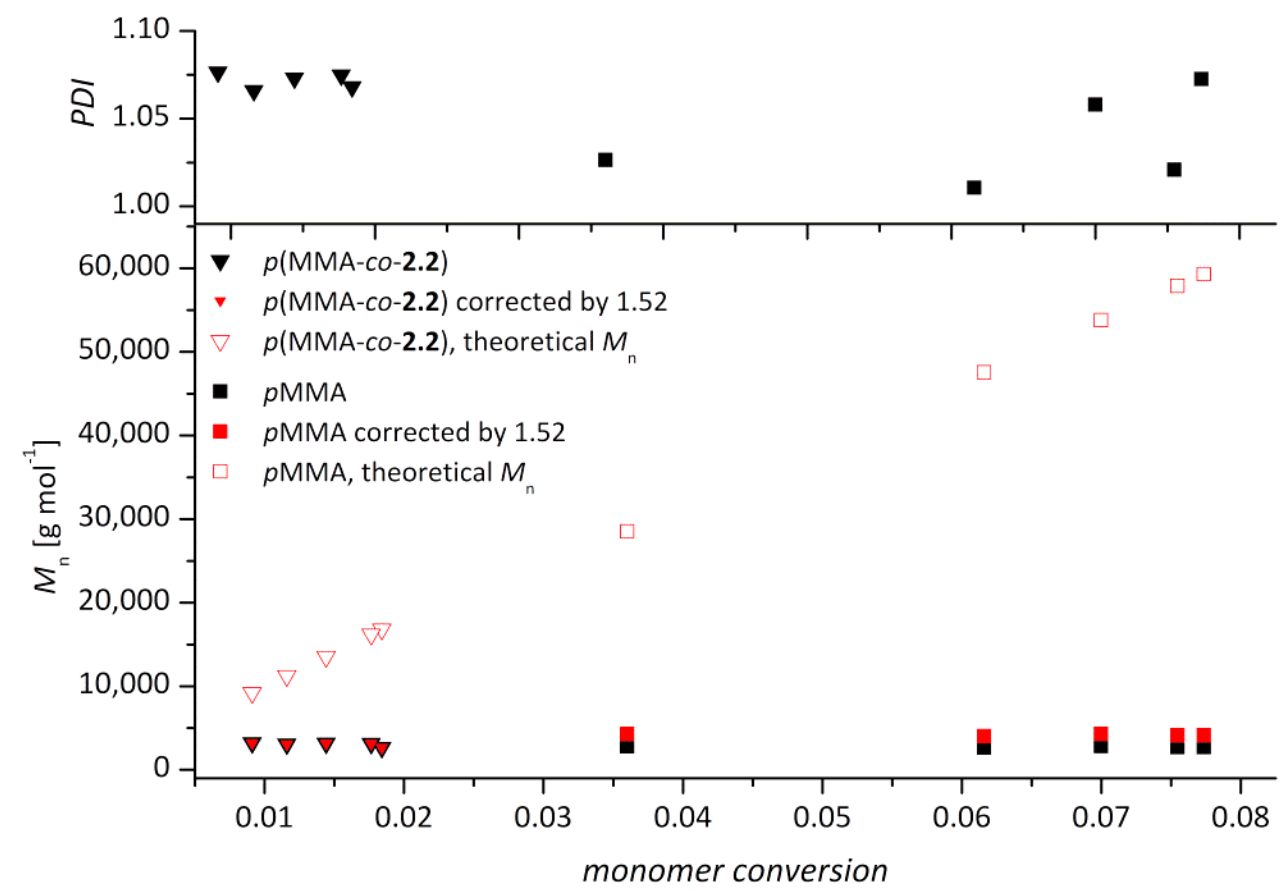

Figure 7-35: Development of molecular mass and PDI, determined by UV-detection on a THF-SEC, with monomer conversion in a homopolymerisation of MMA (squares) and a copolymerisation of MMA with 3 mol\% 2.2 (triangles) using $2 \mathrm{mmol} \cdot \mathrm{L}^{-1} \mathrm{AlBN}, 1 \mathrm{mmol} \cdot \mathrm{L}^{-1} \boldsymbol{R}_{6} 3$ and $10 \mathrm{mmol} \cdot \mathrm{L}^{-1} \boldsymbol{R}_{L} 3$ at $60^{\circ} \mathrm{C}$. 

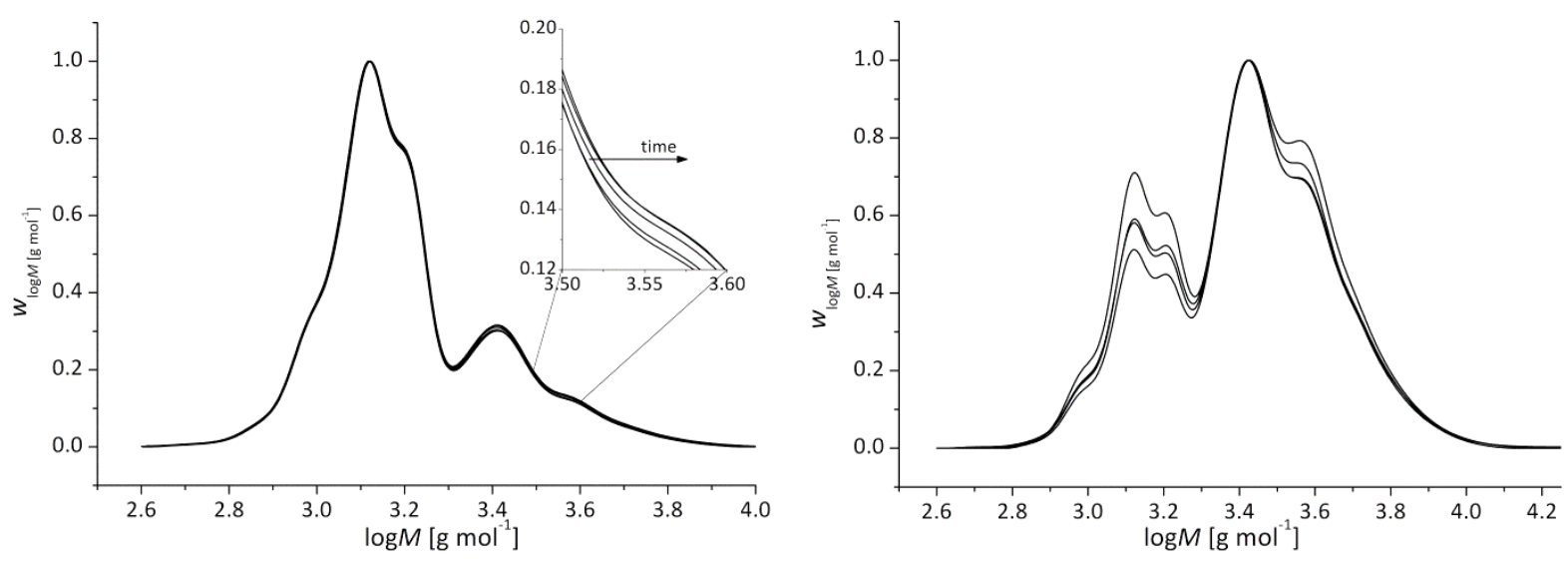

Figure 7-36: SEC-data for the homopolymerisation of MMA (left) and the copolymerisation of MMA with 3 mol\% 2.2 (right) using $2 \mathrm{mmol} \cdot \mathrm{L}^{-1} \mathrm{AlBN}, 1 \mathrm{mmol} \cdot \mathrm{L}^{-1} \boldsymbol{R}_{6} \mathbf{3}$ and $10 \mathrm{mmol} \cdot \mathrm{L}^{-1} \boldsymbol{R}_{L} \mathbf{3}$ at $60^{\circ} \mathrm{C}$.

Both examples show no increase of molecular mass and monomer conversion. Even in the homopolymerisation of MMA no change is observable which indicates that the reaction does not work in general which has to be a consequence of the small $k_{\mathrm{p}}$-value of MMA $\left(k_{\mathrm{p}}{ }^{\mathrm{MMA}}=272 \mathrm{~L} \cdot \mathrm{mol}^{-1} \cdot \mathrm{s}^{-1}\right) \cdot{ }^{(168)}$ It was hypothesised that the initialisation phase of the polymerisation was not completed and that the reaction was going to start on the contrary to the MMA-1.2copolymerisation where only a FRP could be observed, even with the same RAFT-agent. The measured molecular weights correspond with the molecular mass of the RAFT-agent.

\subsubsection{Copolymerisation of MA and Cinnamoyloxyethylmethacrylate}

The copolymerisation of MA with $\mathbf{2 . 2}$, using 3 mol\% of the UV-monomer and the RAFT-agent $\mathbf{R}_{\mathbf{6}} \mathbf{2}$, shows the same results as the one with MMA. Figure 7-37 illustrates the progress of monomer conversion and molecular mass. The reaction time was up to 25 hours<smiles>C=CC(=O)OC</smiles>
( $\equiv 1,500$ minutes) and no change in monomer conversion and molecular mass was observed. The obtained molecular weights correspond again with the mass of the RAFT-agent (Figure 7-38). 


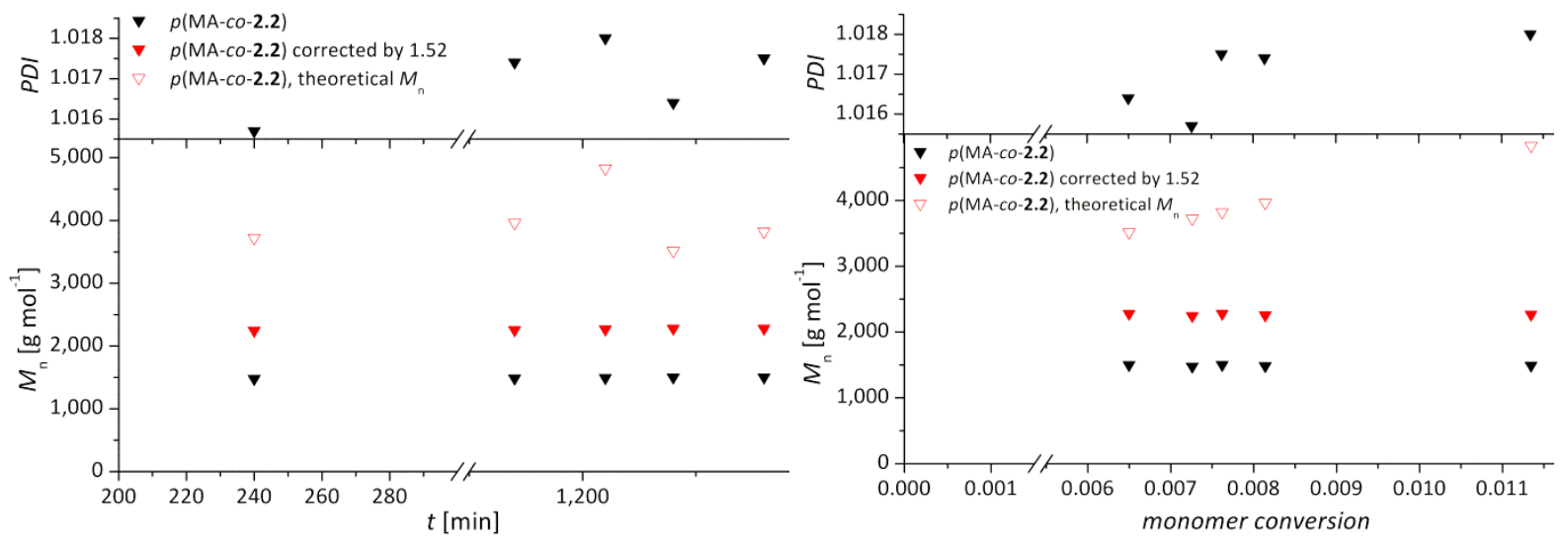

Figure 7-37: Development of molecular weight and PDI versus time (left) and versus monomer conversion (right) for a copolymerisation of MA and $3 \mathrm{~mol} \% \mathbf{2 . 2}$ using $3 \mathrm{mmol} \cdot \mathrm{L}^{-1} \mathrm{AlBN}, 4 \mathrm{mmol} \cdot \mathrm{L}^{-1} \boldsymbol{R}_{6} \mathbf{2}$ and $4 \mathrm{mmol} \cdot \mathrm{L}^{-1} \boldsymbol{R}_{L} \mathbf{2}$ at $60{ }^{\circ} \mathrm{C}$.

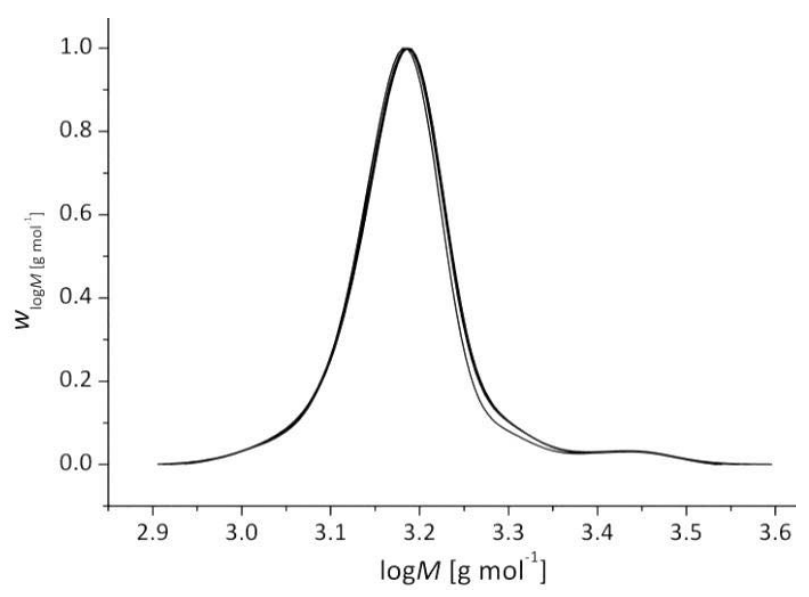

Figure 7-38: SEC-data for a copolymerisation of MA with $3 \mathrm{~mol} \% \mathbf{2 . 2}$ using $3 \mathrm{mmol} \cdot \mathrm{L}^{-1} \mathrm{AIBN}, 4 \mathrm{mmol} \cdot \mathrm{L}^{-1} \boldsymbol{R}_{6} \mathbf{2}$ and $4 \mathrm{mmol} \cdot \mathrm{L}^{-1} \boldsymbol{R}_{L} \mathbf{2}$ at $60^{\circ} \mathrm{C}$.

As this method of polymerisation was not successful, a literature-known procedure ${ }^{(131)}$ was applied and converted to a RAFT-process by addition of a linear RAFT-agent to test whether the monomer interacts at all with such an agent. The reaction was carried out up to 19 hours ( $\equiv 1,140$ minutes) at $80{ }^{\circ} \mathrm{C}$ with dibenzoylperoxide (DBPO) as initiator. The first sample was taken after 16.3 hours (इ 980 minutes). In Figure 7-39 the development of the molecular mass against monomer conversion and time is plotted. 

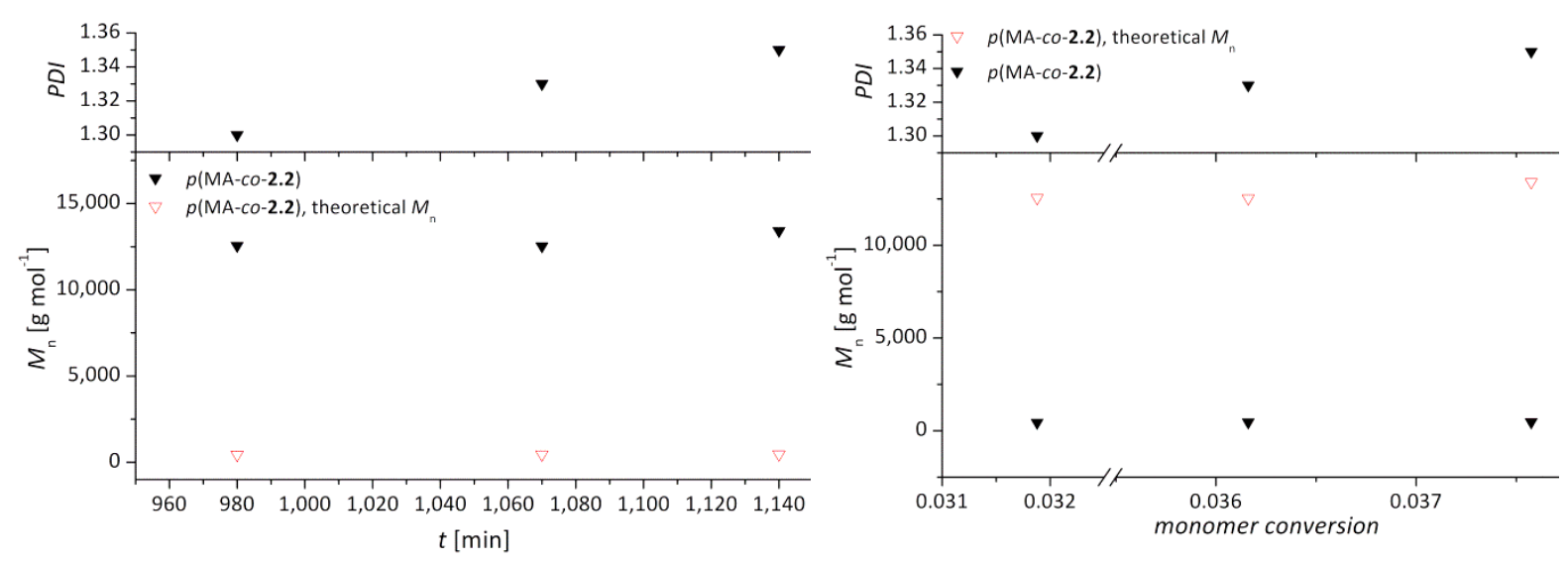

Figure 7-39: Development of molecular weight and $P D I$ versus time (left) and versus monomer conversion (right) for a copolymerisation of MA and $3 \mathrm{~mol} \% \mathbf{2 . 2}$ in toluene using $180 \mathrm{mmol} \cdot \mathrm{L}^{-1} \mathrm{DBPO}$ and $250 \mathrm{mmol} \cdot \mathrm{L}^{-1} \boldsymbol{R}_{L} \mathbf{2}$ at $80^{\circ} \mathrm{C}$.

It can be seen that at the point where the first sample was taken the molecular mass was approximately $12,500 \mathrm{~g} \cdot \mathrm{mol}^{-1}$ while the monomer conversion is only at $3 \%$. Up to the end of the experiment the molecular mass does not change. Although the PDI-values are in the characteristic range for a CRP for these three samples, they rise linearly which indicates that the polymerisation does not proceed in a controlled manner. This is supported by the uncharacteristic shape of the MWDs (Figure 7-40).

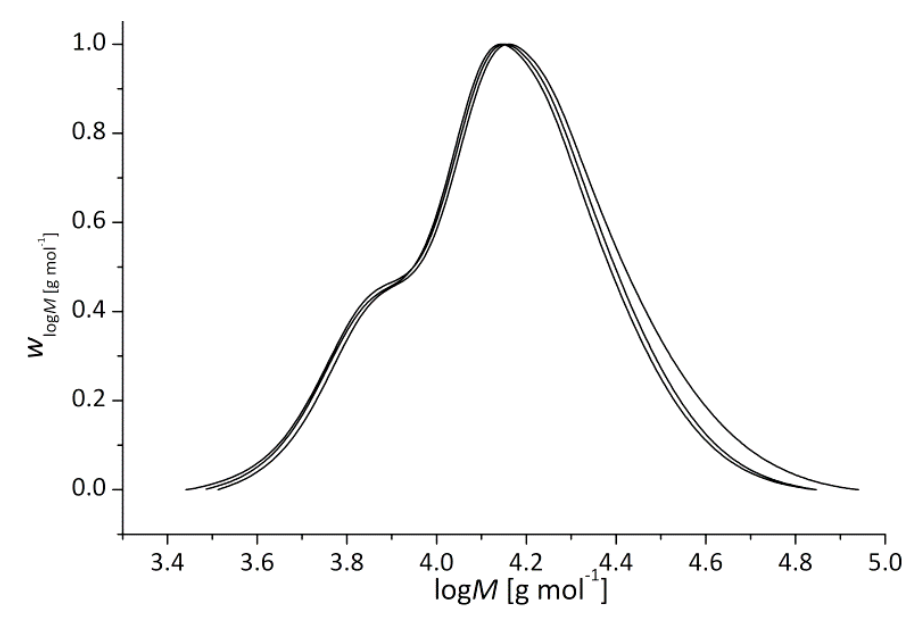

Figure 7-40: UV-SEC-data for a copolymerisation of MA with 3 mol\% 2.2 in toluene using $180 \mathrm{mmol} \cdot \mathrm{L}^{-1}$ DBPO and $250 \mathrm{mmol} \cdot \mathrm{L}^{-1} \boldsymbol{R}_{\mathbf{L}} \mathbf{2}$ at $80^{\circ} \mathrm{C}$.

It can be concluded that a copolymerisation of MMA or MA and $\mathbf{2 . 2}$ does not work under RAFT-conditions. Even if the reaction would start after longer reaction times, this would not be applicable for more step-syntheses as used within this project. These findings are surprising as MApolymerisations are well investigated and known to proceed in short periods of time. ${ }^{(168 ; 169)}$ 


\subsubsection{Homopolymerisation of Cinnamoylethyloxy-Monomers}

Different monomer systems were tested to synthesise a statistical copolymer from cinnamoyloxy-monomers and another basic monomer. All these experiments (chapter 7.2.1 - 7.2.3) failed; therefore the possibility of a triblock star polymer was under discussion. In that case the outer block of the star polymer is connected to the inner block via a block of UV-monomer units which serve as cross-linked belt to gain covalent stability in the system as it was published for vesicle-based systems. ${ }^{(170)}$ In this subchapter the homopolymerisation of the UV-monomers $\mathbf{2 . 1}$ and $\mathbf{2 . 2}$ under RAFT-conditions is investigated as this is the foundation for such a stabilising belt.

\subsubsection{Homopolymerisation of 2.1}

In a first experiment the polymerisation of $\mathbf{2 . 1}$ was mediated by the linear RAFT-agent $\mathbf{R}_{\mathbf{L}} \mathbf{2}$ to check whether this monomer interacts with the RAFT-agent to gain a controlled process. From the literature it can be seen that cinnamoyloxy-based monomers are known to polymerise under FRPconditions. ${ }^{(130 ; 131)}$

The reaction was carried out with $200 \mathrm{mmol} \cdot \mathrm{L}^{-1} \mathbf{2 . 1}, 10 \mathrm{mmol} \cdot \mathrm{L}^{-1} \mathrm{AIBN}, 8 \mathrm{mmol} \cdot \mathrm{L}^{-1} \mathbf{R}_{\mathbf{L}} \mathbf{2}$ in DMF at $80^{\circ} \mathrm{C}$ for up to 23 hours. In Figure 7-41 the development of the molecular weight versus the monomer conversion (left) and the MWDs (right) obtained by measuring the UV-signal (at $310 \mathrm{~nm}$ ) on a THF-SEC-set up are shown. After 17 hours the monomer conversion is below $1 \%$ and does not increase any further up to 23 hours. The molecular weights are all around 3,500 $\mathrm{g} \cdot \mathrm{mol}^{-1}$ which corresponds to the mass of the RAFT-agent plus 13 added monomer units.
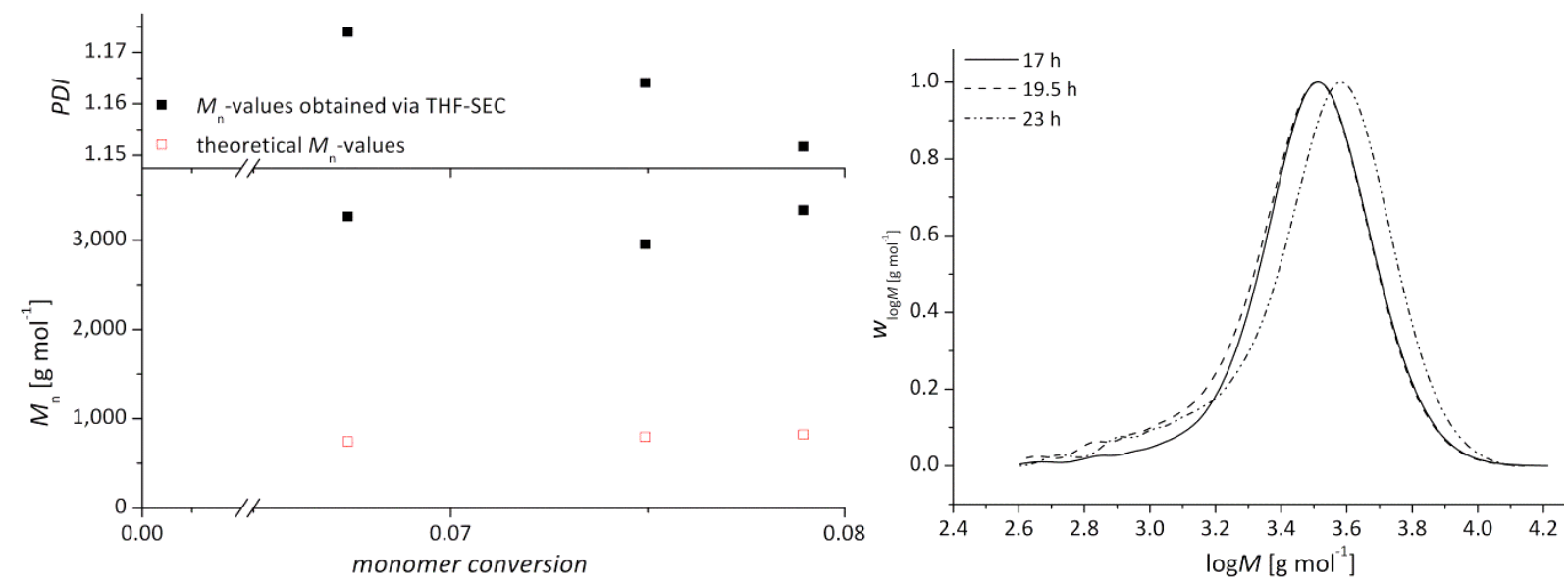

Figure 7-41: Development of the molecular weights with the monomer conversion (left) and the corresponding SEC-UVtraces (right) of a homopolymerisation of 2.1 with $200 \mathrm{mmol} \cdot \mathrm{L}^{-1} \mathbf{2 . 1}, 10 \mathrm{mmol} \cdot \mathrm{L}^{-1} \mathrm{AlBN}, 8 \mathrm{mmol} \cdot \mathrm{L}^{-1} \boldsymbol{R}_{L} \mathbf{2}$ in DMF at $80{ }^{\circ} \mathrm{C}$. 
Even though the PDI-values are in the characteristic range for a RAFT-polymerisation, monomer conversion and molecular mass indicate that the system does not work under RAFTconditions.

\subsubsection{Homopolymerisation of 2.2}

In parallel the homopolymerisation of $\mathbf{2 . 2}$ mediated by $\mathbf{R}_{\mathbf{L}} \mathbf{2}$ was investigated. The reaction was carried out once at $60^{\circ} \mathrm{C}$ in toluene and once at $80^{\circ} \mathrm{C}$ in DMF. Figure 7-42 shows the plot of molecular mass and PDI against the monomer conversion (left) and against the reaction time (right). The corresponding SEC-spectra are included in Figure 7-43.
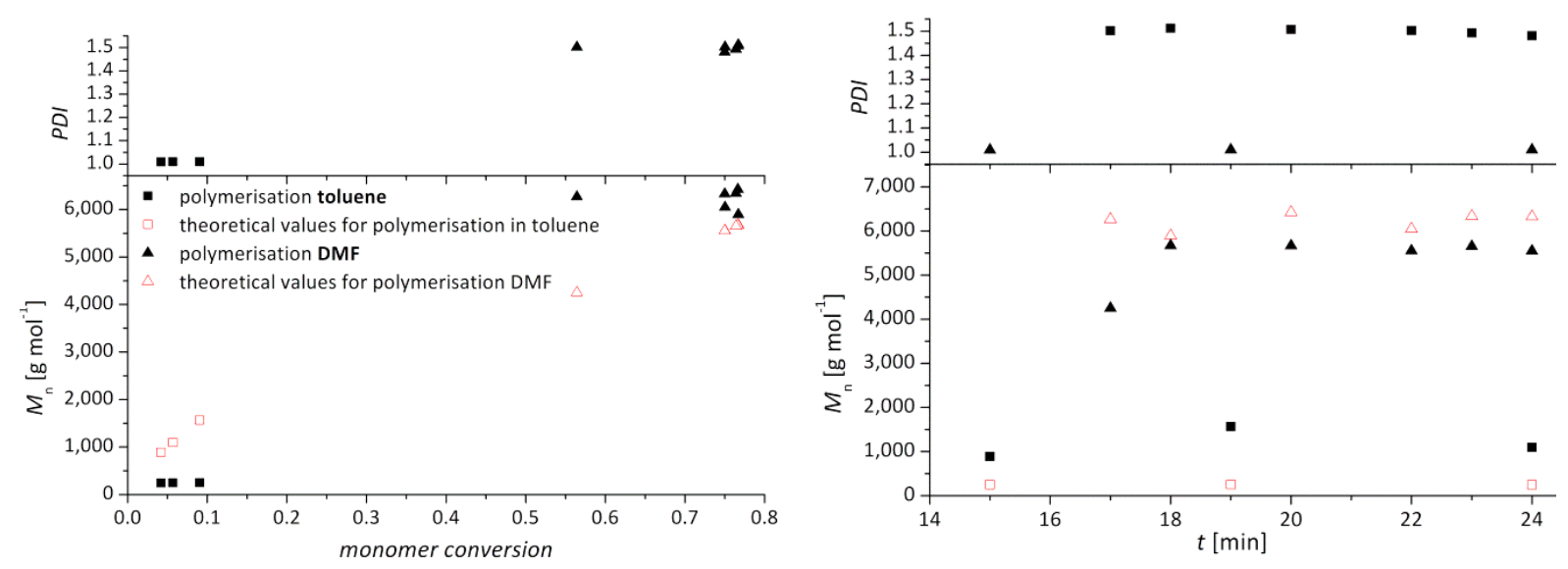

Figure 7-42: Developments of the molecular mass and the PDI with monomer conversion (left) and with reaction time (right) for homopolymerisations of 2.21 ) with $5,000 \mathrm{mmol} \cdot \mathrm{L}^{-1} \mathbf{2 . 2}, 6 \mathrm{mmol} \cdot \mathrm{L}^{-1} \mathrm{AlBN}, 90 \mathrm{mmol} \cdot \mathrm{L}^{-1} \boldsymbol{R}_{L} \mathbf{2}$ in toluene at $60{ }^{\circ} \mathrm{C}$ (squares) and 2) $190 \mathrm{mmol} \cdot \mathrm{L}^{-1} \mathbf{2 . 2}, 8 \mathrm{mmol} \cdot \mathrm{L}^{-1} \mathrm{AIBN}$ and $7 \mathrm{mmol} \cdot \mathrm{L}^{-1} \boldsymbol{R}_{L} \mathbf{2}$ in DMF at $80{ }^{\circ} \mathrm{C}$ (triangles).
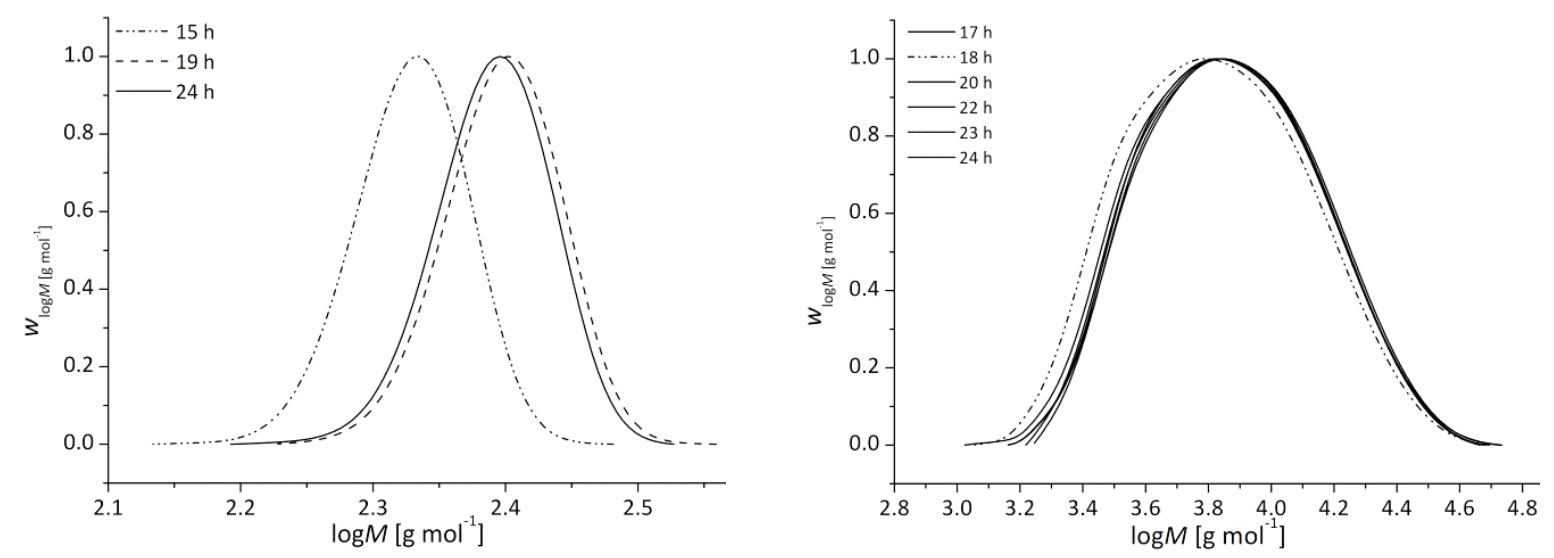

Figure 7-43: SEC-UV-data for the homopolymerisations of 2.2 1) with $5,000 \mathrm{mmol} \cdot \mathrm{L}^{-1} \mathbf{2 . 2}, 6 \mathrm{mmol} \cdot \mathrm{L}^{-1} \mathrm{AlBN}, 90 \mathrm{mmol} \cdot \mathrm{L}^{-1} \boldsymbol{R}_{L} \mathbf{2} \mathrm{in}$ toluene at $60^{\circ} \mathrm{C}$ (left) and 2) $190 \mathrm{mmol} \cdot \mathrm{L}^{-1} \mathbf{2 . 2}, 8 \mathrm{mmol} \cdot \mathrm{L}^{-1} \mathrm{AlBN}$ and $7 \mathrm{mmol} \cdot \mathrm{L}^{-1} \boldsymbol{R}_{L} \mathbf{2}$ in DMF at $80{ }^{\circ} \mathrm{C}$ (right). 
The polymerisation carried out at $60{ }^{\circ} \mathrm{C}$ does not start at all. The obtained molecular weights (ca $250 \mathrm{~g} \cdot \mathrm{mol}^{-1}$ ) correspond with the molecular weight of the RAFT-agent $\left(M=298.53 \mathrm{~g} \cdot \mathrm{mol}^{-1}\right)$. The polymerisation at $80^{\circ} \mathrm{C}$ starts but the molecular weight reaches a plateau at a monomer conversion of approximately $80 \%$. It cannot be proven that the polymerisation proceeds in a controlled manner as the SEC-curves show an untypical shape and a linear growth of the molecular weights could not be observed. Against a controlled behaviour stands the sample with a monomer conversion of $55 \%$. Already at that point of the polymerisation the molecular weight is $6,000 \mathrm{~g} \cdot \mathrm{mol}^{-1}$ and stays in this range until a conversion of $80 \%$ is reached.

To check whether an increase of primary radicals accelerates the start of the reaction and improves the controlled process, a homopolymerisation of $\mathbf{2 . 2}$ mediated by a six-functional RAFTagent was carried out. Figure 7-44 displays the development of the molecular weight and the PDIvalues versus monomer conversion (left) and reaction time (right) for the 2.2-polymerisation with a fourfold increased amount of AIBN.
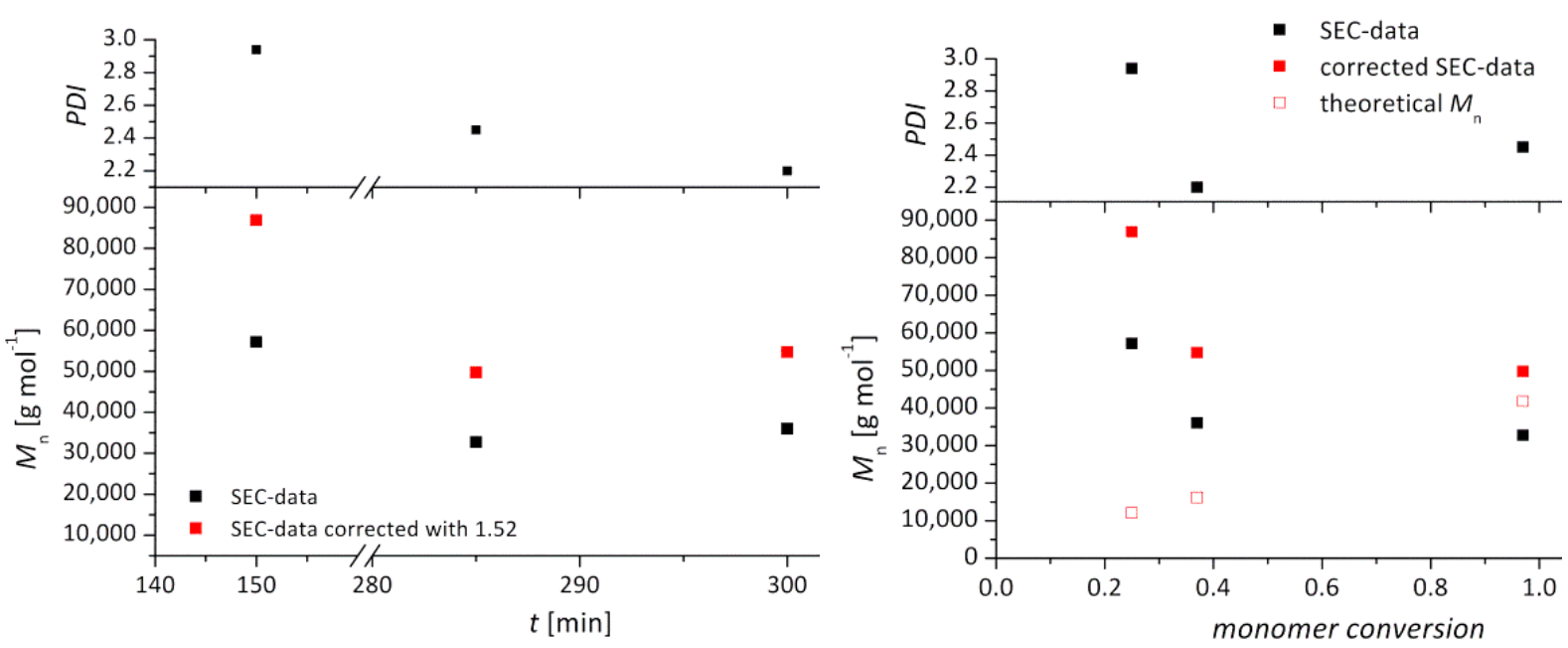

Figure 7-44: Development of the molecular weight with reaction time (left) and monomer conversion (right) for a homopolymerisation of 2.2 with $25 \mathrm{mmol} \cdot \mathrm{L}^{-1} \mathrm{AIBN}, 11 \mathrm{mmol} \cdot \mathrm{L}^{-1} \boldsymbol{R}_{6} \mathbf{3}$ and $1,800 \mathrm{mmol} \cdot \mathrm{L}^{-1} 2.2 \mathrm{in} \mathrm{DMF}$ at $60{ }^{\circ} \mathrm{C}$.

The reaction shows no controlled behaviour at all. The PDI-values are not in the range of a CRP. No linear growth of the molecular weight with reaction time or monomer conversion can be observed. The SEC-data (Figure 7-45) support this finding. In these SEC-spectra the signals of the six-functional RAFT-agent and of the monomer itself can be seen. It also shows the large error in the determination of the monomer conversion as even in the spectrum of the sample with determined complete monomer conversion the signal of the monomer $\mathbf{2 . 2}$ was found. This is a consequence of the difficult work-up of those polymers due to the high boiling point of the monomer. 


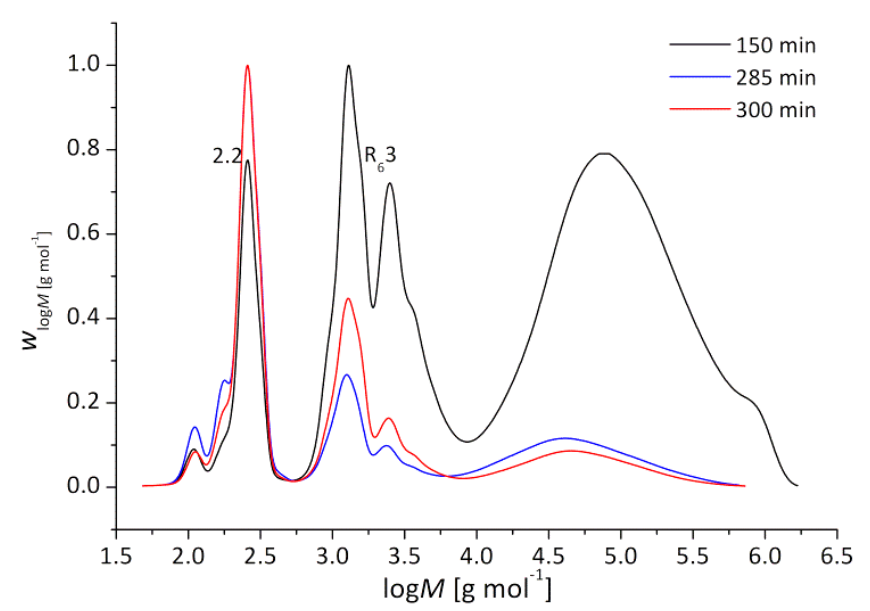

Figure 7-45: SEC-data of the homopolymerisation of 2.2 with $25 \mathrm{mmol} \cdot \mathrm{L}^{-1} \mathrm{AlBN}, 11 \mathrm{mmol} \cdot \mathrm{L}^{-1} \boldsymbol{R}_{6} \mathbf{3}$ and $1,800 \mathrm{mmol} \cdot \mathrm{L}^{-1} \mathbf{2 . 2} \mathrm{in}$ DMF at $60^{\circ} \mathrm{C}$.

It can be summarised that the RAFT homopolymerisation of $\mathbf{2 . 2}$ is not a reliable system to work with. Besides the incomputable reaction behaviour the work-up of these polymers is rather difficult. Therefore the idea of triblock star polymers is excluded from this project.

\subsection{Copolymerisation with Courmarin-based Monomers}

\subsubsection{Copolymerisation of BA and 7-(2-methacryloyloxyethoxy)-4-methylcourmarin}

The third group of tested UV-monomers are courmarin-based monomers. As in the tests before, the copolymerisations were performed as bulk polymerisation. $\mathbf{3 . 1}$ is only sparingly soluble in BA. It was discovered that the solubility limit of $\mathbf{3 . 1}$ is $60 \mathrm{mmol} \cdot \mathrm{L}^{-1}$ in $\mathrm{BA}$ at room temperature. Therefore, polymerisations were carried out with 0.5 and 0.9 mol\%<smiles>C=CC(=O)OCCCC</smiles><smiles>C=C(C)C(=O)OCCOc1ccc2c(C)cc(=O)oc2c1</smiles>
of $\mathbf{3 . 1}$ regarding the initial amount of BA. The RAFT-agent $\mathbf{R}_{6} \mathbf{2}$ was used as mediating agent. Again the copolymerisation is compared with the homopolymerisation of BA and the red symbols correspond with the corrected values of the molecular mass. ${ }^{(93)}$ The Figures 7-45 and 7-46 show the resulting developments of these copolymerisations at $60^{\circ} \mathrm{C}$ with a reaction time of up to 115 minutes. 


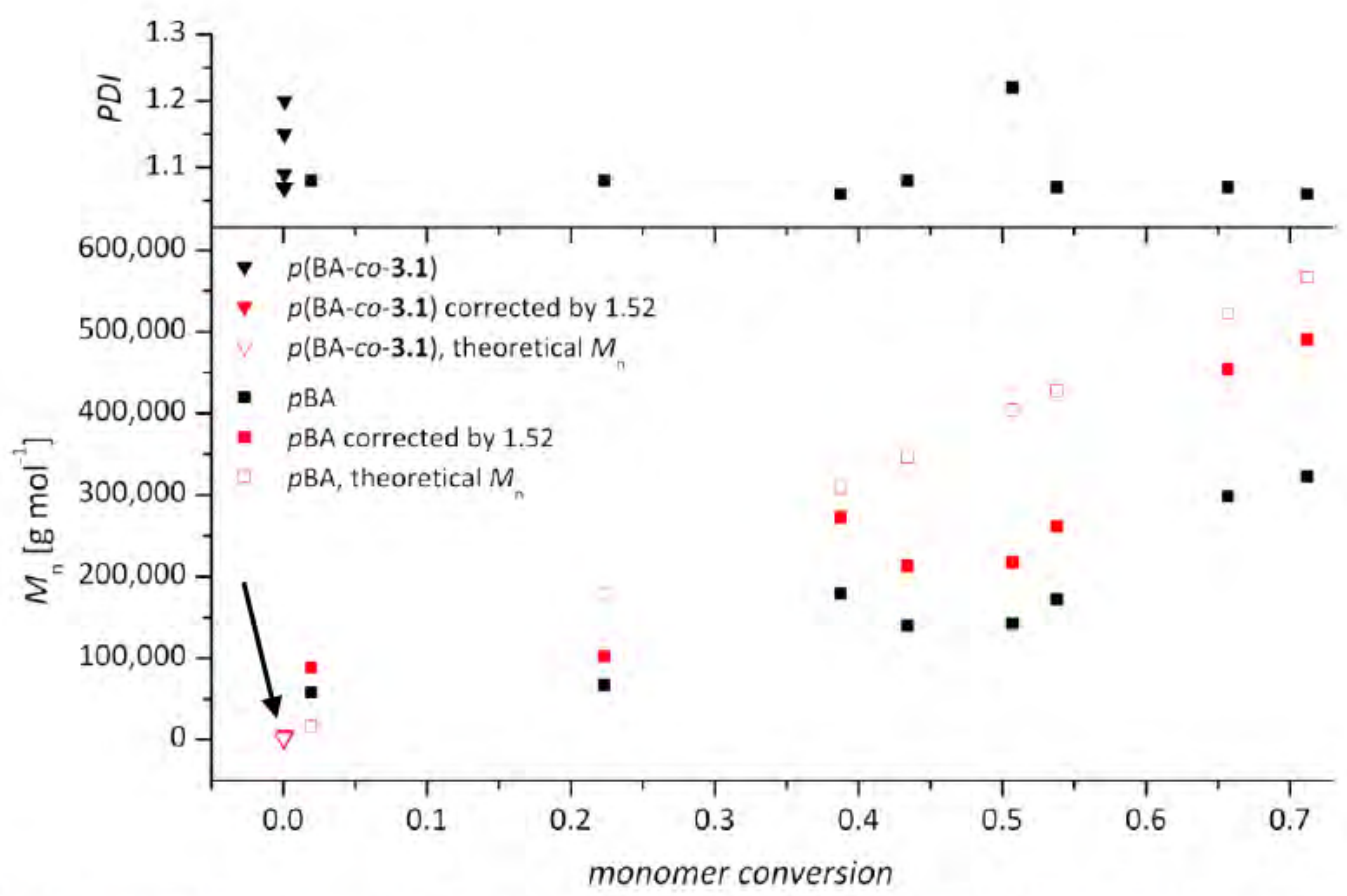

Figure 7-46: Development of molecular mass and PDI, determined by UV-detection on a THF-SEC, with monomer conversion in a homopolymerisation of BA (squares) and a copolymerisation of BA with $0.5 \mathrm{~mol} \% 3.1$ (triangles) using $3 \mathrm{mmol} \cdot \mathrm{L}^{-1} \mathrm{AlBN}$, $1 \mathrm{mmol} \cdot \mathrm{L}^{-1} \boldsymbol{R}_{6} \mathbf{2}$ and $3 \mathrm{mmol} \cdot \mathrm{L}^{-1} \boldsymbol{R}_{L} \mathbf{2}$ at $60^{\circ} \mathrm{C}$. Only the data of the star polymers are shown here.

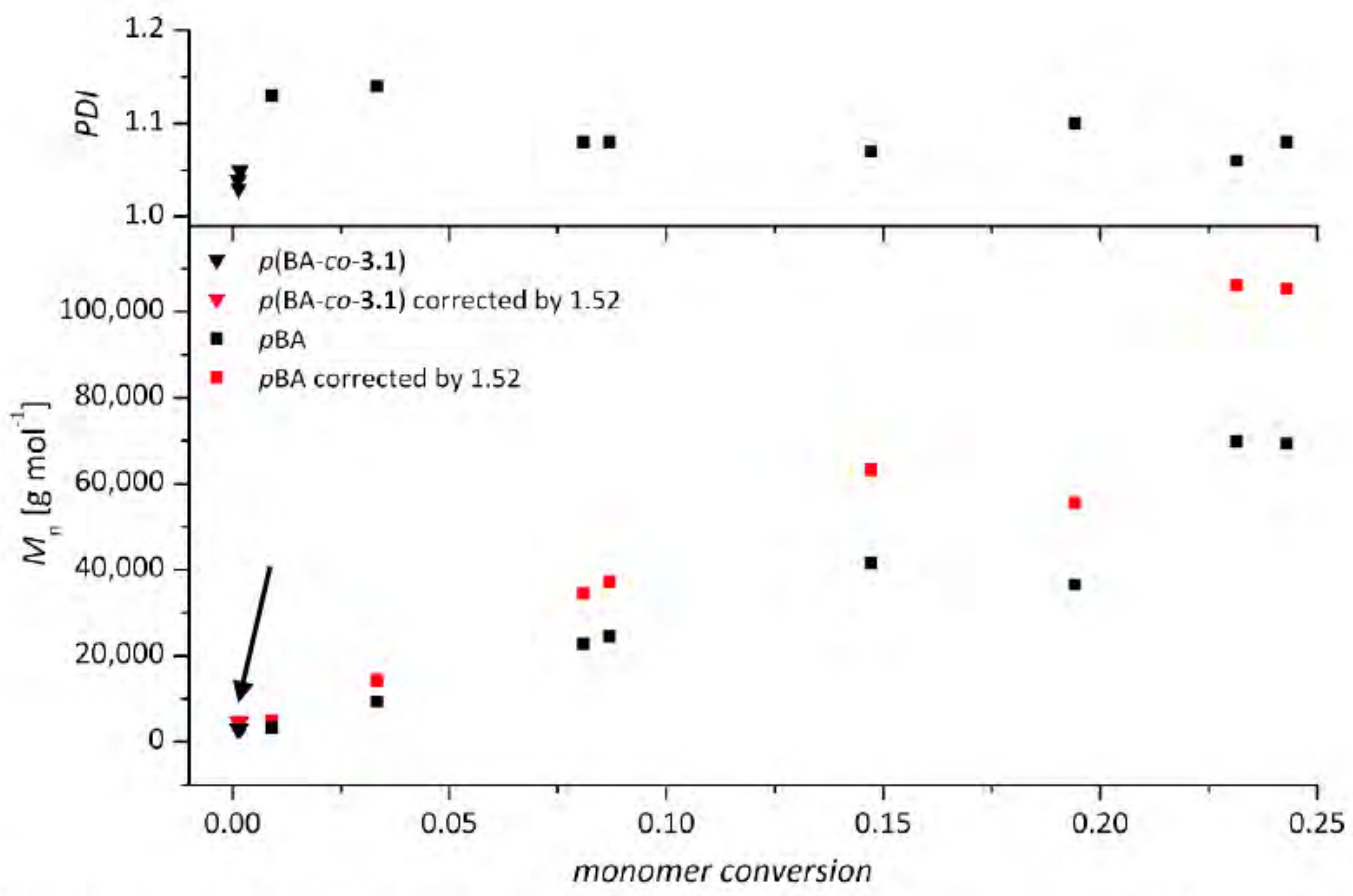

Figure 7-47: Development of molecular mass and PDI, determined by UV-detection on a THF-SEC, with monomer conversion in a homopolymerisation of $B A$ (squares) and a copolymerisation of $B A$ with $0.9 \mathrm{~mol} \% 3.1$ (triangles) using $2 \mathrm{mmol} \cdot \mathrm{L}^{-1} \mathrm{AlBN}$, $1 \mathrm{mmol} \cdot \mathrm{L}^{-1} \boldsymbol{R}_{6} 2$ and $9 \mathrm{mmol} \cdot \mathrm{L}^{-1} \boldsymbol{R}_{L} 2$ at $60^{\circ} \mathrm{C}$. Shown are only the data corresponding to the star polymer. 
As can be seen from these two examples, the addition of $\mathbf{3 . 1}$ seems to inhibit the polymerisation. SEC-analyses revealed a molecular mass of approximately $2,000 \mathrm{~g} \cdot \mathrm{mol}^{-1}$ for all samples of the copolymerisations, which corresponds with the molecular weight of $\mathbf{R}_{6} \mathbf{2}$ $\left(M=1,864.84 \mathrm{~g} \cdot \mathrm{mol}^{-1}\right)$. To check whether the reaction does not take place at all or induction (chapter 3.2.3) occurs, a long-term experiment was carried out with 0.9 mol\% 3.1 and the same concentrations of RAFT and AIBN as before. The plot of monomer conversion versus reaction time for this experiment is shown in Figure 7-48 left.
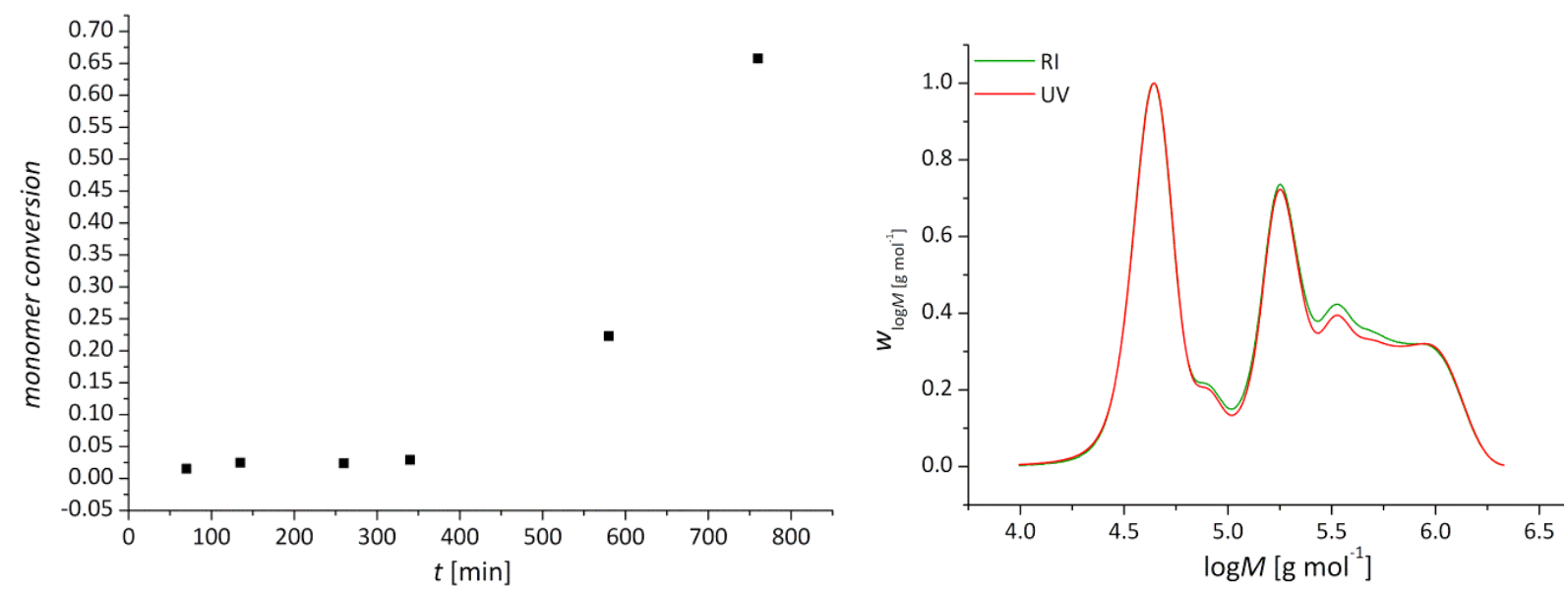

Figure 7-48: Development of monomer conversion with time (left) and SEC-spectra (right) of the copolymerisation of BA with $0.9 \mathrm{~mol} \% 3.1$ using $2 \mathrm{mmol} \cdot \mathrm{L}^{-1} \mathrm{~A} / \mathrm{BN}, 1 \mathrm{mmol} \cdot \mathrm{L}^{-1} \boldsymbol{R}_{6} \mathbf{2}$ and $9 \mathrm{mmol} \cdot \mathrm{L}^{-1} \boldsymbol{R}_{L} \mathbf{2}$.

Again, the molecular weights of the first samples correspond with the molecular weight of the RAFTagent. The polymerisation starts later than anticipated. After approximately 600 minutes the conversion is increasing sharply. The SEC-spectra of the remaining two samples (Figure 7-48, right) show the start of the polymerisation but from the abrupt rise in monomer conversion and the strongly dispersed SEC-spectra it can be concluded that the reaction is not well controlled.

It can be summarised that the copolymerisation of BA and $\mathbf{3 . 1}$ does not work sufficiently under RAFT-conditions and is not applicable for the synthesis of statistical copolymers with BA due to the low control and the long reaction times. It can be speculated that the difference in the polymerisation behaviour between the acrylate and the methacrylate are too high. A copolymerisation with MMA should be more promising as this is literature-known to copolymerise with $\mathbf{3 . 1}$ under FRP-conditions. Furthermore, it was reported that $\mathbf{3 . 1}$ forms statistical copolymers with monomers like 2-(dimethylamino)ethyl methacrylate. ${ }^{(170)}$ 


\subsubsection{Copolymerisation of NIPAam and 7-[4-(Trifluoromethyl)courmarin]methylacrylamide}

7-[4-(Trifluoromethyl)courmarin]methylacrylamide (3.2) is the only UV-monomer used within this project which can be purchased from Sigma-Aldrich. It is assumed that the copolymerisation of NIPAam and $\mathbf{3 . 2}$ works sufficiently as they both hold the same monomeric unit. It is not known to be

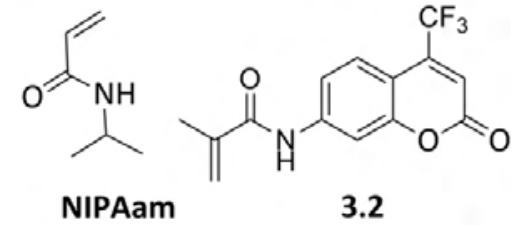
polymerised under RAFT-conditions. The trifluormethyl group next to the UV-reactive double bonds should improve the reactivity of this bond in the [2+2]-cycloaddition step.

The polymerisation of NIPAam and $\mathbf{3 . 2}$ was mediated by $\mathbf{R}_{6} \mathbf{3}$ in DMF at $60{ }^{\circ} \mathrm{C}$ up to 24 hours. From the first few samples it was not possible to precipitate any polymer. After being left to evaporate no oligo- or polymer could be obtained from the residue. After 24 hours reaction time the reaction mixture turned abruptly solid. The resulting polymer is not soluble in THF or DMac; therefore it was not possible to measure a SEC-spectrum. Furthermore it was found to be insoluble in chloroform, methanol, acetone, DMF and DMSO; therefore no NMR-analysis could be performed.

No further investigations were carried out on the NIPAam-3.2-copolymerisation because $\mathbf{3 . 2}$ is expensive and this pre-experiment showed no satisfying results.

\subsection{Conclusion}

In this chapter the results of copolymerisations from different monomer combinations mediated by six-functional RAFT-agents are presented. In general the polymerisations were carried out with AIBN as initiator and at $60^{\circ} \mathrm{C}$. Table 7-1 summarises the findings of these investigations.

Table 7-1: Summary of the different tested copolymerisations and their success by means of RAFT polymerisation. Key: + positive, - negative, / not tested.

\begin{tabular}{c|c|c|c} 
monomers & $\begin{array}{c}\text { DMIs } \\
\text { 1.1 and 1.2 }\end{array}$ & $\begin{array}{c}\text { CAs } \\
\text { 2.1 and 2.2 }\end{array}$ & $\begin{array}{c}\text { Courmarins } \\
\mathbf{3 . 1} \text { and } \mathbf{3 . 2}\end{array}$ \\
\hline BA & + & - & - \\
MA & + & - & $/$ \\
MMA & - & - & $/$ \\
NIPAam & + & $/$ & - \\
homopolymerisation & $/$ & - & $/$
\end{tabular}


It was found that the copolymerisation of BA and the DMI 1.1 works well under RAFTconditions in manageable periods of time yielding defined molecular weights and narrow MWDs. Similar findings were made for the copolymerisation of NIPAam and 1.1. These two systems will be investigated further in this thesis.

The copolymerisation of $\mathbf{1 . 1}$ and MA showed characteristics of a CRP but worked not sufficiently enough in this scope-scanning process to be continued here. For the remaining comonomers no successful RAFT-process could be identified, even though some of them are known to copolymerise with the basic monomers by means of FRP. They were excluded from this project.

The molecular weight correction of the star polymers via the evaluation of linear polymer generated in the same process by addition of mono-functional RAFT-agent yielded in all cases molecular weights below the experimentally obtained weights of the star polymers themselves. Therefore the correction via $K=1.52^{(69)}$ is used throughout the project. 


\section{Copolymerisations with $N$-Ethylacrylate-3,4-dimethylmaleimide}

In this chapter, the copolymerisations of $n$-butylacrylate (BA) and $N$-isopropylacrylamide (NIPAam) with the UV-reactive monomer $N$-ethylacrylate-3,4-dimethylmaleimide (1.1) will be more closely examined (Figure 8-1): The scope-scanning, discussed in the previous chapter, revealed that both monomers polymerise well with $\mathbf{1 . 1}$ under RAFT-conditions in manageable periods of time and with very good yields in monomer conversion and molecular weight.
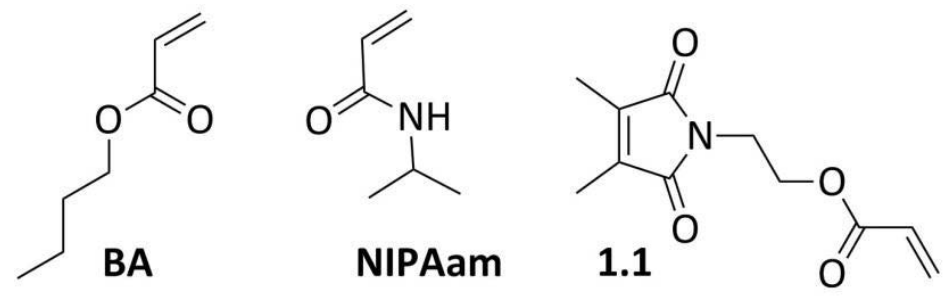

Figure 8-1: The monomers $n$-butylacrylate, $\mathrm{N}$-isopropylacrylamide and $\mathrm{N}$-ethylacrylate-3,4-dimethylmaleimide

$p \mathrm{BA}$ was chosen as the hydrophobic moiety of the star polymer as it is known to be used as encapsulate of hydrophilic drugs. ${ }^{(171-174)}$ Additionally, it is easily purified through removal of the stabiliser via filtration with aluminium oxide, is well known in RAFT polymerisations ${ }^{(175)}$ and polymerises in manageable periods of time due to its high $k_{\mathrm{p}}$-value $\left(k_{\mathrm{p}}^{\mathrm{BA}}=1.5 \cdot 10^{4} \mathrm{~L} \cdot \mathrm{mol}^{-1} \cdot \mathrm{s}^{-1}\right) \cdot{ }^{(168)}$ RAFT-star polymerisation of BA was investigated by Boschmann in his dissertation ${ }^{(21)}$ and various publications. ${ }^{(47 ; 93)}$

pNIPAam, ${ }^{(176)}$ on the other hand, is a hydrophilic polymer whose properties are strongly depended on the surroundings. Below a temperature of $32{ }^{\circ} \mathrm{C}$ the polymer is water-soluble. Rising the temperature above this critical barrier, the so-called lower critical solution temperature (LCST), the coil collapses. ${ }^{(177)}$ The polymer is no longer soluble in water. The same holds true for $\mathrm{pH}$-values above $5 .{ }^{(156 ; 178 ; 179)}$ The swelling behaviour in different media as well as the transition at the LCST were intensely investigated by Kuckling et al. ${ }^{(102 ; 114 ; 118 ; 175)}$ Despite an LCST of $32{ }^{\circ} \mathrm{C}$ it finds application in the establishing of nano-scaled drug delivery systems, as shown by the works of Xu et al. ${ }^{(157)}$ and Chang et al. ${ }^{(156)}$ NIPAam can be purified easily by re-crystallisation from hexane, is also known in RAFT-polymerisations ${ }^{(175 ; 180)}$ and proved in the scope-scanning experiments that polymerisations of NIPAam $\left(k_{\mathrm{p}} \approx 2 \cdot 10^{4} \mathrm{~L} \cdot \mathrm{mol}^{-1} \cdot \mathrm{s}^{-1}\right)^{(181)}$ are successful with the applied RAFT-agents and 1.1. Furthermore, both polymers are known to form blockcopolymers, e.g. Vega-Rios and LiceaClaveríe showed a successful build-up of NIPAam-BA-blockcopolymers by means of RAFTpolymerisation. ${ }^{(181)}$ 


\subsection{Copolymerisation of BA and 1.1}

Firstly the copolymerisations with BA are investigated regarding the influence of the addition of $\mathbf{1 . 1}$ on the reaction. Here reactions carried out under FRP- and RAFT-conditions are considered. All determinations of the molecular weights of BA-based polymer samples were carried out on a THFSEC set up using a universal calibration with linear $p$ Sty standards and the $\mathrm{MH}$ parameters of $p \mathrm{BA} .{ }^{(182)}$

In the following example the FRP of BA and of BA with 1 mol\% $\mathbf{1 . 1}$ $\left(c_{1.1}=35 \mathrm{mmol} \cdot \mathrm{L}^{-1}\right)$ are compared. The polymerisations were conducted with $0.9 \mathrm{mmol} \cdot \mathrm{L}^{-1} \mathrm{AlBN}$ at $60{ }^{\circ} \mathrm{C}$ in toluene as the reaction mixture is getting very fast solid which inhibits a determination of the monomer conversion. Figure 8-2 displays the molecular weights and the PDI-values determined with the SEC-RI set up with monomer conversion for this FRP. The blue symbols correspond with the data of the copolymerisation, the red with the homopolymerisation.

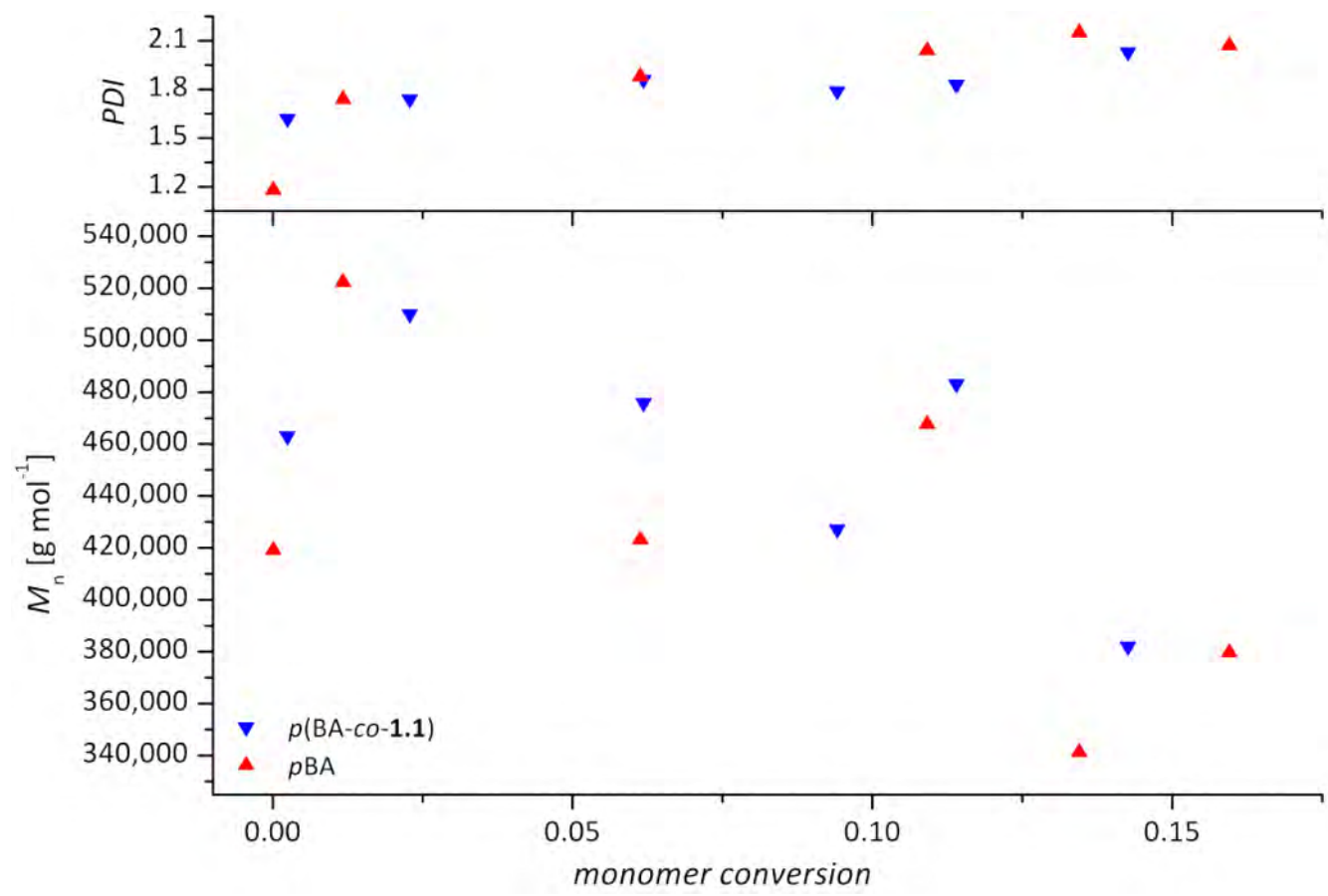

Figure 8-2: Development of the molecular weight (obtained by RI-detection) and the PDI versus the monomer conversion of a FRP of BA (red) and BA with $35 \mathrm{mmol} \cdot \mathrm{L}^{-1} 1.1$ (blue) using $0.9 \mathrm{mmol} \cdot \mathrm{L}^{-1}$ AlBN in toluene at $60^{\circ} \mathrm{C}$.

As noted in chapter 7.1.1, the developments of both values correspond with the expectations of a FRP. No linear increase of the molecular weights with the monomer conversion can be observed. Furthermore the PDI-values are in the characteristic range for a FRP-process (chapter 3.4). An influence of $\mathbf{1 . 1}$ on the polymerisation cannot be observed from this diagram. In both cases the PDIvalues are approximately 1.8 and the molecular weights scatter around $450,000 \mathrm{~g} \cdot \mathrm{mol}^{-1}$. To have a measure to testify the influence of $\mathbf{1 . 1}$ on the reaction, the plot of monomer conversion versus reaction time can be used. This plot indicates the consumed monomer molecules per defined time 
unit. If $\mathbf{1 . 1}$ has an influence on the reaction behaviour, the change has to be observed in this plot (Figure 8-3). In the case that the two reactions proceed with different velocities the slopes of the lines would be dissimilar.

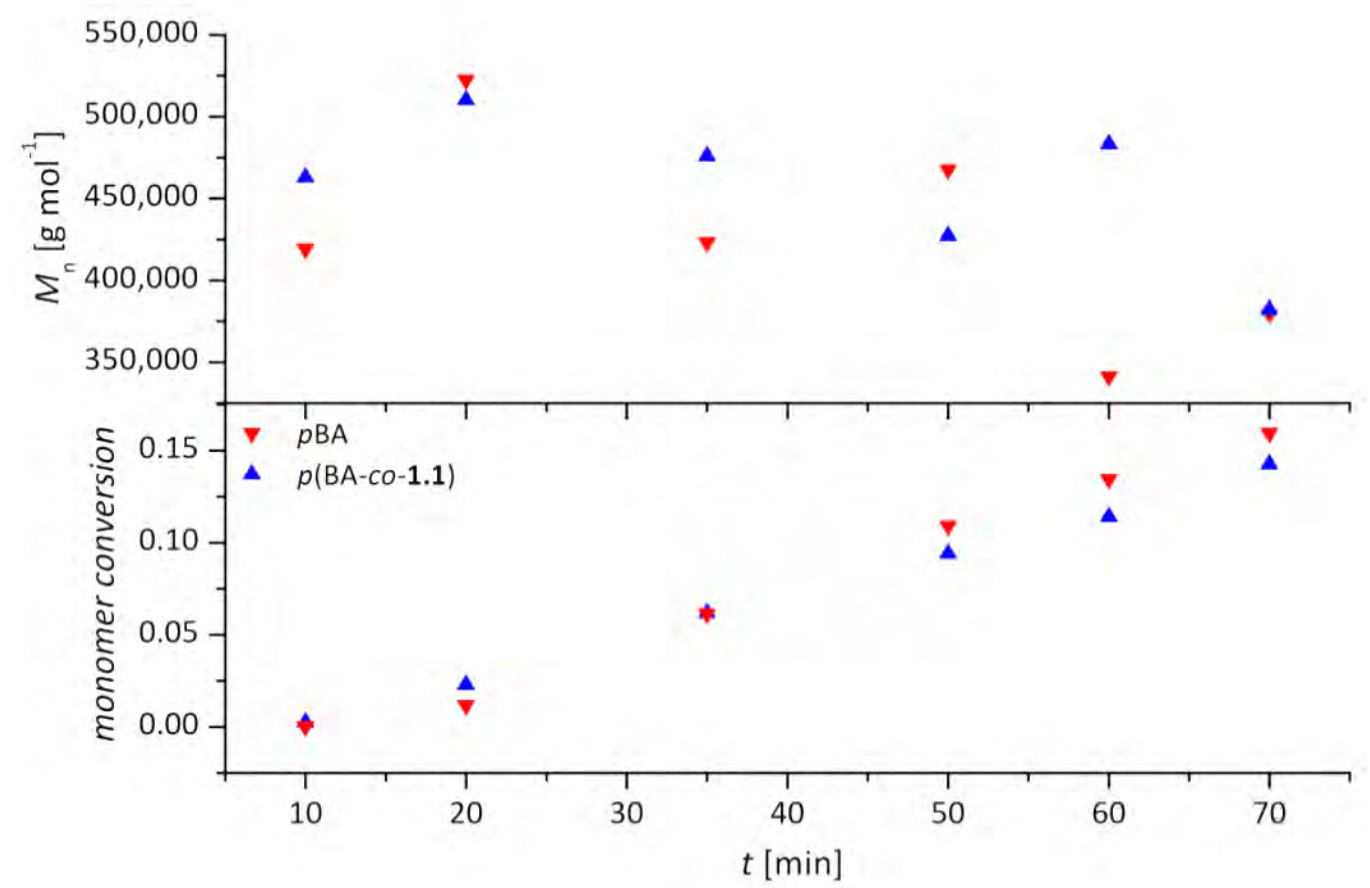

Figure 8-3: Development of the molecular weight (obtained by RI-detection) and the monomer conversion with reaction time of a FRP of BA (red) and BA with $1 \mathrm{~mol} \% 1.1$ (blue) using $0.9 \mathrm{mmol} \cdot \mathrm{L}^{-1} \mathrm{AIBN}$ in toluene at $60^{\circ} \mathrm{C}$.

In the top part of this diagram the molecular weights are plotted versus the reaction time. The FRPcharacter is confirmed. Besides the similar molecular weights for all aliquots, it can be noticed that the difference between the plots of monomer conversion versus time (bottom plot of Figure 8-3) is very small. With regard to the tolerance of the balance and the failure in the determination of the monomer conversion, it can be assumed that the plots of those two polymerisations are equal. To verify that the addition of $\mathbf{1 . 1}$ has no influence on the FRP and even higher co-monomer concentrations do not change the reaction behaviour, higher amounts of $\mathbf{1 . 1}$ in the polymerisation mixture were also tested. $4.5 \mathrm{~mol} \%$ and $7 \mathrm{~mol} \%$ of 1.1 regarding the initial amount of BA were used along with $1.9 \mathrm{mmol} \cdot \mathrm{L}^{-1}$ of AIBN. The Figures 8-4 and 8-5 summarise the findings for those FRPs. The black triangles show the copolymerisation with $4.5 \mathrm{~mol} \%$ of $1.1\left(c_{1.1}=164 \mathrm{mmol} \cdot \mathrm{L}^{-1}\right)$ and the blue triangles the copolymerisation with $7 \mathrm{~mol} \%$ of $1.1\left(c_{1.1}=259 \mathrm{mmol} \cdot \mathrm{L}^{-1}\right)$. 


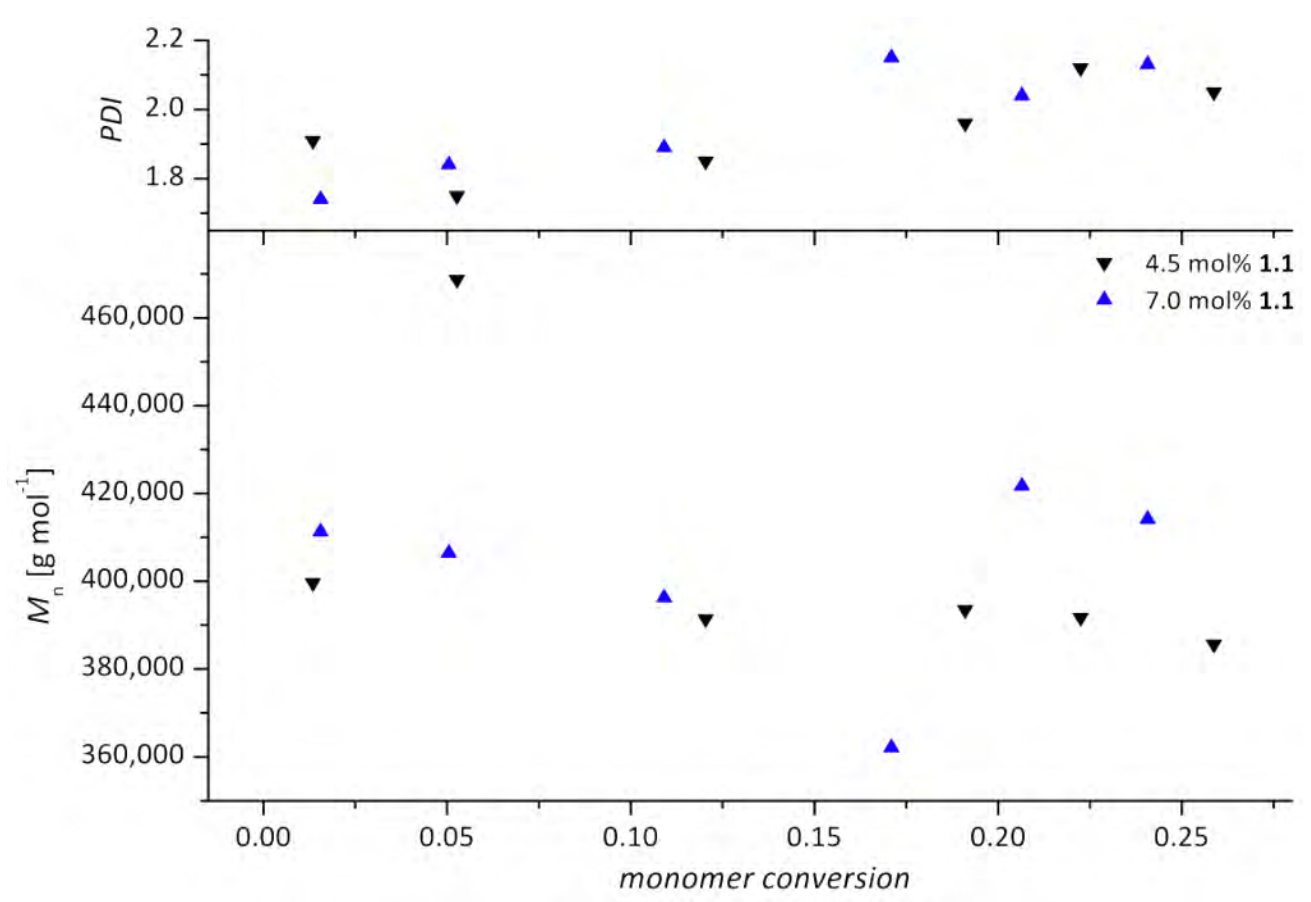

Figure 8-4: Development of the molecular weight (obtained by RI-detection) and the PDI versus the monomer conversion of a FRP of BA with $4.5 \mathrm{~mol} \% 1.1$ (black) and $7.0 \mathrm{~mol} \% 1.1$ (blue) using $1.9 \mathrm{mmol} \cdot \mathrm{L}^{-1} \mathrm{AlBN}$ at $60^{\circ} \mathrm{C}$.

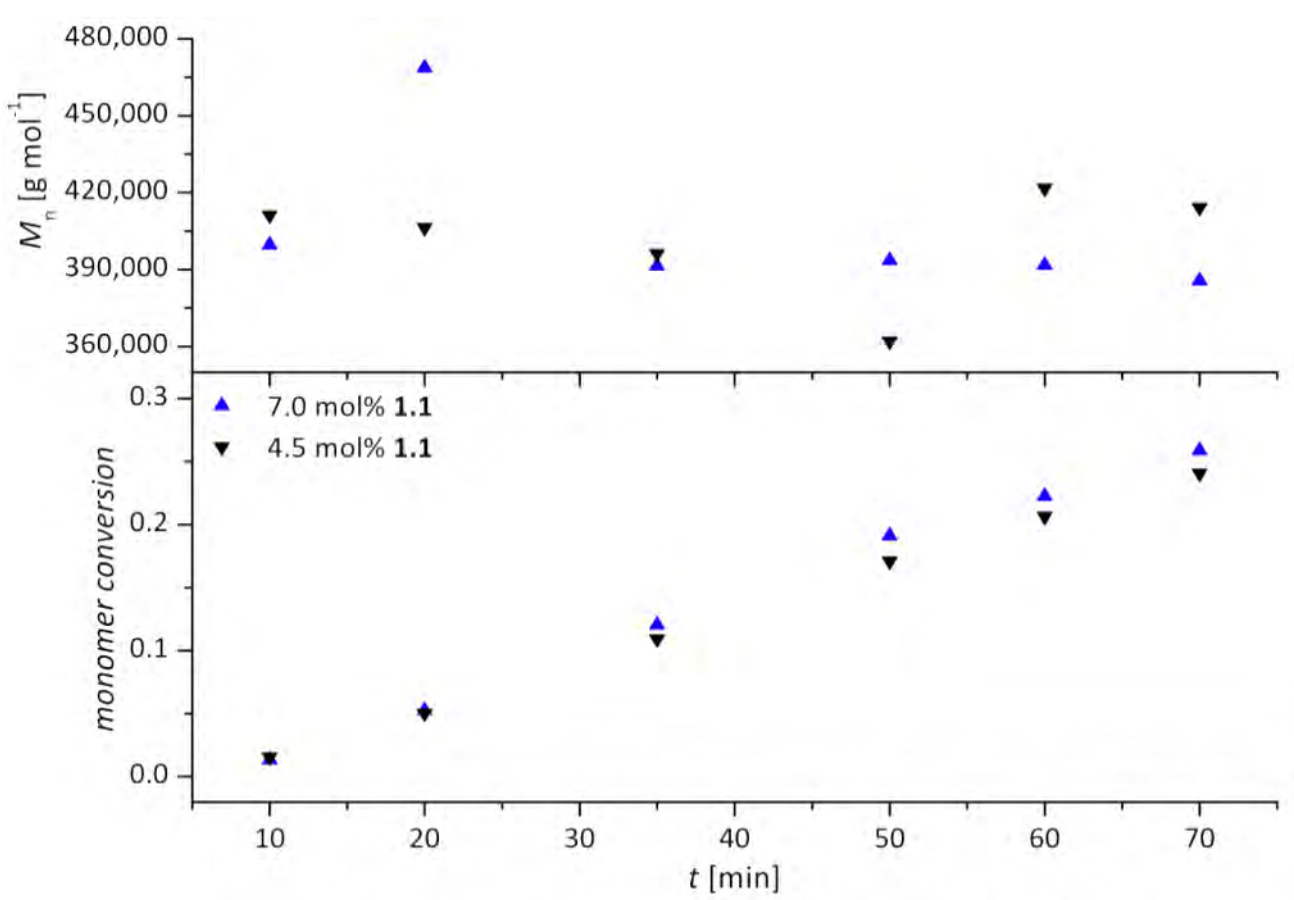

Figure 8-5: Development of the molecular weight (obtained via RI-detection) and the monomer conversion with reaction time of a FRP of BA with $4.5 \mathrm{~mol} \% 1.1$ (black) and $7 \mathrm{~mol} \% 1.1$ (blue) using $1.9 \mathrm{mmol} \cdot \mathrm{L}^{-1} \mathrm{AlBN}$ at $60^{\circ} \mathrm{C}$. 
Again similar molecular weights, PDI-values and equal plots in monomer conversion versus reaction time can be observed for both reactions. It can be concluded that the addition of $\mathbf{1 . 1}$ has no influence on the reaction behaviour in the FRP-case.

As mentioned in chapter 7.1.1, 1.1 influences the polymerisation of BA under RAFTconditions. In contrary to the FRP-case, in the RAFT-polymerisations significantly smaller molecular weights and smaller monomer conversions are obtained for the copolymerisation in bulk than in the corresponding homopolymerisation, illustrated by the SEC-traces in Figure 8-6. Copolymerisations were conducted in bulk with $34 \mathrm{mmol} \cdot \mathrm{L}^{-1}(0.5 \mathrm{~mol} \%) \mathbf{1 . 1}, 2 \mathrm{mmol} \cdot \mathrm{L}^{-1} \mathrm{AlBN}, 3 \mathrm{mmol} \cdot \mathrm{L}^{-1} \mathbf{R}_{\mathrm{L}} \mathbf{2}$ and $1 \mathrm{mmol} \cdot \mathrm{L}^{-1} \mathbf{R}_{6} \mathbf{2}$ as well as with $224 \mathrm{mmol} \cdot \mathrm{L}^{-1}$ (3 mol\%) $1.1,2 \mathrm{mmol} \cdot \mathrm{L}^{-1} \mathrm{AlBN}, 4 \mathrm{mmol} \cdot \mathrm{L}^{-1} \mathbf{R}_{\mathbf{L}} \mathbf{2}$ and $3 \mathrm{mmol} \cdot \mathrm{L}^{-1} \mathbf{R}_{6} \mathbf{2}$ at $60{ }^{\circ} \mathrm{C}$. The comparison of the SEC-curves resulting from aliquots taken after the same time period confirm smaller molecular weights in case of copolymer stars (blue) than of the homopolymerisation (black). In the CRP-case this equals less monomer conversion as a result of the controlled mechanism (chapter 3.2.3).
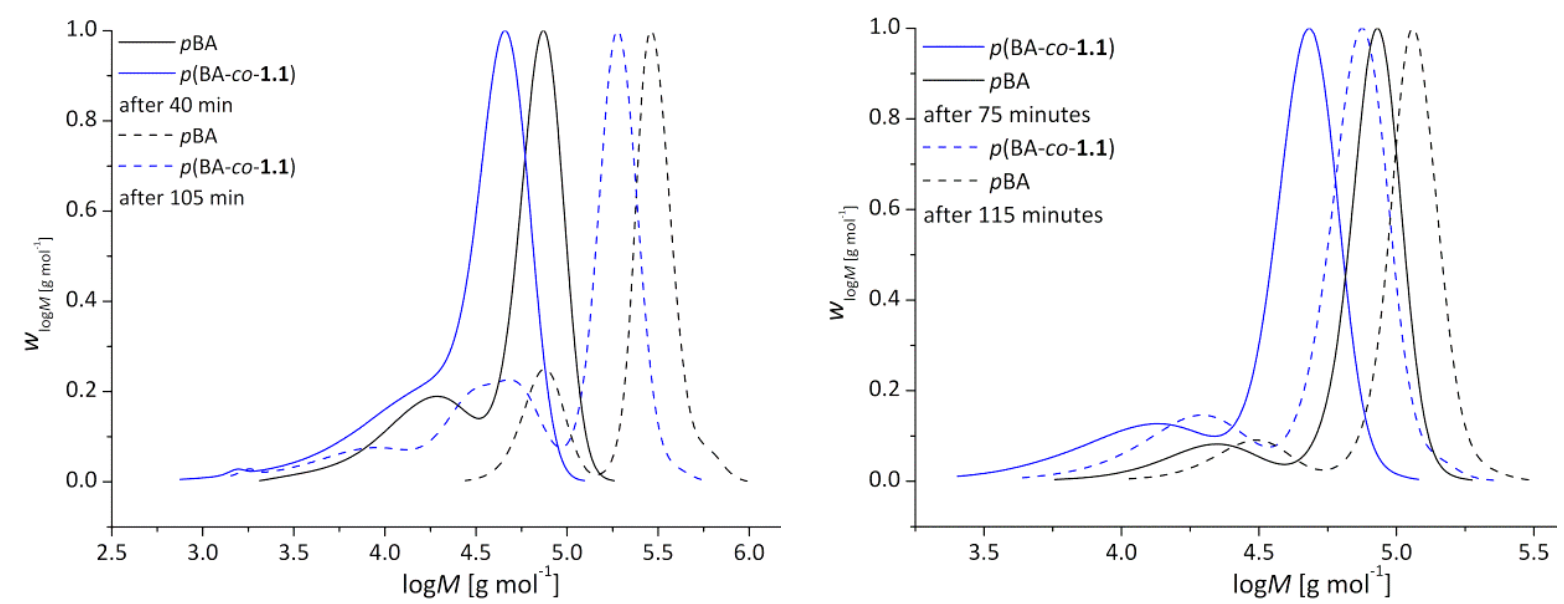

Figure 8-6: SEC-UV-data (corrected) of two samples of the copolymerisation of BA with 0.5 mol\% 1.1 (blue) compared to the homopolymerisation of BA (black) after 40 and 105 minutes (left) and of two samples of the copolymerisation of BA with 3 mol\% 1.1 (blue) compared to the homopolymerisation of BA (black) after 75 and 115 minutes (right).

Smaller molecular weights and smaller monomer conversions in the copolymerisation compared to the according homopolymerisation indicate a delay at the start of the copolymerisation. This effect is called induction (chapter 3.2.3). The following figure displays this observation comparing the previously introduced homo- and copolymerisations. The filled symbols correspond with the data of the copolymerisations whereas the empty symbols belong to the corresponding homopolymerisation. 


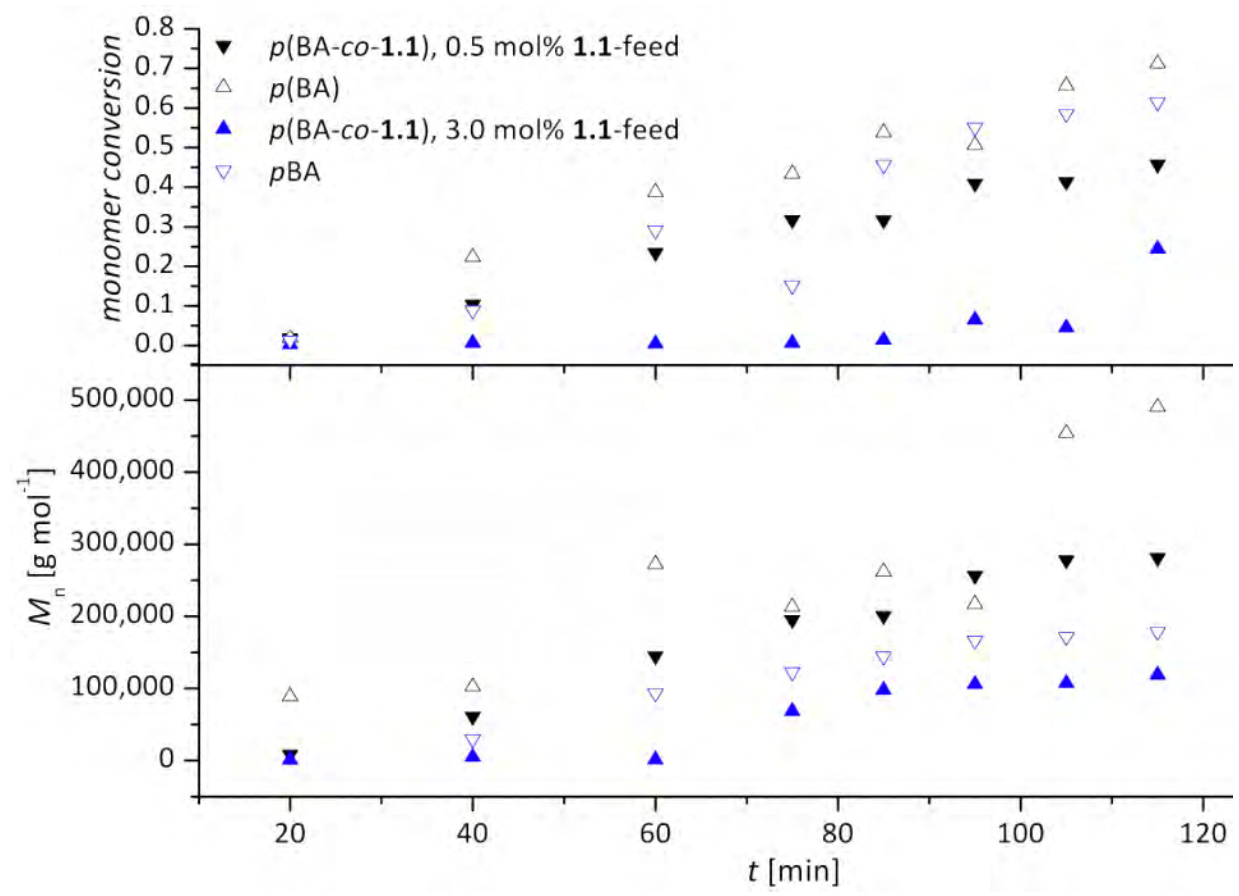

Figure 8-7: Developments of monomer conversion and molecular weight (obtained by UV-detection, corrected by 1.52) with time of a homopolymerisation of $B A$ and a copolymerisation of $B A$ with $0.5 \mathrm{~mol} \% 1.1$ using $2 \mathrm{mmol} \cdot \mathrm{L}^{-1} \mathrm{AlBN}, 1 \mathrm{mmol} \cdot \mathrm{L}^{-1} \boldsymbol{R}_{6} \mathbf{2}$ and $3 \mathrm{mmol} \cdot L^{-1} \boldsymbol{R}_{L} \mathbf{2}$ (black) and of a homopolymerisation of $B A$ and a copolymerisation of $B A$ with 3 mol\% 1.1 using $2 \mathrm{mmol} \cdot \mathrm{L}^{-1} \mathrm{AIBN}, 3 \mathrm{mmol} \cdot \mathrm{L}^{-1} \boldsymbol{R}_{6} \mathbf{2}$ and $4 \mathrm{mmol} \cdot \mathrm{L}^{-1} \boldsymbol{R}_{L} \mathbf{2}$ (blue) in bulk.

Here a difference between the plots can be found, in contrast to the FRP case. For the copolymerisation with 0.5 mol\% 1.1 and the corresponding homopolymerisation (black) their course is parallel to each other which indicates a similar progress of the polymerisation but an induction period in the case of the copolymerisation. As mentioned in chapter 7.1.1, the copolymerisation with the high co-monomer load does not proceed as well as the others. Figure 8-7 supports these findings but the tendency that the induction in the copolymerisation is more pronounced with increasing comonomer concentration can be seen.

Polymerisations of BA and $\mathbf{1 . 1}$ were also conducted in solution with different feeds of 1.1. The reactions were carried out in a BA:toluene mixture with a ratio of 7:3, which corresponds to $4,920 \mathrm{mmol} \cdot \mathrm{L}^{-1}$ BA. $0.6 \mathrm{mmol} \cdot \mathrm{L}^{-1} \mathbf{R}_{6} \mathbf{2}$ and $1.1 \mathrm{mmol} \cdot \mathrm{L}^{-1}$ AlBN were applied and the reactions were carried out at $60{ }^{\circ} \mathrm{C}$ for up to 155 minutes. The concentration of 1.1 was varied between 29 and $279 \mathrm{mmol} \cdot \mathrm{L}^{-1}$. In Figure 8-8 the molecular weights and the PDI-values of these different polymerisations are plotted against the monomer conversion. 


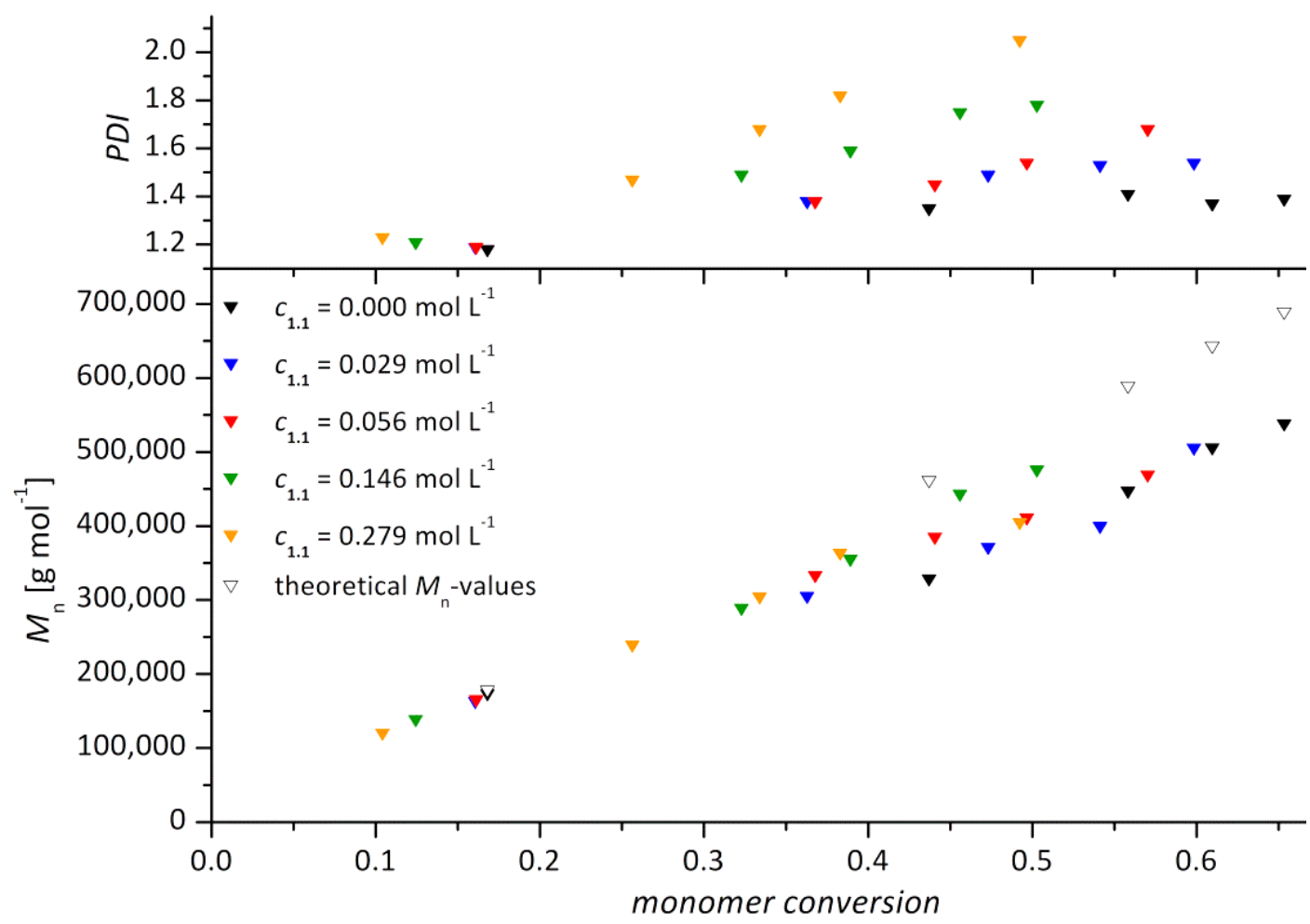

Figure 8-8: Development of the molecular weights (determined by UV-detection, corrected by 1.52) and the PDI with the monomer conversion for a homopolymerisation of $B A$ and four copolymerisations of $B A$ and $\mathbf{1 . 1}$ with $0.6 \mathrm{mmol} \cdot \mathrm{L}^{-1} \boldsymbol{R}_{6} \mathbf{2}$ and $1.1 \mathrm{mmol} \cdot \mathrm{L}^{-1} \mathrm{~A} / B \mathrm{~N}$ in toluene at $60^{\circ} \mathrm{C}$.

In general it has to be noted that the PDI-values of these solvent polymerisations are higher than in the bulk polymerisations where values below 1.25 were obtained (chapter 7.1.1). This is due to the good solvent quality of toluene, as explained in chapter 6 for solvent polymerisations with styrene. Because of the good solubility of BA, $\mathbf{1 . 1}$ and the polymer in toluene the compounds are evenly distributed in the solvent. Diffusion pathways are much longer, than it would be in a poor solvent, and the penetration of the growing chain through the polymer shell of the star polymer results in minimisation of control. Furthermore, the PDI-values are getting higher for higher concentrations of 1.1. The corresponding SEC-spectra show bimodal distributions which cause the high PDI-values (Figure 8-9). It is noticeable that the formation of material with higher molecular weight causing bimodality depends on the co-monomer concentration. This phenomenon could not be observed for the bulk process or the FRP and will be discussed later on. A controlled process of the polymerisation can be recorded for all copolymerisations in toluene proven by the linear development illustrated in Figure 8-8 and the SEC-traces in Figure 8-9. Even for high co-monomer concentrations this holds true, in contrast to the findings in the bulk polymerisation shown before. The experimentally obtained values agree with the theoretical values. The small deviation results from the comparison of the theoretical values with the data obtained by UV-detection. The detection of the UV-signal yields a distribution of numbers which is smaller than the distribution of weights obtained by evaluation of 
the RI-signal. The equation for the theoretical values bases on the molecular weights and would therefore equal the values obtained via the RI-signal. Furthermore uncertainties in the determination of the monomer conversion due to the tolerance of the balance and in the SEC-measurements because of the application of linear polymer calibration standards and $\mathrm{MH}$-coefficients for $p \mathrm{BA}$ also for the copolymer cause deviation from the theoretical value.

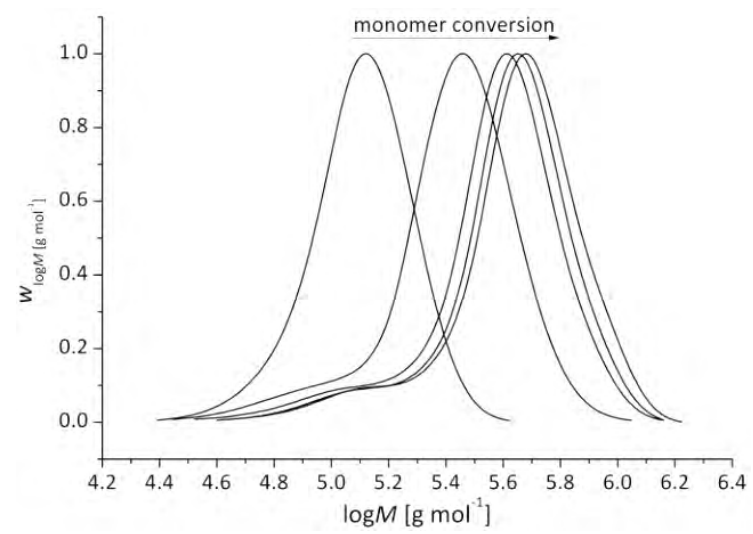

Homopolymerisation of $B A$

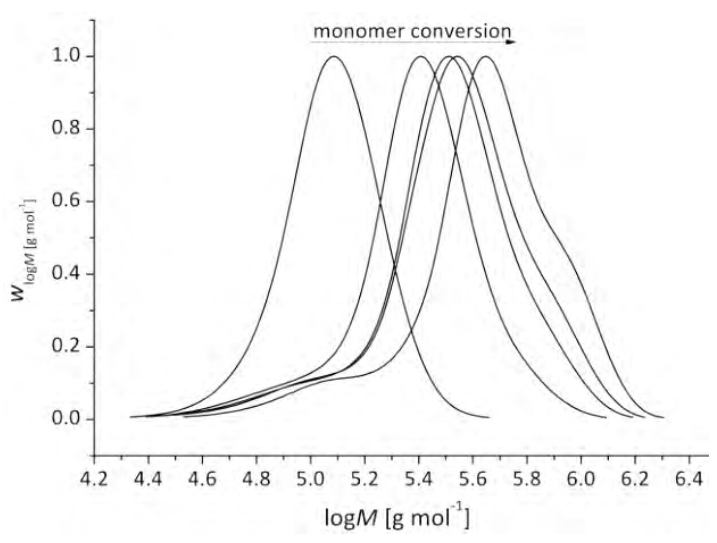

Copolymerisation with $0.029 \mathrm{~mol} \cdot \mathrm{L}^{-1} 1.1$

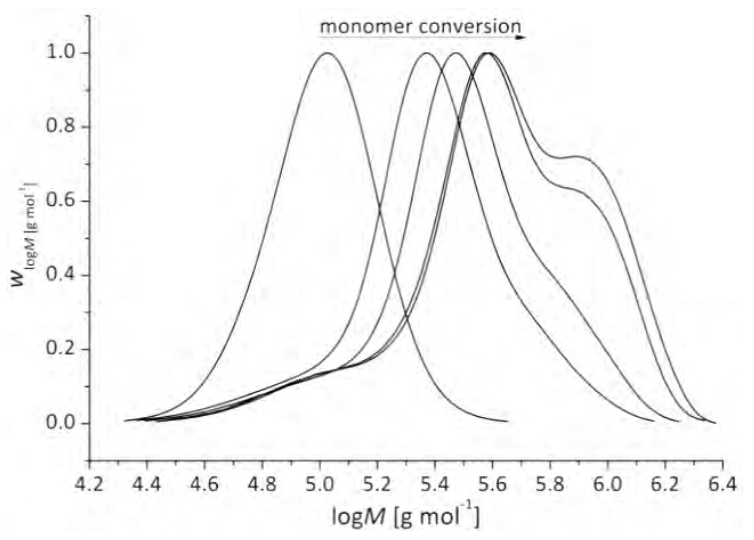

Copolymerisation with $0.146 \mathrm{~mol} \cdot \mathrm{L}^{-1} 1.1$

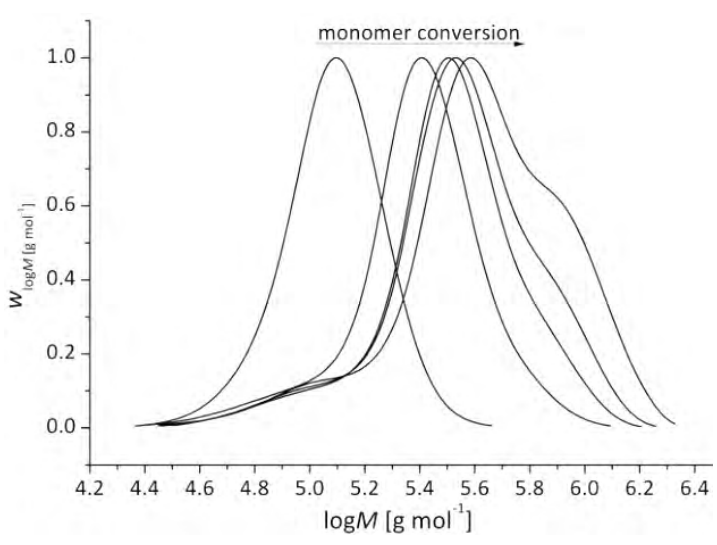

Copolymerisation with $0.056 \mathrm{~mol} \cdot \mathrm{L}^{-1} 1.1$

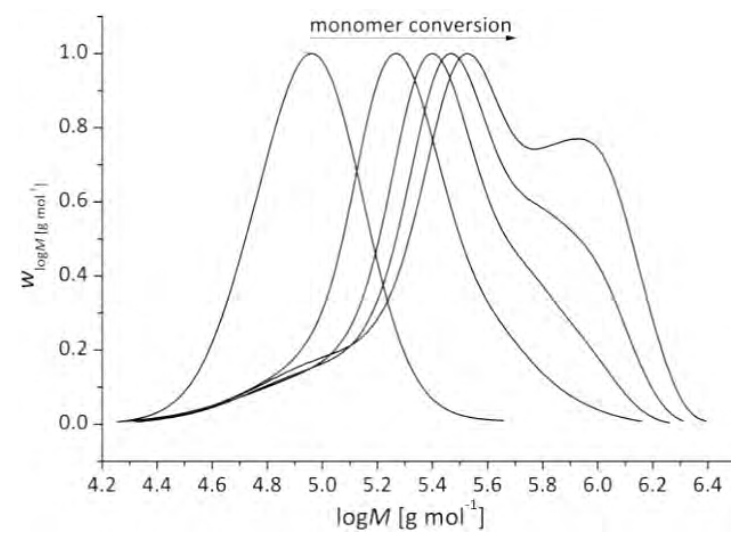

Copolymerisation with $0.279 \mathrm{~mol} \cdot \mathrm{L}^{-1} 1.1$

Figure 8-9: SEC-RI-spectra of different copolymerisations of BA with 1.1 up to a reaction time of 155 minutes. 
As in the bulk polymerisations, the monomer conversions and the molecular masses are smaller for the copolymerisations than for the homopolymerisation for aliquots taken after the same time period, as shown in the next collections of SEC-spectra (Figure 8-10) for the different copolymerisations. Shown are the chromatograms obtained by RI-detection. In the spectra of long reaction times (Figure 8-10 right) a peak in the regime of smaller molecular weights is observed. It results from dead material which is generated during the polymerisation process due to continuous termination.
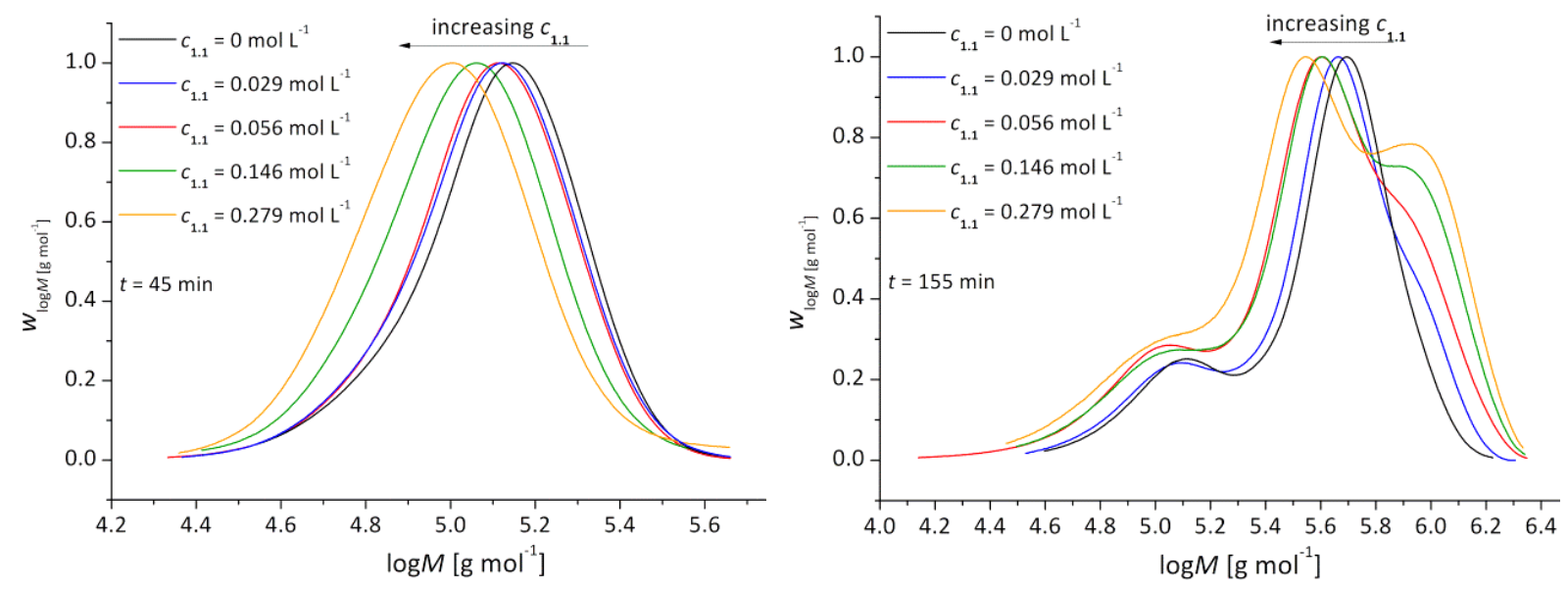

Figure 8-10: Comparison in time of SEC-RI-spectra of different copolymerisations with BA with 1.1: 45 minutes (left), 155 minutes (right).

With increasing concentration of the co-monomer decreasing molecular weights are obtained whereas the formation of dead polymer proceeds approximately in the same extent for all cases. Furthermore the formation of a shoulder in the high molecular weight range was observed for copolymer samples obtained after longer reaction times. This will be discussed later.

The plots of monomer conversion with reaction time (Figure 8-11) confirm the findings of the bulk polymerisation, i.e. with increasing co-monomer concentration the induction in the copolymerisation is getting more pronounced. 


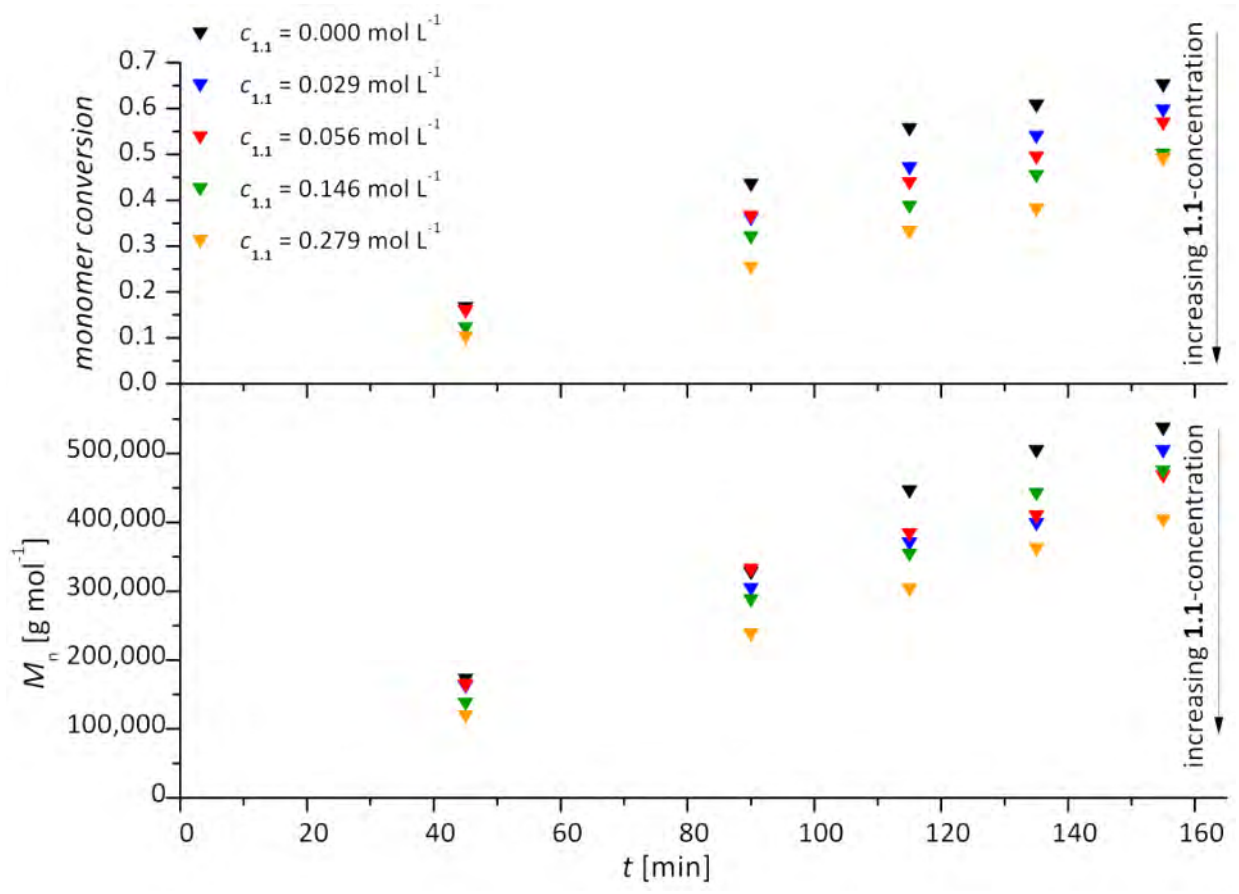

Figure 8-11: Developments of monomer conversion and molecular weight (obtained by UV-detection, corrected with 1.52) with time of a homopolymerisation of $B A$ and different copolymerisations of $B A$ and 1.1 in toluene using $0.6 \mathrm{mmol} \cdot L^{-1} \boldsymbol{R}_{6} \mathbf{2}$ and $1.1 \mathrm{mmol} \cdot \mathrm{L}^{-1} \mathrm{AlBN}$ at $60^{\circ} \mathrm{C}$.

It can be concluded that the addition of co-monomer influences the RAFT-process and that the effect of the induction is dependent on the co-monomer concentration. Nevertheless statistical copolymer stars with defined molecular weights can be obtained. The polymerisation should be investigated in poor solvents. It can be assumed that the control is increased, as explained in chapter 6 , which would be worth striving for especially for the copolymerisations with high 1.1-feeds.

The appearance of multimodal MWDs in copolymerisations with 1.1 should be discussed in more detail. At first the homopolymerisation of $B A$ is examined which is the reference system to compare with. Figure 8-12 shows the MWDs of the first (45 minutes) and the last (155 minutes) aliquot of the homopolymerisation carried out with $0.6 \mathrm{mmol} \cdot \mathrm{L}^{-1} \mathbf{R}_{6} \mathbf{2}$ and $1.1 \mathrm{mmol} \cdot \mathrm{L}^{-1} \mathrm{AlBN}$ in toluene, discussed previously. The here shown MWDs are the experimentally obtained values from the measurements of the samples on a THF-SEC set up using the RI- and the UV-detector $(\lambda=310 \mathrm{~nm})$. The MWDs obtained via UV-detection are usually smaller than the corresponding values of the RI-detection. In the case of the UV-detection a number distribution is obtained which gives smaller molecular weights due to the correlation between retention time and absorbance depending on the number of UV-active centres. This is a result of Lambert-Beer's Law and is therefore valid only for small concentrations of the UV-active substance. In the polymers synthesised here the UV-active species absorbing at $310 \mathrm{~nm}$ are the RAFT-units. During the reaction the number of RAFT-units stays constant at six for every star molecule. Hence, the absorbance for all samples of 
six RAFT-units is measured and correlated with the retention time. In contrast to this the refractive index of a polymer sample is directly dependent on the number of monomer units the polymer consists of, giving a distribution of different weights. It changes with the growing of the polymer. Therefore the RI correlated to the retention time is a direct measure of the molecular weight. In the UV-detection, on contrary, the apparent molecular weight is smaller than the real molecular weight. For the correction of the experimental UV-data every data point of the measured UV-signal is multiplied with the molecular mass corresponding with this data point. The correction of the UV-data should overcome the difference between the experimentally obtained MWDs of the UV- and RIsignal and yield similar chromatograms.

In Figure 8-12 the black chromatogram represents the obtained RI-data. In red the uncorrected UV-data are plotted and in green the corrected UV-data.
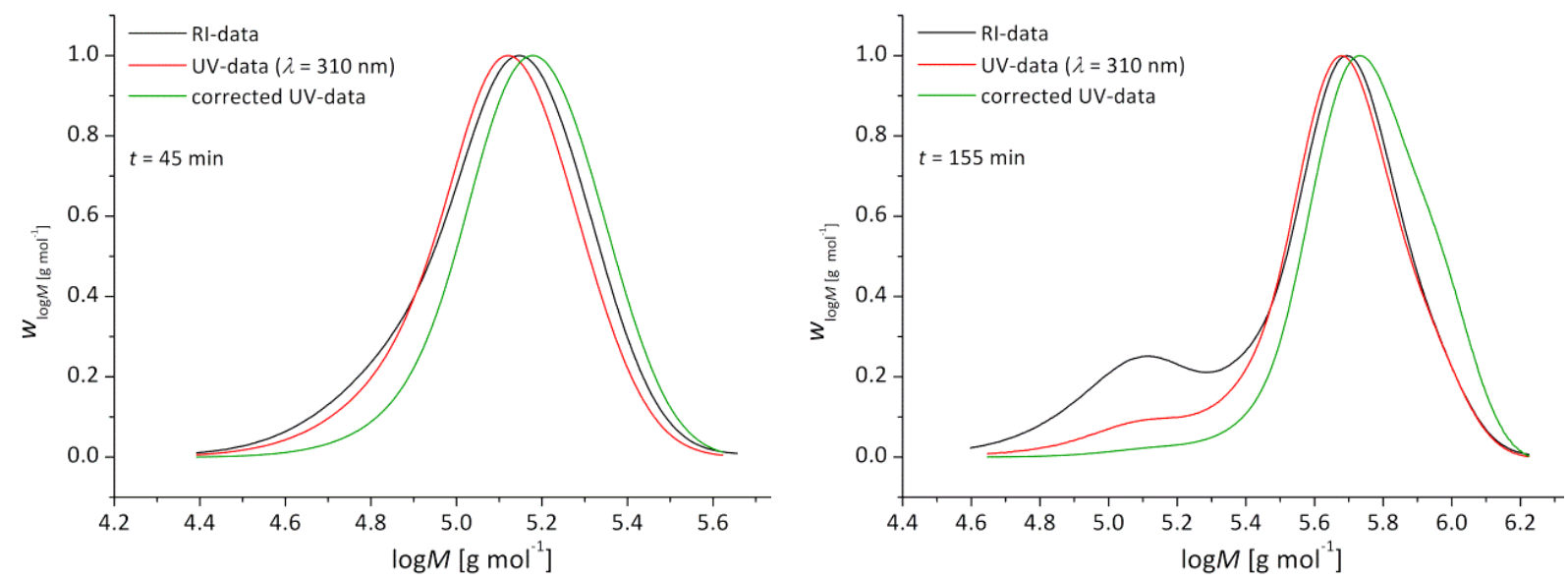

Figure 8-12: MWDs of a BA-homopolymerisation in toluene using $4,920 \mathrm{mmol} \cdot \mathrm{L}^{-1} B A, 0.6 \mathrm{mmol} \cdot \mathrm{L}^{-1} \boldsymbol{R}_{6} \mathbf{2}$ and $1.1 \mathrm{mmol} \cdot \mathrm{L}^{-1} \mathrm{AlBN}$ at $60^{\circ} \mathrm{C}$ : left $45 \mathrm{~min}$ reaction time, right $155 \mathrm{~min}$.

It has to be noted that the experimentally obtained UV-data equal approximately the RI-data. The corrected UV-data yield consequently higher values than the RI-detection. This phenomenon was found for all BA and BA-1.1 polymerisations in this project. A contribute of the phenyl rings of the RAFT-agent or the co-monomer to the absorbance resulting higher values than expected can be excluded. The absorbance of these compounds is in the range below $250 \mathrm{~nm}$. It can be assumed that the concentration of RAFT units, here six, is too high to fulfil the requirements of the validity of Lambert-Beer's Law. Therefore the method of correction basing on this law loses its validity.

Nevertheless from Figure 8-12 the formation of dead polymer chains during the polymerisation process can be observed on the basis of the shoulder in the regime of lower molecular weights. As this material is formed by termination of propagating radicals, it does not consist of RAFT-units, therefore no UV-detection is possible at $310 \mathrm{~nm}$. The corrected UVchromatogram confirms this. After 45 minutes of reaction time hardly any dead chains were formed. 
The amount of dead material increases consequently during the process of polymerisation. For higher monomer conversion, i.e. here after 155 minutes, besides the main signal which results from the star polymer and the dead material in the corrected UV-chromatogram a shoulder in the region of higher molecular weight can be found even though it is not very pronounced in this example. This is the first sign for the formation of a side product with higher molecular weights. In general this shoulder is caused by double stars formed by star-star coupling due to back-biting reactions which are well investigated for BA polymerisations. ${ }^{(183)}$ Boschmann investigated the phenomenon of starstar coupling by back-biting regarding RAFT-star polymerisations with BA more closely. ${ }^{(21 ; 47)} \mathrm{By} \beta$-Habstraction at the polymer backbone through a propagating radical an intermolecular transfer of the radical functionality takes place. A tertiary radical, a so called Mid Chain radical, is formed and dead polymer is generated. As this Mid Chain radical cannot undergo the RAFT-equilibrium it propagates forming a secondary radical which then can participate in the RAFT equilibrium yielding coupled stars.

When now examining the aliquots obtained after 155 minutes of the set of copolymerisations introduced previously (Figure 8-13), the formation of the higher molecular weight material can be observed more clearly. Furthermore it stands out that in polymerisations with higher co-monomer concentrations the caused multimodal distribution is much more pronounced.

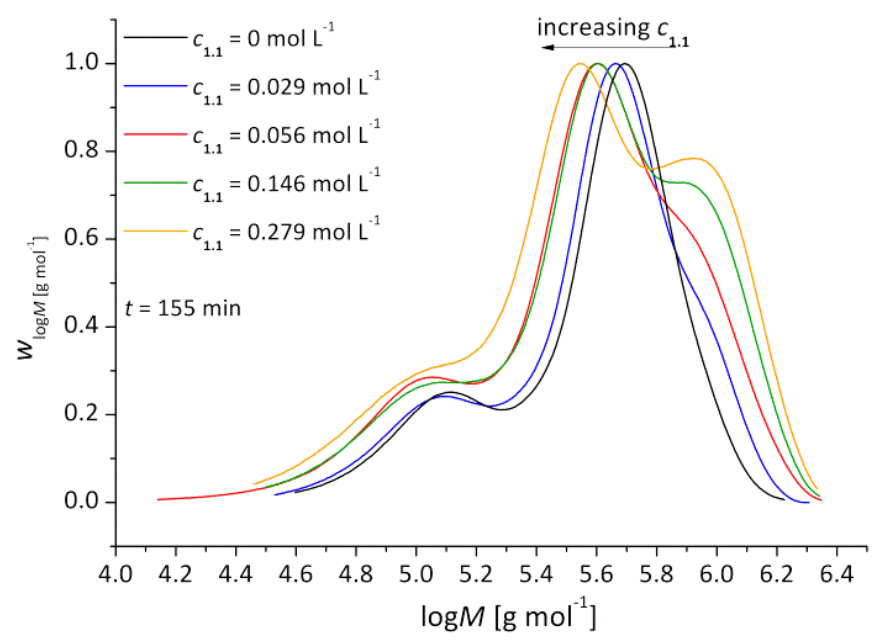

Figure 8-13: SEC-RI-spectra of a homopolymerisation of BA (black) and different copolymerisations of BA and 1.1 in toluene using $0.6 \mathrm{mmol} \cdot \mathrm{L}^{-1} \boldsymbol{R}_{6} \mathbf{2}$ and $1.1 \mathrm{mmol} \cdot \mathrm{L}^{-1} \mathrm{AlBN}$ at $60^{\circ} \mathrm{C}$ quenched after 155 minutes.

The sample of $p B A$ (black) has the highest molecular weight but even after 155 minutes of polymerisation the shoulder in the higher molecular weight region is hardly audible in this comparison. For small 1.1-concentrations this shoulder formation is not as distinctive as for higher concentrations. The examination of the chromatograms resulting from the corrected UV-data (Figure 8-14) confirms a drastic increase of the high molecular weight side product with increasing co- 
monomer concentration and reaction time. It also becomes clear that the undesired side product is getting the main product after longer reaction times.

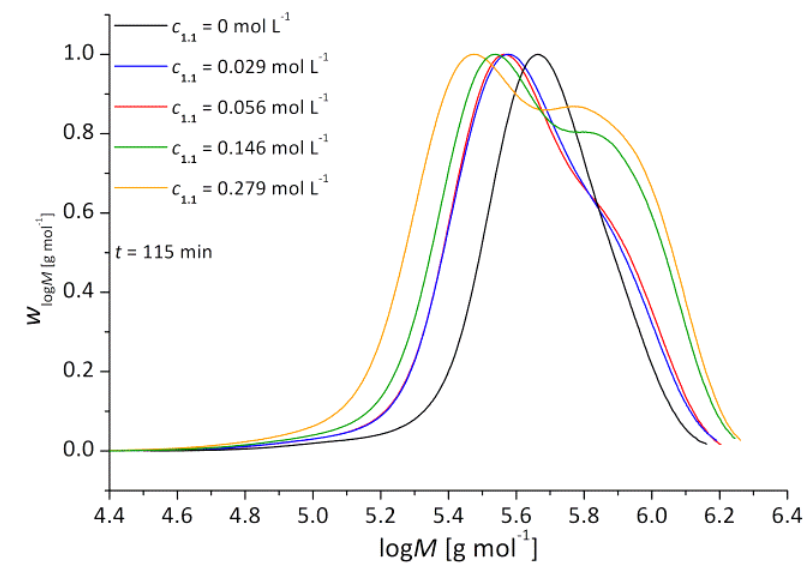

Corrected SEC-UV-chromatograms of the aliquots quenched after 115 minutes

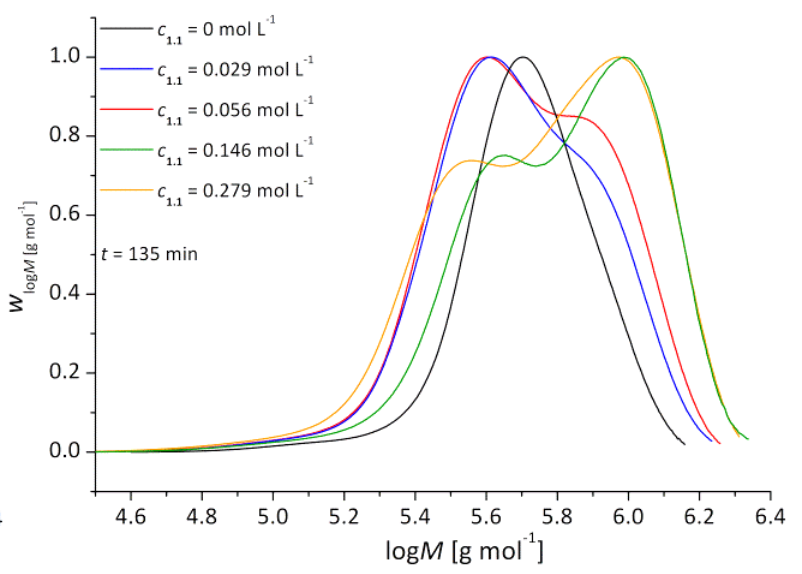

Corrected SEC-UV-chromatograms of the aliquots quenched after 135 minutes

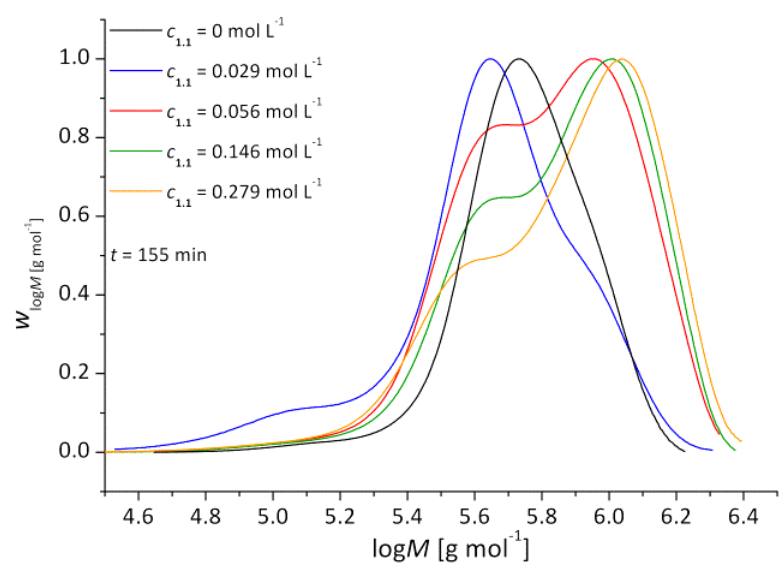

Corrected SEC-UV-chromatograms of the aliquots

quenched after 155 minutes

Figure 8-14: Corrected SEC-UV-chromatograms of the aliquots quenched after 115, 135 and 155 minutes of all copolymerisations with $B A$ and different amounts of 1.1 using $0.6 \mathrm{mmol} \cdot \mathrm{L}^{-1} \boldsymbol{R}_{6} \mathbf{2}$ and $1.1 \mathrm{mmol} \cdot \mathrm{L}^{-1} \mathrm{AlBN}$ at $60{ }^{\circ} \mathrm{C}$.

After 115 minutes of polymerisation the material of the smaller molecular weights, corresponding to the mono-star, is the main product in all cases. With increasing reaction time the formation of high molecular weight material is more pronounced in mixtures with a high co-monomer concentration. After 155 minutes the side product is the main product in the systems for polymerisations with comonomer concentrations over $56 \mathrm{mmol} \cdot \mathrm{L}^{-1}$.

It can be concluded that the DMI moiety is prone to undergo side reactions which yield polymer material with higher molecular weights than a single star polymer. The assumption seems plausible that probability of star-star coupling is intensified by these units. Of the copolymerisations with $0.056,0.146$ and $0.279 \mathrm{~mol} \cdot \mathrm{L}^{-1} 1.1$ each fourth and fifth aliquot are checked for star-star- 
coupling which results in doubling of the molecular mass (Table 8-1). Here the molecular weights of the peak maxima $\left(M_{\mathrm{P}}\right)$ of both peaks were compared. " $M_{\mathrm{P}}$, high" corresponds to the molecular weight at the peak maximum of the high molecular weight material, " $M_{\mathrm{p}}$, low" to the peak maximum of the peak of the material with the smaller molecular weight.

Table 8-1: Comparison of the molecular weights by the two maxima of the MWDs of selected aliquots resulting from the copolymerisation with $B A$ and different amounts of 1.1 using $0.6 \mathrm{mmol} \cdot \mathrm{L}^{-1} \boldsymbol{R}_{6} \mathbf{2}$ and $1.1 \mathrm{mmol} \cdot \mathrm{L}^{-1} \mathrm{AlBN}$ at $60{ }^{\circ} \mathrm{C}$

\begin{tabular}{c|c|c|c} 
aliquot & $M_{\mathrm{P}}\left[\mathrm{g} \cdot \mathrm{mol}{ }^{-1}\right]$, high & $M_{\mathrm{P}}\left[\mathrm{g} \cdot \mathrm{mol}{ }^{-1}\right]$, low & ratio \\
\hline $4\left(0.056 \mathrm{~mol} \cdot \mathrm{L}^{-1}\right)$ & 740,000 & 398,000 & 1.86 \\
\hline $5\left(0.056 \mathrm{~mol} \cdot \mathrm{L}^{-1}\right)$ & 890,000 & 490,000 & 1.82 \\
\hline $4\left(0.146 \mathrm{~mol} \cdot \mathrm{L}^{-1}\right)$ & 980,000 & 447,000 & 2.19 \\
\hline $5\left(0.146 \mathrm{~mol} \cdot \mathrm{L}^{-1}\right)$ & $1,000,000$ & 470,000 & 2.12 \\
\hline $4\left(0.279 \mathrm{~mol} \cdot \mathrm{L}^{-1}\right)$ & 933,000 & 355,000 & 2.63 \\
\hline $5\left(0.279 \mathrm{~mol} \cdot \mathrm{L}^{-1}\right)$ & $1,100,000$ & 407,000 & 2.69
\end{tabular}

The molecular weight of the material being the mono-star polymer $\left(M_{\mathrm{p}}, \mathrm{low}\right)$ has in all cases a molecular weight of the same scale which seems to be a critical size. It can be assumed that this critical size is a consequence of the shielding effect as the polymerisations were carried out in a good solvent (chapter 6). With increasing length of the star's arms the hindrance of the macro radicals' penetration towards the RAFT-unit is more pronounced. The assumption of double star formation was proven to be true as $\left(M_{\mathrm{p}}\right.$, low) is in all cases approximately about a factor 2 smaller than $\left(M_{\mathrm{p}}\right.$, high). Furthermore a trend can be observed as the ratio between the molecular weights is increasing with increasing co-monomer concentration. For the highest co-monomer concentration the ratio is almost threefold larger which indicated that partially aggregates consisting of more than two stars are formed. Due to the high polymer concentration in the polymerisation mixture after 115 minutes and the well extended arms of the star polymer due to the good solvent the contact probability between the star polymers is increased. As the DMI-units reinforce a coupling reaction, the probability for these is much higher in systems with higher co-monomer concentrations. From these two factors the formation of larger formations than double stars seems plausible for systems with a large amount of $\mathbf{1 . 1}$ and long reaction times. A thermal initiation of the [2+2]-cycloaddition is thermodynamically impossible. Therefore the only conceivable pathway is by reaction of the in the process of back-biting formed radical. It could attack the DMI-unit forming a product similar to the by Kuckling et al. proposed product B (chapter 3.6.3). ${ }^{(107)}$ As the formed tertiary radical cannot 
participate in the RAFT-equilibrium two transfer pathways are conceivable: Under $\mathrm{H}$-abstraction at the methyl group of the DMI itself, as proposed, the radical could be transferred intramolecularly to propagate further. Another possibility is $\mathrm{H}$-abstraction at the polymer backbone; the radical could be transferred to another polymer chain leaving an arm coupled to a saturated DMI-unit behind. Figure 8-15 illustrates the mechanism of star-star coupling presented by Boschmann (b) and the proposed coupling reaction via the DMI units (a).

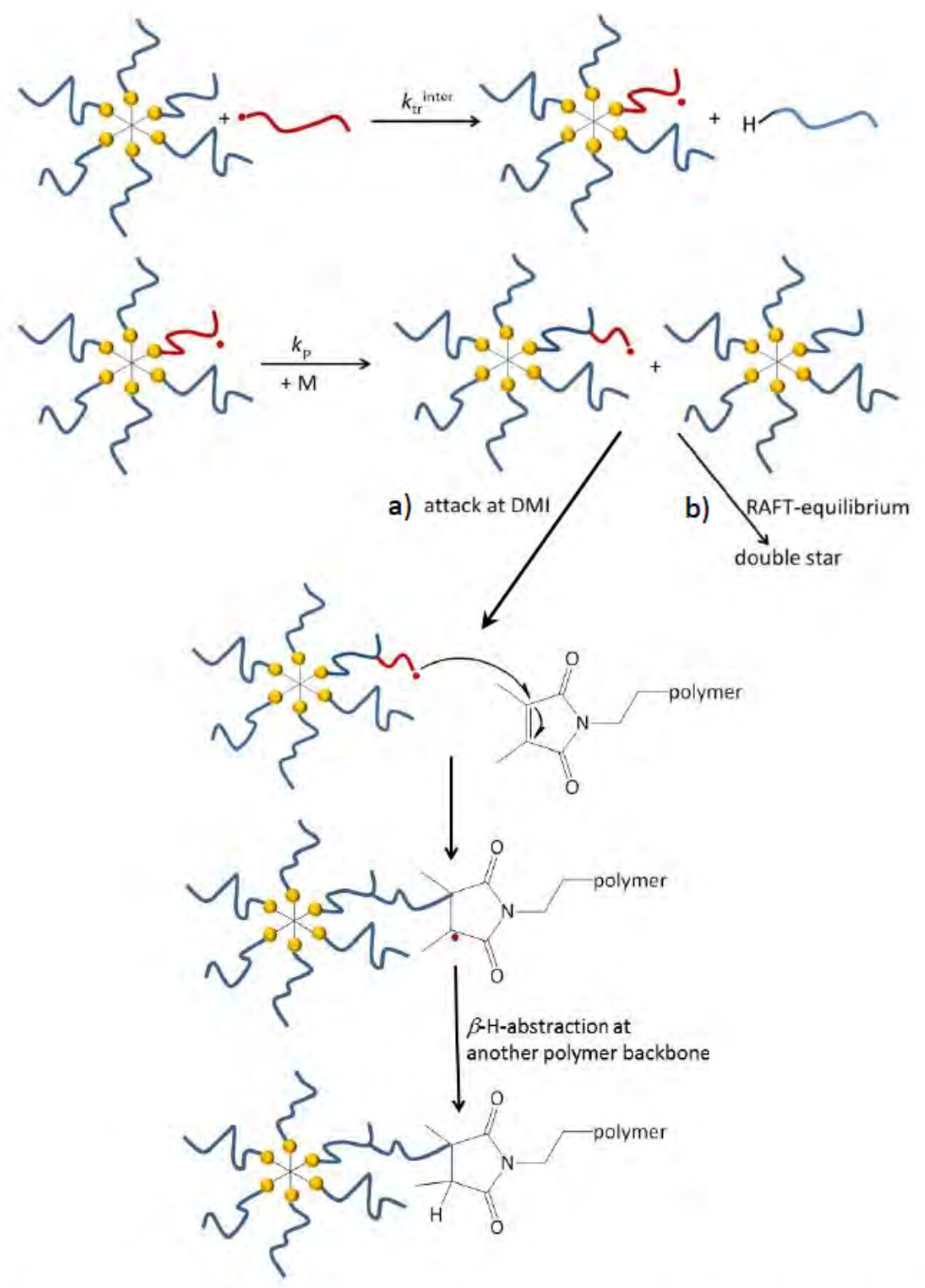

Figure 8-15: Double star formation by $\beta$-H-abstraction: a) via radical attack at the DMI units, b) via the RAFT-equilibrium. 
Mechanism (a) initiates a chain reaction as the Mid Chain Radical formed by the abstraction step propagates further and can then participate in the RAFT equilibrium step or attack another DMI unit. With increasing amount of DMI units in the system the probability for coupling via these units increases. There is a chance to reduce star-star coupling by addition for non-solvents as the arms coil and consequently a reduced contact probability between stars.

Another effect of $\mathbf{1 . 1}$ on the polymerisation of BA and the resulting materials was found: The copolymerisation yields higher molecular weights than the homopolymerisations at the point of same monomer conversion. The monomer conversion is a measure for the consumed monomer units per time period. Independent from the nature of the monomer or the composition of the monomer mixture at the same monomer conversion equally many monomer units were integrated into a polymer strand.

In Figure 8-16 the corrected SEC-UV-spectra of a bulk copolymerisation of BA and $0.5 \mathrm{~mol} \%$ 1.1 is compared with the according homopolymerisation with $2 \mathrm{mmol} \cdot \mathrm{L}^{-1} \mathrm{AIBN}$ and $1 \mathrm{mmol} \cdot \mathrm{L}^{-1} \mathbf{R}_{6} \mathbf{2}$ (left). Also such a comparison of a bulk copolymerisation with $3 \mathrm{~mol} \% 1.1$ and $2 \mathrm{mmol} \cdot \mathrm{L}^{-1}$ AlBN plus $3 \mathrm{mmol} \cdot \mathrm{L}^{-1} \mathbf{R}_{6} \mathbf{2}$ with the corresponding homopolymerisation is shown in the right plot of this figure.
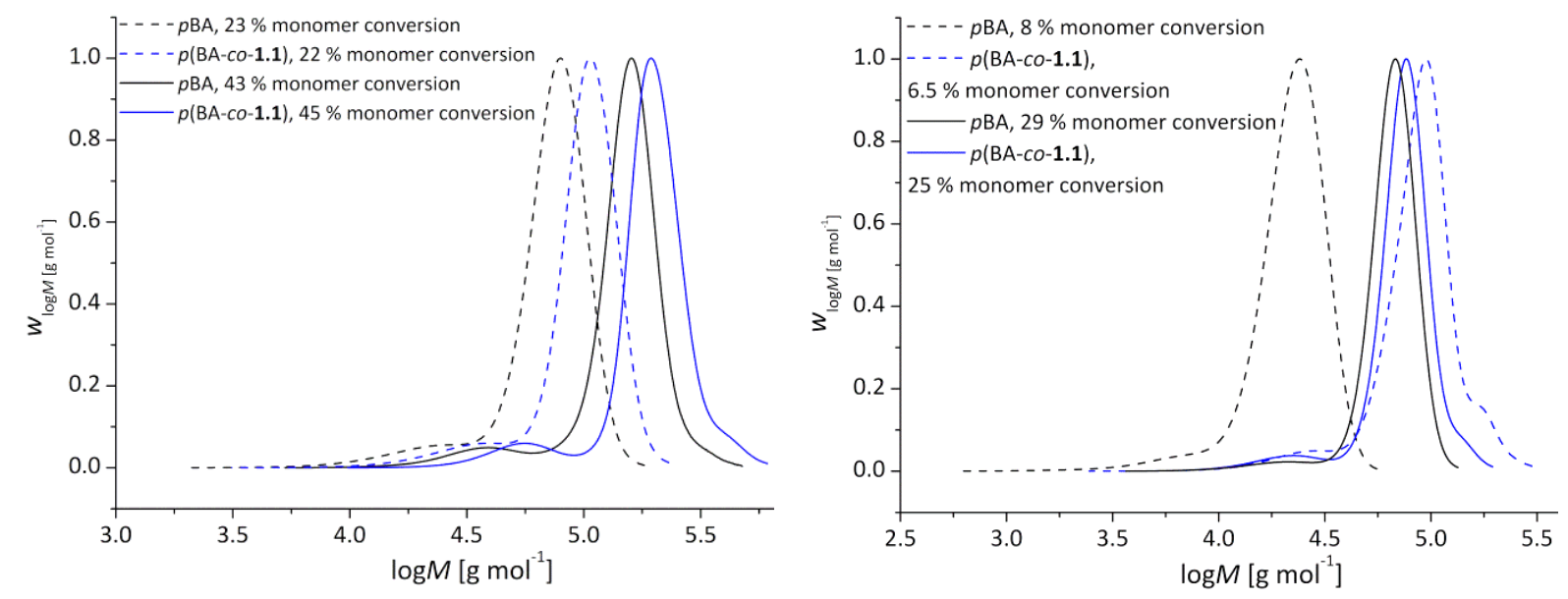

Figure 8-16: Corrected SEC-data of two samples of the copolymerisation of BA with 0.5 mol\% 1.1 (blue) compared to the homopolymerisation of BA (black) at ca 23 and $45 \%$ monomer conversion (left) and of two samples of the copolymerisation of $B A$ with 3 mol\% 1.1 (blue) compared to the homopolymerisation of BA (black) at ca 8 and $29 \%$ conversion (right).

In all cases, this discrepancy in weight cannot be proven arithmetically if an equal consumption of BA $\left(M=128.17 \mathrm{~g} \cdot \mathrm{mol}^{-1}\right)$ and $\mathbf{1 . 1}\left(M=223.23 \mathrm{~g} \cdot \mathrm{mol}^{-1}\right)$ during the reaction is adopted. The following table (Table 8-2) presents two examples of calculations. From the corrected molecular mass $\left(M_{n}{ }^{\text {UV.1.52 }}\right)$ of the samples the molecular weight of the RAFT-agent is subtracted. For the copolymer samples the share of $\mathbf{1 . 1}$ in the polymer is calculated under the assumption that the 1.1-share of the monomer feed is the same as in the resulting polymer. 
Table 8-2: Comparison of the theoretical composition of polymers of the same monomer conversion

\begin{tabular}{c|c|c|c|c|c|c|c} 
sample & $\begin{array}{c}\mathbf{1 . 1} \text {-feed } \\
{[\mathrm{mol} \%]}\end{array}$ & $\begin{array}{c}M_{\mathrm{n}}^{\mathrm{UV} \cdot 1.52} \\
{\left[\mathrm{~g} \cdot \mathrm{mol}^{-1}\right]}\end{array}$ & $\begin{array}{c}M_{\mathrm{n}} \text { minus } \\
M_{\text {RAFT }}\end{array}$ & $\Delta M_{\mathrm{n}}$ & $\begin{array}{c}\text { share 1.1 of } \\
M_{\mathrm{n}}\left[\mathrm{g} \cdot \mathrm{mol}^{-1}\right]\end{array}$ & BA-units & 1.1-units \\
\hline$p$ BA & 0 & 102,000 & 100,500 & & 0 & 784 & 0 \\
\hline$p$ (BA-co-1.1) & 0.5 & 145,000 & 143,040 & 42,540 & 715 & 1,110 & 3 \\
\hline$p$ BA & 0 & 93,000 & 91,440 & & 0 & 713 & 0 \\
\hline$p$ (BA-co-1.1) & 3 & 119,000 & 116,940 & 25,500 & 3,510 & 885 & 16
\end{tabular}

As can be seen for this assumption, the impact of the integration of $\mathbf{1 . 1}$ should not be very large in these ranges of 1.1-quantities in the monomer feed as 16 units of $1.1\left(\approx 3,510 \mathrm{~g} \cdot \mathrm{mol}^{-1}\right)$ do not compensate a mass difference of $25,500 \mathrm{~g} \cdot \mathrm{mol}^{-1}$. Under the assumption that $\mathbf{1 . 1}$ is favoured over BA in the polymerisation and is consumed completely before any BA is which is very unlikely resulting in the following values:

Table 8-3: Comparison of the theoretical composition of polymers of the same monomer conversion

\begin{tabular}{c|c|c|c|c|c|c|c} 
sample & $\begin{array}{c}\mathbf{1 . 1} \text {-feed } \\
{[\mathrm{mol} \%]}\end{array}$ & $\begin{array}{c}M_{\mathrm{n}}^{\mathrm{UV} \cdot 1.52} \\
{\left[\mathrm{~g} \cdot \mathrm{mol}^{-1}\right]}\end{array}$ & $\begin{array}{c}M_{\mathrm{n}} \text { minus } \\
M_{\text {RAFT }}\end{array}$ & $\Delta M_{\mathrm{n}}$ & $\begin{array}{c}\text { share 1.1 of } \\
M_{\mathrm{n}}\left[\mathrm{g} \cdot \mathrm{mol}^{-1}\right]\end{array}$ & BA-units & 1.1-units \\
\hline$p$ BA & 0 & 102,000 & 100,500 & & 0 & 784 & 0 \\
\hline$p$ (BA-co-1.1) & 0.5 & 145,000 & 143,040 & 42,540 & 6,862 & 1,063 & 31 \\
\hline$p$ BA & 0 & 93,000 & 91,440 & & 0 & 713 & 0 \\
\hline$p$ (BA-co-1.1) & 3 & 119,000 & 116,940 & 25,500 & 14,800 & 797 & 66
\end{tabular}

Again the discrepancy cannot be explained via the assumptions made. Also FRP experiments support the finding that the molecular weights of copolymers are higher than homopolymers at the same point of monomer conversion (Figure 8-17). 


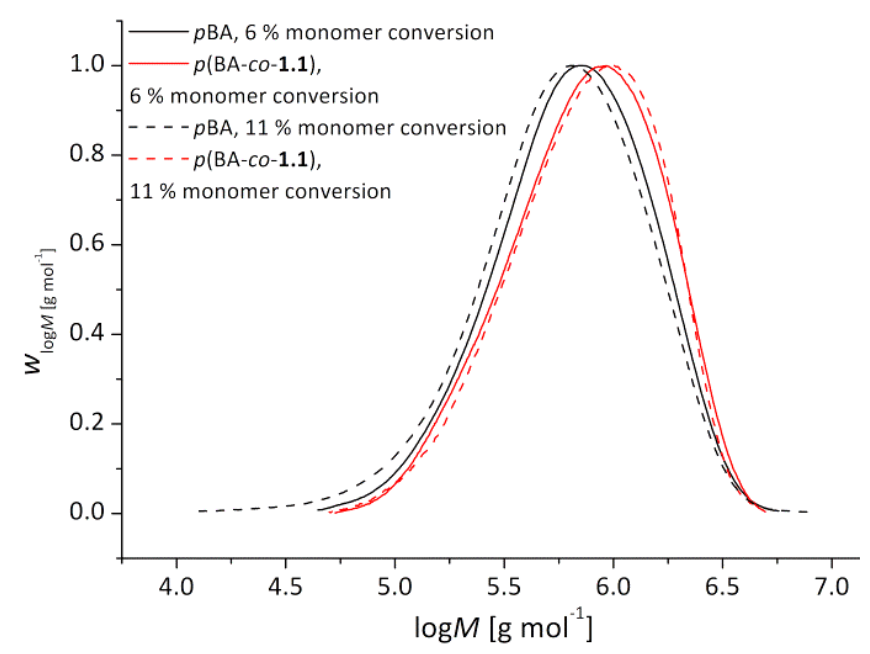

Figure 8-17: SEC-data of two samples of the FRP of BA with 1 mol\% 1.1 (blue) compared to the FRP of BA (black) using $0.9 \mathrm{mmol} \cdot \mathrm{L}^{-1} \mathrm{AIBN}$ at 6 and $11 \%$ monomer conversion.

As there is no arithmetical solution for this phenomenon, investigations via NMR-spectroscopy were conducted to clarify whether chemical reasons cause this discrepancy. The ${ }^{1} \mathrm{H}$ NMR spectra of the $p B A$ and the $p$ (BA-Co-1.1) sample (SECs shown in Figure 8-16 right), with approximately $25 \%$ monomer conversion were recorded. Using acetonitrile as internal standard the peak areas, which correspond directly with the number of monomer units in the polymer, could be standardised. The integration was compiled of the triplet caused by the BA's terminal methyl group and the singlet of the DMIs methyl groups. For the homopolymer the area under the peak corresponds with 1.16 relative monomer units. In the case of the copolymer an area of 1.13 resulting from BA and an area of 0.02 from 1.1 could be determined which sum up to 1.15 relative monomer units. It shows that equally many monomer units were added to form the star polymer. It can be concluded that no effect of the polymerisation itself caused the difference in molecular weights but that the steric demanding DMI moiety distorts the results obtained by SEC-measurements. Due to the bulky side chain of this co-monomer the hydrodynamic radius of the star polymer increases and the molecules appear larger in the SEC-determination. Figure 8-18 illustrates the difference schematically (red spheres $=\mathrm{BA}$, orange spheres $\mathbf{= 1 . 1}$ ). The more 1.1-units are integrated the more pronounced is the steric effect as the co-monomer units are integrated firstly (chapter 8.2) which leads to a stericly demanding outer sphere of the star polymer. 

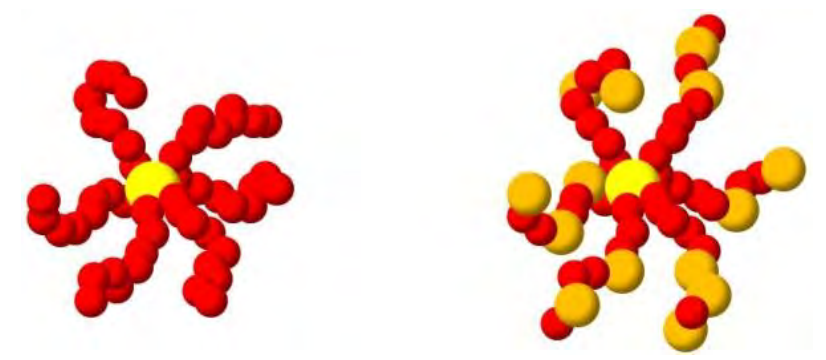

Figure 8-18: Schematic comparison regarding the steric demand of $p B A$ and $p(B A-c o-1.1)$ stars.

It also has to be mentioned that there are no standards to calibrate the SEC set up for the copolymers applied within this project nor are $\mathrm{MH}$ coefficients known for this system. All these measurements were carried out against a universal calibration with $\mathrm{MH}$ parameters for $p \mathrm{BA}$. The NMR investigation verified that both polymers consist of the same number of monomer units and therefore that the copolymers have approximately the same molecular weight as the homopolymers at the point of same monomer conversion.

It can be concluded that the addition of $\mathbf{1 . 1}$ has no influence on the FRP of BA but results in distinctive changes in the RAFT-copolymerisation: induction delays the start of the copolymerisation compared to the equivalent homopolymerisation and leads therefore to smaller molecular weights and smaller monomer conversions. The induction is an effect resulting from interactions between $\mathbf{1 . 1}$ and the RAFT-agent as RAFT homopolymerisations of BA proceed without delay. Furthermore, with increasing 1.1-feed side reactions are supported, i.e. star-star coupling which could be proven by bimodal MWDs and consequently larger PDI-values. Additionally the hydrodynamic radius of the molecules is increased by introduction of the steric demanding co-monomer which leads to apparent higher molecular weights at the same point of monomer conversion.

\subsection{Determination of the Cross-Linkable Units in a pBA Star}

A special interest throughout this work was in determining the copolymer composition of the eventual product. The final star polymer consists of six diblock copolymer arms which are composed of a polymer block of one monomer and a second block synthesised of two statistically distributed monomers. In this chapter the composition of this statistical copolymer block is discussed.

The first method investigated to determine the composition of the copolymer arms was UVVis spectroscopy as it has many advantages: The co-monomer $\mathbf{1 . 1}$ is UV-active, which is the property used in the cross-linking step. $p B A$ is UV-transparent and does therefore not interfere with the measurement. Besides determining the amount of co-monomer in the polymer, it is possible to follow the cross-linking procedure. UV-Vis spectroscopy is easily available in the laboratories and therefore, along with the fast sample preparation and short measuring times, results can be obtained 
quickly. Only small amounts of polymer are needed to measure a spectrum so even samples with small monomer conversions can be examined.

All spectra were recorded on a spectrophotometer Cary 300 at a wavelength range from 190 to $600 \mathrm{~nm}$. Figure 8-19 shows the recorded UV-Vis spectra of the UV-sensitive monomer 1.1 and the six-functional RAFT-agents $\mathbf{R}_{6} \mathbf{2}$ and $\mathbf{R}_{\mathbf{6}} \mathbf{3}$. The data points above $400 \mathrm{~nm}$ were excluded as no absorbance occurred in this region.

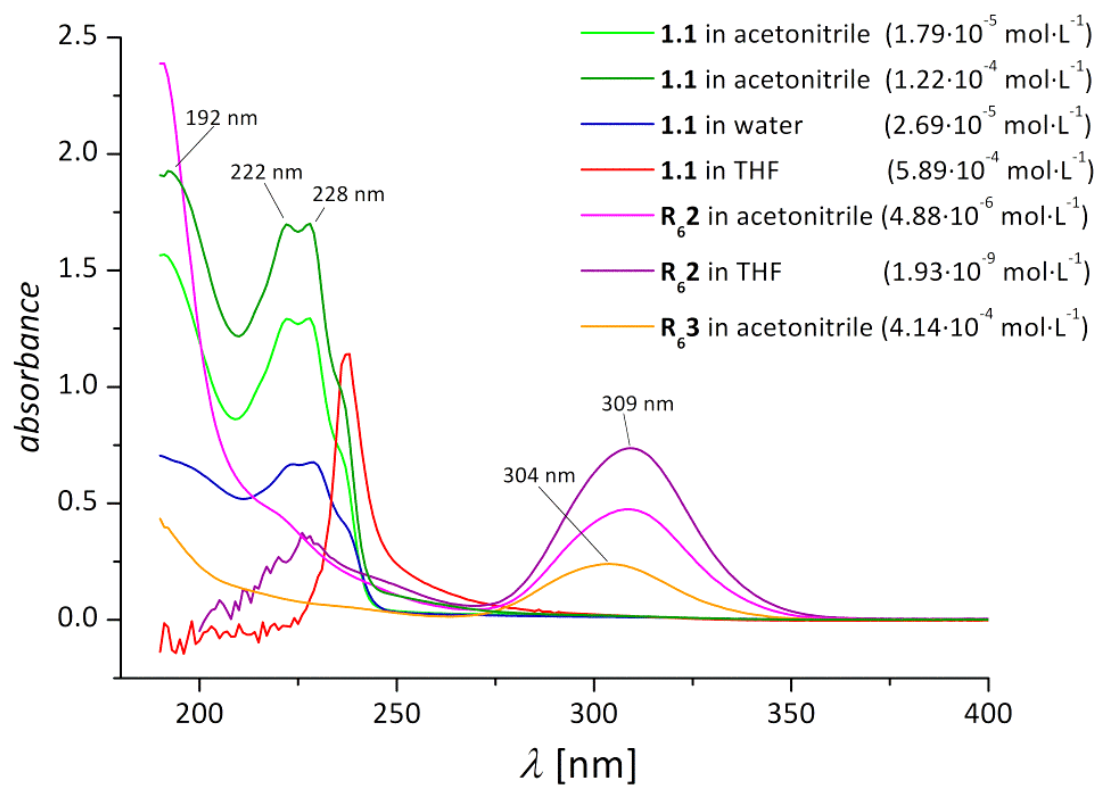

Figure 8-19: UV-Vis spectra of $1.1, \boldsymbol{R}_{6} \mathbf{2}$ and $\boldsymbol{R}_{6} \mathbf{3}$ in various solvents.

As it is known from the literature, ${ }^{(167)}$ the absorbance maximum of the trithio units, the UV-active part of the RAFT-agents, is at approximately $310 \mathrm{~nm}$ (spectra in pink, orange and purple) and the absorbance of the DMI moiety occurs at approximately $224 \mathrm{~nm}$ (spectra in blue and green). ${ }^{(107)}$ For small RAFT-concentrations, which are given in polymer samples, these two signals are suitably separated and hence allow a clean integration of the 1.1-signal over the half-peak width to determine the 1.1-concentration in the polymer. However, a large drawback can be taken from this diagram: THF has its cut-off wavelength at $220 \mathrm{~nm}$, as can be<smiles>C=CC(=O)OCCCC(=O)OCCN1C(=O)C(C)=C(C)C1=O</smiles>
seen from the abrupt change in the spectra recorded in THF (red and purple). Therefore THF cannot be used in this method. Unfortunately $p B A$ is, from all tested solvents, only soluble in THF and chloroform (cut-off at $260 \mathrm{~nm}$ ). As a result UV-Vis spectrometry cannot be used to investigate the polymer composition of BA-co-1.1-polymers or the process of the cross-linking reaction.

A more challenging method to determine the polymer composition is NMR spectroscopy. A known method ${ }^{(147)}$ is the comparison of specific signals belonging to the various monomers which build up the polymer (here method II). To verify those results a second method was designed 
(method I). Here the integrals of the signals belonging to the DMI-unit of the comonomer in the polymer were determined and compared via an internal standard (here acetonitrile). A requirement to apply these methods is the full characterisation of the co-monomer 1.1. Alongside Ann-Christin Pöppler from the research group of Prof. Dr. D. Stalke of the Institute of Inorganic Chemistry, GeorgAugust University Göttingen different NMR-experiments $\left({ }^{15} \mathrm{~N}-\mathrm{HMBC}, \mathrm{H}, \mathrm{H}-\mathrm{COSY},{ }^{1} \mathrm{H}\right.$ NMR) were conducted on a Bruker Avance III $400 \mathrm{MHz}$ spectrometer (BrukerBiospin, Rheinstetten) with a broadband-observe probe, z-gradient and temperature unit. All measurements were performed in $\mathrm{CDCl}_{3}$ with acetonitrile as internal standard applying a relaxation time $d_{1}$ of $0.1 \mathrm{~s}$ and a pulse length $p_{1}$ of $10 \mu \mathrm{s}$. As no literature data was available of the chemical shift of the co-monomer 1.1 in ${ }^{15} \mathrm{~N} N M R$, as a first step the co-monomer $\mathbf{1 . 1}$ had to be characterised. Furthermore the chemical shifts of the ethyl-bridge protons in the ${ }^{1} \mathrm{H}$ NMR spectrum had to be clarified. A two-dimensional NMR method, ${ }^{15} \mathrm{~N}-\mathrm{HMBC}$ (Heteronuclear Multiple Bond Correlation), was applied to yield straightforward data despite the low natural abundance $(0.37 \%)$ of ${ }^{15} \mathrm{~N}$. In a ${ }^{15} \mathrm{~N}-\mathrm{HMBC}$ experiment the interactions between the ${ }^{15} \mathrm{~N}$ and ${ }^{1} \mathrm{H}$ nuclei in proximity to each other are detected.

To determine these values and to check whether the RAFT-agent or the initiator disturb the measurement, ${ }^{15} \mathrm{~N}-\mathrm{HMBC}$ experiments were carried out with the reaction mixture $\left(c_{1.1}=105 \mathrm{mmol} \cdot \mathrm{L}^{-1}, c_{\mathrm{AlBN}}=0.85 \mathrm{mmol} \cdot \mathrm{L}^{-1}, c_{\mathrm{R} 62}=0.55 \mathrm{mmol} \cdot \mathrm{L}^{-1}\right.$ in a toluene:BA mixture $1: 1$, equals $\left.c_{B A}=351 \mathrm{mmol} \cdot \mathrm{L}^{-1}\right)$. In Figure 8-20 first the ${ }^{1} \mathrm{H}$ spectrum of this mixture is shown to get an overview of the different signals.

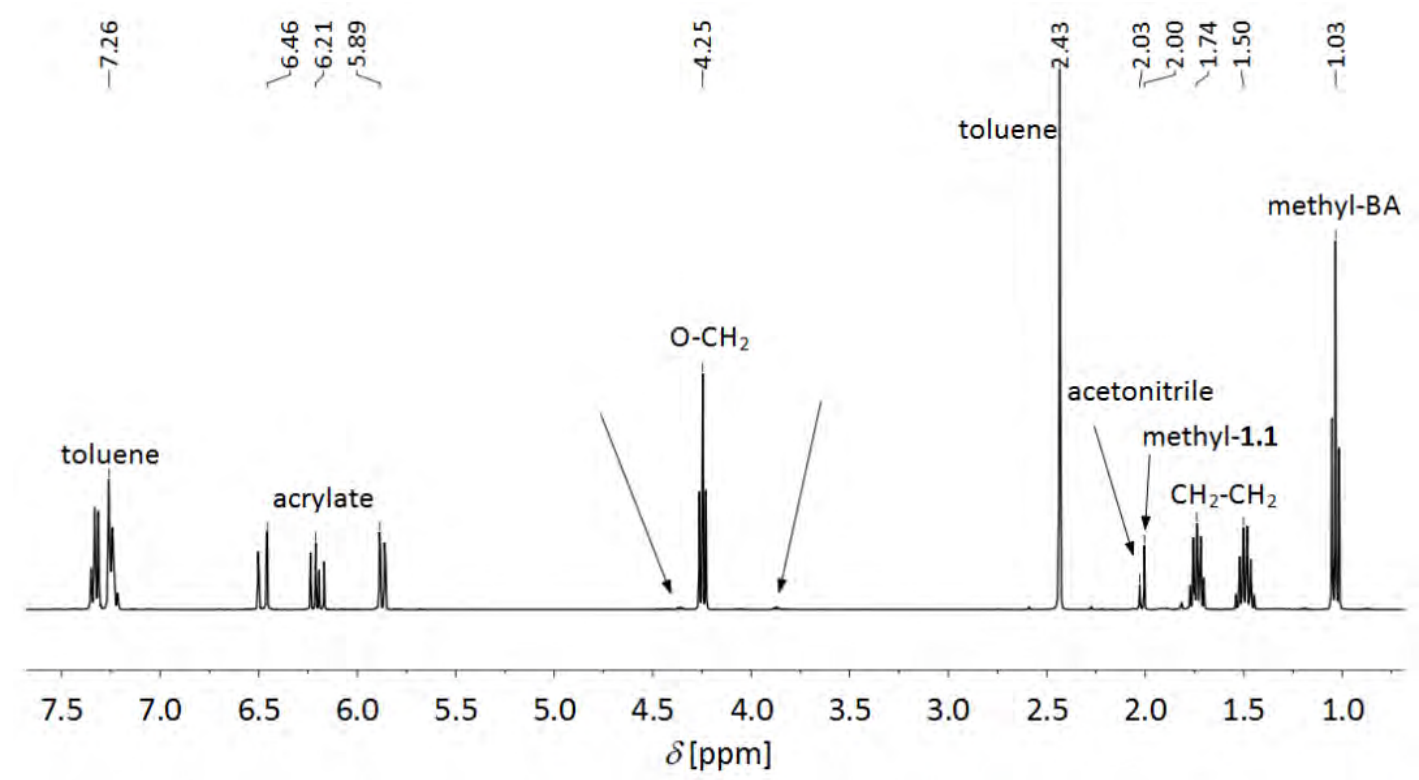

Figure 8-20: ${ }^{1} \mathrm{H}$ NMR spectrum of the polymerisation mixture consisting of $c_{1.1}=105 \mathrm{mmol} \cdot \mathrm{L}^{-1}$, $c_{A I B N}=0.85 \mathrm{mmol} \cdot \mathrm{L}^{-1}, c_{R 62}=0.55 \mathrm{mmol} \cdot \mathrm{L}^{-1}, c_{B A}=351 \mathrm{mmol} \cdot \mathrm{L}^{-1}$ in toluene recorded in $C D \mathrm{Cl}_{3}$. 
From left to right the different signals can be assigned to the compounds in the mixture: In the aromatic region above $7.00 \mathrm{ppm}$ the multiplet of the solvent toluene is found which overlaps with the singlet of residual $\mathrm{CHCl}_{3}$ at $7.26 \mathrm{ppm}$. From 6.46 to $5.89 \mathrm{ppm}$ the signals of the acrylic unit, which is similar in BA and 1.1, appear. The doublets of doublets caused by the terminal protons of the double bond are detected at 5.89 and $6.46 \mathrm{ppm}$, respectively. The quartet in between belongs to the proton next to the carbonyl group. At $4.25 \mathrm{ppm}$ the $\mathrm{CH}_{2}$-group directly adjacent to the BA's ester group shows a triplet. Left and right from this signal, quintets of low intensity, marked by the black arrows, can be seen. These correspond to the protons of the ethyl bridge in $\mathbf{1 . 1}$ connecting the UVsensitive and the monomeric units and have to be categorized. Further in the up field region of the spectrum the methyl group of the toluene can be found as well as the singlet of the DMI's methyl groups at $2.00 \mathrm{ppm}$ and the singlet of the internal standard acetonitrile. The quintet at $1.74 \mathrm{ppm}$ and the sextet at $1.48 \mathrm{ppm}$ result from the protons of the $\mathrm{CH}_{2}-\mathrm{CH}_{2}$-group in the butyl moiety of $\mathrm{BA}$ and the terminal methyl group of this rest causes a triplet at $1.03 \mathrm{ppm}$. As can be seen, neither the RAFTagent nor the initiator can be identified. The concentrations of those compounds in the mixture are small enough to have no influence on the measurement which also ensures that the RAFT-agent does not interfere with the measurements of the polymer samples later on. Figure 8-21 shows the ${ }^{15} \mathrm{~N}-\mathrm{HMBC}$ of this polymerisation mixture. On the $\mathrm{y}$-scale the ${ }^{15} \mathrm{~N}$ NMR spectrum and on the $\mathrm{x}$-scale the ${ }^{1} \mathrm{H}$ NMR spectrum, seen above, are plotted. The observed cross-peaks are the result of a transfer of magnetisation between the ${ }^{1} \mathrm{H}$ and ${ }^{15} \mathrm{~N}$ nuclei in proximity to each other. Interactions between two cores can maximal occur via two or three bonds so that structural conclusions could be drawn by these cross-peaks. 


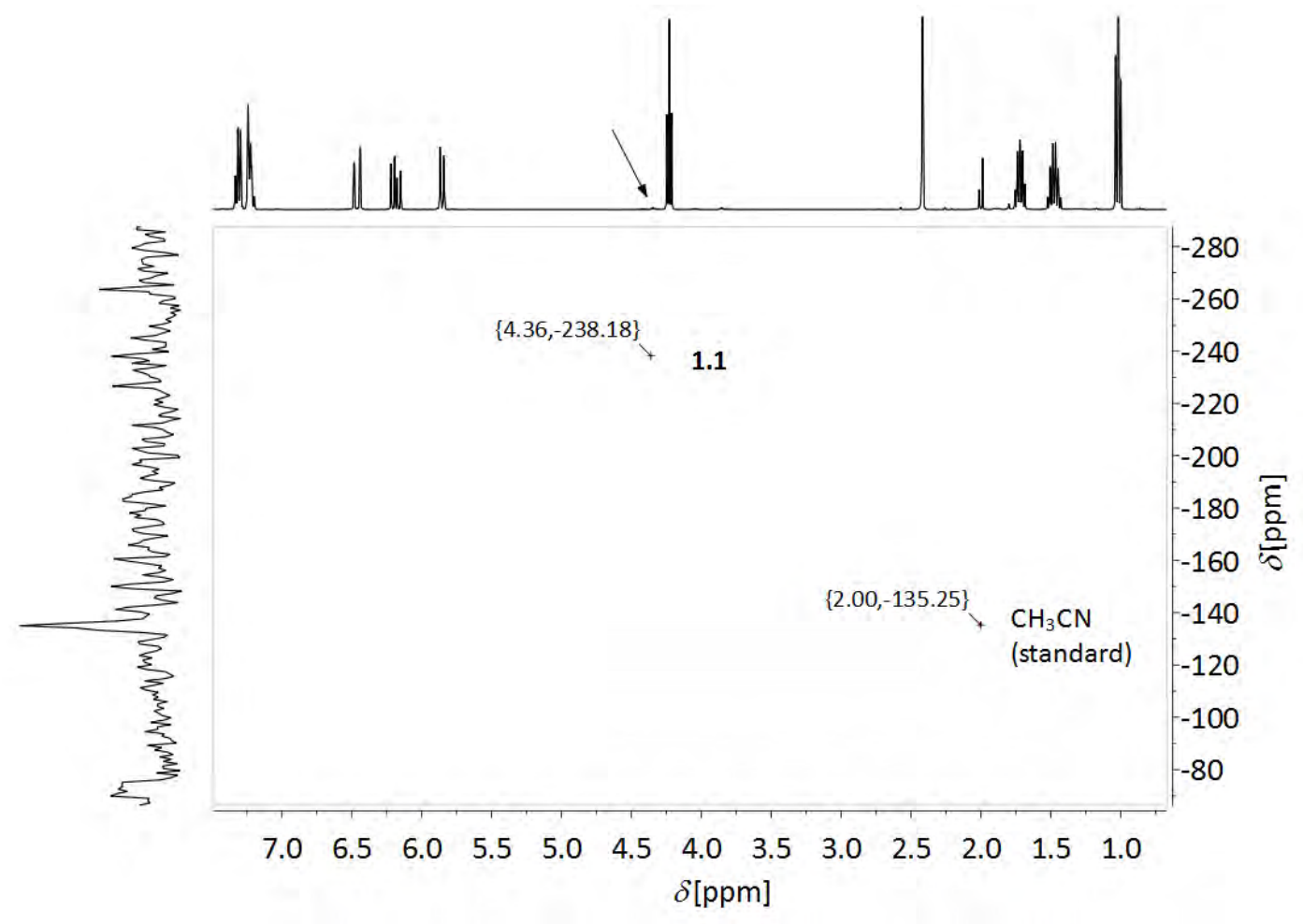

Figure 8-21: ${ }^{15} \mathrm{~N}-\mathrm{HMBC}$ spectrum of the reaction mixture recorded in $\mathrm{CDCl}_{3}\left({ }^{15} \mathrm{~N}\right.$ versus $\left.{ }^{1} \mathrm{H}\right)$.

The chemical shift of $\mathbf{1 . 1}$ in the ${ }^{15} \mathrm{~N}-\mathrm{HMBC}$ could be determined at $4.36 \mathrm{ppm}$ in the ${ }^{1} \mathrm{H}$ dimension and $-238.18 \mathrm{ppm}$ in the ${ }^{15} \mathrm{~N}$ dimension. For acetonitrile values of $2.00 \mathrm{ppm}$ and $-135.25 \mathrm{ppm}$ were found which are confirmed by literature. ${ }^{(184)}$ Accordingly the triplet in the up field region at approximately $3.8 \mathrm{ppm}$ should result from the protons of the $\mathrm{CH}_{2}$-group directly adjacent to the oxygen atom. To confirm this statement $\mathrm{H}, \mathrm{H}-\mathrm{COSY}$ measurements were performed. COSY spectra show the spin-spin-coupling of equal nuclei mediated through bonds and give therefore information about the direct interactions of connected groups. Such a $\mathrm{H}, \mathrm{H}-\mathrm{COSY}$ plot for a $p(\mathrm{BA}-\mathrm{CO}-\mathbf{1 . 1})$ sample is shown below (Figure 8-22). 


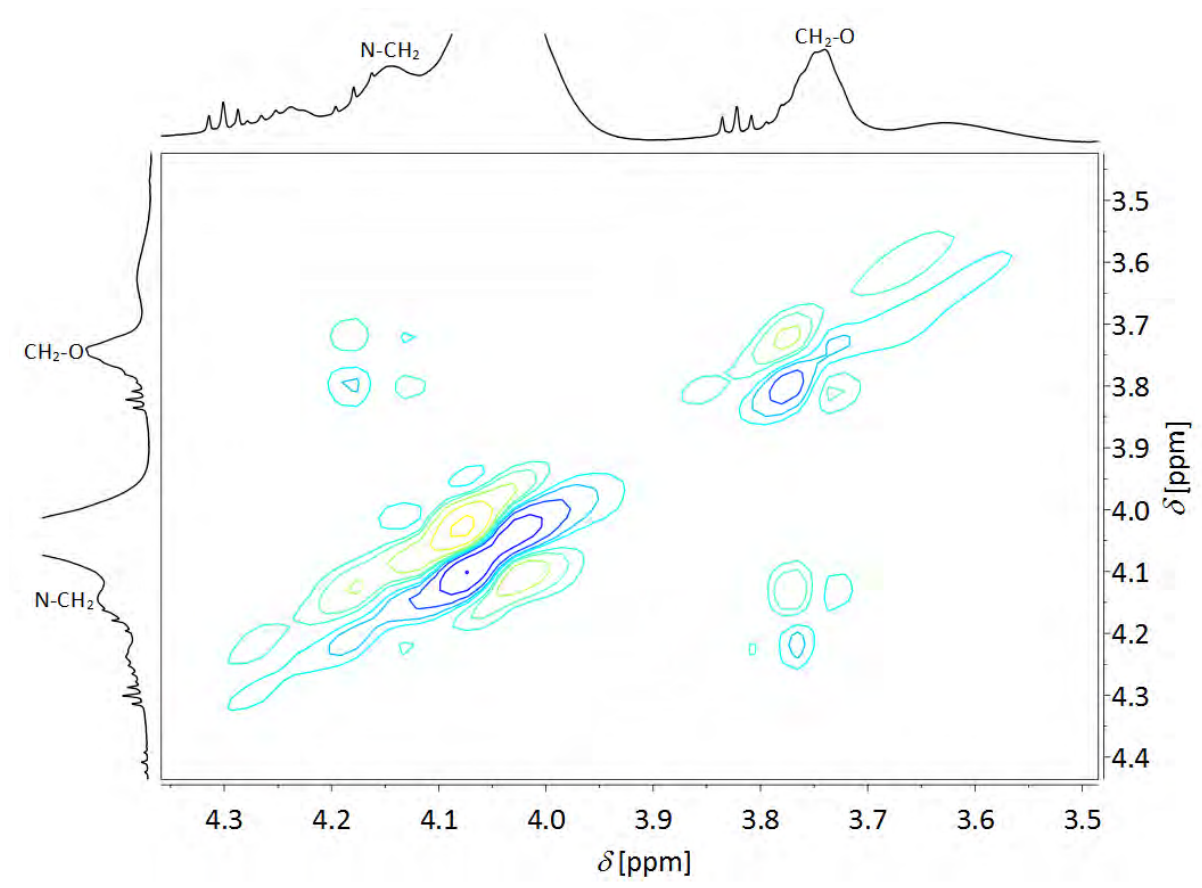

Figure 8-22: Extract from the $\mathrm{H}, \mathrm{H}$-COSY spectrum of a $\mathrm{p}(\mathrm{BA}-\mathrm{CO}-1.1)$ sample recorded in $\mathrm{CDCl}_{3}$.

Only a partial spectrum, from 3.50 to $4.40 \mathrm{ppm}$, is shown here (Figure 8-22) as the other $\mathrm{H}, \mathrm{H}$ coupling processes are not relevant for the structure elucidation of 1.1's ethyl bridge. On basis of the cross-peaks it could be proved that the protons of the $\mathrm{CH}_{2}$-group adjacent to the nitrogen, which was classified previously in the ${ }^{15} \mathrm{~N}$-HMBC experiment, are interacting with the protons of the $\mathrm{CH}_{2}$-group adjacent to the oxygen atom, i.e. they are directly connected. With these data in hand, looking back at Figure 8-20, it can be stated that the triplet of the ethyl bridge in $\mathbf{1 . 1}$ in the down field region at $4.36 \mathrm{ppm}$ results from the protons which are directly adjacent to the nitrogen atom of the DMI moiety. Furthermore the triplet at $3.78 \mathrm{ppm}$ arises from the protons which are directly adjacent to the oxygen atom. This information helps in the determination of the amount of 1.1-units in the copolymer by integration of the peaks. It has become apparent that it is not feasible to use HMBC investigations for the polymer samples as the broadening of the signals caused by the polymer environment often inhibits the detection of the cross-peaks. ${ }^{1} \mathrm{H}$ NMR spectra were recorded and analysed instead. The following figure shows an example of such a spectrum. The different signals are explained for a better overview later on. 


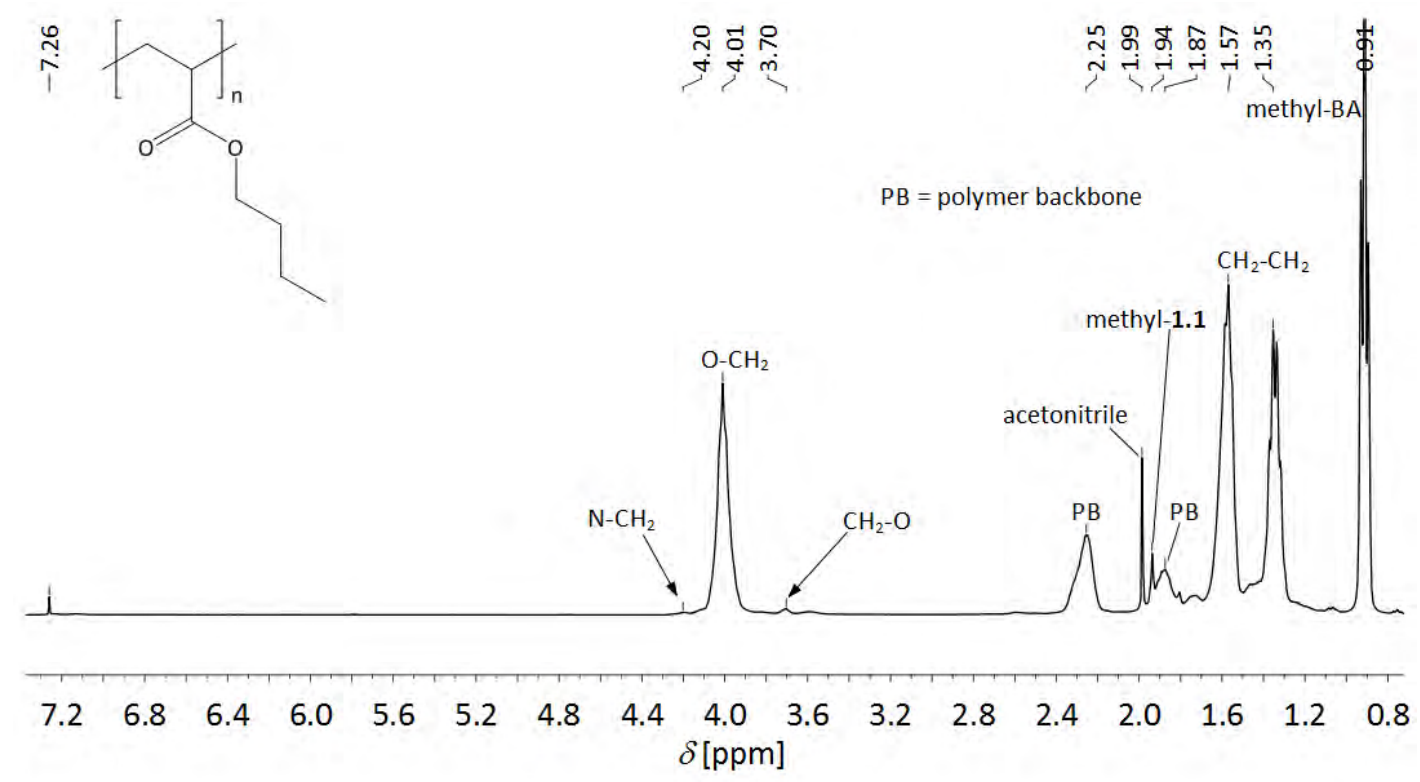

Figure 8-23: ${ }^{1} \mathrm{H} N M R$ spectrum of $p(B A-c o-1.1)$ in $\mathrm{CDCl}_{3}$ with acetonitrile as internal standard.

Regarding the down field region of the spectrum (Figure 8-23) only the $\mathrm{CHCl}_{3}$ peak can be found, as the polymer was precipitated from the reaction mixture. All signals around $6.00 \mathrm{ppm}$ have disappeared as these signals arose due to the acrylate unit. The polymerisation took place at this position and hence, the signal has shifted into the up field region. It can be seen that no monomer remains. At $4.01 \mathrm{ppm}$ again the signal of the $\mathrm{CH}_{2}$-group directly adjacent to $\mathrm{BA}$ 's oxygen atom is found. The signal is broadened due to the amount of BA-units which are connected to one polymer strand. The resolution of the signal is also weakened. Left and right this apparent peak the signals of the now categorized ethyl bridge of $\mathbf{1 . 1}$ are found with the signal from the $\mathrm{CH}_{2}$-group connected to the nitrogen atom arising at $4.20 \mathrm{ppm}$. The signal resulting from the $\mathrm{CH}_{2}$-group connected to the oxygen atom accordingly appears at $3.75 \mathrm{ppm}$. In the range of 2.40 to $2.00 \mathrm{ppm}$ the signals resulting from the polymer backbone appear newly between the already known signals of the methyl groups of 1.1 and acetonitrile. The only change of the signals in the up field region below $2.00 \mathrm{ppm}$ was the signal broadening due to the increasing number of monomer units combined to form the polymer chains, i.e. arms of the star polymer.

In the following the results of those investigations are presented. Figure 8-24 shows the superimposed ${ }^{1} \mathrm{H}$ NMR spectra of a copolymerisation with $4,920 \mathrm{mmol} \cdot \mathrm{L}^{-1} \mathrm{BA}$ and $280 \mathrm{mmol} \cdot \mathrm{L}^{-1} 1.1$ using $0.59 \mathrm{mmol} \cdot \mathrm{L}^{-1} \mathbf{R}_{6} \mathbf{2}\left(M_{\mathrm{R} 62}=1,864.84 \mathrm{~g} \cdot \mathrm{mol}^{-1}\right)$ and $1.09 \mathrm{mmol} \cdot \mathrm{L}^{-1} \mathrm{AIBN}$ in toluene at $60{ }^{\circ} \mathrm{C}$. 


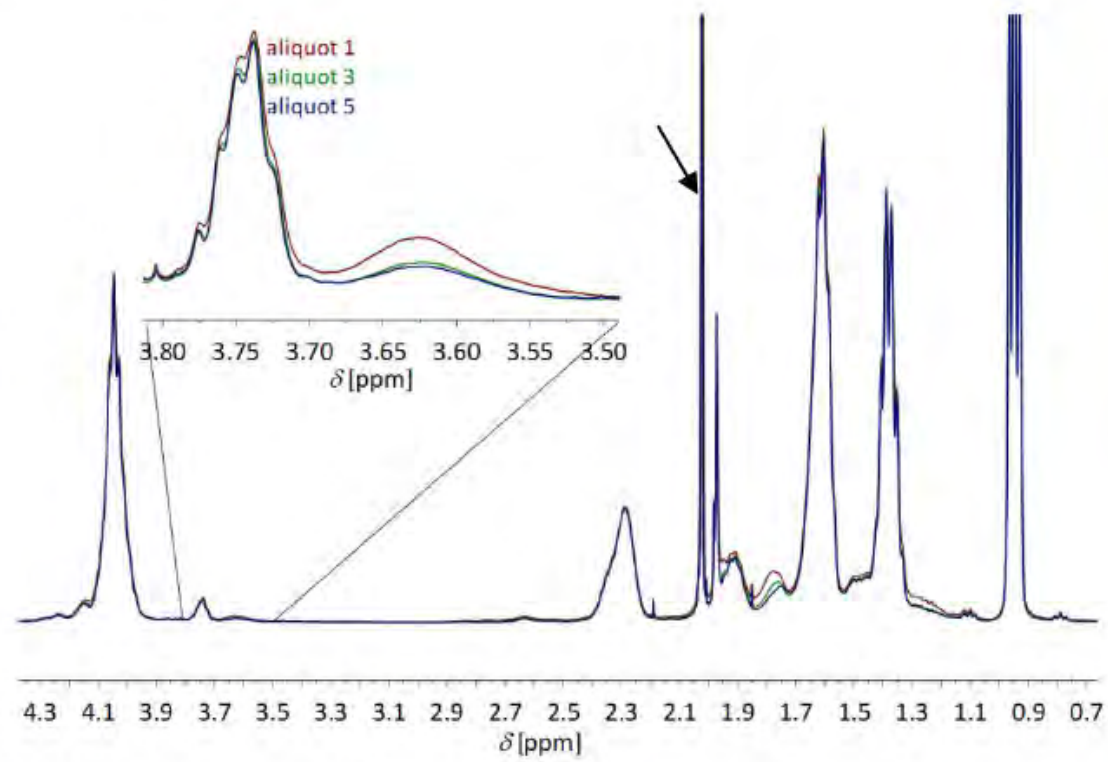

Figure 8-24: Superimposed, standardised ${ }^{1} \mathrm{H} N \mathrm{NR}$ spectra measured in $\mathrm{CDCl}_{3}$ of the first, third and fifth aliquot of a copolymerisation of $B A$ and 1.1 using $4,920 \mathrm{mmol} \cdot \mathrm{L}^{-1} \mathrm{BA}, 280 \mathrm{mmol} \cdot \mathrm{L}^{-1} 1.1,0.59 \mathrm{mmol} \cdot \mathrm{L}^{-1} \boldsymbol{R}_{6} 2$ and $1.09 \mathrm{mmol} \cdot \mathrm{L}^{-1} \mathrm{AlBN}$ in toluene at $60^{\circ} \mathrm{C}$.

The signal at $3.75 \mathrm{ppm}$ (magnified) and the singlet of the standard (arrow) were integrated over their half-peak width due to overlapping with other signals. The integrals have to be standardised with the protons causing the signal, here three protons of acetonitrile and two of 1.1. Via the known concentration of acetonitrile the concentration of 1.1 can be calculated.

$$
\frac{I_{1.1}}{I_{\mathrm{ac}}} \cdot \frac{3}{2}=\frac{c_{1.1}}{c_{\mathrm{ac}}}
$$

with $I_{\mathrm{ac}}$ and $I_{1.1}$ as integrals of the peaks and $c$ the concentrations of the corresponding compound in the sample. Calculating the amount of $\mathbf{1 . 1}$ in the sample by the known sample volume and with the molecular weight of the substance $\left(M_{1.1}=223.23 \mathrm{~g} \cdot \mathrm{mol}^{-1}\right)$ this yields the mass of 1.1 in the sample. As the mass of the polymer $\left(m_{\text {polymer }}\right)$ was known, the weight percentage of $1.1(x w \%)$ related to the polymer can be calculated.

$$
\frac{m_{1.1}}{m_{\text {polymer }}}=\frac{x w \%}{100 w \%}
$$

From the molecular weight of the polymer itself the share of 1.1 in the polymer was calculated and gave, divided by the molecular mass of $\mathbf{1 . 1}$ the number of UV-sensitive units in a star polymer molecule. For the polymer molecular weight the $M_{n}$ determined via UV-detection and corrected by $1.52\left(M_{\mathrm{n}}^{\mathrm{UV} \cdot 1.52}\right)$ is used, as here the contribution of the dead polymer is excluded. The molecular weight of the RAFT-agent applied has to be subtracted. 
An example of calculation is shown here for $0.018 \mathrm{~g}$ of the first aliquot of the polymerisation with a numeric average molecular weight of $121,000 \mathrm{~g} \cdot \mathrm{mol}^{-1}$ dissolved in $0.5 \mathrm{~mL} \mathrm{CDCL}$ with an acetonitrile concentration of $0.099 \mathrm{~mol} \cdot \mathrm{L}^{-1}$ :

$$
\begin{gathered}
I_{1.1} \cdot \frac{3}{2}=a_{1.1} \rightarrow 0.186 \cdot \frac{3}{2}=0.279 \\
a_{1.1} \cdot c_{\text {standard }}=c_{1.1} \rightarrow 0.279 \cdot 0.099 \mathrm{~mol} \cdot L^{-1}=0.0276 \mathrm{~mol} \cdot L^{-1}
\end{gathered}
$$

with $a_{1.1}$ being the area of the relative integral of the monomer to the integral of acetonitrile. Converting back to the sample volume of $0.5 \mathrm{~mL}$ gives $1.38 \cdot 10^{-5} \mathrm{~mol} 1.1$ in the sample which corresponds with $0.0031 \mathrm{~g}$.

$$
\begin{gathered}
\frac{m_{1.1} \cdot 100 w \%}{m_{\text {polymer }}}=w \%_{1.1} \rightarrow \frac{0.0031 \mathrm{~g} \cdot 100 w \%}{0.018 \mathrm{~g}}=17.1 \mathrm{w} \% \\
\frac{M_{\mathrm{n}(\mathbf{1 . 1})}}{\left(M_{\mathrm{n}}^{\mathrm{UV} \cdot 1.52}-M_{\mathrm{R} 62}\right)}=\frac{17.1 \mathrm{w} \%}{100 \mathrm{w} \%} \\
\rightarrow M_{\mathrm{n}(\mathbf{1 . 1})}=\frac{\left(M_{\mathrm{n}}^{\mathrm{UV} \cdot 1.52}-M_{\mathrm{R} 62)}\right) \cdot 17.1 \mathrm{w} \%}{100 \mathrm{w} \%}= \\
\frac{\left(121,000 \mathrm{~g} \cdot \mathrm{mol}^{-1}-1,864 \mathrm{~g} \cdot \mathrm{mol}^{-1}\right) \cdot 17.1 \mathrm{w} \%}{100 \mathrm{w} \%}=20,343 \mathrm{~g} \cdot \mathrm{mol}^{-1} \\
\frac{M_{\mathrm{n}(1.1)}}{M_{1.1}}=\frac{20,343 \mathrm{~g} \cdot \mathrm{mol}^{-1}}{223.23 \mathrm{~g} \cdot \mathrm{mol}^{-1}} \approx 92 \\
\frac{M_{\mathrm{n}(\mathrm{BA})}}{M_{\mathrm{BA}}}=\frac{98,434 \mathrm{~g} \cdot \mathrm{mol}^{-1}}{128.17 \mathrm{~g} \cdot \mathrm{mol}^{-1}} \approx 768
\end{gathered}
$$

with $M_{\mathrm{n}(\mathrm{BA})}$ and $M_{\mathrm{n}(1.1)}$ as share of the polymer's molecular weight corresponding with the monomers. Every star of this polymer aliquot consists of 92 UV-reactive units and 768 units of BA. As all arms show the same reactivity a similar composition can be assumed, therefore every arm of this star polymer has approximately 15 UV-reactive units. These values should be verified by method II via the comparison of monomer-specific signals in the ${ }^{1} \mathrm{H}$ NMR spectrum of the polymer sample. The triplet caused by the methyl group of $\mathrm{BA}$ at $0.91 \mathrm{ppm}$ and the signal at $3.70 \mathrm{ppm}$ causing by the $\mathrm{CH}_{2}$-group of $\mathbf{1 . 1}$ applied before were chosen. The integral $\left(I_{\mathrm{BA}}, I_{1.1}\right)$ of the single peaks is referred back to the protons causing the signals (equation 8-6). 


$$
\begin{gathered}
I_{\mathrm{BA}}=1=3 \text { protons } \rightarrow \frac{I_{\mathrm{BA}}}{3 \text { protons }}=\frac{1}{3 \text { protons }}=0.333 \overline{3} \\
I_{1.1}=0.0061=2 \text { protons } \rightarrow \frac{I_{1.1}}{2 \text { protons }}=\frac{0.069}{2 \text { protons }}=0.0345
\end{gathered}
$$

These values are put in relation to each other yielding the molar share ( $F[\mathrm{~mol} \%])$ of each monomer in the polymer.

$$
\begin{aligned}
F_{1.1} & =\frac{\frac{I_{1.1}}{2 \text { protons }}}{\frac{I_{\mathrm{BA}}}{3 \text { protons }}+\frac{I_{1.1}}{2 \text { protons }}}=\frac{0.0345}{0.368}=0.094 \mathrm{~mol} \% \\
F_{\mathrm{BA}} & =\frac{\frac{I_{\mathrm{BA}}}{3 \text { protons }}}{\frac{I_{\mathrm{BA}}}{3 \text { protons }}+\frac{I_{1.1}}{2 \text { protons }}}=\frac{0.33}{0.368}=0.906 \mathrm{~mol} \%
\end{aligned}
$$

With molecular weights of the monomers $\left(M_{1.1}=223.23 \mathrm{~g} \cdot \mathrm{mol}-1, M_{\mathrm{BA}}=128.17 \mathrm{~g} \cdot \mathrm{mol}^{-1}\right)$ the molecular share is converted to the weight share $(w \%)$ of each monomer.

$$
\begin{aligned}
& F_{\mathrm{BA}} \cdot M_{\mathrm{BA}}=m_{\mathrm{BA}} \rightarrow 0.906 \mathrm{~mol} \% \cdot 128.17 \mathrm{~g} \cdot \mathrm{mol}^{-1}=116.15 \mathrm{~g} \\
& F_{1.1} \cdot M_{1.1}=m_{1.1} \rightarrow 0.094 \mathrm{~mol} \% \cdot 223.23 \mathrm{~g} \cdot \mathrm{mol}^{-1}=20.94 \mathrm{~g} \\
& m_{\mathrm{BA}}+m_{1.1}=m_{100 w \%} \rightarrow 116.15 g+20.94 g=137.09 g \equiv 100 w \% \\
& m_{\mathrm{BA}} \equiv 84.73 w \% \\
& m_{1.1} \equiv 15.27 w \%
\end{aligned}
$$

From the molecular weight of the polymer $\left(M_{\mathrm{n}}\right.$ determined by UV-detection, corrected by 1.52) minus the molecular weight of the RAFT-agent the weight of one star polymer molecule $\left(w_{\text {star }}\right)$ is obtained by applying the Avogadro constant. One star without its RAFT-core of the here introduced aliquot has a weight of $1.97 \cdot 10^{-19} \mathrm{~g}$. Determining the polymer share of $\mathbf{1 . 1}$ with the weight share $\left(w \%_{1.1}\right)$ and divided by the weight of one monomer molecule the number of monomer units in one star molecule is determined. Here only the determination of the number of 1.1-units is shown exemplary. The calculation for the BA-units was carried out accordingly. 


$$
\begin{gathered}
\frac{\left(M_{\mathrm{n}}^{\mathrm{UV} \cdot 1.52}-M_{\mathrm{R} 62}\right)}{N_{\mathrm{A}}}=w_{\text {star }} \rightarrow \frac{\left(121,000 \mathrm{~g} \cdot \mathrm{mol}^{-1}-1,864 \mathrm{~g} \cdot \mathrm{mol}^{-1}\right)}{6.022 \cdot 10^{23} \mathrm{~mol}^{-1}}=1.97 \cdot 10^{-19} \mathrm{~g} \\
15.27 \mathrm{w} \%=3.01 \cdot 10^{-20} \mathrm{~g} \\
\frac{M_{1.1}}{N_{\mathrm{A}}}=w_{1.1} \rightarrow \frac{223.23 \mathrm{~g} \cdot \mathrm{mol}^{-1}}{6.022 \cdot 10^{23} \mathrm{~mol}^{-1}}=3.71 \cdot 10^{-22} \mathrm{~g} \\
\text { number of 1.1-units }=\frac{3.01 \cdot 10^{-20} \mathrm{~g}}{3.71 \cdot 10^{-22} \mathrm{~g}} \approx 81
\end{gathered}
$$

Here 81 1.1-units and 785 BA-units were calculated. These values distinctively differ from those determined by the first method. Table 8-4 summarises the polymer composition in monomer units for the three aliquots of the copolymerisation of BA and 1.1 using $4,920 \mathrm{mmol} \cdot \mathrm{L}^{-1} \mathrm{BA}, 280 \mathrm{mmol} \cdot \mathrm{L}^{-1}$ 1.1, $0.59 \mathrm{mmol} \cdot \mathrm{L}^{-1} \mathbf{R}_{6} \mathbf{2}$ and $1.09 \mathrm{mmol} \cdot \mathrm{L}^{-1} \mathrm{AlBN}$ in toluene at $60{ }^{\circ} \mathrm{C}$ shown in Figure 8-24: From the integration of the signal at $3.75 \mathrm{ppm}$ and referencing to acetonitrile as standard the polymer compositions under method I were obtained. Also the polymer composition via the integration of the 1.1- and BA-signal was determined (method II).

Table 8-4: Determined numbers of BA- and 1.1-units in different polymer samples of a polymerisation with $4,920 \mathrm{mmol} \cdot \mathrm{L}^{-1}$ $B A, 280 \mathrm{mmol} \cdot \mathrm{L}^{-1} 1.1,0.59 \mathrm{mmol} \cdot \mathrm{L}^{-1} \boldsymbol{R}_{6} 2$ and $1.09 \mathrm{mmol} \cdot \mathrm{L}^{-1} \mathrm{AlBN}$ in toluene at $60{ }^{\circ} \mathrm{C}$.

\begin{tabular}{c|c|c|c|c|c|c|c}
\multicolumn{2}{l|}{} & \multicolumn{4}{c|}{ Method I } & \multicolumn{3}{c}{ Method II } \\
\hline $\begin{array}{c}\text { time } \\
{[\mathrm{min}]}\end{array}$ & $\begin{array}{c}M_{\mathrm{n}} \\
{\left[\mathrm{g} \cdot \mathrm{mol}^{-1}\right]}\end{array}$ & $\begin{array}{c}\text { number of } \\
1.1 \text {-units in } \\
\text { the star }\end{array}$ & $\begin{array}{c}\text { number of } \\
\text { BA-units in } \\
\text { the star }\end{array}$ & $\begin{array}{c}1.1 / \text { BA-units } \\
\text { per } \\
\text { star }\end{array}$ & $\begin{array}{c}\text { number of } \\
\text { 1.1-units in } \\
\text { the star }\end{array}$ & $\begin{array}{c}\text { number of } \\
\text { BA-units in } \\
\text { the star }\end{array}$ & $\begin{array}{c}\text { 1.1/BA-units } \\
\text { per } \\
\text { star }\end{array}$ \\
\hline 45 & 121,000 & 91 & 768 & 0.12 & 81 & 785 & 0.10 \\
115 & 305,000 & 207 & 2,002 & 0.10 & 190 & 2,032 & 0.09 \\
155 & 405,000 & 313 & 2,602 & 0.12 & 246 & 2,718 & 0.09
\end{tabular}

A strong discrepancy between the results in monomer units of those methods can be seen. To obtain better results the measuring parameters were changed. The values shown above were derived from spectra recorded with a short relaxation delay $\left(d_{1}=0.1 \mathrm{~s}\right)$ and a large pulse length $\left(p_{1}=10 \mu \mathrm{s}\right)$. Now the experiment was carried out with a longer relaxation delay $\left(d_{1}=10.5 \mathrm{~s}\right)$ and a smaller pulse length $\left(p_{1}=2 \mu \mathrm{s}\right)$ as it was suspected that the relaxation of the nuclei in the polymer is much slower than in smaller molecules. 
For a polymer sample $\left(M_{\mathrm{n}}^{\mathrm{UV} \cdot 1.52}=139,000 \mathrm{~g} \cdot \mathrm{mol}^{-1}\right)$ synthesised with $4,920 \mathrm{mmol} \cdot \mathrm{L}^{-1} \mathrm{BA}, 150 \mathrm{mmol} \cdot \mathrm{L}^{-1}$ 1.1, $0.59 \mathrm{mmol} \cdot \mathrm{L}^{-1} \mathbf{R}_{6} \mathbf{2}$ and $1.09 \mathrm{mmol} \cdot \mathrm{L}^{-1} \mathrm{AIBN}$ in toluene at $60{ }^{\circ} \mathrm{C}$ for 45 minutes following values with both methods and the different experimental settings were obtained:

Table 8-5: Determined numbers of BA- and 1.1-units in a polymer sample of a polymerisation with 4,920 $\mathrm{mmol} \cdot \mathrm{L}^{-1} B A$, $150 \mathrm{mmol} \cdot \mathrm{L}^{-1} \mathbf{1 . 1}, 0.59 \mathrm{mmol} \cdot \mathrm{L}^{-1} \boldsymbol{R}_{6} \mathbf{2}$ and $1.09 \mathrm{mmol} \cdot \mathrm{L}^{-1} \mathrm{AlBN}$ in toluene at $60{ }^{\circ} \mathrm{C}$ for 45 minutes.

\begin{tabular}{c|c|c|c|c} 
& \multicolumn{2}{|c|}{ Method I } & \multicolumn{2}{c}{ Method II } \\
\hline $\begin{array}{c}\text { experimental } \\
\text { settings }\end{array}$ & number of 1.1- & number of BA- & number of 1.1- & number of BA- \\
units in the star & units in the star & units in the star & units in the star \\
\hline$d_{1}=0.1 \mathrm{~s} ; p_{1}=10 \mu \mathrm{s}$ & 43 & 1,052 & 31 & 1,073 \\
$d_{1}=10.5 \mathrm{~s} ; p_{1}=2 \mu \mathrm{s}$ & 28 & 1,079 & 31 & 1,074
\end{tabular}

It can be seen that there is a better agreement between the values obtained by the longer relaxation time. The difference between those values results, on the one hand, from inaccuracies in the integration of the signals. On the other hand, as already mentioned in the previous chapter, the molecular weights of the polymers which were determined by SEC measurements are imperfect causing here a deviation of the results. The experiment carried out with shorter relaxation times and larger pulse angle and with an external standard generates too large values. Furthermore it can be seen that the determination of the polymer composition by the comparison of polymer signals gains same results, independent on the experimental settings. Finally, it could be shown that both methods can verify each other.

From the magnified spectrum of the peak at $3.75 \mathrm{ppm}$ (Figure 8-24) the impression can be gained that the amount of built-in co-monomer decreases with the reaction time regarding the amount of BA-units, i.e. $\mathbf{1 . 1}$ is preferably consumed as it is more frequently integrated into the polymer in the beginning of the polymerisation. This assumption cannot be proven with the data of this polymerisation as the calculation of the polymer composition reveals no change in the $\mathbf{1 . 1}$ to BAratio (Table 8-4) and it is not guaranteed that any co-monomer is left after 115 or 155 minutes of reaction time due to the small co-monomer concentration. To investigate this, a copolymerisation with a much higher amount of 1.1 was performed. $4,920 \mathrm{mmol} \cdot \mathrm{L}^{-1}$ of $\mathrm{BA}$ were polymerised with $1,350 \mathrm{mmol} \cdot \mathrm{L}^{-1} \mathbf{1} .1,0.48 \mathrm{mmol} \cdot \mathrm{L}^{-1} \mathbf{R}_{6} \mathbf{2}$ and $1.22 \mathrm{mmol} \cdot \mathrm{L}^{-1} \mathrm{AlBN}$ in toluene at $60^{\circ} \mathrm{C}$. Figure $8-25 \mathrm{shows}$ the superimposed and standardised ${ }^{1} \mathrm{H}$ NMR spectra of these polymer samples. Again the region between 3.45 and $3.80 \mathrm{ppm}$ was magnified. A decrease of the signal at $3.75 \mathrm{ppm}$ is observed with increasing reaction time as aliquot 3 was taken after 80 minutes of reaction time, aliquot 4 after 125 minutes and aliquot 6 after 215 minutes. The triplet on the left of the signal belongs to 
unconsumed monomer which shows that unconsumed $\mathbf{1 . 1}$ were available in the polymerisation process until the reaction was quenched after 215 minutes.

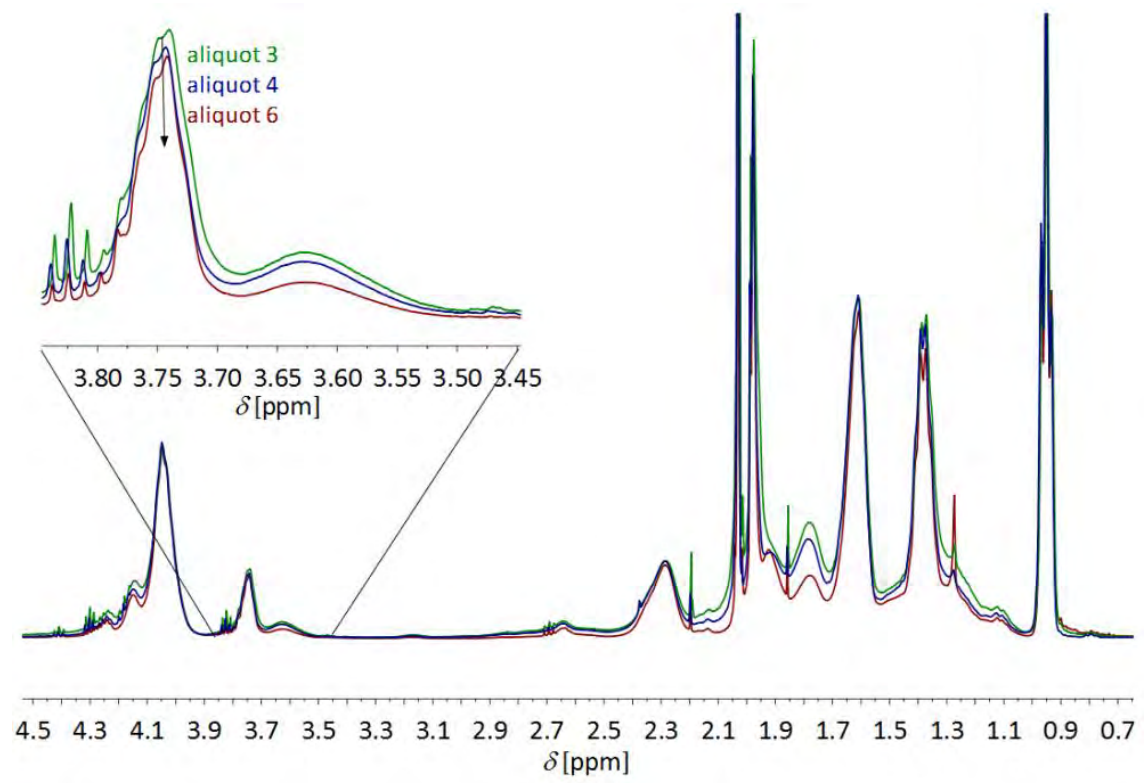

Figure 8-25: Superimposed, standardised ${ }^{1} \mathrm{H} N M R$ spectra measured in $\mathrm{CDCl}_{3}$ of the third, fourth and sixth aliquot of a copolymerisation of BA and 1.1 using $4,920 \mathrm{mmol} \cdot \mathrm{L}^{-1} B A, 1,350 \mathrm{mmol} \cdot \mathrm{L}^{-1} \mathbf{1 . 1}, 0.48 \mathrm{mmol} \cdot \mathrm{L}^{-1} \boldsymbol{R}_{6} \mathbf{2}$ and $1.22 \mathrm{mmol} \cdot \mathrm{L}^{-1} \mathrm{AlBN}$ in toluene at $60^{\circ} \mathrm{C}$.

In the magnified ${ }^{1} \mathrm{H}$ NMR spectra in Figure 8-25 the phenomenon of assumed favoured 1.1consumption mentioned in the example before is observed more clearly. The determination of the polymer composition was executed as described above. Table 8-6 summarises the values for this polymerisation.

Table 8-6: Determined numbers of BA- and 1.1-units in different polymer samples of a polymerisation with $4,920 \mathrm{mmol} \cdot \mathrm{L}^{-1}$ $B A, 1,350 \mathrm{mmol} \cdot \mathrm{L}^{-1} \mathbf{1 . 1}, 0.48 \mathrm{mmol} \cdot \mathrm{L}^{-1} \boldsymbol{R}_{6} \mathbf{2}$ and $1.22 \mathrm{mmol} \cdot \mathrm{L}^{-1} \mathrm{AlBN}$ in toluene at $60{ }^{\circ} \mathrm{C}$.

\begin{tabular}{c|c|c|c|c|c|c}
$\begin{array}{c}\text { time } \\
\text { [min] }\end{array}$ & $\begin{array}{c}M_{\mathrm{n}} \\
{\left[\mathrm{g} \cdot \mathrm{mol}^{-1}\right]}\end{array}$ & $\begin{array}{c}F_{1.1} \\
{[\mathrm{~mol} \%]}\end{array}$ & $\begin{array}{c}F_{\mathrm{BA}} \\
{[\mathrm{mol} \%]}\end{array}$ & $\begin{array}{c}\text { number of } \\
\text { 1.1-units in } \\
\text { the star }\end{array}$ & $\begin{array}{c}\text { number of } \\
\text { BA-units in } \\
\text { the star }\end{array}$ & $\begin{array}{c}\text { 1.1/BA-units } \\
\text { per star }\end{array}$ \\
\hline 80 & 88,000 & 37.9 & 62.1 & 182 & 318 & 0.57 \\
125 & 162,000 & 33.6 & 66.4 & 407 & 709 & 0.57 \\
215 & 306,000 & 30.1 & 69.9 & 910 & 1,585 & 0.57
\end{tabular}

The ratio of the monomer-units shows that there is no change in integration of one of the two monomers over the whole polymerisation process. In this case here with a 3.6:1 ratio of the monomers in the polymerisation mixture, the consumption of the monomers proceeds in a ratio of 
1.7 BA-monomers per 1.1-monomer. It can be concluded that the impression gained from Figure 8-25 is correct, 1.1 is more reactive than BA in this mixture which is supported by the molecular share $F$ of 1.1 in the polymer.

The reactivity of monomers participating in a copolymerisation can be measured and expressed as copolymerisation parameters. Via NMR spectroscopy it is possible to determine these for a defined system. The Lewis-Mayo-equation (equation 8-11) describes the polymer composition $F$ (in mol\%) via the copolymerisation parameters $r$, which result from the different reaction ratios as explained in chapter 3.3.3, and the composition of the monomer mixture $f$.

$$
F_{1}=\frac{r_{1} \cdot f_{1}^{2}+f_{1} \cdot f_{2}}{r_{1} \cdot f_{1}^{2}+2 \cdot f_{1} \cdot f_{2}+r_{2} \cdot f_{2}^{2}}
$$

with $F_{1}$ as the amount of monomer 1 in the polymer, $r_{1}$ and $r_{2}$ the copolymerisation parameters of the two monomers, $f_{1}$ and $f_{2}$ as the shares of the two monomers in the monomer feed. The share of a specific monomer $\left(n_{1}, n_{2}\right)$ in the monomer feed $f$ can be calculated by

$$
\begin{aligned}
& f_{1}=\frac{n_{1}}{n_{1}+n_{2}} \\
& f_{2}=\frac{n_{2}}{n_{2}+n_{1}}
\end{aligned}
$$

$F_{1}$-values result from the integration of specific signals in the polymer ${ }^{1} \mathrm{H}$ NMR spectra of a set of copolymerisations. These copolymerisations are carried out with different monomer compositions. Figure 8-26 shows the superimposed, standardised ${ }^{1} \mathrm{H}$ NMR spectra of such a set of copolymerisations using $1,460 \mathrm{mmol} \cdot \mathrm{L}^{-1}, 760 \mathrm{mmol} \cdot \mathrm{L}^{-1}, 360 \mathrm{mmol} \cdot \mathrm{L}^{-1}, 150 \mathrm{mmol} \cdot \mathrm{L}^{-1}$ and $110 \mathrm{mmol} \cdot \mathrm{L}^{-1}$ of 1.1 with $4,920 \mathrm{mmol} \cdot \mathrm{L}^{-1}$ of $\mathrm{BA}, 0.59 \mathrm{mmol} \cdot \mathrm{L}^{-1} \mathbf{R}_{6} 2$ and $0.97 \mathrm{mmol} \cdot \mathrm{L}^{-1} \mathrm{AlBN}$ in toluene at $60{ }^{\circ} \mathrm{C}$. 


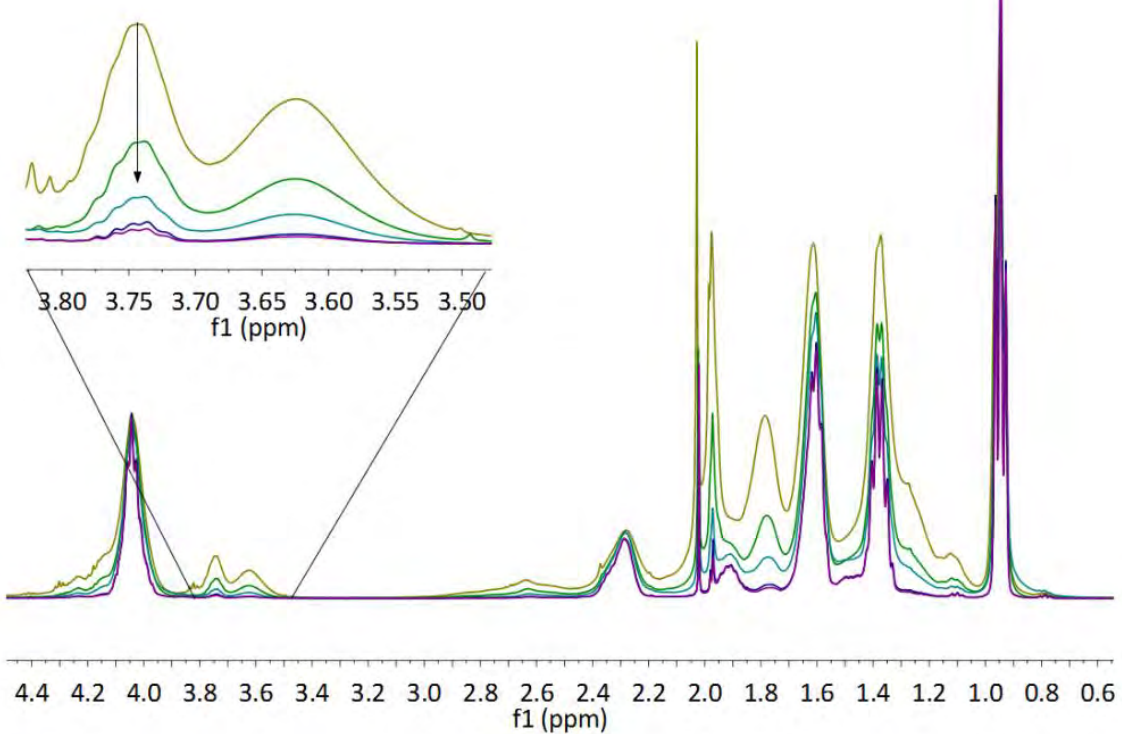

Figure 8-26: Superimposed, standardised ${ }^{1} \mathrm{H} N \mathrm{NR}$ spectra recorded in $\mathrm{CDCl}_{3}$ of copolymerisations with different concentrations of $1.1\left[1,460 \mathrm{mmol} \cdot \mathrm{L}^{-1}\right.$ (yellow), $760 \mathrm{mmol} \cdot \mathrm{L}^{-1}$ (green), $360 \mathrm{mmol} \cdot \mathrm{L}^{-1}$ (aqua), $150 \mathrm{mmol} \cdot \mathrm{L}^{-1}$ (blue), $110 \mathrm{mmo} \cdot \mathrm{L}^{-1}$ (purple)] using $4,920 \mathrm{mmol} \cdot \mathrm{L}^{-1} \mathrm{BA}, 0.59 \mathrm{mmol} \cdot \mathrm{L}^{-1} \boldsymbol{R}_{6} \mathbf{2}$ and $0.97 \mathrm{mmol} \cdot \mathrm{L}^{-1} \mathrm{AlBN}$ in toluene at $60^{\circ} \mathrm{C}$ and 90 minutes reaction time.

The black arrow in Figure 8-26 indicates the decrease in the co-monomer concentration. The properties of these polymers strongly differ: $p B A$ is a sticky, half-fluid gel, depending on the molecular weight. With increasing 1.1-content, the polymer gets more solid even for smaller molecular weights. Samples generated with less than $150 \mathrm{mmol} \cdot \mathrm{L}^{-1}$ of 1.1 in the monomer feed are elastic and rubber-like. Co-monomer concentrations of 360 and $760 \mathrm{mmol} \cdot \mathrm{L}^{-1}$ yield solid rubber compounds that have to be parted by scissors. For even higher co-monomer concentrations brittle plastics are obtained. As expected, polymer samples resulting from polymerisations with less comonomer concentration have less 1.1-units integrated and the properties characteristic for $p \mathrm{BA}$ dominate. As shown previously, the copolymer composition can be determined. Table 8-7 summarises the data for this set of reactions. 
Table 8-7: Determined numbers of BA- and 1.1-units of polymer samples of various copolymerisations with 1.1 and $4,920 \mathrm{mmol} \cdot \mathrm{L}^{-1} B A, 0.59 \mathrm{mmol} \cdot \mathrm{L}^{-1} \boldsymbol{R}_{6} \mathbf{2}$ and $0.97 \mathrm{mmol} \cdot \mathrm{L}^{-1} \mathrm{AlBN}$ in toluene at $60^{\circ} \mathrm{C}$ and 90 minutes (method II).

\begin{tabular}{c|c|c|c|c}
$\begin{array}{c}c_{1.1} \\
{\left[\mathrm{mmol} \cdot \mathrm{L}^{-1}\right]}\end{array}$ & $\begin{array}{c}M_{\mathrm{n}}^{\mathrm{UV} \cdot 1.52} \\
{\left[\mathrm{~g} \cdot \mathrm{mol}^{-1}\right]}\end{array}$ & $\begin{array}{c}\text { number of } \\
\text { 1.1-units in } \\
\text { the star }\end{array}$ & $\begin{array}{c}\text { number of } \\
\text { BA-units in } \\
\text { the star }\end{array}$ & $\begin{array}{c}\mathbf{1 . 1 / B A - u n i t s} \\
\text { per star }\end{array}$ \\
\hline 110 & 418,000 & 58 & 3,146 & 0.018 \\
150 & 396,000 & 84 & 2,927 & 0.029 \\
360 & 330,000 & 175 & 2,259 & 0.077 \\
760 & 306,000 & 347 & 1,774 & 0.195 \\
1,460 & 200,000 & 368 & 907 & 0.406
\end{tabular}

As expected the amount of $\mathbf{1 . 1}$ in the copolymer is higher for samples resulting from polymerisation mixtures with a higher 1.1-feed. Furthermore, the effect of the induction, discussed in chapter 8.1, can be observed in the decrease of the molecular weight with increasing co-monomer concentration. A drawback of this method is the low sensitivity. For quantitative results high 1.1-loads are necessary otherwise the intensity of the signals is too low to perform an accurate integration. Here only samples were investigated resulting from polymerisations with at least $110 \mathrm{mmol} \cdot \mathrm{L}^{-1} 1.1$ in the monomer feed. As can be seen in Figure 8-26, even here the evaluation of the data resulting from smaller 1.1-loads is imperfect and that the evaluation of samples from polymerisations with $360 \mathrm{mmol} \cdot \mathrm{L}^{-1} \mathbf{1 . 1}$ can be accepted as the lower limit for this method.

In addition to determining the polymer composition in monomer units, the $F$-value can be used to apply the Lewis-Mayo-equation and obtain the copolymerisation parameters. To do so, the integral of the BA's methyl group at approximately $0.91 \mathrm{ppm}$ is compared with the integral at $3.75 \mathrm{ppm}$ belonging to the $\mathrm{CH}_{2}$-group of the co-monomer, as shown in method II for the determination of the copolymer composition. The integrals are converted per protons which cause the signal, as shown before. Here this calculation is shown for the copolymerisation with $110 \mathrm{mmol} \cdot \mathrm{L}^{-1}$ 1.1. $I_{\mathrm{BA}}$ and $I_{1.1}$ are the integrals of the peaks. These values are referred back to the sum of protons of both monomers, giving the amount of the monomer $F_{1.1}$ in the polymer at this specific point of polymerisation resulting from the defined composition for the monomer mixture.

$$
\begin{gathered}
I_{\mathrm{BA}}=1=3 \text { protons } \rightarrow \frac{I_{\mathrm{BA}}}{3 \text { protons }}=\frac{1}{3 \text { protons }}=0.333 \overline{3} \\
I_{\mathbf{1 . 1}}=0.0061=2 \text { protons } \rightarrow \frac{I_{1.1}}{2 \text { protons }}=\frac{0.0122}{2 \text { protons }}=0.0061
\end{gathered}
$$




$$
F_{1.1}=\frac{\frac{I_{1.1}}{2 \text { protons }}}{\frac{I_{\mathrm{BA}}}{3 \text { protons }}+\frac{I_{1.1}}{2 \text { protons }}}=\frac{0.0061}{0.3394}=0.0179
$$

Now the $F$-and the $f$-values for all reaction mixtures are plotted against each other and fitted via the Lewis-Mayo-equation (8-11) in OriginPro 8.1 giving $r_{1.1}(\mathrm{a})$ and $r_{\mathrm{BA}}(\mathrm{b})$.

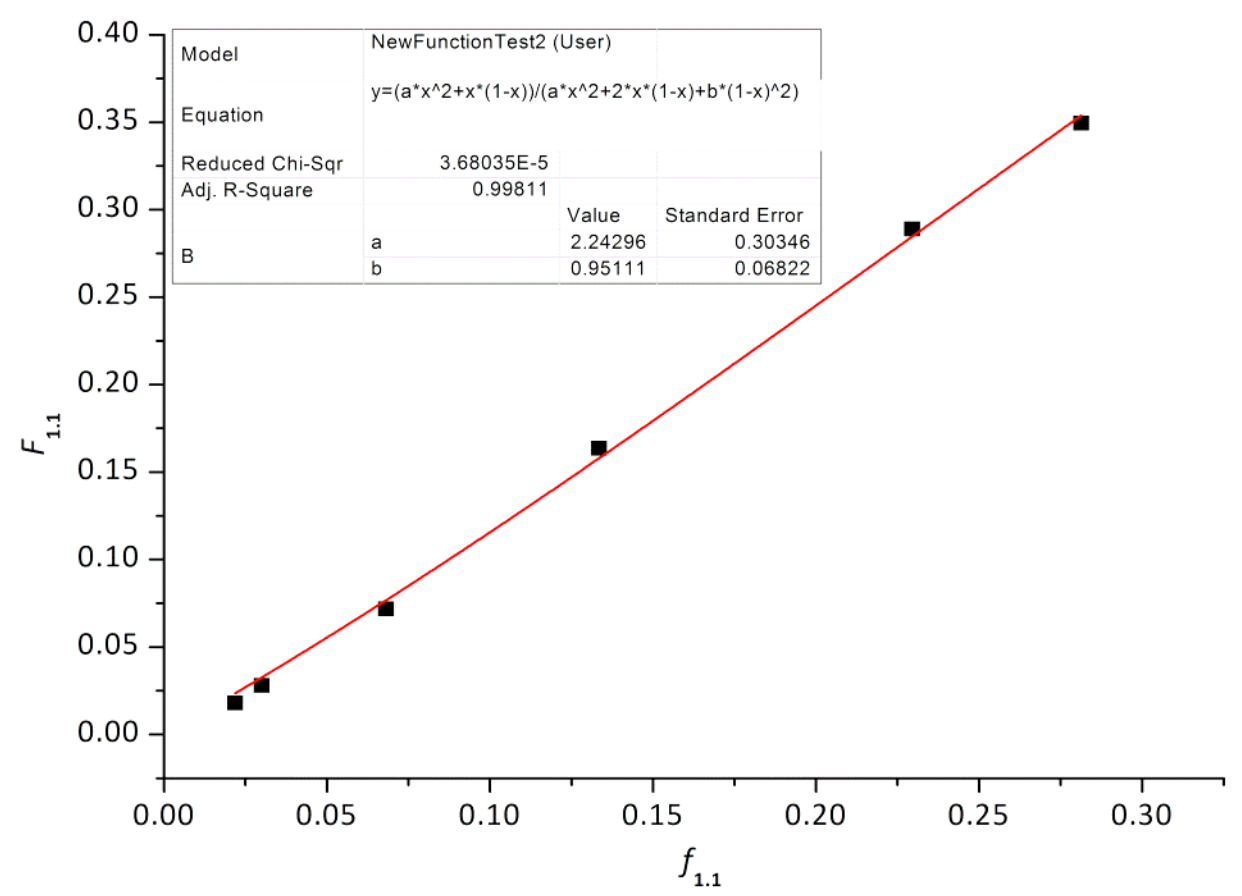

Figure 8-27: Copolymerisation plot for a set of copolymerisations with $B A$ and 1.1 using $0.59 \mathrm{mmol} \cdot \mathrm{L}^{-1} \boldsymbol{R}_{6} \mathbf{2}$ and $0.97 \mathrm{mmol} \cdot \mathrm{L}^{-1}$ AIBN in toluene at $60^{\circ} \mathrm{C}$ and 90 minutes reaction time

Copolymerisation parameters of 2.24 for $\mathbf{1 . 1}$ and 0.95 for BA could be determined. These values are reasonable as they are in an equal range as those of copolymerisations with $\mathbf{1 . 2}$ and EA or MA (chapter 3.6.3). The deviation towards higher values results as the Lewis-Mayo-equation is only valid for polymerisations with small monomer conversions. In this case these were with 23 up to $45 \%$ comparatively high. Copolymerisation parameters above 1 are a signal that the growing radicals favour the monomer equal to themselves, monomers with copolymerisation parameters below 1 favour the opposite monomer. Here the integration of the co-monomer is favoured by both monomers which is supported by the findings in the comparison of the molecular percentage of both monomers in the polymers (Table 8-8). In the beginning 1.1 is consumed solely as it is favoured by

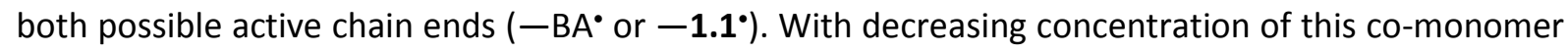
the probability of consumption decreases, hence BA is stronger consumed due to the high contact probability. 
Table 8-8: Comparison of the molecular percentage of monomer units in copolymerisations of BA and 1.1.

\begin{tabular}{|c|c|c|c|c|c|}
\hline \multicolumn{3}{|c|}{$\begin{array}{c}\text { Copolymerisation with } \\
4,920 \mathrm{mmol} \cdot \mathrm{L}^{-1} \mathrm{BA} \text { and } 1,350 \mathrm{mmol} \cdot \mathrm{L}^{-1} 1.1\end{array}$} & \multicolumn{3}{|c|}{$\begin{array}{c}\text { Copolymerisation with } \\
4,920 \mathrm{mmol} \cdot \mathrm{L}^{-1} \mathrm{BA} \text { and } 280 \mathrm{mmol} \cdot \mathrm{L}^{-1} \mathbf{1 . 1},\end{array}$} \\
\hline $\begin{array}{c}M_{\mathrm{n}} \\
{\left[\mathrm{g} \cdot \mathrm{mol}^{-1}\right]}\end{array}$ & $\begin{array}{c}F_{1.1} \\
{[\mathrm{~mol} \%]}\end{array}$ & $\begin{array}{c}F_{\mathrm{BA}} \\
{[\mathrm{mol} \%]}\end{array}$ & $\begin{array}{c}M_{\mathrm{n}} \\
{\left[\mathrm{g} \cdot \mathrm{mol}^{-1}\right]}\end{array}$ & $\begin{array}{c}F_{1.1} \\
{[\mathrm{~mol} \%]}\end{array}$ & $\begin{array}{c}F_{\mathrm{BA}} \\
{[\mathrm{mol} \%]}\end{array}$ \\
\hline 88,000 & 37.9 & 62.1 & 120,000 & 9.4 & 90.6 \\
\hline 162,000 & 33.6 & 66.4 & 305,000 & 8.6 & 91.4 \\
\hline 306,000 & 30.1 & 69.9 & 405,000 & 8.3 & 91.7 \\
\hline
\end{tabular}

As the copolymerisation parameters do not show a very large difference, it can be concluded that a gradient copolymer is formed (e.g. in a polymerisation system of MMA and VAc at $60{ }^{\circ} \mathrm{C}$ the copolymerisation parameters differ distinctively with $r_{\mathrm{MMA}}=20$ and $\left.r_{\mathrm{VAC}}=0.015^{(185)}\right)$. A much larger difference would lead to block copolymers, more equal copolymerisation parameters to strictly alternating copolymers.

\subsection{Copolymerisation of NIPAam and 1.1}

In this chapter a more detailed look should be taken on polymerisations of NIPAam with $\mathbf{1 . 1}$ mediated by the six-functional RAFT agent $\mathbf{R}_{6} \mathbf{3}$. The determination of the molecular weights of pNIPAam samples were carried out in N,N-dimethylacrylamid (DMac) on a SEC set up calibrated with linear $p M M A$ standards as McKee et al. proved that $p$ NIPAam and $p M M A$ are chemically equivalent in solution (chapter 12.1.2). ${ }^{\text {(186) }}$

As the addition of $\mathbf{1 . 1}$ caused an induction period in the polymerisations with BA, the same is to be expected here because the effect of induction is connected to the RAFT-equilibrium, i.e. interactions with the RAFT-agent. In chapter 7.1.4 polymerisations with NIPAam and $\mathbf{1 . 1}$ were introduced. A delay in the start of the RAFT copolymerisation

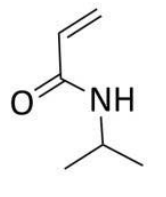

NIPAam

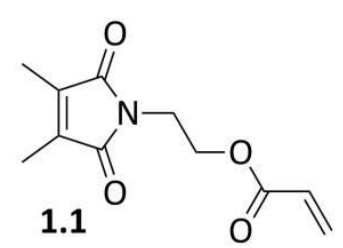
compared to the corresponding homopolymerisation of NIPAam had been noticed. The findings of these polymerisations are shown in the Figures 8-28 and 8-29 to discuss them in more detail. First the plots of the molecular weight and the PDI-values versus the monomer conversion are shown, then the plots of the monomer conversion and the molecular weight with reaction time. In both diagrams the data of the copolymerisation with $3,600 \mathrm{mmol} \cdot \mathrm{L}^{-1}$ of NIPAam and $107.2 \mathrm{mmol} \cdot \mathrm{L}^{-1}$ of 1.1 using $1.3 \mathrm{mmol} \cdot \mathrm{L}^{-1}$ of AIBN and $0.5 \mathrm{mmol} \cdot \mathrm{L}^{-1}$ of $\mathbf{R}_{6} \mathbf{3}$ in dimethylformamide (DMF) are represented by blue symbols, the corresponding homopolymerisation is shown by black symbols. 


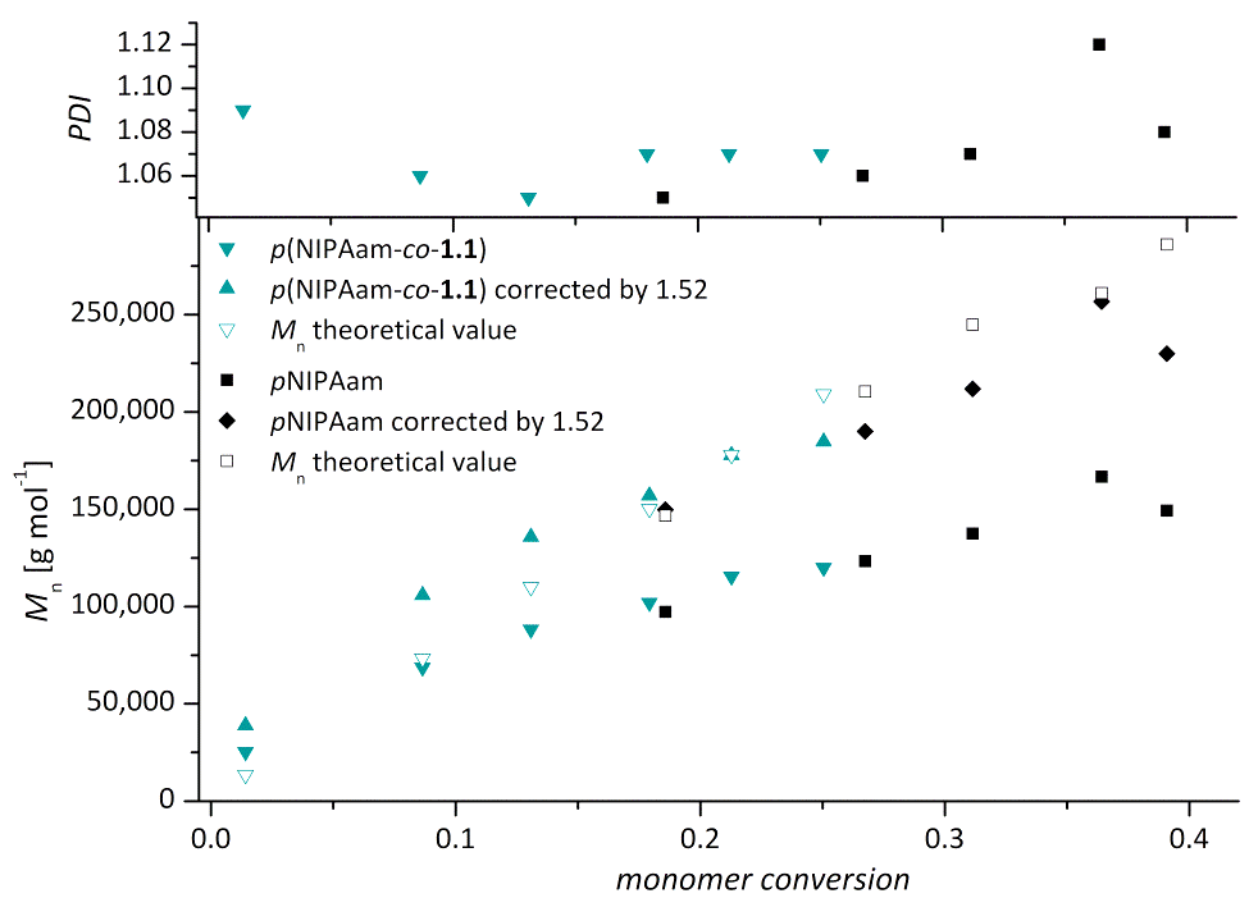

Figure 8-28: Developments of the corrected molecular weights and the PDI, determined by UV-detection on a DMac-SEC with a pMMA calibration, with the monomer conversion for a homopolymerisation of NIPAam (black) and a copolymerisation of NIPAam (aqua) with $107.2 \mathrm{mmol} \cdot \mathrm{L}^{-1} 1.1 \mathrm{using} 0.5 \mathrm{mmol} \cdot \mathrm{L}^{-1} \boldsymbol{R}_{6} 3$ and $1.3 \mathrm{mmol} \cdot \mathrm{L}^{-1} \mathrm{AIBN}$ in DMF at $60{ }^{\circ} \mathrm{C}$.

The development of the molecular weights shows a linear trend with monomer conversions as well as with the reaction times being evidence to support the controlled reaction behaviour. Moreover the theoretically determined values (equations 3-25 and 3-27) of the molecular weights fit well with the experimental, corrected values obtained by the detection and evaluation of the UV-signal of the SEC-measurement. The small deviation is a consequence of the spherical shape of the polymers and uncertainties in the determination via SEC which is calibrated with linear polymer standards. The equation for the theoretical molecular mass does not take dead chains into account, i.e. only living material is compared. Comparing with the data from the RI-detection a larger deviation could be observed, as this corresponds with dead and living material which sums up to smaller average molecular weights. This effect is more pronounced for long reaction times as more dead material is generated. The PDI-values also are in the characteristic range of a CRP (chapter 3.4). As discussed earlier in chapter 8.1 about BA-polymerisations, dead material, which does not consist of RAFT-units and broadens the MWDs observed by RI-detection, does not show any UV-absorbance and is therefore excluded from the determination here. The data shown correspond only with the living material in the system, i.e. the star polymers. Therefore narrow MWDs are obtained which result in small PDI-values. 


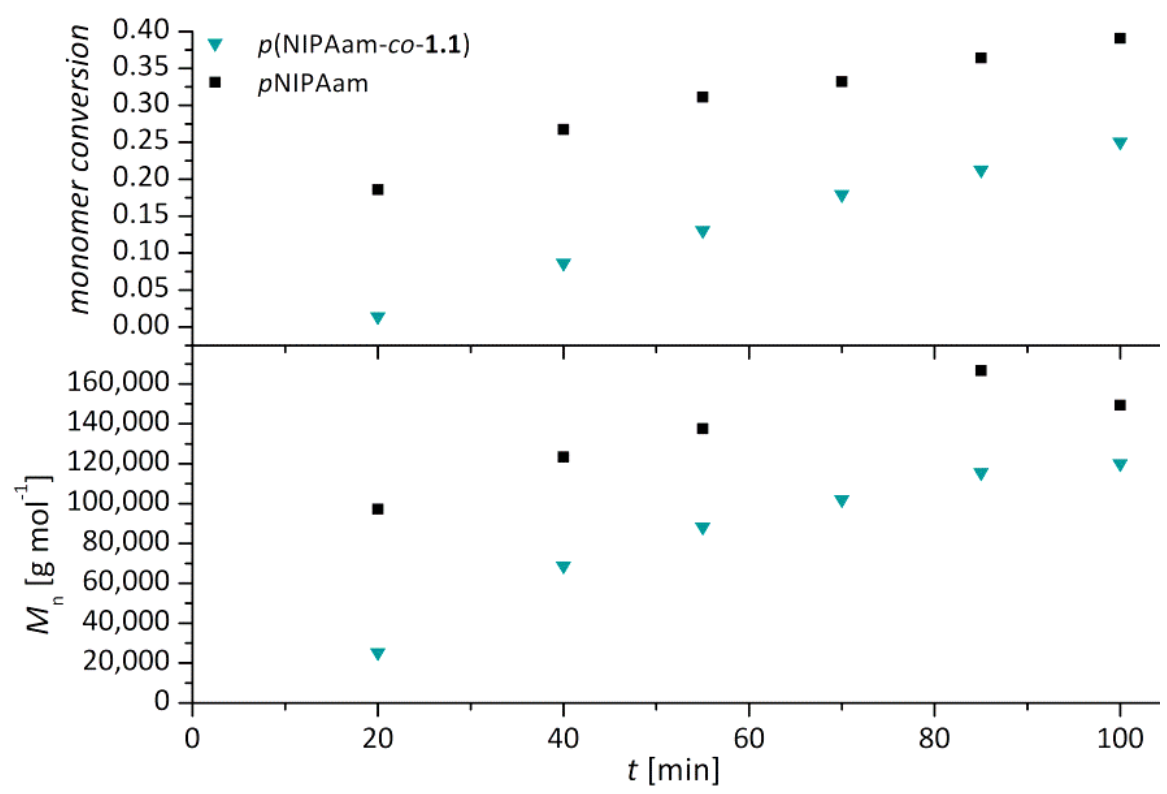

Figure 8-29: Development of monomer conversion with time of a NIPAam homopolymerisation (black) and a copolymerisation of $3,600 \mathrm{mmol} \cdot \mathrm{L}^{-1} \mathrm{NIPAam}$ and $100 \mathrm{mmol} \cdot \mathrm{L}^{-1} 1.1$ (blue) using $1.3 \mathrm{mmol} \cdot \mathrm{L}^{-1} \mathrm{AlBN}$ and $5.2 \mathrm{mmol} \cdot \mathrm{L}^{-1} \boldsymbol{R}_{6} \mathbf{3}$ in DMF.

As expected from the findings in the BA polymerisations, induction due to the addition of $\mathbf{1 . 1}$ takes place, i.e. the copolymerisation starts delayed in comparison with the corresponding homopolymerisation which consequently leads to smaller monomer conversions and smaller molecular weights for aliquots taken after the same period of time. In Figure 8-29's top part the monomer conversion is plotted versus the reaction time, in the bottom part the molecular weight versus reaction time. Both plots support the statement of induction. The polymerisation with 1.1 started delayed but the parallelism of the plots shows a similar process of the reactions.

A set of copolymerisations was performed to verify the dependence of induction on the comonomer concentration. Solutions of $5,000 \mathrm{mmol} \cdot \mathrm{L}^{-1}$ of NIPAam and $28 \mathrm{mmol} \cdot \mathrm{L}^{-1}, 56 \mathrm{mmol} \cdot \mathrm{L}^{-1}$, $140 \mathrm{mmol} \cdot \mathrm{L}^{-1}$ and $280 \mathrm{mmol} \cdot \mathrm{L}^{-1}$ of 1.1 in DMF were polymerised with $0.6 \mathrm{mmol} \cdot \mathrm{L}^{-1}$ of $\mathbf{R}_{6} \mathbf{3}$ and $1.1 \mathrm{mmol} \cdot \mathrm{L}^{-1}$ of $\mathrm{AIBN}$ at $60{ }^{\circ} \mathrm{C}$. In Figure 8-30 the corresponding developments of the molecular weight, determined by detection and evaluation of the UV-signal, corrected by 1.52 , with the monomer conversion are plotted. In the top part of this figure the PDI-values are shown. 


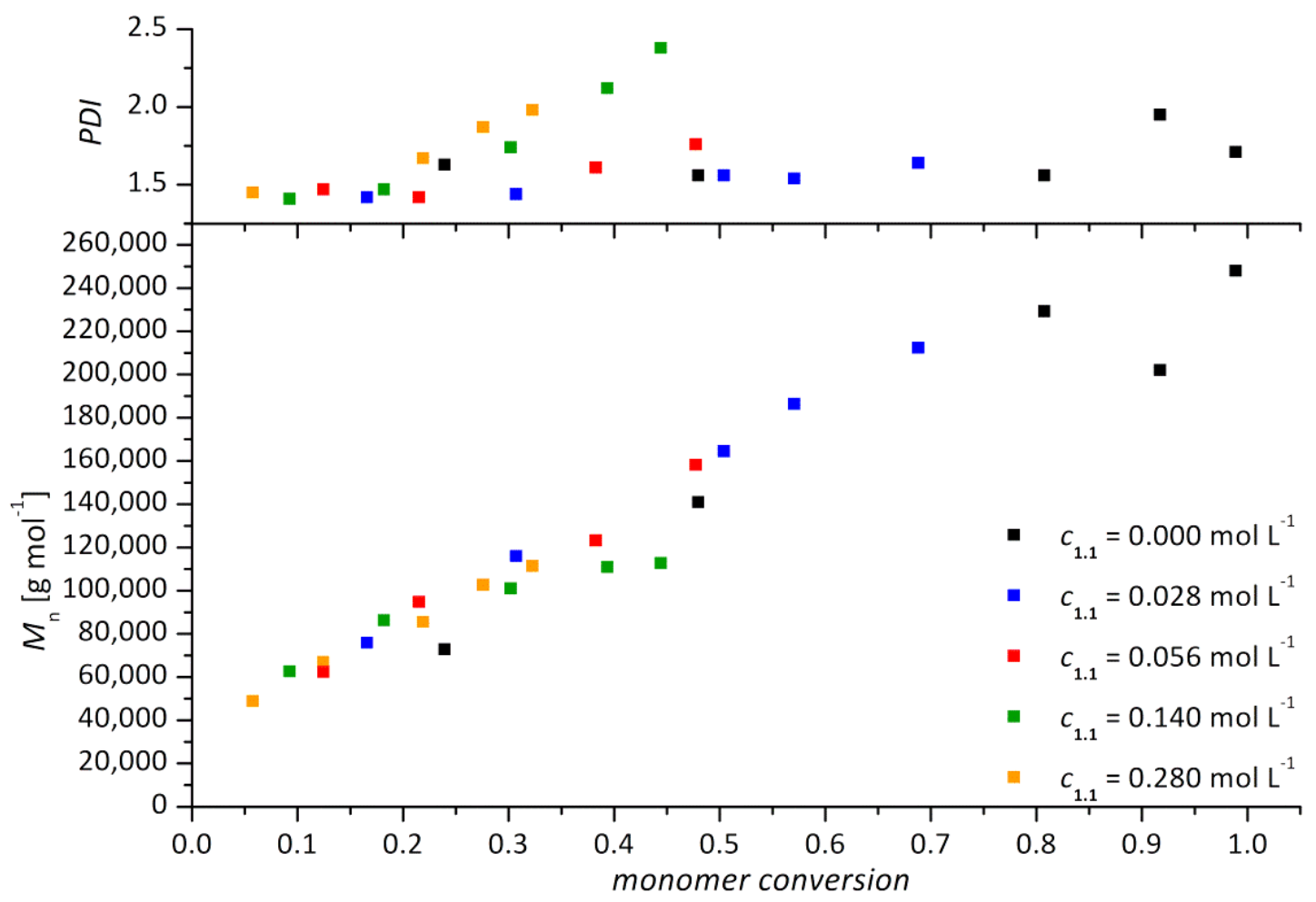

Figure 8-30: Developments of the molecular weight and the PDI, determined by UV-detection on a DMac-SEC with a pMMA calibration, corrected by 1.52, with the monomer conversion for a homopolymerisation of NIPAam (black) and various copolymerisations of NIPAam and 1.1 using $0.6 \mathrm{mmol} \cdot \mathrm{L}^{-1} \boldsymbol{R}_{6} 3$ and $1.1 \mathrm{mmol} \cdot \mathrm{L}^{-1} \mathrm{AIBN}$ in DMF at $60^{\circ} \mathrm{C}$.

It can be observed that the molecular weights increase in a linear fashion with the monomer conversion and the PDI-values are in the characteristic CRP-range for polymerisations with no or a low amount of co-monomer in the monomer feed. For high co-monomer concentrations the PDIvalues increase above values which are characteristic for CRP. This indicates a minimisation in control. In general the PDI-values of 1.5 are comparatively high for a CRP which is a result of the polymerisation in a good solvent. Furthermore the increasing distinctiveness of the induction in dependence on the co-monomer concentration can be observed: The higher the co-monomer feed is in the copolymerisation the smaller the monomer conversions and the smaller molecular weights are obtained. The Figures 8-31 and 8-32 illustrate this effect more clearly. Here the monomer conversion and the molecular weight are plotted versus the reaction time followed by the corresponding SECtraces of each first and last aliquot of the introduced copolymerisations. 


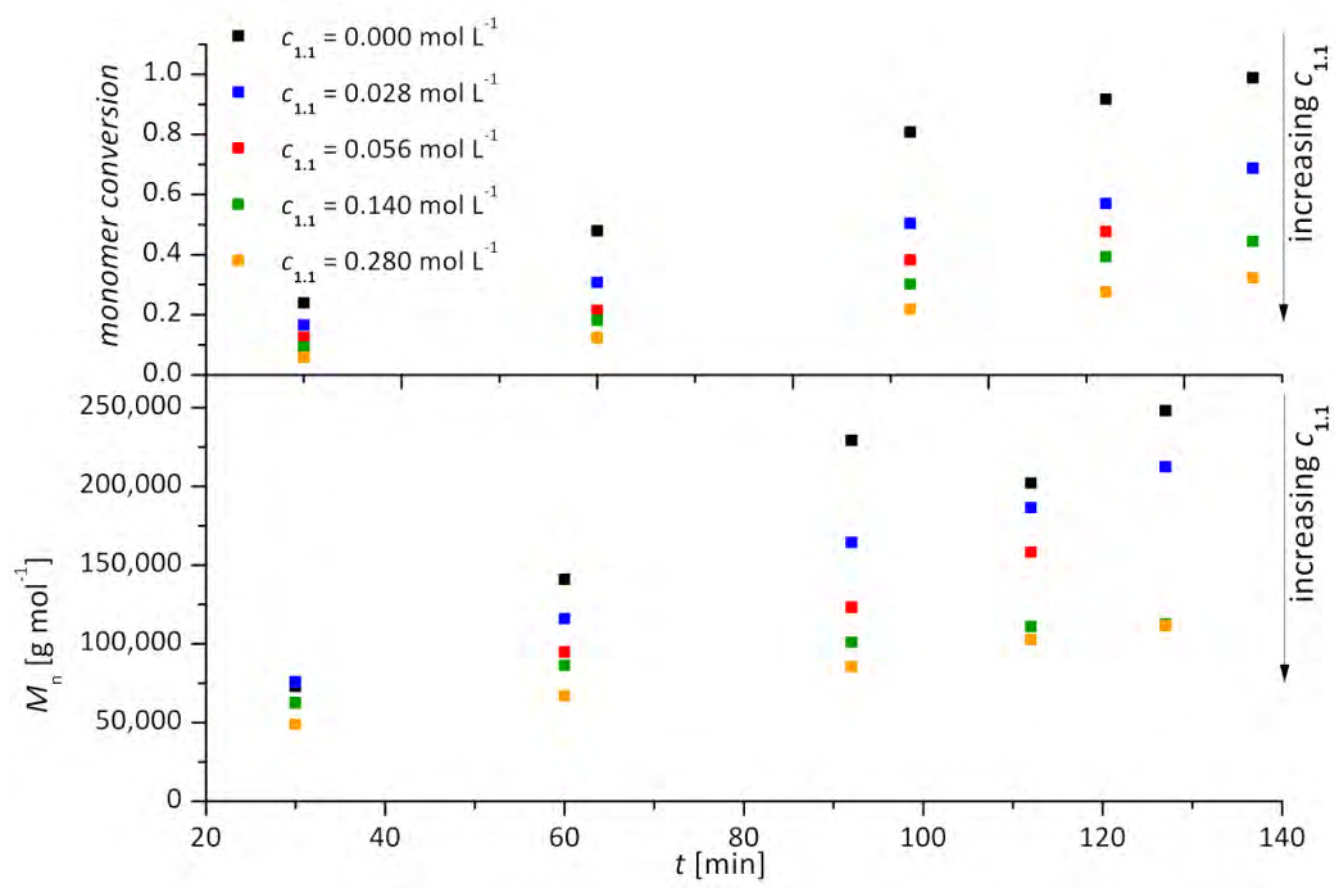

Figure 8-31: Developments of the molecular weight (obtained by UV-detection) and the monomer conversion with the reaction time for a homopolymerisation of NIPAam (black) and various copolymerisations of NIPAam and 1.1 using $0.6 \mathrm{mmol} \cdot \mathrm{L}^{-1} \boldsymbol{R}_{6} 3$ and $1.1 \mathrm{mmol} \cdot \mathrm{L}^{-1} \mathrm{AIBN}$ in DMF at $60^{\circ} \mathrm{C}$.

Similar to the BA-polymerisation it was found that the effect of induction is more pronounced for systems with higher co-monomer loads. Comparing the chromatograms of aliquots taken after the same period of time (Figure 8-32) shows a decrease in the molecular weight with increasing co-monomer concentration.
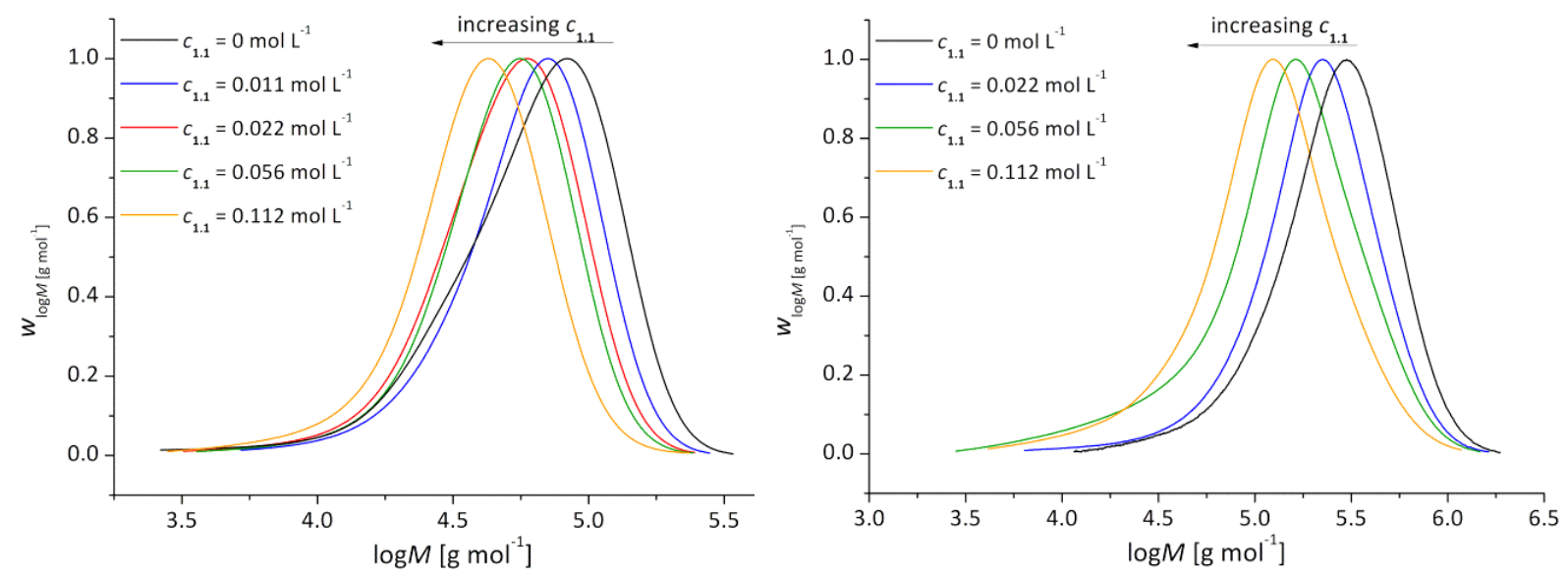

Figure 8-32: Comparison in time of uncorrected SEC-UV-spectra of different copolymerisations with NIPAam with 1.1: 30 minutes (left), 127 minutes (right).

In contrast to the corresponding MWDs of BA polymerisations, introduced in chapter 8.1, no bimodal distributions with shoulders in the region of high molecular weights can be observed. This supports 
the assumption that hardly any star-star coupling occurs in NIPAam polymerisations. This furthermore leads to the assumption that in BA-polymerisations the back biting process is reinforced by the presence of the DMI-unit and that the effect of double star formation by [2+2]-cycloaddition is negligible small.

In the following the MWDs corresponding to the previously mentioned polymerisations will be discussed in more detail. At first three aliquots of the homopolymerisation are examined more closely (Figure 8-33). The correction of the UV-data was conducted in accordance to the procedure described in chapter 8.1 and reference (47).
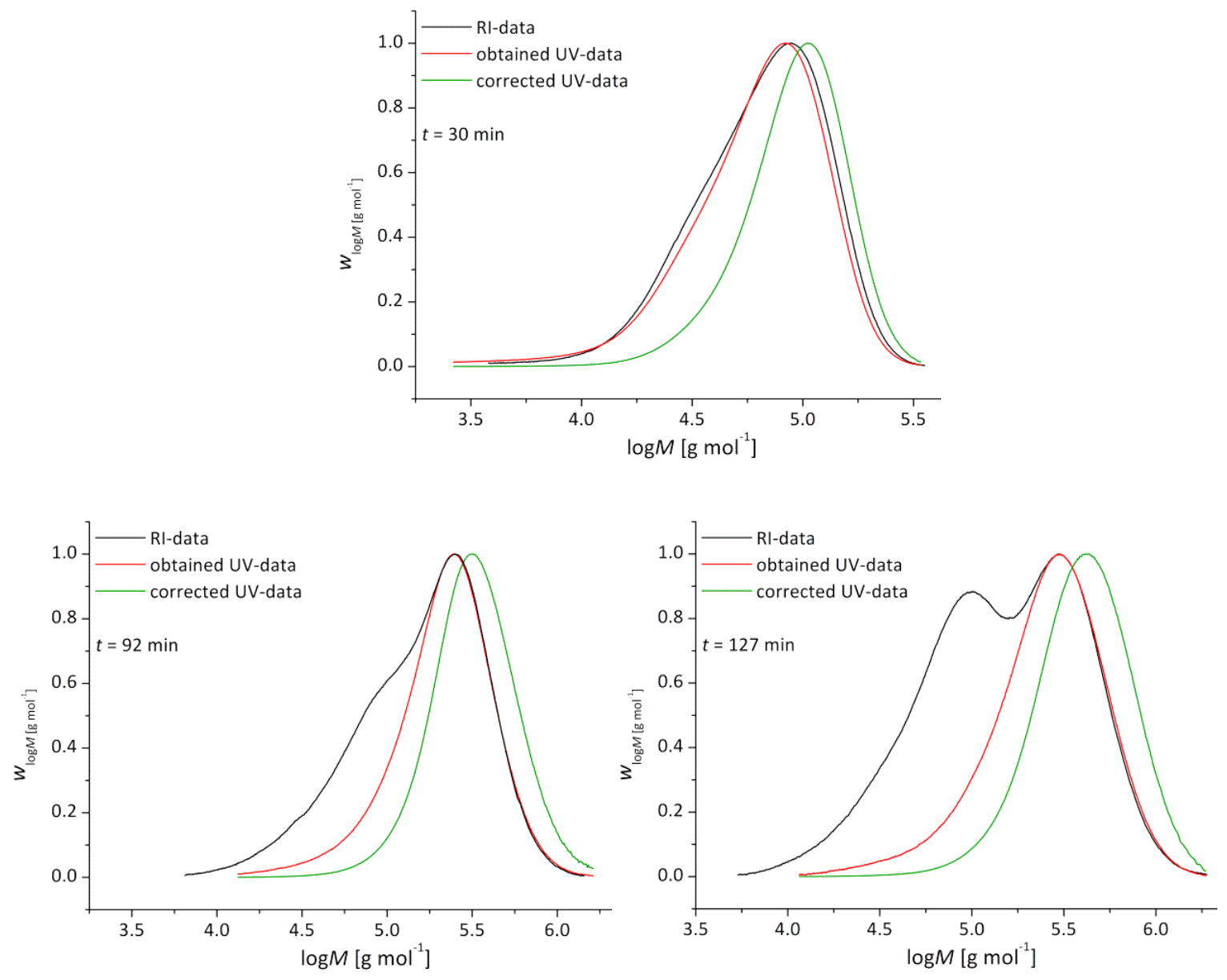

Figure 8-33: Chromatograms of the first, the third and the fifth aliquot of the NIPAam homopolymerisation using $0.6 \mathrm{mmol} \cdot \mathrm{L}^{-1} \boldsymbol{R}_{6} 3$ and $1.1 \mathrm{mmol} \cdot \mathrm{L}^{-1} \mathrm{AIBN}$ in DMF at $60{ }^{\circ} \mathrm{C}$ (black = experimentally obtained $\mathrm{RI}$-data, red = experimentally obtained UV-data and green = corrected UV-data).

The chromatograms obtained by RI-detection reveal very broad MWDs due to dead polymer chains generated in the polymerisation process. In the comparison with the UV-chromatogram it can be seen that a large amount of UV-inactive material, i.e. dead polymer, was formed resulting in high PDI-values from the RI-detection. Furthermore it stands out that the formation of dead polymer material increases with reaction time which is a consequence of continuous termination in the 
system (chapter 3.2.3). The corresponding UV-chromatogram (red and green) shows a more narrow distribution as dead material is excluded. Against the expectations the experimentally obtained UVdata (red) delivers the perfect fit to the experimentally obtained RI-data (black). The correction of those values (green) shifts the chromatogram to much higher values but excludes the artefact of the measurement on the side of the low molecular weight compounds. As explained in chapter 8.2, the absorption maximum of trithio units is at a wavelength of $310 \mathrm{~nm}$. At this wavelength acrylates as well as acrylamides are UV-transparent (Figure 8-36). Therefore only the RAFT-units are detected which usually yields MWDs shifted to smaller molecular weights than the corresponding RI-data as the UV-detection gives only a number distribution and the RI-detection directly a mass distribution. ${ }^{(47)}$ In the case of $p$ NIPAam this statement cannot be supported as seen in Figure 8-33. As already explained of the similar BA-polymerisations the assumption made for the UV-detection bases on Lambert-Beer's Law. It loses validity at higher concentrations of UV-active centres per molecule. As the correction method is based on the validity of the law it becomes incorrect in this case. Furthermore, it has to be speculated that the nitrogen with the free electron pair induces in interaction with the trithio units a promotion process, which leads to end the UV-transparency of the polymer. In this case all monomer units would be detected by the UV-detector resulting in a number distribution that equals the mass distribution.

In Figure 8-34 the chromatograms determined by RI-detection are shown. Very broad MWDs can be observed due to dead polymer generated in the polymerisation process but in the cases of the copolymerisation the formation of low molecular material is not as pronounced as in the homopolymerisation. In the top right corner the example of the RI- and UV-detection for the last aliquot of the homopolymerisation discussed previously is shown again to point the difference to the UV-results out. 


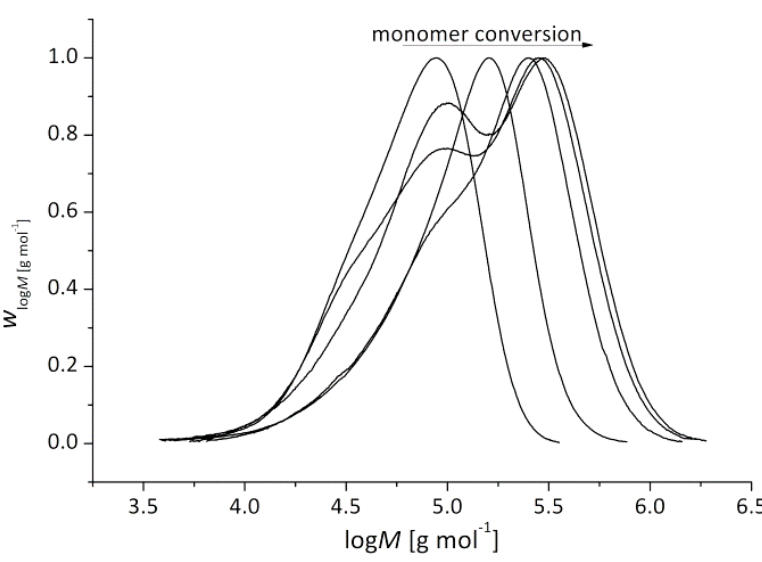

Homopolymerisation of NIPAam

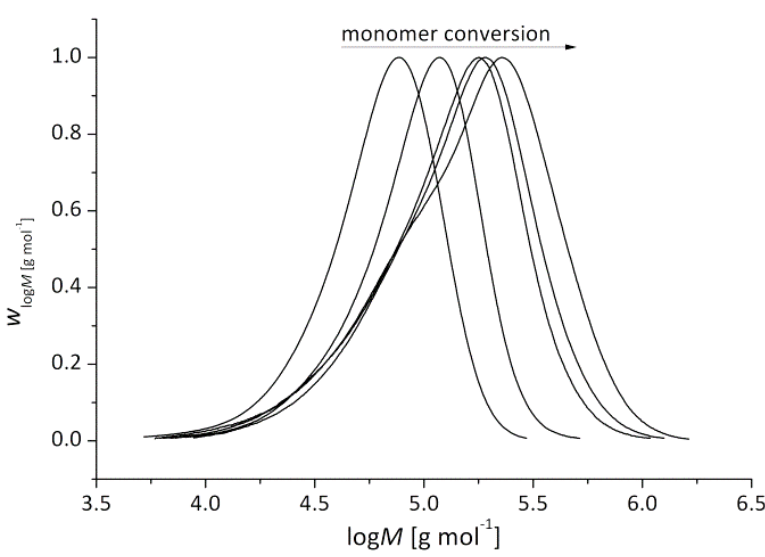

Copolymerisation with $28 \mathrm{mmol} \cdot \mathrm{L}^{-1} 1.1$

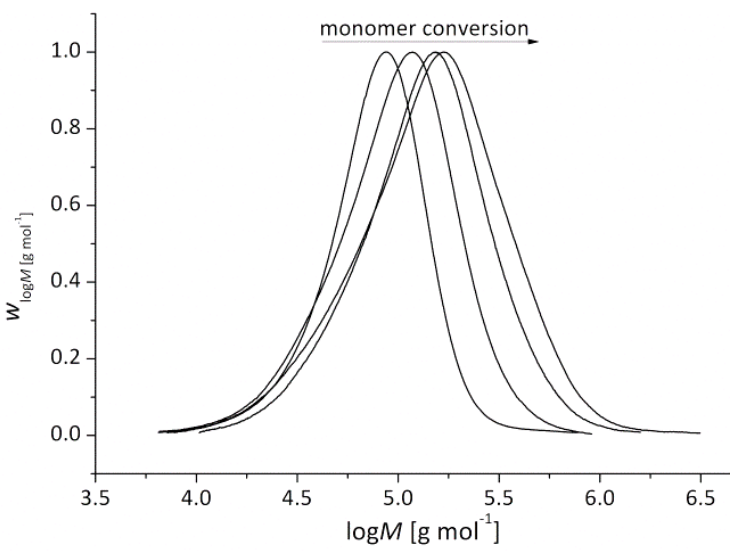

Copolymerisation with $140 \mathrm{mmol} \cdot \mathrm{L}^{-1} 1.1$

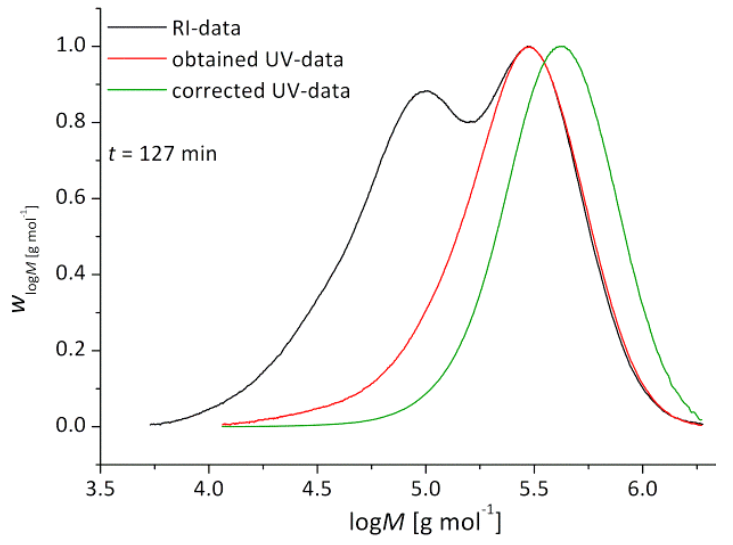

Comparision of RI- and UV-SEC-data of the last aliquot of the homopolymerisation

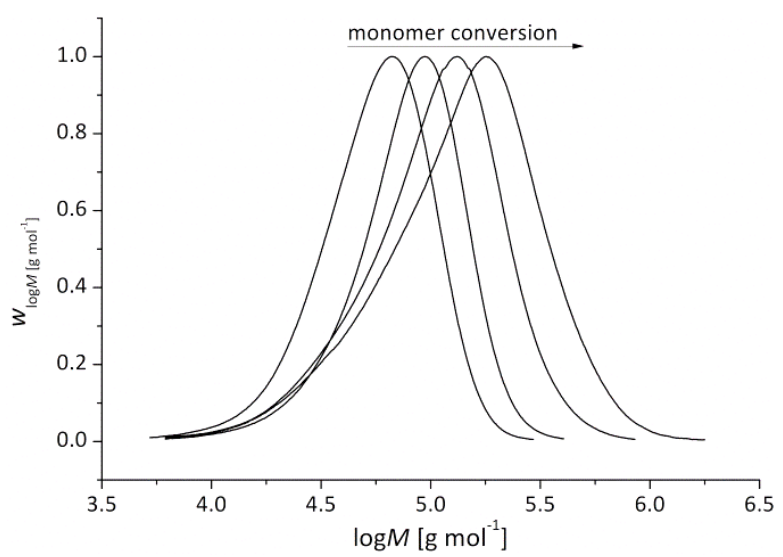

Copolymerisation with $56 \mathrm{mmol} \cdot \mathrm{L}^{-1} 1.1$

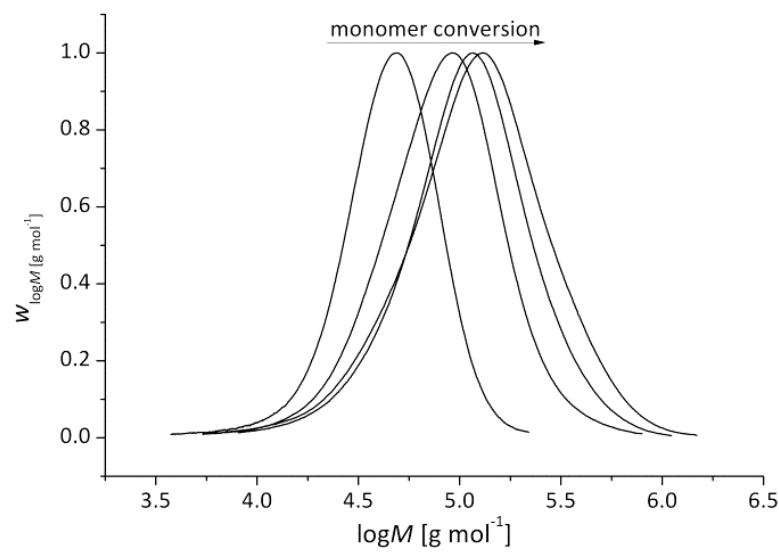

Copolymerisation with $280 \mathrm{mmol} \cdot \mathrm{L}^{-1} 1.1$

Figure 8-34: SEC-RI-spectra of different copolymerisations of NIPAam with 1.1 up to a reaction time of 127 minutes. Topright the SEC-RI-data are compared with the experimentally obtained and the corrected SEC-UV-data.

For the different copolymerisations a broadening of the MWDs in the process of the polymerisation was noticed. In the cases of 28 and $55 \mathrm{mmol} \cdot \mathrm{L}^{-1}$ of co-monomer concentration in the system, the formation of low molecular weight material, i.e. dead polymer, is more pronounced with increasing 
polymerisation time whereas the formation of material with higher molecular weight cannot be observed easily. For higher co-monomer concentrations the broadening appears evidently in the low molecular weight region as well as in the high molecular weight region. The following set of chromatograms shows the corresponding corrected UV-spectra which support that statement.

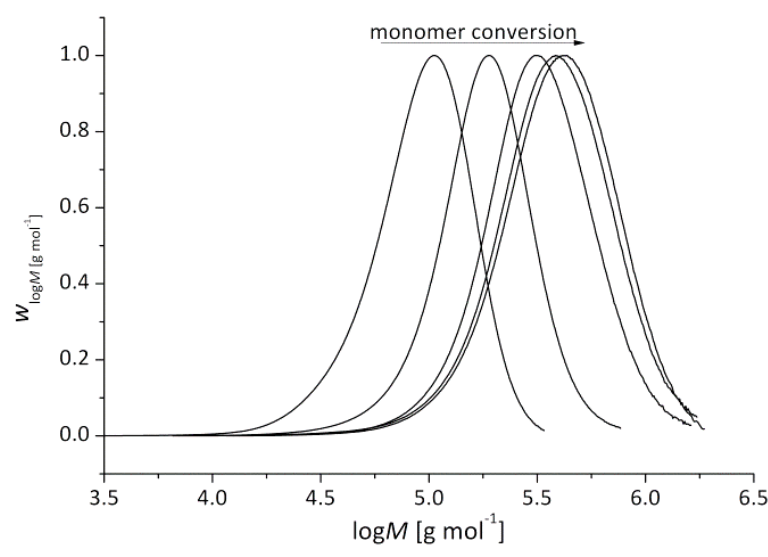

Homopolymerisation of NIPAam

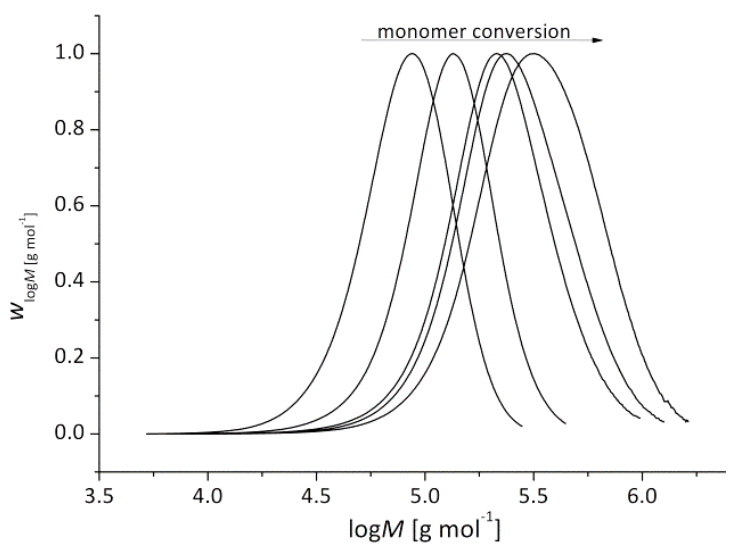

Copolymerisation with $28 \mathrm{mmol} \cdot \mathrm{L}^{-1} 1.1$

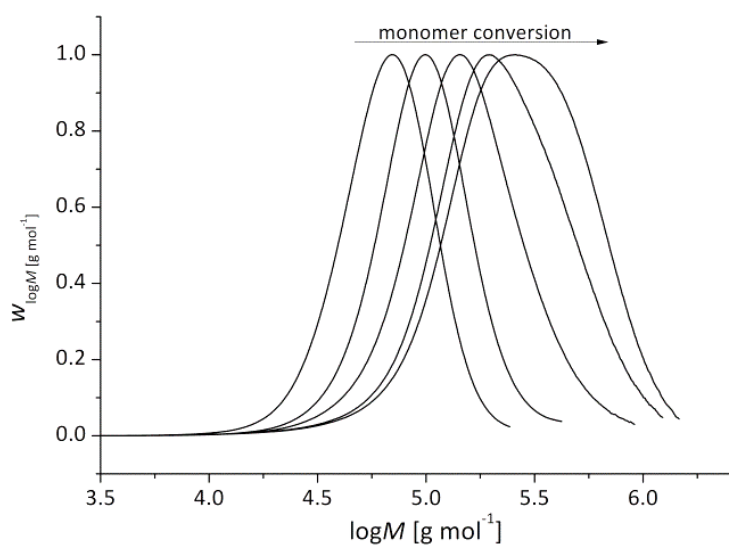

Copolymerisation with $140 \mathrm{mmol} \cdot \mathrm{L}^{-1} 1.1$

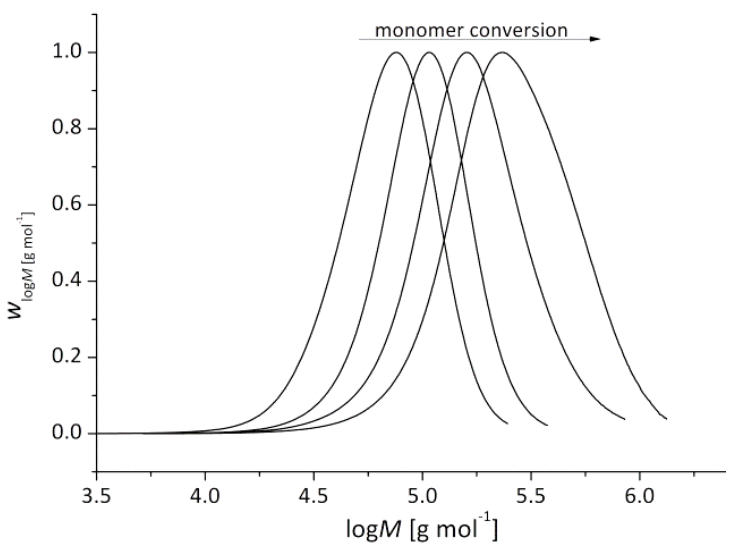

Copolymerisation with $56 \mathrm{mmol} \cdot \mathrm{L}^{-1} 1.1$

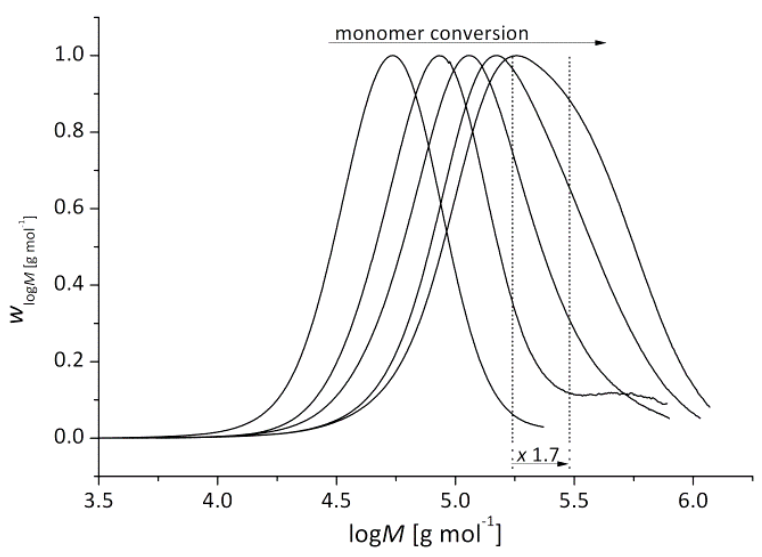

Copolymerisation with $280 \mathrm{mmol} \cdot \mathrm{L}^{-1} 1.1$

Figure 8-35: Corrected SEC-UV-spectra of different copolymerisations of NIPAam with 1.1 up to a reaction time of 127 minutes. 
It can be observed that the MWDs obtained by correction of the UV-signal, i.e. corresponding only with the living polymer in the system, are distinctively narrower than the chromatograms obtained by RI-detection. Furthermore high molecular weight material is formed after 112 minutes, i.e. here the fourth aliquot in all copolymerisations noticeable by the deformation of the distribution curve in the region of higher molecular weights. In the last set of chromatograms of the copolymerisation with the highest co-monomer concentration the peak maxima are marked. They differ approximately by a factor of 2, i.e. double stars are formed. However the formation of high molecular weight material is not as pronounced as in the polymerisations with BA. NIPAam is not known to undergo side reactions as, e.g. back biting. As no formation of high molecular weight polymer is observed in the homopolymerisations, it can be concluded that the DMI units participate occasionally in side reactions. It can be speculated that the cross-linking between DMI units via a radical mechanism takes place as no evidence was found that this reaction can be triggered by increased temperature.

\subsection{Determination of the Cross-Linkable Units in a pNIPAam Star}

In the case of $p \mathrm{BA}$ the investigation of the amount of UV-reactive units in the polymer via UVVis spectroscopy had to be dropped due to the cut-off wavelength of solvents dissolving the polymer. NIPAam polymers have the advantage to be soluble in methanol which has its cut-off wavelength at $205 \mathrm{~nm}$. In Figure 8-36 the UV-spectra of the monomers NIPAam and $\mathbf{1 . 1}$ as well as $\mathbf{R}_{\mathbf{6}} \mathbf{3}$ recorded in methanol are shown.

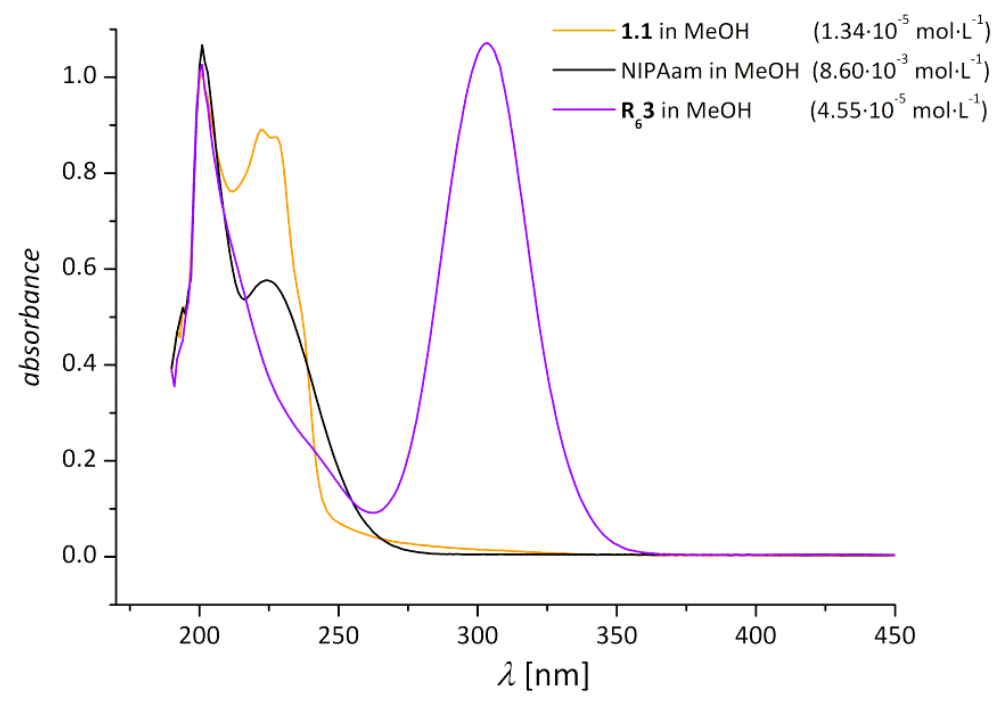

Figure 8-36: UV-Vis spectra of 1.1, NIPAam and $\boldsymbol{R}_{6} \mathbf{3}$ in methanol.

The absorption maximum of $\mathbf{1 . 1}$ at $224 \mathrm{~nm}$ and of the RAFT agent at $310 \mathrm{~nm}$ can be observed. Furthermore it has to be noticed that NIPAam at high concentration, as they are in $p$ NIPAam, shows an absorbance at approximately $225 \mathrm{~nm}$ which corresponds with the characteristic absorbance of 
nitrogen containing groups. It results that a clear determination of the 1.1-amount in the polymer is inhibited.

As UV-Vis spectroscopy had to be excluded, investigations via NMR spectroscopy were carried out. Figure 8-37 shows the ${ }^{1} \mathrm{H}$ NMR spectrum of a $p$ NIPAam sample recorded in $\mathrm{CDCl}_{3}$.

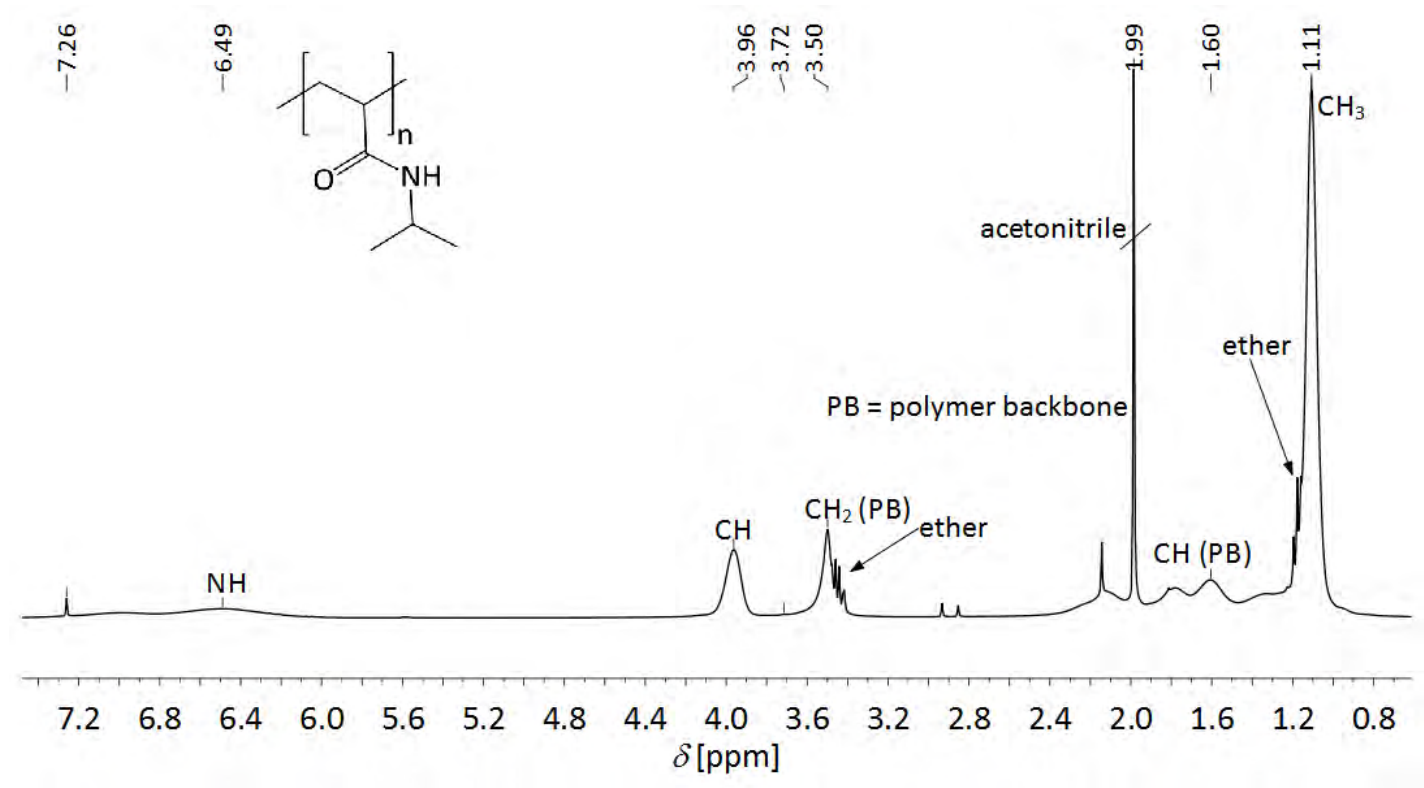

Figure 8-37: ${ }^{1} \mathrm{H}$ NMR spectrum of pNIPAam recorded in $\mathrm{CDCl}_{3}$.

In the down field region at approximately $6.5 \mathrm{ppm}$ the broad signal of the amino group's proton can be seen. The shape and the chemical shift of this group depend strongly on the surrounding regarding other functional groups in spatial proximity or water in the solvent. Further in the up field the broad singlet $(3.96 \mathrm{ppm})$ caused by the proton of the tertiary carbon adjacent to the nitrogen and the signal of the $\mathrm{CH}_{2}$-group (3.72 ppm) of the polymer backbone appear. Between 1.5 and $2.2 \mathrm{ppm}$ various polymer backbone signals can be seen. The different chemical shifts are a consequence of the tacticity of the polymer chains. ${ }^{(187)}$ At $1.11 \mathrm{ppm}$ the singlet caused by NIPAam's terminal methyl groups arise which will be integrated to determine the polymer composition. In the following figure the ${ }^{1} \mathrm{H}$ NMR spectrum of $p$ NIPAam (blue) is superimposed with a ${ }^{1} \mathrm{H}$ NMR spectrum of $p$ (NIPAam-co-1.1) (black). 


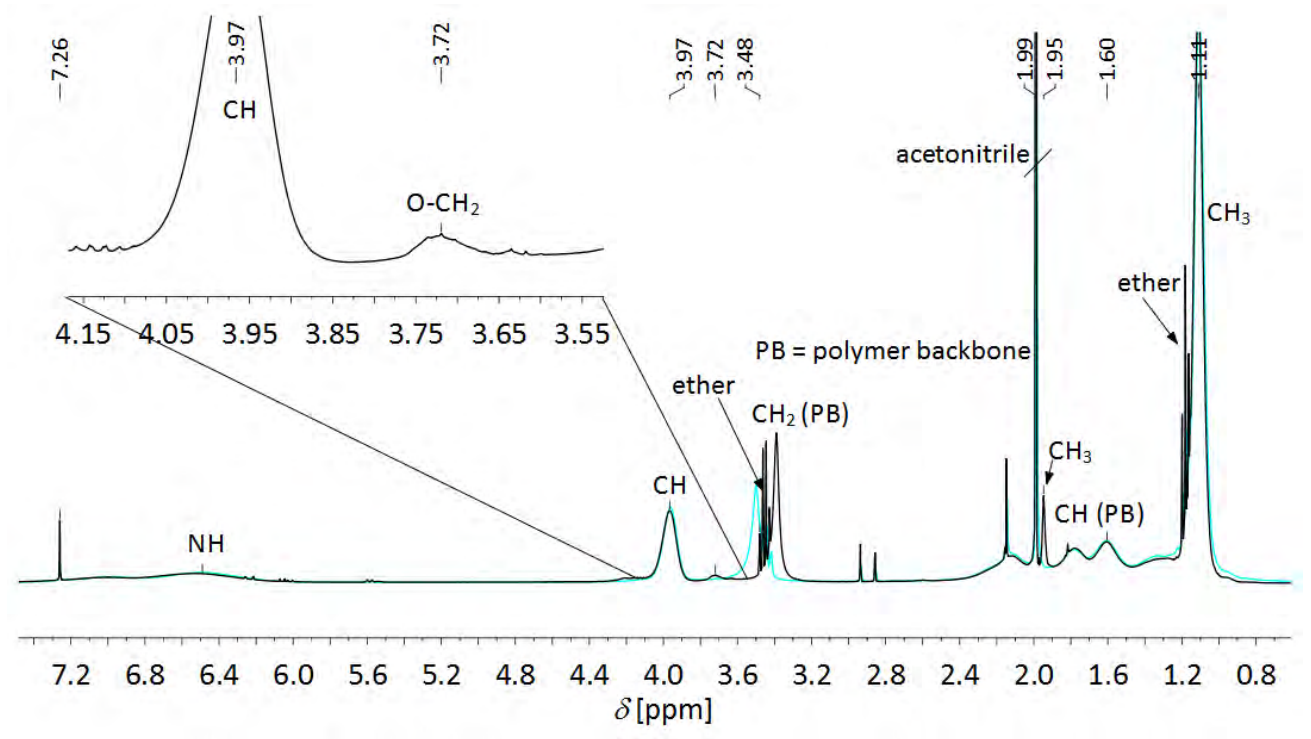

Figure 8-38: Superimposed, standardised ${ }^{1} \mathrm{H}$ NMR spectra of pNIPAam (blue) and p(NIPAam-co-1.1) (black) recorded in $\mathrm{CDCl}_{3}$

The addition of the co-monomer 1.1 causes a singlet at $1.95 \mathrm{ppm}$ resulting from the methyl groups of the DMI unit and a signal at $3.72 \mathrm{ppm}$ which results from the $\mathrm{CH}_{2}$-group adjacent to the oxygen atom of the ethyl bridge in the co-monomer which was classified by ${ }^{15} \mathrm{~N}-\mathrm{HMBC}$ in chapter 8.2 . The second signal of the ethyl bridge is overlapped by the large signal of NIPAam's CH-group. Signals of diethyl ether, the precipitant for $p$ NIPAam, were found in all spectra despite drying of the samples for five days under vacuum at $50^{\circ} \mathrm{C}$. To determine the composition of the copolymer two analytical methods were tested in chapter 8.2, which verified each other. Here method II was applied. For the NIPAamunits the integrals of the half-peak widths of the singlet at $1.11 \mathrm{ppm}$ caused by NIPAam's terminal methyl groups and of the co-monomer signal at $3.72 \mathrm{ppm}$ were used in this method. As in the case of the BA-polymers the error in the determination method due to the low intensity of the co-monomer signal has to be taken in account so the results are imperfect. In the first example of copolymerisation between NIPAam and $\mathbf{1 . 1}$ introduced in this chapter $\left(3,600 \mathrm{mmol} \cdot \mathrm{L}^{-1}\right.$ of NIPAam with $107.2 \mathrm{mmol} \cdot \mathrm{L}^{-1}$ of 1.1 using $1.3 \mathrm{mmol} \cdot \mathrm{L}^{-1}$ of $\mathrm{AlBN}$ and $0.5 \mathrm{mmol} \cdot \mathrm{L}^{-1}$ of $\mathbf{R}_{6} \mathbf{3}$ in DMF) after 55 minutes of reaction time star polymer with $95.2 \mathrm{~mol} \%$ NIPAam and $4.8 \mathrm{~mol} \% 1.1$ and after 85 minutes with 95.7 mol\% NIPAam and 4.3 mol\% 1.1 were obtained. From the set of copolymerisations introduced previously the copolymerisation with the highest co-monomer concentration was investigated more closely. Figure 8-39 shows the superimposed, standardised ${ }^{1} \mathrm{H}$ NMR spectra of this copolymerisation. 


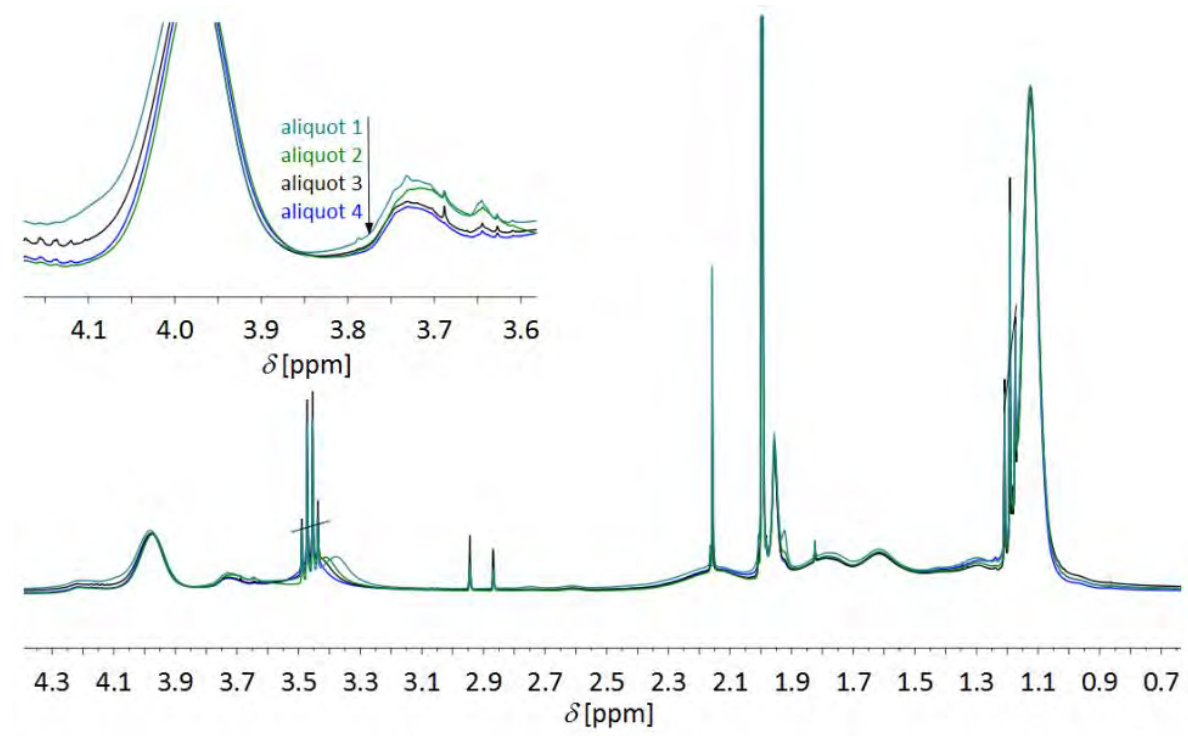

Figure 8-39: Superimposed, standardised ${ }^{1} \mathrm{H} N \mathrm{NR}$ spectra recorded in $\mathrm{CDCl}_{3}$ of the copolymerisation with $5,000 \mathrm{mmol} \cdot \mathrm{L}^{-1}$ NIPAam and $280 \mathrm{mmol} \cdot \mathrm{L}^{-1} 1.1 \mathrm{using} 0.6 \mathrm{mmol} \cdot \mathrm{L}^{-1} \boldsymbol{R}_{6} 3$ and $1.1 \mathrm{mmol} \cdot \mathrm{L}^{-1} \mathrm{AIBN}$ in DMF at $60^{\circ} \mathrm{C}$.

The area between 3.60 and $4.15 \mathrm{ppm}$ was magnified. From this zoomed excerpt it can be observed that the amount of integrated $\mathbf{1 . 1}$ in the polymer decreases regarding the amount of NIPAam. This is supported by the molecular share $F$ determined via method II and can be retraced in Table 8-9.

Table 8-9: Molecular shares of both monomers and number of monomer units per star molecule in the samples of the copolymerisation with $5,000 \mathrm{mmol} \cdot \mathrm{L}^{-1} \mathrm{NIPAam}$ and $280 \mathrm{mmol} \cdot \mathrm{L}^{-1} 1.1 \mathrm{using} 0.6 \mathrm{mmol} \cdot \mathrm{L}^{-1} \boldsymbol{R}_{6} 3$ and $1.1 \mathrm{mmol} \cdot \mathrm{L}^{-1} \mathrm{AIBN}$ in DMF at $60^{\circ} \mathrm{C}$.

\begin{tabular}{c|c|c|c|c|c|c}
$t$ & $\begin{array}{c}M_{\mathrm{n}}^{\mathrm{UV} \cdot 1.52} \\
{\left[\mathrm{~g} \cdot \mathrm{mol}^{-1}\right]}\end{array}$ & $\begin{array}{c}F_{\text {NIPAam }} \\
{[\mathrm{mol} \text { ] }]}\end{array}$ & $\begin{array}{c}F_{1.1} \\
{[\mathrm{~mol} \%]}\end{array}$ & $\begin{array}{c}\text { number of } \\
\text { NIPAam units in } \\
\text { the star }\end{array}$ & $\begin{array}{c}\text { number of 1.1 } \\
\text { units in the } \\
\text { star }\end{array}$ & $\begin{array}{c}\text { 1.1/NIPAam- } \\
\text { units } \\
\text { per star }\end{array}$ \\
\hline 30 & 49,000 & 76.0 & 24.0 & 266 & 84 & 0.32 \\
60 & 70,000 & 80.9 & 19.1 & 404 & 95 & 0.24 \\
92 & 86,000 & 83.9 & 16.1 & 548 & 106 & 0.19 \\
112 & 103,000 & 85.1 & 14.9 & 675 & 118 & 0.17
\end{tabular}

The ratio of 1.1-units to NIPAam-units supports the statement that the integration of $\mathbf{1 . 1}$ in the beginning of the polymerisation is favoured, similar to the findings in the BA-polymerisations. Copolymerisation parameters were obtained in accordance to the procedure shown in chapter 8.2. Values of 0.96 for $\mathbf{1 . 1}$ and 0.05 for NIPAam were determined indicating that the resulting polymer is an alternating copolymer. As $\mathbf{1 . 1}$ has a copolymerisation parameter of almost 1 and NIPAam strongly favours the co-monomer, it can be concluded that $\mathbf{1 . 1}$ is preferably integrated as long as the local concentration is high enough. 


\subsection{AFM-Investigations of $p \mathrm{BA}-$ and $p$ NIPAam Stars}

Atomic Force Microscopy (AFM) is a high resolution technique to create images of samples' surfaces, e.g. of polymers to investigate their structure and possible self-assembly. To record an AFM-image the sample is scanned by an extremely sharp tip which is moved across the object's surface. The tip is mounted on a flexible cantilever and is brought into proximity of the sample's surface. Forces between the tip and the sample lead to a deflection of the cantilever according to Hooke's law. The deflection of the cantilever is measured by a laser and detected via a photodiode.

Here the existence of star shaped polymers should be proven by this depicting method. The experiments here were carried out in an argon-filled glove box on a Scanning Probe Microscope MultiMode 8 by Veeco applying the peak force tapping mode with a silicon-on-nitride cantilever $\left(k=0.4 \mathrm{~N} \cdot \mathrm{m}^{-1}, f_{0}=50-90 \mathrm{kHz}\right.$ ). To gain good results a suitable substrate for the sample has to be chosen. Mica, a sheet silicate, was chosen here in the form of $\mathrm{KAl}_{2}(\mathrm{OH}, \mathrm{F})_{2}\left(\mathrm{AlSi}_{3} \mathrm{O}_{10}\right)$. Mica is a common substrate and well known in AFM-investigations because it provides ultra-flat and clean surfaces as the crystal is freshly split during the sample preparation. ${ }^{(188)}$ The solvent used was chloroform, as this is a good solvent for both polymer kinds and it could be assumed that the polymer was dissolved on a molecular level. The solutions $\left(c_{\text {polymer }} \approx 8 \cdot 10^{-4} \mathrm{~g} \cdot \mathrm{L}^{-1}\right)$ were spin-coated on freshly split mica platelets and left overnight under chloroform atmosphere in a solvent annealing chamber to enable extension of the polymer star's arms. The concentration of the solution has to be very low to avoid overlapping of the single stars.

In Figure 8-40 the AFM image of a $p$ (NIPAam-co-1.1) sample is shown. The sample was synthesised with $\mathbf{3}$ mol\% $\mathbf{1 . 1}$ in the monomer feed and the RAFT-agents $\mathbf{R}_{\mathbf{6}} \mathbf{2}$ and $\mathbf{R}_{\mathbf{L}} \mathbf{2}$ yielding polymer material with a molecular weight of $150,000 \mathrm{~g} \cdot \mathrm{mol}^{-1}$ for the star polymers and $30,000 \mathrm{~g} \cdot \mathrm{mol}^{-1}$ for the linear polymer. First a $15 \mu \mathrm{m} \times 15 \mu \mathrm{m}$ image was recorded with 512 lines, Figure 8-40 left. 


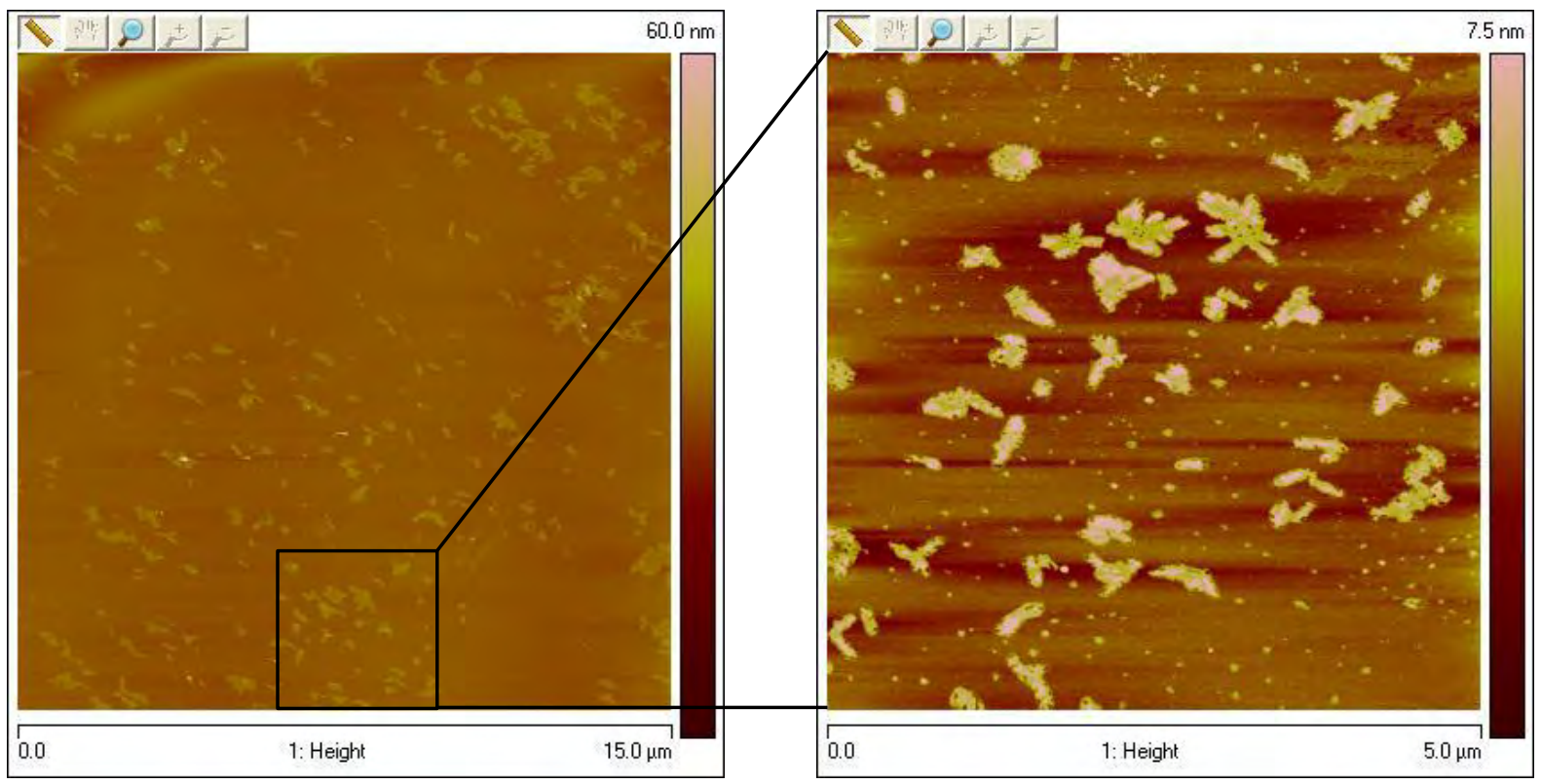

Figure 8-40: $p$ (NIPAam-co-1.1) $M_{n}^{\text {star }}=150,000 \mathrm{~g} \cdot \mathrm{mol}^{1}$ on mica, $c_{\text {polymer }}=8.08 \cdot 10^{-4} \mathrm{~g} \cdot \mathrm{L}^{-1}$.

A gathering of polymer stars and chains can been seen. In the right upper corner the polymer material overlaps and it cannot be distinguished between the single molecules. At the bottom of the image single molecules in star form could be detected. The right-hand picture in this figure shows the $5 \mu \mathrm{m} \times 5 \mu \mathrm{m}$ image (768 lines) of this region. Molecules in star form with six arms can be observed which proves the existence of the desired polymers. Furthermore linear material can be seen which was generated by the linear RAFT-agent as well as coiled polymers which can also be assumed to be six-armed stars. Via the software NanoScope Analysis 1.20 different parameters such as height or density of the molecules can be determined. It has to be noted that the density is given by the program as a pixel unit per area. In the following figure two examples are shown. Density, height, area and diameter were calculated for two six-armed stars marked blue (Figure 8-41). 

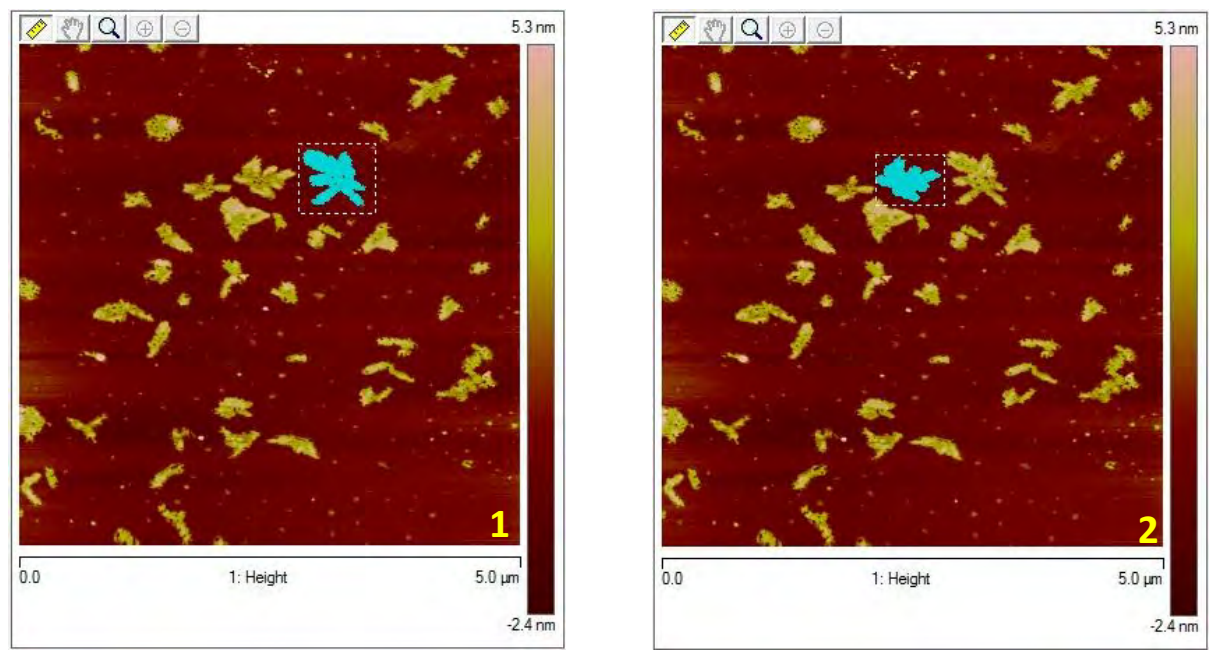

\begin{tabular}{c|c|c} 
Parameter & Star 1 & Star 2 \\
\hline density $\left[{\left.\mu \mathrm{m}^{-2}\right]}^{2}\right.$ & 1.836 & 2.854 \\
maximum height $[\mathrm{nm}]$ & 6.233 & 5.497 \\
area $\left[\mu \mathrm{m}^{2}\right]$ & 0.178 & 0.133 \\
diameter $[\mathrm{nm}]$ & 475 & 412
\end{tabular}

Figure 8-41: p(NIPAam-co-1.1) stretched stars in detail.

Comparing the densities of the different molecules reveals a higher density for some of the polymer stars. This indicates that these polymers are partially or completely coiled compared to stars with lower density (exemplary Figure 8-41). These coiled molecules show consequently smaller diameters and take up less space. Calculation over all polymer molecules of this part of the sample revealed an average height of $5.5 \mathrm{~nm}$. This is confirmed by the following figure which shows cross-sections through various molecules. In the top part the cross-section of the two star molecules introduced previously can be seen. The bottom part presents two different molecules in comparison with Star 2 . It has to be noticed that the $y$-scale start in a negative range which is set up- and programconditioned. 

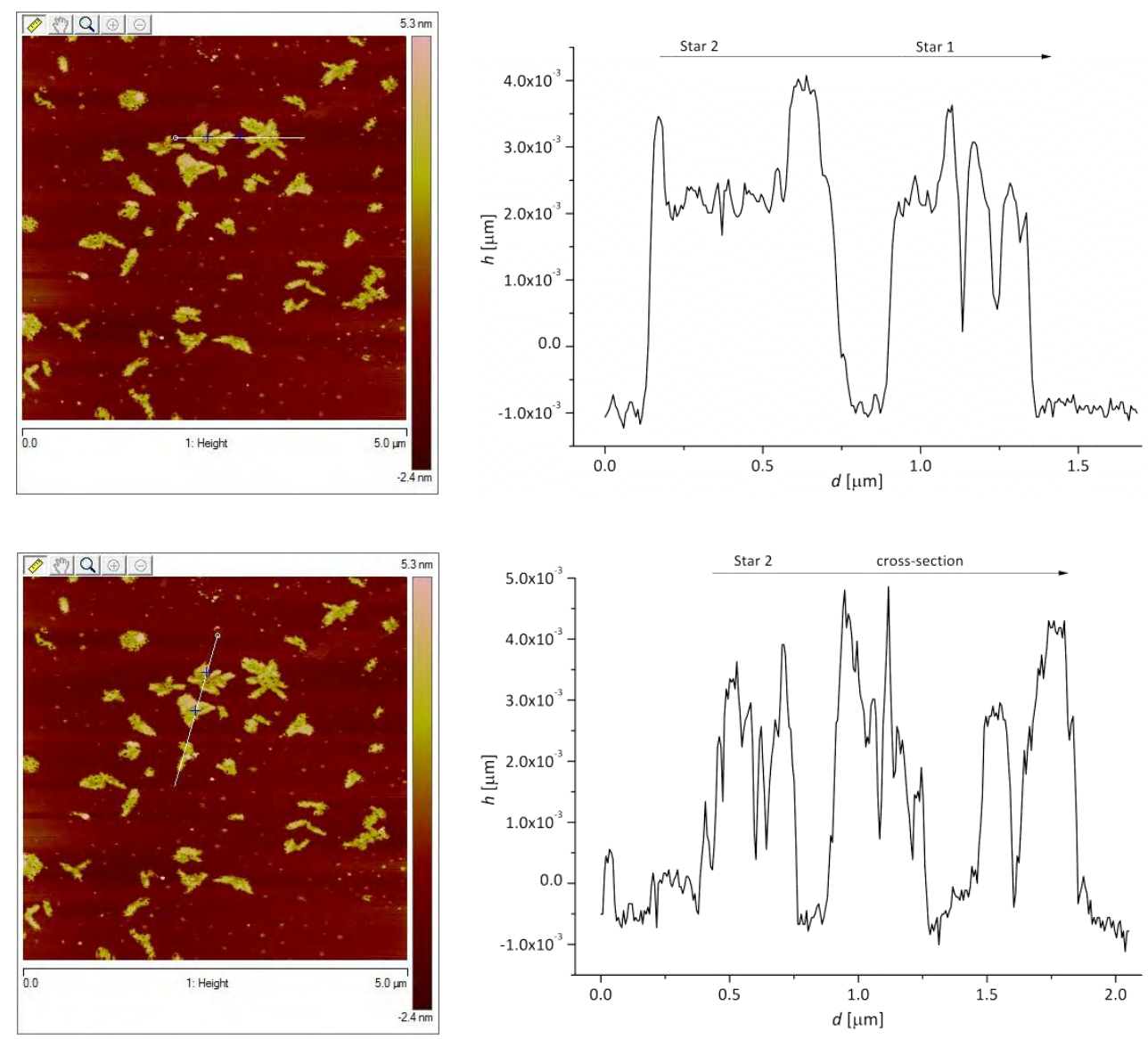

Figure 8-42: Cross-sections of various molecules.

Besides an equal maximum height for all molecules the unevenness of the single star molecules can be seen as the six linear chains which are connected in a central point coil individually on the substrate. Due to the coiling and stacking of chains higher polymer segments than the average can be seen. No regular structure in the assembly can be observed.

The example shown in the following Figure $8-43$ is a $p$ (BA-co-1.1) sample synthesised with 3 mol\% of $\mathbf{1 . 1}$ in the monomer feed, and $\mathbf{R}_{\mathbf{6}} \mathbf{2}$ and $\mathbf{R}_{\mathbf{L}} \mathbf{2}$ with a molecular weight of $167,000 \mathrm{~g} \cdot \mathrm{mol}^{-1}$ for the star polymer and $32,000 \mathrm{~g} \cdot \mathrm{mol}^{-1}$ for the linear polymer. As in the case of $p$ (NIPAam-co-1.1) discussed previously a solution in chloroform $\left(c_{\text {polymer }}=7.14 \cdot 10^{-4} \mathrm{~g} \cdot \mathrm{L}^{-1}\right)$ was spin-coated on mica and left under chloroform atmosphere overnight. On the left side of Figure 8-43 a $15 \mu \mathrm{m} \times 15 \mu \mathrm{m}$-image (512 lines) of this sample is shown. Due to the lower concentration the molecules are broadly distributed on the surface. 


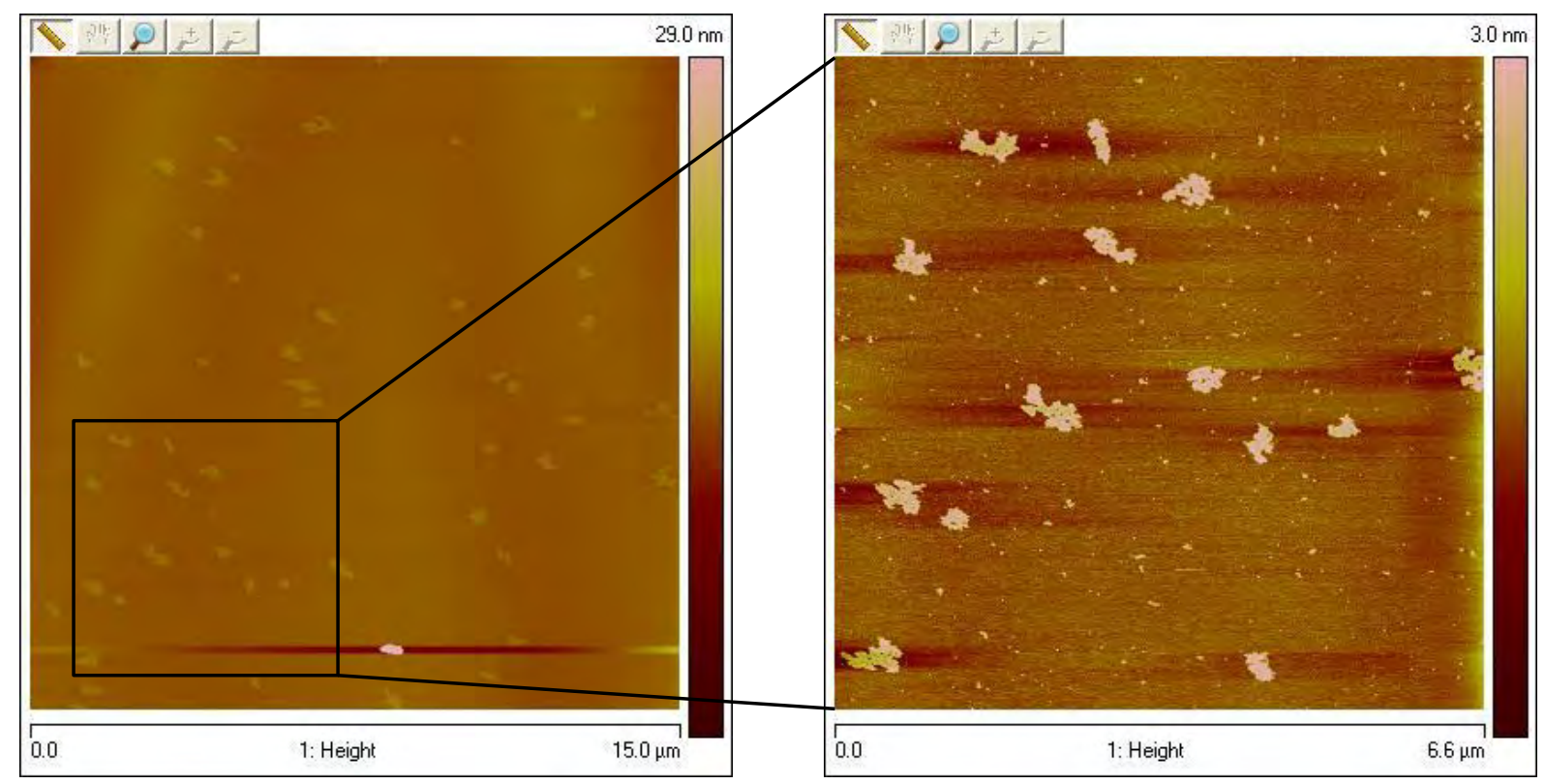

Figure 8-43: $p(B A-c o-1.1) M_{n}^{\text {star }}=167,000 \mathrm{~g} \cdot \mathrm{mol}^{-1}$ on mica, $c_{\text {polymer }}=7.14 \cdot 10^{-4} \mathrm{~g} \cdot \mathrm{L}^{-1}$

It can be observed that the polymer molecules are not extended as in the $p$ NIPAam sample. They are coiled despite the good solvent atmosphere in the solvent annealing chamber which supports the impression of a wider distribution of the molecules. The $6.6 \mu \mathrm{m} \times 6.6 \mu \mathrm{m}$-image (768 lines), Figure 8-43 right-hand side, shows this finding more clearly. A star-shaped form can be distinguished but the molecules appear contracted. The more detailed examination of all polymer molecules of this part of the sample (exemplary Figure 8-44) revealed a maximum height for these polymer molecules of $2.0 \mathrm{~nm}$ which is also supported by the cross-sections in Figure 8-45 below. The particles are more dense than the $p$ NIPAam-particles. Those $p$ NIPAam-particles had a maximum density of $3 \mu \mathrm{m}^{-2}$. This value is the lowest value in the examination of the $p$ BA-particles. 

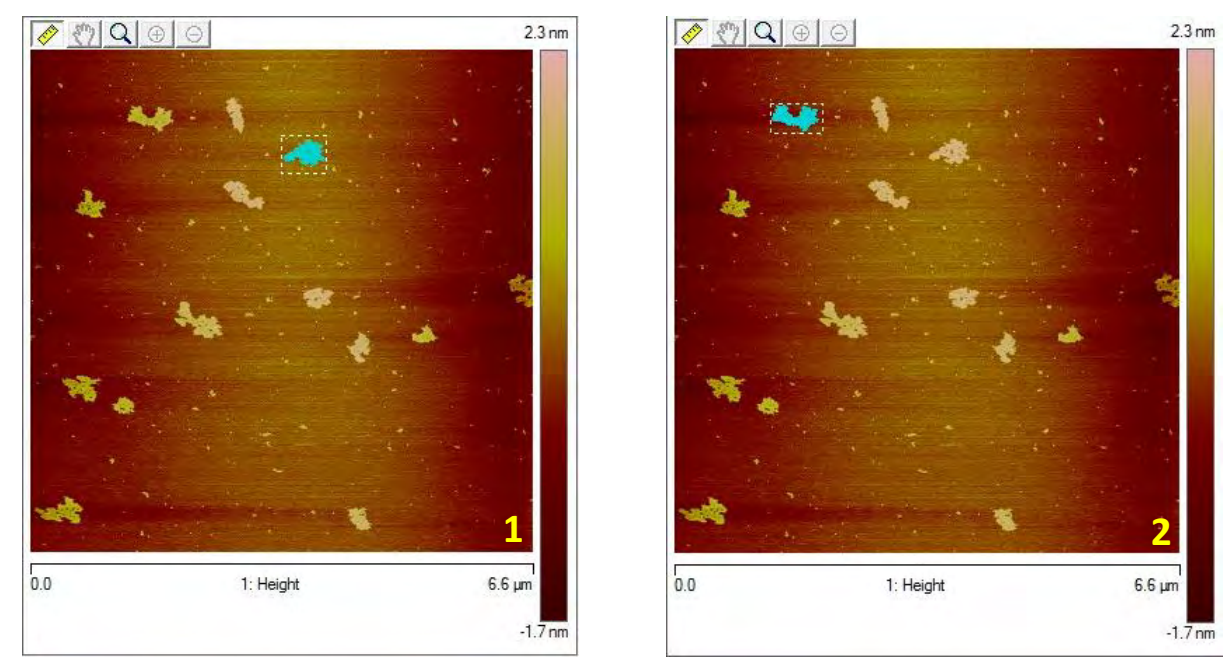

\begin{tabular}{c|c|c} 
Parameter & Star 1 & Star 2 \\
\hline density $\left[{\left.\mu \mathrm{m}^{-2}\right]}_{\text {maximum height }[\mathrm{nm}]}\right.$ & 3.305 & 3.611 \\
area $\left[{\left.\mu \mathrm{m}^{2}\right]}^{2}\right.$ & 2.046 & 2.157 \\
diameter $[\mathrm{nm}]$ & 0.090 & 0.101 \\
\multicolumn{2}{c}{ Figure 8-44: $p(B A-c o-1.1)$ stretched stars in detail. }
\end{tabular}

In contrast to the pNIPAam-particles where fully extended and partially coiled star polymer could be distinguished by obtaining different densities, here all molecules show approximately the same density which is a proof that all molecules are in a completely coiled state. 

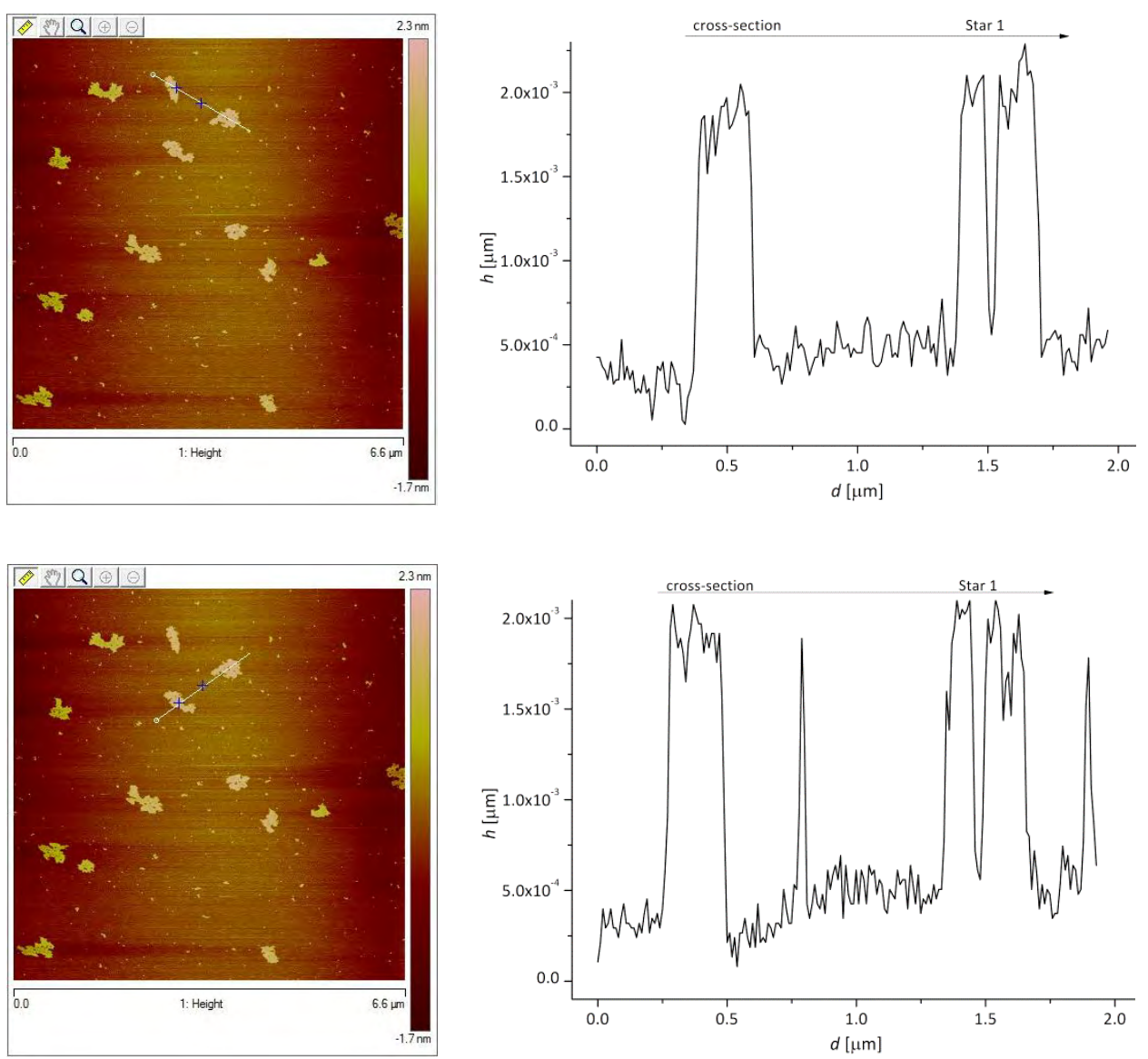

Figure 8-45: Cross-sections of various molecules.

The molecules of this sample are not as uneven as the pNIPAam-molecules and the cross-section proves a much more condensed state of these particles in comparison with the example shown before. Due to this condensed state the particles take up less space and appear much smaller than expected.

Table 8-10 summarises the findings made in this chapter. It was observed that the star polymers basing on NIPAam are more extended than BA-based polymer stars even though the polymers have approximately the same molecular weight which implies an approximate equal size of the molecules. The diameter of the $p$ BA-stars is approximately $100 \mathrm{~nm}$ smaller than the one of $p$ NIPAam-stars. Furthermore BA-polymers appear thinner as the contraction consequently takes place in all three dimensions resulting in more dense material. 
Table 8-10: Summary of the different parameters for the samples of $p$ (NIPAam-co-1.1) and p(BA-co-1.1)

\begin{tabular}{c|c|c|c|c} 
& \multicolumn{2}{|c|}{$p$ (NIPAam-co-1.1) } & \multicolumn{2}{c}{$p($ BA-co-1.1) } \\
Parameter & Star 1 & Star 2 & Star 1 & Star 2 \\
\hline$M_{\mathrm{n}}\left[\mathrm{g} \cdot \mathrm{mol}^{-1}\right]$ & \multicolumn{2}{|c|}{150,000} & \multicolumn{2}{|c}{167,000} \\
density $\left[\mu \mathrm{m}^{-2}\right]$ & 1.836 & 2.854 & 3.305 & 3.611 \\
max. height $[\mathrm{nm}]$ & 6.233 & 5.497 & 2.046 & 2.157 \\
area $\left[\mathrm{\mu m}^{2}\right]$ & 0.178 & 0.133 & 0.090 & 0.101 \\
diameter $[\mathrm{nm}]$ & 475 & 412 & 339 & 359
\end{tabular}

The difference in expansion is caused by the surface of the substrate. As mentioned in the beginning, mica (here $\mathrm{KAl}_{2}(\mathrm{OH}, \mathrm{F})_{2}\left(\mathrm{AlSi}_{3} \mathrm{O}_{10}\right)$ ) is a mineral and belongs to the sheet silicates. Therefore its surface is hydrophilic. ${ }^{(189)}$ pNIPAam is a hydrophilic polymer which interacts well with the surface of the substrate leading to well extended macromolecules. In contrary $p B A$ is hydrophobic and is repelled by the surface. To minimise the surface energy the polymer coils which results in apparently smaller particles. To investigate $p \mathrm{BA}$ samples more thoroughly another substrate has to be used.

It has to be noted that the conformation of polymers on the substrate is strongly depended on the solvent applied and the substrate. The dependence is more pronounced for copolymers. ${ }^{(188)}$ The here obtained data are only valid for the combination of mica with chloroform.

\subsection{Summary and Conclusions}

It can be summarised that the RAFT copolymerisations of the UV-reactive co-monomer $\mathbf{1 . 1}$ with BA and NIPAam proceed in a well-controlled manner. As expected from a RAFT polymerisation, the obtained molecular weights can be influenced by the reaction conditions, i.e. by the RAFT-agent and the initiator concentration. The findings in both polymerisation systems support the assumption that the addition of co-monomer $\mathbf{1 . 1}$ influences the RAFT-process in general, i.e. induction occurs, and that the effect of the induction is more pronounced with increasing co-monomer concentration. It can be concluded that the effect is independent on the basic monomer used to synthesise the star polymer. Despite the induction of the process, star polymers with defined molecular weights and defined compositions could be obtained even though it has to be taken in account that smaller molecular weights than expected are gained. In the copolymerisation case with BA concentrations of 1.1 below $56 \mathrm{mmol} \cdot \mathrm{L}^{-1}$ yield polymers which are $10 \%$ smaller than the corresponding BAhomopolymer, $150 \mathrm{mmol} \cdot \mathrm{L}^{-1}$ yield $15 \%$ and $280 \mathrm{mmol} \cdot \mathrm{L}^{-1} 30 \%$ smaller polymers. In the NIPAam case the deviation is stronger as will be explained later on. 
Besides the effect of induction the addition of $\mathbf{1 . 1}$ evokes the formation of double stars. Here the two systems differ strongly. Whereas in the polymerisations with NIPAam the star-star coupling is not very distinctive and only observable at very high monomer conversions, i.e. longer reaction times, and more pronounced in systems with high co-monomer concentration, the formation of a byproduct with high molecular weight is reinforced in the BA-polymerisations by addition of 1.1. In contrast to NIPAam, BA is known to undergo star-star coupling by means of back-biting. Nevertheless this effect is much stronger in the copolymerisation systems leading to samples with more double than single stars and to coupling products larger than two stars. In chapter 8.1 the mechanism of reinforced back-biting was proposed: The propagating macroBA-radical attacks the DMI unit which then abstracts a proton from another polymer backbone creating a new Mid Chain Radical to propagate. The side reaction becomes a chain reaction. As no back-biting occurs in NIPAam polymerisations, the double star formation there has to be attributed to cross-linking of the DMI units by chemical triggers. Probably the cross-linking proceeds via a radical mechanism as thermal cross-linkage was not observed.

By means of ${ }^{15} \mathrm{~N}-\mathrm{HMBC}$ investigations the chemical shift of 1.1's nitrogen was determined $(-238.18 \mathrm{ppm})$ and the chemical shifts of the ethyl bridge connecting DMI and vinyl unit in the ${ }^{1}$ H NMR spectrum could be classified. Two NMR-based methods to determine the polymer composition were tested verifying each other. Less depending on the chosen measurement settings is the method based on the comparison of polymer signals. It could be shown that BA-based starshaped polymers with molecular weights from 49,000 up to $405,000 \mathrm{~g} \cdot \mathrm{mol}^{-1}$ can be synthesised consisting of 31 up to 910 co-monomer units per star which equals a molecular share in the polymer of up to 38 mol\% depending on the amount of $\mathbf{1 . 1}$ in the monomer feed. It was observed that the polymer properties consequently change with increasing co-monomer amount. Furthermore copolymerisation parameters for the system BA with 1.1 could be determined $\left(r_{\mathrm{BA}}=0.95 ; r_{1.1}=2.24\right)$. For NIPAam-based star polymers $\left(r_{\text {NIPAam }}=0.05 ; r_{1.1}=0.96\right)$ molecular weights from 38,000 up to $250,000 \mathrm{~g} \cdot \mathrm{mol}^{-1}$ could be obtained with 5 to $24 \mathrm{~mol} \% 1.1$ in the polymer which equals up to $130 \mathrm{co}-$ monomer units per star molecule.

In the following the polymerisation of BA and NIPAam should be compared directly. The sets of copolymerisations, introduced in this chapter, were carried out under the same conditions: Solvent polymerisations with approximately $5,000 \mathrm{mmol} \cdot \mathrm{L}^{-1}$ of the basic monomer, from 28 to $280 \mathrm{mmol} \cdot \mathrm{L}^{-1}$ of 1.1 mediated by $0.6 \mathrm{mmol} \cdot \mathrm{L}^{-1}$ of RAFT-agent and $1.1 \mathrm{mmol} \cdot \mathrm{L}^{-1}$ of AIBN at $60{ }^{\circ} \mathrm{C}$. The differences in the procedure of the polymerisation lie in the solvent where toluene as a good solvent was chosen for $\mathrm{BA}$ and $p \mathrm{BA}$ and DMF as a good solvent for NIPAam and $p$ NIPAam. Additionally the Rgroup of the RAFT-agent was different ( $\mathbf{R}_{6} \mathbf{2}$ for BA and $\mathbf{R}_{\mathbf{6}} \mathbf{3}$ for NIPAam), as those gave the best 
results for the current monomer. Figure 8-46 shows the developments of the average molecular weights (determined by UV-detection, corrected; top) and the monomer conversions (bottom) with the reaction time for the homopolymerisations of BA and NIPAam as well as of the copolymerisations with $28 \mathrm{mmol} \cdot \mathrm{I}^{-1}$ and $280 \mathrm{mmol} \cdot \mathrm{L}^{-1}$ of the co-monomer.

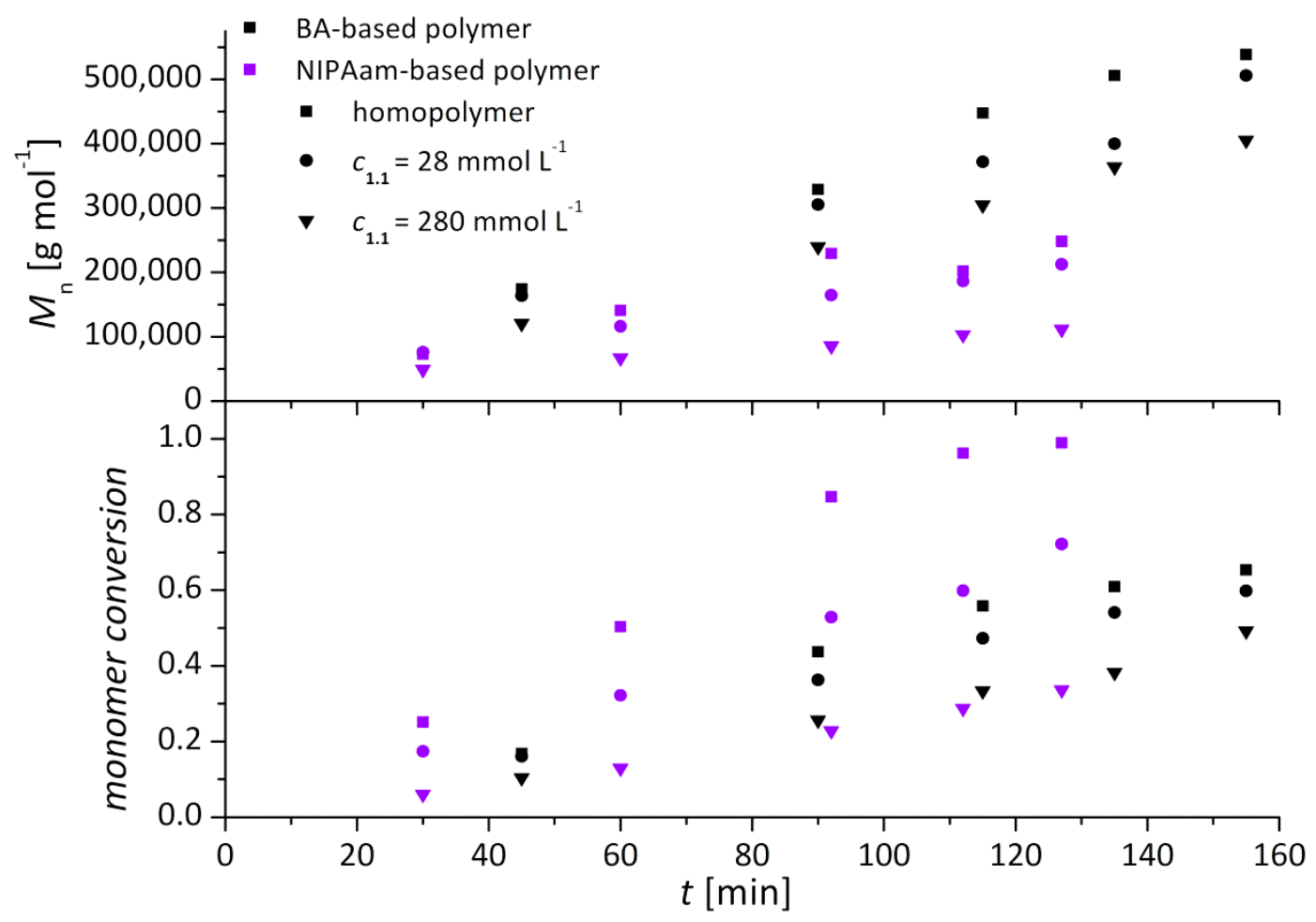

Figure 8-46: Development of the average molecular weights (left) and the monomer conversion (right) with the reaction time of polymerisations with BA (black) or NIPAam (purple) and 28 or $280 \mathrm{mmol} \cdot \mathrm{L}^{-1} 1.1$ mediated by $0.6 \mathrm{mmol} \cdot \mathrm{L}^{-1} R A F T$ agent and $1.1 \mathrm{mmol} \cdot \mathrm{L}^{-1} \mathrm{AlBN}$ at $60^{\circ} \mathrm{C}$.

The plots of the average molecular weights (top) illustrate repeatedly the effect of induction. The molecular weights of the copolymers (circles and triangles) are in all cases smaller than in the homopolymerisation (squares) due to a delayed start of the reaction. Furthermore it can be observed that the NIPAam-polymerisations yield higher monomer conversions (Figure 8-46, bottom) than the BA-polymerisations, except of the polymerisation with the highest co-monomer concentration in the monomer feed. Despite the higher monomer conversions the obtained molecular weights are much smaller for pNIPAam-samples that for pBA-samples. As this is also observed in the homopolymerisation case, hence it can be concluded that this no result from the addition of the comonomer. As here the same concentrations for all reactants were used, it was expected that at the point of same monomer conversion equally many monomer units were consumed which leads to approximately the same molecular weights. NIPAam is $12 \%$ smaller in molecular weight than BA $\left(M_{\mathrm{NIPAam}}=113.16 \mathrm{~g} \cdot \mathrm{mol}^{-1}, M_{\mathrm{BA}}=128.17 \mathrm{~g} \cdot \mathrm{mol}^{-1}\right)$, i.e. it was expected that the molecular weights of the 
resulting polymers should also be $12 \%$ smaller. The found molecular weights are distinctively lower than the expected values. This discrepancy results from two factors: On the one hand NIPAam is known to undergo self-initiation at temperatures above $60{ }^{\circ} \mathrm{C}{ }^{(190)}$ which increases the radical concentration in the system and leads to a higher probability of termination generating dead polymer chains which was shown in the SEC-data in chapter 8.3. Also transfer reactions via the solvent can occur giving the same result. As conclusion, in NIPAam-polymerisations a large amount of monomer is consumed by irreversible termination of growing chains. Therefore the experimentally found molecular weights do not agree with the theoretical values (equation 3-27). On the other hand, as discussed in chapter 8.1, BA is prone to undergo backbiting, which is reinforced by the integrated co-monomer units. In the process of polymerisation the determined, average molecular weights increase stronger due to double star formation. The difference in the molecular weights is consequently a result of continuous loss of growing chains by termination in the NIPAam-case and especially for long reaction times for the formation of multi-star polymers in the case of BA.

When comparing the integration of $\mathbf{1 . 1}$ in both polymers, it can be observed that on a relative basis the copolymer with NIPAam consists of more 1.1 that the corresponding BA-based polymer. Figure 8-47 shows this result for the copolymerisations of NIPAam and BA with
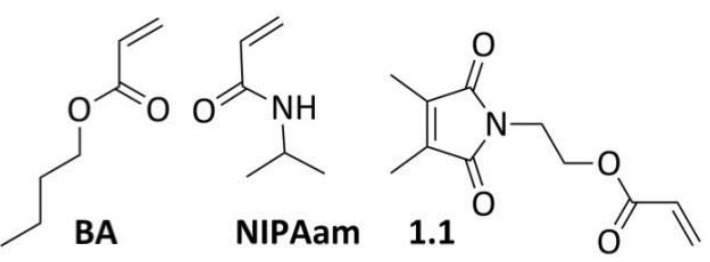
$280 \mathrm{mmol} \cdot \mathrm{L}^{-1}$ 1.1. On the left $\mathrm{y}$-scale the molecular weights are plotted versus the monomer conversion, on the right the molecular share $F$ of the monomers integrated in the copolymers where the pentagons represent the values of the NIPAam-copolymer and the triangles the BA-copolymer. 


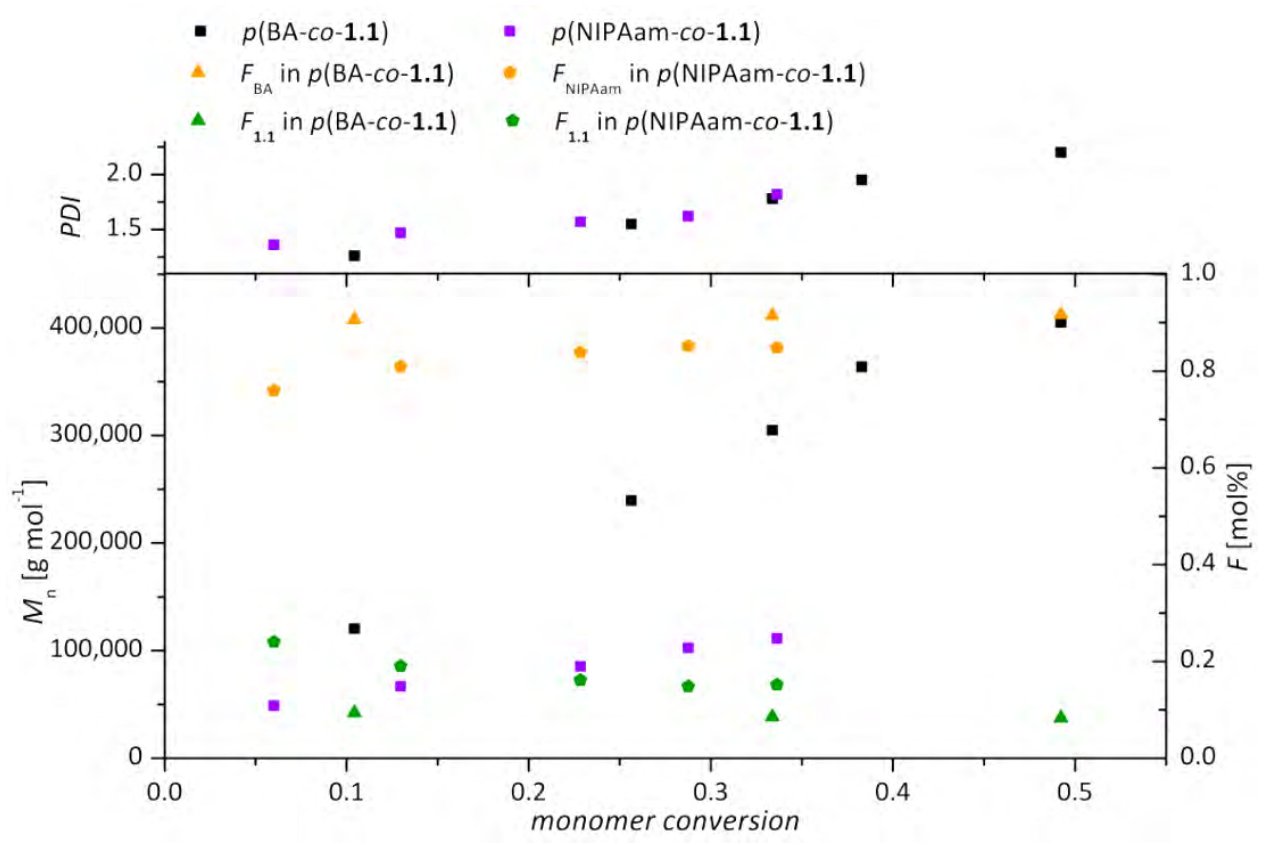

Figure 8-47: Development of the average molecular weights, the PDI-values and the molecular share of the monomers in the polymer with the monomer conversion for the copolymerisations of BA (black) and NIPAam (purple) with $280 \mathrm{mmol} \cdot \mathrm{L}^{-1} \mathbf{1 . 1}$ using $0.6 \mathrm{mmol} \cdot \mathrm{L}^{-1}$ RAFT and $1.1 \mathrm{mmol} \cdot \mathrm{L}^{-1} \mathrm{AlBN}$ at $60^{\circ} \mathrm{C}$.

It can be seen that the molecular share of $\mathbf{1 . 1}$ in all cases is higher in the NIPAam- than in the BA samples which can be explained with help of the copolymerisation parameters (Table 8-11).

Table 8-11: Copolymerisation parameters of 1.1 and the basic monomers

\begin{tabular}{c|c|c} 
& $r_{\text {basic monomer }}$ & $r_{1.1}$ \\
\hline$p$ (NIPAam-CO-1.1) & 0.05 & 0.96 \\
$p$ (BA-co-1.1) & 0.95 & 2.24
\end{tabular}

In the BA-case the copolymerisation parameter of the basic monomer BA is 0.95 which indicates a favoured addition of the co-monomer. As the value is near the critical limit where the monomer similar to the radical chain end $\left(-\mathrm{BA}^{\circ}\right)$ is favoured, it can be assumed that the probability of addition of both monomers to the active chain end is balanced. The copolymer with a unit of $\mathbf{1 . 1}$ as the active chain end $\left(r_{1.1}=2.24\right)$ preferably adds more 1.1-units. In contrary to the parameters of NIPAam, which strongly indicate that the addition of the opposite monomer is favoured. The parameter of the co-monomer is near the critical value of 1 , so it can be assumed that the probability of addition of both monomers to the active chain end is equally high. In the NIPAam-system $\mathbf{1 . 1}$ is integrated exclusively as long as any is available whereas in the BA-system the integration is much more probable yielding in a higher basic monomer share in the polymer compared to the NIPAamsystem. 


\section{Synthesis of Amphiphilic Diblockcopolymer Stars}

The next step of the nano-carrier synthesis is the establishment of the diblock copolymer star. The final carrier should be amphiphilic, therefore, the second block's properties have to be contrary to the first block. In Figure 9-1 the scheme of the process is shown. The first block is coloured red which is a statistical copolymer of either NIPAam yielding a hydrophilic polymer or BA yielding a hydrophobic polymer and the cross-linkable co-monomer $\mathbf{1 . 1}$ (symbolised by the orange spheres). Due to the Z-group approach none of the six arms of the star polymer were terminated in the first polymerisation step synthesising the statistical copolymer. Therefore all six RAFT-units are still in the living state and the star polymer can be applied as marcoRAFT agent in the following step. Furthermore due to the mechanism of the Z-group approach new monomer units are integrated adjacent to the RAFT-units (chapter 3.5). Consequently, the newly formed polymer block (blue in Figure 9-1) is the central part of the polymer star. After the cross-linking of the outer sphere and the removal of the RAFT-core this is the area to store the compound to deliver.

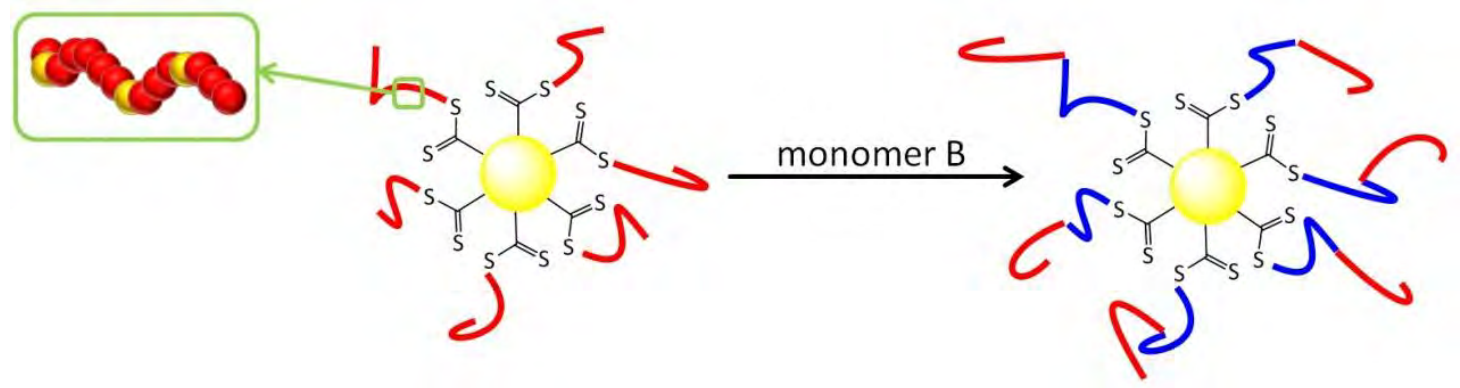

Figure 9-1: Diblock copolymer star synthesis.

Polymer stars with a hydrophobic shell and a hydrophilic centre as well as the reversed case were investigated. It is to be expected that the control of this RAFT-polymerisation is reduced due to the contrasting properties of the monomers and polymers in the system. In the case of a hydrophobic outer sphere, i.e. a BA-block, the NIPAam-radicals in the first step of the polymerisation and later on macro radicals with NIPAam-units at the active chain end have to penetrate the hydrophobic surrounding of the star's centre to reach the RAFT-units

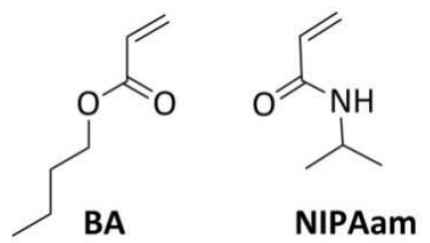
and undergo the, for the control of the process essential, equilibrium step. To increase the probability for the successful penetration a good solvent for all reaction partners has to be used to achieve a wide expansion of the star polymer. If a solvent is used that favours one of the polymers, the other one will coil and close consequently the pathway for the reaction. 
It also has to be mentioned that the determined molecular weights of the copolymer stars are approximate values. On the one hand there are no standards for such copolymer systems to calibrate the SEC set up with. On the other hand it can be assumed that the solubility of the two polymer blocks is different in the two available SEC solvents (THF and DMac). The two blocks of the star polymer will therefore not be equally well expanded which results in deviant hydrodynamic volumes and with that deviant molecular weights. A determination of the molecular weight via mass spectrometry cannot be performed as the upper detection limit of this method is $20,000 \mathrm{~g} \cdot \mathrm{mol}^{-1}$ and the samples synthesised here have a much higher molecular mass.

\subsection{Synthesis of $p$ (BA-co-1.1)-block-NIPAam Stars}

To yield nano-carriers with a hydrophilic core so that water soluble compounds can be stored inside, polymers based on BA were applied as macroRAFT agents. These samples were dissolved in chloroform as this is a good solvent for both, $p \mathrm{BA}$ and $p$ NIPAam.

Subsequently, one example is shown. As NIPAam is a solid monomer any remaining monomer has to be removed from the polymer by precipitation or dialysis. Due to the amphiphilicity of the samples it was not possible to precipitate the polymer. As a consequence no determination of the monomer conversion could be performed. Dialysis as a method to clean the polymer samples was not available at that time. Nevertheless the samples were investigated by SEC. Figure 9-2 shows the SEC-traces of the starting material a $p$ (BA-co-1.1) star with a molecular weight of $64,800 \mathrm{~g} \cdot \mathrm{mol}^{-1}$ (determined in THF, corrected by 1.52) and the amphiphilic polymer star. The red chromatograms were recorded in THF with a universal calibration and $\mathrm{MH}$ parameters for BA. The chromatograms in blue were recorded in DMac with a $p M M A$ calibration. For the synthesis of the $p$ NIPAam block the macroRAFT agent was combined with $0.7 \mathrm{mmol} \cdot \mathrm{L}^{-1} \mathrm{AIBN}$ and $1,290 \mathrm{mmol} \cdot \mathrm{L}^{-1} \mathrm{NIPAam}$ in chloroform. After 180 minutes at $60^{\circ} \mathrm{C}$ the polymerisation was quenched, resulting an amphiphilic star polymer with a molecular weight of approximately $74,200 \mathrm{~g} \cdot \mathrm{mol}^{-1}$ determined in THF or $128,000 \mathrm{~g} \cdot \mathrm{mol}^{-1}$ determined on the DMac-SEC. 


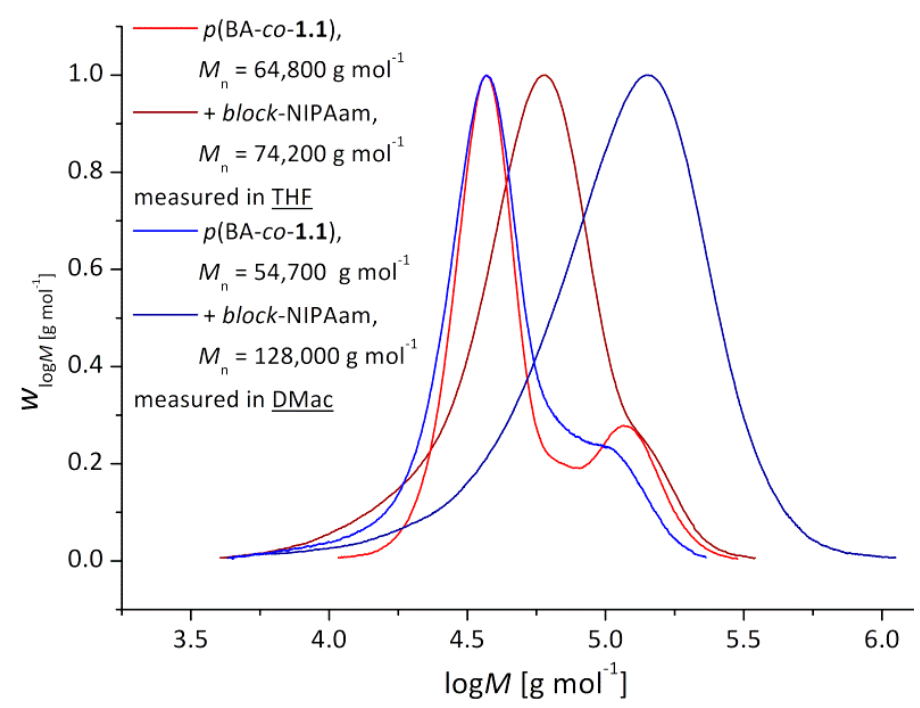

Figure 9-2: SEC-RI-data of a p(BA-co-1.1) star polymer sample and the resulting star[(BA-co-1.1)-block-NIPAam] sample of the polymerisation of $0.3 \mathrm{mmol} \cdot \mathrm{L}^{-1}$ macroRAFT with $0.7 \mathrm{mmol} \cdot \mathrm{L}^{-1} \mathrm{AIBN}$ and $1,290 \mathrm{mmol} \cdot \mathrm{L}^{-1} \mathrm{NIPAam}$ in chloroform at $60^{\circ} \mathrm{C}$ after 180 minutes, measured in THF and DMac.

In both cases the MWD of the diblock copolymer is much broader than the one of the mono-block copolymer independent of the solvent. This is a signal which is typical for a reduced control in the polymerisation process (chapter 3.4). If the SEC-trace of the diblock star polymer recorded in THF (red) is compared with the SEC-trace of the same polymer sample recorded in DMac (blue), it can be noticed that the molecular weight determined in DMac is much higher but also that the MWD is distinctively broader. The higher molecular mass is a result of the fully extended state of the $p$ NIPAam block in DMac due to the good solubility. It can be assumed that $p \mathrm{BA}$ is good soluble in DMac, too, as the chromatograms of the starting material in THF and DMac are almost similar. Therefore in DMac the whole star is well extended and the hydrodynamic radius accordingly higher. As a result a higher molecular weight is determined. pNIPAam is poorly soluble in THF. For the measurements in THF it can therefore be assumed that the pNIPAam-block coils to avoid the contact with the solvent. Due to the contraction of the inner polymer block the molecule shows accordingly a smaller hydrodynamic volume and is eluated at longer retention times which results in smaller molecular weights.

Furthermore a failure in the determination of the molecular weights is caused by the measurements in THF being carried out against the $\mathrm{MH}$ parameters of $p \mathrm{BA}$, as there are no standard parameters for this copolymer system. In DMac the measurements were carried out with a calibration with $p M M A$ standards. This resulted in a failure in determination of the $p B A$ block but it can be seen in Figure 9-2 that this effect is very small.

In both cases an increase in molecular weight can be observed which indicates that a second polymer block is formed. The investigation of the resulting polymer via ${ }^{1} \mathrm{H}$ NMR spectroscopy also 
shows the formation of a new polymer block. In Figure 9-3 the ${ }^{1} \mathrm{H}$ NMR spectrum of a $p$ BA-sample (top) was compared with a ${ }^{1} \mathrm{H}$ NMR spectrum for the diblock copolymer (bottom).

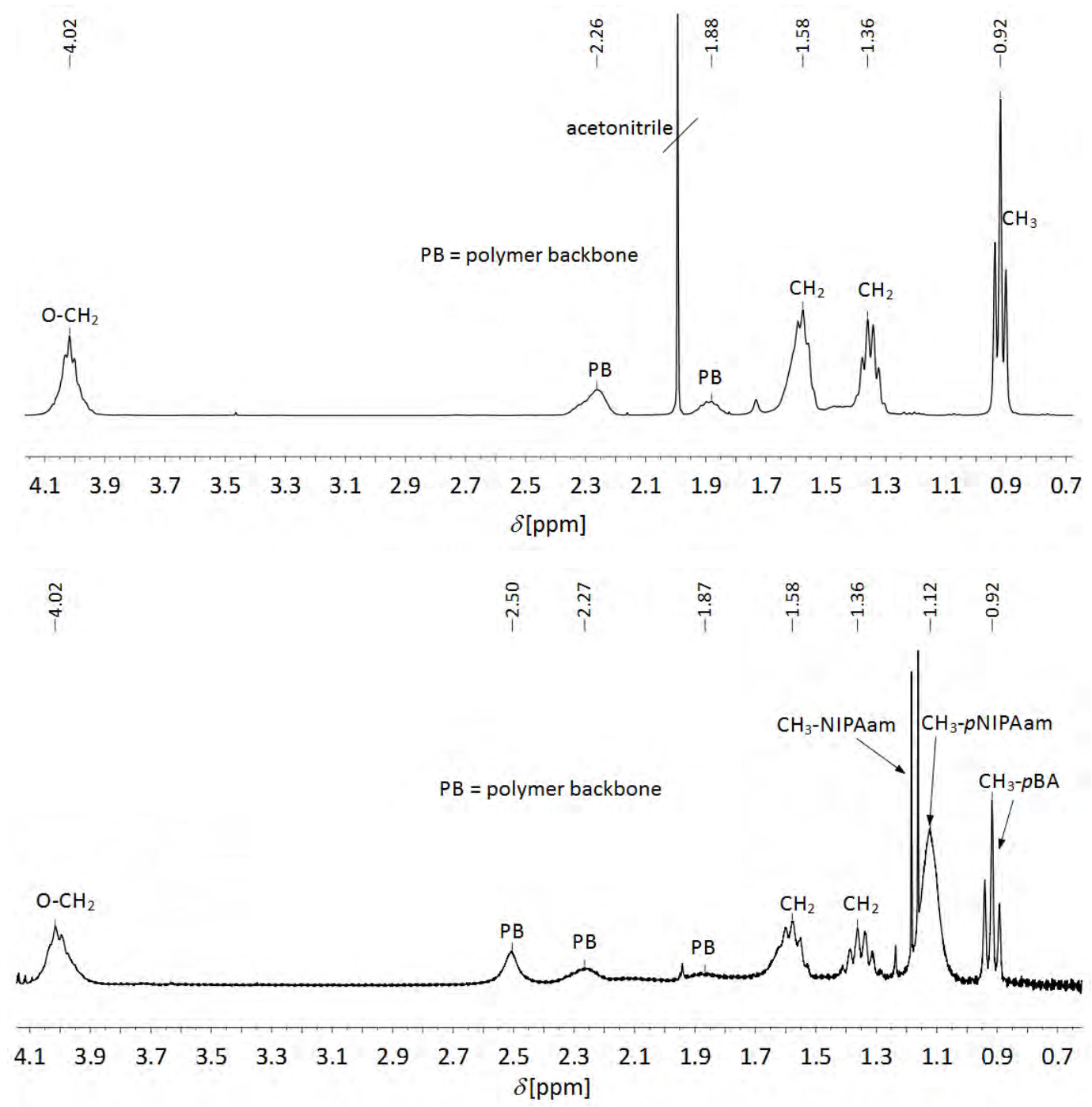

Figure 9-3: ${ }^{1} \mathrm{H} N M R$ spectra of $p(B A)$ (top) and of $p$ [star(BA ${ }_{44}-$-Co-1.1)-block-NIPAam ${ }_{56}$ ] (bottom) recorded in $\mathrm{CDCl}_{3}$.

In the down field regions, not shown here, the singlet of $\mathrm{CDCl}_{3}$ at $7.26 \mathrm{ppm}$ and a very broad signal of the proton of NIPAam's amino group at $8.00 \mathrm{ppm}$ are found. At $4.01 \mathrm{ppm}$ the signal of the $\mathrm{CH}_{2}$-group connected to the carbonyl group in the BA-units and the signal of the $\mathrm{CH}$-group connected to the nitrogen of the NIPAam-units overlap each other. The signals of the polymer backbone are found between 2.20 and $2.60 \mathrm{ppm}$. Further in the up field region the signals resulting from the ethyl group in the BA's side chain appear at 1.58 and $1.36 \mathrm{ppm}$. At $1.12 \mathrm{ppm}$ the signal of the methyl groups of $p$ NIPAam and at $0.92 \mathrm{ppm}$ the signal of the methyl group of $p \mathrm{BA}$ arise. Besides the signal of the $p$ NIPAam's methyl groups a doublet can be seen. This belongs to remains of the monomer NIPAam. It does not interfere with the integrations as the integration in this case is executed over the half-peak width. 
By integrating characteristic signals belonging to the two different monomers, as shown in the previous chapter 8 , it is possible to determine the molecular share of both compounds in the polymer ( $F_{\mathrm{x}}$ in mol\%). The signal of the amino group cannot be used for a reliable integration as it is very broad and is strongly influenced by the surroundings (solvent, water in the solvent, amino groups of other NIPAam-units) which results in shifting of the signals in up field region or change in the signal form. Here the terminal methyl groups of both monomers were used as they are clear and isolated signals which enables a definite integration over their half-peak width. The shift for the BA's methyl group is at $0.92 \mathrm{ppm}$, the shift of the NIPAam's methyl groups at $1.12 \mathrm{ppm}$.

$$
\begin{gathered}
F_{\mathrm{BA}}=\frac{\frac{I_{\mathrm{BA}}}{3 \text { protons }}}{\frac{I_{\mathrm{BA}}}{3 \text { protons }}+\frac{I_{\text {NIPAam }}}{6 \text { protons }}} \\
F_{\text {NIPAam }}=\frac{\frac{I_{\text {NIPAam }}}{6 \text { protons }}}{\frac{I_{\mathrm{BA}}}{3 \text { protons }}+\frac{I_{\text {NIPAam }}}{6 \text { protons }}}
\end{gathered}
$$

The diblock copolymer star introduced in this subchapter is a $p\left[\left(\mathrm{BA}_{44}-\mathrm{CO}-\mathbf{1 . 1}\right)\right.$-block-NIPAam $\left.{ }_{56}\right]$ star sample with an undetermined amount of $\mathbf{1 . 1}$ as the concentration of this compound in the polymer is too low to identify the signals in the ${ }^{1} \mathrm{H}$ NMR spectrum.

With the correct method to clean those polymers, e.g. by dialysis, it is possible to obtain well defined diblock copolymer stars with a hydrophobic surface. 


\subsection{Synthesis of $p$ (NIPAam-co-1.1)-block-BA Stars}

In the reverse case, a pNIPAam-macroRAFT agent mediates the polymerisation of BA to form the second block. Here the determination of the monomer conversion is no problem because monomer and solvent can be removed easily via evaporation.

Figure 9-4 shows the SEC-traces determined in DMac of a copolymerisation with $702 \mathrm{mmol} \cdot \mathrm{L}^{-1}$ BA mediated by a pNIPAam-macroRAFT agent with a molecular weight of $68,400 \mathrm{~g} \cdot \mathrm{mol}^{-1}$ (determined via DMac-SEC, corrected by 1.52 ) initiated by $1.2 \mathrm{mmol} \cdot \mathrm{L}^{-1}$ AIBN in chloroform. In red the chromatogram of the statistical $p$ (NIPAam-co-1.1) is shown, in different shades of blue the resulting diblock copolymer stars after 20,50 and 120 minutes of reaction time.

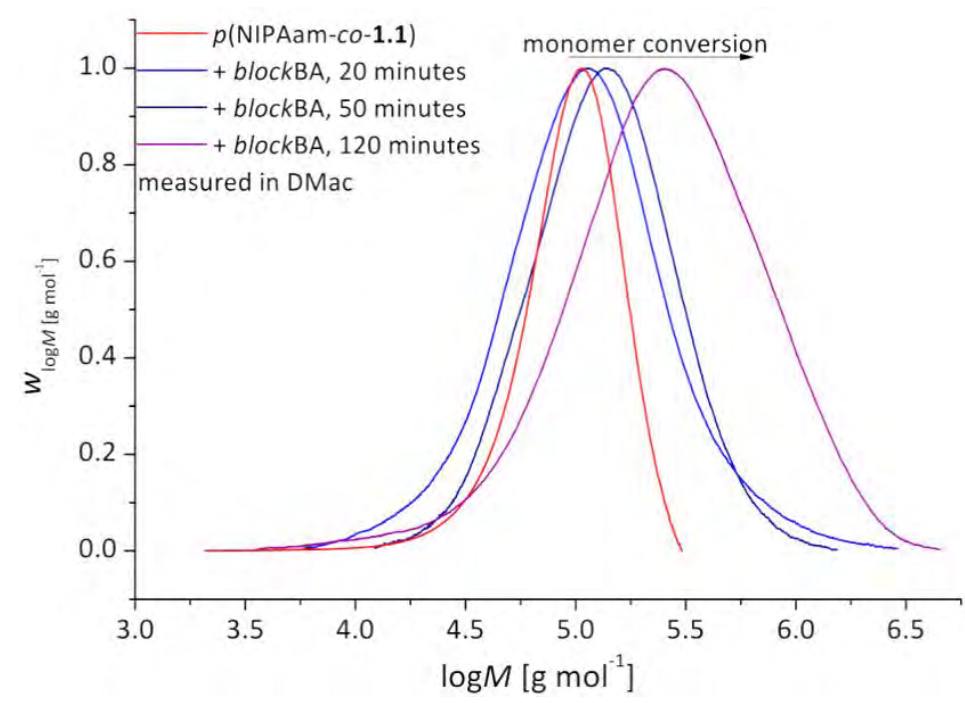

Figure 9-4: SEC-RI-traces determined in DMac of the block copolymerisation of p(NIPAam-co-1.1) $M_{n}=68,400 \mathrm{~g} \cdot \mathrm{mol}^{-1}(\mathrm{red})$ and $702 \mathrm{mmol} \cdot \mathrm{L}^{-1} \mathrm{BA}$ using $1.2 \mathrm{mmol} \cdot \mathrm{L}^{-1} \mathrm{AlBN}$ in chloroform at $60^{\circ} \mathrm{C}$ (blue).

As in the previous example of the [(BA-co-1.1)-block-NIPAam]star polymer, an increase in the molecular weights can be observed as well as a strong broadening of the MWDs compared to the monoblock copolymer star. Despite the high PDI-values, which are plotted versus the monomer conversion in the top part of Figure 9-5, a controlled behaviour of the polymerisation process can be concluded from the development of the molecular weights with the monomer conversion. Besides the data of the copolymerisation, in Figure 9-5 also the molecular weight and the PDI of the starting material are drawn at zero per cent of monomer conversion (black arrow). 


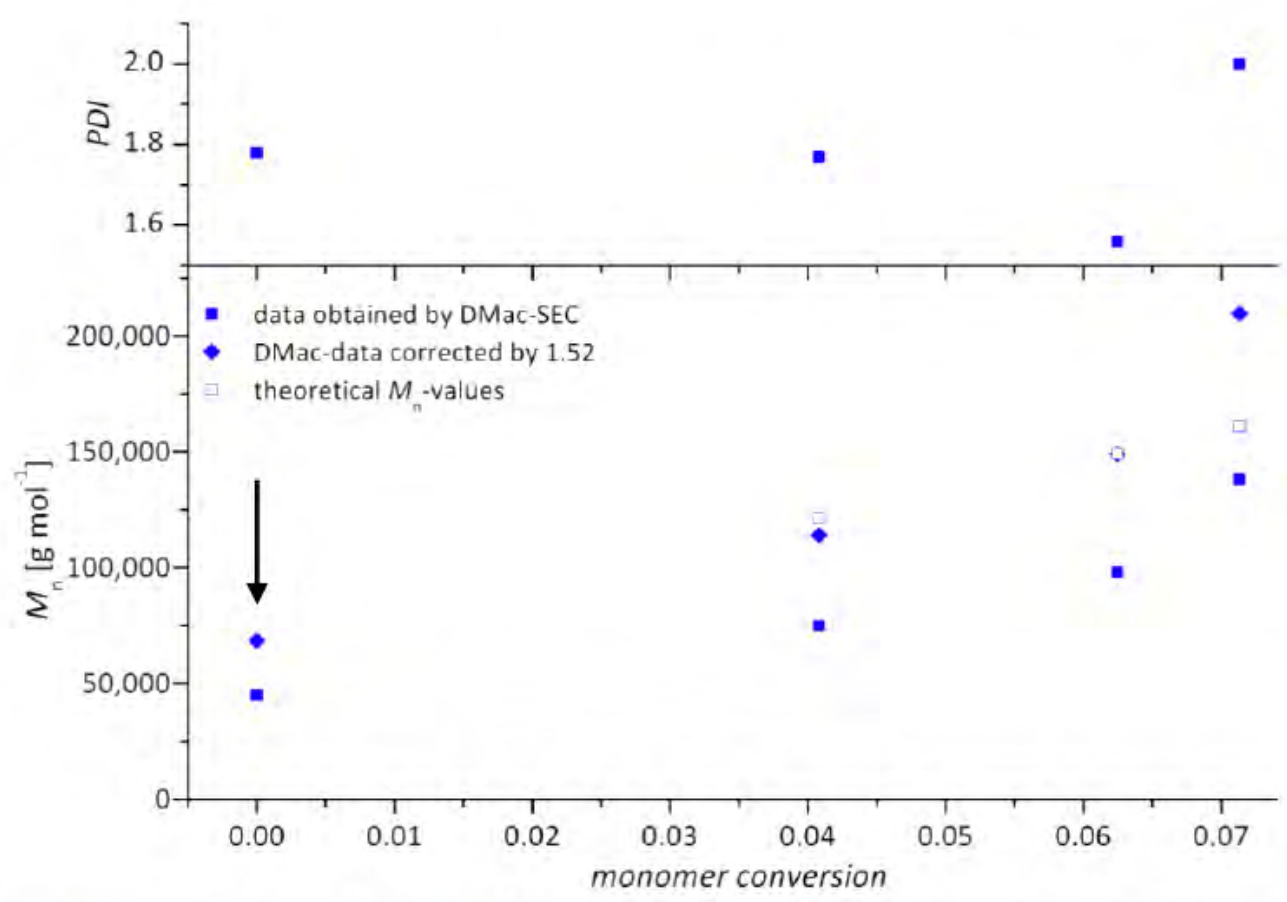

Figure 9-5: Developments of molecular weight and PDI, determined by RI-detection of a DMac-SEC, versus monomer conversion for the block copolymerisation of $p$ (NIPAam-co-1.1) $M_{n}=68,400 \mathrm{~g} \cdot \mathrm{mol}^{1}$ and $702 \mathrm{mmol} \cdot \mathrm{L}^{-1}$ BA using $1.2 \mathrm{mmol} \cdot \mathrm{L}^{-1}$ AlBN in chloroform at $60^{\circ} \mathrm{C}$.

It has to be considered that the high PDI-values are a consequence of a minimisation in the control of the polymerisation which occurs due to the different nature of $p \mathrm{BA}$ and $p$ NIPAam. As mentioned in the introduction of this chapter, it can be expected that the penetration of BA-based macro radicals towards the RAFT-units in the centre of the star polymer is difficult because of the pNIPAam surrounding. Furthermore the reaction is slowed down because of the penetration process. After 120 minutes of reaction time the monomer conversion is only at approximately $7 \%$.

By ${ }^{1} \mathrm{H}$ NMR spectroscopy the composition of the different blocks can be determined, as discussed before. For the copolymerisation, shown in Figure 9-5, following compositions could be calculated (Table 9-1). The $M_{n}$-values were determined by RI-detection on a DMac-SEC set up.

Table 9-1: Polymer compositions of p[star(NIPAam-co-1.1)-block-BA] samples resulting from the block copolymerisation of $0.69 \mathrm{mmol} \cdot \mathrm{L}^{-1} \mathrm{p}$ (NIPAam-co-1.1) $\mathrm{M}_{n}=68,400 \mathrm{~g} \cdot \mathrm{mol}^{1}$ and $702 \mathrm{mmol} \cdot \mathrm{L}^{-1} \mathrm{BA}$ using $1.2 \mathrm{mmol} \cdot \mathrm{L}^{-1} \mathrm{AlBN}$ in chloroform at $60^{\circ} \mathrm{C}$

\begin{tabular}{c|c|c|c}
$t[\mathrm{~min}]$ & $M_{\mathrm{n}}\left[\mathrm{g} \cdot \mathrm{mol}^{-1}\right]$, corrected & $F_{\mathrm{BA}}[\mathrm{mol} \%]$ & $F_{\mathrm{NIPAam}}[\mathrm{mol} \%]$ \\
\hline 20 & 114,000 & 10.1 & 89.9 \\
50 & 150,000 & 38.9 & 61.1 \\
120 & 210,000 & 45.9 & 54.1
\end{tabular}


As expected, the amount of BA in the polymer increases with longer reaction times. Via the controlled mechanism of the process a star polymer with defined blocks of both polymers can be obtained. The properties of the resulting polymer are the consequence of both polymer blocks. However, the larger block has the higher influence: In this example the first aliquot obtained was a white, amorphous polymer comparable to the starting material. With increasing BA-content the polymer was getting film-like and was changing to a transparent material. When larger BA-blocks are obtained $\left(F_{\mathrm{BA}}>70 \mathrm{~mol} \%\right)$, the polymer is a transparent, sticky and rubber-like compound.

Other experiments support the assumption of a controlled process. In Figure 9-6 the chromatograms of the two BA-polymerisations mediated by NIPAam-based macroRAFT agents are shown.
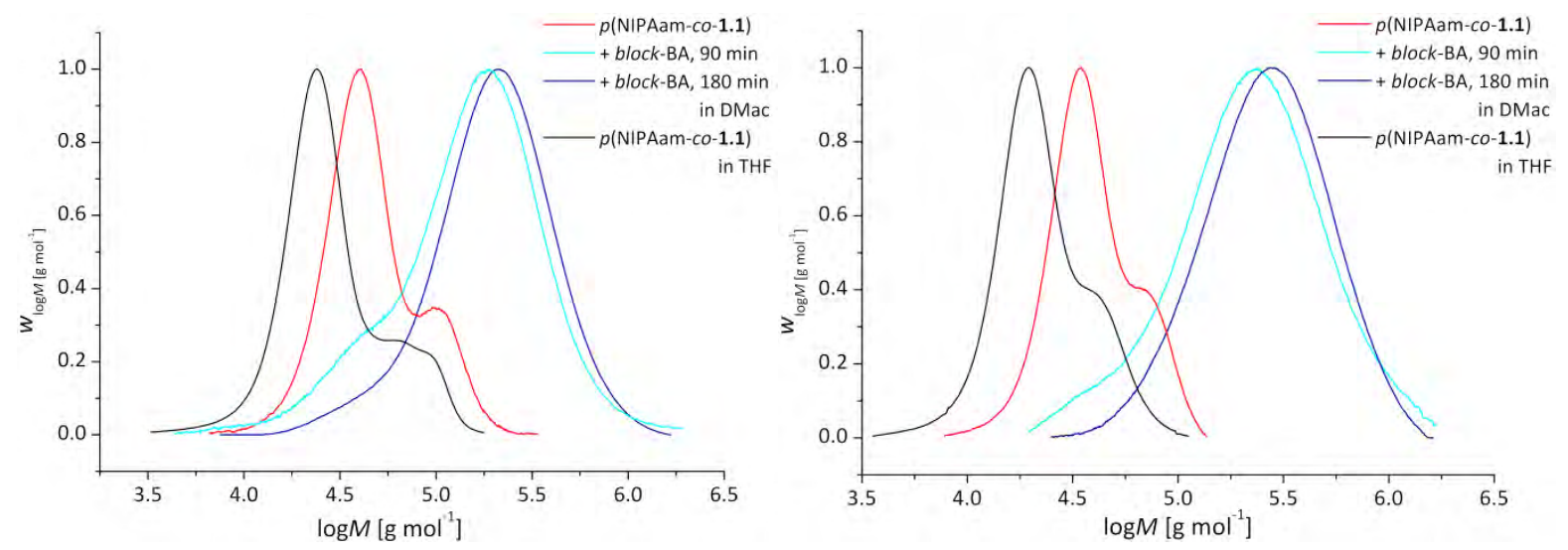

Figure 9-6: SEC-RI traces of the polymerisation of $702 \mathrm{mmol} \cdot \mathrm{L}^{-1}$ BA mediated by a (NIPAam-co-1.1) macroRAFT of $54,000 \mathrm{~g} \cdot \mathrm{mol}^{1}$ (left) and mediated by a (NIPAam-co-1.1) macroRAFT of 102,000 $\mathrm{g} \cdot \mathrm{mol}^{1}$ both with $0.3 \mathrm{mmol} \cdot \mathrm{L}^{-1} \mathrm{AIBN}$ in chloroform at $60^{\circ} \mathrm{C}$.

The chromatograms of the starting material determined in THF (black) are shown to underline the poor solvent quality of THF for pNIPAam. Distinctively smaller molecular weights than in DMac are obtained as the polymer contracts in THF solution giving smaller hydrodynamic volumes. It can be concluded that DMac is the more suitable solvent for this copolymer system even though no calibration is available. This is also supported by Figure 9-2 where the chromatograms shown of the $p$ (BA-co-1.1) starting material are similar in THF and DMac. The chromatograms recorded by UVdetection agree with the chromatograms shown in Figure 9-6. As they have a high signal-to-noise ratio, they are not shown here. This bad signal may be result of the large polymer shell shielding the UV-active RAFT-units.

The following example shows the initial period of the polymerisation. The copolymerisation was conducted with a $p$ (NIPAam-co-1.1)-macroRAFT agent of $212,000 \mathrm{~g} \cdot \mathrm{mol}^{-1}$. The chromatograms obtained in DMac by detection of the RI- and the UV-signals of this monoblock sample were plotted in Figure 9-7. 


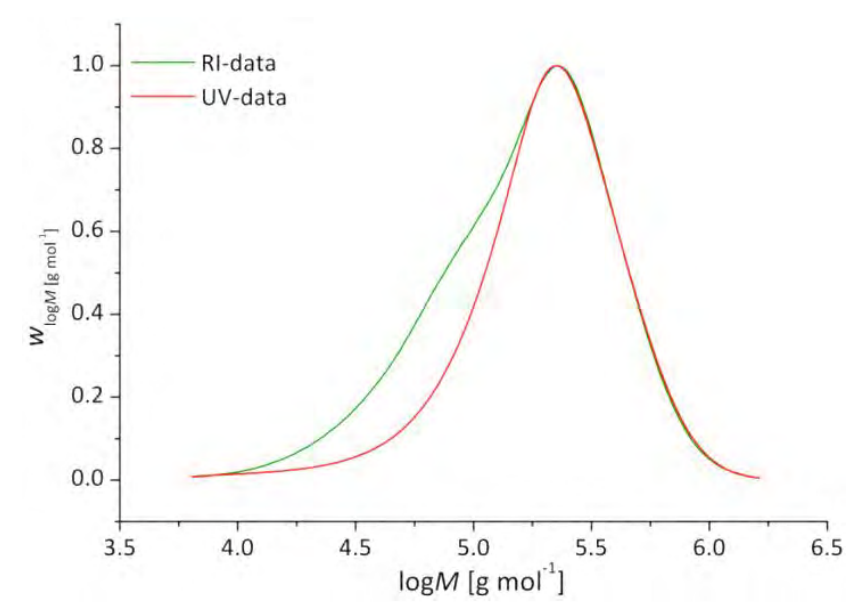

Figure 9-7: SEC-RI- and SEC-UV-data of a p(NIPAam-co-1.1) sample measured in DMac.

The plot reveals that the shoulder in the region of smaller molecular weights results from dead polymer which is generated during the polymerisation. It does not influence the polymerisation process further.

Figure 9-8 shows the RI-chromatograms obtained from measurements on the DMac-SEC set up for the polymerisation with the shown macroRAFT agent and $810 \mathrm{mmol} \cdot \mathrm{L}^{-1} \mathrm{BA}$ using $1.2 \mathrm{mmol} \cdot \mathrm{L}^{-1}$ AIBN in chloroform at $60^{\circ} \mathrm{C}$. In red the SEC-RI-trace of the macroRAFT is plotted again and in different shades of blue the chromatograms of the different aliquots.

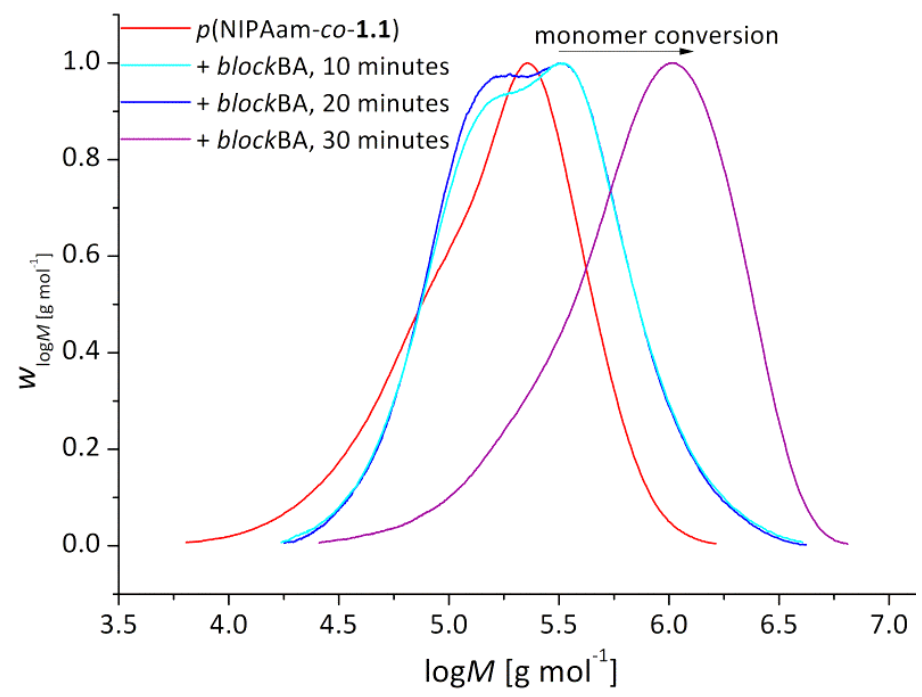

Figure 9-8: SEC-RI-traces determined in DMac of the block copolymerisation of $p\left(\right.$ NIPAam-co-1.1) $M_{n}=212,000 \mathrm{~g} \cdot \mathrm{mol}^{-1}$ and $810 \mathrm{mmol} \cdot \mathrm{L}^{-1} \mathrm{BA}$ using $1.2 \mathrm{mmol} \cdot \mathrm{L}^{-1} \mathrm{AlBN}$ in chloroform at $60^{\circ} \mathrm{C}$.

Aliquots were taken after 10, 20 and 30 minutes. It can be observed that in the initial step the individual arms of the star are re-initiated delayed, resulting in a bimodal distribution for the first two samples. The sample at those points of polymerisation consists therefore of stars which were already completely re-initiated (high molecular weight), stars which are partial re-activated and stars which 
still have to be started (low molecular weights). It has to be noticed that the majority of the stars is already in the growing state. When reaching the main-equilibrium of the RAFT-process for all control units, the bimodality disappears and the controlled polymerisation proceeds as shown in the previous example. Due to the delayed start the PDI-values are comparatively high. Also more dead polymer was generated which leads to a broadening of the MWDs resulting from Rl-detection. Confusing is the high increase in monomer conversion and molecular weight of the third sample (Figure 9-9) within 10 minutes which could be an indicate for a loss of control over the reaction. Is the adjustment of the main-equilibrium too slow, the growth of the polymer chains works freely before those are reversibly terminated by a RAFT-unit. Again at a monomer conversion of zero the data of the starting material are plotted (black arrow).

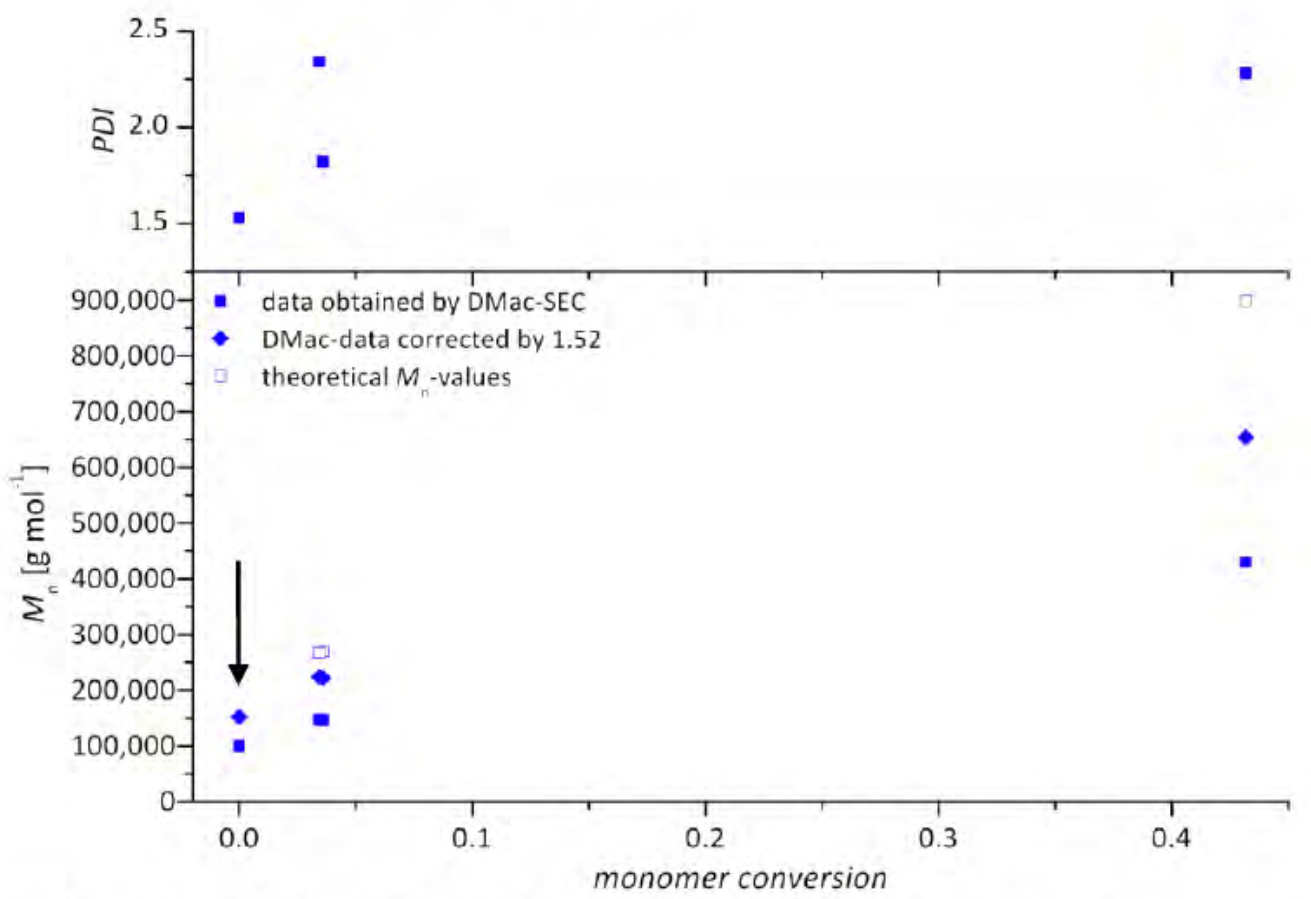

Figure 9-9: Development of molecular weight and PDI with monomer conversion for a block copolymerisation of p(NIPAam-co-1.1) $M_{n}=212,000 \mathrm{~g} \cdot \mathrm{mol}^{-1}$ and $810 \mathrm{mmol} \cdot \mathrm{L}^{-1} \mathrm{BA}$ using $1.2 \mathrm{mmol} \cdot \mathrm{L}^{-1} \mathrm{AlBN}$ in chloroform at $60^{\circ} \mathrm{C}$.

The determination of the polymer composition supports the finding of losing control over the polymerisation. Within 10 minutes the ratio of NIPAam- to BA-units is reversed (Table 9-2).

Table 9-2: Polymer compositions of p[star(NIPAam-co-1.1)-block-BA] samples resulting from the block copolymerisation of $0.65 \mathrm{mmol} \cdot \mathrm{L}^{-1} \mathrm{p}$ (NIPAam-Co-1.1) $\mathrm{M}_{n}=212,000 \mathrm{~g} \cdot \mathrm{mol}^{-1}$ and $810 \mathrm{mmol} \cdot \mathrm{L}^{-1} \mathrm{BA}$ using $1.2 \mathrm{mmol} \cdot \mathrm{L}^{-1} \mathrm{AlBN}$ in chloroform at $60^{\circ} \mathrm{C}$.

\begin{tabular}{c|c|c|c}
$t[\mathrm{~min}]$ & $M_{\mathrm{n}}\left[\mathrm{g} \cdot \mathrm{mol}^{-1}\right]$, corrected by 1.52 & $F_{\mathrm{BA}}[\mathrm{mol} \%]$ & $F_{\text {NIPAam }}[\mathrm{mol}$ ] \\
\hline 10 & 222,000 & 10.5 & 89.5 \\
20 & 223,000 & 7.9 & 92.1 \\
30 & 654,000 & 88.3 & 11.7
\end{tabular}


It seems that the control of the polymerisation depends strongly on the molecular weight of the macroRAFT agent. High molecular weight RAFT agents seem to hinder the equilibrium step and lead to FRP-like results as seen in Figure 9-10 and Table 9-3. Here a polymerisation with a macroRAFT agent of $129,000 \mathrm{~g} \cdot \mathrm{mol}^{-1}$ was carried out.

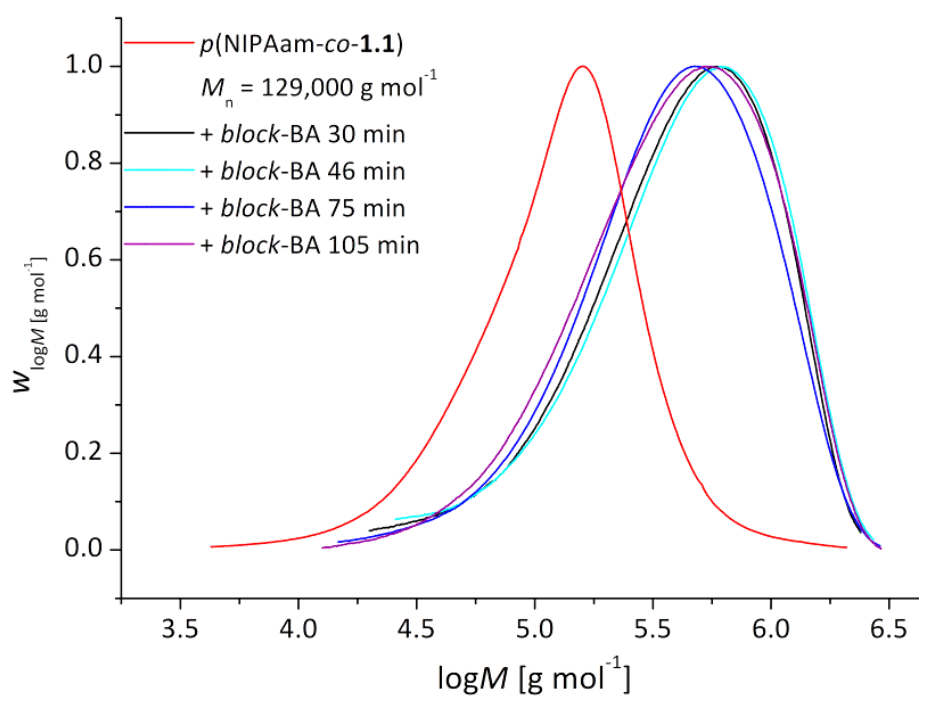

Figure 9-10: UV-chromatograms determined on a THF-SEC of a copolymerisation with $0.61 \mathrm{mmol} \cdot \mathrm{L}^{-1} \mathrm{p}($ NIPAam-co-1.1)macroRAFT agent of $129,000 \mathrm{~g} \cdot \mathrm{mol}^{-1}$ with $2.9 \mathrm{mmol} \cdot \mathrm{L}^{-1} \mathrm{AIBN}$ and $702 \mathrm{mmol} \cdot \mathrm{L}^{-1} \mathrm{BA}$ in chloroform at $60^{\circ} \mathrm{C}$.

Almost similar molecular weights as well as similar polymer compositions were obtained for all aliquots even though there a large difference in the monomer conversion (Table 9-3). The stagnating molecular weights and the PDI-values of approximately 2 are evidence for an FRP-process. Nevertheless, the growing chains have to undergo the equilibrium step as the material consists of the RAFT-units. It can be concluded that a critical size of the star polymer was reached. At this point the FRP-behaviour cannot longer compensated by the RAFT-equilibrium as penetration of the star's polymer surrounding proceeds too slowly.

Table 9-3: Polymer compositions of p[star(NIPAam-co-1.1)-block-BA] samples resulting from the block copolymerisation of $0.61 \mathrm{mmol} \cdot \mathrm{L}^{-1} \mathrm{p}$ (NIPAam-co-1.1) $\mathrm{M}_{n}=129,000 \mathrm{~g} \cdot \mathrm{mol}^{-1}$ and $702 \mathrm{mmol} \cdot \mathrm{L}^{-1} \mathrm{BA}$ using $2.9 \mathrm{mmol} \cdot \mathrm{L}^{-1}$ AlBN in chloroform at $60^{\circ} \mathrm{C}$

\begin{tabular}{c|c|c|c|c}
$t$ & $\begin{array}{c}M_{\mathrm{n}}\left[\mathrm{g} \cdot \mathrm{mol}^{-1}\right], \\
\text { [min] }\end{array}$ & $\begin{array}{c}X \\
\text { corrected by 1.52 }\end{array}$ & $\begin{array}{c}F_{\mathrm{BA}} \\
{[\mathrm{mol} \text { [\%] }}\end{array}$ & $\begin{array}{c}F_{\text {NIPAam }} \\
{[\mathrm{mol} \%]}\end{array}$ \\
\hline 30 & 365,000 & 36.6 & 83.7 & 16.3 \\
46 & 388,000 & 45.1 & 94.9 & 5.1 \\
75 & 346,000 & 60.6 & 89.3 & 10.7 \\
105 & 347,000 & 74.9 & 88.4 & 11.6
\end{tabular}


Considering all BA-polymerisations mentioned which were mediated by a pNIPAam macroRAFT agent, it can be concluded that the controlled polymerisation process works sufficiently with macroRAFT agents with a molecular weight below $100,000 \mathrm{~g} \cdot \mathrm{mol}^{-1}$. In systems with RAFT agents of higher molecular weights the polymer material around the RAFT-core is too dense and shields the core from the attack of the macro radicals which leads to a minimisation in control over the process.

It can be concluded that well defined (NIPAam-co-1.1)-block-BA and (BA-co-1.1)-blockNIPAam star polymers can be generated by use of macroRAFT agents with molecular weights below $100,000 \mathrm{~g} \cdot \mathrm{mol}^{-1}$. Furthermore it was observed that DMac is a suitable solvent to investigate these amphiphil polymer stars as BA- and NIPAam-polymers are well-dissolved. 


\section{Cross-Linking Reactions and De-Coring}

The final steps to obtain nano-scaled hollow spheres from the synthesised star polymers are the shell formation by UV-irradiation and the removal of the RAFT-core. This chapter presents the results of these two steps. For the UV-induced cross-linking step different UV-lamps were tested and the obtained material investigated by SEC-measurements. As the chromatograms obtained by UVdetection are very noisy in all cases, in this chapter only RI-traces are shown. After the removal of the core by aminolysis the final material was compared with all passed states (uncross-linked and crosslinked).

\subsection{UV-Induced Cross-Linking of Dimethymaleimido Units in Star Polymers}

The cross-linking of DMI units as applied here in the co-monomer $\mathbf{1 . 1}$ by irradiation with UVlight was investigated by Kuckling et al. for various solvents and different alkyl groups at the nitrogen atom. ${ }^{(107)}$ A summary of their findings can be found in chapter 3.6.3.1.

The cross-linking is induced by UV irradiation with wavelengths of above $300 \mathrm{~nm} .{ }^{(191)}$ In case of low DMI concentrations sensitizers can be applied to increase the reactivity of the system (chapter 3.6.3). For the investigations here three kinds of UV-lamps were available:

- A Laboratory-UV-Reactor System by Heraeus Noblelight consisting of a medium pressure mercury lamp $(E=150 \mathrm{~W})$, a quartz cooling tube, power supply and a glass reactor vessel with a PTFE circulation pump. Mercury lamps emit a characteristic Hg-line spectrum which reaches from $185 \mathrm{~nm}$ up to the visible wavelengths. The emitter used here has the highest radiation flux at 305 and $360 \mathrm{~nm}$ (manufacturer's data).

- A UV-hand lamp as used for the analysis of thin-layer chromatography by Bender Konrad with a power of $8 \mathrm{~W}$ and monochromatic radiation of $366 \mathrm{~nm}$.

- A UV-lamp system by Müller Elektronik·Optik provided with an external ignition unit and a power supply as well as a mercury short $\operatorname{arc} \operatorname{lamp}(E=100 \mathrm{~W})$ by Osram. With different filters the wavelength range can be reduced to a determined share. Here a filter (coloured glass by Schott) was applied to cut off all wavelengths below $305 \mathrm{~nm}$. Furthermore a light conductor (by Müller Elektronik·Optik) enabled well-directed irradiation of the sample.

To achieve the desired intramolecular cross-linking for the shell formation, it is necessary to have a high dilution of the polymer sample. Is the polymer concentration too high, cross-linking between stars is the consequence and multi stars would be obtained. Concentrations between $10^{-6}$ and $10^{-7} \mathrm{~mol} \cdot \mathrm{L}^{-1}$ should be sufficient. ${ }^{(160)}$ 


\subsubsection{Cross-Linking with the Laboratory-UV-Reactor System}

In the dissertation of Mänz, first preliminary experiments with the Laboratory-UV-Reactor System were carried out with a solution of the co-monomer in ethanol. ${ }^{(160)}$ Three cross-linking products could be isolated: A dimer with the desired coupling between two DMI units, a dimer coupled between a DMI unit and the vinyl double bond of the monomer and a coupling product of three monomer molecules. Here pre-experiments were also carried out to gain knowledge about the reaction times until a complete conversion of the UV-sensitive units is obtained. A solution of $\mathbf{1 . 1}$ in chloroform $\left(c_{1.1}=1.8 \mathrm{~mol} \cdot \mathrm{L}^{-1}\right)$ was irradiated up to 120 minutes. Chloroform was chosen as $p B A$ and $p$ NIPAam are soluble in chloroform and it is therefore a suitable solvent for the amphiphilic star polymers. After different periods of time, samples were taken and analysed by ${ }^{1} \mathrm{H}$ NMR spectroscopy. Figure 10-1 shows a selection of those spectra.

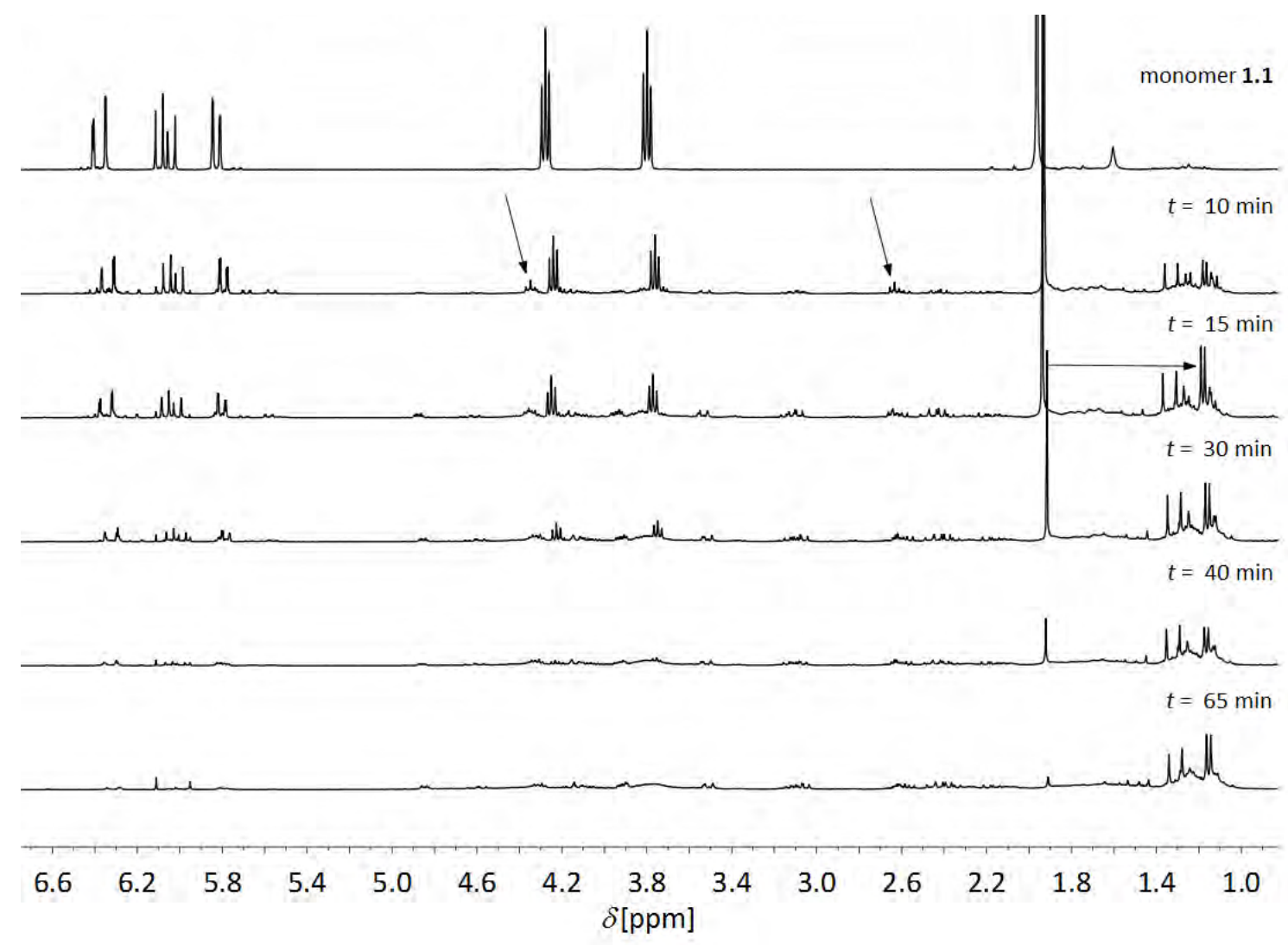

Figure 10-1: ${ }^{1} \mathrm{H}$ NMR spectra of 1.1 after different time intervals of radiation recorded in $\mathrm{CDCl}_{3}$.

The top most spectrum shows the reactant spectrum. From top to the bottom the time of radiation was increased. After ten minutes additional signals can be observed. In the up field region between 1.0 and $1.4 \mathrm{ppm}$ a multitude of peaks arose. Due to the formation of a cyclobutene ring between the DMI units the singlet of those methyl groups is shifted from $2.0 \mathrm{ppm}$ to $1.2 \mathrm{ppm}$ resulting in a doublet which can be seen more clearly in the spectrum of 15 and 30 minutes. The arrow in the 
15 minutes spectrum indicates the shift of the signal due to cross-linking. The cycloaddition of the DMI unit and vinyl double bond yields also a cyclobutane ring. The shifts of these protons are found at approximately $1.3 \mathrm{ppm}$ and at $2.6 \mathrm{ppm}$ (right arrow in the spectrum corresponding to 10 minutes of reaction time). At $4.5 \mathrm{ppm}$ a new triplet arises which is also the result of the undesired cycloaddition and corresponds with the $\mathrm{CH}_{2}$-group adjacent to the nitrogen atom (left arrow in the spectrum corresponding to 10 minutes of reaction time). Up to 30 minutes of reaction time no further changes can be observed. With longer irradiation times the decomposition of the compound begins. After 65 minutes no more change in the spectra could be observed. Over the whole process no complete consumption of the DMI-double bond could be observed which would be indicated by the disappearance of the signal at $2.0 \mathrm{ppm}$.

Experiments were carried out with different polymer samples. It is assumed that side reactions consuming $\mathbf{1 . 1}$ do not occur as the vinyl double bond of the monomer is not available. The polymer concentration has to be low to avoid undesired intermolecular cross-linking. Reactions were carried out between 25 and 120 minutes. To gain a complete conversion and therefore a complete closure of the carrier's shell, shorter reaction times would not be sufficient. In the polymer solution the local concentration of these cross-linking units is much lower than in the pure monomer solution and the groups have to gain a determined spatial proximity to undergo the cross-linking reaction.

Six-armed diblock copolymer stars as well as six-armed monoblock polymer stars were tested. Two examples are shown here. The first one (Figure 10-2), a sample of $p$ (BA-co-1.1) with a molecular weight of $336,000 \mathrm{~g} \cdot \mathrm{mol}^{-1}$ (determined in THF, corrected by 1.52) was dissolved in ethyl acetate $\left(c_{\text {polymer }}=1.79 \cdot 10^{-6} \mathrm{~mol} \cdot \mathrm{L}^{-1}\right)$. The solution was irradiated for 25 minutes resulting in a polymer material with a molecular weight of $50,000 \mathrm{~g} \cdot \mathrm{mol}^{-1}$. The corresponding chromatograms obtained with the THF-SEC are shown in Figure 10-2. The starting material was generated in presence of a six- and a mono-functional RAFT-agent causing the bimodal spectrum (black). 


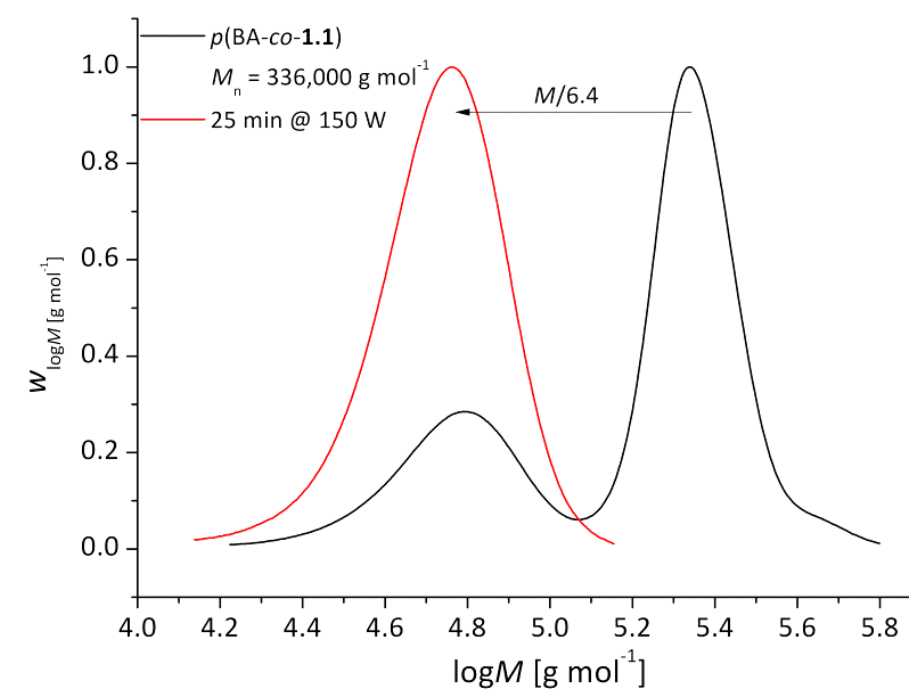

Figure 10-2: SEC-RI-data of a $\mathrm{p}(B A-\mathrm{co}-1.1)$ sample with a molecular weight of $336,000 \mathrm{~g} \cdot \mathrm{mol}^{-1}$ (black)and the resulting polymer $\left(M_{n}=50,000 \mathrm{~g} \cdot \mathrm{mol}^{-1}\right)$ after irradiation (red) for 25 min with $150 \mathrm{~W}$, determined in THF.

A strong reduction in the molecular weight was observed which indicates a reduction in the size of the macromolecule. The material obtained after irradiation (red) is approximately six times smaller than the starting material which indicates a complete destruction of the RAFT-unit in the star polymer which leads to single arms, i.e. polymer chains. This is supported by the signal of the polymer generated through the mono-functional RAFT-agent which shows the same MWD as the material after irradiation.

Similar observations were made for a diblock copolymer sample. Here the polymer of $244,000 \mathrm{~g} \cdot \mathrm{mol}^{-1}$ (determined in THF, corrected) was dissolved in chloroform $\left(c_{\text {polymer }} \approx 8.7 \cdot 10^{-7} \mathrm{~mol} \cdot \mathrm{L}^{-1}\right)$. Samples were taken after 30,60 and 120 minutes. The corresponding SECtraces are shown in Figure 10-3. 


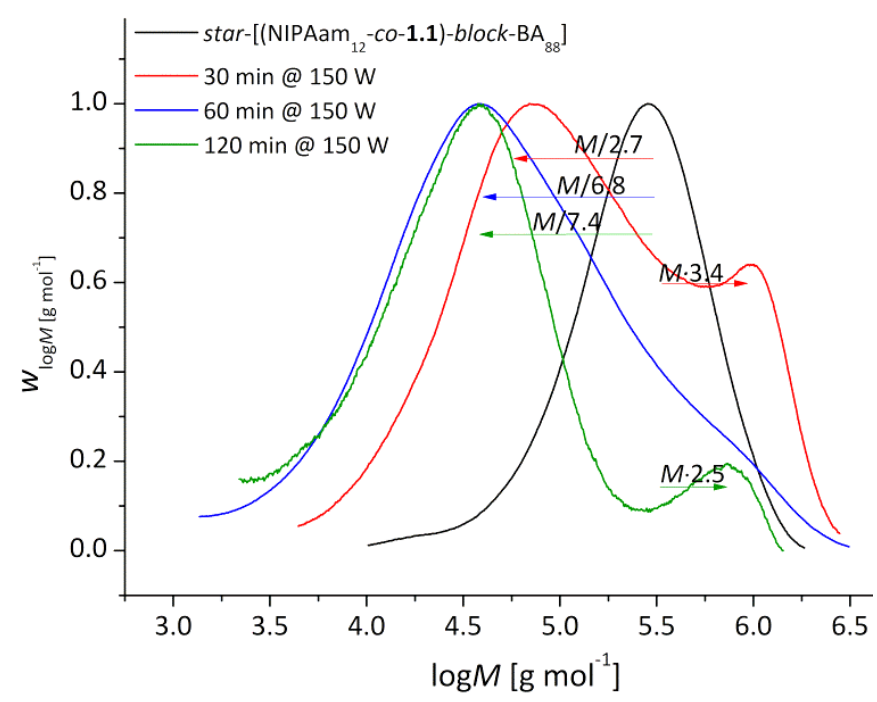

Figure 10-3: SEC-RI-data of a p[NIPAam ${ }_{12}$-co-1.1)-block-BA 88 star sample with a molecular weight of $244,000 \mathrm{~g} \cdot \mathrm{mol}^{-1}$ (black) and the resulting polymers after irradiation for different periods of time at $150 \mathrm{~W}$, determined in THF.

Again a drastic reduction in the molecular weight has to be noted. In this diagram the step-wise destruction of the original star polymer can be observed. In black the chromatogram of the starting material is shown. After 30 minutes of irradiation the chromatogram is bimodal. One part of the resulting polymer is approximately three times smaller (red). The other part indicates the formation of coupling products with higher molecular weight than a single star molecule. Here the material is a threefold larger, i.e. it can be concluded that larger aggregates than double stars were formed. it is evident that the cross-linking reaction takes place in this system. Even longer reaction times (60 minutes blue and 120 minutes green) yield polymer material that is approximately seven times smaller than the reactant, i.e. as in the previous example linear polymer was obtained. The chromatogram of the material obtained after 120 minutes of irradiation shows also a shoulder in the higher molecular weight region. The material has approximately the double molecular weight than the starting material. Double stars are formed. All measurements were carried out on the THF-SEC set up with a universal calibration and $\mathrm{MH}$ parameters of $p \mathrm{BA}$. As explained in chapter 9 , the determination of molecular weights of copolymers is not accurate resulting in approximate values. This is the reason why the molecular weight of the linear polymer obtained is not exactly a sixth of the molecular weights of the star polymer. Furthermore it can be assumed that the molecular weight corresponding with the red curve is half of the molecular weight of the whole star polymer. It is suspected that the comparatively weak ether bond in the backbone of the RAFT-agent, shown in Figure 10-4, may be split up by the UV-light first (purple arrow) resulting in polymer material with half of the original molecular weight. Afterwards the trithio units could be attacked resulting in the release of the arms of the star polymer (blue arrows). The mechanism of the cleavage of the S-Rbond by UV-light is known from UV-induced RAFT-polymerisation. ${ }^{(192)}$ Due to the low concentration 
in the mixture, the contact probability between RAFT-agent and expelled macro radical is too low for the backwards reaction. In addition the cross-linking procedure is carried out under air with not dried solvents, so that the generated radicals are terminated by other compounds, i.e. oxygen.

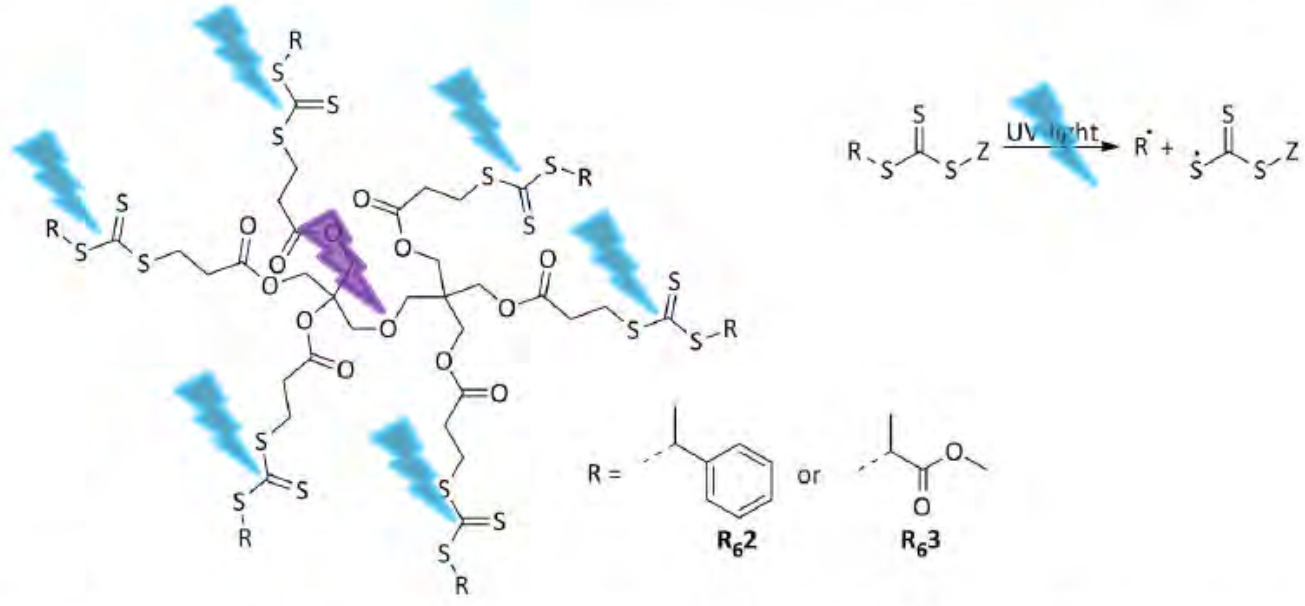

Figure 10-4: Proposed splitting of the six-armed RAFT-agents used within this project (left) and RAFT-initiation by UV-light (top right). ${ }^{(192)}$

The formation of material with higher molecular weights indicated a successful cross-linking procedure. Nevertheless the reaction does not proceed sufficiently. Either the concentration of DMI units in the solution was too low, and the reaction partners were not in spatial proximity for the cross-linking process, or the destruction of the RAFT-agent was much faster than the cross-linking reaction. In that case a formation of a nano-carrier by means of UV-irradiation would not be possible.

In general it can be assumed that the energy introduced was too high and led to the destruction of the polymer.

\subsubsection{Cross-Linking with the UV-Hand Lamp}

To avoid cleaving off the RAFT-agent core, a UV light source with less energy output was tested. The UV-hand lamp provides radiation with a wavelength of $366 \mathrm{~nm}$ at a power of $8 \mathrm{~W}$. Longer reaction times have to be planed as the process is performed by a small energy entry. Experiments were carried out with $p[($ NIPAam-co-1.1)-block-BA $]$ star polymers in chloroform $\left(c_{\text {polymer }} \approx 2.6 \cdot 10^{-6} \mathrm{~mol} \cdot \mathrm{L}^{-1}\right)$. Figure $10-5$ shows the chromatograms measured in THF of a polymer sample before (black) and after the irradiation (red) for 57 hours. The here applied $p\left[\left(\right.\right.$ NIPAam $\left._{12}-c 0-1.1\right)$-block-BA $\left.{ }_{88}\right]$ star has a $M_{\mathrm{n}}$-value of $250,000 \mathrm{~g} \cdot \mathrm{mol}^{-1}$ (determined in THF with universal calibration and $\mathrm{MH}$-coefficients of $p \mathrm{BA}$ ) where the $p$ (NIPAam-co-1.1)-block consists of 64 DMI-units per star ( $\$ 3.5 \mathrm{~mol} \%)$. 


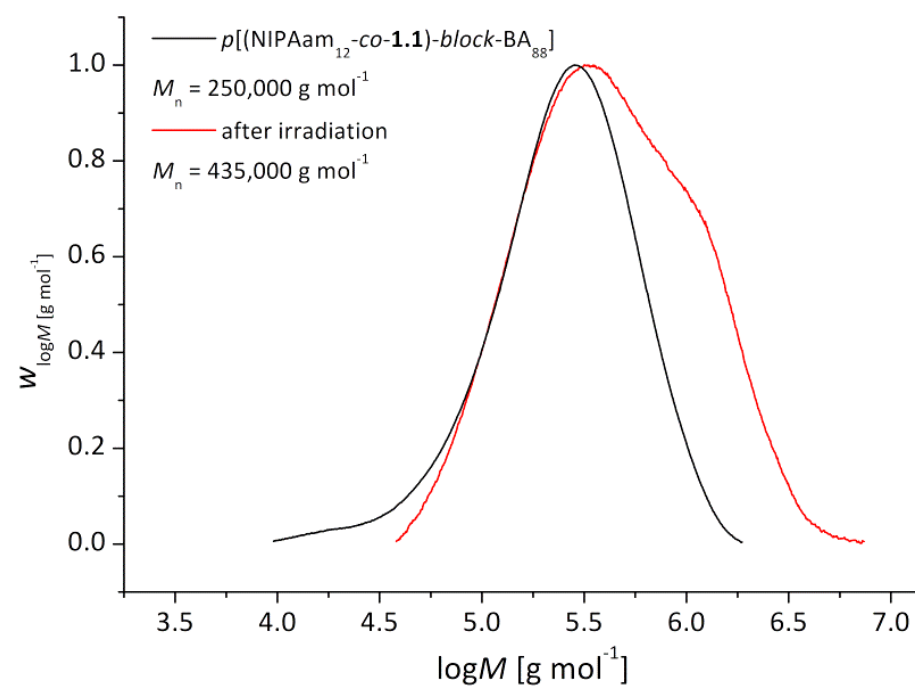

Figure 10-5: SEC-RI-traces of a p[(NIPAam ${ }_{12}$-CO-1.1)-block-BA ${ }_{88}$ ] before and after the irradiation with $366 \mathrm{~nm}$ and $8 \mathrm{~W}$ for 57 hours, measured in THF.

The spectrum of the material obtained after irradiation (red) shows a shoulder in the region of higher molecular weight, similar to those found in polymerisations where star-star coupling occurs. Therefore, it was investigated whether star-star couplings also occur in this case. The molecular weight of the material causing the shoulder is 1.7 times higher than that of the starting material. With regard to the uncertainty in the measurement, due to the calibration of the SEC set up against linear $p$ Sty standards as well as the application of BA's MH parameters, which does not correspond to this copolymer system, it can be concluded that double stars are formed during the irradiation process. That proves that the $[2+2]$-cycloaddition works sufficiently. Furthermore it can be seen that no change in weight and consequently size of the single star material occurred, which indicates that the cross-linking was successful as no change in size is expected. The chemical composition stays the same, thus, only a shell was formed around the star. The block of polymer containing the crosslinkable units is comparatively small and therefore a change in size of the macromolecule, due to compression of this block, as a consequence of the shell-formation can be assumed to be negligibly small. In addition, in order to successfully establish the carrier's shell, it was also found that 1.1-units, which were not consumed in the shell formation process, caused the star-star-coupling described before. Consequently 3.5 mol\% of $\mathbf{1 . 1}$ in the statistical copolymer block were sufficient to perform a complete closure of the carrier's shell.

It can be concluded that the [2+2]-cycloaddition between DMI moieties by application of an $8 \mathrm{~W}$-UV-hand lamp was successful. The reaction proceeds slowly but the stars remain intact throughout the entire process. By application of solvent mixtures consisting of good and poor solvents the intramolecular cross-linking could be enhanced by achieving an increase in the contact 
probability of the 1.1-units. Additionally the contact probability between star molecules should be reduced which prevents the undesired intermolecular cross-linking reaction between consumed DMI-units.

\subsubsection{Cross-Linking with the UV-Lamp}

The third applied radiation system was a UV-lamp system with a mercury lamp, an external ignition unit and a power supply $(E=100 \mathrm{~W})$. The emitted spectrum was reduced by a filter to avoid possible side reactions induced with light of shorter wavelength, e.g. cleavage of the RAFT-agent or the cross-linking product. The filter excluded wavelength below $305 \mathrm{~nm}$. A light conductor enabled directed irradiation of the sample.

Diblock copolymer stars as well as monoblock copolymer stars were tested. Solvent mixtures as mentioned in the previous section were applied. All reactions were carried out in round-bottomed flasks and with three centimetre distance between the light conductor and the surface of the reaction mixture.

$p\left[\left(\right.\right.$ NIPAam $_{90}$-co-1.1)-block-BA $\left.{ }_{10}\right]$ star with a molecular weight of $114,000 \mathrm{~g} \cdot \mathrm{mol}^{-1}$ was dissolved in acetone $\left(c_{\text {polymer }} \approx 2.6 \cdot 10^{-6} \mathrm{~mol} \cdot \mathrm{L}^{-1}\right)$ and combined with diethyl ether (acetone:diethyl ether 3:1). As the NIPAam-block in this polymer is much larger than the BA-block the properties of the NIPAam polymer block dominate, as explained in chapter 9. Therefore the solvent system is adapted to this part of the star polymer. By coiling the larger block of the polymer the reactive centres gain spatial proximity which is necessary to undergo the cross-linking reaction in a fast and intramolecular way. In this case here contraction of the BA-block would not fulfil this requirement. For $p$ NIPAam acetone is a good solvent and diethyl ether is a non-solvent, which was used to precipitate pNIPAam from the polymerisation mixture. The reaction mixture was irradiated for 25 minutes, yielding the following SEC-spectra recorded on the DMac-SEC set up (Figure 10-6). 


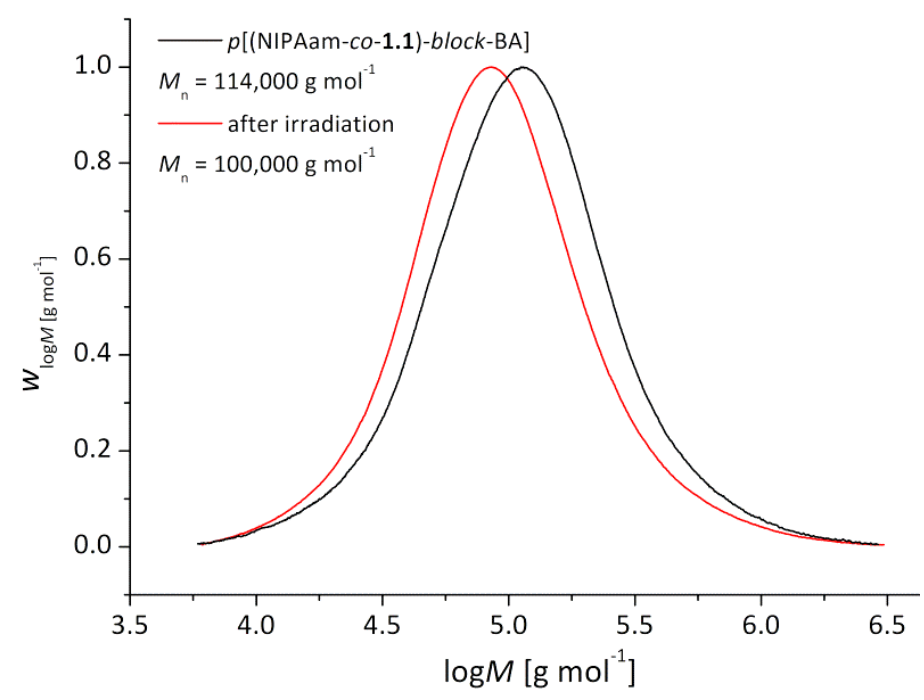

Figure 10-6: SEC-RI-traces of p[(NIPAam ${ }_{90}$-co-1.1)-block-BA $\left.A_{10}\right]$ star and the cross-linked polymer after 25 minutes, measured in DMac.

Here just a small difference in the molecular weight before (black) and after the irradiation process (red) was observed. In contrary to the example in the previous section where the shell-formation was obtained without a change in size, the block containing the co-monomer was much smaller. Furthermore the cross-linking reaction to form the carrier's shell proceeds here in the coiled polymer due to the addition of non-solvent. A reduction in size and therefore in the hydrodynamic radius is expected (depicted schematically in Figure 10-7). In contrast to the cross-linked polymer where the chains are bound around the core, in the starting material the arms can move freely and a well expended state results in larger hydrodynamic radii. It can be concluded that the shell formation was successful.

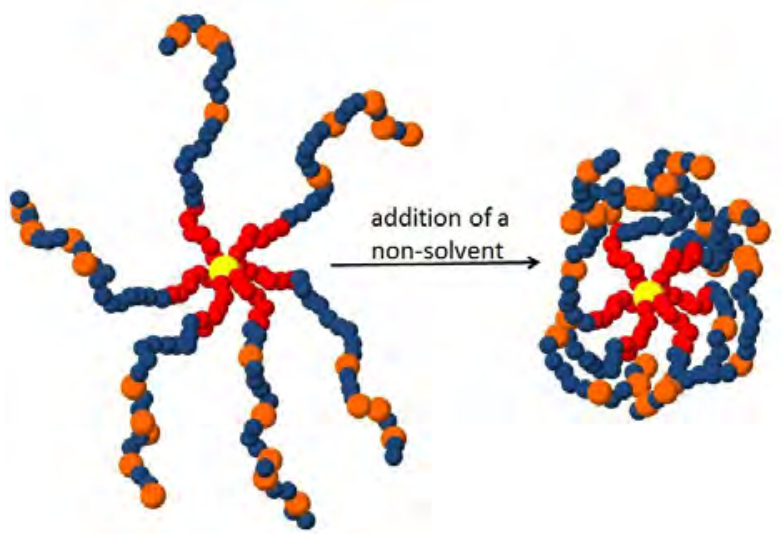

Figure 10-7: Star polymer in the fully extended state (left), coiled star polymer in a solvent/non-solvent mixture, orange spheres represent the DMI units in spatial proximity (right). 
The next examples samples shown are based on the same (NIPAam $96-{ }^{-C o-1.1} \mathbf{1}_{4}$ )-macroRAFT agent. Here the influence of benzophenone (BP) as sensitizer was investigated. Figure 10-8 presents the SEC-traces of the original $p\left(\right.$ NIPAam $_{96}-$ Co-1.1 $\left._{4}\right)$ (black), the $\operatorname{star}\left[\left(\right.\right.$ NIPAam $\left.\left._{92}-c o-1.1\right)-b l o c k-\mathrm{BA}_{8}\right]$ (red), the $\operatorname{star}\left[\left(\right.\right.$ NIPAam $_{89}-$-co-1.1)-block-BA $\left.{ }_{11}\right]$ (blue) and of the corresponding samples after application of UV-radiation for 15 minutes (dashed). In the case of the polymer sample represented by the red chromatograms BP was added.

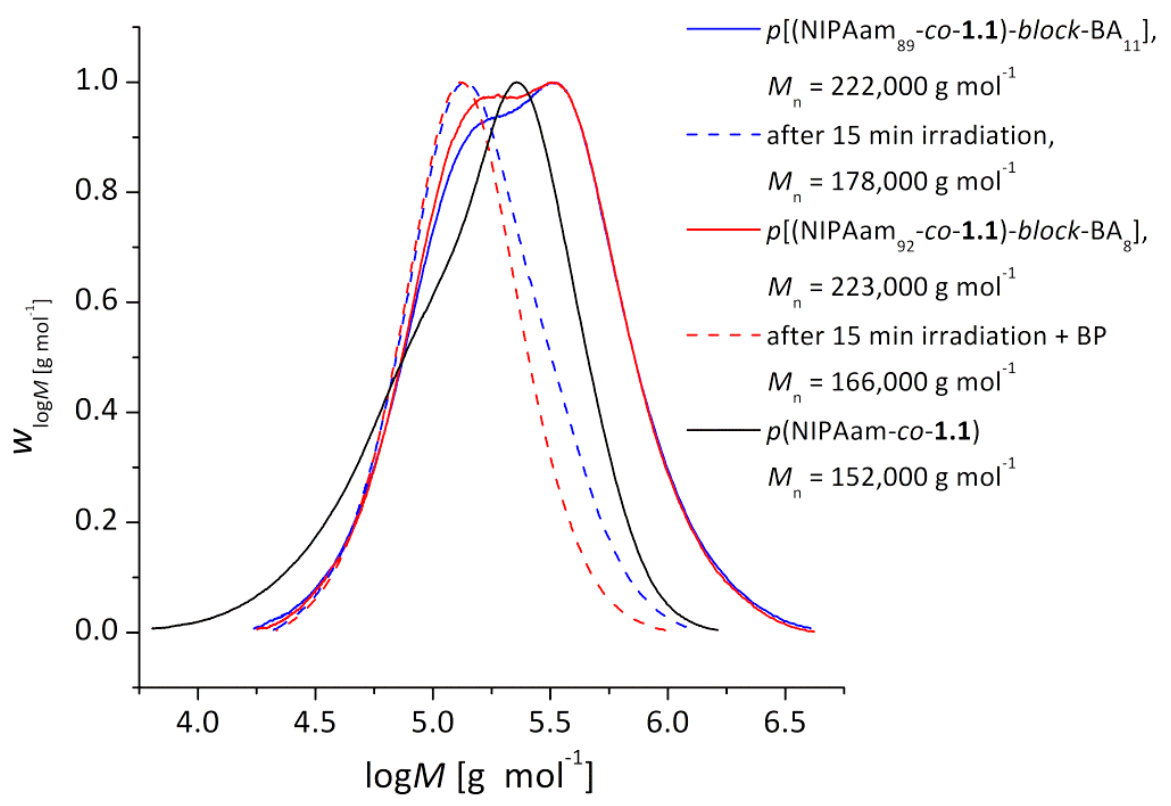

Figure 10-8: SEC-RI-traces of [(NIPAam-co-1.1)-block-BA]star polymer samples and the corresponding cross-linked polymers after 15 min irradiation, measured in DMac.

The bimodal distribution in the starting material (solid lines) results from the delay in re-initiation of several arms, as discussed in chapter 9. For these two examples, the system is composed of star molecules with different sizes and compositions due to quenching of the polymerisation at different states of the initial phase. Nevertheless all arms of the star polymer molecules consist of crosslinkable monomer units as the NIPAam-corona is equal in both cases. Therefore, the shell formation proceeds in all molecules independent of the size of the second block or the successful re-initiation of the single arms. Because of the small size of the inner block the change in size is determined by the NIPAam-block. As this block is equal in all cases as well as the composition of the solvent mixture (acetone:diethyl ether 3:1), the reduction in size is approximately the same. Even though for the sample with the higher amount of BA a slightly broader MWD was found. Here the influence of the core size can be observed: $\operatorname{star}\left[\left(\right.\right.$ NIPAam $\left._{92}-c o-1.1\right)-$ block-BA $\left._{8}\right]$ (red) consists of approximately 160 BAand 1,790 NIPAam-units, star[(NIPAam 89 -co-1.1)-block-BA ${ }_{11}$ ] (blue) is accumulated of approximately 210 BA- and 1,790 NIPAam-units, i.e. the arms of the second polymer are approximately eight BA- 
units longer than the one from the first. From this experiment an influence of the addition of BP cannot be verified.

BA-based monoblock star polymers were tested as well. A sample of $62 \mathrm{~mol} \%$ BA and $38 \mathrm{~mol} \% 1.1$ with a molecular weight of $111,000 \mathrm{~g} \cdot \mathrm{mol}^{-1}$ and a sample of $92 \mathrm{~mol} \% \mathrm{BA}$ and $8 \mathrm{~mol} \% 1.1$ with a molecular weight of $279,000 \mathrm{~g} \cdot \mathrm{mol}^{-1}$, both measured in THF and corrected by a factor of 1.52 , were irradiated for 30 minutes. BP was added to the second sample. The reactions were carried out in a THF:methanol mixture (7:3) where THF acts as solvent and methanol as non-solvent. The corresponding SEC-data of the samples before (black) and after the cross-linking step (blue) can be seen in Figure 10-9.
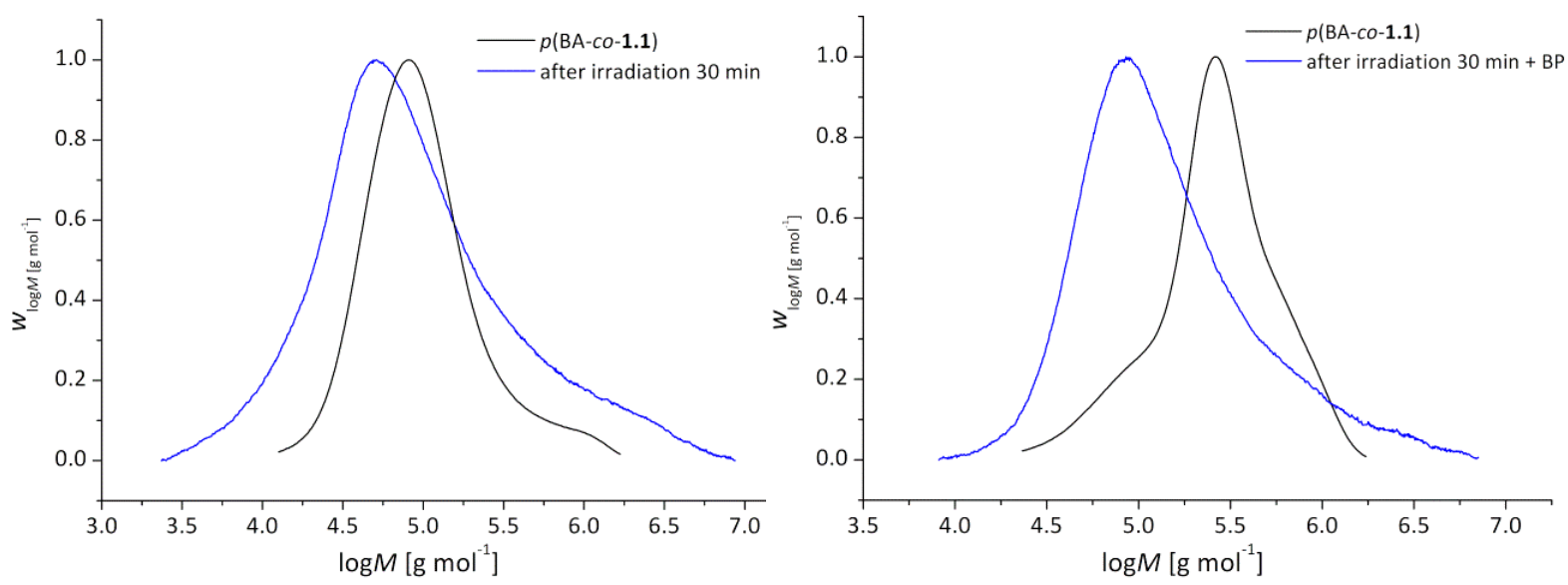

Figure 10-9: SEC-RI-traces of star $\left(B A_{62}-\right.$ Co-1.1 38$)$ and the cross-linked polymer after 30 min of irradiation (left) and of $\operatorname{star}\left(B A_{92}-C_{-1} \mathbf{1}_{8}\right)$ and the corresponding cross-linked polymer after $30 \mathrm{~min}$ of irradiation and under addition of benzophenone (right).

A reduction in molecular weight can be observed. As mentioned before, the change in size for blocks with more cross-linkable units is higher than with fewer integrated 1.1-units (Table 10-1).

Table 10-1: Molecular weights before and after the cross-linking process for different star(BA-co-1.1) samples.

\begin{tabular}{c|c|c|c} 
Sample & $\begin{array}{c}M_{\mathrm{n}} \text { before irradiation } \\
\text { corrected } \\
\text { uncorrected }\end{array}$ & $\begin{array}{c}M_{\mathrm{n}} \text { after irradiation } \\
\text { corrected } \\
\text { uncorrected }\end{array}$ & $\frac{M_{\mathrm{n}}^{\text {before }}}{M_{\mathrm{n}}^{\text {after }}}$ \\
\hline \multirow{2}{*}{$\operatorname{star}\left(\mathrm{BA}_{62}-\operatorname{co}-\mathbf{1 . 1}_{38}\right)$} & 111,000 & 65,400 & 1.69 \\
& 73,000 & 43,000 & \\
$\operatorname{star}\left(\mathrm{BA}_{92}-\operatorname{co-1.1} \mathbf{1}_{8}\right)$ & 279,000 & 179,000 & 1.56
\end{tabular}


The ratio of the average molecular weights before and after irradiation shows that the reduction in size is stronger for molecules with a higher amount of cross-linker. Due to a tight shell formed by a large amount of cross-linking units the structure cannot expand in a good solvent environment. For stars with fewer 1.1-units than the average, this leads to less rigid cross-linking due to more distance between the coiled chains. This results in a more expanded shell formation in a good solvent, e.g. DMac. The reduction in size is dependent on the length of the block containing the cross-linkable unit and the amount of DMI units. In non-solvents short and long blocks of polymer will coil to reduce the contact with the surrounding, i.e. solvent. Here the chains wrap around the star's core. The difference in the size of the macromolecule between extended and collapsed state is much higher for stars with longer arms. The amount of $\mathbf{1 . 1}$ in the polymer chains has an influence on the strength of the shell. If only a few 1.1-units cause the shell-formation the chains are wrapped loosely around the core and fixed in a few points. Therefore, the possibility of expansion in a good solvent is given. In the case of many cross-linkers in the system the chains are bound tightly around the core and the expansion of this polymer part in a good solvent is low. The influence of the solvent mixture on the resulting size of the particle and the strength of the obtained shell has to be investigated more in detail in a future project. Knowledge about the behaviour of the polymer stars in systems with different ratios of solvent to non-solvent would offer a pathway to tune the nano-carrier's for their purpose. The broad MWDs after the cross-linking procedure can be ascribed to un-cross-linked or partial cross-linked macromolecules. Due to a statistical distribution of $\mathbf{1 . 1}$ in the polymerisation mixture and a statistical integration of $\mathbf{1 . 1}$ in the polymer (chapter 8.2) it is possible that the star consist of arms with a very low amount of $\mathbf{1 . 1}$ which yields to partially cross-linked or not cross-linked stars. The application of the sensitizer seems to have no influence on the reaction.

To prove the successful cross-linking the RAFT-cores of these two samples were removed (chapter 10.2). 


\subsection{Removal of the RAFT-core by Aminolysis}

The removal of the RAFT-core was conducted by aminolysis as it was described by Qiu and Winnik. ${ }^{(193)}$ It was expected that the hollow spheres obtained after the aminolysis show approximately the same molecular weight as before as the polymer material stays the same. The RAFT-core is assumed to be negligible small compared to the polymer material surrounding it. The cross-linked samples of $\operatorname{star}\left(\mathrm{BA}_{62}-\mathrm{CO}-\mathbf{1 . 1} \mathbf{1}_{38}\right)$ and $\operatorname{star}\left(\mathrm{BA}_{92}-\mathrm{CO}-\mathbf{1 . 1}_{8}\right)$, introduced in the previous subchapter, were combined with an excess of butylamine and catalytic amounts of tris(2-carboxyethyl) phosphine hydrochloride (TCP.HCl). The resulting SEC-RI-traces are shown in the following diagrams. The chromatograms of the original (black), the cross-linked (blue) and the decored material (green) are plotted together.

For the sample of $\operatorname{star}\left(\mathrm{BA}_{62}-\mathrm{Co}-\mathbf{1 . 1}_{38}\right)$ no change in size due to the core-removal can be observed (Figure 10-10).

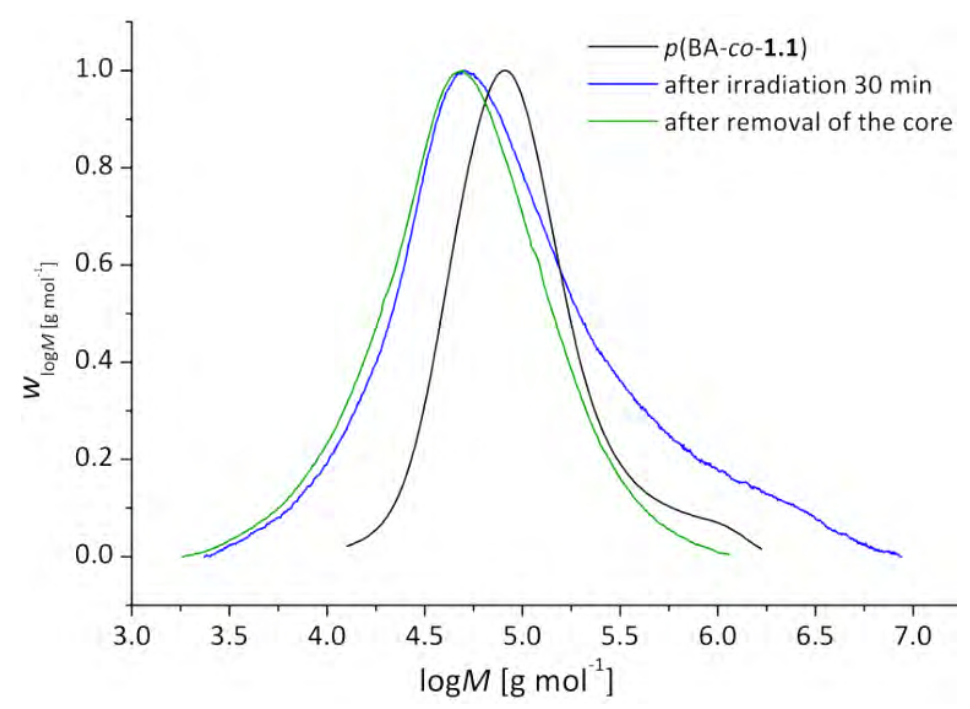

Figure 10-10: SEC-RI-traces of star(BA 62 -Co-1.1 $\left.1_{38}\right)$ before irradiation (black), after irradiation (blue) and after cleavage of the core (green).

The chromatogram of the material after the expulsion of the RAFT-core is identical with the one after the cross-linking apart from the broad shoulder in the region of high molecular weight. It was assumed before that this material of higher molecular weight corresponds to star polymers which are not yet cross-linked. Through aminolysis the RAFT-units decomposed. Arms which were not integrated in the shell during the process of cross-linking were expelled as single chains of smaller molecular weight. Prove of a successful de-coring could be gained by the SEC-UV-traces. In case of complete de-coring no UV-signal could be observed at similar molecular weights. Figure 10-11 shows the chromatograms resulting from RI- and UV-detection for the sample before (left) and after the aminolysis (right). 

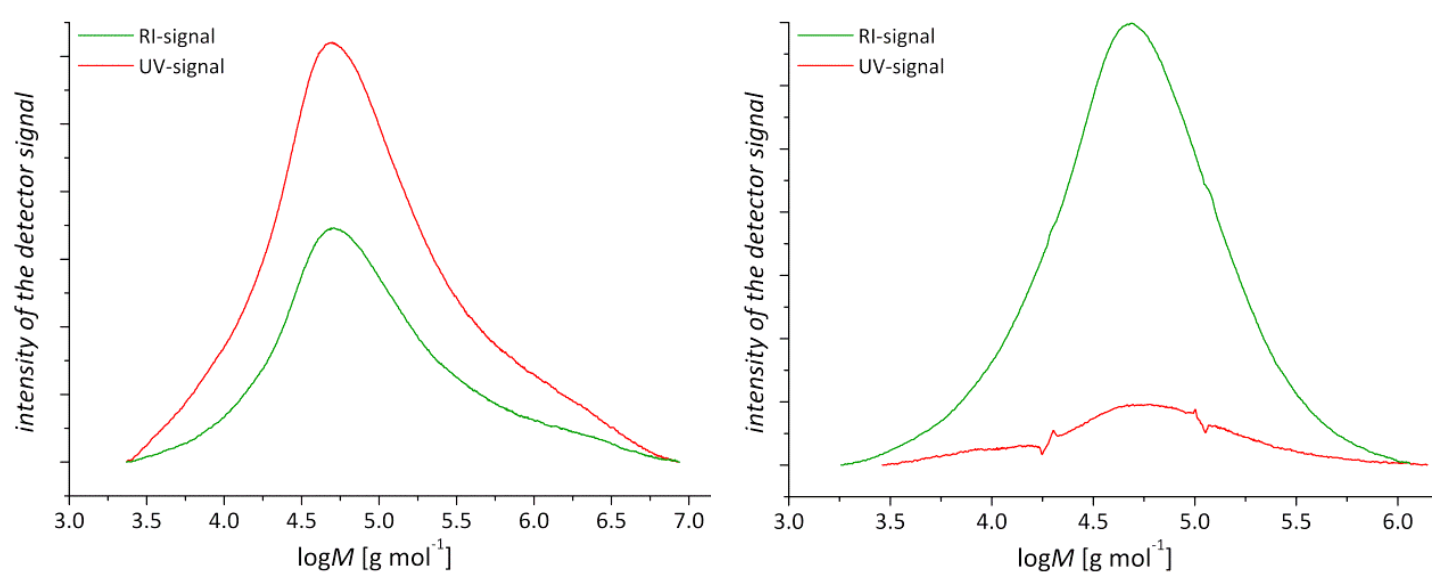

Figure 10-11: Left: RI- and UV-chromatograms after the cross-linking. Right: RI- and UV-chromatograms after the de-coring.

UV-absorbance at low intensity can still be observed, indicating that not all RAFT-units were decomposed. Nevertheless the comparison reveals that most of the units were decomposed and the size of the polymer material stays the same, i.e. nano-spheres without a RAFT-core were obtained.

In the case of $\operatorname{star}\left(\mathrm{BA}_{92}-\mathrm{CO}-\mathbf{1 . 1}_{8}\right)$ the difference between cross-linked material and de-cored material is more pronounced (Figure 10-12, left). The right part of the figure shows the comparison of the UV- and RI-chromatograms. It can be seen that the intensity of the UV-signal is very low, i.e. almost no RAFT-units remained after the aminolysis.
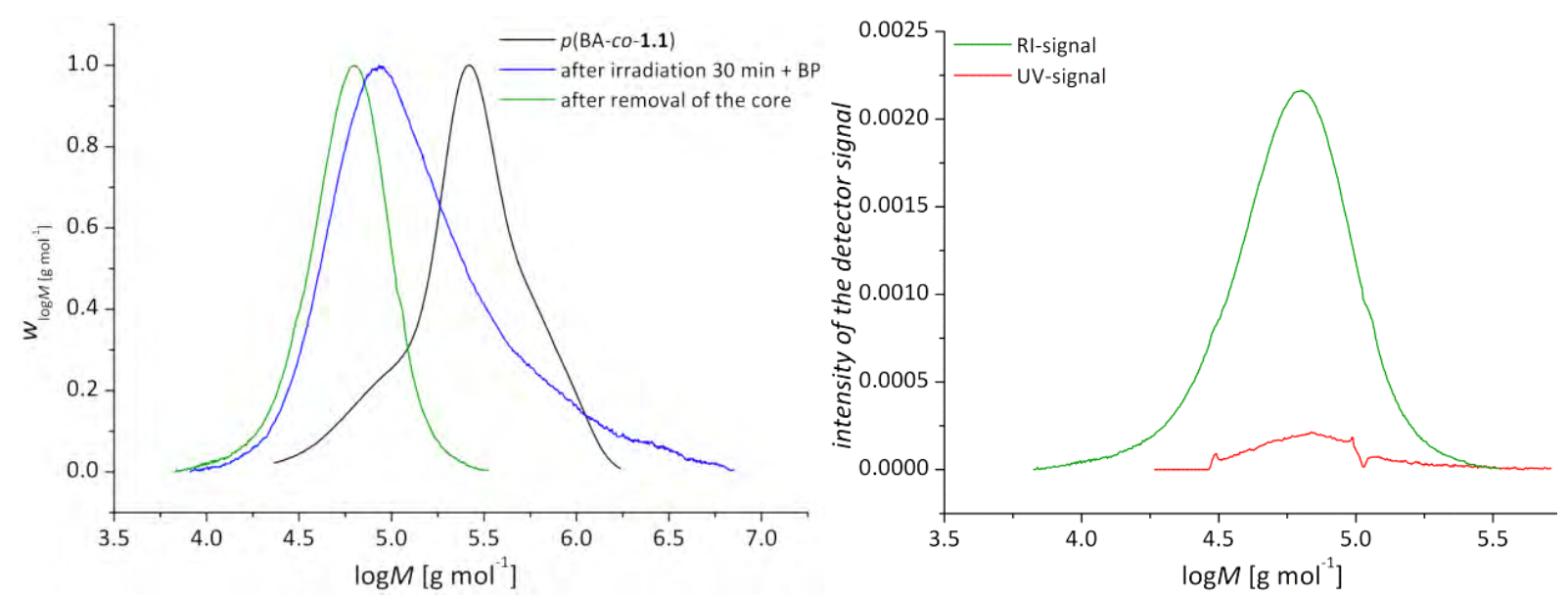

Figure 10-12: Left: SEC-RI-traces of star $\left(B A_{92}-C_{0}-1_{1} 1_{8}\right)$ before irradiation (black), after irradiation (blue) and after cleavage of the core (green). Right: Comparison of the RI- and UV-signal after the de-coring process.

The main difference between the first sample and this sample is the amount of cross-linking units. In the first example the polymer consisted of approximately 77 BA-units and 30 1.1-units per arm which could participate in the cross-linking step. It can be assumed that most arms were integrated in the shell and a comparatively rigid shell was formed. In contrast, the second sample consisted also of approximately 32 1.1-units but 339 BA-units per arm. The closed shell is not as strongly bound 
together as in the first example. The UV-reactive centres are therefore widely distributed within the polymer resulting in arms that are more loosely wrapped around the core. A reduction in the molecular size can consequently be the result of breaking the stabilising backbone, i.e. the RAFTcore. The soft shell shrinks due to the missing RAFT-template. Furthermore, the probability of arms without any 1.1 molecule is much higher. Therefore, partial cross-linked stars are formed and in the disbanding of the RAFT-core these single arms are cleaved off.

No other methods to confirm these findings were compiled as the sample volumes were much too small, e.g. to measure ${ }^{13} \mathrm{C}$ NMR spectra to follow the cross-linking process by shifting of the methyl signals of the DMI moiety.

It can be summarised that the closure of the carrier's shell can be conducted for all kinds of star copolymers within this project in mixtures of solvent and non-solvent for the block of higher molecular weight with UV-lamps with 8 or $100 \mathrm{~W}$ and wavelengths above $300 \mathrm{~nm}$. Depending on the energy input the reaction time has to be adjusted. In case of equal sized blocks, shrinking of the inner block would be more prudent. A high dilution has to be applied to avoid intermolecular coupling.

Besides successful shell formation, the removal of the RAFT-core by aminolysis could be shown which also provided the pathway to eliminate not cross-linked stars and arms. 


\section{Closing Remarks}

This work shows the establishment of nano-sized hollow sphered particles with an amphiphilic corecorona system synthesised in a five-step-procedure. By means of the controlled radical polymerisation process of RAFT, star polymers consisting of six amphiphilic diblock copolymer arms were synthesised. The outer polymer block was composed of a basic monomer and a monomer providing a cross-linkable unit to enable the shell formation via UV-irradiation.

Under discussion were monomers based on $\mathrm{N}$-alkyl-3,4-dimethylmaleimid (DMI), cinnamic acid (CA) and courmarins, which undergo [2+2]-cycloaddition under UV-irradiation, in RAFT copolymerisation with $n$-butylacrylate (BA), $N$-isopropylacrylamide (NIPAam), methylmethacrylate (MMA) and methylacrylate (MA). It was found that the copolymerisations of $N$-ethylacrylate-3,4dimethylmaleimide (1.1) with BA or NIPAam proceed in a controlled manner under RAFT-conditions yielding narrow distributed MWDs. Star polymers with molecular weights from 49,000 up to $405,000 \mathrm{~g} \cdot \mathrm{mol}^{-1}$ could be obtained. Nevertheless the addition of 1.1 evokes an induction period in the polymerisation process leading to smaller monomer conversions and smaller molecular weights than the corresponding homopolymerisation of the basic monomer. Furthermore a reinforcement of starstar coupling by back-biting in BA-polymerisations with 1.1 was detected. With increasing comonomer concentration an increase in the effect of induction and of reinforced star-star coupling had to be noted. In NIPAam polymerisations a loss of monomer units due to self-initiation and therefore increased irreversible termination was observed, here especially in the homopolymerisation.

The compositions of the copolymers and the copolymerisation parameters of these systems were determined by NMR-analysis. To do so, the co-monomer $\mathbf{1 . 1}$ had to be fully classified by different NMR methods (HMBC, COSY and ${ }^{1} \mathrm{H}$ NMR). Star polymers with 31 up to 910 1.1-units per star molecule were synthesised. From the NMR-analysis in accordance with the copolymerisation parameters (BA-Co-1.1 $r_{\mathrm{BA}}=0.95, r_{1.1}=2.24$; NIPAam-co-1.1 $r_{\mathrm{NIPAam}}=0.05, r_{1.1}=0.96$ ) a favoured integration of 1.1 over the polymerisation process was detected resulting in gradient polymers, i.e. the co-monomer units are located at the tips of the star's arms due to the Z-group approach. The properties of the polymer depend strongly on the amount of integrated co-monomer. In the case of $p B A$ the material becomes hard and brittle. Via AFM-investigations the existence of six-armed star polymers could be proven. Here the different nature of the hydrophobic $p B A-b a s e d$ and hydrophilic $p$ NIPAam-based stars became clear as $p$ BA-stars were repelled by the hydrophilic surface of the substrate mica.

By application of those gradient copolymer stars as macroRAFT-agents a second polymer block of opposite polarity was synthesised onto the first. As the Z-group approach was used the new 
polymer block realises the core part of the nano-carrier where the compounds to deliver are stored. With this polymerisation procedure star polymers with hydrophilic core and hydrophobic corona as well as the reversed case basing on BA- and NIPAam-polymers were established. As it the case of the gradient copolymer, the polymer compositions were determined by NMR-analysis. Various copolymers could be obtained; their properties strongly depend on the size of the individual blocks. It was found that the control over the polymerisation is minimised by larger polymer blocks $\left(M_{\mathrm{n}}>100,000 \mathrm{~g} \cdot \mathrm{mol}^{-1}\right)$ in the macroRAFT agent as the penetration of the growing radicals towards the trithio units is impeded. Besides the size of the block, the opposite polarity of growing macro radical and polymer surrounding has an influence on this topic. It has to be further investigated whether there is a critical block length of the macroRAFT's arms to find the optimum balance between this block size and control over the polymerisation. Furthermore other solvents or different solvent mixtures could be applied to increase the penetration towards the RAFT-units. As described in chapter 6 , there is the possibility to used non-solvents under theta-conditions. For block copolymers this would open to achieve optimal solvent conditions for both kinds of polymer during the polymerisation. Polymerisations of Sty in a good solvent (toluene) and a poor solvent (cyclohexane) showed that the control of the polymerisation is improved in the non-solvent. It was assumed that a spatial concentration of polymer and monomer occurs to reduce the contact probability with the unflavoured solvent resulting in short pathways of the reactive centres.

UV-irradiation with wavelengths over $300 \mathrm{~nm}$ was applied in different energies and over various periods of time to achieve the formation of the carrier's shell. Via SEC-measurements the success of the procedure was monitored. It was found that 3.5 mol\% cross-linkable units in the outer block of the star polymer are sufficient to perform the shell closure at energy entries of $100 \mathrm{~W}$ over maximum 30 minutes or $8 \mathrm{~W}$ over 57 hours. The density and strength of the formed shell is depended on the size of the polymer block, i.e. the distribution of cross-linkable units over the star. Long blocks with a small co-monomer share yield carriers with a loose bound shell whereas short blocks gain a tight corona. The difference can be observed by SEC-measurements. Further investigations of the dependence of block length and co-monomer content and distribution are a task for future work.

The de-coring process to yield the prospected nano-carrier was performed by aminolysis. With the comparison of the UV-detection during the SEC-analysis over the whole process the success of the process could be proven as the trithio units, the UV-active part of the polymer at $310 \mathrm{~nm}$, were decomposed during the aminolysis. 
Besides the optimisation of the synthesis procedure, especially regarding the control over the polymerisation in the diblock copolymer synthesis, future investigations have to be done in size determination of the entire carrier regarding different solvent conditions, the sizes of blocks and the strength of the shell due to the 1.1 basic monomer ratio. Also the change of the polymer's properties by integration of co-monomer has to be clarified to yield a polymer with desired properties for the task at hand.

Furthermore the loading mechanism has to be investigated so that the carrier's load capacity and the load-carrying-and-release capacity for the different applications can be determined. Investigations by AFM and Dynamic Light Scattering (DLS) measurements would be favourable. Moreover tests have to be carried out verify the biocompatibility in fields of application for medical purposes. The modification of the carrier's surface to achieve better biocompatibility, e.g. with antibodies, has to be considered. For chemical application, as e.g. catalyst storage, the chemical stability of the carrier under various reaction conditions has to be tested. 


\section{Experimental Part}

\subsection{Chemicals and Equipment}

In this chapter, the experimental procedures and the instrumentation along with the preparation and synthesis of monomers, RAFT-agents and initiators are described.

\subsubsection{Chemicals}

\section{Chemicals for Synthesis}

All chemicals were purchased at least in pro analysi quality with a degree of purity of at least $95 \%$ (from Fluka, ABCR, Sigma-Aldrich, Acros Organics or Fisher Chemicals) and used as received. For purification of crude products via column chromatography silica gel (particle size 0.06-0.2 mm, pore size $60 \AA$ A) was purchased from Sigma-Aldrich.

\section{Monomers}

Before use styrene (Sty; Aldrich, $\geq 99 \%$, stabilised with 4-tert-butylcatechol), $n$-butylacrylate (BA; Aldrich, $\geq 99 \%$, stabilised with hydrochinone monomethyl ether), methylmethacrylate (MMA; Aldrich, $\geq 99 \%$, stabilised with hydrochinone monomethyl ether), and methylacrylate (MA; Aldrich, $\geq 99 \%$, stabilised with hydrochinone monomethyl ether) were passed through a column of neutral aluminium oxide (Aldrich, Brockmann activity I) to remove the inhibitor. $\mathrm{N}$-isopropylacrylamide (NIPAam; Acros Organics, $99 \%$, stabilised) was re-crystallised from $n$-hexane. All monomers were stored at $-18^{\circ} \mathrm{C}$.

\section{Initiators}

Azo-bis-(isobutyronitrile) (AIBN; AkzoNobel, $98 \%$ ) was re-crystallised from methanol. Dibenzoylperoxide (DBPO; AkzoNobel, $98 \%$ ) was precipitated with methanol from chloroform. The so prepared initiators were stored at $-18{ }^{\circ} \mathrm{C}$ before usage. Di(2-ethylhexyl)peroxydicarbonate (DEPC; AkzoNobel, $97 \%$ ) was used as received.

\section{Solvents}

Solvents used in synthesis and solution polymerisations were purchased from different suppliers at least in pro analysi quality and were used as received. Solvents used for preparative column chromatography were bought in minor quality and distilled before use. NMR-solvents were purchased from Deutero or Aldrich (99.8 atom\% D) and used as received. 


\subsubsection{Equipment}

\section{Size Exclusion Chromatography (SEC)}

The molecular weight distributions of Sty-, BA-, MMA- and MA-polymers as well as of the homopolymers of $\mathbf{2 . 1}$ and $\mathbf{2 . 2}$ were determined with a SEC Analysis Systems 1260 Infinity by PSS Agilent. The system consisted of a PSS Agilent Technologies 1260 Iso Pump (HPLC-pump), an Agilent 1260 ALS injector, a pre-column (PSS SDV, $8 \times 50 \mathrm{~mm}$, particle size: $5 \mu \mathrm{m}$ ), three separation columns (PSS SDV, $8 \times 300 \mathrm{~mm}$, particle size: $5 \mu \mathrm{m}$, pore size: $10^{5}, 10^{3}$ and $10^{2} \AA$ A $)$ and the detectors. In this work, the UV-detector PSS Agilent Technologies 1260 VWDVL at a wavelength of $310 \mathrm{~nm}^{(194)}$ and the RI-detector PSS Agilent Technologies 1260 RID were used in parallel. As eluent tetrahydrofuran (THF; HPLC-grade from Sigma-Aldrich) was used with toluene (> $99.7 \%$, dry, from Sigma-Aldrich) as internal standard (flow velocity $1.0 \mathrm{~mL} \cdot \mathrm{min}^{-1}$ at $35^{\circ} \mathrm{C}$ ). The system was calibrated with polystyrene standards of low polydispersity from PSS. The Mark-Houwink-coefficients used to gain access to the molecular masses according to the principles of universal calibration are listed in Table 12-1.

The molecular weight distributions of pNIPAam samples were determined with a SEC Analysis Systems 1260 Infinity by PSS Agilent. The system had the same set up as the THF-SEC mentioned above. As eluent $\mathrm{N}, \mathrm{N}$-dimethylacetamide (DMac; HPLC-grade from Sigma-Aldrich) with $0.1 \mathrm{w} \% \mathrm{LiBr}$ was used (flow velocity $0.8 \mathrm{~mL} \cdot \mathrm{min}^{-1}$ at $30^{\circ} \mathrm{C}$ ). Again toluene was used as internal standard. The system was calibrated with pMMA standards from PSS. McKee et al. proved by using NMR-analysis parallel to the SEC measurements that $p$ NIPAam is chemical equally to $p M M A$ in solution. ${ }^{(186)}$

Table 12-1: Mark-Houwink-coefficients

\begin{tabular}{c|c|c} 
monomer & $K\left[\mathrm{~cm}^{3} \cdot \mathrm{g}^{-1}\right]$ & $a$ \\
\hline$n \mathrm{BA}^{(182)}$ & 0.0122 & 0.700 \\
$\mathrm{MMA}^{(195)}$ & 0.01298 & 0.688 \\
$\mathrm{MA}^{(196)}$ & 0.0168 & 0.696
\end{tabular}

For the evalution of the taken data the program WinGPC 6.20 by PSS was used. All polymer standards to calibrate the systems were purchased from Polymer Standard Service GmbH (PSS).

\section{UV-Vis Spectrometry}

UV-Vis spectra were measured on the spectrophotometer Cary 300 in a wavelength range of $190-800 \mathrm{~nm}$ using QuarzSuprasil cuvettes (110-QS, $d=10 \mathrm{~mm}$ ) from Hellma. Dichlormethane, acetonitrile, methanol or water were used as solvents, as noted. 


\section{Nuclear Magnetic Resonance Spectrometry}

Nuclear magnetic resonance (NMR) spectra were recorded at room temperature on a Varian Unity $300\left(300 \mathrm{MHz} ;{ }^{1} \mathrm{H},{ }^{13} \mathrm{C}\right)$ or a Bruker Avance III (300 MHz; $\left.{ }^{1} \mathrm{H},{ }^{13} \mathrm{C}\right)$ spectrometer, using deuterated chloroform- $d_{1}$ or dimethylsulfoxide- $d_{6}$ as solvent and internal standard. For the evaluation of the spectra the program MestReNova 7.1 was used. ${ }^{15} \mathrm{~N}-\mathrm{HMBC}$ and $\mathrm{H}, \mathrm{H}-\mathrm{COSY}$ spectra as well as ${ }^{1} \mathrm{H}$ NMR spectra were recorded on a Bruker Avance III $400 \mathrm{MHz}$ spectrometer (BrukerBiospin, Rheinstetten with a broadband-observe probe, z-gradient and temperature unit) in $\mathrm{CDCl}_{3}$ with acetonitrile as internal standard. All spectra were processed with Topspin 2.1 and further analysed with MestReNova 7.1.

\section{Mass spectrometry}

The mass spectra were taken with a Bruker Daltonik ESI-ToF-MS (MIRCO-TOF) by the laboratories of the Zentrale Analytik of the Institute of Organic Chemistry at the Georg-August University Göttingen. All measurements were carried out with a temperature of the capillary of $180{ }^{\circ} \mathrm{C}$ and a put-on voltage of $4.5 \mathrm{kV}$.

\section{Elemental Analysis}

All elemental analyses were performed at the analytical laboratory of the Institute of Inorganic Chemistry at the Georg-August University Göttingen. Elemental analyses for carbon, hydrogen, nitrogen and sulfur were carried out on an Elementar Vario EL III elemental analyser. The halogens $\mathrm{Cl}$ and $\mathrm{Br}$ were determined potentiometrically on a 636 Titroprocesso by Methrom.

\section{Atomic Force Microscopy}

The scanning experiments were carried out in an argon-filled glove box (oxygen content below $1 \mathrm{ppm}$ ) on a Scanning Probe Microscope MultiMode 8 by Veeco applying the peak force tapping mode with a silicon-on-nitride cantilever $\left(k=0.4 \mathrm{~N} \cdot \mathrm{m}^{-1}, f_{0}=50-90 \mathrm{kHz}\right)$. The experiments and evaluation were carried out with the software Research NanoScope 8.10.

For the preparation of the sample one drop of the polymer solution in chloroform $\left(c_{\text {polymer }} \approx 8 \cdot 10^{-4} \mathrm{~g} \cdot \mathrm{L}^{-1}\right)$ was set onto a freshly split piece of potash mica $\left(\mathrm{KAl}_{2}(\mathrm{OH}, \mathrm{F})_{2}\left(\mathrm{AlSi}_{3} \mathrm{O}_{10}\right)\right.$; high quality bought from PLANO GmbH) and left to spin for one minute at 1,500 rpm in the Spin Coater WS-650-23NPP by Laurell. Afterwards the samples were left under chloroform atmosphere at $50{ }^{\circ} \mathrm{C}$ over night in a solvent annealing chamber. 


\section{Microwave Synthesis System}

The microwave assisted reactions were carried out in a Discover $S$ microwave reactor from CEM. The reaction conditions and device settings are noted in the syntheses procedure.

\section{Further Equipment}

All substances were weighed out on an Analytic AC 210 S from Sartorius. To degas the solvents and the monomers for the polymerisations an Edwards EXC 120 or a Pfeiffer TMH 071 turbo molecular pump was employed. The stock solutions were all prepared in a glove box Lab Master 130 of the company Braun with an oxygen content below $4 \mathrm{ppm}$. Samples in polymerisation vials were heated in a block heater MBT 250 by Kleinfeld Labortechnik which is fixed onto a shaking device from Edmund Bühler $\mathrm{GmbH}$. To divide off the precipitated polymer from the solvent the laboratory centrifuge 2-16KC from Sigma was applied with the rotor 12151 at $8,800 \mathrm{rpm}$ and $5{ }^{\circ} \mathrm{C}$ for $10-30 \mathrm{~min}$. The polymer samples were dried at $35-55{ }^{\circ} \mathrm{C}$ until weight-constancy in a vacuum drying cabinet Thermo Scientific by Thermo Electron LED GmbH. 


\subsection{Synthesises}

This chapter presents the synthesis of the different UV-sensitive monomers as well as of various RAFT-agents.

\subsubsection{Monomers with UV-Reactive Unit}

All monomers were synthesised in adaption and in combination of the procedures reported in the papers quoted.

\subsubsection{3,4-Dimethylmaleimides 1.1 and 1.2}

The monomers $N$-ethylacrylate-3,4-dimethylmaleimide $\mathbf{1 . 1}$ and $\mathrm{N}$-ethylmethacrylate-3,4-dimethyl-maleimide $\mathbf{1 . 2}$ were synthesised according to the procedure presented by Gupta et al. with acrylic and methacrylic acid. ${ }^{(116)}$ Therefore, as the first step, dimethylmaleimido ethanol 1 was synthesised: $39 \mathrm{mmol}$ 2,3-dimethylmaleic anhydride (4.92 g; $126.11 \mathrm{~g} \cdot \mathrm{mol}^{-1}$ ) were combined with $4 \mathrm{mmol}$ 2-aminoethanol (2.44 g; $2.40 \mathrm{~mL} ; 61.08 \mathrm{~g} \cdot \mathrm{mol}^{-1}$ ) in $200 \mathrm{~mL}$ toluene and heated to the boiling point of toluene. With DeanStark-apparatus $4 \mathrm{mmol}$ water were collected. Afterwards the solution was dried over magnesium sulphate to remove any remaining traces of water which support the reverse reaction. The toluene was removed in vacuo. The
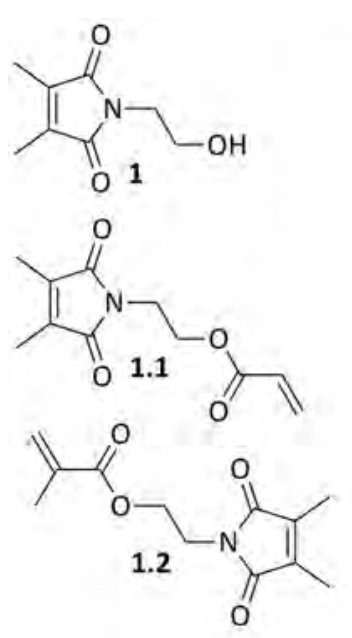
product obtained is a colourless liquid but can also appear as white crystals (98\% yield; $6.09 \mathrm{~g}$; $\left.36 \mathrm{mmol} ; 169.18 \mathrm{~g} \cdot \mathrm{mol}^{-1}\right)$.

${ }^{1} \mathrm{H}$ NMR $\left(300 \mathrm{MHz}, \mathrm{CDCl}_{3}\right) \delta(\mathrm{ppm}): \quad 1.96\left(\mathrm{~s}, 6 \mathrm{H}, \underline{\mathrm{H}}_{3} \mathrm{C}-\mathrm{C}=\mathrm{C}-\right), 2.36$ (s, 1H, -Oㅡ) , 3.68-3.73 (tt, ${ }^{13} \mathrm{C} \mathrm{NMR}\left(75 \mathrm{MHz}, \mathrm{CDCl}_{3}\right) \delta(\mathrm{ppm}): \quad 8.84 \quad\left(\mathrm{H}_{3} \underline{\mathrm{C}}-\mathrm{C}=\mathrm{C}-\right), \quad 41.13 \quad\left(-\mathrm{N}-\mathrm{CH}_{2}-\mathrm{CH}_{2}-\mathrm{O}-\right), \quad 61.32$ $\left(-\mathrm{N}-\underline{\mathrm{CH}}_{2}-\mathrm{CH}_{2}-\mathrm{O}-\right), 137.52(-\mathrm{C}=\mathrm{C}-), 172.75$ (- $\left.\underline{\mathrm{C}}=\mathrm{O}\right)$

ESI-MS: $169.1[\mathrm{M}]^{+}$

1 was used without further purification. To combine this UV-sensitive unit with the monomer-unit a Steglich esterfication was applied. ${ }^{(197)} 7 \mathrm{mmol} 1\left(1.18 \mathrm{~g} ; 169.18 \mathrm{~g} \cdot \mathrm{mol}^{-1}\right)$ were combined with of $0.7 \mathrm{mmol}$ 4-dimethylaminopyridin (DMAP; $0.08 \mathrm{~g} ; \quad 122.17 \mathrm{~g} \cdot \mathrm{mol}^{-1}$ ) and $7 \mathrm{mmol}$ dicyclohexylcarbodiimide (DCC; $1.44 \mathrm{~g} ; 206.33 \mathrm{~g} \cdot \mathrm{mol}^{-1}$ ) in $30 \mathrm{~mL}$ chloroform. The mixture was cooled with an ice bath to $0{ }^{\circ} \mathrm{C} .7 \mathrm{mmol}$ acrylic acid (AA; $0.50 \mathrm{~g} ; 0.50 \mathrm{~mL} ; 72.06 \mathrm{~g} \cdot \mathrm{mol}^{-1}$ ) for monomer 1.1 or methacrylic acid for 1.2 (MAA; $0.60 \mathrm{~g} ; 0.60 \mathrm{~mL} ; 86.06 \mathrm{~g} \cdot \mathrm{mol}^{-1}$ ) were added drop wise. After stirring for 1 hour at $0{ }^{\circ} \mathrm{C}$ the ice bath was removed and the mixture was left to stir over night at room temperature. The converted DCC was removed from the solution via a G4-frit and washed with a 
little amount of diethyl ether. The solvents were removed in vacuo and the crude product was purified via column chromatography (3:1 ratio of $n$-pentane and ethyl acetate for $\mathbf{1 . 1}$ and a 4:1 $n$-pentane and ethyl acetate ratio for 1.2). Both monomers were obtained as white crystals with an overall yield of $80 \%$.

\section{$\underline{1.1}$}

${ }^{1} \mathrm{H}$ NMR $\left(300 \mathrm{MHz}, \mathrm{CDCl}_{3}\right) \delta(\mathrm{ppm}): \quad 1.96\left(\mathrm{~s}, 6 \mathrm{H}, \underline{\mathrm{H}}_{3} \mathrm{C}-\mathrm{C}=\mathrm{C}-\right), 3.75\left(\mathrm{t}, 2 \mathrm{H},-\mathrm{N}-\mathrm{CH}_{2}-\mathrm{C}_{2}-\mathrm{O}-\right)$, 4.28 (t, $\left.2 \mathrm{H},-\mathrm{N}-\mathrm{C}_{2}-\mathrm{CH}_{2}-\mathrm{O}-\right), 5.77\left(\mathrm{~m}, 1 \mathrm{H},-\mathrm{CH}=\underline{\mathrm{C}}_{2}\right), 6.08(\mathrm{~m}, 1 \mathrm{H}$, $\left.-\mathrm{C} \underline{\mathrm{H}}=\mathrm{CH}_{2}\right), 6.37\left(\mathrm{~m}, 1 \mathrm{H},-\mathrm{CH}=\mathrm{C}_{2} \underline{2}\right)$

${ }^{15} \mathrm{~N}$ NMR $\left(400 \mathrm{MHz}, \mathrm{CDCl}_{3}\right) \delta(\mathrm{ppm}):$ $-238.18$

ESI-MS:

$246.1[\mathrm{M}+\mathrm{Na}]^{+}, 469.2[2 \mathrm{M}+\mathrm{Na}]^{+}$

Elemental analysis [w\%]:

C 61.85; H 7.07; N 7.31

theoretical values

C 59.19 ; H 5.87 ; N 6.27

UV-Vis absorbance in acetonitrile:

$222 \mathrm{~nm}, 228 \mathrm{~nm}$ (spectrum see chapter 8.2 and 8.4)

\section{$\underline{1.2}$}

${ }^{1} \mathbf{H}$ NMR (300 MHz, $\left.\mathrm{CDCl}_{3}\right) \delta(\mathrm{ppm})$ :

$1.89\left(\mathrm{~s}, 3 \mathrm{H}, \mathrm{H}_{2} \mathrm{C}=\mathrm{C}-\mathrm{C}_{3}\right), 1.96\left(\mathrm{~s}, 6 \mathrm{H}, \underline{\mathrm{H}}_{3} \mathrm{C}-\mathrm{C}=\mathrm{C}-\right), 3.80(\mathrm{t}, 2 \mathrm{H}$, $\left.-\mathrm{N}-\mathrm{CH}_{2}-\mathrm{CH}_{2}-\mathrm{O}-\right), 4.25$ (t, $\left.2 \mathrm{H},-\mathrm{N}-\mathrm{CH}_{2}-\mathrm{C}_{2}-\mathrm{O}-\right), 5.55(\mathrm{~m}, 1 \mathrm{H}$, $\left.-\mathrm{CH}=\underline{\mathrm{C}}_{2}\right), 6.08\left(\mathrm{~m}, 1 \mathrm{H},-\underline{\mathrm{H}}=\mathrm{CH}_{2}\right)$

ESI-MS: $260.1[\mathrm{M}+\mathrm{Na}]^{+}, 497.2[2 \mathrm{M}+\mathrm{Na}]^{+}$

Elemental analysis [w\%]:

C 60.45; H 6.54; N 6.03

theoretical values

C $60.75 ;$ H 6.37 ; N 5.90

\subsubsection{Cinnamoyloxyethyl-monomers 2.1 and 2.2}

For the synthesis of cinnamoyloxyethyl acrylate $\mathbf{2 . 1}$ and cinnamoyloxyethyl methacrylate $\mathbf{2 . 2}$ the Steglich esterfication (197) with cinnamic acid and 2-hydroxyethylacrylate (HEA) or 2-hydroxyethyl-methacrylate (HEMA) proved to be insufficient as the conversion was not complete and the purification via re-crystallisation and column chromatography was not successful. Therefore, the monomers<smiles>[Z12]COC(=O)/C=C/c1ccccc1</smiles><smiles>[Z20]COC(=O)/C=C/c1ccccc1</smiles>
were synthesised with HEMA or HEA and cinnamoylchloride, as described by Atta et al. ${ }^{(54)}$ In $100 \mathrm{~mL}$ THF $66 \mathrm{mmol}$ HEMA (8.59 g; $\left.8.10 \mathrm{~mL} ; 130.14 \mathrm{~g} \cdot \mathrm{mol}^{-1}\right)$ or HEA (7.66 g; $7.00 \mathrm{~mL}$; $116.12 \mathrm{~g} \cdot \mathrm{mol}^{-1}$ ) and $66 \mathrm{mmol}$ TEA (6.68 g; $9.20 \mathrm{~mL} ; 101.19 \mathrm{~g} \cdot \mathrm{mol}^{-1}$ ) were dissolved under nitrogen atmosphere. The solution was refluxed for $120 \mathrm{~min}$ and then cooled to $0{ }^{\circ} \mathrm{C}$ with an ice bath. A 
solution of $79 \mathrm{mmol}$ cinnamoylchloride (13.16 g; $166.60 \mathrm{~g} \cdot \mathrm{mol}^{-1}$ ) in $15 \mathrm{~mL}$ THF was added drop wise. The mixture was left to stir at $0{ }^{\circ} \mathrm{C}$ for another $120 \mathrm{~min}$ before stirring at room temperature for $240 \mathrm{~min}$. The formed triethylammonium chloride was filtered off with a G4-frit. Afterwards $500 \mathrm{~mL}$ $n$-hexane were added to the filtrate and the resulting precipitate removed via a G4-frit. To prevent polymerisation during the removal of the solvents in vacuo copper(I)-chloride was added. Afterwards copper(I)-chloride was filtered off and the product isolated as a yellow oil (2.1 82 \%; $15.99 \mathrm{~g}$; $65 \mathrm{mmol} ; 246.09 \mathrm{~g} \cdot \mathrm{mol}^{-1} ; 2.260 \%$; $\left.12.22 \mathrm{~g} ; 47 \mathrm{mmol} ; 260.10 \mathrm{~g} \cdot \mathrm{mol}^{-1}\right)$.

\section{$\underline{2.1}$}

${ }^{1} \mathrm{H}$ NMR $\left(300 \mathrm{MHz}, \mathrm{CDCl}_{3}\right) \delta(\mathrm{ppm}): \quad 4.47\left(\mathrm{~s}, 4 \mathrm{H},-\mathrm{C}_{\underline{2}}-\underline{\mathrm{C}}_{2}-{ }_{2}\right), 5.88\left(\mathrm{~m}, 1 \mathrm{H},-\mathrm{CH}=\mathrm{C}_{2} \underline{2}\right), 6.18(\mathrm{~m}, 1 \mathrm{H}$, $\left.-\mathrm{CH}=\underline{\mathrm{C}}_{2}\right), 6.47\left(\mathrm{~m}, 2 \mathrm{H},-\mathrm{CH}=\underline{\mathrm{H}}-\mathrm{CO}_{2}-\right.$ and $\left.-\underline{\mathrm{H}}=\mathrm{CH}_{2}\right)$, 7.39-7.56 (m, $\left.5 \mathrm{H}, \underline{\mathrm{H}}_{\text {aromatic }}\right), 7.73\left(\mathrm{~m}, 1 \mathrm{H},-\mathrm{CH}=\underline{\mathrm{CH}}-\mathrm{C}_{6} \mathrm{H}_{5}\right)$

${ }^{13} \mathrm{C} \mathrm{NMR}\left(75 \mathrm{MHz}, \mathrm{CDCl}_{3}\right) \delta(\mathrm{ppm}): \quad 62.23\left(-\mathrm{CH}_{2}-\mathrm{CH}_{2}-\right), 62.37 \quad\left(-\mathrm{CH}_{2}-\mathrm{CH}_{2}-\right), 117.49\left(\mathrm{CH}_{2}=\mathrm{CH}\right)$, 128.01 ( $\left.\mathrm{C}_{\mathrm{Ar}-\mathrm{C}} \mathrm{Ar}-\mathrm{CH}\right), 128.16$ ( $\left.\mathrm{CH}-\mathrm{CO}\right), 128.92\left(\mathrm{C}_{\mathrm{Ar}}-\mathrm{CH}\right), 130.45$ ( $\left.\mathrm{C}_{\mathrm{Ar}}\right), 131.42\left(\mathrm{C}_{\mathrm{Ar}}-\mathrm{CH}\right), 134.27\left(\mathrm{CH}_{2}=\mathrm{CH}\right), 145.47\left(\mathrm{C}_{\mathrm{Ar}-\mathrm{C}} \mathrm{C}_{\mathrm{Ar}}-\mathrm{CH}\right)$, $165.94\left(\mathrm{CO}-\mathrm{CH}=\mathrm{CH}_{2}\right), 166.68\left(\mathrm{CH}-\mathrm{CO}-\mathrm{O}-\mathrm{CH}_{2}\right)$

ESI-MS: $247.09[\mathrm{M}+\mathrm{H}]^{+}$

\section{$\underline{2.2}$}

${ }^{1} \mathrm{H}$ NMR $\left(300 \mathrm{MHz}, \mathrm{CDCl}_{3}\right) \delta(\mathrm{ppm}): \quad 1.98\left(\mathrm{~s}, 3 \mathrm{H},-\mathrm{C}-\mathrm{C}_{3}\right), 4.47\left(\mathrm{~m}, 4 \mathrm{H},-\underline{\mathrm{C}}_{2}-\underline{\mathrm{C}}_{2}-\right), 5.62(\mathrm{~m}, 1 \mathrm{H}$, $\left.-\mathrm{C}=\mathrm{CH}_{2}\right), 6.17\left(\mathrm{~m}, 1 \mathrm{H},-\mathrm{C}=\mathrm{CH}_{2}\right), 6.48\left(\mathrm{~d}, 1 \mathrm{H},-\mathrm{CH}=\underline{\mathrm{CH}}-\mathrm{CO}_{2}-\right)$,

${ }^{13} \mathrm{C} \mathrm{NMR}\left(75 \mathrm{MHz}, \mathrm{CDCl}_{3}\right) \delta(\mathrm{ppm})$ : 7.40-7.62 (m, $\left.5 \mathrm{H}, \underline{\mathrm{H}}_{\text {aromatic }}\right), 7.74\left(\mathrm{~m}, 1 \mathrm{H},-\mathrm{CH}=\mathrm{C} \underline{\mathrm{H}}-\mathrm{C}_{6} \mathrm{H}_{5}\right)$ $18.28\left(\mathrm{CH}_{3}\right), 62.20\left(-\mathrm{CH}_{2}-\mathrm{CH}_{2}-\right), 62.52\left(\mathrm{CO}-\mathrm{O}-\mathrm{CH}_{2}\right), 117.54$ $\left(\mathrm{CH}_{2}=\mathrm{CH}\right), 126.08\left(\mathrm{Car}_{\mathrm{ar}}-\mathrm{C}_{\mathrm{Ar}}-\mathrm{CH}\right), 128.15\left(\mathrm{C}_{\mathrm{Ar}}-\mathrm{CH}\right), 128.91\left(\mathrm{C}_{\mathrm{Ar}}-\right.$ $\mathrm{CH}), 130.43$ ( $\left.\mathrm{CAr}_{\mathrm{Ar}}\right), 134.29$ ( $\left.\mathrm{Car}_{\mathrm{Ar}}-\mathrm{Car}_{\mathrm{Ar}}-\mathrm{CAr}_{\mathrm{Ar}}-\mathrm{CH}\right), 135.99\left(\mathrm{CH}=\mathrm{CH}_{2}\right)$, $145.41 \quad\left(\mathrm{C}_{\mathrm{Ar}}-\mathrm{C}_{\mathrm{Ar}}-\mathrm{C}_{\mathrm{Ar}}-\mathrm{CH}\right), \quad 166.68 \quad\left(\mathrm{CO}-\mathrm{CH}=\mathrm{CH}_{2}\right), \quad 167.17$ $(\mathrm{CH}=\mathrm{CH}-\mathrm{CO})$

ESI-MS: $283.09[\mathrm{M}+\mathrm{Na}]^{+}$ 


\subsubsection{7-(2-Methacryloyloxyethoxy)-4-methylcourmarin}

\section{Synthesis of the precursor 3 via standard laboratory methods}

To synthesise the precursor 3 for 7-(2-methacryloyloxyethoxy)-4methylcourmarin 3.1 firstly the synthesis of Jiang et al. ${ }^{(113)}$ was applied. Therefore, $10 \mathrm{mmol}$ 7-hydroxy-4-methylcoumarin were mixed with $10 \mathrm{mmol}$ 2-bromoethanol and $10 \mathrm{mmol}$ potassium carbonate and suspended in $50 \mathrm{ml}$ dry ethanol under nitrogen. The

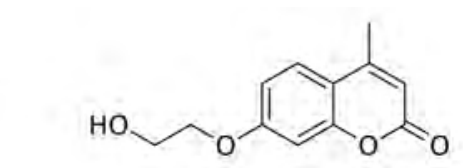

3

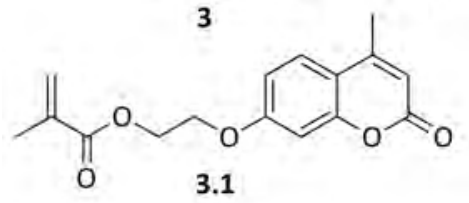
mixture was refluxed for 20 hours. After cooling $50 \mathrm{~mL}$ water was added and the product extracted with diethyl ether. The organic layers were dried over magnesium sulphate and the solvents removed in vacuo. The NMR and mass analysis revealed that the coupling of the ethanol-bridge to the courmarin backbone was not successful.

The experiment was adapted by the addition of 1 mmol 18-crown-6, as reported for other alcohols $^{(198)}$, but again NMR and mass analysis revealed failure of the procedure.

Therefore another pathway, reported by Zhang et al., was tested. ${ }^{(199)}$ In $50 \mathrm{~mL}$ DMF $10 \mathrm{mmol}$ 7-hydroxy-4-methylcoumarin (1.76 g; $\left.176.17 \mathrm{~g} \cdot \mathrm{mol}^{-1}\right)$ were combined with $20 \mathrm{mmol}$ 2-chloroethanol $\left(1.61 \mathrm{~g} ; 1.40 \mathrm{~mL} ; 80.51 \mathrm{~g} \cdot \mathrm{mol}^{-1}\right), 20 \mathrm{mmol}$ potassium carbonate $\left(2.76 \mathrm{~g} ; 138.20 \mathrm{~g} \cdot \mathrm{mol}^{-1}\right)$ and a catalytical amount of potassium iodine $\left(0.083 \mathrm{~g} ; 0.5 \mathrm{mmol} ; 166.00 \mathrm{~g} \cdot \mathrm{mol}^{-1}\right)$. The mixture was stirred at $110{ }^{\circ} \mathrm{C}$ over night. After cooling $50 \mathrm{~mL}$ water was added and the product extracted with ethyl acetate. The organic phase was dried over sodium sulphate. After removal of the solvent the product was isolated as a white, amorphous solid (90\% yield; $\left.1.98 \mathrm{~g} ; 9 \mathrm{mmol} ; 220.07 \mathrm{~g} \cdot \mathrm{mol}^{-1}\right)$.

\section{Synthesis of the precursor 3 under microwave irradiation}

Besides this standard procedure to synthesise the alcohol a microwave assisted synthesis was developed. Here $1 \mathrm{mmol}$ 7-hydroxy-4-methylcoumarin (0.18 g; $\left.176.14 \mathrm{~g} \cdot \mathrm{mol}^{-1}\right), 2.5 \mathrm{mmol}$ 2-chloroethanol $\left(0.16 \mathrm{~g} ; 0.14 \mathrm{~mL} ; 80.51 \mathrm{~g} \cdot \mathrm{mol}^{-1}\right), 4 \mathrm{mmol}$ potassium carbonate $(0.55 \mathrm{~g}$; $\left.138.20 \mathrm{~g} \cdot \mathrm{mol}^{-1}\right)$, and $0.3 \mathrm{mmol}$ potassium iodine $\left(0.049 \mathrm{~g} ; 166.00 \mathrm{~g} \cdot \mathrm{mol}^{-1}\right)$ were combined with $5 \mathrm{~mL}$ DMF in a suitable glass tube for the microwave reactor or a round-bottomed flask. The dynamic mode of the microwave reactor Discover $S$ was used with the settings presented in Table 12-2. 
Table 12-2 Settings for microwave assisted synthesis of $\mathbf{3}$

\begin{tabular}{|c|c|c|c|c|}
\hline & $\begin{array}{c}\text { power } \\
\text { in-put [W] }\end{array}$ & $T_{\max }\left[{ }^{\circ} \mathrm{C}\right]$ & $t[\min ]$ & Device settings \\
\hline 1) & 50 & 100 & 10 & high stirring, 5 bar pressure, $60 \mathrm{sec}$ pre-mixing \\
\hline 2) & 50 & 100 & 20 & high stirring, 5 bar pressure, $60 \mathrm{sec}$ pre-mixing \\
\hline 3) & 50 & 100 & 30 & high stirring, 5 bar pressure, $120 \mathrm{sec}$ pre-mixing \\
\hline 4) & 75 & 100 & 20 & high stirring, 5 bar pressure, $120 \mathrm{sec}$ pre-mixing \\
\hline 5) & 75 & 100 & 30 & high stirring, 5 bar pressure, $120 \mathrm{sec}$ pre-mixing \\
\hline
\end{tabular}

During the running experiment the power entry was kept constant due to permanently cooling. The temperature fluctuated set up conditioned $\left(\Delta T= \pm 5^{\circ} \mathrm{C}\right)$.

After 10 minutes no conversion at all was detected. At $50 \mathrm{~W}$ and 20 minutes the conversion was not complete and at $75 \mathrm{~W}$ and 30 minutes the ${ }^{1} \mathrm{H}$ NMR-spectra revealed peaks besides those of the product which do not result from the educts. Full conversion was observed at $50 \mathrm{~W}$ and 30 minutes as well as at $75 \mathrm{~W}$ and 20 minutes.

${ }^{1} \mathrm{H}$ NMR $\left(300 \mathrm{MHz}, \mathrm{CDCl}_{3}\right) \delta(\mathrm{ppm}): \quad 2.39\left(\mathrm{~s}, 3 \mathrm{H},-\mathrm{C}^{4}-\mathrm{C}_{3}-3\right), 4.01\left(\mathrm{t}, 2 \mathrm{H}, \mathrm{HO}-\mathrm{CH}_{2}-\mathrm{CH}_{2}-\right), 4.14(\mathrm{t}, 2 \mathrm{H}$, $\left.\mathrm{HO}-\mathrm{CH}_{2}-\mathrm{CH}_{2} \underline{2}^{-}\right), 4.52\left(\mathrm{~s}, 1 \mathrm{H}, \underline{\mathrm{HO}}-\mathrm{CH}_{2}-\right), 6.13\left(\mathrm{~s}, 1 \mathrm{H},-\mathrm{C}^{3}-\underline{\mathrm{H}}\right), 6.82$ (d, $\left.1 \mathrm{H},-\mathrm{C}^{7}-\underline{H}\right), 7.26$ (s, $\left.1 \mathrm{H},-\mathrm{C}^{9}-\underline{H}\right), 7.48$ (d, $\left.1 \mathrm{H},-\mathrm{C}^{6}-\underline{H}\right)$

ESI-MS: $220.07[\mathrm{M}]^{+}, 243.1[\mathrm{M}+\mathrm{Na}]^{+}, 463.1[2 \mathrm{M}+\mathrm{Na}]^{+}$

3 was used for the next step without further purification. 3.1 was synthesised in adaption of ref. (113). In $40 \mathrm{~mL}$ dry chloroform under nitrogen atmosphere $3 \mathrm{mmol} 3$ (0.66 g; $220.07 \mathrm{~g} \cdot \mathrm{mol}^{-1}$ ) were suspended and cooled to $0{ }^{\circ} \mathrm{C}$ with an ice bath. Firstly $7 \mathrm{mmol}$ TEA $(0.71 \mathrm{~g} ; 1.00 \mathrm{~mL}$; $\left.101.19 \mathrm{~g} \cdot \mathrm{mol}^{-1}\right)$ and then $8 \mathrm{mmol}$ methylacroyl chloride $\left(0.84 \mathrm{~g} ; 0.80 \mathrm{~mL} ; 104.54 \mathrm{~g} \cdot \mathrm{mol}^{-1}\right)$ were added drop wise. The mixture was stirred over night while the ice bath was left to thaw. The mixture was washed two times with $30 \mathrm{~mL}$ aqueous sodium chloride solution. Afterward the organic phase was dried over magnesium sulphate and the solvent removed in vacuo. The crude product was recrystallised from ethanol at a temperature of $40{ }^{\circ} \mathrm{C}$ and a white powder was obtained $(70 \%$ yield; $\left.0.57 \mathrm{~g} ; 2 \mathrm{mmol} ; 288.10 \mathrm{~g} \cdot \mathrm{mol}^{-1}\right)$.

\section{$\underline{3.1}$}

${ }^{1} \mathrm{H}$ NMR $\left(300 \mathrm{MHz}, \mathrm{CDCl}_{3}\right) \delta(\mathrm{ppm}): \quad 1.87\left(\mathrm{~s}, 3 \mathrm{H}, \mathrm{H}_{2} \mathrm{C}=\mathrm{C}-\mathrm{C}_{\underline{3}}\right), 2.39\left(\mathrm{~s}, 3 \mathrm{H},-\mathrm{C}^{4}-\mathrm{C}_{3} \underline{3}\right), 4.38(\mathrm{t}, 2 \mathrm{H}$, $\left.-\mathrm{O}-\mathrm{C}_{2}-\mathrm{CH}_{2}-\right), 4.45\left(\mathrm{t}, 2 \mathrm{H},-\mathrm{O}-\mathrm{CH}_{2}-\mathrm{C}_{2} \underline{2}^{-}\right), 5.70$ (q, $1 \mathrm{H}$, $\left.\underline{\mathrm{H}}_{2} \mathrm{C}=\mathrm{C}-\mathrm{CH}_{3}\right), 6.03\left(\mathrm{q}, 1 \mathrm{H}, \underline{\mathrm{H}}_{2} \mathrm{C}=\mathrm{C}-\mathrm{CH}_{3}\right), 6.22\left(\mathrm{~s}, 1 \mathrm{H},-\mathrm{C}^{3}-\underline{\mathrm{H}}\right)$, $6.99\left(\mathrm{~d}, 1 \mathrm{H},-\mathrm{C}^{7}-\underline{H}\right), 7.03\left(\mathrm{~s}, 1 \mathrm{H},-\mathrm{C}^{9}-\underline{H}\right), 7.70\left(\mathrm{~d}, 1 \mathrm{H},-\mathrm{C}^{6}-\underline{H}\right)$ 
ESI-MS:

Elementary analysis [w\%]:

theoretical values
$311.1[\mathrm{M}+\mathrm{Na}]^{+}, 599.2[2 \mathrm{M}+\mathrm{Na}]^{+}, 887.3[3 \mathrm{M}+\mathrm{Na}]^{+}$

C $66.45 ; \mathrm{H} 5.70$

C 66.66; H 5.59 


\subsubsection{RAFT-Agents}

The RAFT-agents were synthesised in adaption of the in reference (21) and (160) described procedures.

\subsubsection{Pentaerythriol-tetrakis(3-(S-benzyl-trithio-carbonate))-propanoate}

A solution of $10 \mathrm{mmol}$ pentaerythriol-tetrakis(3-mercaptopropionate) $\left(4.88 \mathrm{~g} ; 488.66 \mathrm{~g} \cdot \mathrm{mol}^{-1}\right.$ ) in $30 \mathrm{~mL}$ dichloromethane was stirred at room temperature, when $40 \mathrm{mmol}$ triethylamine (TEA; $4.05 \mathrm{~g} ; 5.50 \mathrm{~mL} ; 101.19 \mathrm{~g} \cdot \mathrm{mol}^{-1}$ ) in $20 \mathrm{~mL}$ dichloromethane and $80 \mathrm{mmol}$ carbon disulfide $\left(6.10 \mathrm{~g} ; 5.00 \mathrm{~mL} ; 76.14 \mathrm{~g} \cdot \mathrm{mol}^{-1}\right)$ were added. After $90 \mathrm{~min}$ stirring at room temperature $40 \mathrm{mmol}$ benzylbromide (6.84 g; $4.80 \mathrm{~mL} ; 171.04 \mathrm{~g} \cdot \mathrm{mol}^{-1}$ ) were added drop wise. The mixture was left to stir for another $180 \mathrm{~min}$. Then the reaction was

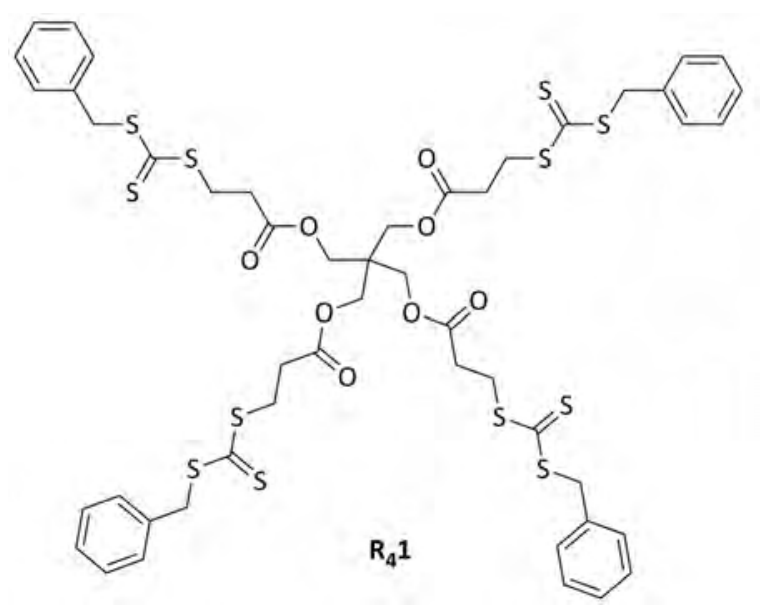
quenched by addition of $50 \mathrm{~mL} 10 \%$ hydrochloric acid. The organic layer was divided off and the water phase extracted three times with $20 \mathrm{~mL}$ ethyl acetate. The combined organic layers were washed with water and then dried of sodium sulphate. The solvents were removed in vacuo and the product was purified by column chromatography ( $n$-pentane: ethyl acetate 2:1) to give a viscous, yellow liquid (33\% yield; $3.83 \mathrm{~g}$; $3.3 \mathrm{mmol} ; 1,153.71 \mathrm{~g} \cdot \mathrm{mol}^{-1}$ ).

${ }^{1} \mathrm{H}$ NMR $\left(300 \mathrm{MHz}, \mathrm{CDCl}_{3}\right) \delta(\mathrm{ppm}): \quad 2.27\left(\mathrm{t}, 8 \mathrm{H}, \mathrm{O}=\mathrm{C}-\underline{\mathrm{C}}_{2}\right), 3.58\left(\mathrm{t}, 8 \mathrm{H}, \mathrm{CH}_{2}-\mathrm{C}_{2}-\underline{2}\right), 4.11(\mathrm{~s}, 8 \mathrm{H}$,

ESI-MS:

Elemental analysis [w\%]: theoretical values $\left.\mathrm{C}_{\text {central }}-\mathrm{C}_{2} \underline{2}\right), 4.57\left(\mathrm{~s}, 8 \mathrm{H}, \mathrm{S}-\underline{\mathrm{C}}_{2}-\mathrm{C}_{6} \mathrm{H}_{5}\right), 7.28\left(20 \mathrm{H}, \underline{\mathrm{H}}_{\text {aromatic }}\right)$

$1175.1[\mathrm{M}+\mathrm{Na}]^{+}$

C 28.45; H 4.73; S 66.05

C 28.57; H 4.80; S 66.63 


\subsubsection{Pentaerythriol-tetrakis(3-(S-phenylethyl-trithio-carbonate))-propanoate}

A solution of $2.5 \mathrm{mmol}$ pentaerythriol-tetrakis(3-mercaptopropionate) $\left(1.22 \mathrm{~g} ; 488.66 \mathrm{~g} \cdot \mathrm{mol}^{-1}\right.$ ) in $60 \mathrm{~mL}$ chloroform was stirred at room temperature, while $3 \mathrm{mmol}$ TEA (0.30 g; $0.42 \mathrm{~mL} ; 101.19 \mathrm{~g} \cdot \mathrm{mol}^{-1}$ ) were added. The mixture was left to stir for $60 \mathrm{~min}$, then $80 \mathrm{mmol}$ carbon disulfide $(6.10 \mathrm{~g} ; 5.00 \mathrm{~mL}$; $76.14 \mathrm{~g} \cdot \mathrm{mol}^{-1}$ ) were added. After stirring for $30 \mathrm{~min}$ stirring at room temperature $10 \mathrm{mmol}$ (1-bromoethyl) benzene (1.85 g; $1.40 \mathrm{~mL}$; $185.06 \mathrm{~g} \cdot \mathrm{mol}^{-1}$ ) were added drop wise and the mixture was left to stir over night. Then the reaction

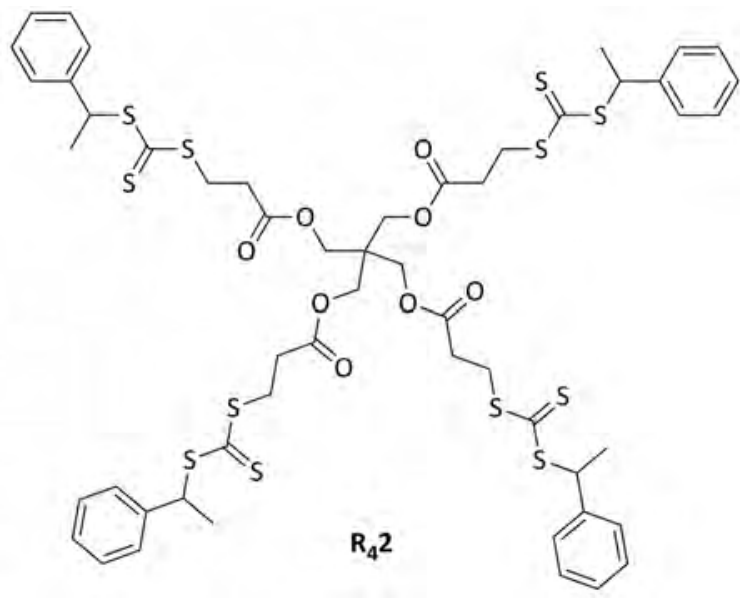
was quenched by addition of $50 \mathrm{~mL} 10 \%$ hydrochloric acid. The organic layer was separated and washed two times with $100 \mathrm{~mL}$ water and then dried with sodium sulphate. The solvent was removed in vacuo resulting a viscous, yellow liquid which required no further purification ( $72 \%$ yield; $\left.2.18 \mathrm{~g} ; 1.8 \mathrm{mmol} ; 1,209.82 \mathrm{~g} \cdot \mathrm{mol}^{-1}\right)$.

${ }^{1} \mathbf{H}$ NMR $\left(300 \mathrm{MHz}, \mathrm{CDCl}_{3}\right) \delta(\mathrm{ppm}):$ $1.76\left(\mathrm{~d}, 12 \mathrm{H}, \underline{\mathrm{H}}_{3} \mathrm{C}-\mathrm{CH}-\right), 2.75\left(\mathrm{t}, 8 \mathrm{H}, \mathrm{O}=\mathrm{C}-\mathrm{C}_{2}\right), 3.55(\mathrm{t}, 8 \mathrm{H}$, $\left.\mathrm{CH}_{2}-\mathrm{CH}_{2}-\mathrm{S}\right), 4.11\left(\mathrm{~s}, 8 \mathrm{H}, \mathrm{C}_{\text {central }}-\underline{\mathrm{C}}_{2}\right), 4.57\left(\mathrm{~s}, 8 \mathrm{H}, \mathrm{S}^{-} \underline{\mathrm{C}}_{2}-\mathrm{C}_{6} \mathrm{H}_{5}\right)$,

${ }^{13} \mathrm{C}$ NMR $\left(75 \mathrm{MHz}, \mathrm{CDCl}_{3}\right) \delta(\mathrm{ppm}):$ $7.31\left(20 \mathrm{H}, \underline{\mathrm{H}}_{\text {aromatic }}\right)$

21.08 (4 C, $-\mathrm{CH}-\underline{\mathrm{CH}}_{3}$ ), 26.44 (4 C, O=C- $\left.\underline{\mathrm{C}} \mathrm{H}_{2}\right), 30.46\left(4 \mathrm{C}, \underline{\mathrm{CH}}_{2}-\mathrm{S}\right)$, 32.61 (1 C, $\left.\underline{\mathrm{C}}_{\text {central }}\right), 50.08$ (4 C, $\left.-\underline{\mathrm{CH}}-\mathrm{CH}_{3}\right), 61.84$ (4 C, $\left.\underline{\mathrm{CH}}_{2}-\mathrm{C}_{\text {central }}\right), 128.33\left(20 \mathrm{C}, \underline{\mathrm{C}}_{\text {aromatic }} 2-6\right), 140.72(4 \mathrm{C}$, $-\mathrm{CH}-\underline{\mathrm{C}}_{\text {aromatic }}$ ) $), 170.43$ (4 C, $\left.\underline{\mathrm{C}}=\mathrm{O}\right), 221.22$ (4 C, $\left.\underline{\mathrm{C}}=\mathrm{S}\right)$

ESI-MS:

$1,231.1[\mathrm{M}+\mathrm{Na}]^{+}$

\subsubsection{3 (Phenylethyl)hexyltricarbonate}

In $100 \mathrm{~mL}$ chloroform $40 \mathrm{mmol}$ hexanthiol (4.72 g; $5.70 \mathrm{~mL} ; 118.24 \mathrm{~g} \cdot \mathrm{mol}^{-1}$ ) were dissolved and combined with $50 \mathrm{mmol}$ TEA $(5.06 \mathrm{~g}$; $7.00 \mathrm{~mL}$; $\left.101.19 \mathrm{~g} \cdot \mathrm{mol}^{-1}\right)$. The mixture was stirred for $60 \mathrm{~min}$ at room temperature. Then $160 \mathrm{mmol}$ carbon disulfide (12.20 g; $9.70 \mathrm{~mL} ; 76.14 \mathrm{~g} \cdot \mathrm{mol}^{-1}$ ) were added drop wise. The yellow solution was left to stir for another $30 \mathrm{~min}$

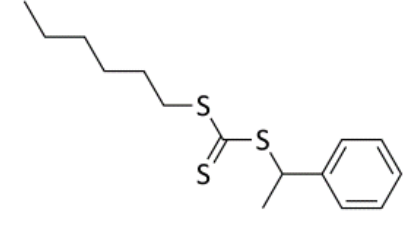

$\mathbf{R}_{1} \mathbf{2}$ before adding $50 \mathrm{mmol}$ (1-bromoethyl) benzene (9.25 g; $7.00 \mathrm{~mL} ; 185.06 \mathrm{~g} \cdot \mathrm{mol}^{-1}$ ) and stirring over night. The reaction was quenched by addition of $100 \mathrm{~mL} 10 \%$ hydrochloric acid. The organic layer was washed three times with $20 \mathrm{~mL}$ water. The combined aqueous phases were extracted with diethyl ether. Afterwards the combined organic phases were dried over sodium sulphate and the 
solvents removed in vacuo. The product was received as a yellow liquid without further purification (95\% yield; $11.34 \mathrm{~g} ; 38 \mathrm{mmol} ; 298.53 \mathrm{~g} \cdot \mathrm{mol}^{-1}$ ).

${ }^{1} \mathrm{H}$ NMR $\left(300 \mathrm{MHz}, \mathrm{CDCl}_{3}\right) \delta(\mathrm{ppm}): \quad 0.90\left(\mathrm{t}, 3 \mathrm{H}, \mathrm{C}_{\underline{3}}-\mathrm{CH}_{2}-\right), 1.29-1.42\left(6 \mathrm{H},-\mathrm{C}_{2}-\underline{\mathrm{C}}_{2}-\mathrm{C}_{2}-\right)$, 1.71 (q, $\left.3 \mathrm{H},-\mathrm{CH}-\underline{\mathrm{C}}_{3}\right), 2.99$ (q, $\left.2 \mathrm{H},-\mathrm{C}_{2}-\mathrm{CH}_{2}-\mathrm{S}-\right), 3.38$ (t, $2 \mathrm{H}$, $\left.-\mathrm{S}-\mathrm{C}_{2}-\mathrm{CH}_{2}-\right), 3.58\left(\mathrm{q}, 1 \mathrm{H}, \mathrm{S}-\mathrm{C} \underline{\mathrm{H}}-\mathrm{C}_{\text {aromatic }}\right), 7.30\left(5 \mathrm{H}, \underline{\mathrm{H}}_{\text {aromatic }}\right)$

${ }^{13} \mathrm{C} \mathrm{NMR}\left(75 \mathrm{MHz}, \mathrm{CDCl}_{3}\right) \delta(\mathrm{ppm}): \quad 14.12\left(-\mathrm{CH}_{2}-\underline{\mathrm{CH}_{3}}\right), 22.61 \quad\left(\mathrm{H}_{3} \underline{\mathrm{C}}-\mathrm{CH}-\right), 28.09-33.02 \quad(3 \mathrm{C}$, $\left.-\underline{C H}_{2}-\underline{C H}_{2}-\underline{\mathrm{C}} \mathrm{H}_{2}-\right), 34.58\left(-\underline{\mathrm{CH}}_{2}-\mathrm{S}\right), 40.66\left(-\mathrm{S}-\underline{\mathrm{C}} \mathrm{CH}-\mathrm{CH}_{3}\right), 128.81$ (5 C, $\left.\underline{\mathrm{C}}_{\text {aromatic } 2-6}\right), 139.54\left(-\mathrm{CH}-\underline{\mathrm{C}}_{\text {aromatic } 1}\right), 224.24$ ( $\left.\underline{\mathrm{C}}=\mathrm{S}\right)$

ESI-MS:

$299.09[\mathrm{M}+\mathrm{Na}]^{+}$

\subsubsection{Dipentaerythriol-hexakis(3-(S-methoxopropionate-trithiocarbonate))-propanoate}

In $150 \mathrm{~mL}$ chloroform $5 \mathrm{mmol}$ dipentaerythritol-hexakis(3-mercaptopropanoate) (3.96 g; $783.05 \mathrm{~g} \cdot \mathrm{mol}^{-1}$ ) were dissolved and $60 \mathrm{mmol}$ TEA $\left(6.10 \mathrm{~g} ; 8.50 \mathrm{~mL} ; 101.19 \mathrm{~g} \cdot \mathrm{mol}^{-1}\right)$ were added. The solution was left to stir for $30 \mathrm{~min}$ at $33^{\circ} \mathrm{C}$. Then $800 \mathrm{mmol}$ carbon disulfide $(60.91 \mathrm{~g} ; 50 \mathrm{~mL}$; $76.14 \mathrm{~g} \cdot \mathrm{mol}^{-1}$ ) were added drop wise over $30 \mathrm{~min}$. After the addition of $18 \mathrm{mmol}$ bromomethylpropionate $(4.68 \mathrm{~g} ; 2.60 \mathrm{~mL}$; $259.95 \mathrm{~g} \cdot \mathrm{mol}^{-1}$ ) the mixture was stirred over night at $33{ }^{\circ} \mathrm{C}$. To quench the reaction $100 \mathrm{~mL} 10 \%$ hydrochloric acid were added and the layers

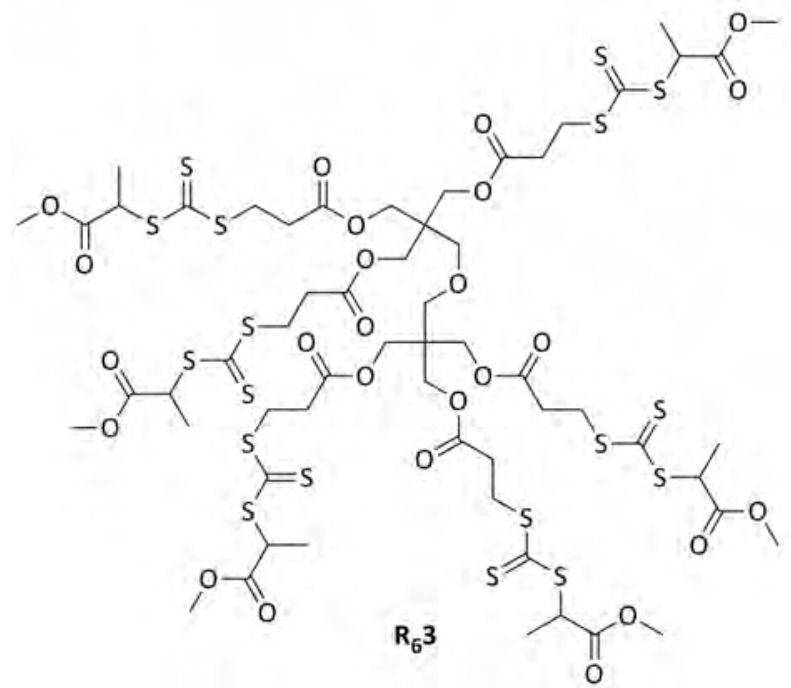
divided. The organic phase was washed two times with water and the aqueous phase extracted two times with ethyl acetate. The combined organic layers were dried over sodium sulphate. The solvents were removed in vacuo resulting a viscous, yellow oil (95 \%; $8.42 \mathrm{~g} ; 4.8 \mathrm{mmol} ; 1,754.01 \mathrm{~g} \cdot \mathrm{mol}^{-1}$ ).

${ }^{1} \mathrm{H}$ NMR $\left(300 \mathrm{MHz}, \mathrm{CDCl}_{3}\right) \delta(\mathrm{ppm}): \quad 1.61\left(\mathrm{~d}, 18 \mathrm{H}, \underline{\mathrm{H}}_{3} \mathrm{C}-\mathrm{CH}-\mathrm{S}-\right), 2.78\left(\mathrm{t}, 12 \mathrm{H}, \mathrm{O}=\mathrm{C}-\mathrm{C}_{\underline{H}}-\mathrm{CH}_{2}-\right)$, $3.60\left(\mathrm{t}, 18 \mathrm{H},-\mathrm{CH}_{2}-\mathrm{CH}_{2}-\mathrm{S}\right), 3.74\left(\mathrm{~s}, 4 \mathrm{H},-\underline{H}_{2} \mathrm{C}-\mathrm{O}-\mathrm{C}_{2}-{ }_{2}\right), 3.78$ (s, $\left.18 \mathrm{H}, \underline{\mathrm{H}}_{3} \mathrm{C}-\mathrm{O}-\mathrm{C}=\mathrm{O}\right), 4.11\left(\mathrm{~m}, 12 \mathrm{H}, \mathrm{C}_{\text {central }}-\mathrm{C}_{2}-\underline{\mathrm{H}}^{-} \mathrm{O}-\right), 4.83$ (q, $\left.6 \mathrm{H},-\mathrm{CS}_{3}-\mathrm{CH}\left(\mathrm{CH}_{3}\right)-\mathrm{CO}_{2} \mathrm{CH}_{3}\right)$

ESI-MS:

$1,779.1[\mathrm{M}+\mathrm{Na}]^{+}$ 


\subsubsection{5 (Methoxopropionate)hexyltricarbonate}

In $50 \mathrm{~mL}$ chloroform $20 \mathrm{mmol}$ hexanthiol (2.36 g; $2.80 \mathrm{~mL} ; 118.24 \mathrm{~g} \cdot \mathrm{mol}^{-1}$ ) were dissolved and $30 \mathrm{mmol}$ TEA (2.02 g; $2.80 \mathrm{~mL} ; 101.1 \mathrm{~g} \cdot \mathrm{mol}^{-1}$ ) were added. The solution was left to stir for $60 \mathrm{~min}$ at room temperature. Then $80 \mathrm{mmol}$ carbon disulfide $\left(6.09 \mathrm{~g} ; 5 \mathrm{~mL} ; 76.14 \mathrm{~g} \cdot \mathrm{mol}^{-1}\right)$ were added<smiles>[R3]SC(=S)SCCCCCC</smiles>
drop wise over $30 \mathrm{~min}$. After the addition of $24 \mathrm{mmol}$ bromomethylpropionate $(6.24 \mathrm{~g} ; 3.50 \mathrm{~mL}$; $259.95 \mathrm{~g} \cdot \mathrm{mol}^{-1}$ ) the mixture was stirred over night. To quench the reaction $50 \mathrm{~mL} 10 \%$ hydrochloric acid were added and the layers separated. The organic phase was washed twice with water and the aqueous phase extracted two times with ethyl acetate. The combined organic layers were dried over sodium sulphate. The solvents were removed in vacuo and a yellow liquid was received (86 \%; $4.82 \mathrm{~g}$; $\left.17 \mathrm{mmol} ; 280.47 \mathrm{~g} \cdot \mathrm{mol}^{-1}\right)$.

${ }^{1} \mathrm{H}$ NMR $\left(300 \mathrm{MHz}, \mathrm{CDCl}_{3}\right) \delta(\mathrm{ppm}): \quad 0.88\left(\mathrm{t}, 3 \mathrm{H}, \mathrm{C}_{\underline{3}}-\mathrm{CH}_{2}-\right), 1.26-1.39\left(\mathrm{~m}, 6 \mathrm{H},-\mathrm{C}_{2}-\underline{\mathrm{C}}_{2}-\underline{\mathrm{C}}_{2}-\right)$, 1.61 (d, $3 \mathrm{H}, \underline{\mathrm{H}}_{3} \mathrm{C}-\mathrm{CH}-\mathrm{S}-$ ), 1.69 (q, $\left.2 \mathrm{H},-\mathrm{S}-\mathrm{CH}_{2}-\mathrm{C}_{2}-{ }^{-}\right), 3.35$ (t, $\left.2 \mathrm{H},-\mathrm{S}-\mathrm{C}_{2}-\mathrm{CH}_{2}-\right), 3.74\left(\mathrm{~s}, 3 \mathrm{H}, \underline{\mathrm{H}}_{3} \mathrm{C}-\mathrm{O}-\mathrm{C}=\mathrm{O}\right), 4.83$ (q, $1 \mathrm{H}$, $\left.-\mathrm{CS}_{3}-\mathrm{C}-\mathrm{H}\left(\mathrm{CH}_{3}\right)-\mathrm{CO}_{2} \mathrm{CH}_{3}\right)$

ESI-MS:

$303.1[\mathrm{M}+\mathrm{Na}]^{+}, 583.1[2 \mathrm{M}+\mathrm{Na}]^{+}$ 


\subsection{Polymerisations}

\subsubsection{Polymerisations in Bulk}

The monomer was degassed via at least three pump-freeze-thaw cycles and was transferred along with a volumetric flask already filled with initiator, RAFT-agents and, where applicable, the comonomer into an argon-filled glove box (oxygen content below $4 \mathrm{ppm}$ ). In the box the stock solutions consisting of initiator, monomers and RAFT-agent were prepared. Then samples of the solution were filled into individual glass vials and sealed with Teflon/rubber lids. The vials were subsequently inserted into a block heater, thermostated at a given temperature (AIBN initiated polymerisations at $60 \pm 0.1^{\circ} \mathrm{C}$, DEPC initiated at $47 \pm 0.1^{\circ} \mathrm{C}$ ). The samples were removed after preset time intervals and cooled in an ice bath to quench the reaction. Monomer-to-polymer conversions were determined gravimetrically. Therefore the polymer was precipitated from the reaction mixture ( $p B A$ with methanol), divided of via centrifugation and dried to constant weight under vacuum in a heating cabinet.

\subsubsection{Polymerisations in Solution}

\section{Solvent Polymerisation in the Block Heater}

Solvent and liquid monomers were degassed via at least three pump-freeze-thaw cycles. Along with the polymerisation vials, the RAFT-agent (macroRAFT in case of block copolymerisation), initiator and the co-monomer the flasks were transferred into an argon-filled glove box. There the stock solutions for the polymerisation were prepared and divided in the glass sample vials and sealed with Teflon/rubber lids. As in the case of bulk polymerisation, the vials are inserted into the block heater at $60 \pm 0.1{ }^{\circ} \mathrm{C}$ and removed after preset time intervals. After cooling in an ice bath to quench the reaction, the monomer-to-polymer conversion was determined gravimetrically by precipitating the polymer from the reaction mixture ( $p \mathrm{BA}$ with methanol, $p$ NIPAam with diethyl ether), as mentioned previously, was divided of via centrifugation and dried under vacuum. In case of block copolymer synthesis with NIPAam-based macroRAFT-agents the remaining solvent and BA were removed by evaporation.

\section{Solvent Homopolymerisation of $\mathbf{2 . 1}$ and $\mathbf{2 . 2}$ in Round-Bottomed Flasks}

This homopolymerisation was adapted from ref. (130). A brown $50 \mathrm{~mL}$ round-bottomed flask was equipped with a magnetic stir bar, RAFT-agent, AIBN, and monomer. Afterwards the DMF was added and the flask sealed with a septum. The mixture was flushed with dry nitrogen for at least $10 \mathrm{~min}$. Then the flask was inserted into a pre-heated oil bath $\left(80^{\circ} \mathrm{C}\right)$ and stirred for 24 hours. After preset time intervals a $1 \mathrm{~mL}$-sample was removed with a syringe and the polymer-to-monomer conversion determined by precipitation with methanol. 


\section{Solvent copolymerisation of MA and 2.2}

Here the procedure of Kim et al. published in 1999 was conformed. ${ }^{(131)}$ In a brown round-bottomed flask $58 \mathrm{mmol}$ MA ( $5 \mathrm{~g} ; 86.09 \mathrm{~g} \cdot \mathrm{mol}^{-1}$ ) along with $3 \mathrm{~mol} \% 2.2$ were dissolved in $50 \mathrm{~mL}$ toluene. The mixture was degassed via at least three pump-freeze-thaw cycles. In another brown round-bottomed flask DBPO and RAFT-agent were weighted-in. Both flasks were transferred into an argon-filled glove box, where the polymerisation mixture was prepared by adding the monomer solution into the initiator/RAFT-flask. This flask was sealed with a septum and inserted in a preheated oil bath $\left(70^{\circ} \mathrm{C}\right)$ and stirred for 24 hours. After preset time intervals a $2 \mathrm{~mL}$-sample was removed with a syringe and the polymer-to-monomer conversion determined by precipitation with methanol. 


\section{Appendix}

\subsection{List of Abbreviations}

\section{Abbreviation Name/Explanation}

1.1

1.2

2.1

2.2

3.1

3.2

$[\ldots]$

$[\eta]$

$a$

AA

AFM

$a_{\mathrm{i}}$

AIBN

APMA

ATRP

BA

BIS

c

CA

CEA

CEMA

CMC

COSY

CRP

CTA

$C_{\mathrm{tr}}$

$d$

$d_{1}$

DA
$\mathrm{N}$-ethylacrylate-3,4-dimethylmaleimide

$\mathrm{N}$-ethylmethacrylate-3,4-dimethyl-maleimide

cinnamoyloxyethyl acrylate

cinnamoyloxyethyl methacrylate

7-(2-methacryloyloxyethoxy)-4-methylcourmarin

7-[4-(trifluoromethyl)courmarin]methylacrylamide

concentration of the named compound

intrinsic viscosity

$\mathrm{MH}-$ parameter describing the hydrodynamic interaction between polymer and solvent

acrylic acid

Atomic Force Microscopy

area of the relative integral

$\mathrm{N}, \mathrm{N}$-azoisobutylnitrile

$N$-(3-aminopropyl)methylacrylamide

Atom Transfer Radical Polymerisation

$n$-butylacrylate

$N, N^{\prime}$-methylene bisacrylamide

concentration

cinnamic acid

cinnamoyloxyethylacrylate

cinnamoyloxyethylmethacrylate

Critical Micelle Concentration

Correlated Spectroscopy

Controlled Radical Polymerisation

chain transfer agent

transfer constant

kind of termination: $d=1$ combination, $d=2$ disproportionation

relaxation time

dodecyl acrylate 


\begin{tabular}{|c|c|}
\hline DBPO & dibenzoylperoxide \\
\hline $\mathrm{DCC}$ & dicyclohexylcarbodiimid \\
\hline DEPC & di(2-ethylhexyl)peroxydicarbonate \\
\hline DLS & Dynamic Light Scattering \\
\hline DMac & $N, N$-dimethylacetamide \\
\hline DMAP & 4-dimethylamionpyridin \\
\hline DMF & dimethylformamide \\
\hline DMI & $N$-alkyl-3,4-dimethylmaleimid \\
\hline$E$ & energy \\
\hline EA & ethyl acrylate \\
\hline EGDMA & ethylene glycol dimethylacrylate \\
\hline ESR & Electron Spin Resonance \\
\hline$f$ & initiator effectiveness \\
\hline$F_{\mathrm{x}}$ & polymer composition \\
\hline$f_{\mathrm{x}}$ & composition of the monomer feed \\
\hline$f_{\text {app. }}$ & apparent number of arms \\
\hline FRP & free radical polymerisation \\
\hline$f_{\text {theor. }}$ & $\begin{array}{l}\text { number of RAFT-units of the multifunctional agent, i.e. number of arms at the } \\
\text { star }\end{array}$ \\
\hline G3 & third generation dendrimer \\
\hline GMA & glycidyl methylacrylate \\
\hline HD-PE & high density polyethylene \\
\hline HEA & 2-hydroxyethylacrylate \\
\hline HEMA & 2-hydroxyethylmethacrylate \\
\hline HMBC & Heteronuclear Multiple Bond Correlation \\
\hline$i$ & number \\
\hline I & integral \\
\hline$i$ & chain length \\
\hline $1^{\bullet}$ & initiator radical \\
\hline $\ln$ & initiator \\
\hline IOA & isooctyl acrylate \\
\hline$K$ & MH-coefficient \\
\hline$K$ & correction factor for star polymers, e.g. for a six-armed star $K=1.52$ \\
\hline$k_{\text {act }}$ & rate coefficient activation \\
\hline
\end{tabular}




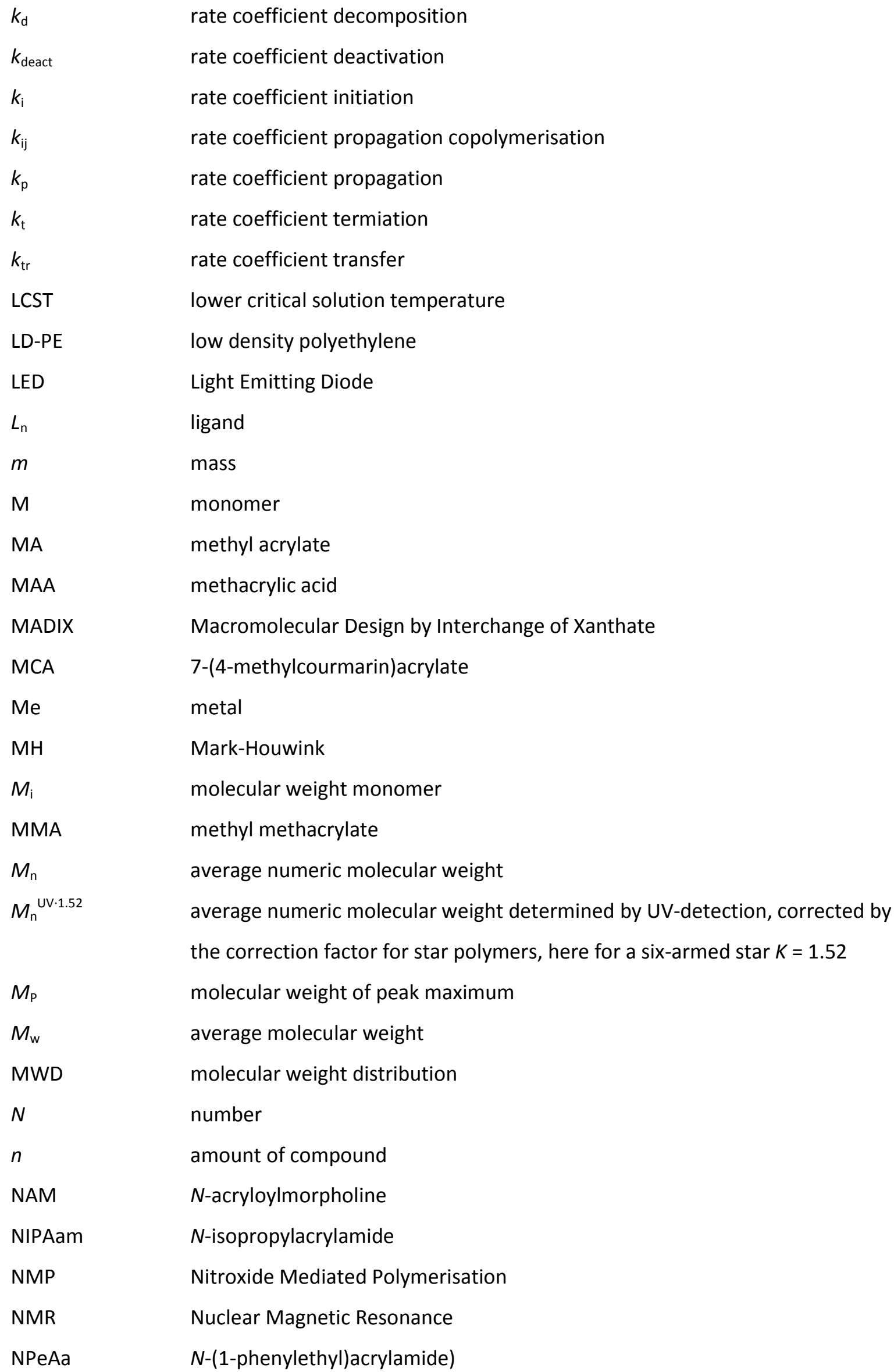




\begin{tabular}{|c|c|}
\hline p... & poly... \\
\hline$p_{1}$ & pulse length \\
\hline PDI & Polydispersity Index \\
\hline PE & polyethylene \\
\hline$p \mathrm{EO}$ & polyethylene oxid \\
\hline PET & polyethyleneterephtalat \\
\hline$P_{m}{ }^{*}, P_{n}^{\cdot}$ & polymer radical \\
\hline$P_{\mathrm{n}}$ & degree of polymerisation \\
\hline POSS & polyhedral oligomeric silsesquioxane \\
\hline PSS & Polymer Standard Service \\
\hline PTFE & polytetrafluorethylene \\
\hline $\mathrm{R}^{\bullet}$ & radical \\
\hline $\mathbf{R}_{\mathbf{4}} \ldots$ & four-functional RAFT-agent \\
\hline $\mathbf{R}_{4} \mathbf{1}$ & Penta-erythritol-tetrakis-(3-(S-benzyl-trithiocarbonate))-propanoate \\
\hline $\mathbf{R}_{6} \ldots$ & six-functional RAFT-agent \\
\hline $\mathbf{R}_{6} \mathbf{2}$ & Dipentaerythriol-hexakis-(3-(phenylethyltricarbonate))-propanoate \\
\hline $\mathbf{R}_{6} \mathbf{3}$ & $\begin{array}{l}\text { Dipentaerythriol-hexakis-(3-(S-methoxopropionate-trithiocarbonate))- } \\
\text { propanoate }\end{array}$ \\
\hline RAFT & Reversible Addition-Fragmentation Transfer \\
\hline$r_{\mathrm{b}}$ & brutto-polymerisation rate \\
\hline RTCP & Reversible chain Transfer Catalysed Polymerisation \\
\hline$r_{\mathrm{d}}$ & rate of decomposition \\
\hline $\mathrm{RI}$ & refractive index \\
\hline$r_{\mathrm{i}}$ & copolymerisation parameter \\
\hline $\mathbf{R}_{\mathbf{L}} \ldots$ & linear, mono-functional RAFT-agent \\
\hline $\mathbf{R}_{\mathbf{L}} \mathbf{2}$ & (Phenylethyl)hexyltricarbonate \\
\hline $\mathbf{R}_{\mathbf{L}} \mathbf{3}$ & (Methoxopropionate)hexyltricarbonate \\
\hline$r_{\mathrm{p}}$ & rate of propagation \\
\hline$r_{\mathrm{t}}$ & rate of termination \\
\hline$r_{\mathrm{tr}}$ & rate of transfer \\
\hline SCM & Shell Cross-linked Micelle \\
\hline SEC & Size Exclusion Chromatography \\
\hline Sty & styrene \\
\hline$T$ & temperature \\
\hline
\end{tabular}


$t$

TA

$T_{\mathrm{c}}$

$\mathrm{TCP} \cdot \mathrm{HCl}$

TEA

$T_{\mathrm{g}}$

THF

UV

VAc

$V_{\mathrm{h}}$

VPr

w

$W_{\log M n}[\mathrm{~g} \cdot \mathrm{mol}-1]$

$w_{\text {star }}$

$x$

$X$ time

transfer agent

phase transition temperature

tris(2-carboxyethyl) phosphine hydrochloride

triethylamine

glass transition temperature

tetrahydrofuran

ultraviolet

vinyl acetate

hydrodynamic volume

vinyl propionate

weight share

probability of occurrence of $M_{\mathrm{n}}$

weight of one star molecule

amount of molecules

monomer conversion 


\subsection{References}

1. H. Gao. Macromol. Rapid Comm. 33, 2012, pp. 722-734.

2. C. N. Goodyear. A 18590816 U.S., 1859. US 25111.

3. C. N. Goodyear. A 18440309 U.S., 1844. US 3462.

4. $\quad$ Plastic - The Facts 2011. Brussels : PlasticsEurope, 2011.

5. F. Xiea, P. J. Halleya, L. Avérousb. Progress in Polymer Science. 37, 2012, pp. 595- 623.

6. Chemistry and polymer science of lignin-based materials . N. R. Washburn, H. Chung. San Diego, CA, United States: Abstracts of Papers, 243rd ACS National Meeting \& Exposition, 2012.

7. D. H. Solomon, E. Rizzardo, P. Cacioli. 19840711 1984. EP0135280A2.

8. G. Moad, E. Rizzardo, D. H. Solomon. Macromolecules. 15, 1982, pp. 909-914.

9. M. K. Georges, R. P. N. Veregin, P. M. Kazmeier, G. K. Hamer. Macromolecules. 26, 1993, pp. 2987-2988.

10. M. Kato, M. Kamigaito, M. Sawamoto, T. Higashimura. Macromolecules. 28, 1995, pp. $1721-1723$.

11. J.-S. Wang, K. Matyjaszewski. J. Am. Chem. Soc. 117, 1995, pp. 5614-5615.

12. J. Chiefari, Y. K. Chong, F. Ercole, J. Krstina, J. Jeffery, T. P. T. Le, R. T. A. Mayadunne, G. F. Meijs, C. L. Moad, G. Moad, E. Rizzardo, S. H. Thang. Macromolecules. 31, 1998, p. $5559-5562$.

13. A. Goto, H. Zushi, N. Hirai, T. Wakada, Y. Tsujii, T. Fukuda. J. Am. Chem. Soc.. 129, 2007, pp. $13347-13357$.

14. A. Jelicic, F. Köhler, A. Winter, S. Beuermann. J. Polym. Sci.: Part A. 48, 2010, pp. 3188-3199.

15. J. M. DeSimone, Z. Guan, C. S. Elsbernd. Science. 257, 1992, pp. 945-947.

16. A. I. Cooper. J. Mater. Chem. 10, 2000, pp. 207-234.

17. M. Buback. Angew. Chem. Int. Ed. 30, 1991, p. 1654.

18. T. Junkers. Dissertation. Göttingen, 2006.

19. Akzo Nobel. Initiators for High Polymers. 2006. 2161 BTB Communication.

20. M. Buback, B. Fischer, S. Hinrichs, S. Jauer, J. Meijer, J. Sandmann. Macromol. Chem. Phys. 208, 2007, pp. 772-783.

21. D. Boschmann. "Sternpolymere mittels RAFT-Polymerisation". Dissertation. Göttingen, 2008.

22. C. Boyer, D. Valade, L. Sauguet, B. Ameduri, B. Boutevin. Macromolecules. 38, 2005, pp. 10353-10362.

23. Danke an Wibke Meiser.

24. G. Moad, E. Rizzardo, D. H. Solomon. Polymer Bulletin. 6, 1982, pp. 589-593. 
25. H. Fischer. Chem. Rev. 101, 2001, pp. 3581-3610.

26. K. Matyjaszewski, J. Xia. Chem. Rev. 101, 2001, pp. 2921-2990.

27. G. Moad, E. Rizzardo, S. H. Thang. Aust. J. Chem. 56, 2006, pp. 669-692.

28. G. Moad, E- Rizzardo, S. H. Thang. Aust. J. Chem. 58, 2005, pp. 379-410.

29. G. Moad, E. Rizzardo, S. H. Thang. Aust. J. Chem. 62, 2009, pp. 1402-1472.

30. R. T. A Mayadunne, E. Rizzardo, J. Chiefari, Y. K. Chong, G. Moad, S. H. Thang. Macromolecules. 32, 1999, pp. 6977-6980.

31. E. Rizzardo, B. Chong, G. Moad, M. Skidmore, S. H. Thang. Macromol. Symp. 248, 2007, p. 104.

32. Y. K. Chong, J. Krstina, T. P. T. Le, G. Moad, A. Postma, E. Rizzardo, S. H. Thang. Macromolecules. 36, 2003, pp. 2256-2272.

33. C. Barner-Kowollik, J. F. Quinn, D. R. Morsley, T. P. Davis. J. Polym. Sci.: Part A. 39, 2001, pp. $1353-1365$.

34. C. Barner-Kowollik, J. F. Quinn, T. L. U. Nguyen, J. P. A. Heuts, T. P. Davis. Macromolecules. 34, 2001, pp. 7849-7857.

35. M. J. Monteiro, H. de Brouwer. Macromolecules. 34, 2001, pp. 349-352.

36. Y. Kwak, A. Goto, Y. Tsujii, Y. Murata, K. Komatsu, T. Fukuda. Macromolecules. 2002, pp. 3026-3029.

37. J. Chiefari, R. T. A. Mayadunne, C. L. Moad, G. Moad, E. Rizzardo, A. Postma, M. A. Skidmore, S. H. Thang. Macromolecules. 36, 2003, pp. 2273-2283.

38. J. B. McLeary, F. M. Calitz, J. M. McKenzie, M. P. Tonge, R. D. Sanderson, B. Klumperman. Macromolecules. 37, 2004, pp. 2383-2394.

39. J. B. McLeary, F. M. Calitz, J. M. McKenzie, M. P. Tonge, R. D. Sanderson, B. Klumperman. Macromolecules. 38, 2005, pp. 3151-3161.

40. T. Arita, S. Beuermann, M. Buback, P. Vana. e-Polymers. 003, 2004, pp. 1-14.

41. C. Barner-Kowollik, P. Vana, T. P. Davis. Kinetics of Free Radical Polymerization. Book Author: T. P. Davis K. Matyjaszewski. Handbook of Radical Polymerization. Hoboken : WileyInterscience, 2002.

42. A. A. Toy, P. Vana, T. P. Davis, C. Barner-Kowollik. Macromolecules. 37, 2004, pp. 744-751.

43. Y. Kwak, A. Goto, T. Fukuda. Macromolecules. 37, 2004, pp. 1219-1225.

44. M. Buback, P. Vana. Macromol. Rapid Commun. 27, 2006, pp. 1299-1305.

45. M. Buback, O. Janssen, R. Oswald, S. Schmatz, P. Vana. Macromol. Symp. 248, 2007, pp. 158-167.

46. C. Barner-Kowollik, M. L. Coote, T. P. Davis, L. Radom, P. Vana. J. Polym. Sci.: Part A. 41, 2003, pp. 2828-2832. 
47. D. Boschmann, P. Vana. Macromolecules. 40, 2007, pp. 2683-2693.

48. X. Huang, D. Appelhans, P. Formanek, F. Simon, B. Voit. Macromolecules. 44, 2011, pp. 8351-8360.

49. Y. Chen. Macromolecules. 45, 2012, pp. 2619-2631.

50. W. Zhang, A. H. E. Müller. Polymer. 51, 2010, pp. 2133-2139.

51. Y.-S. Ye, W.-C. Shen, C.-Y. Tseng, J. Rick, Y.-J. Huang, F.-C. Chang, B.-J. Hwang. ChemComm. 47, 2011, pp. 10656-10658.

52. 3MTM Solutions. [http://solutions.3m.com/wps/portal/3M/en_US/dyneon_fluoropolymers/ Home/?WT.mc_id=www.dyneon.com] St. Paul, MN 55144-1000, USA : Dyneon, 10.09.2012.

53. $F A V O R^{\circledast}$ - Unser Superabsorber für moderne Hygieneprodukte. [www.evonik.de/superabsorber] Krefeld : Evonik, Evonik Industries, 06.03.2012.

54. A. M. Atta, R. A. El-Ghazawy, R. K. Farag, A. F. El-Kafrawy, A. A. Abdel-Azim. Polymer International. 54, 2005, pp. 1088-1096.

55. E. Lejeune, M. Drechsler, J. Jestin, A. H. E. Müller, C. Chassenieux, O. Colombani. Macromolecules. 43, 2010, pp. 2667-2671.

56. Y. Liu, X. Cao, M. Luo, Z. Le, W. Wu. Journal of Colloid and Interface Science. 329, 2009, pp. 244-252.

57. S. Förster, T. Plantenberg. Angew. Chem. Int. Ed. 41, 2002, pp. 688-714.

58. I. Astafieva, X. F. Zhong, A. Eisenberg. Macromolecules. 26, 1993, pp. 7339-7352.

59. S. Förster, M. Antonietti. Avanced Materials. 10, 1998, pp. 195-217.

60. S. Luo, X. Hu, C. Ling, X. Liu, S. Chen, M. Han. Polymer International. 60, 2011, pp. 717-724.

61. B. Mendrek, B. Trzebicka, W. Walach, A. Dworak. European Polymer Journal. 46, 2010, pp. 2341-2351.

62. A. Kowalczuk-Bleja, B. Sierocka, J. Muszynski, B. Trebicka, A. Dworak. Polymer. 46, 2005, pp. $8555-8564$.

63. W. Burchard. Advances in Polymer Science. 143, 1999, pp. 113-194.

64. R. Seghrouchni, G. Petekidis, D. Vlassopoulos, G. Fytas, A. N. Semenov, J. Roovers, G. Fleischer. Europhys. Lett. 42, 1998, pp. 271-276.

65. M. Stepánek, M. Uchman, K. Procházka. Polymer. 50, 2009, pp. 3638-3644.

66. F. R. Mayo, F. M. Lewis. J. Am. Chem. Soc. 66, 1944, pp. 1594-1601.

67. Matyjaszewski, K., T. P. Davis. General Concepts and History of Living Radical Polymerization. Book Author: K. Matjaszewski. Handbook of Radical Polymerization. Hoboken : Wiley-Interscience, 2002.

68. P. Vana, T. P. Davis, C. Barner-Kowollik. Aust. J. Chem. 55, 2002, p. 379.

69. D. Boschmann, R. Edam, P. J. Schoenmakers, P. Vana. Polymer. 49, 2008, pp. 5199-5208. 
70. Z. Grubsici, P. Rempp, H. Benoit. J. Polym. Sci. Part B: Polym. Letters. 5, 1967, pp. 753-759.

71. A. Lederer, D. Voigt, D. Appelhans, B. Voit. Polymer Bulletin. 57, 2006, pp. 329-340.

72. A. L. Spatorico, B. Coulter. J. Polym. Sci.: Polymer Physics Edition. 11, 1973, pp. 1139-1150.

73. T. Gruendling, T. Junkers, M. Guilhaus, C. Barner-Kowollik. Macromol. Chem. Phys. 211, 2010, pp. 520-528.

74. C. J. Hawker, E. E. Malmström, C. W. Frank, J. P. Kampf. J. Am. Chem. Soc. 119, 1997, pp. 9903-9904.

75. J. F. Douglas, J. Roovers, K. F. Freed. Macromolecules. 23, 1990, pp. 4168-4180.

76. W. H. Stockmayer, M. Fixmann. Ann. N. Y. Acad. Sci. 57, 1953, pp. 334-352.

77. J. R. Schaefgen, P. J. Flory. J. Am. Chem. Soc. 70, 1948, pp. 2709-2718.

78. R. C. Ball, T. C. B. McLeish. Macromolecules. 22, 1989, pp. 1911-1913.

79. $\quad$ S. M. Grayson, J. M. J. Fréchet. Chem. Rev. 101, 2001, pp. 3819-3867.

80. A. W. Bosman, H. M. Janssen, E. W. Meijer. Chem. Rev. 99, 1999, pp. 1665-1688.

81. J. F. G. A. Jansen, E. W. Meijer. J. Am. Chem. Soc. 117, 1995, pp. 4417-4418.

82. B. Voit, D. Beyerlein, K.-J. Einhorn, K. Grundke, D. Schmaljohann, T. Loontjens. Chemie Ingenieur Technik. 73, 2001, pp. 1592-1596.

83. D. A. Tomalia, J. M. J. Fréchet. J. Polym. Sci. 40, 2002, pp. 2719-2728.

84. M. Pittelkow, J. B. Christensen. Org. Lett. 7, 2005, pp. 1295-1298.

85. N. G. Lemcoff, T. A. Spurlin, A. A. Gewirth, S. C. Zimmermann, J. B. Beil, S. L. Elmer, H. G. Vandeveer. J. Am. Chem. Soc. 126, 2004, pp. 11420-11421.

86. M. A. R. Meier, J.-F. Gohy, C.-A. Fustin, U. S. Schubert. J. Am. Chem. Soc. 126, 2004, pp. 11517-11521.

87. L. Xue, U. S. Agarwal, M. Zhang, B. B. P. Staal, A. H. L. Müller, C. M. E. Bailly, P. J. Lemstra. Macromolecules. 38, 2005, pp. 2093-2100.

88. P.-E. Dufils, N. Chagneux, D. Gigmes, T. Trimaille, S. R.A. Marque, D. Bertin, P. Tordo. Polymer. 48, 2007, pp. 5219-5225.

89. M. H. Stenzel, T. P. Davis. J. Polym. Sci.: Part A. 40, 2002, pp. 4498-4512.

90. D. Boschmann, M. Drache, M. Fröhlich, G. Zifferer, P. Vana. Polymer Preprints. 49 (2), 2008, pp. 189-190.

91. C. Barner-Kowollik, T. P. Davis, J. P. A. Heuts, M. H. Stenzel, P. Vana, M. Whittaker. J. Polym. Sci.: Part A. 41, 2003, pp. 365-375.

92. M. A. Stenzel-Rosenbaum, T. P. Davis, A. G. Fane, V. Chen. J. Polym. Sci.: Part A. 39, 2001, pp. $2777-2783$.

93. D. Boschmann, P. Vana. Polymer Bulletin. 53, 2005, pp. 231-242. 
94. J. Bernard, A. Favier, L. Zhang, A. Nilasaroya, T. P. Davis, C. Barner-Kowollik, M. H. Stenzel. Macromolecules. 38, 2005, pp. 5475-5484.

95. H. Chaffey-Millar, M. H. Stenzel, T. P. Davis, M. L. Coote, C. Barner-Kowollik. Macromolecules. 39, 2006, pp. 6406-6419.

96. R. T. A. Mayadunne, J. Jeffery, G. Moad, E. Rizzardo. Macromolecules. 36, 2003, pp. 1505-1513.

97. M. C. Parrott, J. C. Luft, J. D. Byrne, J. H. Fain, M. E. Napier, J. M. DeSimone. J. Am. Chem. Soc. 132, 2010, pp. 17928-17932.

98. L.-P. Lv, J.-P. Xu, X.-S. Liu, G.-Y. Liu, X. Yang, J. Ji. Macromol. Chem. Phys. 211, 2010, pp. 2292-2300.

99. H. C. Kolb, M. G. Finn, K. B. Sharpless. Angew. Chem. Int. Ed. 40, 2001, pp. 2004-2021.

100. W. Lv, L. Liu, Y. Luo, X. Wang, Y. Liu. Journal of Colloid and Interface Science. 356, 2011, pp. 16-23.

101. M. Hales, C. Barner-Kowollik, T. P. Davis, M. H. Stenzel. Langmuir. 20, 2004, pp. $10809-10817$.

102. D. Singh, D. Kuckling, V. Choudhary, H.-J. Adler, V. Koul. Polym. Adv. Technol. 17, 2006, pp. 186-192.

103. D. Kafouris, C. S. Patrickios. European Polymer Journal. 45, 2009, pp. 10-18.

104. G. Zheng, C. Pan. Polymer. 46, 2005, pp. 2802-2810.

105. L. M. Van Renterghem, M. Lammens, B. Dervaux, P. Viville, R. Lazzaroni, F. E. Du Prez. J. Am. Chem. Soc. 130, 2008, pp. 10802-10811.

106. E. Drockenmuller, L. Y. T. Li, D. Y. Rye, E. Harth, T. P. Russell, H.-C. Kim, C. J. Hawker. Journal of Polymer Sciene: Part A: Polymer Chemistry. 43, 2005, pp. 1028-1037.

107. X. Yu, C. Corten, H. Görner, T. Wolff, D. Kuckling. Journal of Photochemistry and Photobiology A: Chemistry. 198, 2008, pp. 34-44.

108. H. Bouas-Laurent, A. Castellan, J.-P. Desvergnea, R. Lapouyadec. Chem. Soc. Rev. 30, 2001, pp. 248-263.

109. A. Schütz, T. Wolff. Journal of Photochemistry and Photobiology A: Chemistry. 109, 1997, pp. 251-258.

110. S. Menon, R. Thekkayil, S. Varghese, S. Das. J. Polym. Sci. Part A: Polymer Chem. 49, 2011, pp. 5063-5073.

111. A. Lendlein, H. Jiang, O. Jünger, R. Langer. Nature. 434, 2005, pp. 879-882.

112. M. Tominaga, K. Konishi, T. Aida. Chemistry Letters. 29, 2000, Vol. 4, pp. 374-375.

113. J. Jiang, B. Qi, M. Lepage, Y. Zhao. Macromolecules. 40, 2007, pp. 790-792. 
114. C. Duan Vo, D. Kuckling, H.-J. P. Adler, M. Schönhoff. Colloid Polym. Sci. 208, 2002, pp. 400-409.

115. K. Meier, H. Zweifel. Journal of Photochemistry. 35, 1986, pp. 353-366.

116. S. Gupta, D. Kuckling, K. Kretschmer, V. Choudhary, H.-J. Adler. J. Polym. Sci.: Part A. 45, 2007, pp. 669-679.

117. P. Pareek, H.-J. Adler, D. Kuckling. Progr. Colloid Polym. Sci. 132, 2006, pp. 145-151.

118. D. Kuckling, H.-J. Adler, L. Ling, W. D. Habicher, K.-F. Arndt. Polymer Bulletin. 44, 2000, pp. 269-276.

119. M. Baumann, H. Bosshard. Helv. Chim. Acta. 61, 1978, pp. 2751-2753.

120. J. Berger, H. Zweifel. Angew. Makro. Chem. 115, 1983, pp. 163-181.

121. C. D. Eisenbach. Makromol. Chem. 179, 1978, pp. 2489-2506.

122. K. Fujimori, G. B. Butler. J. Macromol. Sci. Chem. 7, 1973, pp. 415-432.

123. G. O. Schenk, W. Hartmann, S. P. Mannsfeld, W. Metzner, C. H. Krauch. Chem. Ber. 95, 1962, pp. 1642-1647.

124. F. C. de Schryver, N. Boenis, G. Smets. J. Polym. Sci.: Part A-1. 10, 1972, pp. 1687-1699 .

125. L. M. Minsk. US 27253771955.

126. M. Tsuda. J. Polym. Sci.: Part A. 2, 1964, pp. 2907-2916.

127. H. Tanaka, K. Honda. J. Polym. Sci. 15, 1977, pp. 2685-2689.

128. P. L. Egerton, E. Pitts, A. Reiser. Macromolecules. 14, 1981, pp. 95-100.

129. K. Nakamura, S. Kikuchi. Bull. Chem. Soc. Jpn. 40, 1967, pp. 2684-2685.

130. A. H. Ali, K. S. V. Srinivasan. J. Appl. Polym. Sci. 67, 1998, pp. 441-448.

131. S.-H. Kim, I.-D. Chung, C.-S. Ha, K.-J. Kim, W.-J. Cho. J. Appl. Polym. Sci. 73, 1999, pp. 2349-2357.

132. Y. Yu, T. Ikeda. Macromol. Chem. Phys. 206, 2005, pp. 1705-1708.

133. H. Yang, L. Jia, Z. Wang, A. Di-Cicco, D. Lévy, P. Keller. Macromolecules. 44, 2011, pp. 159-165.

134. D. Hernanz, V. Nunez, A. I. Sancho, C. B. Faulds, G. Williamson. J. Agric. Food Chem. 49, 2001, pp. 4884-4888.

135. D. Shi, M. Matsusaki, M. Akashi. Journal of Controlled Release. 149, 2011, pp. 182-189.

136. D. Shi, T. Kaneko, M. Akashi. Langmuir. 23, 2007, pp. 3485-3488.

137. D. Shi, M. Matsuaki, T. Kaneko, M. Akashi. Macromolecules. 41, 2008, pp. 8167-8172.

138. S. R. Trenor, A. R. Shultz, B. J. Love, T. E. Long. Chem. Rev. 104, 2004, pp. 3059-3077.

139. G. Ciamician, P. Silber. Ber. Chem. Dtsch. Ges. Berlin. 35, 1902, pp. 4128-4131.

140. R. Zhang, H. Zheng, J. Shen. Synthetic Metals. 106, 1999, pp. 157-160.

141. E. Stathatos, P. Lianos, U. L.Stanger, B. Orel. Chem. Phys. Lett. 345, 2001, pp. 381-385. 
142. C. E. Wheelock. J. Am. Chem. Soc. 81, 1959, pp. 1348-1352.

143. F. D. Lewis, S. V. Barancyk. J. Am. Chem. Soc. 111, 1989, pp. 8653-8661.

144. T. J. Brett, J. M. Alexander, J. J. Stezowski. J. Chem. Soc., Perkin Trans. 2, 2000, Vol. 6, pp. $1105-1111$.

145. Y. Chen, J.-D. Wu. J. Polym. Sci.: Part A. 32, 1994, pp. 1867-1875.

146. Y. Chen, C.-F. Chou. J. Polym. Sci.: Part A. 33, 1995, pp. 2705-2714.

147. C. Sinkel, A. Greiner, S. Agarwal. Macromolecul. Chem. Phys. 211, 2010, pp. 1857-1867.

148. K. Landfester, A. Musyanovych, V. Mailänder. J. Polym. Sci.: Part A. 48, 2010, pp. 493-515.

149. Y. Wang, A. S. Angelatos, F. Caruso. Chem. Mater. 20, 2008, pp. 848-858.

150. C. Boyer, M. R. Whittaker, C. Nouvel, T. P. Davis. Macromolecules. 43, 2010, pp. 1792-1799.

151. C. L. McCormick, B. S. Sumerlin, B. S. Lokitza, J. E. Stempka. Soft Matter. 4, 2008, pp. 1760-1773.

152. J. Xu, L. Tao, C. Boyer, A. B. Lowe, T. P. Davis. Macromolecules. 44, 2011, pp. 299-312.

153. J. Xu, S. Liu. Soft Matter. 4, 2008, pp. 1745-1749.

154. K. B. Thurmond, T. Kowalewski, K. L. Wooley. J. Am. Chem. Soc. 118, 1996, pp. 7239-7240.

155. K. B. Thurmond, T. Kowalewski, K. L. Wooley. J. Am. Chem. Soc. 119, 1997, pp. 6656-6665.

156. C. Chang, H. Wei, D.-Q. Wu, B. Yang, N. Chen, S.-X. Cheng, X.-Z. Zhang. International Journal of Pharmaceutics. 420, 2011, pp. 333-340.

157. X. Xu, J. D. Flores, C. L. McCormick. Macromolecules. 44, 2011, pp. 1327-1334.

158. J.-T. Sun, Z.-Q. Yu, C.-Y. Hong, C.-Y. Pan. Macromol. Rapid Commun. 33, 2012, pp. 811-818.

159. M. H. Stenzel, T. P. Davis, C. Barner-Kowollik. Chem. Commun. 2004, pp. 1546-1547.

160. M. Mänz. "Wege zu makromolekularen Nanotransportern aus Sternpolymeren mittels RAFTPolymerisation". Dissertation. Göttingen, 2010.

161. D. Boschmann, M. Mänz, A.-C. Pöppler, N. Sörensen, P. Vana. J. Polym. Sci.: Part A. 46, 2008, pp. $7280-7286$.

162. W. Shen, Q. Qiu, Y. Wang, M. Miao, B. Li, T. Zhang, A. Cao, Z. An. Macromol. Rapid Commun. 31, 2010, pp. 1444-1448.

163. M. G. Fröhlich, P. Vana, G. Zifferer. J. Chem. Phys. 127, 2007, p. 164906.

164. M. G. Fröhlich, P. Vana, G. Zifferer. Macromol. Theory Simul. 16, 2007, pp. 610-618.

165. M. G. Fröhlich, M. M. Nardai, N. Förster, P. Vana, G. Zifferer. Polymer. 51, 2010, pp. 5122-5134.

166. Müller, E. "Detailed Investigations into the Propagation and Termination Kinetics of Bulk Homo- and Copolymerization of (Meth)Acrylates". Dissertation. Göttingen, 2005.

167. M. Buback, T. Junkers, P. Vana. Macromol. Rapid. Commun. 26, 2005, p. 796. 
168. J. M. Asua, S. Beuermann, M. Buback, P. Castignolles. B. Charleux, R. G. Gilbert, R. A. Hutchinson, J. R. Leiza, A. N. Nikitin, J.-P. Varion, A. M. van Herk. Macromol. Chem. Phys. 205, 2004, pp. 2152-2160.

169. M. Buback, C. H. Kurz,C. Schmaltz. Macromol. Chem. Phys. 199, 1998, pp. 1721-1727.

170. J. He, X. Tong, L. Tremblay, Y. Zhao. Macromolecules. 42, 2009, pp. 7267-7270.

171. M. H. Stenzel, C. Barner-Kowollik, T. P. Davis, H. M. Dalton. Macromol. Biosci. 4, 2004, pp. 445-453.

172. J. C. Garay-Jimenez, D. Gergeres, A. Young, S. Dickey, D. V. Lim, E. Turos. Nanomedicine. 5, 2009, pp. 443-453.

173. J. Garay-Jimenez, A. Young, D. gergeres, K. Greenhalgh, E. Turos. Nanomedicine. 4, 2008, pp. 98-105.

174. X. Gao, Y. Cui, R. M. Levenson, L. W. K. Chung, S. Nie. Nature Biotechnology. 22, 2004, pp. 969-976.

175. D. Seifert, M. Kipping, H.-J. P. Adler, D. Kuckling. Macromol. Symp. 254, 2007, pp. 386-391.

176. N. A. Peppas. Polymer Data Handbook. Oxford University Press, 1999, pp. 625-626.

177. G. Yohannes, J. Shan, M. Jussila, M. Nuopponen, H. Tenhu, M.-L. Riekkola. J. Sep. Sci. 28, 2005, pp. 435-442.

178. H. Wei, S.-X. Cheng, X.-Z. Zhang, R.-X. Zhuo. Progress in Polymer Science. 34, 2009, pp. 893-910.

179. J. Hu, X. Zhang, D. Wang, X. Hu, T. Liu, G. Zhang, S. Liu. Journal of Material Chemistry. 21, 2011, pp. 19030-19038.

180. Y. Cao, X. X. Zhu, J. Luo, H. Liu. Macromolecules. 40, 2007, pp. 6481-6488.

181. A. Vega-Rios, Licea-Claveríe. J. Mex. Chem. Soc. 55, 2011, pp. 21-32.

182. S. Beuermann, D. A. Paquet, J. H. McMinn, R. A. Hutchinson. Macromolecules. 29, 1996, pp. 4206-4215.

183. M. Buback, P. Hesse, T. Junkers, P. Vana. Macromol. Rapid. Commun. 27, 2006, pp. 182-187.

184. P. S. Pregosin. NMR in Organometallic Chemistry. Wiley-VCH, 2012.

185. E. A. Grulke, J. Brandrup, E. H. Immergut. Polymer Handbook 4th ed. Wiley-Intersciene, 1999.

186. J. R. McKee, V. Ladmiral, J. Niskanen, H. Tenhu, S. P. Armes. Macromolecules. 44, 2011, pp. 7692-7703.

187. H.-G. Elias. Chemical Structure. An Introduction to Polymer Science. Weinheim : VCH, 1997.

188. A. Kiriy, G. Gorodyska, S. Minko, M. Stamm. Macromolecules. 36, 2003, pp. 8704-8711. 
189. Willett, Jason Christopher. U.S Geological Survey. http://minerals.usgs.gov/minerals/pubs/ commodity/mica/. [Online] 273 2012. [Cited: 1308 2012.]

http://minerals.usgs.gov/minerals/pubs/commodity/mica/index.html .

190. H. Bouchékif, R. Narain. J. Phys. Chem. B. 111, 2007, pp. 11120-11126.

191. F. C. De Schryver, N. Boens, G. Smets. J. Am. Chem. Soc. 96:20, 1974, pp. 6463-6469.

192. J. F. Quinn, L. Barner, C. Barner-Kowollik, E. Rizzardo, T. P. Davis. Macromolecules. 35, 2002, pp. 7620-7627.

193. X.-P. Qiu, F. M. Winnik. Macromol. Rapid Commun. 27, 2006, pp. 1648-1653.

194. M. Buback, T. Junkers, P. Vana. Macromol. Rapid Commun. 26, 2005, pp. 796-802.

195. Polymer Standard Service GmbH.

196. M. Buback, A. Feldermann, Ch. Barner-Kowollik, I. Lacik. Macromolecules. 34, 2001, pp. 5439-5448.

197. B. Neises, W. Steglich. Angew. Chem. 90, 1978, pp. 556-557.

198. G. Pintér, G. Batta, S. Kéki, A. Mándi, I. Komáromi, K. Takács-Novák, F. Sztaricskai, E. Roth, E. Ostorházi, F. Rozgonyi, L. Naesens, P. Herczegh. J. Med. Chem. 52, 2009, pp. 60-53-6061.

199. B.-L. Zhang, F.-D. Wang, J.-M. Yue. Synthetic Communications. 37, 2007, pp. 63-70. 


\section{Acknowledgments}

Ich danke meinem Doktorvater Prof. Dr. Philipp Vana für die Möglichkeit, in seiner Arbeitsgruppe diese Dissertation anzufertigen, für das interessante und fordernde Thema, bei dem ich meine Fähigkeiten einsetzen, ausprobieren und erweitern sowie mein Wissen ausbauen konnte, was mir ohne die mir gewährte Freiheit in der Arbeit sowie die stete Unterstützung und Beratung nicht möglich gewesen wäre. Gedankt wird auch der Deutschen Forschungsgemeinschaft, die dieses Projekt finanziell unterstützt hat. Des Weiteren gilt mein Dank Prof. Dr. Michael Buback und Prof. Dr. Dietmar Stalke, die das Betreuungskomitee dieser Arbeit vervollständigten und mir mit fachlichen Diskussionen zur Seite standen.

Ich danke allen Mitgliedern der Arbeitsgruppen Makromolekulare Chemie und Technische und Makromolekulare Chemie für ihre Unterstützung bei der Entstehung dieser Arbeit: Dr. Martin Mänz für die erste, grundlegende Einweisung in die Geräte, die für die Durchführung von Polymerisationen nötig waren, Sebastian Primpke und Sandra Lotze für Einweisung und Unterstützung im Umgang mit den GPC-Anlagen, Dr. Florian Ehlers für die Einweisung am AFM, Katharina Tietz für die an der Lösungsmittelbedampfungszelle, Cathrin Conrad für die am UV Cary und Wibke Meiser für die Bereitstellung einiger Predici-Schaubilder in dieser Arbeit.

Dr. Björn Springer, Cathrin Conrad, Sonja Schmidt, Vanessa Koch und Patrick Drawe danke ich für die entspannte und unterhaltsame Laboratmosphäre. Außerdem danke ich meinen zahlreichen Bürokollegen Björn Sölter, Sebastian Primpke, Jan-Hendrik Schütz, Sonja Schmidt, Dr. Gregory Thomas Russell und Cathrin Conrad für die gute, produktive und zugleich amüsante Büroatmosphäre. Weiterhin möchte ich allen Arbeitsgruppenmitgliedern für ihre stete Diskussionsbereitschaft danken, die zu neuen Ansätzen und guten Lösungen geführt hat, für das angenehme Betriebsklima und unterhaltsame gemeinsame Abende. Ich danke Dr. Hans-Peter Vögele für aufschlussreiche arbeitsspezifische und-unspezifische Gespräche.

Großer Dank gilt Ann-Christin Pöppler aus dem Arbeitskreis Stalke, die viele, viele NMR-Spektren für mich gemessen, die Ergebnisse mit mir diskutiert und die entsprechenden Abschnitte der Arbeit Korrektur gelesen hat. Weiterhin bin ich Sonja Schmidt zu großem Dank verpflichtet, die ihre Bachelorarbeit bei mir angefertigt und in diesem Zusammenhang die Copolymerisationen mit Zimtsäurederivaten untersucht hat. Auch Felix Lohmann und Willi Smolan sei für ihr Mitwirken an Synthesen und Polymerisationen gedankt.

Weiterhin möchte ich den Mitarbeitern der NMR-Abteilung des Instituts für Organische Chemie, unter der Leitung von Reinhard Machinek, für das Messen meiner NMR-Spektren und die Diskussion 
zu NMR-spezifischen Problemen und ebenso den Mitarbeitern der NMR-Abteilung des Institutes für Anorganische Chemie, unter der Leitung von Dr. Michael John, für die Bereitstellung und Instandhaltung des NMR-Gerätes im AC-Praktikum danken. Ich danke Dr. Holm Frauendorf und seinem Team für das Messen der Massenspektren. Weiterer Dank gebührt den Damen des Analytischen Labors in der AC, Angelika Wraage, Diana Kumpart und Susanne Petrich, für zahlreiche durchgeführte Elementaranalysen. Bei der Glaswerkstatt, unter der Leitung von Joachim Schlette und Annika von Roden, möchte ich mich für viele große und kleine Reparaturen, aber vor allem für den Bau der kühlbaren Säule bedanken.

Für die fürsorgliche Pflege und Wartung meines Computers gilt mein Dank Norbert Neisen.

Sehr großer Dank gebührt meinen äußerst fleißigen Korrekturleserinnen Dr. Ev Stöckel, Dr. Diana Thomas und Cathrin Conrad, die so manche Sonnenstunde dieses Sommers für die Perfektionierung dieser Arbeit geopfert haben.

Ich danke im Speziellen Cathrin Conrad, in der ich hier in Göttingen eine sehr gute Freundin gefunden habe, für viele gemeinsame Stunden.

Des Weiteren danke ich meiner Omi sehr für unzählige Päckchen, die die nötige Arbeitsenergie lieferten, meinen Freunden, die mich mein gesamtes Studium und die Zeit meiner Promotion begleitet und unterstützt haben, und André Winterfeld, der mir zuliebe viel zu viel Zeit in den Zügen der Deutschen Bahn verbringen musste.

Mein ganz besonderer Dank jedoch gilt meinen Eltern, die mich seit jeher in jeder Lebenslage und bei jeder Entscheidung unterstützt und gefördert haben. Ohne euch hätte ich dieses Ziel hier nicht erreichen können. 


\section{Curriculum Vitae}

Diplom Chemist

Nadja Förster

born the 30. January 1984 in Berlin, Germany

nationality: German

\section{Studies}

Since 04.2009

PhD-studies at the Georg-August-University Göttingen

Member of the study program Catalysis for Sustainable Synthesis (CaSuS)

Research topic "Synthesis of Nano-Carriers via RAFT-star polymerisation" in the working group Macromolecular Chemistry of Prof. Dr. Philipp Vana

17.09.2012 Submission of the Thesis

“Nano-Carrier Synthesis via Z-RAFT Star Polymerisation"

18.10.2012 Day of Defence

$10.2003-03.2009$ Chemistry studies at the University of Potsdam

$03.2007 \quad$ Vordiplomprüfung (1.3)

06.2008 Diplomprüfung (2.0)

08.2008-03.2009

Diploma thesis (1.0) in the working group Polymer Chemistry of Prof. Dr. Sabine Beuermann

Research topic "Click-Chemistry on Vinylenfluorid polymers"

Qualification: Diplom Chemist (1.7)

\section{School}

$07.1996-06.2003$

Rosa-Luxemburg-Oberschule (secondary school) in Berlin-Pankow

$06.2003 \quad$ Allgemeine Hochschulreife (Abitur) 